
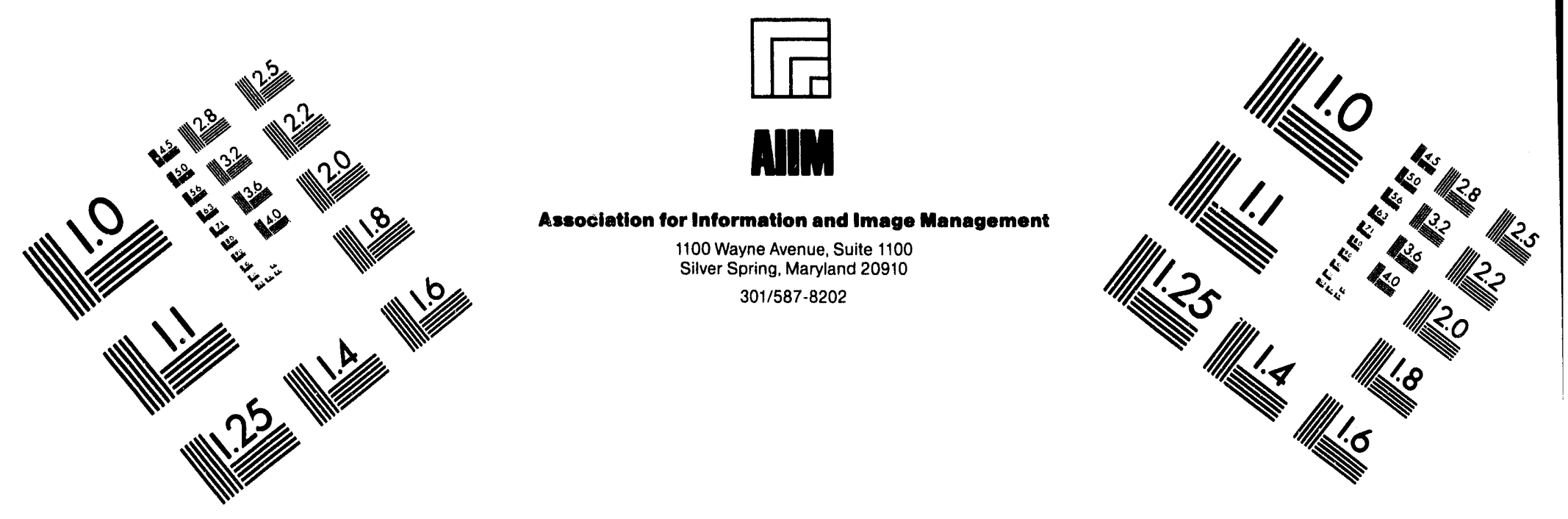

\title{
Centimeter
}

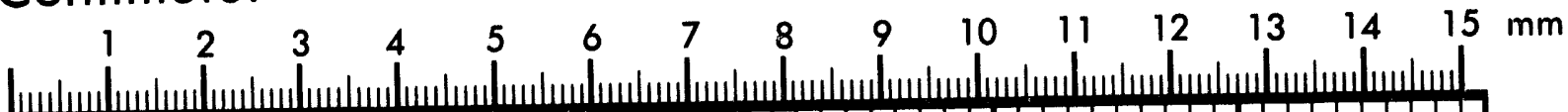

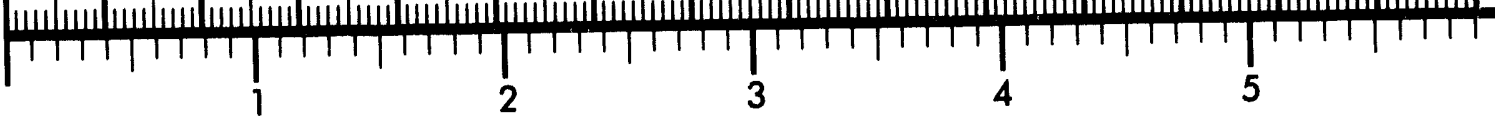
Inches

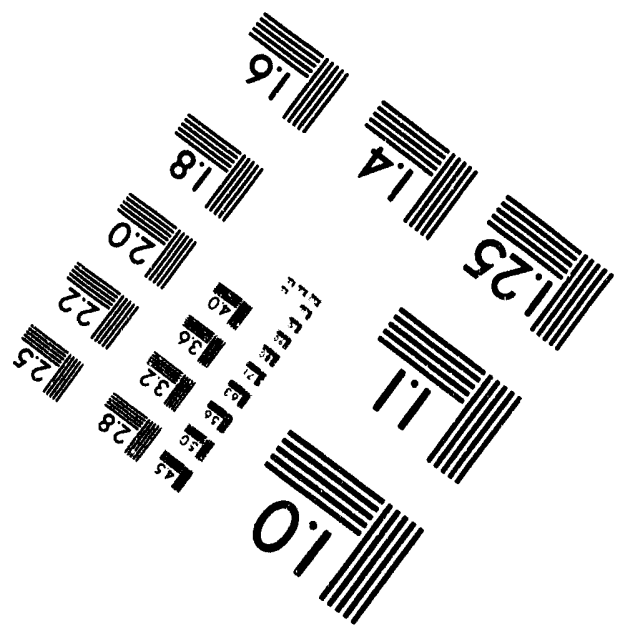

MANUFACTURED TO AIIM STANDARDS

BY APPLIED IMAGE, INC.

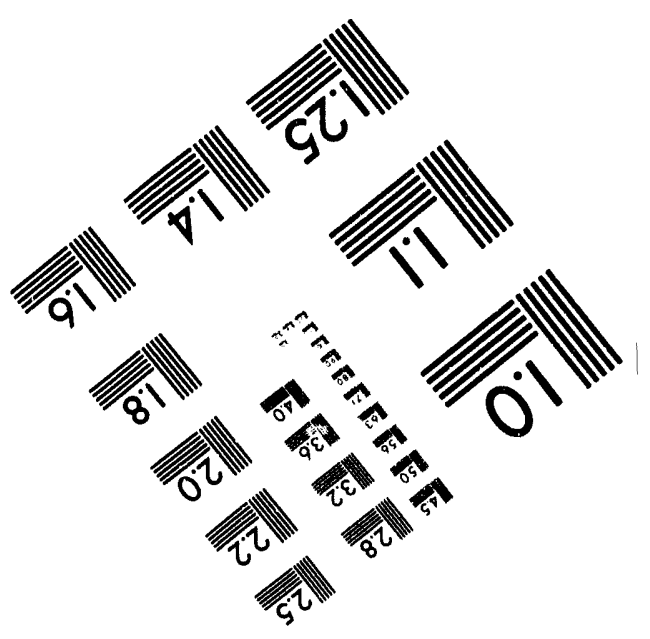



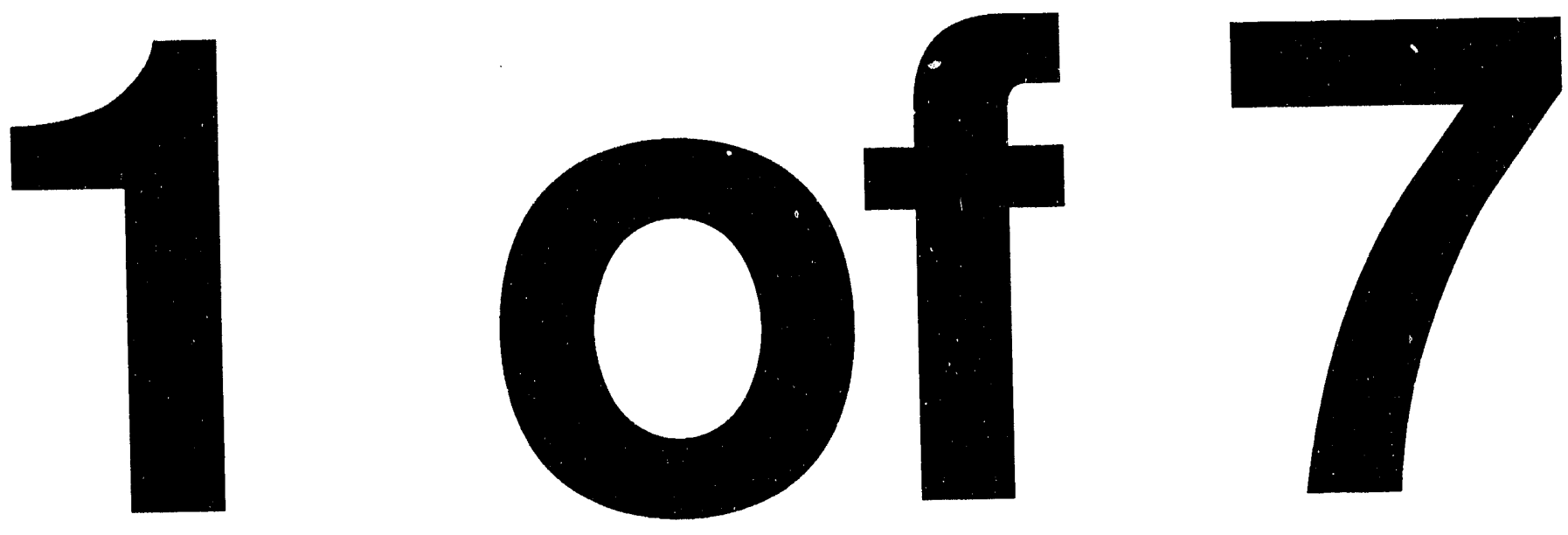


\section{Characterization of Past and Present Waste Streams from the 325 Radiochemistry Building}

J. A. Pottmeyer

M. I. Weyns-Rollosson

K. D. Dicenso

D. S. DeLorenzo

Los Alamos Technical Associates, Incorporated

D. R. Duncan

Westinghouse Hanford Company

Date Published

December 1993

Prepared for Westinghouse Hanford Company by Los Alamos Associates

8633 Gage Blvd.

Kennewick, Washington 99336

Prepared for the U.S. Department of Energy

Assistant Secretary for Environment, Safety and Health

(2) Westinghouse $\begin{aligned} & \text { P.O. Box } 1970 \\ & \text { Hantord Company } \\ & \text { Richland, Washington } 99352\end{aligned}$

Hanford Operations and Engineering Contractor for the

U.S. Department of Energy under Contract DE-AC06-87RL10930

Approved for Public Release 


\section{LEGAL DISCLAIMER}

This report was prepared as an account of work sponsored by an agency of the United States Government. Neither the United States Government nor any agency thereof, nor any of their employees, nor any of their contractors, subcontractors or their employees, makes any warranty, express or implied, or assumes any legal liability or responsibility for the accuracy, completeness, or any third party's use or the results of such use of any information, apparatus, product, or process disclosed, or represents that its use would not infringe privately owned rights. Reference herein to any specific commercial product, process, or service by trade name trademark, manufacturer, or otherwise, does not necessarily constitute or imply its endorsement, recommendation, or favering by the United States Government or any agency thereof or its contractors or subcontractors. The views and opinions of authors expressed herein do not necessarily state or reflect those of the United States Government or any agency thereof.

This report has been reproduced from the best available copy. Available in paper copy and microfiche.

Available to the U.S. Department of Energy

and its contractors from

Office of Scientific and Technical Information

P.0. Box 62

Oak Ridge, TN 37831

(615) $576-840$

Available to the public from the U.S. Department of Commerce

National Technical Information Service

5285 Port Royal Road

Springfield, VA 22161

(703) $487-4650$

Printed in the United States of America

DISCLM-1.CHP $(1-91)$ 


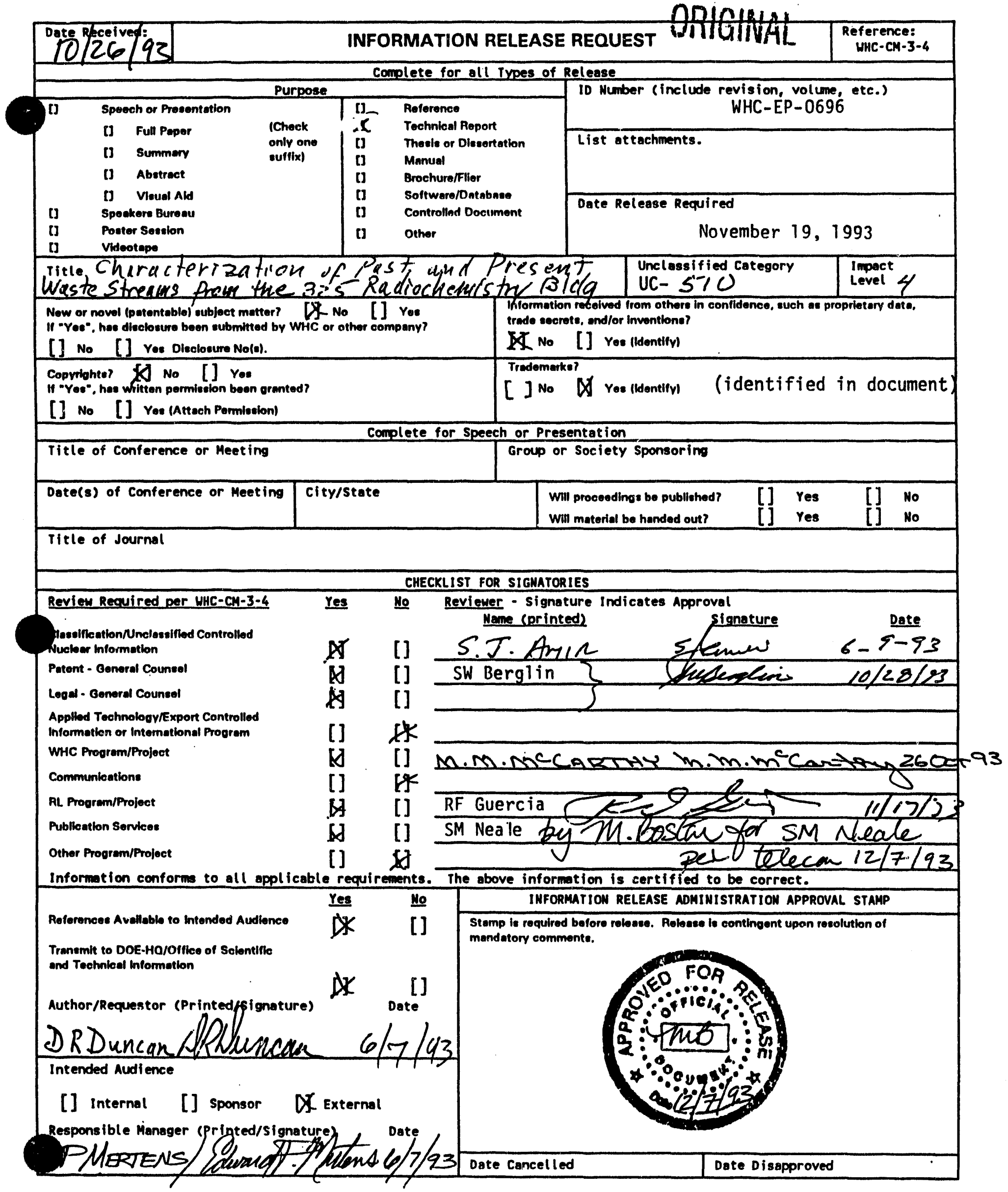


WHC-EP-0696

\section{EXECUTIVE SUMMARY}

During the next two decades the transuranic wastes now stored in the burial trenches and storage facilities at the Hanford Site are to be retrieved, processed at the Waste Receiving and Processing Facility, and shipped to the Waste Isolation Pilot Plant near Carlsbad, New Mexico for final disposal. Over $6.8 \%$ of the transuranic waste to be retrieved for shipment to the Waste isolation Pilot Plant has been generated at the 325 Radiochemistry Building by the Westinghouse Hanford Company (Westinghouse Hanford) and Battelle's Pacific Northwest Laboratories (PNL).

The purpose of this report is to characterize the radioactive solid wastes generated at the 325 Building by Westinghouse Hanford Company and PNL using process knowledge, existing records, and oral history interviews. The 325 Building is currently operated by PNL for the U.S. Department of Energy.

The 325 Radiochemistry Building, also called the Applied Chemistry Building, was originally built to safely house and handle multi-curie level chemical development work with high activity substance. Tasks performed at this facility have included chemistry and analysis, radioactive materials processing, safeguards and materials management, waste management, technical research and development, and operational health physics. Programs supported by the 325 Facility have included, but are not limited to, the following:

- Reclamation of Uranium and Plutonium by Extraction (RECUPLEX) process

- Reduction-Oxidation (REDOX) process

- Plutonium-Uranium Extraction (PUREX) process

- Plutonium Reclamation Facility (PRF)

- Nuclear Waste Vitrification Project (NWVP)

- Fast Flux Test Facility (FFTF)

- Liquid Metal Fast Breeder Reactor (LMFBR) program.

Section 2.0 provides further details of the 325 Facility's physical plant and the operations that occurred there.

Because the 325 Building is a research facility, the work performed in this building has been extremely varied. Section 3.0 discusses the analyses done at 325 and the types of solid waste generated. A brief description of the systems currently in place to handle $\mathbf{3 2 5}$ gaseous and liquid wastes also is included in this section.

All solid wastes originating at the $\mathbf{3 2 5}$ Building are packaged for onsite/offsite storage or disposal. The waste packages are designed to safely contain the waste during transportation and storage, and to meet the criteria of the storage/disposal unit. Waste packaging and reporting requirements have undergone significant changes throughout the history of the 325 Building. Current and historical packaging and handling procedures for radioactive wastes at the 325 Building are provided in Section 4.0.

Information on the radioactive wastes generated at the 325 Radiochemistry Building can be found in a number of existing documents and databases. The most important of these are the Solid Waste Information Tracking System database and Solid Waste Burial Records. 


\section{WHC-EP-0696}

Facility personnel also provide excellent information about past waste generation and the procedures used to handle that waste. Sections 5.0 through 8.0 were compiled using these sources to characterize the radioactive wastes, especially TRU wastes, generated at the 325 Radiochemistry Building.

Between 1970 and 1992, 77,494 containers of radioactive solid waste were generated by Westinghouse Hanford and PNL at the 325 Building. These containers represent over almost 3.3 million $\mathrm{kg}(7.2$ million $\mathrm{lb})$ of waste occupying $14,730 \mathrm{~m}^{3}\left(520,190 \mathrm{ft}^{3}\right)$ of space. Sections $\mathbf{5 . 0}$ through $\mathbf{8 . 0}$ provide an in depth look at the waste generated by Westinghouse Hanford and PNL in the analytical laboratories, the 325A Annex and the 325B Annex, respectively. Spesific information about the radioactive waste generated includes the following:

- Weight and volume of the waste

- Container types and numbers

- Physical description of the waste

- Radiological Components

- Hazardous Constituents

- Current Storage/Disposal Locations. 
WHC-EP.0696

CONTENTS

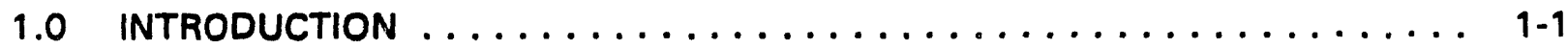

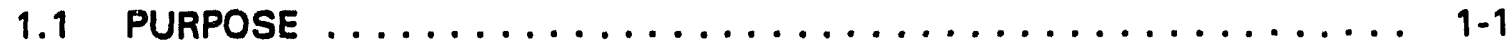

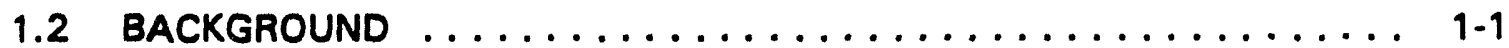

1.3 SOURCES .......................... 1-2

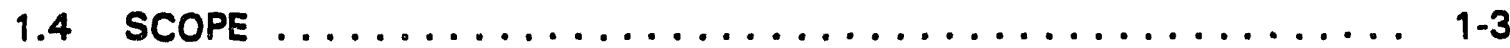

2.0 DESCRIPTION OF THE 325 RADIOCHEMISTRY BUILDING $\ldots \ldots \ldots \ldots \ldots \ldots$.

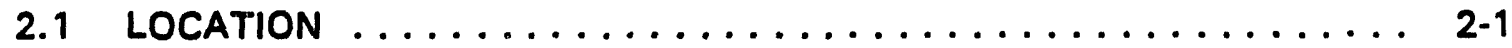

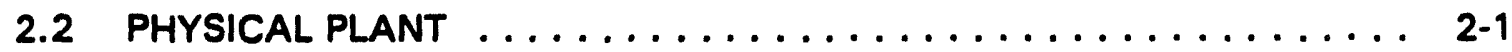

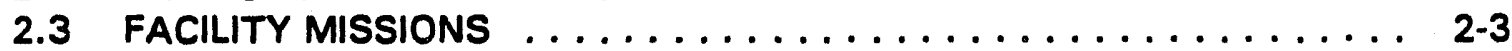

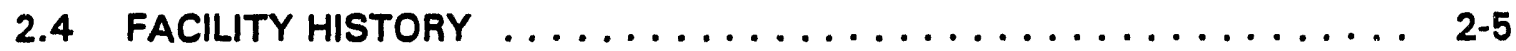

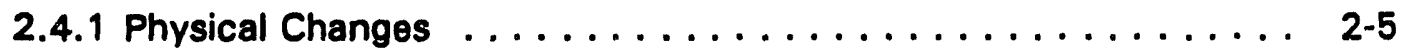

2.4.2 Activity Changes .................... 2-6

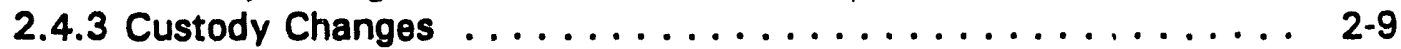

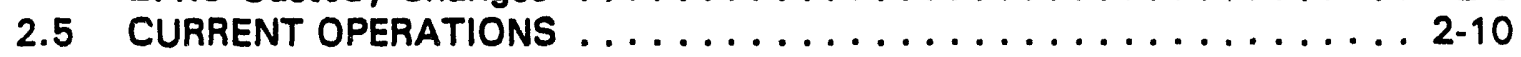

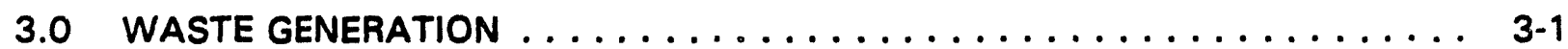

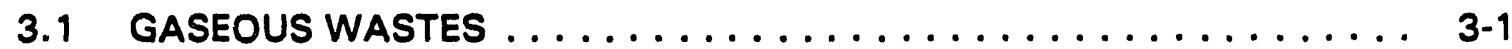

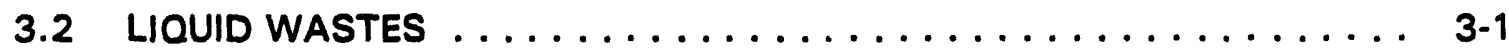

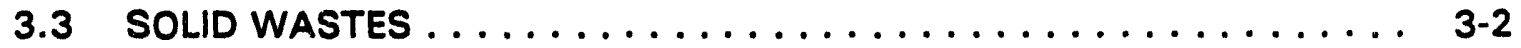

3.4 SOLID WASTE GENERATING ACTIVITIES $\ldots \ldots \ldots \ldots \ldots \ldots \ldots \ldots$. $\ldots \ldots$

4.0 SOLID WASTE HANDLING PRACTICES AND PROCEDURES $\ldots \ldots \ldots \ldots \ldots$. . . . 4

4.1 HISTORICAL WASTE PACKAGING AND HANDLING

REQUIREMENTS AT HANFORD . . . . . . . . . . . . . 4-1

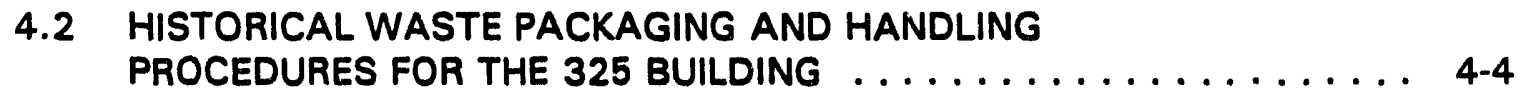

4.2.1 Transuranic Waste . . . . . . . . . . . . . . . . . . 4-4

4.2.2 Low-Level Waste ... . . . . . . . . . . . . . . . 4-5

4.3 HANDLING AND PACKAGING OF CAISSON WASTE FROM THE
325 HOT CELLS $\ldots \ldots \ldots \ldots \ldots \ldots \ldots \ldots \ldots \ldots \ldots \ldots \ldots \ldots \ldots$

4.3.1 Pre-1970 Handling and Packaging Procedures . . . . . . . . . 4-6

4.3.2 Post-1970 Packaging and Handling Procedures . . . . . . . . . . . 4-6

5.0 CHARACTERIZATION OF RETRIEVABLY STORED SOLID WASTE

GENERATED AT 325 BY WESTINGHOUSE HANFORD . . . . . . . . . . . 5-1

5.1 SUMMARY OF 325 WASTE GENERATION BY WESTINGHOUSE

HANFORD . . . . . . . . . . . . . . . . . . . . . $5-2$

5.1.1 Waste Stored in 55-Gallon Steel Drums . . . . . . . . . . . . 5-2

5.1.2 Waste Stored in Containers Other Than 55-Gallon

Steel Drums .......................... 5-3

5.1.3 Summary of Waste Generation Rates at Westinghouse

Hanford 325 Between 1970 and $1992 \ldots \ldots \ldots \ldots \ldots$. . . . . . . .

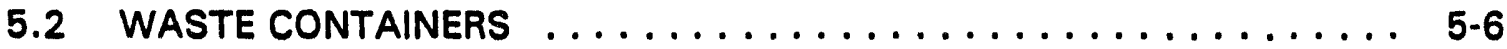

5.2.1 TRU Waste Containers . . . . . . . . . . . . . . . . . 5-6

5.2.2 Non-TRU Waste Containers . . . . . . . . . . . . . . . . 5-6 
WHC-EP-0696

CONTENTS (cont)

5.3 TRU WASTE STORAGE LOCATIONS $\ldots \ldots \ldots \ldots \ldots \ldots \ldots \ldots \ldots$

5.4 PHYSICAL CONTENTS OF TRU WASTE CONTAINERS $\ldots \ldots \ldots \ldots \ldots \ldots$

5.5 RADIOLOGICAL DESCRIPTION OF SOLID WASTE AT 325 BY WESTINGHOUSE HANFORD . . . . . . . . . . . . . . . . 5-7

5.6 RADIOACTIVE ISOTOPES PRESENT IN SOLID WASTE AT 325 BY WESTINGHOUSE HANFORD . . . . . . . . . . . . . . . . . 5-7

5.7 HAZARDOUS CONSTITUENTS OF SOLID WASTE GENERATED

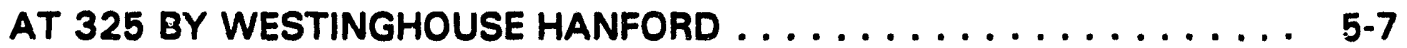

5.7.1 Chemical Inventories . . . . . . . . . . . . . . . . . . . 5-7

5.7.2 Solid Waste Information and Tracking System (SWITS) . . . . . . 5-8

5.7.3 Burial Records ........................ 5-8

5.7.4 Interviews with 325 Building Personnel . . . . . . . . . . 5-8

6.0 CHARACTERIZATION OF RETRIEVABLY STORED SOLID WASTE

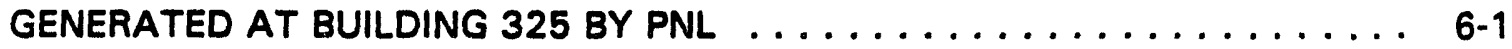

6.1 SUMMARY OF BUILDING 325 WASTE GENERATION BY PNL . . . . . . 6 6-2

6.1.1 Waste Stored in 55-Gallon Steel Drums . . . . . . . . . . . . . 6. 6-2

6.1.2 Waste Stored in Containers Other Than 55-Gallon

Steel Drums .......................... 6-3

6.1.3 Summary of Waste Generation Rates at 325 Between

1970 and 1992 by PNL . . . . . . . . . . . . . . . . 6-5

6.2 WASTE CONTAINERS ..................... 6-6

6.2.1 TRU Waste Containers . . . . . . . . . . . . . . . 6-6

6.2.2 Non-TRU Waste Containers ... . . . . . . . . . . . . 6. 6-6

6.3 TRU WASTE STORAGE LOCATIONS $\ldots \ldots \ldots \ldots \ldots \ldots \ldots \ldots \ldots \ldots$

6.4 PHYSICAL CONTENTS OF TRU WASTE CONTAINERS . . . . . . . . 6 6-7

6.5 RADIOLOGICAL DESCRIPTION OF BUILDING 325 SOLID

WASTE BY PNL ... . . . . . . . . . . . . . . . . . . 6-7

6.6 RADIOACTIVE ISOTOPES PRESENT IN BUILDING 325 SOLID

WASTE BY PNL . . . . . . . . . . . . . . . . . . . . . . 6-8

6.7 HAZARDOUS CONSTITUENTS OF SOLID WASTE GENERATED AT

325 BY PNL ............................ 6-8

6.7.1 Chemical Inventories . . . . . . . . . . . . . . . . . . . 6-8

6.7.2 Solid Waste Information and Tracking System (SWITS) . . . . . . 6-9

6.7.3 Burial Rec.ords ........................ 6-9

6.7 .4 Interviews with 325 Building Personnel . . . . . . . . . . . 6-9

7.0 CHARACTERIZATION OF RETRIEVABLY STORED SOLID WASTE

GENERATED BY 325 A HOT CELLS ANNEX $\ldots \ldots \ldots \ldots \ldots \ldots \ldots \ldots$ 7-1

7.1 SUMMARY OF 325A WASTE GENERATION $\ldots \ldots \ldots \ldots \ldots \ldots \ldots$. . . . .

7.1.1 Waste Stored in 55-Gallon Steel Drums . . . . . . . . . . . . 7-1

7.1.2 Waste Stored in Containers Other Than 55-Gallon

Steel Drums ........................ 7-2

7.1.3 Summary of Waste Generation Rates at the 325A

Annex Between 1970 and $1992 \ldots \ldots \ldots \ldots$ 7-4 
WHC-EP-0696

CONTENTS (cont)

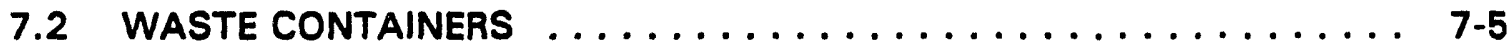

7.2.1 TRU Waste Containers $\ldots \ldots \ldots \ldots \ldots \ldots \ldots \ldots \ldots \ldots$ 7-5

7.2.2 Non-TRU Waste Containers .................. 7-5

7.3 TRU WASTE STORAGE LOCATIONS $\ldots \ldots \ldots \ldots \ldots \ldots \ldots \ldots$

7.4 PHYSICAL CONTENTS OF TRU WASTE CONTAINERS $\ldots \ldots \ldots \ldots \ldots$ 7-6

7.5 RADIOLOGICAL DESCRIPTION OF THE 325A ANNEX

7.6 RADIOACTIVE IS $\mathrm{T}$ TOPES PRESENT IN THE $325 \mathrm{~A}$ ANNEX

SOLID WASTE . . . . . . . . . . . . . . . . . . . 7-7

7.7 HAZARDOUS CONSTITUENTS OF SOLID WASTE GENERATED BY

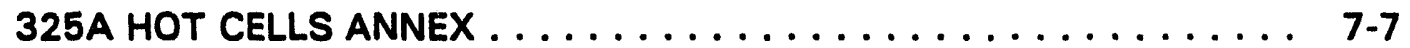

7.7.1 Chemical Inventories . . . . . . . . . . . . . . . . . . . 7-7

7.7.2 Solid Waste Information and Tracking System (SWITS) . . . . . 7 7-7

7.7.3 Burial Records . . . . . . . . . . . . . . . . . 7-7

7.7.4 Interviews with 325 Building Personnel . . . . . . . . . 7-8

8.0 CHARACTERIZATION OF RETRIEVABLY STORED SOLID WASTE

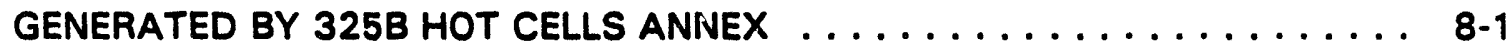

8.1 SUMMARY OF 325B WASTE GENERATION $\ldots \ldots \ldots \ldots \ldots \ldots \ldots$ 8-1

8.1.1 Waste Stored in 55-Gallon Steel Drums . . . . . . . . . . . . 8-1

8.1.2 Waste Stored in Containers Other Than 55-Gallon

Steel Drums ........................ 8-1

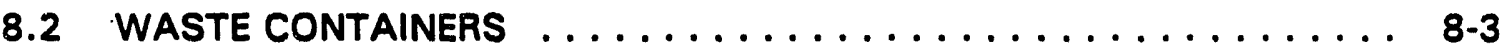

8.2.1 TRU Waste Containers . . . . . . . . . . . . . . . 8-3

8.2.2 Non-TRU Waste Containers . . . . . . . . . . . . . . 8 8-3

8.3 TRU WASTE STORAGE LOCATIONS $\ldots \ldots \ldots \ldots \ldots \ldots \ldots \ldots$

8.4 PHYSICAL CONTENTS OF TRU WASTE CONTAINERS $\ldots \ldots \ldots \ldots \ldots . . .$.

8.5 RADIOLOGICAL DESCRIPTION OF 325B SOLID WASTE $\ldots \ldots \ldots \ldots \ldots \ldots$.

8.6 RADIOACTIVE ISOTOPES PRESENT IN 325B SOLID WASTE $\ldots \ldots \ldots \ldots$.

8.7 HAZARDOUS CONSTITUENTS OF SOLID WASTE GENERATED

BY 325B HOT CELLS ANNEX . . . . . . . . . . . . . . . . . . 8 8-4

8.7.1 Chemical Inventories . . . . . . . . . . . . . . . . . . 8-4

8.7.2 Solid Waste Information and Tracking System (SWITS) . . . . . 8 8-5

8.7.3 Burial Records ....................... 8-5

8.7.4 Interviews with 325 Building Personnel . . . . . . . . . . 8 8-5

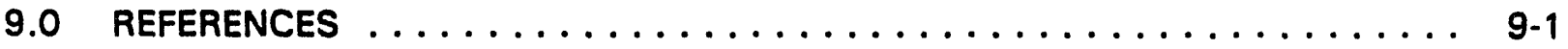


WHC-EP-0696

CONTENTS (cont)

APFENDICES:

A. DATA ON BUILDING 325 WASTE GENERATION BY WESTINGHOUSE HANFORD FROM THE SOLID WASTE INFORMATION TRACKING SYSTEM $\ldots \ldots \ldots \ldots \ldots \ldots \ldots \ldots$ A-1

B. DATA ON BUILDING 325 WASTE GENERATION BY

PNL FROM THE SOLID WASTE INFORMATION

TRACKING SYSTEM . . . . . . . . . . . . . . . . . . . . . B-1

C. DATA ON BUILDING 325A WASTE GENERATION BY

PNL FROM THE SOLID WASTE INFORMATION

TRACKING SYSTEM ..................... $\mathrm{C}-1$

D. DATA ON BUILDING 325B WASTE GENERATION BY

WESTINGHOUSE HANFORD FROM THE SOLID WASTE

INFORMATION TRACKING SYSTEM

D-1

E. DATA ON BUILDING 325B WASTE GENERATION BY

PNL FROM THE SOLID WASTE INFORMATION

TRACKING SYSTEM .

E-1 


\section{LIST OF FIGURES}

$2-1$ Location of the Hanford Site .................... 2-13

2-2 Hanford Site Map .......................... 2-14

2-3 Photograph of the 325 Building. . . . . . . . . . . . $\ldots \ldots$

2-4 Building 325A High-Level Radiochemistry Facility . . . . . . . . . . . 2-16

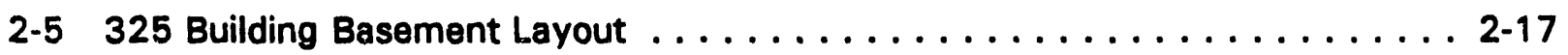

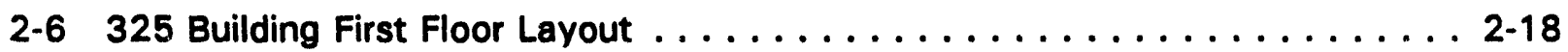

2-7 Layout of the 325 Building Second and Third Floors . . . . . . . . . . 2-19

4-1 Post-1970 Underground Caisson TRU Storage $\ldots \ldots \ldots \ldots \ldots \ldots \ldots \ldots$. . . . . . .

5-1 55-Gallon Drum Waste Count Generated by Westinghouse Hanford Building 325 Annually . . . . . . . . . . . . . . . 5-11

5-2 Percentage of Westinghouse Hanford Building 325 TRU/Non-TRU $55-$ Gallon Drums . . . . . . . . . . . . . . . . . . . 5-12

5-3 Weight of 55-Gallon Drum Waste Generated by Westinghouse Hanford Building 325 Annually . . . . . . . . . . . . . . . . . 5-13

5-4 Weight Percentage of Westinghouse Hanford Building 325 55-Gallon Drums . . . . . . . . . . . . . . . . . . . . . 5-14

5-5 Volume of 55-Gallon Drum Waste Generated by Westinghouse Hanford Building 325 Annually . . . . . . . . . . . . . . . . . . 5-15

5-6 Volume Percentage of Westinghouse Hanford Building 325 55-Gallon Drums . . . . . . . . . . . . . . . . . . . 5-16

5-7 Other Container Waste Count Generated by Westinghouse Hanford Building 325 Annually . . . . . . . . . . . . . . . . . 5-17

5-8 Percentage of Westinghouse Hanford Building 325 TRU/Non-TRU Other Containers ............................ 5-18

5-9 Weight of Other Containers Generated by Westinghouse Hanford Building 325 Annually . . . . . . . . . . . . . . . . . . 5-19

5-10 Weight Percentage of Other Containers Generated by Westinghouse Hanford Building 325 Annually . . . . . . . . . . . . . . . . 5-20 
WHC-EP-0696

LIST OF FIGURES (cont)

5-11 Volume of Other Containers Generated by Westinghouse Hanford

Building 325 Annually . . . . . . . . . . . . . . . . . . 5-21

5-12 Volume Percentage of Other Containers Generated by Westinghouse Hanford Building 325 Annually . . . . . . . . . . . . . . . . . 5 5-22

5-13 Total Number of Waste Containers Generated by Westinghouse Hanford Building 325 Annually . . . . . . . . . . . . . . . . . . . 5-23

5-14 Totai Weight of Waste Generated by Westinghouse Hanford Building 325 Annually ............................... 5-24

5-15 Total Volume of Waste Generated by Westinghouse Hanford Building 325 Annually ............................... 5-25

5-16 Grams of TRU Waste Generated by Westinghouse Hanford Building 325 Annually .............................. 5-26

6-1 55-Gallon Drum Waste Count Generated at Building 325 by PNL Annually . . . 6-10

6-2 Percentage of Building 325 TRU/Non-TRU 55-Gallon Drums by PNL . . . . . 6-11

6-3 Weight of 55-Gallon Drum Waste Generated at Building 325 by PNL Annually ............................. 6-12

6-4 Weight Percentage of Building 325 55-Gallon Drums by PNL $\ldots \ldots \ldots \ldots$. . . . 3

6-5 Volume of 55-Gallon Drum Waste Generated at Building 325 by PNL Annually ............................... 6-14

6-6 Volume Percentage of Building 325 55-Gallon Drums by PNL . . . . . . . . 6-15

6-7 Other Container Waste Count Generated at Building 325 by PNL Annually . . . 6-16

6-8 Percentage of Building 325 TRU/Non-TRU Other Containers by PNIL $\ldots \ldots$. . 6-17

6-9 Weight of Other Containers Generated at Building 325 by PNL Annually . . . 6-18

6-10 Weight Percentage of Other Containers Generated at Building 325 by PNL Annually . . . . . . . . . . . . . . . . . . . 6-19

6-11 Volume of Other Containers Generated at Building 325 by PNL Annually . . . 6-20

6-12 Volume Percentage of Other Containers Generated at Building 325 by PNL Annually . . . . . . . . . . . . . . . . . . . . 6-21 


\section{WHC-EP-0696}

\section{LIST OF FIGURES (cont)}

6-13 Total Number of Waste Containers Generated at Building 325 by PNL Annually . . . . . . . . . . . . . . . . . . . . 6-22

6-14 Total Weight of Waste Generated at Building 325 by PNL Annually . . . . . 6-23

6-15 Total Volume of Waste Generated at Building 325 by PNL Annually . . . . . . 6-24

6-16 Grams of TRU! Waste Generated at Building 325 by PNL Annually . . . . . . 6-25

7-1 55-Gallon Drum Waste Count Generated by the 325 A Annex Annually . . . . 7-9

7-2 Percentage of 325A Annex TRU/Non-TRU 55-Gallon Drums . . . . . . . . . . 7-10

7-3 Weight of 55-Gallon Drum Weste Generated by the 325A Annex Annually .......................... 7-11

7-4 Weight Percentage of 325A Annex 55-Gallon Drums $\ldots \ldots \ldots \ldots \ldots \ldots$. . . . 12

7-5 Volume of 55-Gallon Drum Waste Generated by the 325A Annex Annually .......................... 7-13

7-6 Volume Percentage of 325A Annex 55-Gallon Drums . . . . . . . . . . 7-14

7.7 Other Container Waste Count Generated by the 325A Annex Annually ........................ 7-15

7-8 Percentage of 325A Annex TRU/Non-TRU Other Containers $\ldots \ldots \ldots \ldots$. . . . .

7-9 Weight of Other Containers Generated by the 325A Annex Annually $\ldots \ldots$. . 7-17

7-10 Weight Percentage of Other Containers Generated by the 325A Annex Annually ........................ 7-18

7-11 Volume of Other Containers Generated by the 325A Annex Annually ... . . 7-19

7-12 Volume Percentage of Other Containers Generated by the 325A Annex Annually ............................. 7-20

7-13 Total Number of Waste Containers Generated by the 325A Annex Annually .......................... 7-21

7-14 Total Weight of Waste Generated by the 325A Annex Annually . . . . . . . 7-22

7-15 Total Volume of Waste Generated by the 325A Annex Annually . . . . . . 7-23

7-16 Grams of TRU Waste Generated by the 325A Annex Annually . . . . . . . . . 7-24 
WHC-EP-0696

LIST OF FIQURES (cont)

8-1 Other Container Waste Count Generated by the Westinghouse

Hanford 325B Annex Annually . . . . . . . . . . . . . . . . . . 8-6

8-2 Weight of Other Containers Generated by the Westinghouse

Hanford 325B Annex Annually . . . . . . . . . . . . . . . . 8-7

8-3 Volume of Other Containers Generated by the Westinghouse

Hanford 325B Annex Annually . . . . . . . . . . . . . . . . . . 8-8

8-4 Grams of TRU Waste Generated by the Westinghouse Hanford

325B Annex Annually ......................... 8-9 
WHC-EP-0696

\section{LIST OF TABLES}

4-1 TRU Storage Requirements for the Hanford Site . . . . . . . . . . . . 4-9

5-1 Transuranic Waste Attributed to Westinghouse Hanford Building 325

but Originating at Other Facilities . . . . . . . . . . . . . . 5-27

5-2 Low-Level Wastes Attributed to Westinghouse Hanford Building 325

but Originating at Other Facilities . . . . . . . . . . . . . . 5-28

5-3 TRU Waste Generated at Building 325 Westinghouse Hanford by

Container Type and Year . . . . . . . . . . . . . . . . . . . 5 5-31

5-4 Non-TRU Waste Generated at Building 325 Westinghouse Hanford by

Container Type and Year . . . . . . . . . . . . . . . . . . 5-35

5-5 Building 325 Westinghouse Hanford Waste Summary Data for

55-Gallon Drums . . . . . . . . . . . . . . . . . . . 5-45

5-6 Building 325 Westinghouse Hanford Waste Summary Data for

Containers Other Than 55-Gallon Drums . . . . . . . . . . . . . . 5-46

5-7 Building 325 Westinghouse Hanford TRU Waste in 55-Gallon Drums:

Drum Count by Storage Location . . . . . . . . . . . . . . . 5-47

5-8 Building 325 Westinghouse Hanford TRU Waste in Containers Other Than

55-Gallon Drums: Container Count by Storage Location . . . . . . . . . . . 5-47

5-9 Distribution and Percentage for Building 325 Westinghouse Hanford of TRU 55-Gallon Drum Physical Contents by Storage Location - 218-W-3A . . . . . . 5-47

5-10 Distribution and Percentage for Building 325 Westinghouse Hanford of TRU 55-Gallon Drum Physical Contents by Storage Location - 218-W-4B . . . . . . 5-48

5-11 Distribution and Percentage for Building 325 Westinghouse Hanford of TRU 55-Gallon Drums Physical Contents by Storage Location - 218-W-4C . . . . . 5-49

5-12 Distribution and Percentage for Building 325 Westinghouse Hanford of TRU Containers Other Than 55-Gallon Drums Physical Contents by Storage Location - 218-W-3A . . . . . . . . . . . . . . . . . . . . . 5-51

5-13 Distribution and Percentage for Building 325 Westinghouse Hanford of TRU Containers Other Than 55-Gallon Drums Physical Contents by Storage Location - 218-W-4B . . . . . . . . . . . . . . . . . . . . . . .

5-14 Distribution and Percentage for Building 325 Westinghouse Hanford of TRU Containers Other Than 55-Gallon Drums Physical Contents by Storage Location - 218-W-4C 


\section{WHC-EP-0696}

\section{LIST OF TABLES (cont)}

5-15 Building 325 Westinghouse Hanford TRU Waste in 55-Gallon Drums:

Total Grams TRU by Storago Location . . . . . . . . . . . . . . . . . . . . 5-53

5-16 Building 325 Westinghouse Hanford TRU Waste in Containers Other than

55-Gallon Drums: Total Grams by Storage Location . . . . . . . . . . . . . . . 5-53

5-17 Isotopes in 55-Gallon Drums for Westinghouse Hanford Building $325 \ldots$. . . 5-54

5-18 Isotopes in Other Containers for Westinghouse Hanford Building $325 \ldots \ldots$ 5-54

5-19 Building 325: Chemicals Used at Westinghouse Hanford Company

Development Laboratory (1970-1976) . . . . . . . . . . . . . . . . . 5-55

5-20 Building 325: Chemicals Used at Analytical Laboratory (1953-1976) . . . . . 5-58

5-21 Summary of Information from Solid Waste Storage Disposal

Records (SWSDR) for TRU Wastes with Hazardous Contents from

Building 325 Westinghouse Hanford . . . . . . . . . . . . . . . . 5-60

5-22 Summary of Information from Solid Waste Storage Disposal Records (SWSDR) for Low-Level Wastes with Hazardous Contents from Building 325

Westinghouse Hanford

6-1 TRU Wastes Attributed to PNL Building 325 but Generated at

Other Facilities

6-2 Low-Level Wastes Attributed to PNL Building 325 but Generated

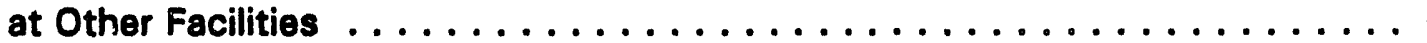

6-3 TRU Waste Generated at Building 325 by PNL by Container

Type and Year . . . . . . . . . . . . . . . . . . . . . . . . . . . . 6 6-29

6-4 Non-TRU Waste Generated at Building 325 by PNL by Container

Type and Year .............................. 6-.33

6-5 Building 325 Waste Summary Data for 55-Gallon Drums by PNL $\ldots \ldots \ldots$. . 6-42

6-6 Building 325 Waste Summary Data for Waste Containers Other

Than 55-Gallon Drums by PNL . . . . . . . . . . . . . . . . . . 6-43

6-7 Building 325 TRU Waste in 55-Gallon Drums by PNL:

Drum Count by Storage Location . . . . . . . . . . . . . . . . . . . . . . 6-44

6-8 Building 325 TRU Waste in Containers Other Than 55-Gallon Drums

by PNL: Container Count by Storage Location . . . . . . . . . . . . . . . . . . 6-44 
WHC-EP-0696

LIST OF TABLES (cont)

6-9 Distribution and Percentage of TRU 55-Gallon Drum Physical Contents by Storage Location - 218-W-3A . . . . . . . . . . . . . . . . . . 6-45

6-10 Distribution and Percentage of TRU 55-Gallon Drum Physical Contents by Storage Location - 218-W-4B . . . . . . . . . . . . . . . . . . 6-45

6-11 Distribution and Percentage of TRU 55-Gallon Drum Physical Contents by Storage Location - 218-W-4C . . . . . . . . . . . . . . . . . 6-46

6-12 Distribution and Percentage of TRU 55-Gallon Drum Physical Contents by Storage Location - 224T . . . . . . . . . . . . . . . . . . 6-48

6-13 Distribution and Percentage of TRU 55-Gallon Drum Physical Contents by Storage Location - 2403-WA . . . . . . . . . . . . . . . 6-51

6-14 Distribution and Percentage of TRU 55-Gallon Drum Physical Contents by Storage Location - 218-W-3A . . . . . . . . . . . . . . . . . . . 6-52

6-15 Distribution and Percentage of TRU 55-Gallon Drum Physical Contents by Storage Location - 218-W-4B . . . . . . . . . . . . . . . . 6-52

6-16 Distribution and Percentage of TRU 55-Gallon Drum Physical Contents by Storage Location - 218-w-4C . . . . . . . . . . . . . . . . 6 6-53

6-17 Distribution and Percentage of TRU 55-Gallon Drum Physical Contents by Storage Location - 2402-WE

6-18 Building 325 TRU Waste by PNL in 55-Gallon Drums: Total Grams TRU by Storage Location

6-19 Building 325 TRU Waste by PNL in Containers Other Than 55-Gallon Drums:

Total Grams TRU by Storage Location . . . . . . . . . . . . . . . . 6-55

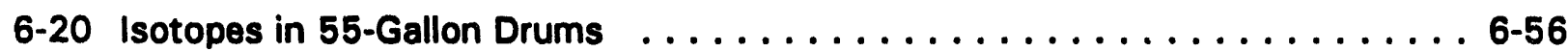

6-21 Isotopes in Other Containers than 55-Gallon Drums for $1970-1992 \ldots 6-57$

6-22 Chemicals Used at Building 325: Pacific Northwest Laboratory Development and Production Laboratory (1955-1976) . . . . . . . . . . . . 6-58

6-23 Building 325 TRU 55-Gallon Drums Containing Hazardous Constituents by PNL: Drum Count by Storage Location . . . . . . . . . . . . . . . 6-60

6-24 Number and Percentage of Building 325 TRU 55-Gallon Drums Containing Hazardous Constituents by Storage Location - 224T $6-60$ 


\section{LIST OF TABLES (cont)}

6-25 Summary of Information from Solid Waste Storage Disposal

Records (SWSDR) for TRU Wastes with Hazardous Contents from

PNL at Building $325 \ldots \ldots \ldots \ldots \ldots \ldots \ldots \ldots \ldots \ldots \ldots$. . . . . . . . . . . . . . . .

6-26 Summary of Information from Solid Waste Storage Disposal

Records (SWSDR) for LLW Wastes with Hazardous Contents from

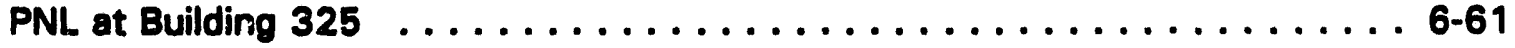

7-1 TRU Waste Generated at the 325A Annex by Container Type and Year . . . . 7-25

7-2 Non-TRU Waste Generated at the 325A Annex by Container

Type and Year ........................... 7-27

7-3 The 325A Annex Waste Summary Data for $55-$ Gallon Drums . . . . . . . . . 7-30

7-4 The 325A Annex Waste Summary Data for Waste Containers Other than 55-Gallon Drums . . . . . . . . . . . . . . . . . . . . . 7-31

7-5 The 325A Annex TRU Waste in 55-Gallon Drums: Drum Count by Storage Location . . . . . . . . . . . . . . . . . 7-32

7-6 The 325A Annex TRU Waste in Containers Other Than 55-Gallon Drums: Container Count by Storage Location . . . . . . . . . . . . . . 7-32

7-7 Distribution and Percentage of TRU 55-Gallon Drum Physical Contents by Storage Location - 218-W-3A . . . . . . . . . . . . . . . . 7-32

7-8 Distribution and Percentage of TRU 55-Gallon Drum Physical Contents by Storage Location - 218-W-4C . . . . . . . . . . . . . . . . . 7-33

7-9 Distribution and Percentage of TRU Containers Other than 55-Gallon Drums Physical Contents by Storage Location - 218-W-3A . . . . . . . . . . 7-33

7-10 Distribution and Percentage of TRU Containers Other than 55-Gallon Drums Physical Contents by Storage Location - 218-W-4B . . . . . . . . . . . . 7-34

7-11 The 325A Annex TRU Waste in 55-Gallon: Total Grams TRU by Storage Location . . . . . . . . . . . . . . . . . . . . . . . . 7-34

7-12 The 325A Annex TRU Waste in Containers Other than 55-Gallon Drums: Total Grams TRU by Storage Location . . . . . . . . . . . . . . . . . . 7-35

7-13 Isotopes in 55-Gallon Drums for the 325A Annex for 1970-1992 . . . . 7-36

7-14 Isotopes in Other Containers for the 325A Annex for 1970-1992 . . . . 7-36 
WHC-EP-0696

LIST OF TABLES (cont)

7-15 Summary of Information from Solid Waste Burial Records (SWBR) for TRU Wastes with Hazardous Cor:tents from 325A

Hot Cell Annex by PNL . . . . . . . . . . . . . . . . 7-37

7-16 Summary of Information from Solid Waste Burial Records (SWBR)

for Low-Level Wastes with Hazardous Contents from 325A

Hot Cell Annex by PNL . . . . . . . . . . . . . . . . . 7-38

8-1 TRU Waste Generated at the Westinghouse Hanford 325B Annex by Container Type and Year . . . . . . . . . . . . . . . . 8-10

8-2 The 325B Annex Westinghouse Hanford Waste Summary Data for Waste Containers Other than $55-G a l l o n$ Drums . . . . . . . . . . 8-15

8-3 The 325B Annex Westinghouse Hanford TRU Waste in Containers Other Than 55-Gallon Drums: Container Count by Storage Location . . . . . . . 8-16

8-4 Distribution and Percentage of TRU Containers Other Than 55-Gallon Drum Physical Contents by Storage Location - 218-W-4B . . . . . . . . . 8-17

8-5 The 325B Annex Westinghouse Hanford TRU Waste in Containers Other Than 55-Gallon Drums: Total Grams TRU by Storage Location . . . . . . 8-18

8-6 Characterization of TRU Waste from the PNL 325B Annex in 1 Gallon Containers Stored at 218-W-4B . . . . . . . . . . . . . . 8-19

8-7 Isotopes in Other Containers for the 325B Annex from 1970 to $1992 \ldots \ldots$. . 8-19 
WHC-EP-0696

This page intentionally left blank. 
WHC-EP-0696

\section{LIST OF TERMS}

325

$325 A$

325B

$325 \mathrm{C}$

325D

AEC

AMU

Bldg

BNWL

CEPOD

CERCLA

$D \& D$

DOE

DOT

Ecology

EIS

EPA

FFTF

FRP

FSAR

GE

HEDL

HEPA

HLRF

$\mathrm{HNO}_{3}$

IAD

LLW

LMFBR

MFP

$M W$

NDA

NRC

NWVP

PCB

PNL

PRF

PSAR

PSE

PUREX

R-SWIMS

RCRA

RECUPLEX

REDOX

RHO

RLWS
Radiochemistry Building (Applied Chemistry Laboratory)

High Level Radiochemistry Facility

Shielded Analytical Laboratory

Fluorine Gas Storage

Maintenance Shop

Atomic Energy Commission (now DOE)

Aqueous Makeup

Building

Battelle Pacific Northwest Laboratories

Catalyzed Electrochemical Plutonium Oxide Dissolution

Comprehensive Environmental Response, Compensation, and

Liability Act

Decontamination and Decommissioning

Department of Energy

U.S. Department of Transportation

Washington State Department of Ecology

Environmental Impact Statement

U.S. Environmental Protection Agency

Fast Flux Test Facility

Fiberglass Reinforced Plywood

Final Safety Analysis Report

General Electric

Hanford Engineering Development Laboratory

High Efficiency Particulate Air

High Level Radiochemistry Facility (325A)

nitric acid

Immediate Action Directive

Low Level Waste

Liquid Metal Fast Breeder Reactor

Mixed Fission Products

Mixed Waste

Nondestructive Assay

Nuclear Regulatory Commission

Nuclear Waste Vitrification Project

Polychlorinated biphenyls

Pacific Northwest Laboratories

Plutonium Reclamation Facility

Preliminary Safety Analysis Report

Preliminary Safety Evaluation

Plutonium Uranium Extraction Plant

Richland Solid Waste Information Management Systems

Resource Conservation and Recovery Act

Reclamation of Uranium and Plutonium by Extraction

Reduction-Oxidation

Rockwell Hanford Operations

Radioactive Liquid Waste System 


\section{LIST OF TERMS (cont)}

SAL
SAR
SARP
SDAR
SGSAS
SNM
SWBR
SWITS
SWSDR
TRU
TRUSAF
WAC
WADCO
WEStinghouse Hanford
WIPP
WRAP

Shielded Analytical Laboratory

Safety Analysis Report

Safety Analysis Report for Packaging

Storage/Disposal Approval Record

Segmented Gamma Scan Assay System

Special Nuclear Material

Solid Waste Burial Record

Solid Waste Information and Tracking System

Solid Waste Storage and Disposal Record

Transuranic

Transuranic Storage and Assay Facility

Waste Acceptance Criteria

Westinghouse Hanford Company (used in early 1970's)

Westinghouse Hanford Company

Waste Isolation Pilot Plant

Waste Receiving and Processing Facility 


\section{WHC-EP-0696}

\subsection{INTRODUCTION}

\subsection{PURPOSE}

The purpose of this report is to characterize, as far as possible, the solid waste generated by the 325 Radiochemistry Building since its construction in 1953. Solid waste as defined in this document is any containerized or self-contained material that has been declared waste. This characterization is of particular interest in the planning of transuranic (TRU) waste retrieval operations including the Waste Receiving and Processing (WRAP) Facility. Westinghouse Hanford Company (Westinghouse Hanford) and Battelle Pacific Northwest Laboratory (PNL) activities at Building 325 have generated approximately $4.4 \%$ and $2.4 \%$, respectively, of the totz: volume of TRU waste currently stored at the Hanford Site.

\subsection{BACKGROUND}

Since 1944, the production of defense related materials at the Hanford Site has generated radioactive wastes. The bulk of these wastes have been disposed of or stored in the 200 East and 200 West Area burial grounds and waste storage facilities.

In the period between 1944 and 1970, both TRU and low-level wastes (LLW) were disposed of in shallow land trenches with no attempt to segregate these materials by their chemical or radioactive natures. In 1970 the Atomic Energy Commission (AEC, now the U.S. Department of Energy [DOE]) directed that AEC sites segregate "waste with known or detectable contamination of transuranium nuclides" from other waste types //mmediate Action Directive 0511-21 (AEC 19701). Transuranic radionuclides are those with an atomic number greater than 92. The AEC further directed that these wastes be packaged and stored as contamination-free packages for at least 20 years. The 20 -year interim storage period was to allow time to study permanent disposal options for TRU contaminated wastes.

The Immediate Action Directive (IAD) did not provide a detailed definition for TRU waste in 1970. AEC contractors implemented the IAD to the best of their ability with the instrumentation then available. In 1973, the Atomic Energy Commission Manual (AEC 1973) further defined TRU waste as material contaminated with certain alpha-emitting radionuclides with half-lives greater than 20 years and activity greater than $10 \mathrm{nCi} / \mathrm{g}$. The radionuclides included were ${ }^{233} \mathrm{U}$ and its daughter products, as well as plutonium and transplutonium nuclides with the exception of ${ }^{238} \mathrm{Pu}$ and ${ }^{241} \mathrm{Pu}$. In 1982, the TRU waste segregation limits were raised to $100 \mathrm{nCi} / \mathrm{g}$ and included ${ }^{238} \mathrm{Pu}$ and ${ }^{241} \mathrm{Pu}$ as stated in DOE Order 5820.1 , Management of Transuranic Material (DOE 1982).

In addition to radioactive materials, Hanford production plants and support operations used a wide variety of chemicals. Many of these chemicals are currently classified as dangerous or hazardous by the U.S. Environmental Protection Agency (EPA) and the Washington State Department of Ecology (Ecology). When dangerous or hazardous wastes are found in radioactive wastes, they are termed "mixed" wastes.

During the time much of the mixed waste at the Hanford Site was generated, there were no detinitions or regulations governing the storage, disposal, or documentation of mixed 
wastes. In 1987, the DOE issued a mixed byproduct ruling stating that the hazardous components of mixed waste are regulated by the Resource Conservation and Recovery Act of 1976 (RCRA) (DOE 1987). In November 1987, the EPA authorized Ecology to regulate the hazardous constituents of mixed waste at Hanford.

During the next two decades, the TRU waste now stored in the 200 Area burial trenches and storage facilities is to be retrieved, processed at the WRAP Facility, and shipped to the Waste Isolation Pilot Plant (WIPP) near Carlsbad, New Mexico for final disposal. Approximately 6.8 percent of the total volume of TRU waste to be retrieved for shipment to WIPP has been generated by the 325 Facility.

\subsection{SOURCES}

Data for this study were compiled from a variety of sources. Each of the major sources used is listed below with a few explanatory notes. Greater detail on each of the data sources can be found in the body of this document as the information from each is discussed.

Documents that describe the Building 325 processes and experiments, including the Safety Analysis Report for the 325 Radiochemistry Building (Anderson and Hanthorn 1977), Past Practices Technical Characterization Study - 300 Area Hanford Site (Gerber 1992), and Analytical Chemistry Laboratory Manual, Volumes 2 and 3 (HEDL 1975a and 1975b) were used to determine the solid wastes that may have been generated at Building $\mathbf{3 2 5}$ during its 40 year history.

Documents that describe Building 325 Wasto Packaging and Handling Procedures, including the Hanford Site Radioactive Solid Waste Acceptance Criteria (Willis and Triner 1991) and the Waste Management Manual (WHC 1987), were used to describe packaging and handling practices.

Interviews with current and former Building 325 personnel were used to gather information regarding unusual waste contents and historical waste handling and packaging procedures.

Characterization data was primarily derived from the Solid Waste Information and Tracking System (SWITS) database. The SWITS database was created in 1991, primarily with the solid waste data from the Richland Solid Waste Information Management System (R-SWIMS). The SWITS database contains information about radioactive wastes, both TRU and LLW, buried or stored in the 200 Areas since 1970. Data in SWITS were originally taken from Solid Waste Burial Records (SWBRs) and their replacements, the Solid Waste Storage and Disposal Records (SWSDRs). Both SWBRs and SWSDRs, which will be jointly referred to as "burial records" in this document, often contain supplementary forms such as shipment manifests, NRC 741 Forms, etc.

Hazardous waste components were determined using information from the SWITS database, SWBRs, SWSDRs, and personnel interviews. Additional information was obtained from Reactive and Unstable Chemicals in Transuranic Retrievable Waste at the Hanford Site, WHC-EP-0603 (Reddinger 1992) and an internal report examining hazardous components of solid waste. 


\subsection{SCOPE}

The major sections of this document and the topics they cover are outlined briefly below. Because of the many tables and figures included in this report, they appear at the end of each section.

Section 2.0 provides a brief description of the Building 325 physical plant and the operations that occurred there. A short history of the significant occurrences and changes at Building 325 follows this description.

Section 3.0 identifies the Solid Waste Streams that arose from Building 325 activities. The types and amounts of solid waste that may have been generated from each of the waste streams are estimated.

Section 4.0 discusses the waste handling and packaging procedures used at Building 325. Historical changes in waste handling, packaging, and record keeping are also reviewed in this section.

Section 5.0 through 8.0 contain the results of a search for actual waste container data including database searches, literature reviews, and personnel interviews. These sections describe what is known about the physical, radiological, and hazardous characteristics of the radioactive solid waste generated in the 325 laboratories by Westinghouse Hanford (Section 5.0) and PNL (Section 6.0), as well as waste generated in the hot cells located in 325A (Section 7.0) and 325B (Section 8.0).

Section 9.0 lists the references used in the compilation of this report.

Appendix A contains the original SWITS data runs that form the basis for most of the tables and figures found in Section 5.0. Appendices $B$ and $C$ provide the same data for the tables and figures found in Sections 6.0 and 7.0, respectively; Appendices $D$ and $E$ provide the data for the tables and figures in Section 8.0 
WHC-EP-0696

This page intentionally left blank. 


\section{WHC-EP-0696}

\section{$\therefore$ \\ 2.0 DESCRIPTION OF THE 325 RADIOCHEMISTRY BUILDING (APPLIED CHEMISTRY LABORATORY)}

Originally built to safely house and handle multicurie level chemical development work with high activity substances, construction of the 325 Radiochemistry Building was completed in 1953. In the years since 1953, the building has been modified to support a variety of tasks and organizations. The tasks performed here have included chemical analysis, radioactive materials processing, safeguards and materials management, waste management, technical research and development, laboratory maintenance, and operational health physics. Programs supported by the 325 Facility have included support of the Reclamation of Uranium and Plutonium by Extraction (RECUPLEX) process, the Reduction-Oxidation (REDOX) process, the Plutonium !Jranium Extraction (PUREX) process, the Plutonium Reclamation Facility (PRF), the Nuclear Waste Vitrification Project (NWVP), the Fast Flux Test Facility (FFTF), the Liquid Metal Fast Breeder Reactor (LMFBR) Program, and many others (Anderson and Hanthorn 1977).

The 325 Building is now called the Applied Chemistry Laboratory and consists of five sections; 325 main Radiochemistry Building, 325-A High Level Radiochemistry Facility, 325-B Shielded Laboratory Annex, 325-C Fluorine Gas Storage, and 325-D Mainta 'nce Shop. The purpose of this section is to describe the 325 Building facilities and to summarize its history, so that solid waste generating activities can be compared to the status of the facility.

\subsection{LOCATION}

The 325 Radiochemistry Building is located within the 300 Area of the DOE's Hanford Site. The Hanford Site occupies 148,000 hectares $(365,000$ acres) of arid steppe on the southeastern part of Washington State, approximately $72 \mathrm{~km}$ (45 miles) north of the Oregon Border (Figure 2-1). The 300 Area is located about a mile north of the Richland city limits or: the bank of the Columbia River which bounds it on the east (Figure 2-2). It is bounded on the west by Stevens Drive (shown on map, but not labeled). Roughly rectangular in shape, the 300 Area included about 152 hectares ( 375 acres) in 1975. Subsequently, the 300 Area was expanded northward for added waste management facilities.

\subsection{PHYSICAL PLANT}

The 325 Building has about $13,000 \mathrm{~m}^{2}\left(140,000 \mathrm{ft}^{2}\right)$ of floor area, $5,200 \mathrm{~m}^{2}$ $\left(56,000 \mathrm{ft}^{2}\right)$ of which are used for laboratory space. The basement and three upper floors house several shielded hot cells and numerous laboratories. First floor laboratories are separated by moveable metal partitions, while basement and second floor laboratories are separated by concrete block partitions. Ventilation roods and gloveboxes are common equipment in the building. A photograph of the 325 Building is provided in Figure 2-3.

In general, the 325 Radiochemistry Building consists of (1) a central portion containing general purpose laboratories modified for low-level radiochemical work by provision of special ventilation and work enclosures, (2) a south (front) wing containing office space, locker rooms and lunch rooms, and (3) east and west wings containing shielded enclosures with remote manipulators for high level radiochemical work. Some of the low level laboratories house a 
variety of standard and special research instruments. For example, emission spectrographs, mass spectrometers, spectrophotometers, flame photometers, electron microscopes, and radiation measuring instruments can be found. The process study and analytical laboratories are equipped with some $\mathbf{4 0}$ hoods and 30 gloveboxes for safe work with radioactive materials (Anderson and Hanthorn 1977).

The central portion of the building is $59.1 \mathrm{~m} \times 59.8 \mathrm{~m}(194 \mathrm{ft} \times 196 \mathrm{ft}$ ) overall, on three floors (basement, ground, and second), and contains over 100 laboratories and offices. The south wing is $22.6 \mathrm{~m} \times 40.5 \mathrm{~m}$ (74 ft $\times 133 \mathrm{ft}$ ) on two floors and contains offices, a conference room, a machine shop, a lunch room, and rest rooms. The east wing (325-A), housing the shielded process research hot cells, is $14.6 \mathrm{~m} \times 39.6 \mathrm{~m}$ (48 ft $\times 130 \mathrm{ft}$ ), and the west wing (325-B), the shielded analytical facility, is $16.2 \mathrm{~m} \times 16.5 \mathrm{~m}$ (53 ft $\times 54 \mathrm{ft}$ ).

A breakdown of the current space usage of the building is es follows:

\begin{tabular}{lrr} 
Laboratories & $4,314 \mathrm{~m}^{2}$ & $\left(46,415 \mathrm{ft}^{2}\right)$ \\
Offices & $1,312 \mathrm{~m}^{2}$ & $\left(14,114 \mathrm{ft}^{2}\right)$ \\
Shops & $171 \mathrm{~m}^{2}$ & $\left(1,840 \mathrm{ft}^{2}\right)$ \\
Storage & $103 \mathrm{~m}^{2}$ & $\left(1,113 \mathrm{ft}^{2}\right)$ \\
Common & $6,561 \mathrm{~m}^{2}$ & $\left(70,593 \mathrm{ft}^{2}\right)$ \\
Light Industrial & $931 \mathrm{~m}^{2}$ & $\left(10,017 \mathrm{ft}^{2}\right)$ \\
\hline \multicolumn{1}{c}{ Total } & $13,392 \mathrm{~m}^{2}$ & $\left(144,092 \mathrm{ft}^{2}\right)$
\end{tabular}

Standard laboratories are multiples of $3 \mathrm{~m} \times 3.7 \mathrm{~m}$ (10 ft $\times 12 \mathrm{ft}$ ) bays. Steel, freestanding, Formica -topped laboratory benches are arranged along the walls with peninsulas in large laboratories. Many of the laboratories have aluminum downdraft hoods, with round corner construction, coated with chemical resistant paint. The hoods have heavy stainless steel tray bottoms and steel bases capable of supporting 3 tons. Besides the hoods, many stainless steel gloveboxes are provided for work with moderate quantities of alpha emitters. The gloveboxes have individual high efficiency particulate air (HEPA) filters located at the exhaust port. Hoods exhaust through HEPA filters located in boxes in the exhaust ductwork system (stainless steel or polyvinyl chloride, depending on the service), then via the exhaust fans of the general ventilation system to the stack.

The 325-A High Level Radiochemistry Facility (HLRF) has three hot cells, shielded with 1.2-m (4 ft-thick high density concrete walls. The cells are $4.6 \mathrm{~m}(15 \mathrm{ft}$ ) high by $2.1 \mathrm{~m}(7 \mathrm{ft})$ deep inside dimensions; two are $1.8 \mathrm{~m}(6 \mathrm{ft}$ ) wide, and one is $4.6 \mathrm{~m}(15 \mathrm{ft}$ ) wide.

There is an underground vault for storage tanks (capacities: $1,040 \mathrm{~L}$ to $68,130 \mathrm{~L}$ [275 to 18,000 gal]) adjacent to the HLRF. Remote operation of the cell equipment is

\footnotetext{
'Trademark of Formica Corporation.
} 
performed in a "front face" operating gallery; movement of materials in the cells takes place in a rear face gallery. The cells are ventilated by air drawn from the rear face gallery and exhausted through HEPA filters to the general ventilation exhaust. Figure 2-4 is a cut-a-way view of the 325-A High Level Radiochemistry Facility.

The 325-B Shielded Analytical Laboratory (SAL) has six compartments, each about $0.5 \mathrm{~m}(5.5 \mathrm{ft})$ square, enclosed in shield walls either of $66 \mathrm{~cm}(26-\mathrm{in})$ magnetite concrete or $30.5 \mathrm{~cm}$ (12-in) Meehanite iron. Four-inch lead shielding divides the compartments into three groups of two. The compartments are served by front face and rear face galleries as above. The cells are ventilated by air drawn in from the rear face, exhausted through two HEPA filter banks in series and an activated charcoal absorber, and finally released to a separate $13 \mathrm{~m}$ $(42.5-\mathrm{ft})$ tall stack. There are three exhaust fans, two normally operating and one on standby.

Figure 2-5 shows the basement which underlies the main building and the 325-A and 325-B wings. The basement includes rooms numbered 2 through 98 . Rooms 40 and $40 A$, $B$, and $C$ are in 325-A; rooms 23 and 32 are in 325-B.

The first floor layout is shown in Figure 2-6. The portion labeled 325-C is the central part of the building. Hot cells are shown in the East (325-A) and West (325-B) wings. The electrical machine shop and instrument shop, 325-D, is shown in the southwest corner of the building. The 325-C fluorine gas storage enclosure is located just outside the building in the southeast quadrant. First floor rooms are numbered 101 through 604.

The second floor plan is shown in Figure 2-7. Four rooms are located in the 325-A wing: 606, 607, 609 and 610. The second floor rooms, located in the central part of the building, are numbered 910 through 971 . This floor also includes an equipment room, power operating room, and change room.

Also shown in Figure 2-7 is the third floor, which extends only above the 325-A wing. The top of the A-wing cells and the Aqueous Makeup (AMU) area, which is labeled room 611, are located on this floor.

\subsection{FACILITY MISSIONS}

Over the years, a wide variety of chemical and physical analyses have been performed in the 325 Building. A listing of some of the missions and techniques used over the years is presented below.

Original Missions. Initial building missions included production support and process improvement for the REDOX separations process, improvements for a uranium metal recovery process (especially in handling wastes and supernatants), and studies of separations waste treatment aimed at developing techniques to reduce activity levels in high level wastes. Additional early missions included production development for radioactive lanthanum, temporary technical support for the bismuth phosphate separations process, support studies for tritium production, and basic investigations of plutonium chemistry. The 325 Building was also used to support development of the PUREX, RECUPLEX, and PRF processes during its first decade of operation, 1953-1963 (Gerber 1992). 
Isotope Campaigns. The high level radiochemistry addition (325-A) was completed in time to support work done in the 1960's to separate isotopes from high level waste. These isotopes were produced for medical use and for use in the space program. Methods employed to separate specific isotopes in the waste included ion exchange, carrier precipitation, and solvent extraction, among others. Strontium-90, cesium-137, curium-244, americium-241, and promethium-147 were the isotopes in highest demand. In fact, Hanford was the only supplier in the world of promethium-147, which was used in the development of the artificial heart.

Promethium-147, strontium-90, americium-241 and curium-244 were all purified in the A-cell (325-A wing), while the cesium and strontium separations process used in B-Plant encapsulation work was developed in the 325 Building's B-Cell (325-A wing). Strontium fluoride and cesium chloride encapsulation chemistry for the B Plant mission also was developed in B-Cell. In the same period, compatibility studies for strontium fluoride capsule development experiments in plutonium recovery from special irradiated neptunium-237/aluminum target material were performed in C-Cell (also 325-A wing) (Gerber 1992).

Waste Vitrification and Other Projects. From 1962-1964, A-Cell (325-A wing) was used to support waste solidification and vitrification tests, including calcining and glass melting. Studies that were part of a large vitrification project took place in the A, B, and $C$ cells from 1977 to 1980 . Other projects in this period included high level waste behavior and fuel dissolution tests performed in B-cell. At the same time, demonstrations of molten salt electrodeposition of uranium oxide and plutonium oxide were conducted in C-cell, as were solvent degradation tests used as part of FFTF development.

The vitrification mission was placed on standby by the DOE in 1980. Because budget authorizations did not provide for cell and equipment decontamination and removal, space in the A, B, and C cells in 325-A has been partially occupied with contarninated material ever since. Since 1980, the remaining available cell space has been used for materials characterization leach tests of waste glass and of spent nuclear fuel, post irradiation examination of the boron thermal shield from $\mathrm{N}$ Reactor, and characterization of neutralized cladding removal waste (along with the testing of a potential method of converting it to non-TRU wastel. Other radiochemical work conducted in these cells has included characterization of double-shell tank slurry, tests of fuel iodine control and fuel uranium dissolution methods for $\mathbf{N}$ Reactor, and experiments in the recovery of strontium using antimonic acid (Gerber 1992).

Liquid Metal Fast Breeder Reactor Program. During the 1970's much of the work performed by Westinghouse Hanford in the 325 Building was done in support of the LMFBR Program, especially the FFTF. Activities under the LMFBR program included characterization studies of mixed-oxide fuel, fuel cladding, absorber, and sodium metal coolant. 


\subsection{FACILITY HISTORY}

Over the past 40 years the 325 Building has been occupied by several different contractors working on a wide variety of projects. This has resulted in numerous changes to the physical plant. This section first discusses the physical changes, followed by a review of the activities performed in the 325 Building. Finally, there is a brief section outlining contractor occupancy and custody changes between 1953 and 1993.

\subsubsection{Physical Changes}

A review of an index of project documentation shows many changes to the building and its laboratories. Topics and dates are summarized below.

\begin{tabular}{|c|c|c|}
\hline Date & Contractor & Iopic \\
\hline 1951 & $\cdot$ & Specifications for 325 Radiochemistry Building, Project C381. \\
\hline 1960 & GE & $\begin{array}{l}\text { Specifications for 325-B Shielded Analytical Lab Project } \\
\text { CAH } 866 \text {. }\end{array}$ \\
\hline 1960 & - & 352-A, High Level Radiochemistry Annex completed. \\
\hline 1970 & BNWL & $\begin{array}{l}\text { Modifications, rooms } 411 \text { and } 415,404,701,515,416 \text {, } \\
325 \text {-A basement, } 54,504,506 \text {. }\end{array}$ \\
\hline 1970 & WADCO & $\begin{array}{l}\text { Modifications, rooms } 971,945,947,55,94,94 A, 417 \text {, } \\
419,414,52,510,22,527,520,312 \text {. }\end{array}$ \\
\hline 1971 & WADCO & $\begin{array}{l}\text { Modifications, rooms } 48,101,102,117,118,55,522 \text {, } \\
325 \text { basement, } 972,109,504,525 .\end{array}$ \\
\hline 1972 & WADCO & $\begin{array}{l}\text { Modifications, rooms 409, 972, sodium glove box, 605, 109, } \\
422,530 A, 528,409 A, 401,22,316,325,326,330,421, \\
425,700,539,504,309,529,400,206,325 B \text { hot cell. }\end{array}$ \\
\hline 1973 & WHC & $\begin{array}{l}\text { Rm 313: remove old style filter box and replace, hood } 1 \text {. } \\
\text { Modifications, room } 307 \text {, and fry pans } A, B \text { and } C \text { Cell in the } \\
\text { high level radiochemistry facility. }\end{array}$ \\
\hline 1974 & WHC & $\begin{array}{l}\text { Modifications, rooms } 505 A, 94,95,96 A, 97 A, 316,52,56 \text {, } \\
24,54,950,22,30,318,524,313,702,12,32,24,5 .\end{array}$ \\
\hline 1975 & - & $\begin{array}{l}\text { Major modifications to the shielded analytical complex in the } \\
\text { 325-B Building. }\end{array}$ \\
\hline 1975 & WHC & $\begin{array}{l}\text { Hood/equipment relocation. Modifications, rooms } 511 \text {, } \\
\text { mezzanine, 524A, 52, 48, 12, 21, 21A, } 401,950 \text {. }\end{array}$ \\
\hline 1976 & WHC & $\begin{array}{l}\text { Modifications, rooms } 971 \text {, storage cage partitions in } \\
\text { basement, } 40,327 \mathrm{~A}, 327 \mathrm{~B}, 58,50,50 \mathrm{~A} \text {. }\end{array}$ \\
\hline 1977 & - & $\begin{array}{l}\text { Final exhaust ventilation HEPA filters, Hand dig vitrification } \\
\text { project utilities. }\end{array}$ \\
\hline
\end{tabular}




\begin{tabular}{|c|c|c|}
\hline Date & Contractor & Iopic \\
\hline 1977 & WHC & $\begin{array}{l}\text { Modifications, rooms 110,603, 21A, 30, 524A, basement, } \\
\text { Air } 325 \text { bldg, dock, 54, 327B, 501, 93. }\end{array}$ \\
\hline 1977 & BNWL & Modifications room 422, upper C-Cell liner. \\
\hline 1978 & WHC & Install mini hot cell in room 23. \\
\hline 1978 & BNWL & 325-A Security modifications. Modifications, room 119. \\
\hline 1979 & WHC & $\begin{array}{l}\text { Replace corroded HVAC ducts. Modifications, rooms } 308 \text {, } \\
110,110 A, 525,970,23,102,103,48 \text {, }\end{array}$ \\
\hline 1979 & BNWL & $\begin{array}{l}\text { Modifications, room } 504 \text {, add HVAC to } 325-A \text {, Remove } \\
15 \text { ton crane, replace with } 30 \text { ton crane. }\end{array}$ \\
\hline 1980 & - & Install additional criticality howlers. \\
\hline 1980 & WHC & $\begin{array}{l}\text { Modifications, rooms } 200,209,91,312,313,504 \text {, kitchen, } \\
58,90,326 \text {. }\end{array}$ \\
\hline 1981 & WHC & $\begin{array}{l}\text { Modifications, rooms } 303,23,(505,517,510,506 \text {, and } \\
601 \text { joint with BNWL), 405A, 23B, men's change room, 30A, } \\
400,404 \mathrm{~A} \text {. }\end{array}$ \\
\hline 1982 & $\cdot$ & $\begin{array}{l}\text { Utility changes, temporary modification for high voltage } \\
\text { power supply. }\end{array}$ \\
\hline 1982 & WHC & Modifications, rooms 40, 50A, 23, 500, 50 \\
\hline 1983 & - & Relocated radioactive liquid waste line. \\
\hline 1983 & WHC & Modifications, rooms 209, 200, 306, 400, 110, 202, 200. \\
\hline 1984 & WHC & Modifications, rooms $32,202,530 \mathrm{~A}$. \\
\hline 1985 & WHC & Modifications, rooms 55A, 111A, 112, 103A, 416 . \\
\hline 1986 & WHC & $\begin{array}{l}\text { Modifications, rooms } 430,91,111 \mathrm{~A}, 409,303,506,422, \\
500,524,(521,525, \text { PNL related), 504, } 603 .\end{array}$ \\
\hline 1987 & WHC & Modifications, rooms 520, 32 . \\
\hline
\end{tabular}

\subsubsection{Activity Changes}

The 325 Building activities have changed considerable with time. As described in facility Safety Analysis Reports (McCormack 1969, Anderson and Hanthorn 1975 and 1977). operations can best be described by dividing the building into several working facilities. The following sections address changes in the operations in each facility over time. Many of the dates used in the following sections correspond to the dates that the source safety analysis documents were published. 
The 325 Building houses laboratories and specialized facilities for activities ranging from work with nonradioactive materials to work with gram to kilogram quantities of fissionable materials and up to megacurie quantities of other radionuclides. The building is divided into several working facilities, which are addressed below.

\section{Genoral Purpose Chemical Laboratories}

In 1969 the general purpose laboratories were located on the main floor and in the basement and were used for research, development and analytical operations requiring little or no radiation shielding. Most labs were equipped with hoods and many with gloveboxes. Studies of actinide elements, the chemistry of fission products and other radionuclides, nonradioactive organic and physical chemistry operations, and analytical services of all types were conducted in the general purpose laboratories.

In 1975-1977 the general purpose laboratories were located on the main floor but also included Room 52 in the basement. The chemical, radiochemical and microstructural laboratories consisted of 55 rooms with a combined area of approximately $1,860 \mathrm{~m}^{2}$ $\left(20,000 \mathrm{ft}^{2}\right)$. Installed facilities included radiochemistry hoods, gloveboxes for transuranic materials, inert atmosphere gloveboxes, and laminar flow hoods for the analysis of metallic sodium. These laboratories were required for characterization studies of fuel, absorber, sodium metal coolant, and other materials for quality assurance and for the evaluation of developmental tests.

Three laboratories, Rooms 52, 312 and 318 in the 325 Building, were used for the analysis of metallic sodium. Analytical samples normally contained between one and $100 \mathrm{~g}$ of sodium.

Room 52 is a $9.1 \mathrm{~m} \times 9.1 \mathrm{~m}$ ( $30 \mathrm{ft} \times 30 \mathrm{ft}$ ) laboratory in the basement of the building. Sodium handling was carried out in a $0.9 \mathrm{~m} \times 2.4 \mathrm{~m}$ ( $3 \mathrm{ft} \times 8 \mathrm{ft}$ ) inert atmosphere glovebox. No radioactive materials were permitted or handled in the room.

Rooms 312 and 318 are both standard $6.1 \mathrm{~m} \times 7.3 \mathrm{~m}(20 \mathrm{ft} \times 24 \mathrm{ft}$ ) laboratories on the main floor of the building; each contained two inert atmosphere gloveboxes for the handling of sodium. Radioactive materials were not normally handled in these laboratories. Occasionally sodium samples that had been in contact with either activated corrosion products or fission products were received and analyzed. Maximum amounts of radioactive material were under 10 millicuries. When beta-gamma contaminated samples were handled, a temporary radiation zone was established, and a Radiation Work Permit prepared. Alphacontaminated materials were not permitted in these laboratories.

Between 1975 and 1977, the description of sodium handling became more detailed. In 1975, the sodium inventory was limited to 100 grams or under in a single piece. In 1977 , the sodium inventory was limited to less than $4.5 \mathrm{~kg}(10 \mathrm{lb})$ per lab and 100 grams or less in any single container, except for reservoirs of up to $3.6 \mathrm{~kg}(8 \mathrm{lbs})$ in inert atmosphere. In 1977 all other laboratories also had a limit on reagent alkali metals of $.9 \mathrm{~kg}(2 \mathrm{lb})$ per lab with additional amounts requiring special evaluation.

These laboratories are currently performing the operations described in Section 2.5. 


\section{Shlolded Analytical Laboratory (325-B Annex)}

The 325-B Annex contains six interconnecting compartments (cells) inside thick shielding walls equipped with lead glass viewing windows. Operations are conducted using manipulators or other remote equipment.

In 1969, the 325-B cells were used for examining small amounts of radioactive materials and for preparing high-activity samples for analysis, as well as for carrying out several routine analytical determinations.

In 1975 and 1977, the cells continued to be used for analytical chemistry operations on small amounts of highly radioactive materials, such as irradiated mixed-oxide fuels and fuel cladding samples. Typical operations included analytical weighing, sample dissolution, sample dilution and aliquoting, emission spectrographic analysis, titrimetric analyses, solvent extraction, and ion exchange separations.

\section{High Level Radiochemistry Facillty (325-A Annax)}

The 325-A Annex contains three interconnected multi-curie cells, which are identified as A, B, and C. The cells are heavily shielded and equipped with lead glass windows. Auxiliary to the cells are vaults containing tanks for radioactive solution storage; facilities for handling shielded, large volume shipping casks; and solution transfer facilities.

A-Cell is equipped with ion exchange columns and auxiliary equipment for the separation and purification of large quantities of various fission products. It was used routinely to produce multi-kilocurie quantities of promethium-147 but was also used for isolating and purifying americium and curium. Processing included the use of ion exchange resins and complexing agents.

B-Cell was used for miscellaneous, short term chemical studies involving small amounts of highly radioactive materials.

C-Cell is equipped for 'separating and purifying 100-g quantities of plutonium-238 and neptunium-237 from experimental irradiated fuel elements. Processing included the use of mercury-catalyzed nitric acid, caustic solutions, and ion exchange resins.

In 1978, A-Cell and C-Cell were cleaned out, renovated, and re-equipped to process light-water reactor fuel as part of the NWVP. The equipment included dissolvers, feed preparation tanks, a battery of four pulse columns, a concentrator, ion-exchange columns for the recovery of plutonium, and supporting tanks and instrumentation. The equipment extracted the fission product waste and transferred it to the 324 Building for conversion to borosilicate glass. The project was shut down in 1980, but no funds were provided for cleanup. Some unused equipment still remains. Space has been made in front of each window to conduct other research. Currently, that space is used for the tank sludge characterization program. B-Cell was not used during the NWVP program and currently contains a core extruder and analytical measuring equipment used for the sludge characterization program. 


\section{Shielded Clove Boxes}

In 1969, Room 604 contained two shielded glove-boxes used for converting pure promethlum-147 solution to the dry oxide in multicurie amounts and for conversion to promethium metal. Hundreds of grams of pure promethium oxide powder, averaging about $750 \mathrm{Cl} / 9$, were stored in sealed stainless steel containers in these glove boxes.

\section{Fiealle Matorial Storage Room}

Since 1969, Room 530 has been used for storing fissile material. Storage of material is controlled by a criticality safety specification.

\section{Basement and Mezzanine}

In 1969, the "Fuels and Materials Department Facility" occupled several thousand square feet of the 325 Building basement and mezzenine. It included storage cages for enriched uranium; laboratories for fuel element assembly, high temperature research, thoria preparation, uranium dioxide preparation, and nuclear poisons handling; a high temperature research facility; and a welding area and machine shop. Experimental fuels and fuel elements were fabricated in these laboratories, but more active alpha emitters, such as plutonium, were not allowed in the facility. High-temperature research studies were conducted occasionally upon encapsulated radioactive heat sources.

In 1975, the mezzanine area provided space for offices and a few laboratories equipped with special laboratory apparatus for nonradioactive work. The north end of the basement area contained several analytical laboratories. The south end of the basement contained a laboratory area, Room 30, wherein pressed and sintered $\mathrm{UO}_{2}$ pellets were ground and finished to size. This work was done on $\mathrm{UO}_{2}$ pellets with enrichments varying from normal to fully enriched. A maximum of about one kilogram of material could be processed in one day depending on enrichment. Some work was also done on the blending, pressing, and binding operations with normal or depleted $\mathrm{UO}_{2}$ or with normal $\mathrm{UO}_{2}$ with zirconia added.

Currently, the basement contains approximately 25 laboratories for low-level radioactive work. There is one miniature, all-metal hot cell in Room 23 of the basement. The basement also houses the primary high efficiency particulate air (HEPA) filters (for all for the building's hoods, gloveboxes, room exhaust vents, etc.), the exhaust ductwork for the building, the normal and standby electrical power switchgear and panels, the laboratory vacuum and airsampling vacuum pumps, the backup air compressor, the sanitary and process water piping, and other required building services.

\subsubsection{Custody Changes}

The 325 Building has been operated by several contractors. From 1953 to 1960, the operator was General Electric (GE); later operators were Westinghouse Hanford and Battelle's Pacific Northwest Laboratories (PNL). Westinghouse Hanford Company has also been called 


\title{
WHC-EP-0696
}

"HEDL" for Hanford Engineering Development Laboratory and "WADCO". Battelle Pacific Northwest Laboratories is also known as "BNWL" and has been both the operator of the building and a tenant. Custody changes are described below:

Date

Contractor

\section{3-1965 General Electric}

1968

1970

1987

\author{
BNWL (Battelle)
}

Joint occupation by WADCO (Westinghouse) \& BNWL (Battello). WADCO was the managing contractor. WADCO occupied $2880 \mathrm{~m}^{2}$ $\left(31,000 \mathrm{ft}^{2}\right)$ of the building; BNWL occupied $4560 \mathrm{~m}^{2}\left(49,000 \mathrm{ft}^{2}\right)$.

PNL (Battelle) assumed landlord responsibilities; WHC retained 41 rooms. WHC space includes:

Rooms 941, 942, 943, 944, 945, 946, $36\left(\mathrm{UO}_{2} \mathrm{Cage}\right)$ for Safoguards \& Security;

Room 108 for Quality Assurance;

Room 522, 524A, 528, $529 \& 530$ A for Waste Operations;

Rooms 2, 3, 11, 12A, 12B, 12C, 13, 14, 15, 16, 17, 1819 , 19A, 21, 21A, 226, 27, 28, 29, 43, 43A, 57, 58, 97, 97A, 105, 420, \& Welding Development Laboratory for Engineering Development.

\subsection{CURRENT OPERATIONS}

Because the 325 Building is a research facility, the work conducted in the building is constantly changing according to programmatic needs. Typically, from 20 to 40 projects are being conducted simultaneously in the building. Work recently performed, currently underway, or planned includes process research, development and demonstration; analytical chemistry research and services; and treatment of radioactive, hazardous, or mixed hazardous wastes. This section describes typical work in the building, although the description is not intended to be comprehensive.

Work is divided among the two hot cell complexes, gloveboxes, fume hoods, and laboratory benches, depending on the radioactive or hazardous nature of the work. Projects frequently involve working in more than one of these locations, such as sample preparation or dilution in a hot cell or glovebox, followed by analytical measurements in a fume hood. Analytical services are provided for all projects in the 325 Building as well as services for outside customers.

General chemical laboratories are typically equipped with work space on laboratory benches and fume hoods and often include gloveboxes or large equipment such as 
spectrometers. Some laboratories are devoted exclusively to housing specialized instruments such as spectrometers. These laboratories do not contain any fume hoods and have few or no laboratory benches. Laboratories also may be devoted exclusively or almost exclusively to housing gloveboxes. The arrangement of laboratories and equipment in laboratories is constantly changing as some projects are completed and others are started or as project needs change.

Some typical work in the 325 Building is briefly described below. Most of this work is in progress, and the projects selected for description represent a wide range of activities.

- Development and demonstrate the Catalyzed Electrochemical Plutonium Oxide Dissolution (CEPOD) process for processing plutonium-rich materials for plutonium recovery of recycle operations and for processing plutonium-lean scrap or waste to convert TRU waste to non-TRU waste.

- Characterize chemical, radiochemical, and physical properties of wastes from double-shell waste tanks with particular emphasis on waste stream suitability for processing at the Hanford Waste Vitrification Plant (HWVP). Similarly, characterize waste from single-shell tanks. Treat some of the wastes and evaluate the effects of the treatment.

- Provide a treatment service for hazardous waste or mixed (hazardous and radioactive) waste generated by PNL laboratory activities. Include grouting, neutralization, and distillation, and perhaps demonstrate new and emerging technologies for chemical waste treatment and destruction.

- Provide analytical chemistry support for fuels-related programs such as the Materials Characterization Center and tuff repository programs.

- Provide, on an as-requested basis, RCRA and Comprehensive Environmental Response, Compensation, and Liability Act (CERCLA) analyses of liquids and solids associated with the Hanford Site characterization and remediation effort and with plant operations.

- Analyze performance evaluation samples submitted by the EPA, the Environmental Monitoring Laboratory, and other organizations as a routine part of the laboratory quality control program.

- Develop flowsheets for processing selected Hanford wastes.

- Develop and test flowsheets for the removal of transuranics and other selected radionuclides from dissolved fuel from light-water reactors in support of a process being developed by Westinghouse Hanford.

- Determine the potential for rapid exothermic reactions between ferrocyanide and nitrate or nitrite in waste from single-shell tanks.

- Evaluate ion-exchange resins for the recovery of selected radionuclides from vitrification waste produced by the HWVP. 
- Evaluate the performance of a resorcinol/iormaldehyde ion-exchange resin for removal of cesium-137 from Savannah River alkaline waste.

- Develop and demonstrate a process for dissolving highly radioactive europium. oxide pellets in acetic acid, separating gadolinium-153 from the europium, and preparing the recovered gadolinium for shipment to Oak Ridge National Laboratory or return to the FFTF.

- Development and demonatrate a process for preparing waste sludges from West Valley Nuclear Services Company for vitrification feeds. Develop processes for secondary treatment of wash or supernatant liquids to reduce level of radioactivity to as low as reasonably achievable.

- Store natural or depleted uranium and thorium as a service for Westinghouse Hanford.

- Perform nonradioactive research work on waste volume reduction and compaction.

- Prepare samples for x-ray diffraction, scanning electron microscope, or Auger analysis; analyze samples. Include samples of oxidized and nonoxidized spent fuel from light-water reactors and fission product deposits from fuel rods.

- Prepare standard solutions of radionuclides from stock batches for use in research and development of analytical procedures and for quality control.

- Investigate tritium release from tritium-containing materials such as beryllium and lithium oxide. Examine releases by both carrier gas extraction and vacuum extraction.

- Perform chemical and physical experiments involving sodium and lithium metals. Provide chemical analyses of these materials including FFTF primary sodium.

- Prepare organic standards and investigate organic extractions. (Work may involve gallon quantities of solvents such as acetone, hexane, toluene, isooctane, carbon tetrachloride, freon 112, and methylene chloride).

- Perform autoclave tests up to and including 5,000 psi on rock samples with various waste environments to simulate geologic conditions pertinent to the Yucca Mountain repository project.

- Investigate oxidation kinetics of spent light-water reactor fuel.

- Study tritium permeation through reactor target rods.

- Separate and process medically usable radionuclides. 
WHC-EP-0696

Figure 2-1. Location of the Hanford Site.

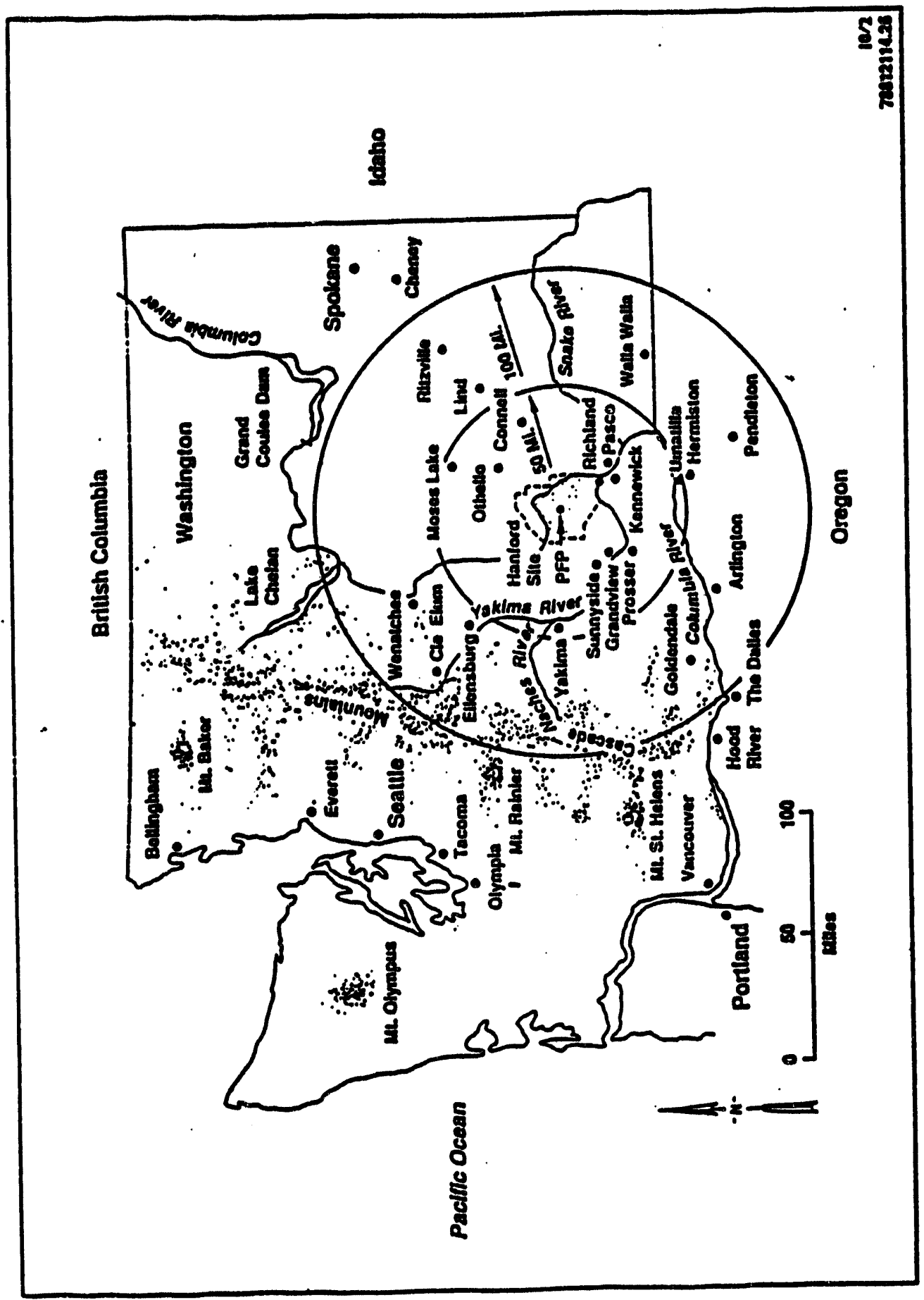


Figure 2-2. Hanford Site Map.

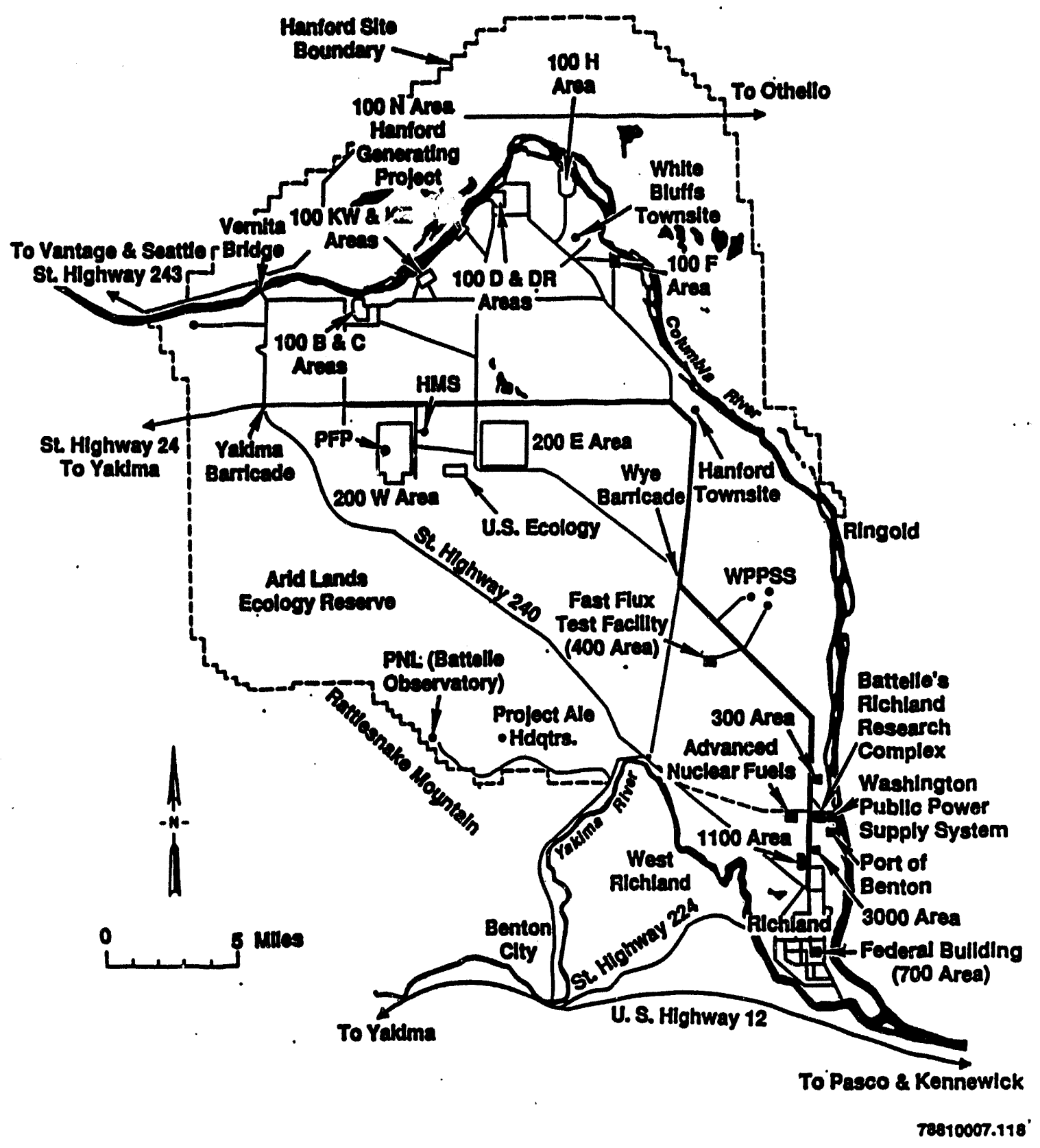


WHC-EP-0696

Figure 2.3. Photograph of the 325 Building.

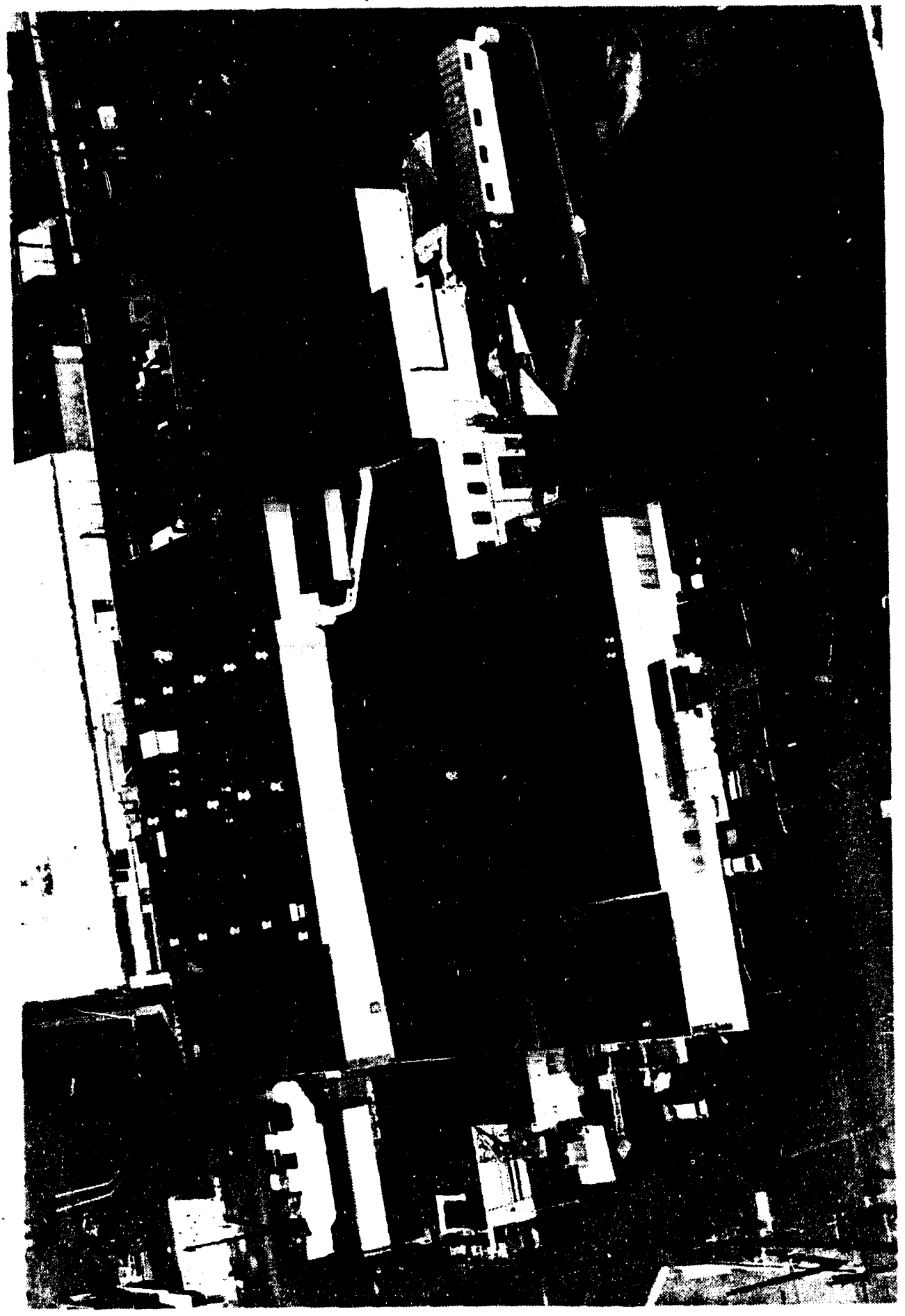




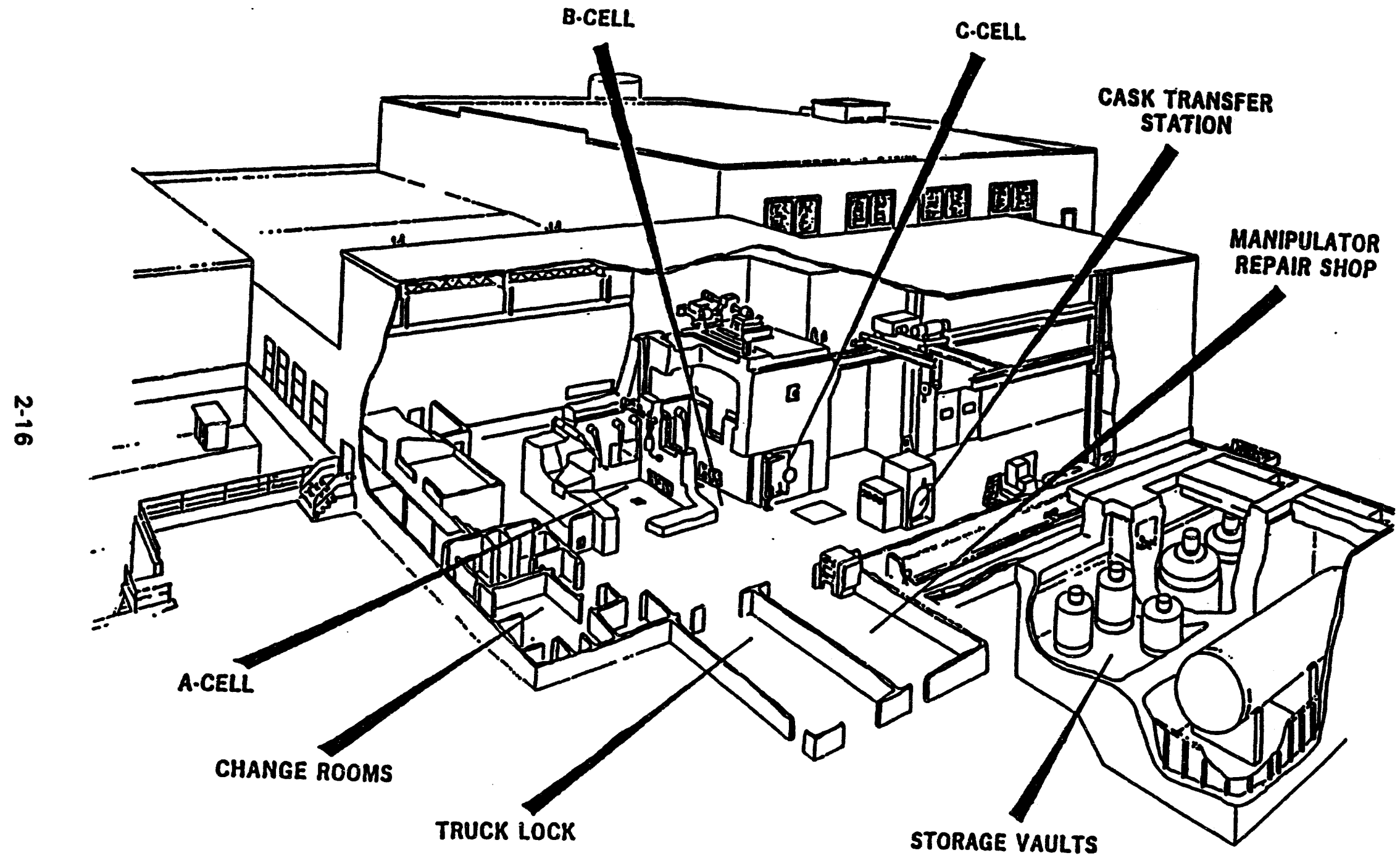

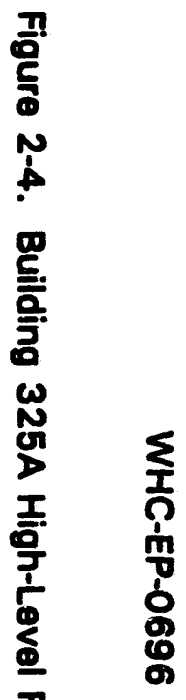

STORAGE VAULTS 
Figure 2-5. 325 Building Basement Layout.

(not to scale)

4
$N$

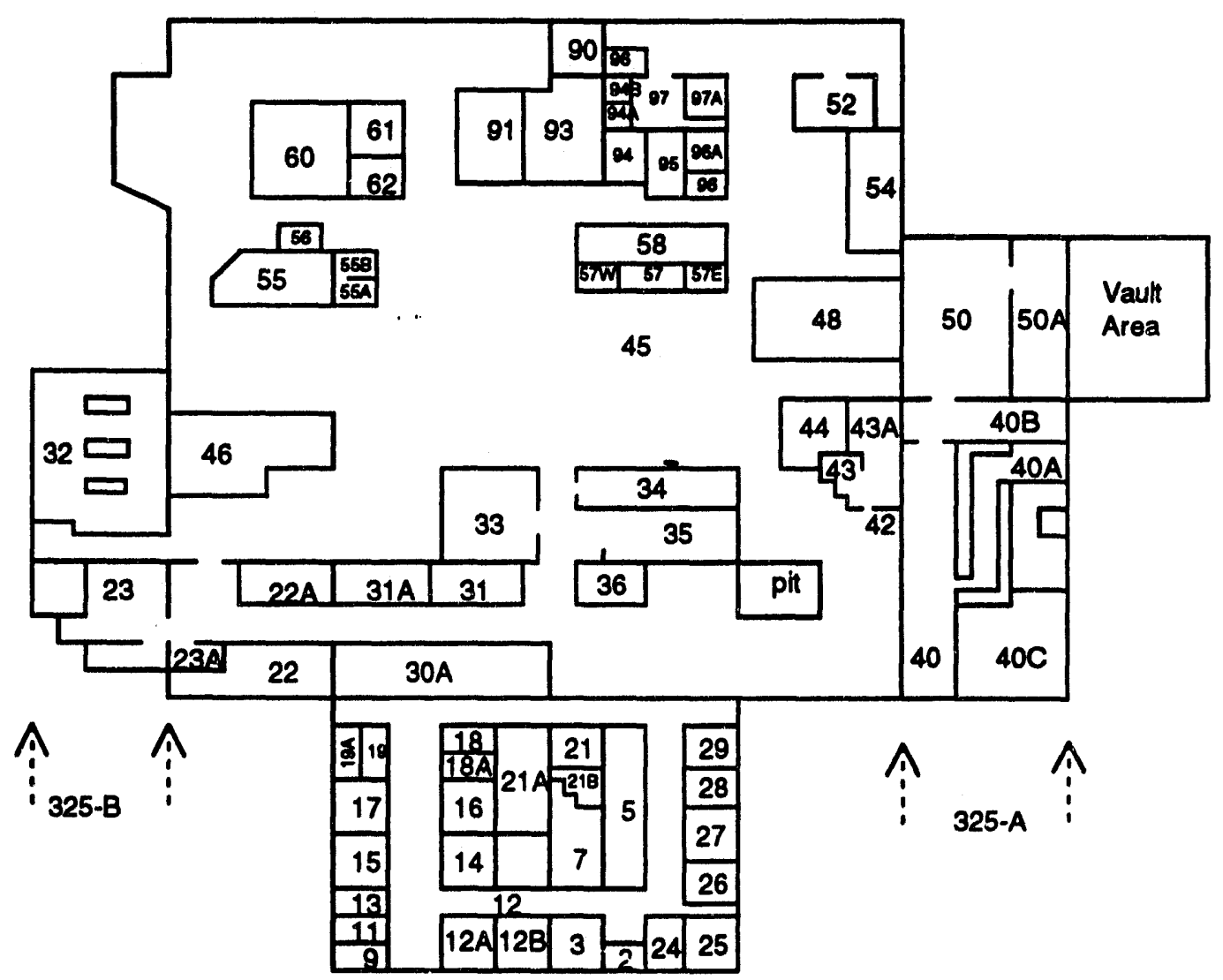


Figure 2-6. 325 Building First Floor Layout. (not to scale)

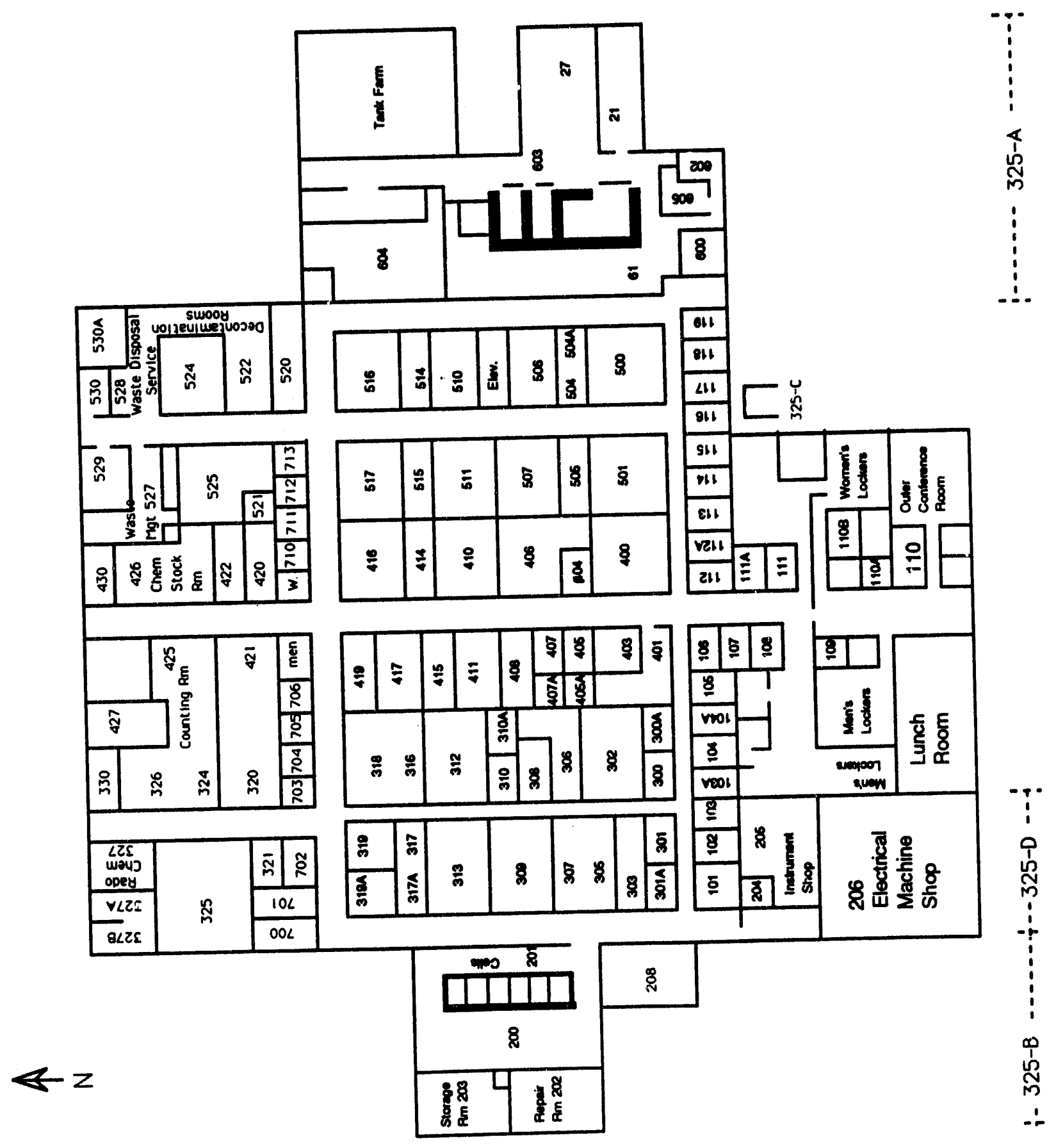


WHC-EP-0696

Figure 2-7. Layout of the 325 Building Second and Third Floors.

$A$
$N$
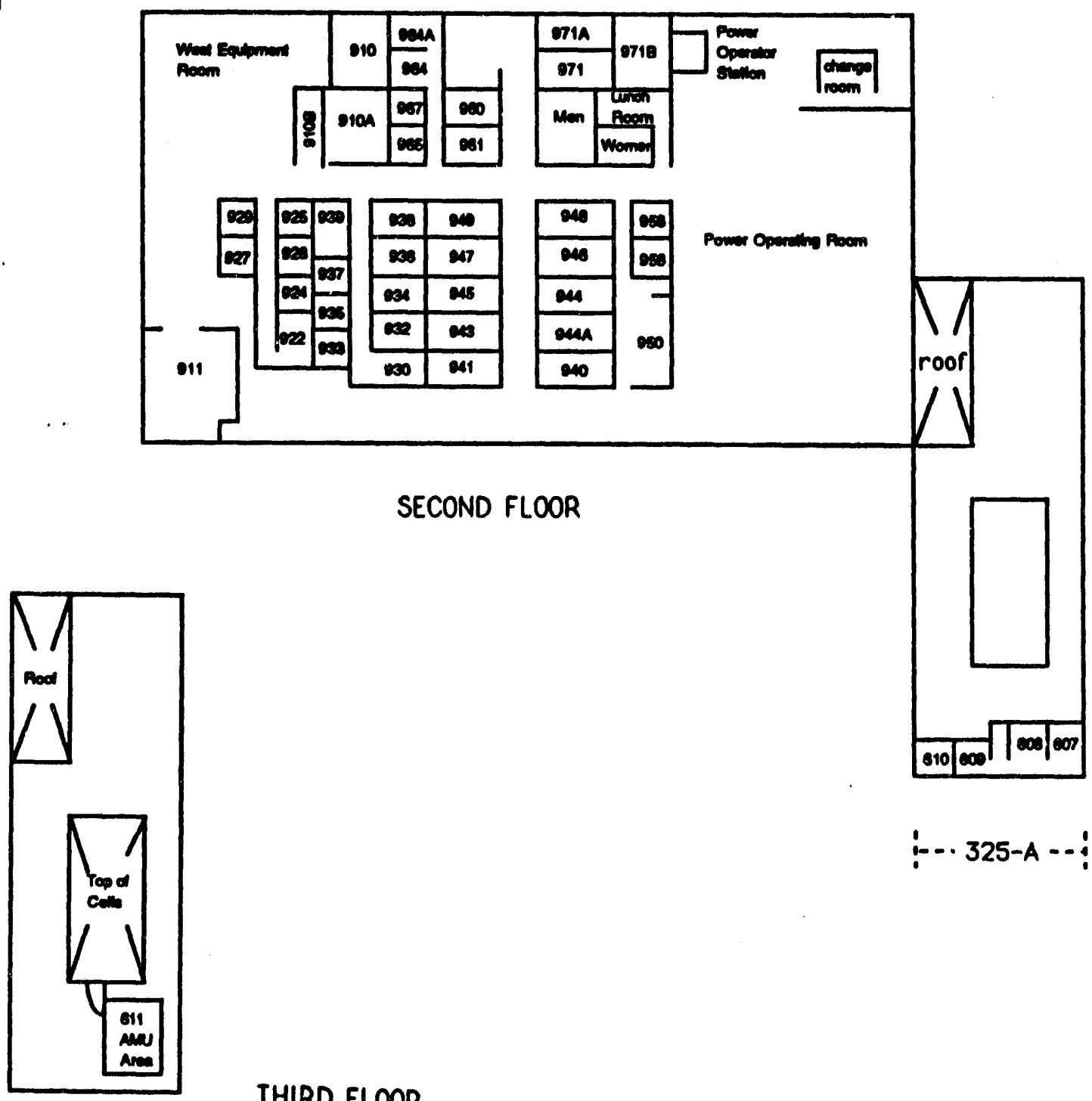

THIRD FLOOR

:-. 325-A - - : 
WHC-EP-0696

This page intentionally left blank. 
WHC-EP-0696

\subsection{WASTE GENERATION}

The wastes generated at the 325 Building can be segregated into radiological and nonradiological wastes in gaseous, liquid, and solid forms.

\subsection{GASEOUS WASTES}

The 325 Building ventilation system was designed to assist in the control of radioactive contamination by maintaining pressure differentials between zones. Air flow is always from zones of lower contamination potential to zones of higher potential (Anderson and Hanthorn 1977). Gaseous and particulate wastes are exhausted through cell, hood, and stack filters. These wastes have included iodine-131 and -132, cerium-144, ruthenium-103 and -106 , strontium-90, and yttrium-90.

All gloveboxes are equipped with small high efficiency particulate air (HEPA) filters near the glovebox, in series with the larger HEPA filter further downstream. The main stack and the 325-B Shielded Analytical Laboratory (SAL) stack are equipped with continuous, calibrated, alarming stack monitors that respond to alpha, particulate beta, and iodine-131 radiations. An alarming monitor, the same as the stack monitors, samples the exhaust from the 325-A cells. This monitor serves to give an advance warning of emissions from this megacurie facility (Anderson and Hanthorn 1977).

\subsection{LIQUID WASTES}

Currently, the liquid effluents generated by the 325 Building are handled by one of three drainage systems: the sanitary sewer, the retention process sewer, and the radioactive liquid waste system (RLWS).

The sanitary sewer handles liquid waste from drinking fountains, the lunchroom, lavatory areas, service, and other similar areas. This waste stream is essentially free of radioactivity and is discharged into the $\mathbf{3 0 0}$ Area sanitary sewer system.

Cooling water, sink wastes, floor drains, and laboratory chemical waste water systems that might accidentally contain radioactivity are discharged through the retention process sewer system. This sewer is routed through a diversion station in the 325 Building basement that is equipped with a radioactivity monitor. In the event that the stream contains radioactivity above a present level, the flow is diverted to the 300 Area radioactive liquid waste system. Until 1975, uncontaminated liquid chemical wastes were piped to the 300 Area process ponds.

The RLWS within the 325 Building consists of three sections. Radioactive liquid wastes from these sections exits the 325 Building at two locations to join the 300 Area RLWS. The waste is then routed to the 340 Building.

Specific routing of radioactive liquid wastes from the three sections of the 325 Building is outlined below. 


\begin{tabular}{|c|c|}
\hline Section & $\begin{array}{l}\text { Specific } \\
\text { Source(s) }\end{array}$ \\
\hline $\begin{array}{l}\text { Central Portion } \\
\text { Building (Bldg) } 325\end{array}$ & $\begin{array}{l}\text { Hood, Room } 528 \\
\text { Floor Drain (Plugged), } \\
\text { Room } 529\end{array}$ \\
\hline $\begin{array}{l}\text { Bldg 325B } \\
\text { (West Wing) }\end{array}$ & cells and 2 sinks \\
\hline \multirow[t]{3}{*}{$\begin{array}{l}\text { Bldg 325A } \\
\text { (East Wing) }\end{array}$} & cells \\
\hline & - $\quad \operatorname{sinks}$ and floor drains \\
\hline & $\begin{array}{l}\text { cooling water from } \\
\text { process tanks, a } \\
\text { glovebox, and cells. }\end{array}$ \\
\hline
\end{tabular}

Waste

Routing

To one of the two waste lines leaving Bldg 325, then to Bldg 340.

To 760-1 holding tank in Room 32, then jetted to RLWS line to Bidg 340.

To Room 40A tanks (4 tanks ranging in size from 3401 to 4001 1), then can be transferred to several other locations in 325A.

To second RLWS line existing the building.

To diverter station in Room 50. Valve diverting flow to RLWS vault tank (WT-1) is locked open. The vault tank is the central collection point for Annex 325A radioactive liquid waste. Transfer from WT-1 is done either by jetting to the RLWS line exiting Annex 325A or by vacuum transfer to a cask in the liquid transfer hood. Shielded casks can be used to transport waste to the 200 Area for disposal.

\subsection{SOLID WASTES}

Historically, several types of dry wastes have been segregated and packaged at the 325 Building. These types are: trash, salvageable scrap, toxic nonradioactive waste, pyrophoric nonradioactive waste, low dose rate radioactive waste, high dose rate radioactive waste, and transuranic waste (Anderson and Hanthorn 1977).

The new 325 Waste Treatment Unit will provide storage (less than 90 days) and has applied for a permit for treatment of hazardous and radioactive mixed wastes. It will provide routine treatment of wastes, research and development, and new treatment processes. Space limitations do not allow extensive waste segregation using physical barriers, but wastes are administratively segregated into low-level, transuranic, and mixed wastes. Waste handled by the 325 Waste Treatment Unit are packaged and shipped to, Westinghouse Hanford the current waste-handling contractor for the Hanford Site. 


\subsection{SOLID WASTE GENERATINO ACTIVITIES}

Because the 325 Facillty has supported 80 many different projects and missions, it is impossible to describe the solid wastes that each of them might have generated. Therefore, in lieu of describing specific projects, some of the analytical procedures commonly performed in the 1970's and 1980's are listed below with brief descriptions. More complete information on each can be found in MG-28, Analytical Chomistry Laboratory Manual, Vols. 2 and 3 (HEDL 1975a and 1975b).

- Sulfur by Combustion and Idometry

Sulfur is determined by igniting the sample between $1600^{\circ} \mathrm{C}$ and $1700^{\circ} \mathrm{C}$ in a stream of oxygen. The sulfur dioxide formed is swept from the combustion chamber into an acid solution containing potassium iodide and the blue lodinestarch complex. The sulfur dioxide, which reduces iodine, is automatically titrated with standard potassium iodate solution using a colorimetric end point monitor.

- Sulfur by Combustion and Turbidimetry

Sulfur is oxidized to sulfur trioxide by combustion of the sample mixed with vanadium pentoxide. The sulfur trioxide is swept from the combustion tube into a trap of dilute caustic, after which hydrogen peroxide is added to ensure that all sulfur is in the $6+$ valence state. The sulfate is precipitated as barium sulfate and the resulting turbidity is measured spectrophotometrically.

- Tungsten by Spectrophotometry

Tungsten(VI) is reduced to tungsten(V) with titanium(III) and the tungsten(V) is extracted from a hot hydrochloric acid solution into a solution of dithiol in amyl acetate. The absorbance of the tungsten-dithiol complex is measured at $640 \mathrm{~nm}$. Molybdenum interference is removed by a preliminary extraction as molybdenum(VI). If tungsten is present as oxide in the samples, it is not completely dissolved by acid digestion. Therefore, the sample solution is filtered and the residue is dissolved by fusion with potassium pyrosulfate.

- Water by Constant Voltage Coulometry

Water is released from the sample by heating. A carrier gas sweeps the water into an electrolytic cell containing phosphorus pentoxide to absorb the water. A potential applied across the cell causes the absorbed water to electrolyzed. The total current is integrated and displayed digitally as micrograms of water.

- Impurities by Emission Spectroscopy: Photographic Method

This spectrographic method is based upon the carrier-distillation technique using carriers of gallium oxide and sodium fluoride. The concentrations of 24 impurity elements are determined by visual comparison of selected-line densities from the sample and standards. 
- Impuritios by Emlssion Spectroscopy: Direct Reader

Impurity elements are determined by a direct reading emission spectrometer using the carrier distillation technique to suppress the complex spectra from uranium and plutonium. A carrier mixture of gallium oxide and strontium fluoride is used to determine 24 elements whose concentrations are printed out directly in $\mu \mathrm{g} / \mathrm{g}$ after computer calculation of the data supplied from the spectrometer.

\section{Rare Earth by Emission Spectroscopy}

Samples are dissolved in a hydrofluoric-nitric acid mixture. Uranium and plutonium are separated from the rare earths by a tri-n-octylamine extraction into xylene, and the rare earths are determined by copper-spark spectrographic analysis of the aqueous phase.

- Impurities by Spark Source Mass Spectroscopy

The sample material is vaporized, and the elements present are ionized by a high voltage if spark. The ions formed are separated according to their mass to charge ratios. The exposure of a photographic plate to the ion beams produce lines that are characteristic of the elements producing the ions. The positions of the lines indicate the elements present, and the densities of the lines relate to concentrations. The impurity elements determined quantitatively are tungsten, tantalum, dysprosium, europium, gadolinium, and samarium. Other impurities are determined semiquantitatively.

- Americium-241 by Anion Exchange and Alpha Analysis

Americium is determined from a total alpha count and an alpha spectrometric analysis after separation from plutonium and uranium by anion exchange.

Americium is determined by direct gamma spectrometric analysis of the dissolved sample using a Ge(Li) jetector and multichannel analyzer. No separation of americium from plutr, nium or uranium is required. The 59.6-keV ${ }^{241} \mathrm{Am}$ gamma peak is corrected for minor plutonium gamma interference by making a simple leading edge correction.

\section{- Gas Content by Vacuum Outgassing}

The sample is heated by induction heating in a tungsten crucible at $1600^{\circ} \mathrm{C}$. The pressure and temperature of the evolved gas are measured at a known volume. From these data, the $\mathrm{cm}^{3} / \mathrm{g}$ of gas at standard temperature and pressure is calculated. The apparatus is designed so that a sample of the gas can be taken for an analysis of composition. 
Samples are ignited with a tungsten accelerator between $1600^{\circ} \mathrm{C}$ and $1700{ }^{\circ} \mathrm{C}$ in a stream of oxygen. The carbon and sulfur, as their oxides, are swept from the furnace into an infrared detector. The oxides absorb a portion of the infrared radiation, and this absorption is used to measure the amount of those gases evolved from the sample simultaneously.

\section{Mixed Uranium - Plutonium Oxide Fuel Sample Dissolution}

Mixed oxide and plutonium dioxide samples are dissolved in a hot solution of nitric and hydrofluoric acids. The fluoride concentration is reduced by a boil-down with nitric acid after which the solution is diluted to volume with $0.5 \mathrm{M}$ nitric acid. Uranium dioxide is dissolved in nitric acid.

Plutonium by Controlled Potential Coulometry

Plutonium is determined by a coulometric titration in a hydrochloric acid electrolyte. A preliminary adjustment of valence states is made as follows: any trace of Pu(VI) that may be present is reduced to Pu(III) by electrolytically generated Fe(II) after which Fe(II) and Pu(III) are oxidized to Fe(III) and Pu(IV) at a platinum cathode by applying a potential of $+0.900 \mathrm{~V}$ (versus a saturated calomel electrode). Then the measurement is made by reducing the Pu(IV) to Pu(III) at $+0.550 \mathrm{~V}$. The plutonium content of the sample is calculated from the integrated current required for the titration, which is displayed on a digital voltmeter as milligrams of plutonium.

\section{- Plutonium by Amperometric Titration}

A solid sample is dissolved either by fusion with sodium bisulfate or by heating in nitric acid with a trace of hydrofluoric acid. The fused sample is dissolved in sulfuric acid, after which the plutonium is oxidized to plutonium(IV) with excess silver(II) oxide, and the excess silver(II) is reduced by heating. The nitric acid dissolved sample and other plutonium nitrate solutions are fumed with sulfuric acid to remove the nitrate before the plutonium is oxidized. After the oxidation of plutonium and reduction of excess silver(II), the solution is adjusted to about $6 \mathrm{M}$ in sulfuric acid.

\section{- Uranium by Controlled Potential Coulometry}

Uranium is determined by a coulometric titration in which $U(V I)$ is reduced to $U(I V)$ at a mercury cathode using a potential of $-0.300 \mathrm{~V}$ (versus a saturated calomel electrode) in a sulfuric acid electrolyte. A pre-reduction at $+0.080 \mathrm{~V}$ eliminates interferences from more readily reduced substances. The integrated current of the titration is displayed on a digital voltmeter as milligrams of uranium, from which the uranium content of the sample is calculated. 
Samples are weighed and then heated to constant weight at 800 to $900^{\circ} \mathrm{C}$ in an atmosphere of hydrogen-helium saturated with water vapor at $0^{\circ} \mathrm{C}$. The $0 / \mathrm{M}$ is calculated from the difference between the initial and final weights. Procedures for macroanalysis ( $20.5-9$ samples) and microanalysis ( $100-\mathrm{mg}$ samples) are included in this method.

- Isotopic Composition of Plutonium and Uranium by Mass Spectrometry

The isotopic abundances of plutonium and uranium are determined by thermal ionization mass spectrometry. Purified portions of these elements are prepared using ion exchange chromatography. The separation of americium and uranium from plutonium is complete enough to permit the unbiased measurements of ${ }^{241} \mathrm{Pu}$ and ${ }^{230} \mathrm{Pu}$. Ions are produced from a carbonized, single rhenium filament. An ion current measuring technique is used in the analysis.

- Weight Loss Upon Ignition

Weight loss is determined by measuring the change in weight of a sample when heated to constant weight at $950^{\circ} \mathrm{C}$ in air.

- Uranium by Potentiometric Titration

Uranium is reduced to U(IV) by excess Fo(II) in strong phosphoric acid and sulfamic acid. Excess Fe(II) is selectively oxidized by nitric acid in the presence of Mo(VI) catalyst. The U(IV) is titrated using standard potassium dichromate solution to a potentiometric end point. Vanadium(IV) solution is added before the titration to sharpen the end point. About 90 percent of the dichromate titrant is added by weight and the remainder is added by volume from a digital buret.

- Fluoride and Chloride Separation From Samples by Pyrohydrolysis

Water vapor is passed over a sample heated at $1000^{\circ} \mathrm{C}$. The pyrohydrolytic reaction quantitatively released fluoride and chloride as hydrofluoric and hydrochloric acids, which volatilize and collect in a trap solution. The trap solution is analyzed for fluoride and chloride using Methods 40.2 and 40.3 or 40.4 .

- Fluoride by Ion Selective Electrode Measurement

The solution obtained from pyrohydrolysis (Method 40.1) is analyzed for fluoride with a fluoride-selective electrode in an acetate buffered solution.

- Chloride by Constant Current Coulometry

The solution obtained from pyrohydrolysis (Method 40.1) is analyzed for chloride with an automatic chloride titrator, which coulometrically generates silver ion to form insoluble silver chloride. The end point is sensed amperometrically and the 
generating current is stopped automatically at a preset increment of indicator current. Because the rate of generating silver ion is constant, the titration time is proportional to the amount of chloride precipitated.

- Chloride by Spectrophotometry

The solution obtained from pyrohydrolysis (Method 40.1) is analyzed for chloride using a spectrophotometric procedure in which the absorbance of a ferric thiocyanate complex is measured at $460 \mathrm{~nm}$. The red-colored complex is formed from chloride, mercuric thiocyanate, and ferric ammonium sulfate by a reaction in which the chloride complexes mercury, releasing an equivalent amount of thiocyanate to react with iron.

- Carbon by Combustion and Gas Chromatography

Carbon, as carbon dioxide, is quantitatively released from powdered samples by heating at $1000^{\circ} \mathrm{C}$ in a stream of pure oxygen. The oxygen carries the carbon dioxide to a trap where the carbon dioxide is isolated by adsorption on molecular sieve at $0^{\circ} \mathrm{C}$. Subsequently, the carbon dioxide is desorbed by flash heating the trap and is carried in helium to gas chromatograph for measurement.

- Nitride by Kjeldahl-Spectrophotometric Method

Samples are dissolved in an acid mixture to convert nitride nitrogen to ammonium salts. Ammonia is released quantitatively by steam distillation from a strongly basic solution and is collected in a dilute acid solution. The ammonia is measured spectrophoto-metrically using the color developed from the reaction between ammonia and Nessler reagent.

- Total Nitrogen by Fusion and Gas Chromatography

Powdered or small pieces of sample are fused at about $2600{ }^{\circ} \mathrm{C}$ in a graphite crucible. The nitrogen is released in its elemental form and it is measured by gas chromatography, which is based upon the differences in thermal conductivity of gases.

- Surface Area by a Modified BET (Brunauer, Emmett, and Teller) Method

The surface area is determined using a commercially available analyzer that measures the amount of nitrogen gas adsorbed as a monomolecular layer on the surface on a sample held at the temperature of liquid nitrogen. Total surface area in square meters is displayed digitally on the analyzer.

- Particle Size Distribution by Sedimentation and X-Ray Scattering

The sample material is dispersed ultrasonically and with stirring in a liquid. The particle size analyzer measures the settling rates of the suspended particles by determining the concentration of particles remaining in suspension at various 
depths as a function of time. The data is automatically presented as a curve relating a cumulative percent distribution to the equivalent spherical diameters of the particles.

Density and Open Porosity by Mercury Displacement

A mercury-intrusion porosimeter is used to immerse a sample in mercury and to force mercury into open pores of the sample at various pressures ranging up to 5,000 psi. Density is determined by measuring the amount of mercury displaced by the sample and porosity is determined by measuring the amount of mercury penetrating pores at various pressures.

\section{- Boron Carbide Sample Preparation}

Boron Carbide Pellet samples are crushed, sieved, and mixed to produce a fine, homogeneous powder for various analyses.

- Total Boron by A Volumetric Method

Powdered nuclear-grade boron carbide is mixed with sodium carbonate and this mixture is fused to decompose the boron carbide. The melt is dissolved in water, filtered to remove the insoluble residue, acidified, and heated to remove carbon dioxide. The boron as boric acid is titrated with standardized sodium hydroxide using the mannitoboric acid procedure.

\section{- Total Carbon by Combustion and Gravimetry}

The sample mixed with a flux material is burned in oxygen at a temperature not lower than $1400{ }^{\circ} \mathrm{C}$. Traces of carbon monoxide are oxidized to carbon dioxide and traces of dust, sulfur dioxide, and moisture are removed prior to the gravimetric measurement of carbon dioxide.

- Isotopic Composition by Mass Spectrometry

Boron isotopic ratios are measured in boron carbide by thermal ionization mass spectrometry without prior chemical separation of boron. Boron is converted to sodium borate by fusion of the boron carbide with sodium carbonate directly on a tantalum filament. The loaded filament is transferred to the mass spectrometer where boron isotopic ratios are measured using the $\mathrm{Na}_{2} \mathrm{BO}_{2}{ }^{+}$ion.

- Fluoride and Chloride Separation from Boron Carbide

Water vapor is passed over a sample heated at about $1000{ }^{\circ} \mathrm{C}$. The pyrohydrolytic reaction quantitatively releases fluoride and chloride as hydrofluoric and hydrochloric acids, which volatilize and collect in a trap solution. The trap solution is analyzed for fluoride and chloride using Methods 40.2 and 40.3. 
The solution obtained from pyrohydrolysis (Method 40.1) is analyzed for fluoride with a fluoride-selective electrode in an boric acid buffered solution.

The solution obtained from pyrohydrolysis (Method 40.1) is analyzed for chloride with an automatic chloride titrator, which coulometrically generates silver ion to form insoluble silver chloride. The end point is sensed amperometrically and the generating current is stopped automatically at a preset increment of indicator current. Since the rate of generating silver ion is constant, the titration time is proportional to the amount of chloride precipitated.

- Soluble Carbon by A Manometric Measurement

The sample in powder form is treated with a solution of sodium dichromate in sulfuric acid at a temperature of $100^{\circ} \mathrm{C}$. The soluble carbon is oxidized to carbon dioxide, which is measured manometrically.

- Soluble Boron by Extraction and Titration

Soluble boron is extracted from two portions of sample by refluxing separately with hydrochloric and nitric acids. The method is based upon the assumption that hydrochloric acid extracts only boron oxide, and nitric acid extracts both boron oxide and elemental boron. Each extract is purified from hydrolyzable metals by precipitation, and the filtrates are analyzed for boron by titration with standardized sodium hydroxide using the mannitoboric acid procedure. This method is empirical; therefore, it is important that the apparatus and analysis conditions used are exactly as described in this method.

- Total Nitrogen by Fusion and Gas Chromatography

Powdered or small pieces of boron carbide are fused with nickel at about $2600{ }^{\circ} \mathrm{C}$ in a graphite crucible. The nitrogen is released in its elemental form and it is measured by gas chromatography, which is based upon the differences in thermal conductivity of gases.

- Nitrogen by A Fusion - Spectrophotometric Method

The sample is mixed with lithium hydroxide and fused to decompose the boron carbide. The nitride nitrogen is converted to ammonia, which is trapped in a hydrochloric acid solution. The ammonium ion is measured spectrophotometrically using the colored complex produced by Nessler reagent.

- Total Oxygen by Inert Gas Fusion

Powdered boron carbide is fused with iron at $2600{ }^{\circ} \mathrm{C}$ in a graphite crucible. Oxygen evolves as carbon monoxide, which is converted to carbon dioxide when 
passed over heated rare earth oxide. The carbon dioxide is measured by thermalconductivity gas chromatography. A water analysis is required to correct the oxygen results for any water absorbed by the powdered material.

- Gas Content by Vacuum Outgassing

The sample is heated at $1000^{\circ} \mathrm{C}$ by induction heating in a tungsten crucible. The pressure and temperature of the evolved gas are measured while the gas occupies a known volume. From these data the $\mathrm{cm}^{3} / \mathrm{g}$ of gas at standard temperature and pressure (STP) is calculated. The apparatus is designed so that a sample of the gas can be taken for an analysis of composition.

- Impurities by Direct Reading Emission Spectroscopy

Impurity elements are determined by a direct reading emission spectrometer using a mixture of germanium oxide and graphite as a buffer. Concentrations of $\mathrm{Al}, \mathrm{Ca}$, $\mathrm{Cr}, \mathrm{Fe}, \mathrm{Mg}, \mathrm{Mn}, \mathrm{No}, \mathrm{Si}$, and Ti are printed out directly in $\mu \mathrm{g} / \mathrm{g}$ after computer calculation of the data supplied from the spectrometer.

- Titanium by Emission Spectrography

Titanium is determined by emission spectrography using a mixture of germanium oxide and graphite as a buffer. This method is used when the Direct Reader Analysis for titanium exceeds $100 \mu g / g$ (Method 40.11).

- Tungsten by Spectrophotometry

Tungsten is reduced to cungsten(V) with titanium(III) and the tungsten is extracted from a hot hydrochloric acid solution into a solution of dithiol in amyl acetate. The absorbance of the tungsten-dithiol complex is measured at $640 \mathrm{~nm}$. Molybdenum interference is removed by a preliminary extraction as molybdenum(IV).

- Total Carbon by Combustion and Gas Chromatography

The sample, mixed with an accelerator, is burned in oxygen at greater that $1600{ }^{\circ} \mathrm{C}$. The $\mathrm{CO}_{2}$ product is selectively adsorbed on molecular sieve. When the combustion is complete, the molecular sieve is heated and the desorbed $\mathrm{CO}_{2}$ is measured by thermal conductance gas chromatography. 


\subsection{SOLID WASTE HANDLING PRACTICES AND PROCEDURES}

Solid radioactive waste handling, packaging, and characterization at the Hanford Site progressed through four general phases:

1. pre -1969

2. $1970-1972$

3. $1973-1982$

4. 1982 - present.

Before 1969, radioactive solid waste received very little attention as to characterization, packaging, sorting, or treating. Marginal records were maintained with emphasis on the Special Nuclear Materials (SNM) operating laws. As waste retrieval was not a factor for consideration, the radioactive waste burial containers were not chosen for their resistance to long-term corrosion, weathering, or degradation. The solid radioactive waste burial during this time period is not very definable with respect to today's intricate waste classification system. The tables and graphs in this report do not include pre-1970 data; however, packaging and handling information is provided for this time period.

The AEC directive establishing the basis for TRU waste and the requirement for 20 year retrievable storage was issued in 1970. Solid Waste Burial Records (SWBR) were kept for all containers, although the information on physical contents and hazardous constituents was limited.

During the next period, 1973 to 1979, the AEC further defined TRU waste as material contaminated with certain alpha-emitting radionuclides with half-lives greater than 20 years and activity greater than $10 \mathrm{nCi} / \mathrm{g}$. Record keeping and waste packaging improved during this time period.

In the early 1980's, the ability to assay waste packages accurately was improved with the addition of several Segmented Gamma Scan Assay Systems (SGSAS). This improved the ability to characterize TRU waste.

Since 1982, the $100 \mathrm{nCi} / \mathrm{g}$ TRU limit has been in effect. With this change in the TRU requirements as well as improved recording, packaging, and sorting practices, waste classified as TRU waste under the previous requirements now may be categorized as low-level. Waste acceptance criteria were developed and improved at this time also. RCRA requirements and other more strict compliance criteria, especially for waste containing hazardous chemicals, have been applied to radioactive solid waste handling, packaging, characterization, and record keeping practices.

\subsection{HISTORICAL WASTE PACKAGING AND HANDLING REQUIREMENTS AT HANFORD}

Eleven historical waste packaging manuals were reviewed for information regarding packaging and labeling requirements for TRU waste in retrievable storage at the Hanford Site. These manuals covered requirements from 1974 through 1988. 


\section{WHC-EP-0696}

Packaging requirements have changed over time and have become more stringent. Table 4-1 summarizes TRU storage requirements for the Hanford Site from 1974 through 1988. The columns in Table 4-1 are not symmetrical; when a definition or requirement is consistent between manuals, text is shared under several document number columns. A blank spot indicates that no requirements or definition are found in that manual. Definitions (i.e., hazardous and toxic materials, etc.) change from manual to manual and so require reference to each particular manual.

Some of the more significant changes that have occurred in the past twenty years with regard to the packaging, handling, and record keeping for radioactive wastes from the 325 Building follow:

1. The definition of TRU waste has changed since 1970, and the designation of waste packages as TRU has also changed. During the period from 1970 to 1973 TRU segregation was based on generator practice. From 1974 until 1982, TRU waste was segregated if the concentration of TRU was greater than $10 \mathrm{nCi} / \mathrm{g}$. In 1982, the current $100 \mathrm{nCi} / \mathrm{g}$ definition for TRU waste was implemented by the DOE.

2. Because of the varied contents of the waste containers, chemical reactions can occur. Gases, including hydrogen, that can contribute to fire and/or explosion may accumulate. Since 1982, waste materials that generate $\mathrm{H}_{2}, \mathrm{O}_{2}$, or $\mathrm{NO}_{x}$ are prohibited unless drums have been permanently fitted with vent clips to allow continual release of gases or include catalyst packs to recombine any hydrogen that may be produced.

3. Criticality specifications limited the amount of TRU to less than $250 \mathrm{~g}$ per 55-gallon drum from 1975 through 1978 and to $200 \mathrm{~g} /$ drum after that time. Prior to 1975 the criticality limit on drums and cartons was 400 grams.

4. Individual container weights were not required prior to 1977; however, estimated weights for pre-1977 containers were added to the records in the R-SWIMS database during the data reentry program in the mid-1980's. Standard weights were given for each container type. All 55-gallon drums, for example, were given a value of $68 \mathrm{Kg}(150 \mathrm{lbs})$.

5. Originally, waste burial records for TRU were not done for individual containers but for entire shipments. In 1982, TRU burial and storage records began to be based on an individual container basis. The entry of unique data for each waste container into the R-SWIMS database was not fully implemented until 1984. During the R-SWIMS reentry program, historical TRU records were converted to an individual basis so that today there is one SWITS record for each TRU container stored at Hanford. 


\section{WHC-EP-0696}

The conversion from group data to individual container data required some assumptions about the containers in the group to be made. Chief among these assumptions was that an even distribution of radiological and hazardous constituents existed among the members of the group.

LLW records are still kept for multiple containers rather than for individual containers.

6. Information on the hazardous constituents of waste containers was not required prior to 1986. During the R-SWIMS reentry program, any available information from the SWBRs was added, however, this information is limited.

7. Physical descriptions of the waste contents were not required on the SWBR prior to 1978. SWITS records for waste buried prior to 1978 list the contents as "miscellaneous" in most cases. In more recent years, the physical contents description has become more detailed. Many of the records now include the weight and volume percentages of a given component in the waste container.

8. Between 1972 and 1978, combustible and noncombustible waste components were segregated. Although the SWITS database did not retain a data field for this information, it was added to the physical contents description field.

9. The most common TRU waste container used at the Hanford Site is the 55-gallon steel drum. U.S. Department of Transportation (DOT) 17C and 17H drums, with minimum wall thicknesses of 0.053 in. and 0.043 in., respectively, were both used. Prior to 1982 most of the drums were painted, so there is approximately 0.005 in. of paint on both the exterior and interior of the drums. In 1982, galvanized drums replaced the painted drums. Recycled 55-gallon drums were also permitted for the storage of TRU waste from 1973 to 1978.

10. Data for wastes generated prior to 1970 are not included in the SWITS database.

Over the years, solid radioactive wastes generated at the 325 Building have been handled in a variety of ways. A variety of containers, including "gunk catchers," "paint cans," "milk pails," "lard cans," and "potato chip cans," have been used to hold radioactive waste. These waste were stored at collection points in room $52 \mathrm{~A}$ and in several basement rooms (rooms 32, 40A, 40B, 40C, and 50) prior to disposal at the various burial grounds designated for use by 300 Area buildings: 618-10, 300 North, Wye, and the 200 Area Burial Grounds.

Prior to 1970, radioactive dry wastes were segregated into low-level wastes and highlevel wastes based on their activity. Low-level solid wastes from all potentially contaminated areas were sealed in plastic-lined cardboard cartons and buried. High-level solid wastes from the multicurie "hot" cells were sealed in leak-proof metal cans and transported to the burial grounds in shielded casks. Small quantities of high-level wastes, such as analytical samples and glovebox wastes, were encapsulated in concrete prior to burial. Before 1968 some contaminated, used resins from 325 Building ion-exchange columns were placed in $30-$ gallon paper drums and disposed aboveground west of the 300 Area west gates. 
In 1970, the Hanford Site implemented the requirement to segregate TRU waste from LLW. During the mid-1970's dry solid wastes were segregated into seven categories at 325. They were as follows:
- Trash
- Salvageable scrap
- Toxic non-radioactive waste
- Pyrophoric nonradioactive waste
- Low dose rate radioactive waste
- High dose rate radioactive waste
- Transuranic waste.

Presently, radioactive wastes are administratively segregated into low-level, transuranic, and mixed wastes.

\subsection{HISTORICAL WASTE PACKAGING AND HANDLING PROCEDURES FOR THE 325 BUILDING}

\subsubsection{Transuranic Waste}

During the 1970 to 1987 time frame, TRU waste was generated by five to six separate glovebox lines. The waste was primarily contaminated laboratory supplies that was put into 5-quart paint cans. For a time, the paint cans were placed in a larger "potato chip" can, which were then stacked in a 55-gallon drum. This practice was abandoned because of the large bulk of waste the overpacking created.

The accumulation limit for 5-quart paint cans in an individual glovebox was four. Once this limit was reached, the cans had to be bagged out. It took an average of 15 paint cans to fill a 55-gallon drum, as the cans were generally arranged in three stacks of five cans each. In order to meet requirements for waste accumulation and holding times in the laboratory, several gloveboxes were bagged out at the same time so that drums could be filled as quickly as possible. Therefore, an individual 55-gallon drum generally will contain solid wastes from several gloveboxes.

Glovebox operators were responsible for the bagout of their respective gloveboxes after 1980 or so. Prior to that time, the Waste Management Group was responsible for waste collection and decontamination work.

Catalyst packs were placed on the drums beginning in the mid-1980's. If a drum was vented, it also had a catalyst pack.

Acid solutions containing uranium and plutonium, acid digestion wastes, and uranyl nitrate from the digestion of fuel pins were mixed with Portland Cement in a 5-quart paint can to effectively neutralize them. There was a special glovebox dedicated to this process. Beginning in the mid-1980's the procedure changed, and acid solutions had to be neutralized prior to mixing with the cement. Frequently used acid solutions included phosphoric acid and hydroxylamine hydrochloride. 
Prior to about 1987, old gloveboxes were packaged in burial boxes intact. For a brief period of time, they were sent to the $231-\mathrm{Z}$ Facility to be cut up into smaller pieces. The pieces were packaged in steel culverts, steel boxes, plywood boxes, and some of the smaller pieces were placed in 55-gallon drums.

In the mid-1980's time frame, mercury was packaged in culverts about 8 in. in diameter and $2.4 \mathrm{ft}$ long. The culverts were then put into drums with approximately 6 in. of concrete on the top and the bottom. It was common to fill the space around the culverts with bagged poly bottles and other items. These drums are extremely heavy; facility personnel estimate that these drums weighed between 600 and $800 \mathrm{lb}$ each.

Beginning in 1980 or so, solid waste containers were sent from 325 to 324 for nondestructive assay (NDA). The NDA instrument used was Segmented Gamma Scanner Assay System (SGSAS). These containers were then shipped out from 324 to the burial grounds. The SWBRs for these drums lists 324 , not 325 , as the generating facility. A significant number of containers were also staged at the 340 facility prior to shipping. These containers list 340 , not 325 , as the generating facility.

Waste volume fluctuated from year to year according to facility personnel. One truck could hold about 23 drums, so that many ware accumulated prior to sending them to the 200 Area burial ground. Sometimes there were several shipments of TRU waste per year. The normal holding time for a drum in the 300 Area during the 1980's was less than a year except in unusual circumstances. Drums that exceeded this holding time were special case drums that, in most cases, had to be brought into compliance with new regulations.

A typical TRU waste drum would probably be made up of the following items:

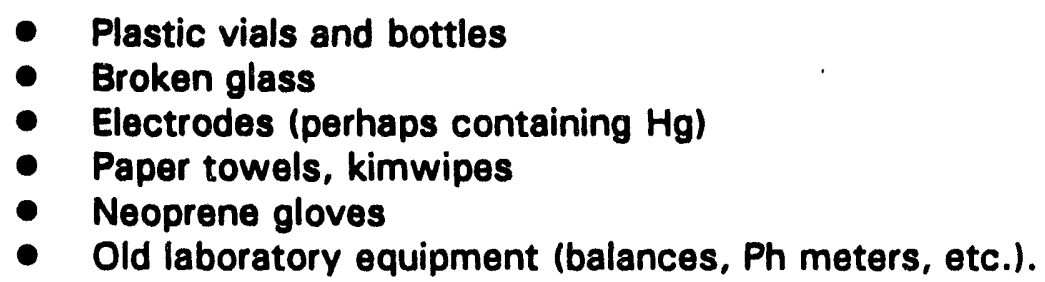

\subsubsection{Low-Level Waste}

Facility personnel indicated that there was a significant amount of low-level waste generated at Building 325 during the 1980's. It was not unusual for two truckloads of LLW to be shipped in a given week. Typical LLW drums contained housekeeping and room waste only. All maintenance waste was placed in burial boxes and were handled by JA Jones or Kaiser.

According to facility personnel, the nominal generation rate for drums from 325 since PNL assumed landlord responsibilities is $\mathbf{3 6}$ drums of compacted waste per year. (In 1990 , there were 36 drums of compacted waste produced; in 1991, 35 drums of compacted waste ware produced.) 


\subsection{HANDLINO AND PACKAGING OF CAISSON WASTE FROM THE 325 HOT CELLS}

\subsubsection{Pre-1970 Handiling and Packaging Procedures}

Prior to 1966, wastes generated in the hot cells were collected in extruded aluminum cans with a riveted bail for a handle. These cans were referred to as "milk pails" and were approximately the size of an imperial gallon. Although liquids were placed into the pails, most solvents evaporated quickly due to the high flow rate through the hot cells. When the milk pails were full, within about two inches of the top, they were covered with a "gelatin" layer, since they had no lids of their own. These pails were then disposed of at the Wye Burial Ground.

During 1966 and 1967, solid waste disposal caissons and containers used for 300 Area disposal changed in two ways. A new type of caisson (sometimes called a "silo"), with its top opening angled at $\mathbf{4 5}$ degrees to give better radiation shielding to disposal workers, was designed and fabricated in Hanford's 1100 Area Shops. At the same time, the milk pail system was replaced by the paint can system. Radioactive wastes from operations buildings were collected in 1-gallon paint cans with metal lids fastened on by two sets of clips. Eight paint cans together fit into a new cylindrical casks with 6 inches of lead shielding surrounded by a stainless steel shell. Thus, more securely sealed and heavily shielded, solid wastes traveled to the Wye Burial Grounds.

\subsubsection{Post-1970 Packaging and Handling Procedures}

After 1970, caisson waste was sent to the 200 Areas Burial Grounds for retrievable storage in underground caisson TRU storage containers. Casks from the 325B Hot Cell Annex were lined with 2-in. lead shielding and held three 1-gallon buckets. The casks themselves were not disposed but were reused many times. The paint can system continued in use for many years and is still used to some extent today. Later the system was augmented by the use of $50 \mathrm{lb}$ "lard cans." These 10-gallon containers are used for high-activity solid radioactive wastes generated in the 325 Building and in other 300 Area structures (Gerber 1992). A diagram of the underground caisson TRU storage container is shown in Figure 4-1.

Interviews with 325B Hot Cells Annex Personnel were conducted to gather additional details regarding the handling of caisson waste. Personnel indicated that unused fuels from the Hot Cells were reacted with nitric acid in glass beakers. The liquid was then allowed to evaporate into a solidified waste form resulting in a saltcake that was crushed along with the beaker and placed in 1-gallon buckets to be sent to the caissons for disposal. In addition to the fuel, articles used in the analytical work became grossly contaminated with fuel to a level high enough that they were put in the same can as the fuel and sent to the alpha caissons. Considerable effort was made to keep the buckets externally clean or to fix any contamination externally. On a few occasions the cask boat that was used for lowering the cans into the caisson was lost in the process. It is expected that some of these cask boats will be encountered during retrieval efforts. 
WHC-EP-0696

Figure 4-1. Post-1970 Underground Caisson TRU Storage (218-W-4B Burial Ground).

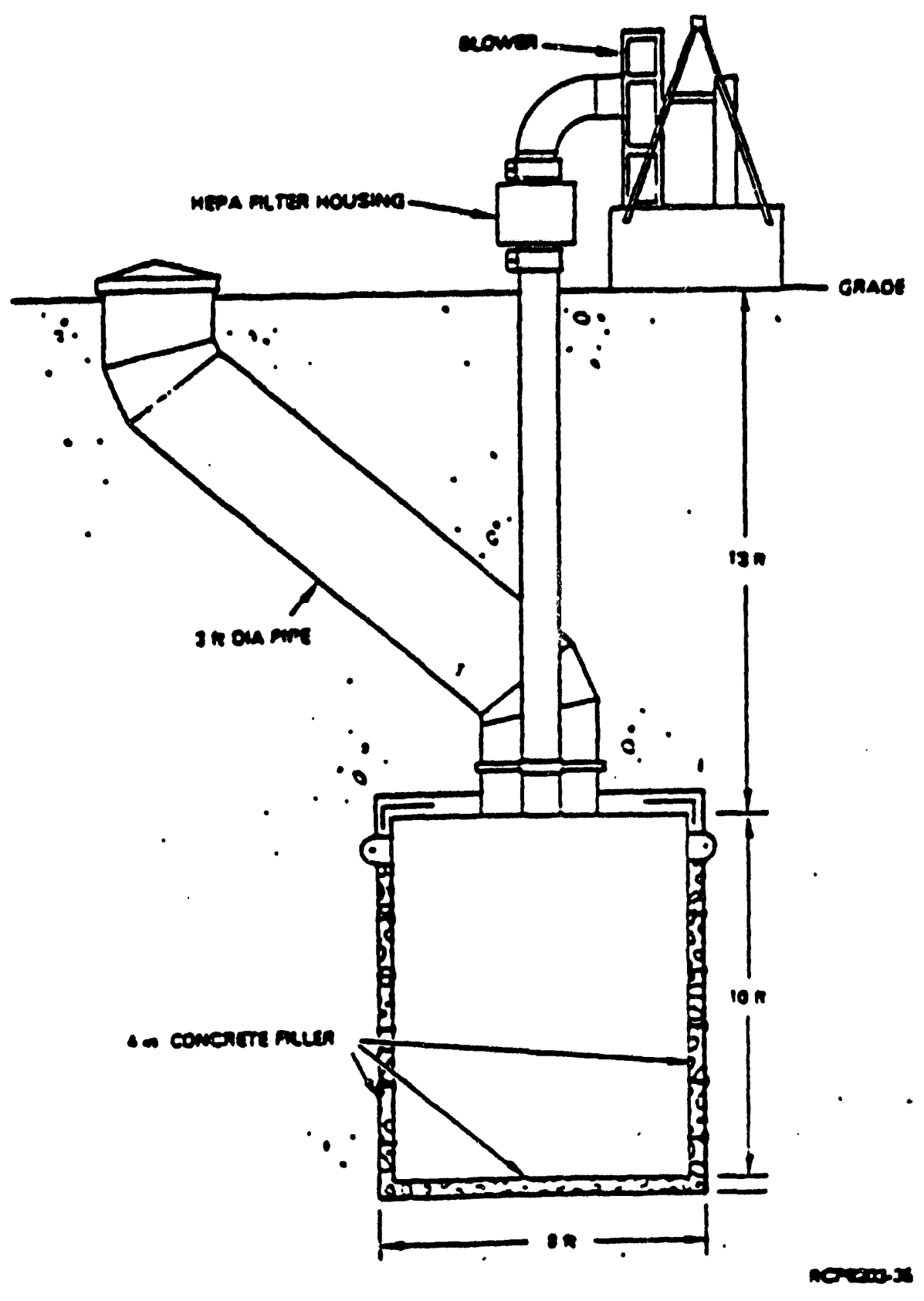

4-7 


\section{WHC-EP-0696}

This page intentionally left blank. 
Table 4-1. TRU Storage Requirements for the Hanfor

NOTE: For the pariod of 1970 through April 1974, document numbers are unknown.

\begin{tabular}{|c|c|c|c|c|c|}
\hline Des: & $\begin{array}{l}414 \cdot 3932 \\
4 / 74 \cdot 4 \sqrt{77}\end{array}$ & $\begin{array}{c}A n+3002, \pi n n_{1} 1 \\
477+7776\end{array}$ & 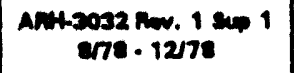 & $\begin{array}{c}\text { Ant-30032 Row. } 1 \text { supp. } 2 \\
12 / 79 \cdot \text { Breo }\end{array}$ & 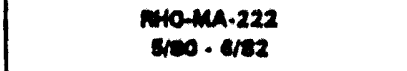 \\
\hline TrW Dofinition & $\begin{array}{l}\text { Weote conteining Pu } \\
\text { endler other trensurenium } \\
\text { nuelides in concentustions } \\
\text { oreater then } 10 \mathrm{nCV} \text {. }\end{array}$ & \multicolumn{3}{|c|}{ 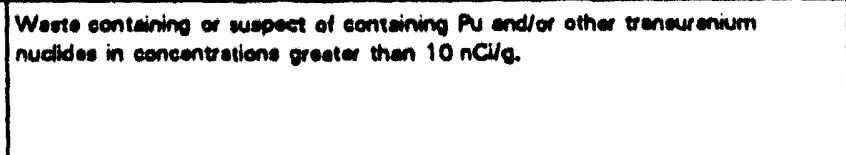 } & 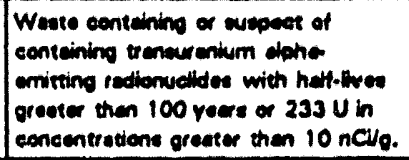 \\
\hline \multirow{6}{*}{$\begin{array}{l}\text { Prohibited westue types } \\
\text { and Peckeging } \\
\text { Requirements }\end{array}$} & \multicolumn{3}{|c|}{ 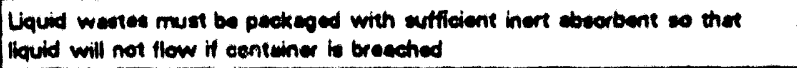 } & No tree liquids & $\begin{array}{l}\text { No fres llouide - except haud orgenie } \\
\text { westes. }\end{array}$ \\
\hline & & \multicolumn{2}{|c|}{ 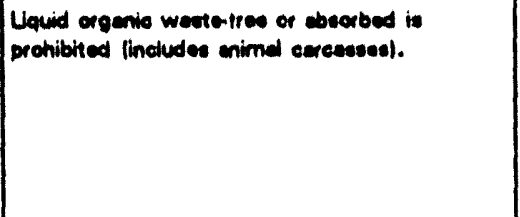 } & 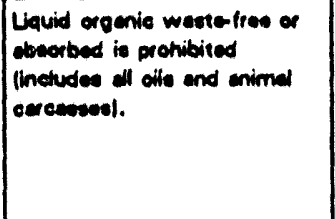 & 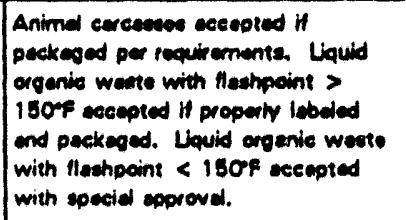 \\
\hline & & \multicolumn{2}{|c|}{$\begin{array}{l}\text { Flemmeble sesorbed liquids prohibited from } \\
\text { dieposel in ceriesone. }\end{array}$} & $\begin{array}{l}\text { Demp of wot weaten with } \\
\text { highty combustible of } \\
\text { explowive liquide prohibited. }\end{array}$ & $\begin{array}{l}\text { Fiemmable liquide free of ebeorbed } \\
\text { with flachpoints below } 150 \text { f } \\
\text { prohibited }\end{array}$ \\
\hline & & \multicolumn{3}{|c|}{ Unreseted Aketi Motels prohibited. } & Une oeeted Alkell Metels prohibited. \\
\hline & & & & Explosives Prohibited & Explosives prohibrted \\
\hline & & & & Pyrophorics prohibited & Pyrophorice prohibited \\
\hline & & & \multicolumn{2}{|c|}{ 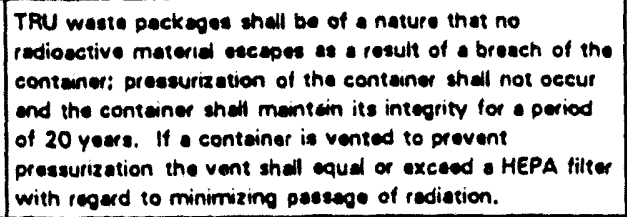 } & $\begin{array}{l}\text { Matorids that generote significent } \\
\text { omounts of gas ere orohibited. }\end{array}$ \\
\hline & & \multicolumn{3}{|c|}{ Hazardous and toxic materiels must be treated prior to buriel. } & $\begin{array}{l}\text { Hezerdous and toxic meterials } \\
\text { ecceoted on case by case basis. }\end{array}$ \\
\hline & & \multicolumn{3}{|c|}{ Exterior surfaces must be free from smeerdole contamination-fixation allowed. } & $\begin{array}{l}\text { Exterior surtace must be from } \\
\text { smonably contemination. }\end{array}$ \\
\hline
\end{tabular}




\section{Site. (3 sheets)}

\begin{tabular}{|c|c|c|c|}
\hline 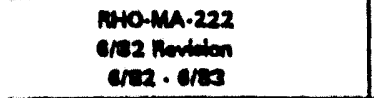 & 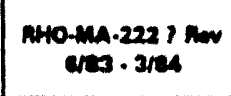 & $\begin{array}{c}\text { mo.MA.222 Rov. } 1 \\
324 \cdot 7 m 4\end{array}$ & 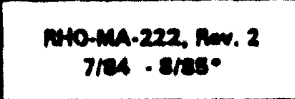 \\
\hline 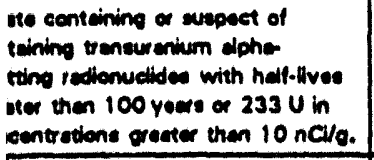 & $\begin{array}{l}\text { Doeumant } \\
\text { Uneveileble }\end{array}$ & \multicolumn{2}{|c|}{ 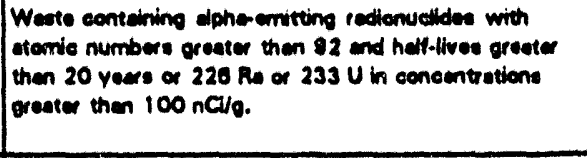 } \\
\hline $\begin{array}{l}\text { tree llavide - oxeept laquid ongenie } \\
\text { tien }\end{array}$ & & $\begin{array}{l}\text { No tres laquide - exeept } \\
\text { Hopid orgenid weoten }\end{array}$ & No tree linquids \\
\hline \multirow[t]{2}{*}{ 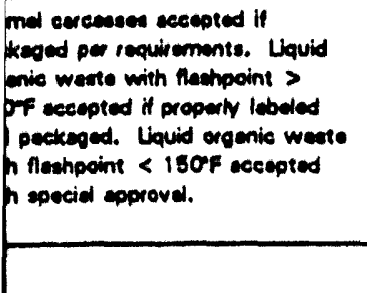 } & & \multicolumn{2}{|c|}{$\begin{array}{l}\text { Animed cerceseses eceepted if peckesond per } \\
\text { requinements. }\end{array}$} \\
\hline & & \multicolumn{2}{|c|}{ Shudges emnot contein tree llequide. } \\
\hline eacted Alkati Motals prohibited. & & \multicolumn{2}{|c|}{ Unreected Alkell metels prohibited with exeeptions. } \\
\hline Sosives prohibited & & \multirow{2}{*}{$\begin{array}{l}\text { Prroohorice and } \\
\text { explosives prohibited } \\
\text { oxcept metel fines. }\end{array}$} & Explosives prohibited. \\
\hline ophorics prohibited & & & Prrophorice prohibited. \\
\hline & & $\begin{array}{l}\text { Qee cylinders conteining } \\
\text { redioective gases } \\
\text { prohibited. Emory } \\
\text { oylinders accepted if } \\
\text { permenenty vented. }\end{array}$ & $\begin{array}{l}\text { Compreased gesese } \\
\text { prohibited. }\end{array}$ \\
\hline $\begin{array}{l}\text { ste materids that generate } \mathrm{H}_{2}, \mathrm{O}_{2} \\
\text { NOx wo prohibited unless } \\
\text { menently vented or catalyst } \\
\text { tege in conteiner. }\end{array}$ & & 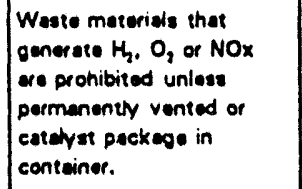 & $\begin{array}{l}\text { Weste conteiners thet could } \\
\text { repressurize to greater then } \\
7 \text { psi within } 25 \text { yeers } \\
\text { requirs venting through } \\
\text { HEPA filters. }\end{array}$ \\
\hline $\begin{array}{l}\text { turdous and toxic meterials } \\
\text { septed on case by case basis. }\end{array}$ & & \multicolumn{2}{|c|}{$\begin{array}{l}\text { Hezadous and Toxic materials must be treated to meat } \\
\text { the waste ecceptence criterie for disposal. }\end{array}$} \\
\hline $\begin{array}{l}\text { ferior surfaces free from smear able } \\
\text { ptamination fixation allowed. }\end{array}$ & & $\begin{array}{l}\text { Exterior surfaces fres from } \\
\text { smemeble conternination } \\
\text { fixation ellowed. }\end{array}$ & $\begin{array}{l}\text { Removable surface } \\
\text { contemination limits } \\
\text { fixetion is not ecceptable. }\end{array}$ \\
\hline
\end{tabular}


Table 4-1. TRU Storage Requirements for the Hanfor

NOTE: For the period of 1970 through April 1974, document numbers are unknown.

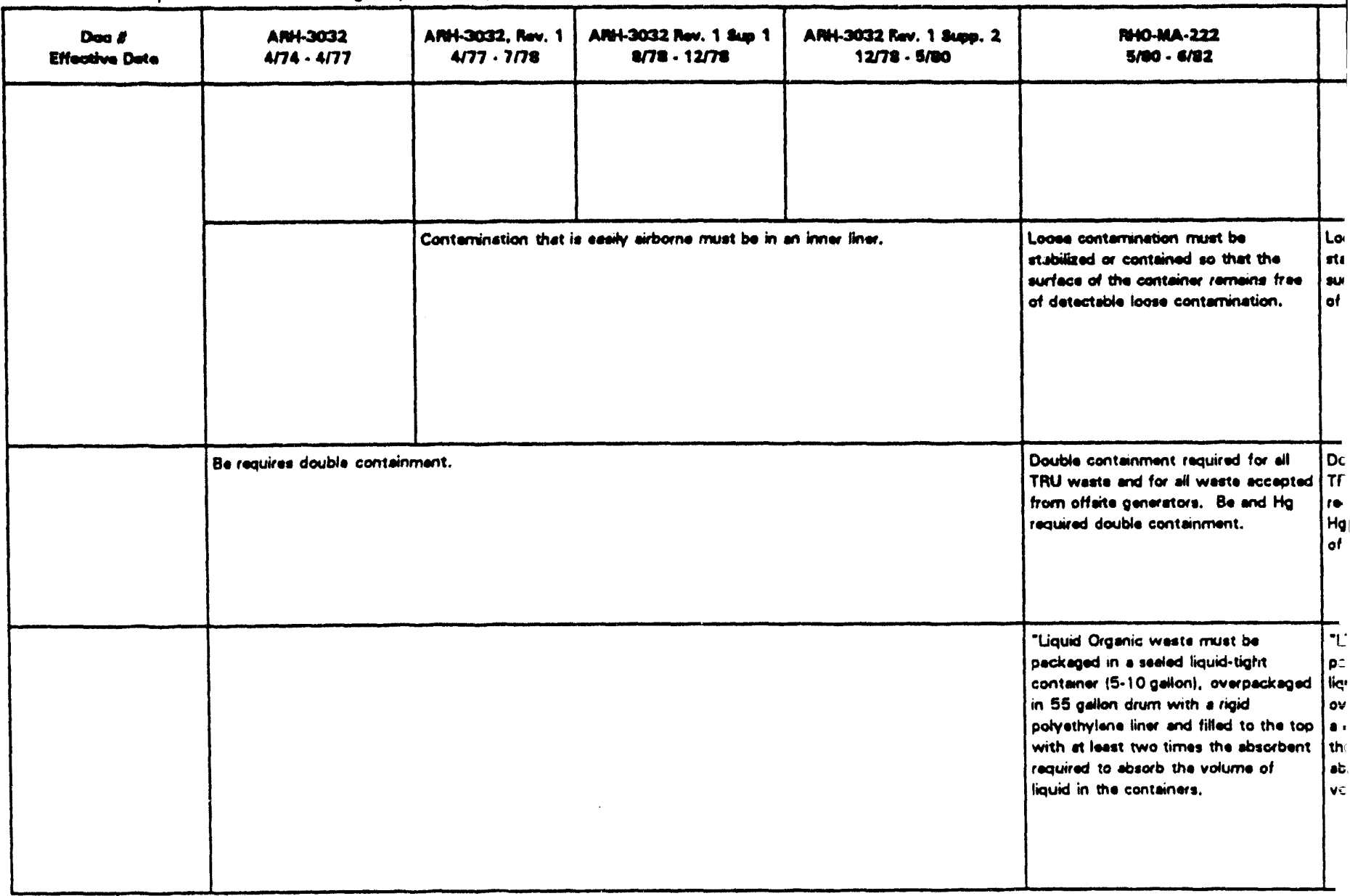


WHC-EP-0696

| Site. (3 sheets)

\begin{tabular}{|c|c|c|c|}
\hline 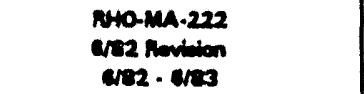 & 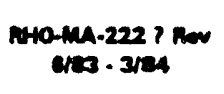 & 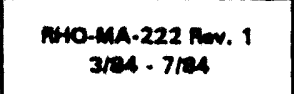 & $\begin{array}{c}\text { Homa-222, Rew. } 2 \\
\text { ImA - }\end{array}$ \\
\hline & & & 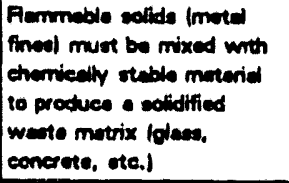 \\
\hline 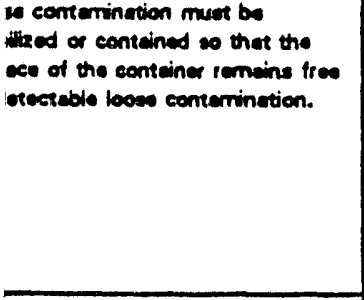 & & 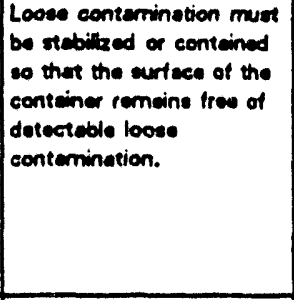 & 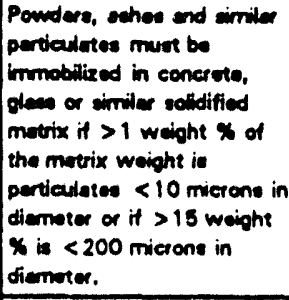 \\
\hline 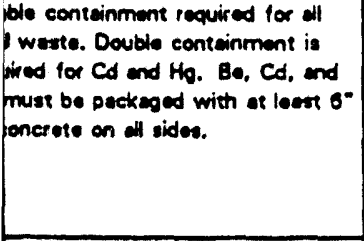 & & 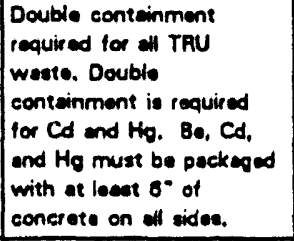 & 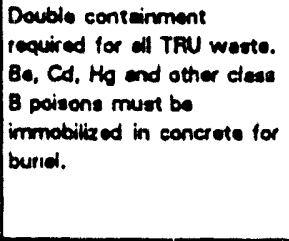 \\
\hline 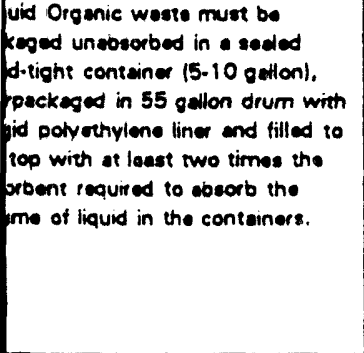 & & 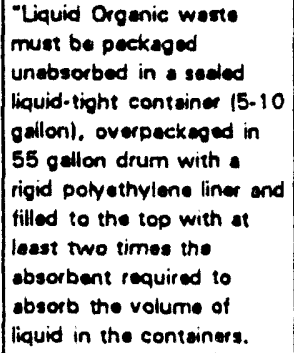 & $\begin{array}{l}\text { Liquid orgenic weste must } \\
\text { be solidified for storege or } \\
\text { dispoed. Exceptions ate } \\
\text { coneidered on a case by } \\
\text { case besis. }\end{array}$ \\
\hline
\end{tabular}


Table 4-1. TRU Storage Requirements for the Hanfor

NOTE: For the period of 1970 through April 1974, document numbers aro unknown.

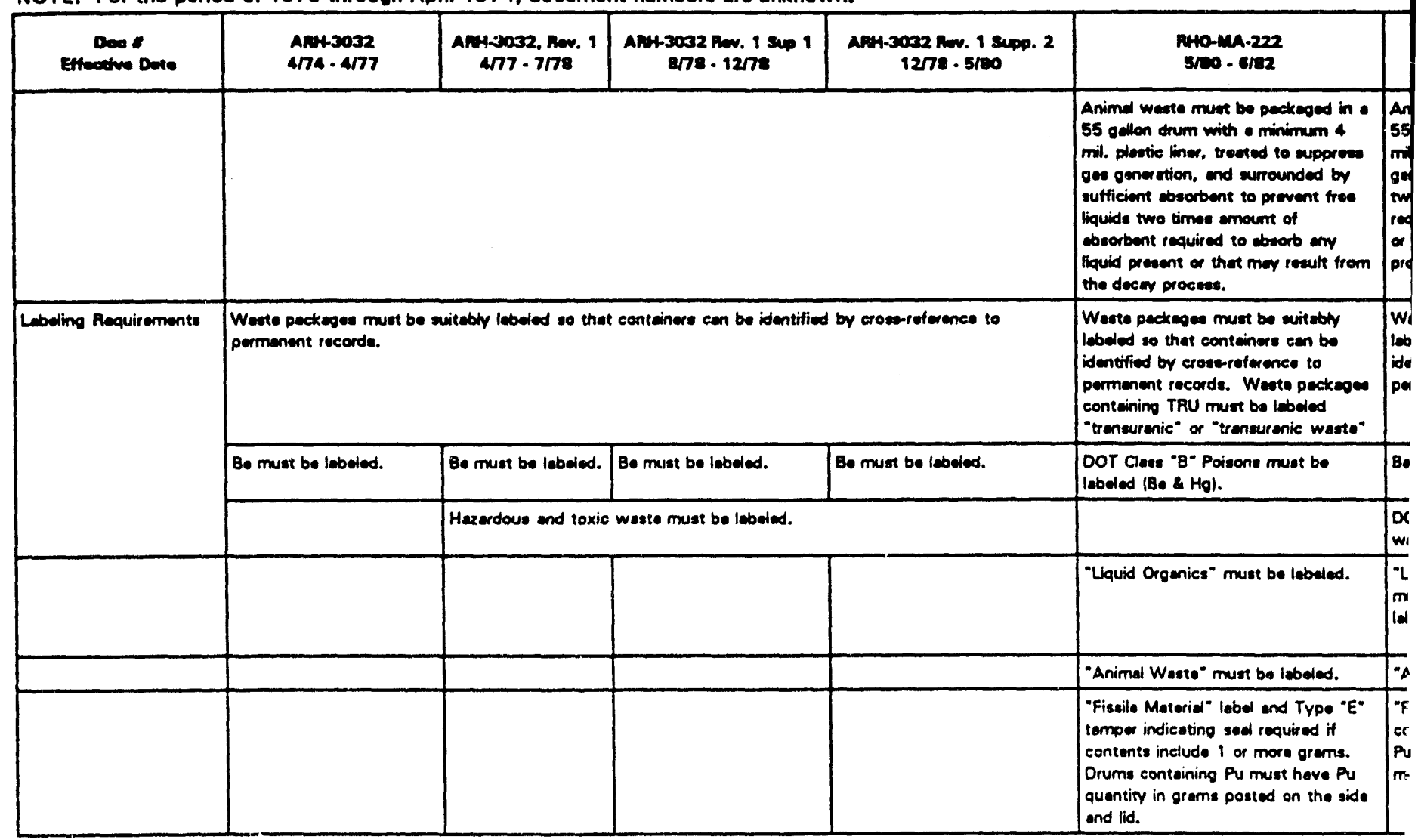

-Includes RHO-MA.222, Rev. 3, 3A and 4. The changes mede in these revisions ore genered and do not affect the packeging, storege or disposel requirements of TRU unste' offective dete of WHC-EP-OOB2, Rev. 0 . 
Site. (3 sheets)

\begin{tabular}{|c|c|c|c|}
\hline 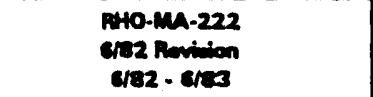 & $\begin{array}{c}\text { RHO-MA-222 i Mov } \\
\text { O/83 - 3/84 }\end{array}$ & $\begin{array}{l}\text { MHO-MA.222 Rew. } 1 \\
\text { 3/24. } 7 \text { TReA }\end{array}$ & $\begin{array}{c}\text { Ho-mA-222, Rov. } 2 \\
\text { T/8R - 8/85" }\end{array}$ \\
\hline 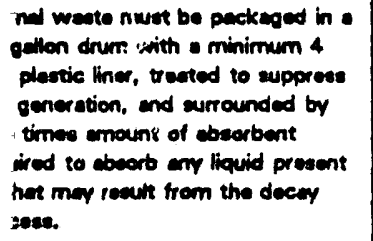 & & \multicolumn{2}{|c|}{ 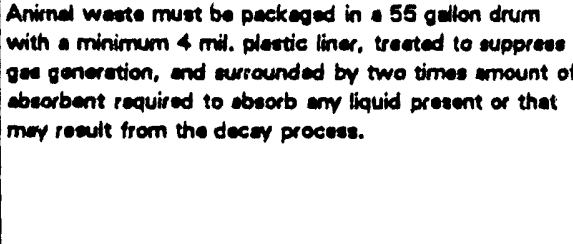 } \\
\hline 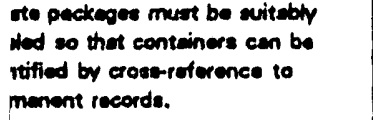 & & \multicolumn{2}{|c|}{$\begin{array}{l}\text { Werte peckeses must bo surtebly lebeled so that } \\
\text { conteinere cen be identificd by crosereference to } \\
\text { permenent rocorda. }\end{array}$} \\
\hline $\mathrm{Cd}$ and $\mathrm{Hg}$ must be lebolied. & & \multicolumn{2}{|c|}{ Bo, Cd and $\mathrm{Hg}$ must to laberied. } \\
\hline $\begin{array}{l}\text { Th lebols required for all clessose of } \\
\text { to that apply to the peckego. }\end{array}$ & & \multicolumn{2}{|c|}{$\begin{array}{l}\text { DOT lebole and color codine required on ell wasto } \\
\text { peckeges. }\end{array}$} \\
\hline $\begin{array}{l}\text { wuid Orgemies" must be labeled and } \\
\text { tet have fleshooint renge lieted on } \\
\text { t. }\end{array}$ & & $\begin{array}{l}\text { Liquid Orgenics" must bo } \\
\text { laboled and must have } \\
\text { floehpoint rangs lieted on } \\
\text { labol. }\end{array}$ & \\
\hline himed Wasto" murt be laboled. & & \multicolumn{2}{|c|}{ ["Animel Wasto" munt bo loboled. } \\
\hline 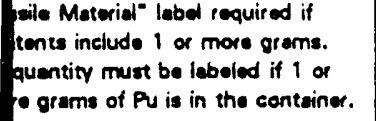 & & \multicolumn{2}{|c|}{$\begin{array}{l}\text { "Fissile Matorial" label required if concents include } 1 \text { or } \\
\text { more grems. Pu quentity mure be laboled if } 1 \text { or more } \\
\text { greme of Pu is in the conteinor. }\end{array}$} \\
\hline
\end{tabular}

- or resctive wastes specifically. The requirements liated under Rov. 2 of RHO-MA-222 are affective up to the 
WHC-EP-0696

\section{INTRODUCTION TO SECTIONS 5.0 THROUGH 8.0}

Because the main 325 Radiochemistry Building was jointly occupied by Westinghouse Hanford and PNL throughout the 1970's and 1980's, the waste that was generated there was designated by the company producing it. In this report we have retained this separation; Section 5.0 contains information on waste designated as Westinghouse Hanford and Section 6.0 contains information on waste designated as PNL. It should be noted, however, that Westinghouse Hanford had the responsibility for the waste handling operations at 325 from 1970 until 1987, at which time PNL assumed this role. During the earlier period it is likely that some PNL waste was designated as Westinghouse Hanford waste. It is also possible that some Westinghouse Hanford waste was designated as PNL waste after 1987.

The 325 Building A and B annexes contain three and six "hot" cells, respectively. The activities performed in these hot cells were quite different from the activities performed in the analytical laboratories. The wastes generated were often designated more specifically as '325A' and '325B'. The 325A annex has been operated solely by PNL since the mid-1960's. The waste designated as being from 325A is discussed in Section 7.0. The 325B annex was operated by PNL until the middle of 1970, when Westinghouse Hanford took occupancy of the hot cells. Waste designated as being from 325B is discussed in Section 8.0. Please note that it is extremely likely that some wastes from both $325 \mathrm{~A}$ and $325 \mathrm{~B}$ were designated as 325. 
WHC-EP-0696

This page intentionally left blank.

Intro-ii 


\subsection{CHARACTERIZATION OF RETRIEVABLY STORED SOLID WASTE GENERATED AT 325 BY WESTINGHOUSE HANFORD}

The information found in this section is based primarily on data from the Solid Waste Information and Tracking System (SWITS) database. This database, which incorporated the older R-SWIMS database, is used to track information on radioactive and other wastes stored or disposed at the Hanford Site. Transuranic solid waste packages have been tracked since 1970, however, changes in the requirements and regulations governing radioactive wastes have left their mark in the quantity and quality of the data contained in this database. Caveats are included in the text to alert the reader to changes that may affect the interpretation of the data provided.

Solid Waste Burial Records (SWBRs) are the original source of data found in the SWITS database and were used as a secondary source of information in this section. Between 1970 and 1980, the 325 Building was used as a collection point for all waste in the 300 Area. Although waste originated from other buildings in the 300 Area, it's not uncommon for the SWITS database to list the originating source as 325. Review of the original SWBRs clearly indicates a different facility as the originating source for some of the waste listed under 325. The majority of the tables and figures in this section are based on all waste listed in the SWITS database as 325 waste even though some of this waste did originate elsewhere. The summary, Tables 5-1 and 5-2, however, show the breakdown of waste designated as coming from 325 but known to have originated at other facilities.

Beginning in 1980 solid waste originating from 325 by Westinghouse Hanford was sent to $\mathbf{3 2 4}$ or $\mathbf{3 4 0}$ for interim storage and assay. Subsequently, waste containers from 325 were recorded in SWITS as originating from 324 or 340 , although the original SWBRs clearly indicate the 325 Building as the originating source of the waste. These waste containers are included in the tables and figures in this section, and data obtained from SWBRs rather than SWITS is clearly labeled.

The bulk of the data provided in this section is limited to information about the TRU waste that was generated at 325 by Westinghouse Hanford; however, some general information on the non-TRU waste is included for completeness. The term non-TRU waste is used instead of LLW because a small percentage of the unsegregated waste is designated only as not TRU. Tables 5-3 and 5-4 present TRU and non-TRU waste generation summaries by container type and year. As the initial retrieval efforts and WRAP 1 will focus on 55-gal drums, these containers are considered separately from all other container types. Therefore, the term "other containers" in this report will refer to all container types except 55-gal drums. The term "drum" refers to 55-gal drums only, unless specified otherwise.

The original SWITS data that form the basis for most of the tables and figures in this section can be found in Appendix A. Each computer run is preceded by the query used to generate the data.

Because of the large number of figures and tables in this section, they are placed at the end of the chapter. 
WHC-EP-0696

\subsection{SUMMARY OF 325 WASTE GENERATION BY WESTINGHOUSE HANFORD}

\subsubsection{Waste Stored in 55-Callon Steel Drums}

The most common waste container for TRU waste stored at the Hanford Site is the 55-gal steel drum. The drums used are either DOT 17C or 17H drums made from carbon sheet steel with minimum thicknesses of $0.053 \mathrm{in}$. and 0.043 in., respectively. Prior to 1982 most of the drums were painted, so these drums have approximately 0.005 in. of paint on both the exterior and interior of the drums. In 1982, galvanized drums replaced painted drums. Recycled 55-gal drums were also permitted for the storage of TRU waste between 1973 and 1978.

The 1970 Immediate Action Directive (AEC 1970) stipulated that TRU wastes be packaged and stored as contamination-free packages for at least 20 years. The 20 year interim period was to allow time to study permanent disposal options for TRU contaminated wastes.

As more of the 55-gal drums reach and exceed the twenty year storage mark, more attention is being given to ascertaining the condition of these drums. A discussion of the previous studies of steel drum corrosion and degradation can be found in WHC-0225, Rev. 1 (Anderson et al. 1991) and WHC-SA-1450FP (Duncan et al. 1992).

Table 5-5 cointains waste summary data for 55-gal drums of radioactive waste generated at 325 by Westinghouse Hanford between 1970 and 1992. The upper portion of this table presents the number, total weight, and total volume of TRU waste drums. The number of TRU mixed waste drums are also indicated. The same information for non-TRU waste follows. At the bottom of the table are the relative percentages of TRU and non-TRU wastes by container number, weight, and volume. Figures 5-1 through 5-6 present this information graphically.

5.1.1.1 Number of Drums. Between 1970 and 1992, 3,803 55-gal drums of radioactive waste were generated by Westinghouse Hanford at 325. This total includes 1,069 drums of non-TRU and 2,414 drums of TRU waste. The total number of radioactive mixed waste drums is $\mathbf{4 5}$ which are all non-TRU MW drums. Figure 5-1 provides a graph of the number of 55-gal drums of TRU and non-TRU generated by Westinghouse Hanford at 325 annually. The major features of this graph are directly related to historical events that took place at 325.

The Commercial Alpha Waste Program was developed in the early 1970's to study and implement an alpha waste reduction plan. Six tasks provided objectives for the treatment and disposal of alpha waste through waste segregation, waste product criterion and evaluation, and waste container criterion and evaluation. Results from the programs are seen in Figure 5-1, which indicates a general decline in TRU waste during the mid-1970's.

The large TRU peaks in 1978 and 1979 are probably due to expanded support of the Fast Flux Test Facility (FFTF) prior to its startup. 
Figure 5-2 shows the relative percentage of TRU and non-TRU by year.

5.1.1.2 Weight. In the period between 1970 and 1990 approximately $186,999 \mathrm{~kg}$ of TRU waste and $138,092 \mathrm{~kg}$ of non-TRU waste generated by Westinghouse Hanford at 325 were stored in 55-gal drums. These numbers are estimates since container weights were not required for individual containers prior to 1977. During the R-SWIMS data reentry program in the mid-1980's, all pre-1977 containers were assigned standard weights. All 55-gal drums, for instance, were assigned a value of $68 \mathrm{~kg}$ (150 lbs). Since 1979, the average vieight of all 325 drums generated by Westinghouse Hanford has been $89 \mathrm{~kg}$. However, while the average weight of TRU drums from that period was $68 \mathrm{~kg}$, the average weight of non-TRU drums was $143 \mathrm{~kg}$, making these drums over $100 \%$ heavier than the TRU drums.

Figure 5-3 presents the total weight of both TRU and non-TRU radioactive wastes stored annually in 55-gal drums. The relative weight percentages for TRU and non-TRU drums generated annually by Westinghouse Hanford at 325 are shown in Figure 5-4.

The large TRU peak in 1979 is probably a result of the removal and replacement of corroded HVAC ducts. This and other TRU wastes in 1979 were stored and buried in 55-gal drum containers only.

5.1.1.3 Volume. The total volume of waste generated by Westinghouse Hanford at 325 and stored in 55-gal drums is $774 \mathrm{~m}^{3}$, of which $507 \mathrm{~m}^{3}$ is TRU and $267 \mathrm{~m}^{3}$ is non-TRU. Annual waste generation information is shown in Figure 5-5; the volume percentages of radioactive waste stored in 55-gal drums are provided in Figure 5-6. Since all 55-gal drums have a volume of $0.21 \mathrm{~m}^{3}$, the patterns shown in Figures 5-5 and 5-6 are the same as those described for Figures 5-1 and 5-2 (drum number), respectively. They are included here so that comparisons with later figures can be made (Figures 5-11 and 5-12).

\subsubsection{Waste Stored in Containers Other Than 55-Gallon Steel Drums}

Radioactive solid wastes have been stored or disposed of in a wide variety of containers other than 55-gal steel drums. Other container types used for solid waste generated at 325 by Westinghouse Hanford include the following:

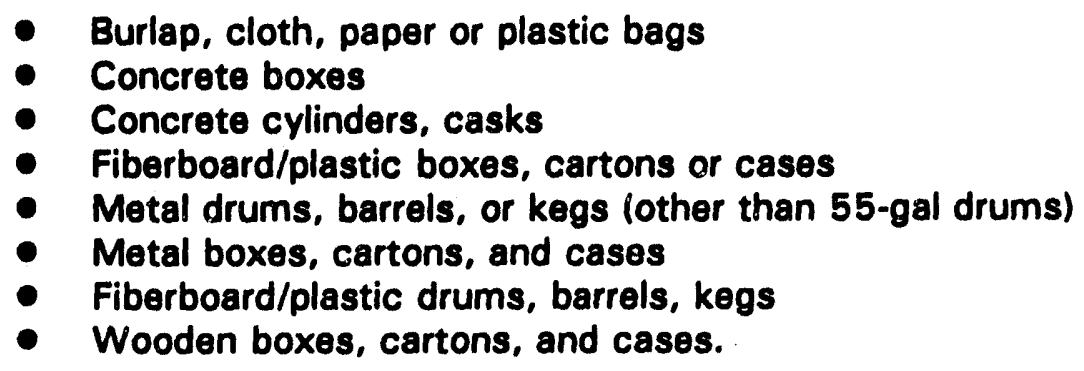

Some items were disposed of or stored without additional packaging, such as dump trucks; gloveboxes; HEPA filters; miscellaneous scrap; trucks, flatbeds, compactors, loadluggers; self-contained equipment; portable tanks, and metal cylinder casks. These items are also included in the "Other Container" category. 
Table 5-6 summarizes the waste data for containers of radioactive waste other than 55-gal drums that were generated at 325 by Westinghouse Hanford between 1970 and 1992. The upper portion of this table indicates the number, total weight and total volume of TRU waste stored in other containers. The number of other containers of TRU mixed waste is also provided. The same information for non-TRU waste follows. At the bottom of this table are the relative percentages of TRU and non-TRU wastes by container number, weight and volume. Figures 5-7 through 5-12 present these data graphically.

5.1.2.1 Number of Other Containers. Between 1970 and 1992, 19,887 other containers of radioactive waste were generated at 325 by Westinghouse Hanford. Of these containers, 97 hold TRU waste, while 19,790 contain non-TRU waste. For reference, during the same period of time a total of 3,483 55-gal drums of radioactive solid waste were generated at 325 by Westinghouse Hanford. Figure 5-7 is a graph of the number of other containers of TRU and non-TRU waste generated at 325 by Westinghouse Hanford by year.

The largest number of other containers containing TRU waste was generated in 1972 , with 25 containers. This is the result of a large number of concrete boxes. The vast majority of other containers contain non-TRU waste. In the years 1970 to 1992 , over $99 \%$ of the waste is non-TRU with fiberboard/plastic boxes, cartons, and cases accounting for over $91 \%$ of the non-TRU total.

Figure 5-8 presents the relative percentages of TRU and non-TRU containers other than 55-gal drums. Two things are apparent from this figure: no TRU waste was packaged in other containers from 1986 to 1992 , almost all of the waste in other containers was nonTRU.

5.1.2.2 Weight. In the period between 1970 and 1992, approximately $1,142,956 \mathrm{~kg}$ of radioactive solid waste was generated at 325 by Westinghouse Hanford and placed in containers other than 55-gal drums. This total includes approximately $95,127 \mathrm{~kg}$ of TRU and $1,047,829 \mathrm{~kg}$ of non-TRU contaminated material. These weights are approximations because individual container weights were not required to be recorded for individual containers prior to 1977. During an update of the R-SWIMS database in the mid-1980's, all waste containers without recorded weights were assigned standard values. For example, all $2 \mathrm{ft}^{3}$ boxes were given a standard weight of $5.5 \mathrm{~kg} \mathrm{(12} \mathrm{lb)} \mathrm{and} 5$ gal lard cans were assigned a standard weight of $22.7 \mathrm{~kg}(50 \mathrm{lb})$.

Figure 5-9 depicts the weight of TRU and non-TRU waste stored in containers other than 55-gal drums on an annual basis. The most notable TRU peaks in 1972, 1974, and 1975 are due mainly to 16 concrete boxes, one $7 \mathrm{ft} \times 10 \mathrm{ft} \times 16 \mathrm{ft}$ metal box, and one $1,250 \mathrm{ft}^{3}$ metal box, respectively. Some of the weight represented by the large non-TRU peaks in 1976 and 1979 is a result of the disposal of trucks, flatbeds, compactors and/or loadluggers.

Figure 5-10 shows the percent contribution of TRU and non-TRU waste to the total annual weight of waste packaged in other containers. Because of the practice of storing TRU waste in drums when possible, proportionately less of the other container waste is TRU.

5.1.2.3 Volume. The volume of waste generated at 325 by Westinghouse Hanford and packaged in containers other than 55-gal drums during the period from 1970 through 1992 
is $4,758 \mathrm{~m}^{3}$. Transuranic wastes account for $195 \mathrm{~m}^{3}$ of the total volume; non-TRU wastes account for $4,563 \mathrm{~m}^{3}$. Figure 5-11 shows annual volumes of TRU and non-TRU wastes generated at 325 by Westinghouse Hanford and packaged in containers other than 55-gal drums. Figure 5-12 shows the relative contribution of TRU and non-TRU wastes to the total volume of waste in other containers for each year.

\subsubsection{Summary of Waste Ceneration Rates at Westinghouse Hanford 325 Botween 1970 and 1992}

5.1.3.1 Number of Waste Containers. Between 1970 and 1992, there were 23,370 containers of radioactive solid waste generated at 325 by Westinghouse Hanford. Fifty-five gallon drums account for 3,483 of the total number of containers. Containers of transuranics comprise only $11 \%$ of the total container numbers.

Figure 5-13 shows the total numbers of both TRU and non-TRU waste containers generated on an annual basis during this period. The difference in the amount of waste characterized as TRU and non-TRU from 1970 to 1992 is most evident in this graph. As mentioned previously, the Commercial Alpha Waste Program helped to reduce the amount of TRU waste during the mid-1970's. In addition, changes in the DOE (formerly AEC) regulations and disposal practices directly affected the proportion of the waste determined to be TRU and non-TRU. A significant portion of the waste stored as TRU prior to 1982 may be redesignated as LLW upon characterization.

5.1.3.2 Total Weight. Approximately $1,468,047 \mathrm{~kg}$ of radioactive solid waste was generated at 325 by Westinghouse Hanford between 1970 and 1992. Waste packaged in 55-gal drums accounts for about $22 \%$ of the total weight of waste from Westinghouse Hanford 325 , with $78 \%$ of the total weight comprised of waste packaged in some other type of waste container.

Waste designated as TRU makes up $19 \%$ of the total weight while waste designated as non-TRU makes up the remainder. Figure 5-14 provides a graph of the total weight of TRU and non-TRU waste generated at 325 by Westinghouse Hanford on a yearly basis between 1970 and 1992.

The majority of the weight, $66 \%$, of TRU waste is contained in 55-gal drums followed by metal boxes, cartons, cases with $44,203 \mathrm{~kg}$ or $16 \%$ of the TRU waste weight. Concrete boxes with $8 \%$ of the waste accounted for $23,354 \mathrm{~kg}$ of TRU waste.

5.1.3.3 Total Volume. In the period between 1970 and $1992,5532 \mathrm{~m}^{3}$ of radioactive solid wastes were generated at 325 by Westinghouse Hanford. Only $14 \%$ of this volume is comprised of $55-\mathrm{gal}$ drums; the remaining $86 \%$ of the volume is made up of other container types. Transuranic wastes account for $13 \%$ of the waste volume, while non-TRU wastes make up the remaining $87 \%$. Figure 5-15 shows the total volume of TRU and non-TRU waste generated at 325 by Westinghouse Hanford on a yearly basis.

One interesting fact observable in this figure shows all TRU waste generated in 1973, 1979,1984 , and 1987 was disposed of in 55-gal drums oniy. 


\subsection{WASTE CONTAINERS}

\subsubsection{TRU Waste Containers}

Table 5-3 provides a summary of the TRU waste generated annually at 325 by Westinghouse Hanford from 1970 to 1992 sorted by container type. The greatest number of containers are 55-gal drums, 2,414 by count. The percentages for all container types and the weight and volume of these containers are shown for each year in this table.

\subsubsection{NON-TRU Waste Containers}

Table 5-4 provides a summary of the non-TRU waste generated at 325 by Westinghouse Hanford from 1970 to 1992 by container type. Fiberboard/plastic boxes, cartons, and cases were the most common containers used accounting for more than $86 \%$ of the total non-TRU waste count. Significant numbers of metal drums, barrels, and kegs were also generated including 55-gal and other container sizes.

\subsection{TRU WASTE STORAGE LOCATIONS}

Table 5-7 provides the storage locations for TRU waste packaged in 55-gal drums by year. Storage locations for TRU waste packaged in other containers are shown in Table 5-8. In general $58 \%$ of the drums and $56 \%$ of other containers generated at 325 by Westinghouse Hanford are stored at burial ground $218-W-4 B$. A summary of 325 waste by PNL by storage location follows:

- 218-W-3A - Burial Ground 218-W-3A consists of 14 earthen-bottom, gravel filled trenches with waste emplaced from May 1970 to April 1988. Drums and other containers from 325 by Westinghouse Hanford were disposed here in the years between 1970 and 1978; they contain no hazardous constituents according to the SWITS database search.

- 218-W-4B - Burial Ground 218-W-4B, consisting of 14 trenches and 5 trenches, accepted retrievable waste through September of 1978. One thousand four hundred forty-five drums and other containers from 325 by Westinghouse Hanford were buried here from 1971 to 1978.

- 218-W-4C - Burial Ground 218-W-4C consists of six trenches with the first waste emplaced in March 1978. Seven-hundred-eighty-six 55-gal drum TRU waste was sent to this area from 1978 to 1987 with four other containers emplaced between 1980 and 1985.

\subsection{PHYSICAL CONTENTS OF TRU WASTE CONTAINERS}

The physical contents of 55-gal drums are shown in Tables 5-9 through 5-11. There is a separate table for each storage location. The top portion of each table indicates the 
number of drums for which a given component is listed; the bottom part of the table indicates the percentage of the total drums from 325 by Westinghouse Hanford in that burial location that contain the listed constituent. The physical contents for TRU waste containers other than 55-gal drums can be found in Tables 5-12 through 5-14.

It should be noted that prior to 1978 , physical contents were not required to be listed on the burial records. A great many of the contents on early records are listed only as "Miscellaneous".

\subsection{RADIOLOGICAL DESCRIPTION OF SOLID WASTE AT 325 BY WESTINOHOUSE HANFORD}

Table 5-15 shows the number of grams of transuranics contained in waste stored in 55-gal drums each year by storage location; Table 5-16 provides the same information for containers other than 55-gal drums. Figure 5-16 combines the information on these tables in a graph that shows the total amount of transuranics present in waste packages from Westinghouse Hanford 325 by year. The noticeable peak in the quantity of transuranics present in the 325 waste from the late 1970's reflects the high level of analytical support for the FFTF in these years.

\subsection{RADIOACTIVE ISOTOPES PRESENT IN SOLID WASTE AT 325 BY WESTINGHOUSE HANFORD}

The information on radioactive isotopes found in Table 5-17 and 5-18 was obtained from the SWITS database searches. Table 5-17 lists the isotopes found in 55-gal drums along with their half lives, quantity, and radioactive emission type. Table 5-18 contains this information for waste from 325 stored in containers other than 55-gal drums.

\subsection{HAZARDOUS CONSTITUENTS OF SOLID WASTE GENERATED AT 325 BY WESTINGHOUSE HANFORD}

This section provides a review of the data on the hazardous components known or suspected to be in the solid waste stream from Westinghouse Hanford at 325 . Information was gathered from several sources, including: the SWITS database, SWBRs, personnel interviews, an internal report examining hazardous components of solid waste, Inventory of Chemicals Used at Hanford Site Production Plants and Support Operations (Klem 1990), and Unstable and Reactive Chemicals in Transuranic Retrievable Waste at the Hanford Site (Reddinger 1992).

\subsubsection{Chemical Inventories}

Tables 5-19 and 5-20 provide lists of chemicals used at the Westinghouse Hanford Company Development Laboratory from 1970 to 1976 and the 325 Building Analytical Laboratory from 1953 to 1976, respectively. These lists were developed by Klem (1990) to support waste characterization efforts for the single-shell tanks. Although most liquids from 
the 325 Building did end up in the tanks, it may be possible to find small amounts of these chemicals in solid waste containers. These lists are based on chemical process flow sheets, essential materials consumption records, letters, reports, and other historical data.

The final two columns in both of the tables cited above contain information on the toxicity and hazards associated with each of the chemicals listed. The first of these columns lists the EPA toxicity category, which was determined using the Registry of Toxic Effects of Chemical Substances Quarterly Issue (NIUSH 1992) and the EPA "Spill Table" (EPA 1989). In cases where the two sources differed, the more recent source, the Registry of Toxic Effects, was used. Category $X$ marks the most toxic chemicals, followed by Categories A, B, $C$, and D, listed in order of decreasing toxicity. The EPA "Spill Table" is incorporated by reference into the Washington State Administrative Code, Chapter 173-303, Dangerous Waste Regulations. The Registry of Toxic Effects is a quarterly update to the EPA "Spill Table." The final column lists the U.S. Department of Transportation (DOT) designation found in the DOT Hazardous Materials Table (49 CFR 172.101).

\subsubsection{Solld Waste Information and Tracking System (SWITS)}

The hazardous constituent field in the SWITS database was searched for all containers from Westinghouse Hanford Building 325 that contained radioactive mixed waste. The database search revealed no TRU containers from Westinghouse Hanford Building 325 with mixed waste.

\subsubsection{Burial Records}

The SWBR or SWSDR for a given container is the source of waste container information abstracted for the SWITS database. Often these records will have more detailed information on the hazardous components of a waste containers than is found in SWITS. Additional data can also be found on the supplementary forms often attached to the SWBR or SWSDR. These supplementary documents include Uniform Hazardous Waste Manifests, Contents Inventory Sheets, NRC 741 Forms, and Storage/Disposal Approval Records (SDARS).

A review of the Westinghouse Hanford 325 burial records for all TRU mixed waste containers was made. Table 5-21 contains a summary of the information obtained. Table 5-24 is a summary of the low-level mixed waste containers. The presence of asbestoscontaining waste material was included in the summary tables despite the fact that it is not considered to be mixed waste. Lead shielding is also not considered to be waste at the current time.

\subsubsection{Interviews with 325 Building Personnel}

From the information gained in interviews with 325 Building personnel, a list of known or suspected components in the solid waste stream has been compiled. As much information as possible concerning the quantity, form, packaging, and relevant use of each of the 
constituents was provided by the interviewees. It is important to note that the liquids below were typically disposod of in a nonliquid form, often absorbed by kitty litter, rags, cotton swabs, lab coats, or other absorbents.

Mercury (Hg)

Mercury was used in large quantities in the 325 laboratory. Mercury was used in diffusion pumps and up to $15 \mathrm{~cm}^{3}$ was used as an electrode in coulometry cells. Although mercury was recycled whenever possible, both free and concreted mercury are expected to be found in the waste from 325 . It is expected that the maximum amount of free mercury in a drum might exceed $15 \mathrm{~cm}^{3}$.

Boron Carbide Boron carbide was used as a poison for the FFTF core.

Sodium Bisulfite

Sodium bisulfite was used by the pound at Building 325 . It is believed most of this solution went to liquid waste; it is possible that there is some in the solid waste.

Zirconium (Zr)

There were small amounts of zirconium metal used in Building 325 , so not much is expected to be in the waste from this facility. In the 1988 time frame there was some uranium-zirconium alloy work that was going on, but it was felt that most of the waste would have been liquid, and any waste of this type that was sent to solid waste would have been concreted first.

Asbestos

Asbestos sheet, approximately $5 \mathrm{ft}$ by $3 \mathrm{ft}$, was used to line the gloveboxes because of the furnaces. In addition, asbestos was used in the gaskets. Since it was in the gloveboxes, any asbestos encountered may be corrosive due to absorbed acid. Not much of the asbestos disposed of at Building 325 was believed to be in friable form. If it was friable, it was wetted and sealed in 5 quart cans.

Nitric Acid

Potassium/Sodium Hydroxide

Propane Cylinders

Nitric acid was used little in the processing of fuel. Most of the liquid waste was neutralized and mixed with Portland cement.

Potassium and sodium hydroxide was neutralized and concreted.

One interviewee remembered three or four propane cylinders that were disposed of at Building 325. These cylinders were definitely empty and had their valves extracted prior to packaging.

Sodium Metal (Na) All sodium waste was reacted prior to disposal.

Uranyl Nitrate

One respondent thought that there was a possibility of uranyl nitrate precipitate in the waste from Building 325 , but added that it was usually grouted with cement.

Metal fines

No metal fines are expected to be in the drums from Building 325 according to facility personnel. 
Aerosol cans

Lead (Pb)

Nitrated Rags

Scintillation Liquids
Unvented aerosol cans are potentially present in the drums from Building 325. Most commonly these cans would contain Windex' or WD- $40^{2}$.

Lead shielding and bricks are expected.

It is possible that rags and paper towels used to clean up nitric acid spills are present in the waste from Building 325 . Facility personnel did not recall any effort being made to dry these items prior to disposal. After 1980, such items would have been neutralized and grouted prior to disposal.

Organic solvents commonly used in scintillation cocktails (toluene, xylene, etc.) were disposed of in scintillation vials. Vial contents were poured into absorbent, usually kitty litter, in a metal can. Conwed ${ }^{3}$ fiber was used as an absorbent in the most recent years. The metal cans were usually one quart "ice cream" cans, which had a slip lid, although 5 quart and other size cans were sometimes used. When filled, the cans were placed in double lined bags. These bags, in turn, were packaged in 55-gal drums with more kitty litter. The amount of kitty litter was determined by assuming that the entire volume of the can was liquid. In the early 1980's an absorbent to liquid ration of $1: 1$ was required; later this ratio was changed to 2:1. Approximately five to eight cans were packed into each 55-gal drum.

'Trademark of Drackett Company.

${ }^{2}$ Trademark of WD-40 Company.

${ }^{3}$ Trademark Conwed Plastics. 


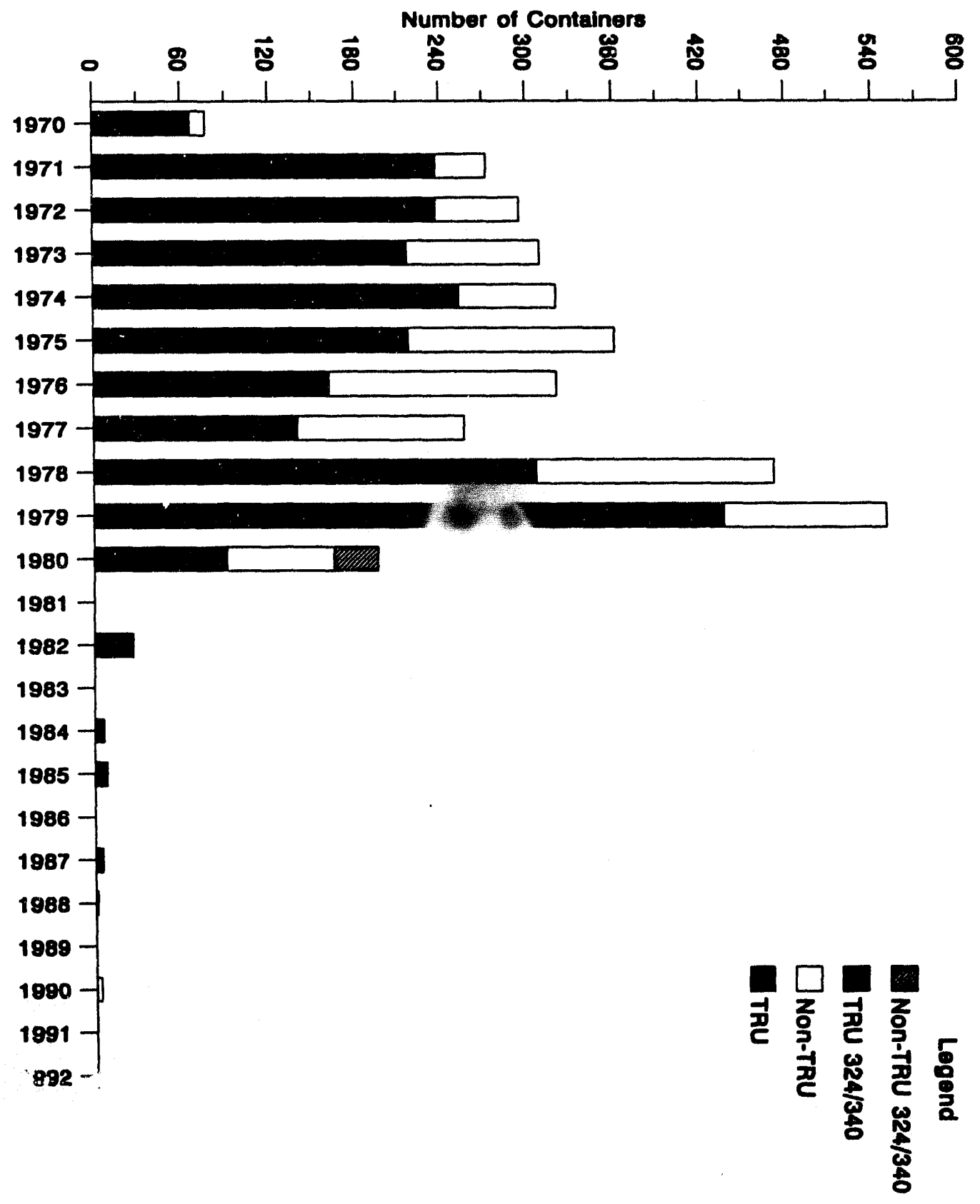

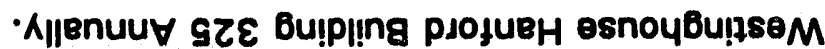

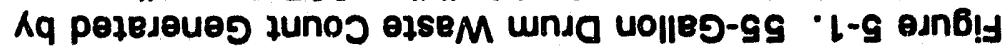


Figure 5-2. Percentage of Westinghouse Hanford Building 325 TRU/ Non-TRU 55-Gallon Drums.
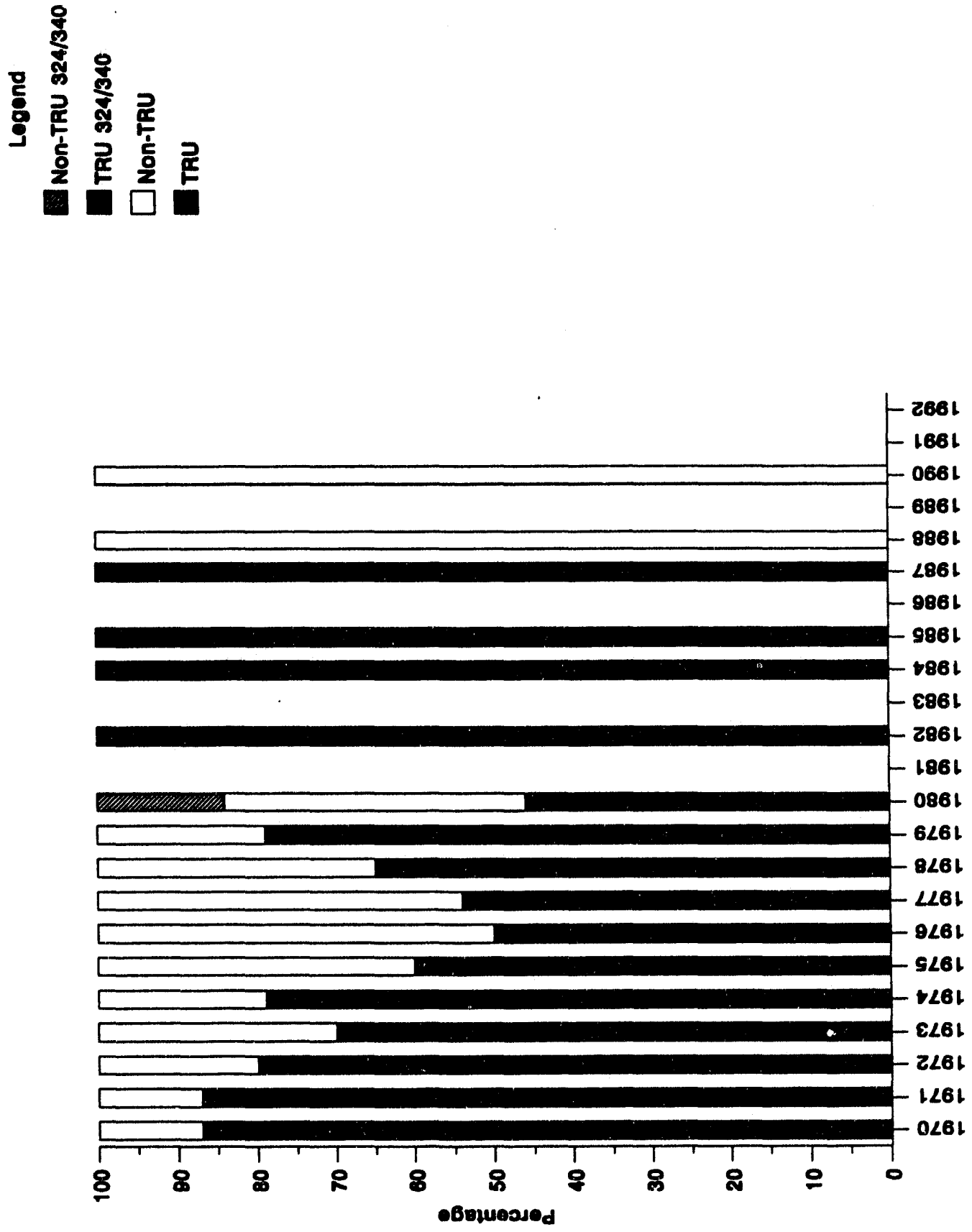


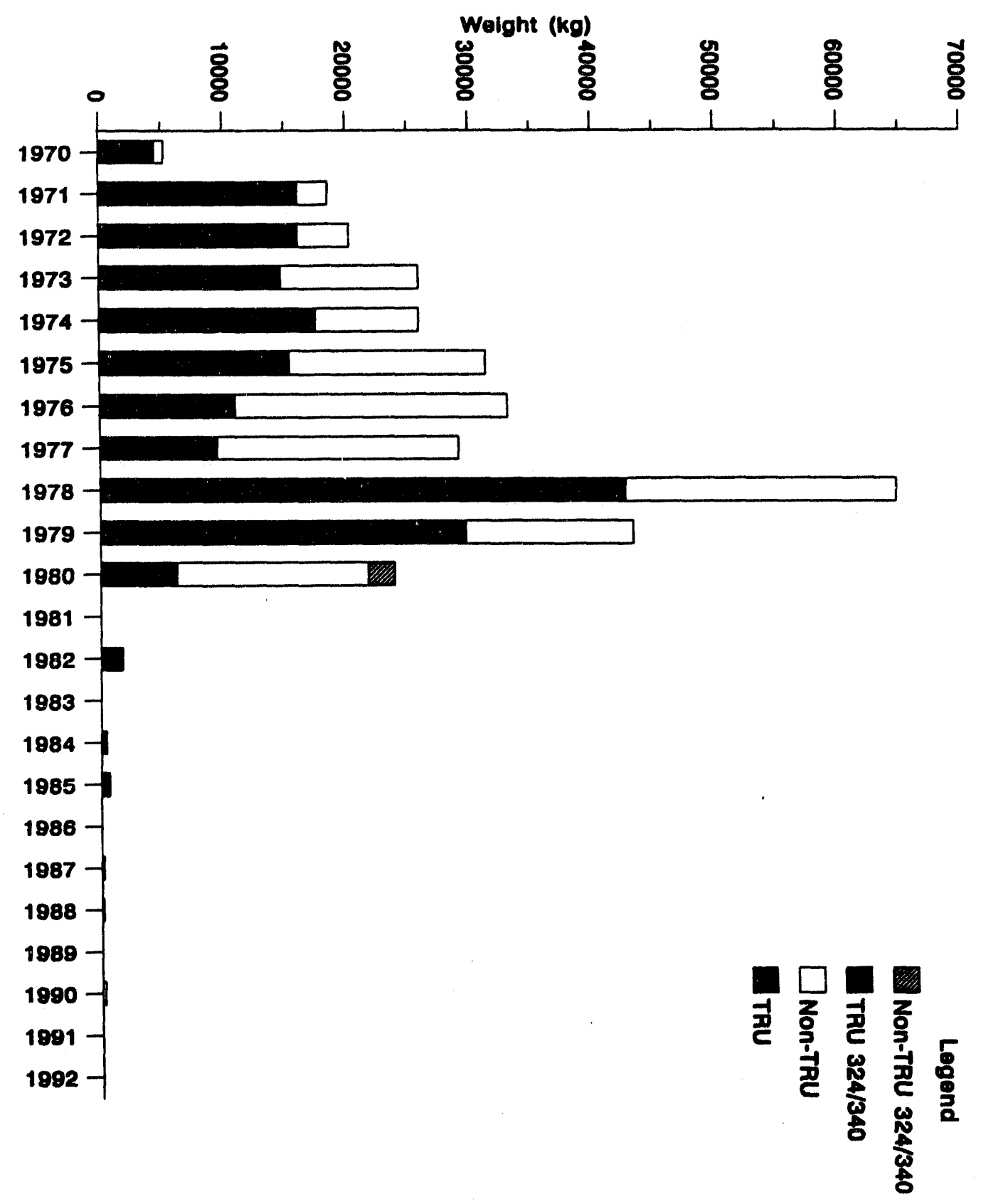

- Aा|enuU

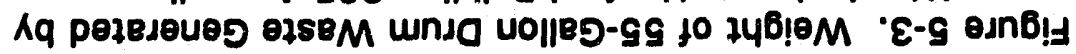




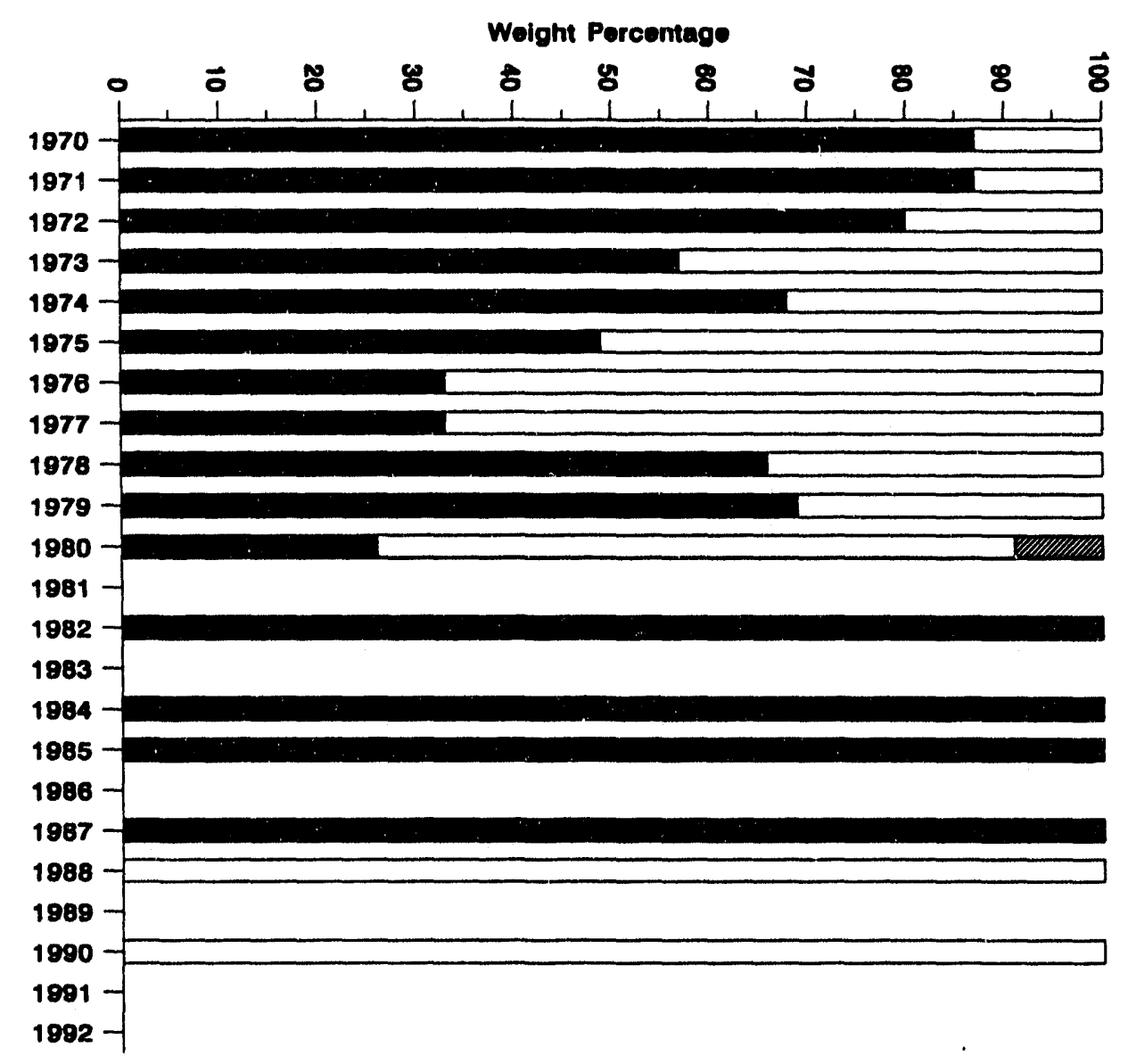




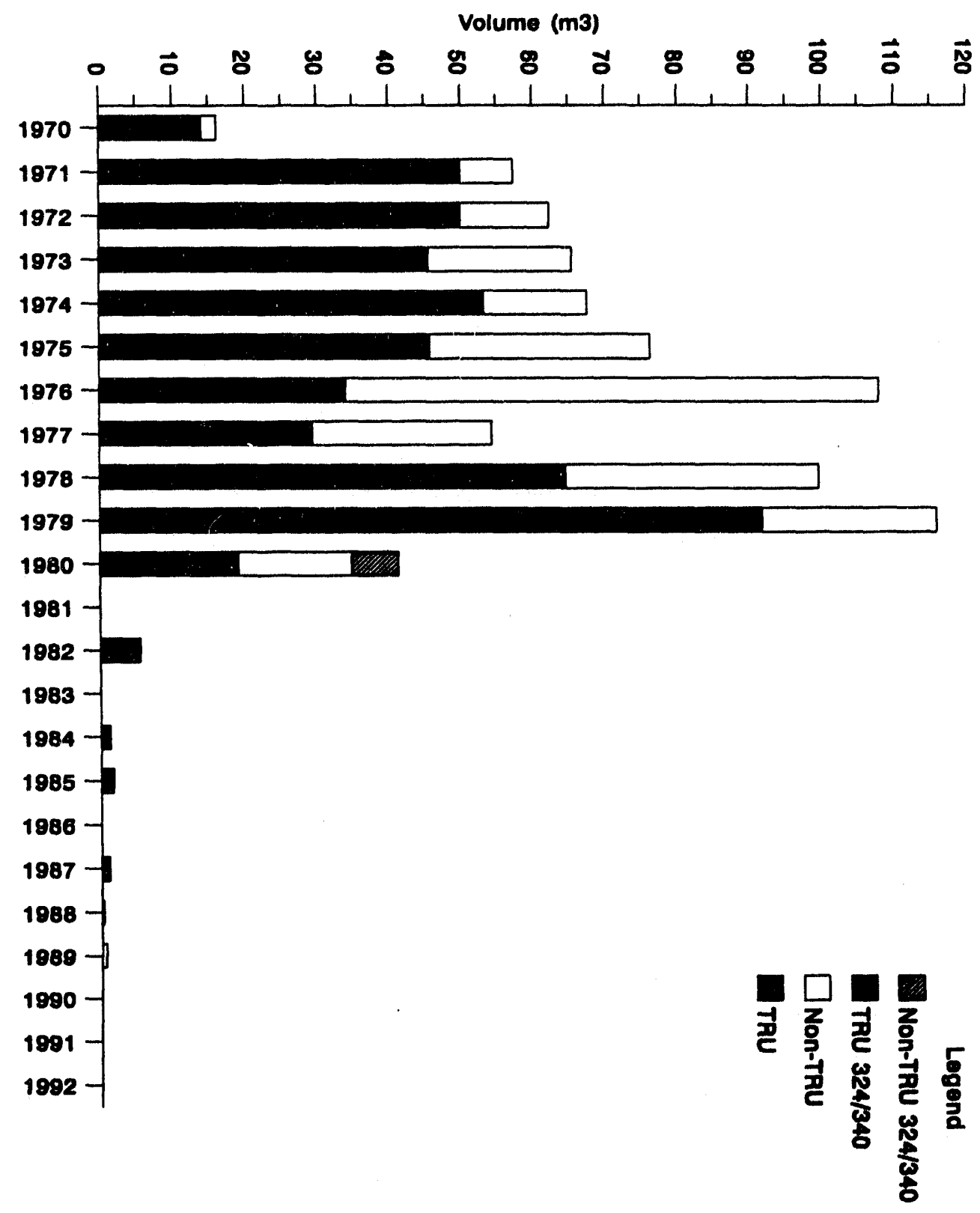

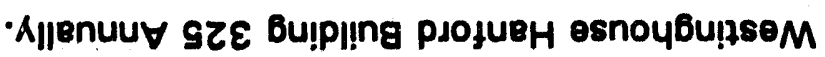

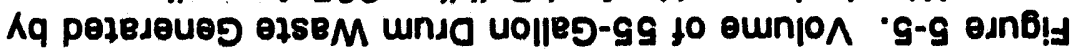


8
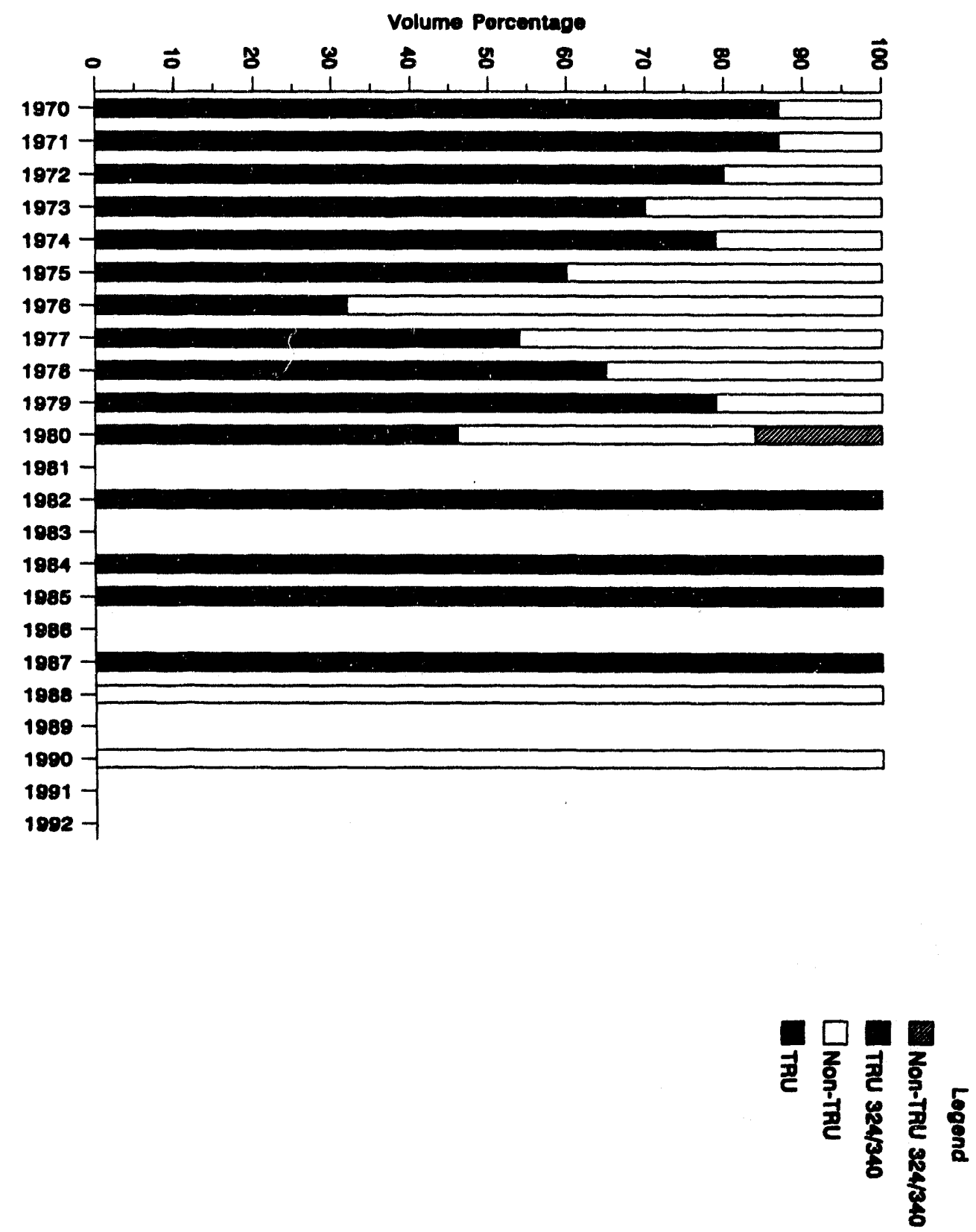

-sundg Uo||85-9g

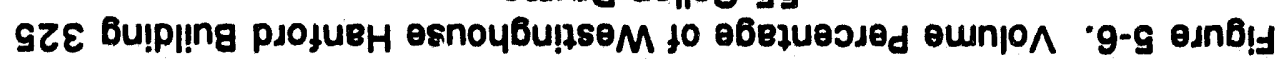




\section{$\angle L-9$}

点

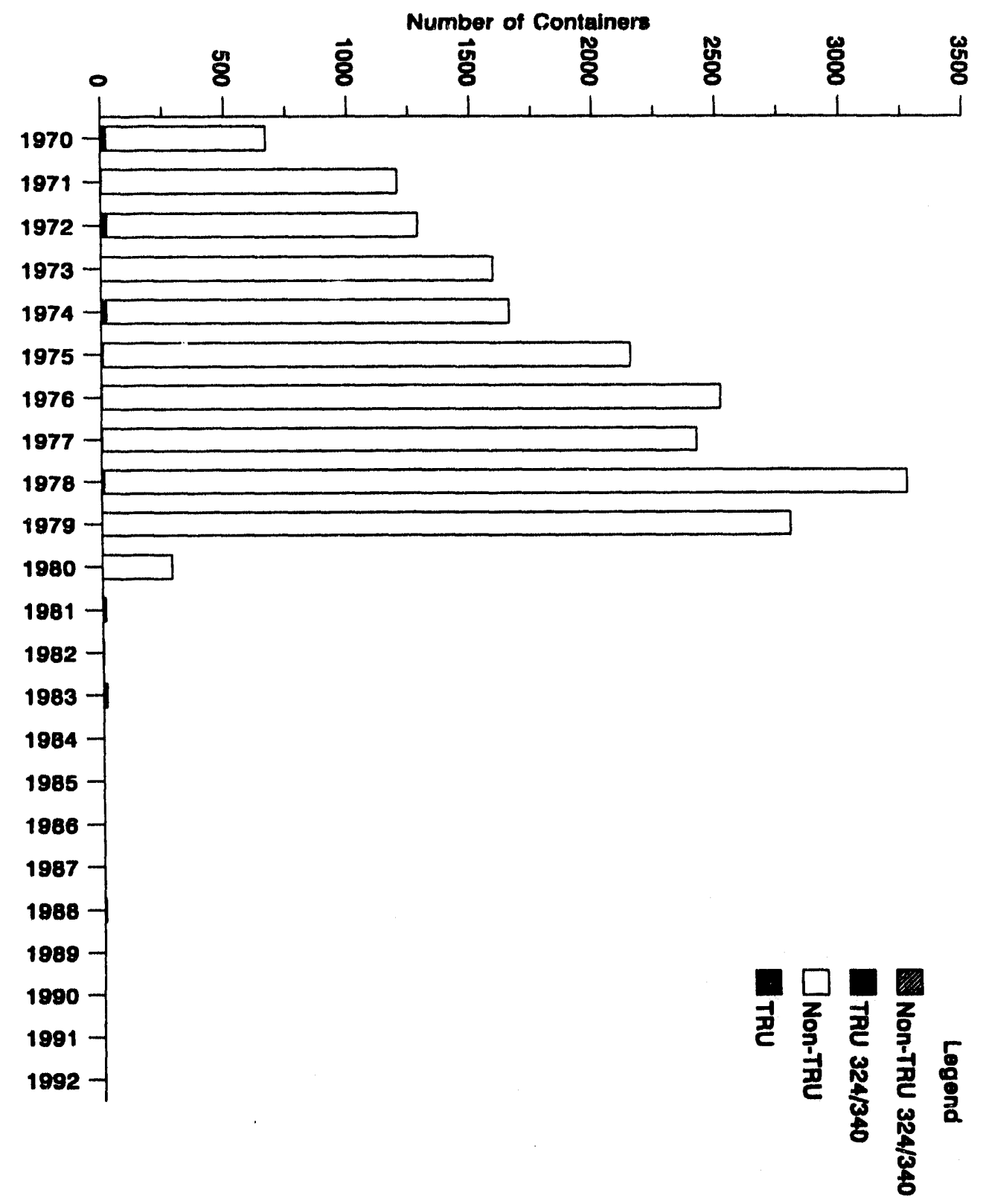

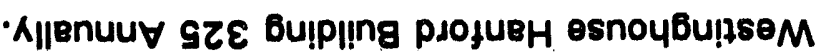

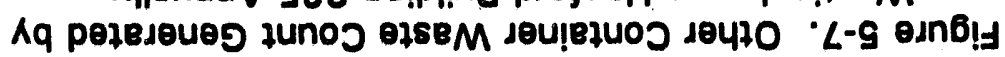




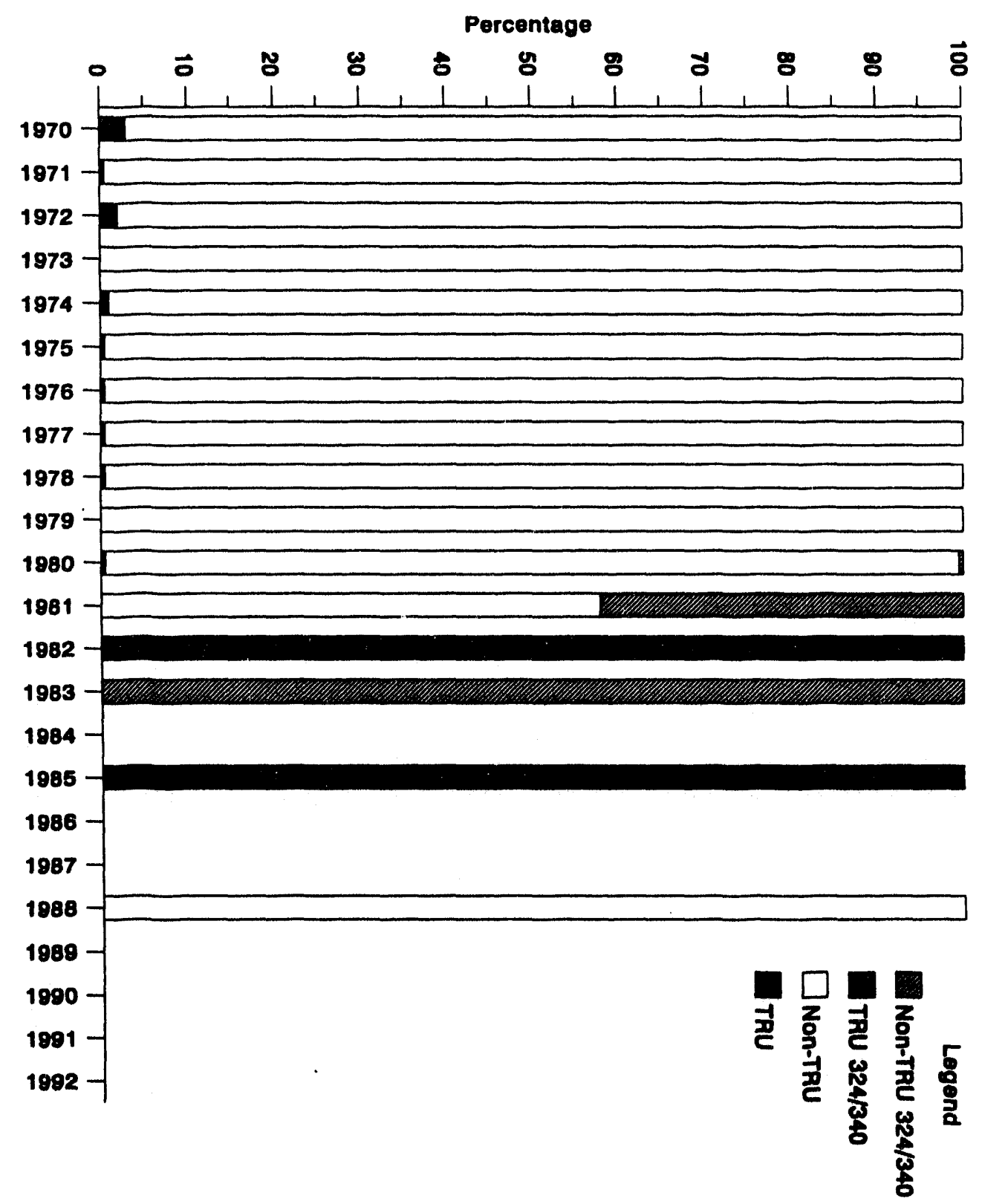




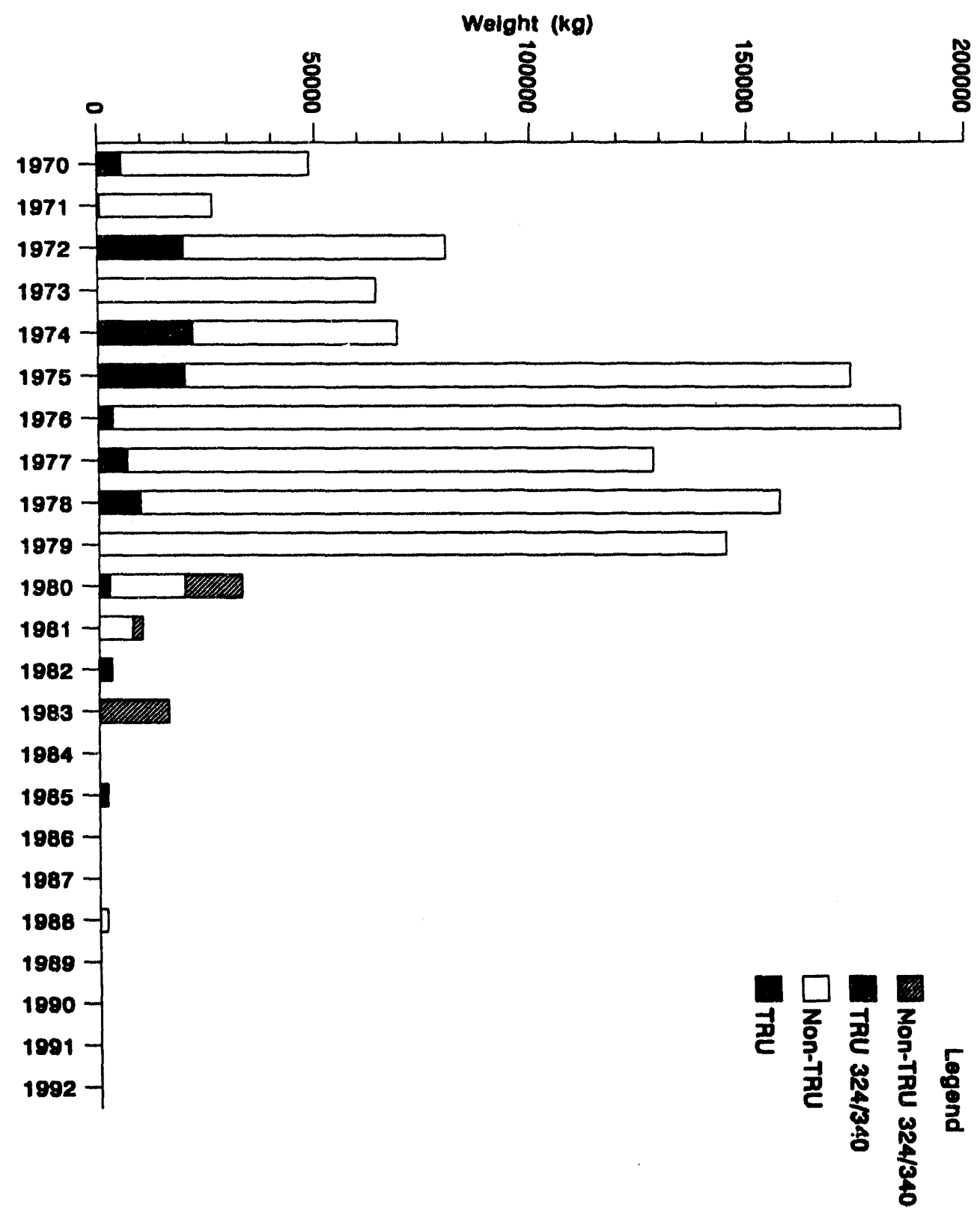

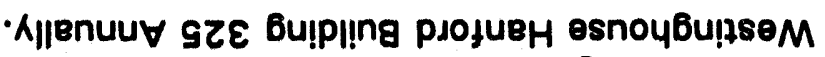

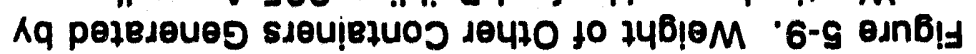


Figure 5-10. Weight Percentage of Other Containers Generated by Westinghouse Hanford Building 325 Annually.

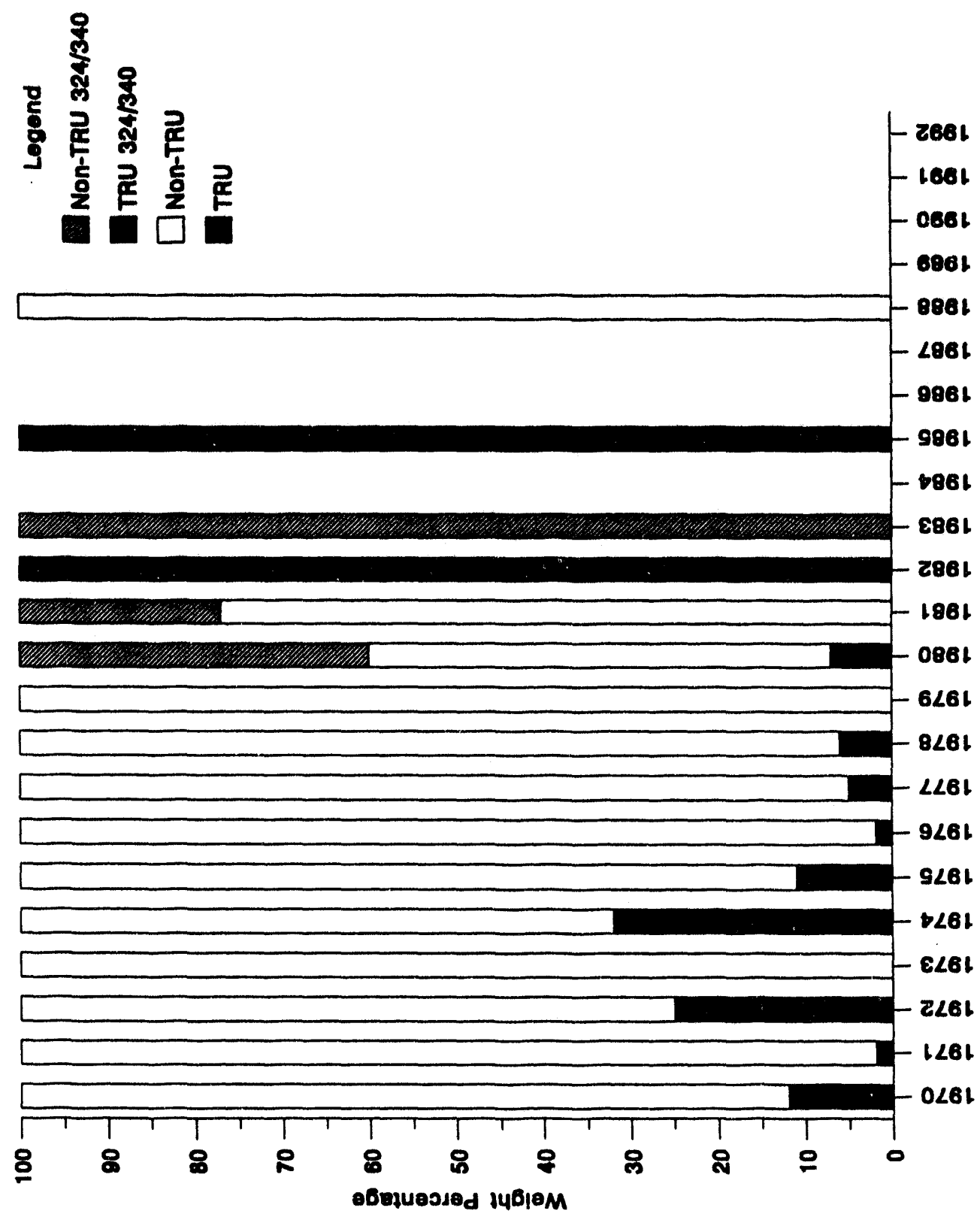




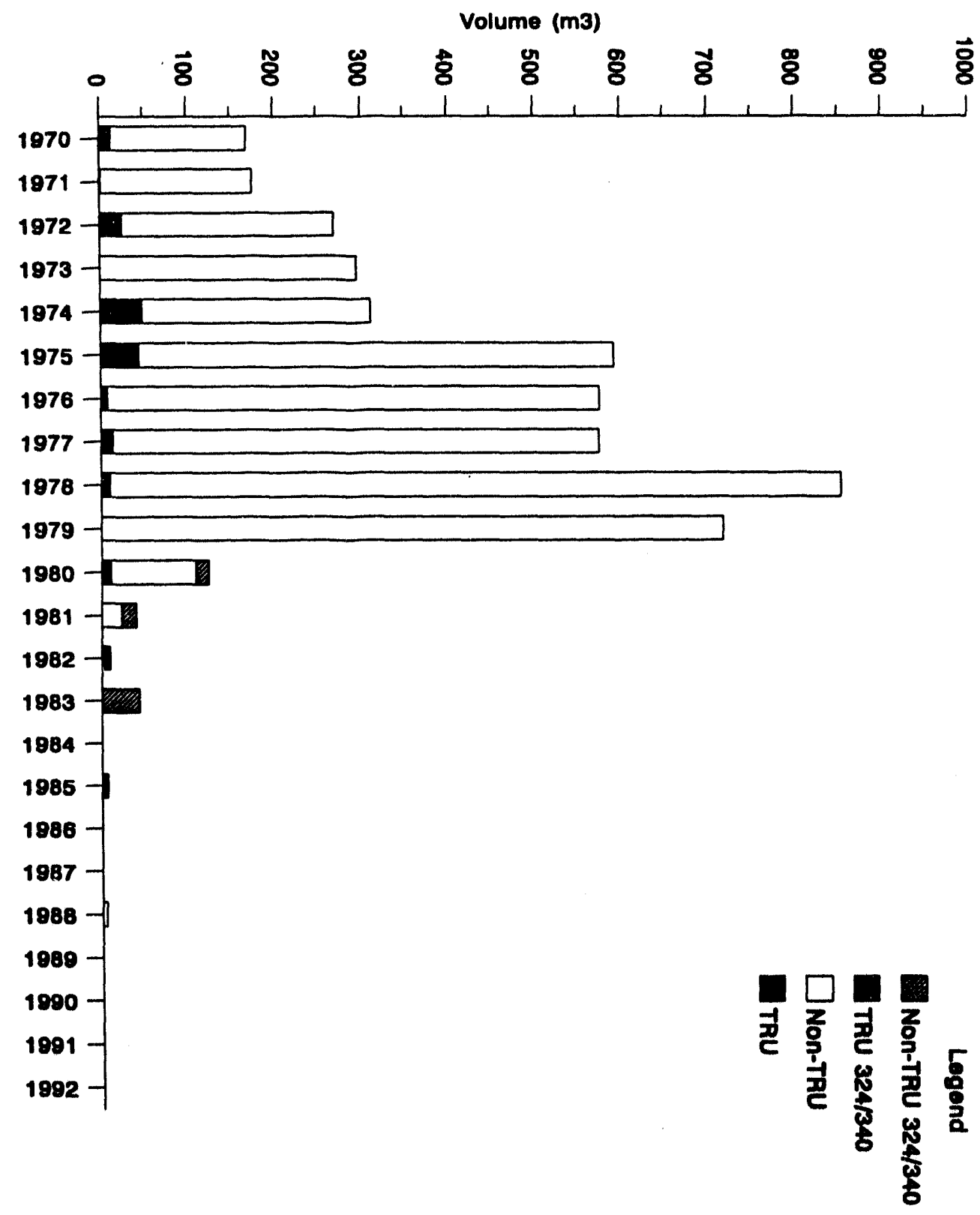

- Aןвnuu

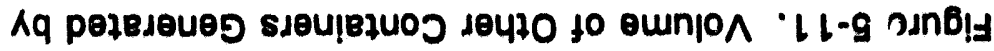




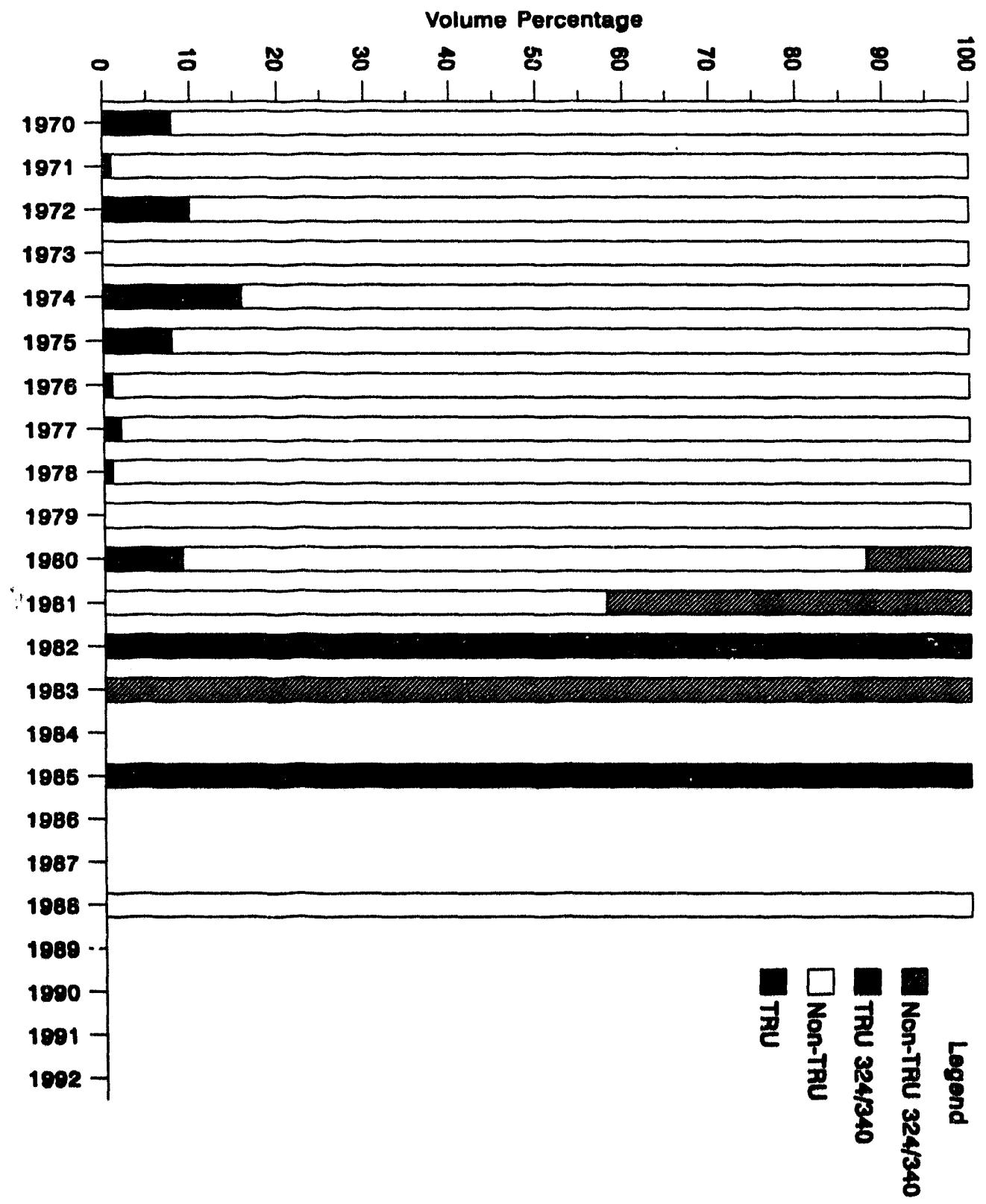

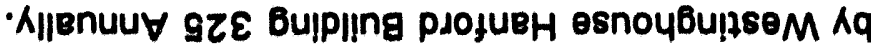

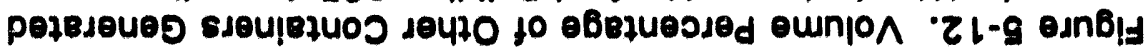


$\varepsilon 乙-9$

产

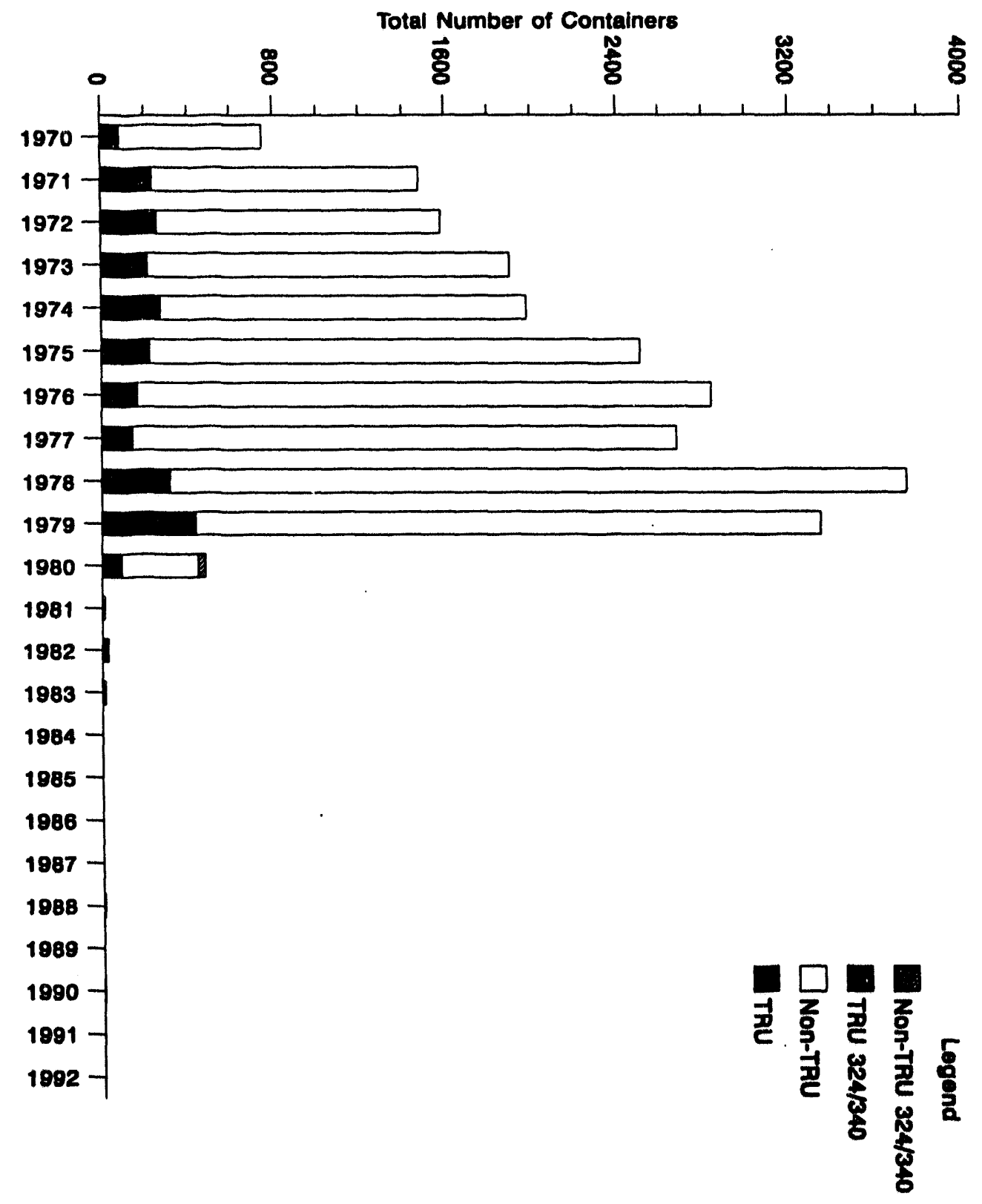

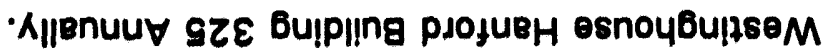

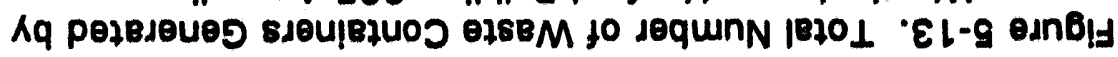


Figure 5-14. Total Weight of Waste Generated by Westinghouse Hanford Building 325 Annually.

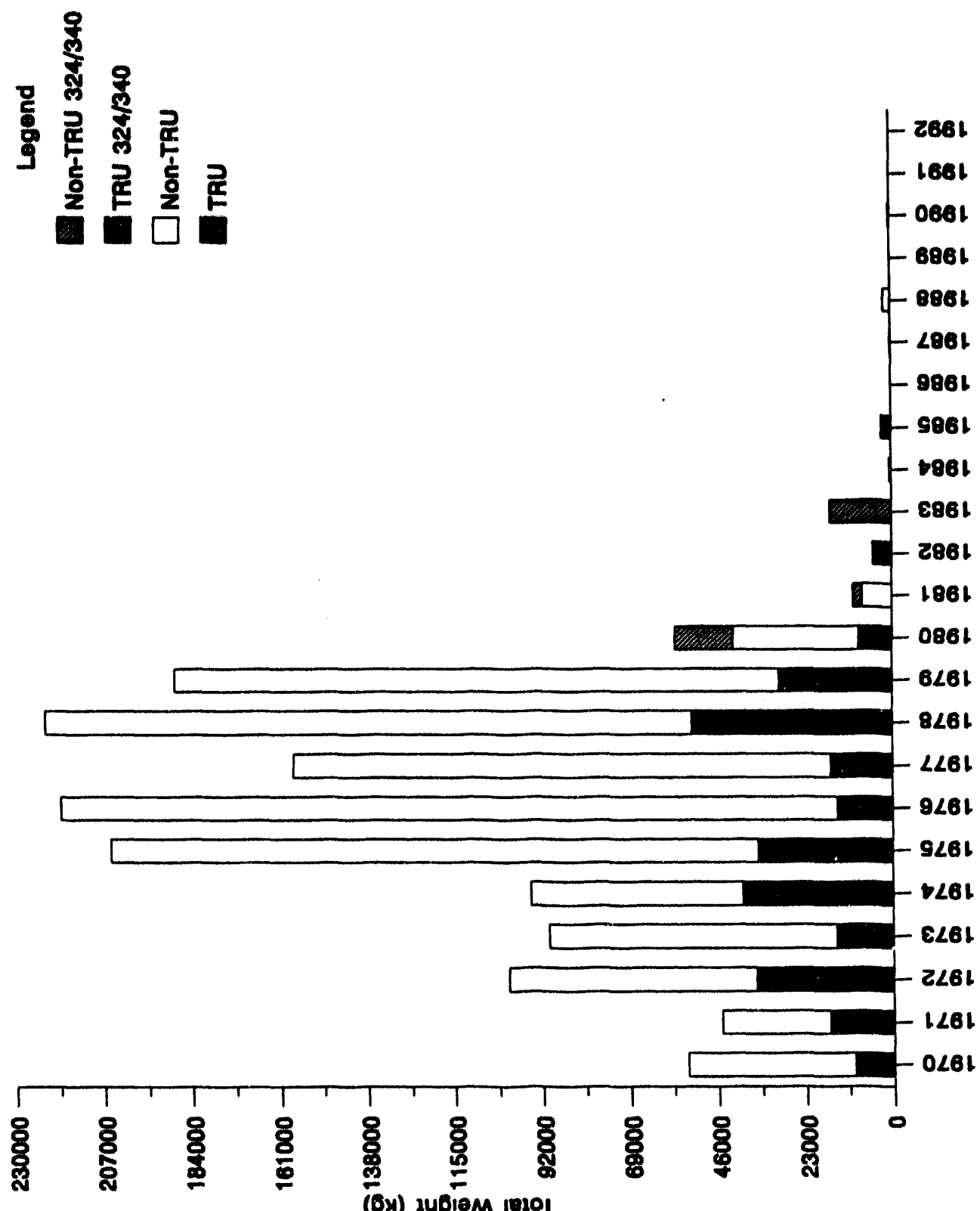

(6y) 34010M 10301 

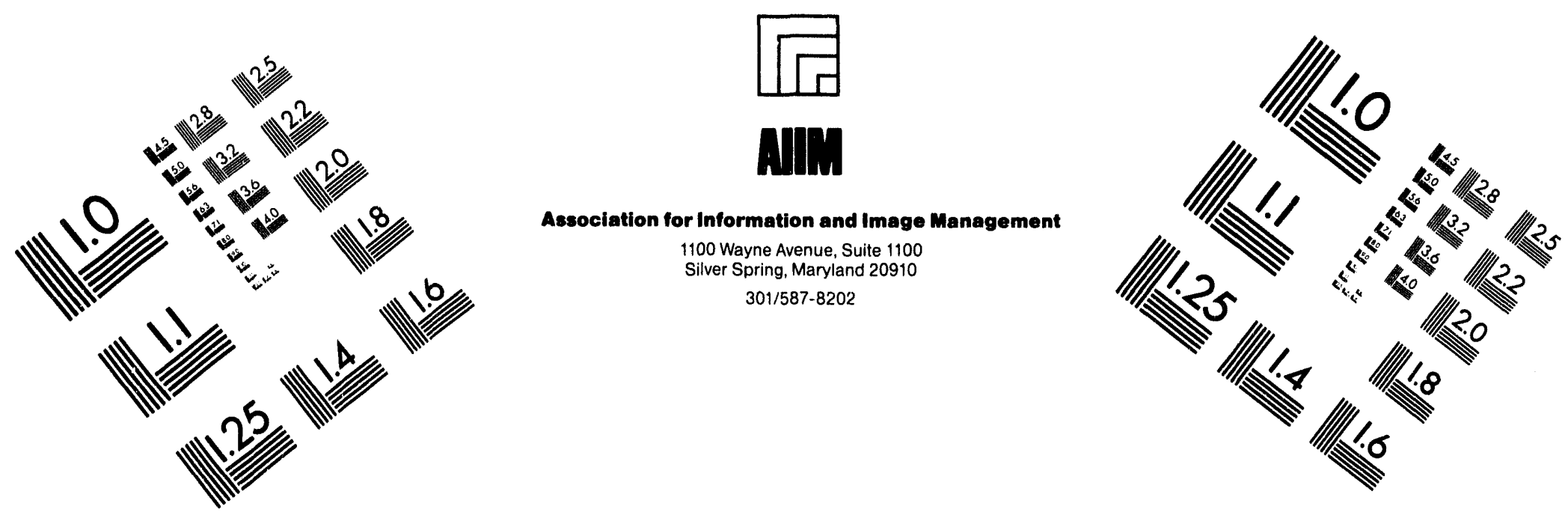

\section{Centimeter}

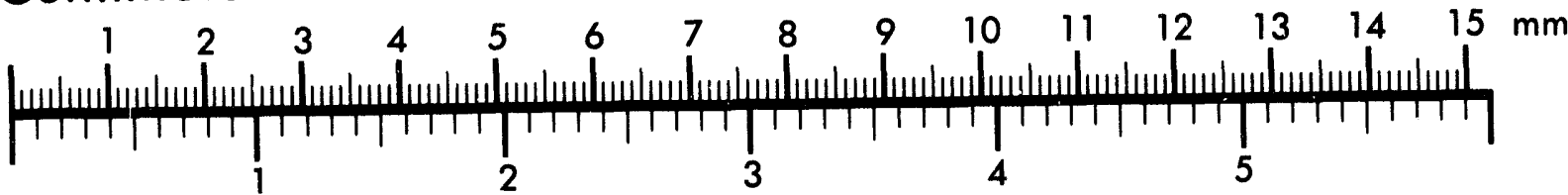
Inches
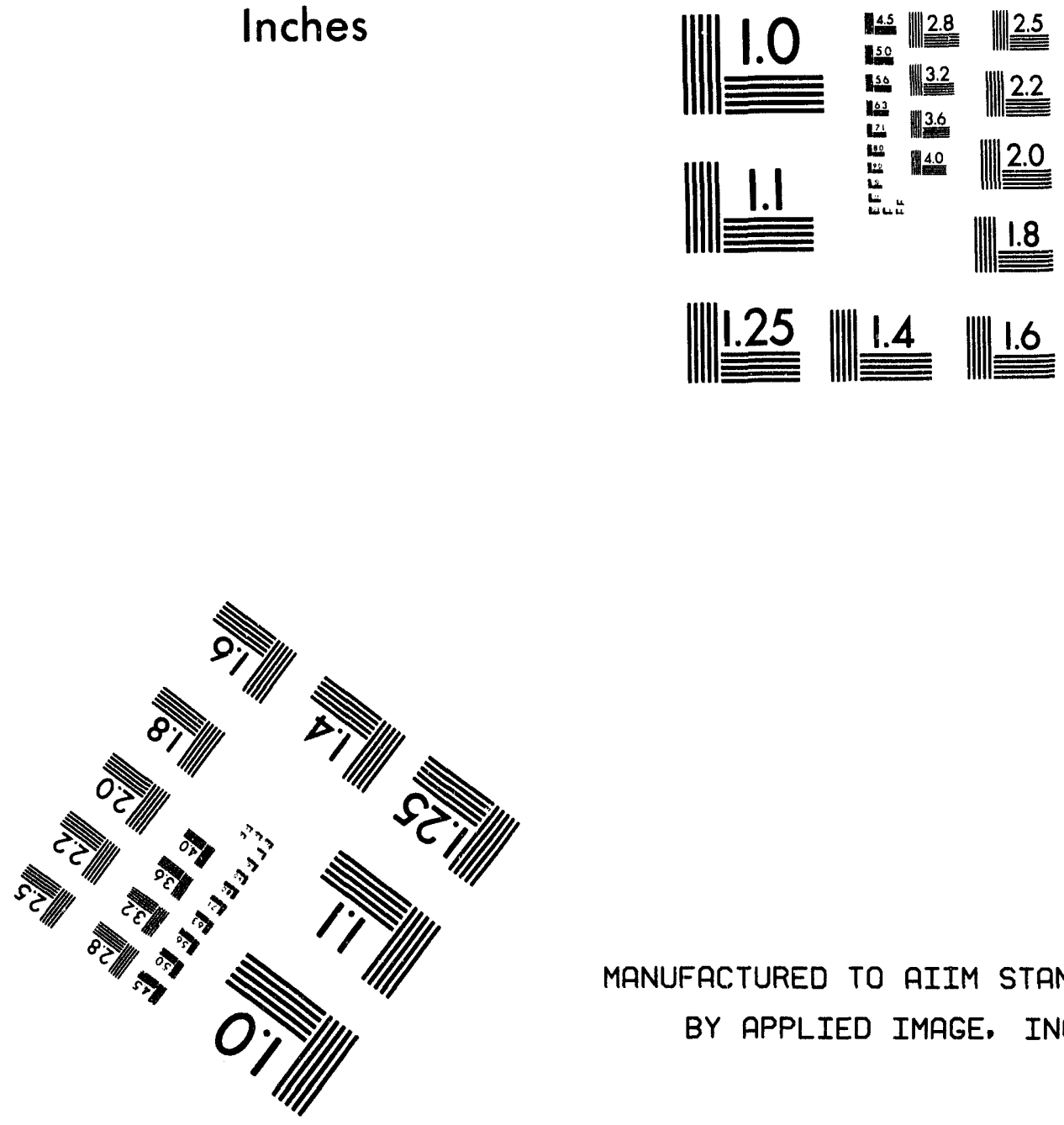

MANUFACTURED TO AIIM STANDARDS

BY APPLIED IMAGE, INC.

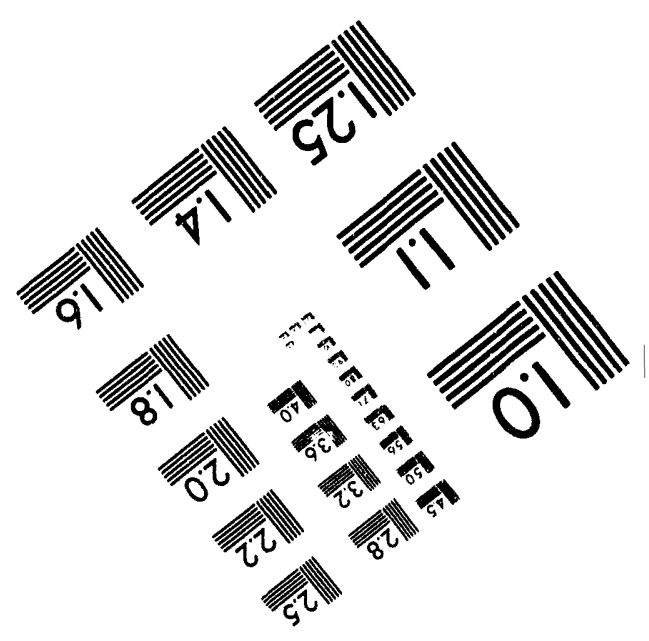



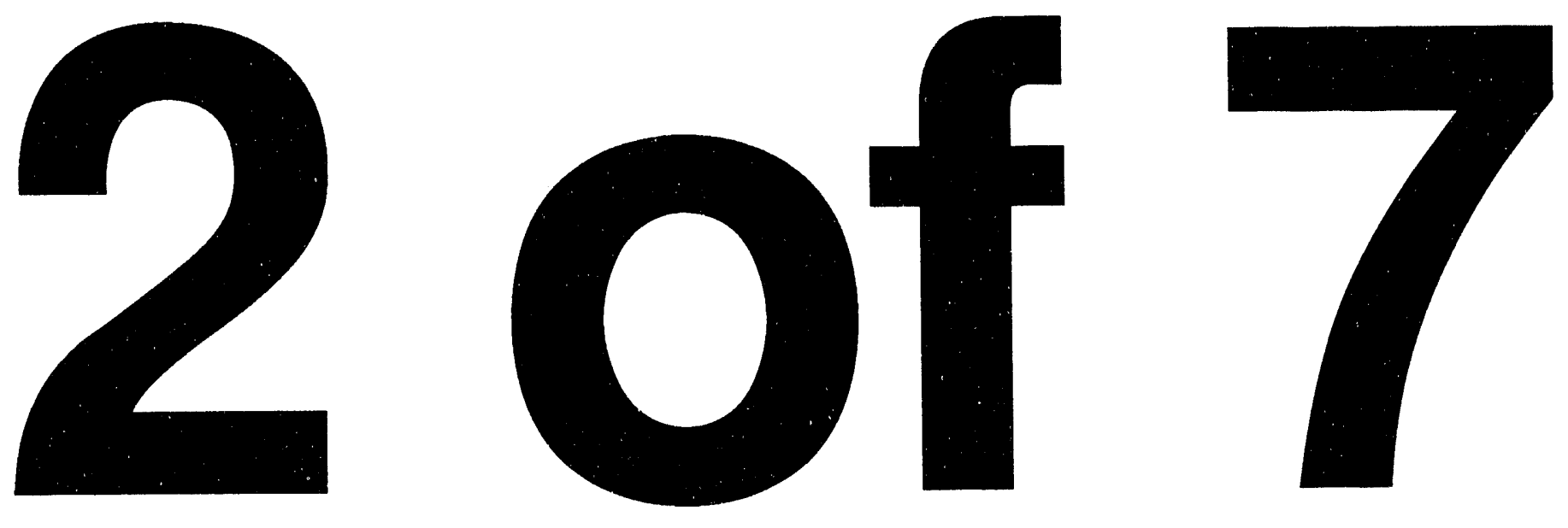


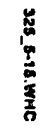

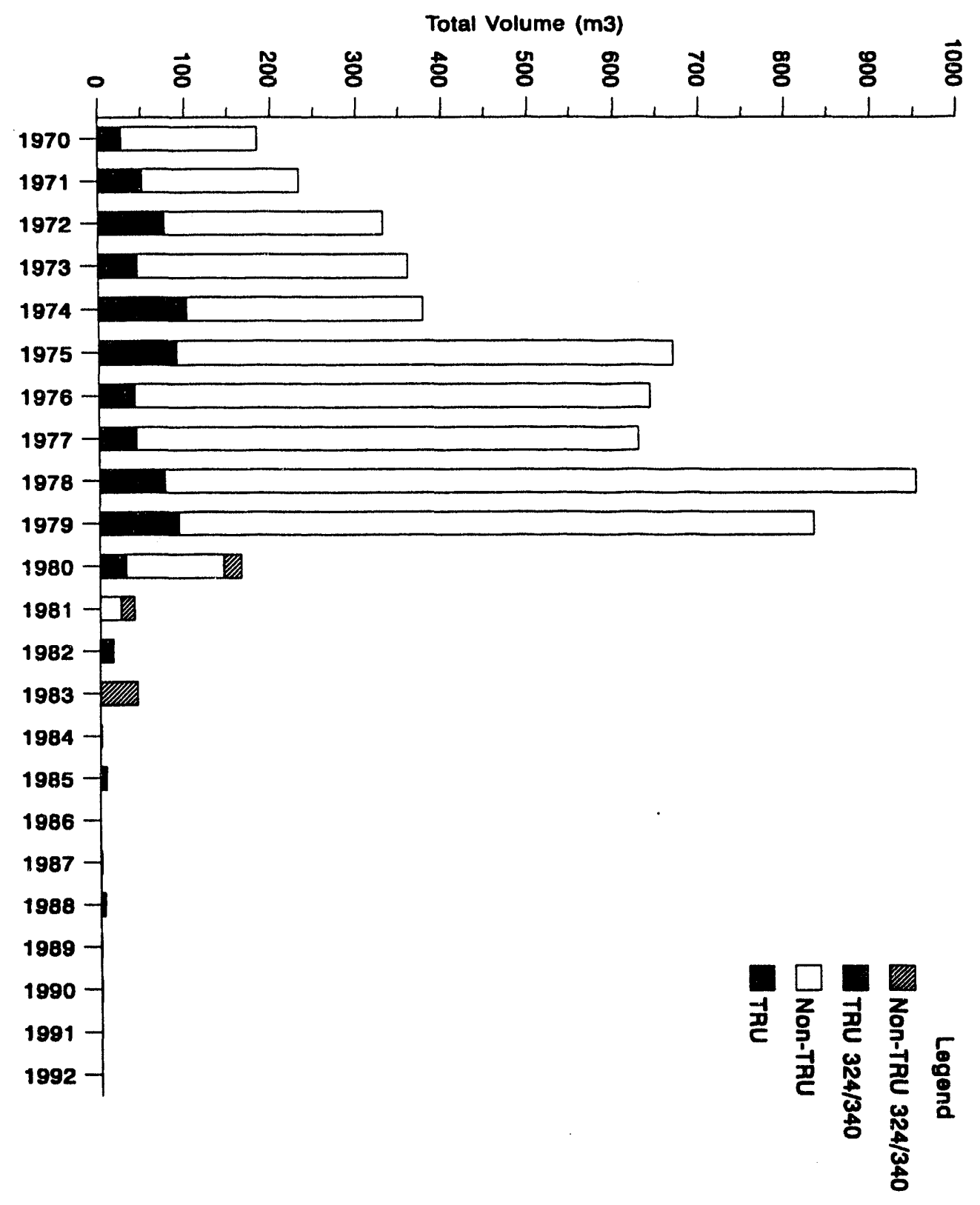

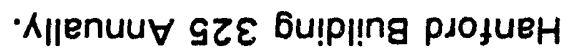

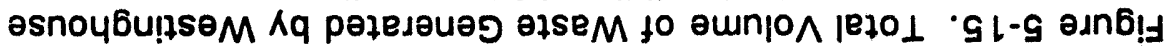




\section{$92-9$}

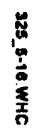

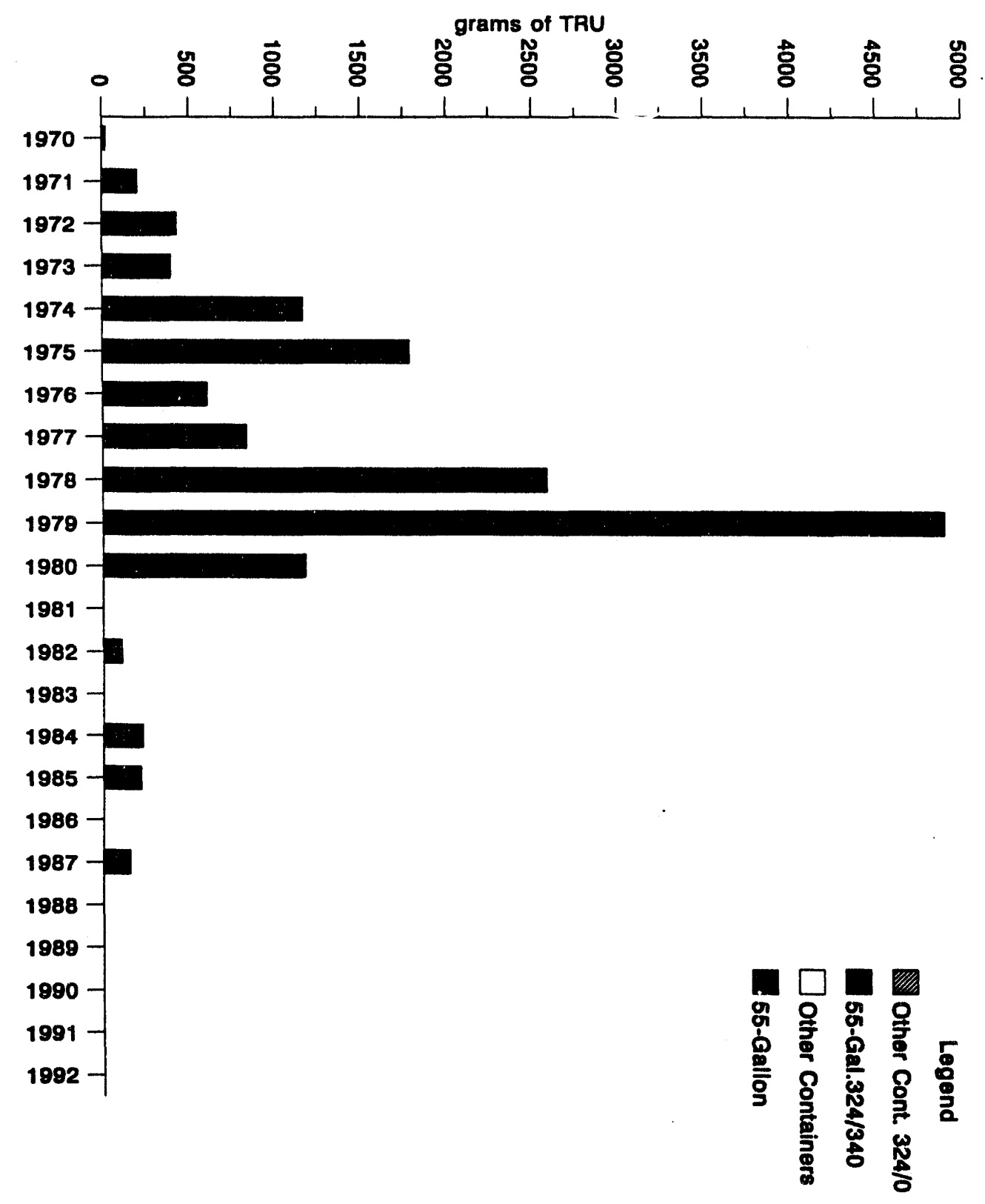

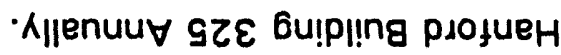

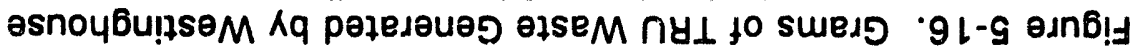


Table 5-1. Transuranic Wastes Attributed to Westinghouse Hanford Building 325 but Originating at Other Facilities.

\begin{tabular}{|c|c|c|c|c|c|c|c|}
\hline Year & Source & $\begin{array}{l}\text { Container } \\
\text { Number }\end{array}$ & $\begin{array}{l}\text { Percent } \\
\text { of Total }\end{array}$ & $\begin{array}{l}\text { Weight } \\
\text { (kg) }\end{array}$ & $\begin{array}{l}\text { Weight } \\
\text { Percent }\end{array}$ & $\begin{array}{c}\text { Volume } \\
\left(\mathrm{m}^{3}\right)\end{array}$ & $\begin{array}{l}\text { Volume } \\
\text { Percent }\end{array}$ \\
\hline \multirow[t]{3}{*}{1974} & 308 & 20 Drums & $8 \%$ & 1,360 & $8 \%$ & 4.1 & $8 \%$ \\
\hline & 309 & 6 Others & $26 \%$ & 506 & $2 \%$ & 1.1 & $2 \%$ \\
\hline & Total & $\begin{array}{l}20 \text { Drums } \\
6 \text { Others }\end{array}$ & $8 \%$ & 1,360 & $8 \%$ & $\frac{4}{1,1}, 1$ & $8 \%$ \\
\hline 1975 & No other sources listed & & & & & & \\
\hline \multirow[t]{2}{*}{1976} & 308 & 14 Drums & $9 \%$ & 779 & $7 \%$ & 2.9 & $9 \%$ \\
\hline & Total 1. & 14 Drums & $9 \%$ & 779 & $7 \%$ & 5.2 & $9 \%$ \\
\hline \multirow[t]{2}{*}{1977} & 308 & 20 Drums & $14 \%$ & 1,024 & $11 \%$ & 4.2 & $14 \%$ \\
\hline & Total & 20 Drums & $14 \%$ & 1.024 & $11 \%$ & $4.2 \%$ & $14 \%$ \\
\hline \multirow[t]{4}{*}{1978} & 308 & $\begin{array}{l}4 \text { Drums } \\
1 \text { Other }\end{array}$ & $\begin{array}{r}1 \% \\
10 \%\end{array}$ & $\begin{array}{r}202 \\
1,998\end{array}$ & $\begin{array}{r}5 \% \\
21 \%\end{array}$ & $\begin{array}{l}0.8 \\
4.6\end{array}$ & $\begin{array}{r}1 \% \\
42 \%\end{array}$ \\
\hline & 340 & $\begin{array}{l}86 \text { Drums } \\
1 \text { Other }\end{array}$ & $\begin{array}{l}28 \% \\
10 \%\end{array}$ & $\begin{array}{r}22,378 \\
5,996\end{array}$ & $\begin{array}{l}52 \% \\
62 \%\end{array}$ & $\begin{array}{c}17.8 \\
5.2\end{array}$ & $\begin{array}{l}28 \% \\
48 \%\end{array}$ \\
\hline & Babcock \& Wilcox & 62. Drums & $20 \%$ & 3427 & $8 \%$ & 13.0 & $20 \%$ \\
\hline & Total & $\begin{array}{l}152 \text { Drums } \\
2 \text { Other }\end{array}$ & $49 \%$ & 26,007 & $\begin{array}{l}61 \% \\
83 \%\end{array}$ & 91.9 & $99 \% \%$ \\
\hline \multirow[t]{3}{*}{1979} & 327 & 4 Drums & $1 \%$ & 1907 & $6 \%$ & 0.8 & $1 \%$ \\
\hline & Babcock \& Wilcox & 221 Drums & $51 \%$ & 14,261 & $49 \%$ & 46.4 & $51 \%$ \\
\hline & Total & 225 Drums & $51 \%$ & 16,528 & $56 \%$ & 47.2 & $51 \%$ \\
\hline \multirow[t]{2}{*}{1980} & Babcock \& Wilcox & 12 Drums & $13 \%$ & 627 & $10 \%$ & 2.5 & $13 \%$ \\
\hline & Total & 12 Drums & $13 \%$ & 627 & $10 \%$ & 2.5 & $13 \%$ \\
\hline
\end{tabular}


Table 5-2. Low-Level Wastes Attributed to Westinghouse Hanford Building 325 but Originating at Other Facilities.

(3 sheets)

\begin{tabular}{|c|c|c|c|c|c|c|c|}
\hline Year & Source & $\begin{array}{l}\text { Container } \\
\text { Number }\end{array}$ & $\begin{array}{l}\text { Percent } \\
\text { of Total }\end{array}$ & $\begin{array}{c}\text { Weight } \\
\text { (kg) }\end{array}$ & $\begin{array}{l}\text { Weight } \\
\text { Percent }\end{array}$ & $\begin{array}{l}\text { Volume } \\
\left(\mathrm{m}^{3}\right)\end{array}$ & $\begin{array}{l}\text { Volume } \\
\text { Percent }\end{array}$ \\
\hline \multirow[t]{2}{*}{1971} & 327 & 54 Others & $4 \%$ & 1157 & $4 \%$ & 7.3 & $4 \%$ \\
\hline & Total & 54 Others. & $4 \%$ & 1157 & $4 \%$ & 7.3. & $4 \%$ \\
\hline \multirow[t]{5}{*}{1972} & 306 & 51 Others & $4 \%$ & 1792 & $3 \%$ & 8.1 & $3 \%$ \\
\hline & 327 & 98 Others & $8 \%$ & 5333 & $9 \%$ & 21.0 & $9 \%$ \\
\hline & 340 & 26 Others & $2 \%$ & 3534 & $6 \%$ & 10.1 & $4 \%$ \\
\hline & 3706 & 40 Others & $3 \%$ & 508 & $1 \%$ & 5.0 & $2 \%$ \\
\hline & Total & 215 others & $17 \%$ & 11167 & $18 \%$ & $44 \% 2$ & $18 \%$ \\
\hline \multirow[t]{4}{*}{1973} & 306 & $\begin{array}{l}9 \text { Drums } \\
1 \text { Other }\end{array}$ & $\begin{array}{l}10 \% \\
<1 \%\end{array}$ & $\begin{array}{r}846 \\
2511\end{array}$ & $\begin{array}{l}8 \% \\
4 \%\end{array}$ & $\begin{array}{l}1.9 \\
5.5\end{array}$ & $\begin{array}{r}10 \% \\
2 \%\end{array}$ \\
\hline & 308 & 24 Others & $1 \%$ & 3833 & $6 \%$ & 10.0 & $3 \%$ \\
\hline & 340 & $\begin{array}{l}13 \text { Drums } \\
4 \text { Others }\end{array}$ & $\begin{array}{l}14 \% \\
<1 \%\end{array}$ & $\begin{array}{l}4449 \\
3836\end{array}$ & $\begin{array}{r}.40 \% \\
6 \%\end{array}$ & $\begin{array}{l}7.3 \\
2.7\end{array}$ & $\begin{array}{r}37 \% \\
1 \%\end{array}$ \\
\hline & Total & $\begin{array}{l}22 \text { Drums } \\
29 \text { Others }\end{array}$ & $\begin{array}{r}24 \% \\
2 \%\end{array}$ & $\begin{array}{r}5295 \\
10180\end{array}$ & $\begin{array}{l}47 \% \\
16 \%\end{array}$ & $\begin{array}{r}9.2 \\
18.2\end{array}$ & $46 \%$ \\
\hline \multirow[t]{5}{*}{1974} & 306 & 41 Others & $3 \%$ & 2495 & $5 \%$ & 8.0 & $3 \%$ \\
\hline & 308 & 50 Others & $3 \%$ & 636 & $1 \%$ & 6.2 & $2 \%$ \\
\hline & 309 & 10 Others & $1 \%$ & 1240 & $3 \%$ & 3.2 & $1 \%$ \\
\hline & 324 & 2 Others & $<1 \%$ & 2237 & $5 \%$ & 4.9 & $2 \%$ \\
\hline & 340 & $\begin{array}{l}10 \text { Drums } \\
16 \text { Others }\end{array}$ & $\begin{array}{r}15 \% \\
1 \%\end{array}$ & $\begin{array}{l}2315 \\
1545\end{array}$ & $\begin{array}{r}15 \% \\
3 \%\end{array}$ & $\begin{array}{l}2.1 \\
4.7\end{array}$ & $\begin{array}{l}7 \% \\
2 \%\end{array}$ \\
\hline
\end{tabular}


Table 5-2. Low-Level Wastes Attributed to Westinghouse Hanford Building 325 but Originating at Other Facilities. (3 sheets)

\begin{tabular}{|c|c|c|c|c|c|c|c|}
\hline Year & Source & $\begin{array}{l}\text { Container } \\
\text { Number }\end{array}$ & $\begin{array}{l}\text { Percent } \\
\text { of Total }\end{array}$ & $\begin{array}{l}\text { Weight } \\
\text { (kg) }\end{array}$ & $\begin{array}{l}\text { Weight } \\
\text { Percent }\end{array}$ & $\begin{array}{c}\text { Volume } \\
\left(\mathrm{m}^{3}\right)\end{array}$ & $\begin{array}{l}\text { Volume } \\
\text { Percent }\end{array}$ \\
\hline \multirow[t]{2}{*}{1974 (cont) } & 3706 & 48 Others & $3 \%$ & 3966 & $8 \%$ & 13.2 & $5 \%$ \\
\hline & Total & $\begin{array}{l}10 \text { Drums } \\
167 \text { Others }\end{array}$ & $\begin{array}{l}15 \% \\
10 \% \text {. }\end{array}$ & $\begin{array}{l}2315 \\
12119\end{array}$ & $\begin{array}{l}15 \% \\
26 \%\end{array}$ & $40.2 \%$ & $15 \%$ \\
\hline \multirow[t]{6}{*}{1975} & 324 & 37 Others & $2 \%$ & 470 & $<1 \%$ & 4.7 & $1 \%$ \\
\hline & 327 & 11 Others & $1 \%$ & 2136 & $1 \%$ & 4.7 & $1 \%$ \\
\hline & 340 & 1 Other & $<1 \%$ & 1889 & $1 \%$ & 1.8 & $<1 \%$ \\
\hline & 3706 & 3 Others & $<1 \%$ & 13125 & $9 \%$ & 28.9 & $5 \%$ \\
\hline & 3760 & 1 Other & $<1 \%$ & 4977 & $3 \%$ & 11.0 & $2 \%$ \\
\hline & Total & 53 Others & $2 \%$ & 22597 & $15 \%$ & 51 ? & $9 \%$ \\
\hline \multirow[t]{4}{*}{1976} & 309 & 1 Other & $<1 \%$ & 2554 & $1 \%$ & 2.1 & $<1 \%$ \\
\hline & 340 & $\begin{array}{l}1 \text { Drum } \\
42 \text { Others }\end{array}$ & $\begin{array}{l}1 \% \\
2 \%\end{array}$ & $\begin{array}{r}94 \\
852\end{array}$ & $\begin{array}{l}<1 \% \\
<1 \%\end{array}$ & $\begin{array}{l}0.2 \\
2.6 \\
\end{array}$ & $\begin{array}{l}<1 \% \\
<1 \%\end{array}$ \\
\hline & 3706 & 1 Others & $<1 \%$ & 2314 & $1 \%$ & 5.1 & $1 \%$ \\
\hline & Total & $\begin{array}{l}1 \text { Drum } \\
45 \text { Others }\end{array}$ & $\begin{array}{l}1 \% \\
2 \%\end{array}$ & $\begin{array}{r}94 \\
5720\end{array}$ & $3 \%$ & 9.2 & $=1 \%$ \\
\hline \multirow[t]{3}{*}{1977} & 308 & 1 Other & $<1 \%$ & 2802 & $2 \%$ & 6.3 & $1 \%$ \\
\hline & $\begin{array}{l}\text { North Bone } \\
\text { Yard }\end{array}$ & 1 Other & $<1 \%$ & 2059 & $2 \%$ & 4.5 & $1 \%$ \\
\hline & Total & 2 Others & $4 \%$ & 4861 & $4 \%$ & 10.8 & $2 \%$ \\
\hline \multirow[t]{2}{*}{1978} & 306 & 2 Others & $<1 \%$ & 908 & $1 \%$ & 5.6 & $1 \%$ \\
\hline & 324 & 3 Others & $<1 \%$ & 4881 & $3 \%$ & 10.8 & $1 \%$ \\
\hline
\end{tabular}


Table 5-2. Low-Level Wastes Attributed to Westinghouse Hanford Building 325 but Originating at Other Facilities. (3 sheets)

\begin{tabular}{|c|c|c|c|c|c|c|c|}
\hline & Source & $\begin{array}{c}\text { Container } \\
\text { Number }\end{array}$ & $\begin{array}{l}\text { Percent } \\
\text { of Total }\end{array}$ & $\begin{array}{c}\text { Weight } \\
\text { (kg) }\end{array}$ & $\begin{array}{l}\text { Weight } \\
\text { Percent }\end{array}$ & $\begin{array}{l}\text { Volume } \\
\left(\mathrm{m}^{3}\right)\end{array}$ & $\begin{array}{l}\text { Volume } \\
\text { Percent }\end{array}$ \\
\hline \multirow[t]{2}{*}{1978 (cont) } & 3730 & $\begin{array}{l}1 \text { Drum } \\
1 \text { Other }\end{array}$ & $\begin{array}{r}1 \% \\
<1 \%\end{array}$ & $\begin{array}{l}227 \\
454\end{array}$ & $\begin{array}{r}1 \% \\
<1 \%\end{array}$ & $\begin{array}{l}0.2 \\
0.4\end{array}$ & $\begin{array}{r}1 \% \\
<1 \%\end{array}$ \\
\hline & Total: & $\begin{array}{l}1 \text { Drum } \\
6 \text { Others }\end{array}$ & $\begin{array}{l}1 \% \\
<1 \%\end{array}$ & $\begin{array}{r}227 \\
6243\end{array}$ & $\begin{array}{l}1 \% \\
4 \%\end{array}$ & 10.2 .8 & $1 \%$ \\
\hline \multirow[t]{5}{*}{1979} & 308 & 21 Others & $1 \%$ & 14982 & $10 \%$ & 83.7 & $12 \%$ \\
\hline & 324 & 13 Others & $<1 \%$ & 7970 & $5 \%$ & 59.1 & $8 \%$ \\
\hline & 327 & 8 Others & $<1 \%$ & 6538 & $5 \%$ & 16.9 & $2 \%$ \\
\hline & 340 & 2 Others & $<1 \%$ & 14528 & $10 \%$ & 15.1 & $2 \%$ \\
\hline & Total & 44 Others & $2 \%$ & 44018 & $30 \%$. & 17488 & $24 \%$. \\
\hline \multirow[t]{3}{*}{1980} & 324 & 9 Others & $3 \%$ & 6211 & $36 \%$ & 32.3 & $33 \%$ \\
\hline & 327 & $\begin{array}{l}20 \text { Drums } \\
46 \text { Others }\end{array}$ & $\begin{array}{l}27 \% \\
16 \%\end{array}$ & $\begin{array}{r}3588 \\
725\end{array}$ & $\begin{array}{r}23 \% \\
4 \%\end{array}$ & $\begin{array}{l}4.2 \\
6.0\end{array}$ & $\begin{array}{r}27 \% \\
6 \%\end{array}$ \\
\hline & Total & $\begin{array}{l}20 \text { Drums } \\
55 \text { Others }\end{array}$ & $\begin{array}{r}27 \% \\
8 \%\end{array}$ & $\begin{array}{l}3588 \\
6936\end{array}$ & $\begin{array}{l}23 \% \\
40 \%\end{array}$ & $\begin{array}{l}4,2 \\
38,3\end{array}$ & $\begin{array}{l}27 \% \\
39 \%\end{array}$ \\
\hline
\end{tabular}




\section{WHC-EP-0696}

Table 5-3. TRU Waste Generated at Building 325 Westinghouse Hanford by

Container Type and Year. (4 sheets)

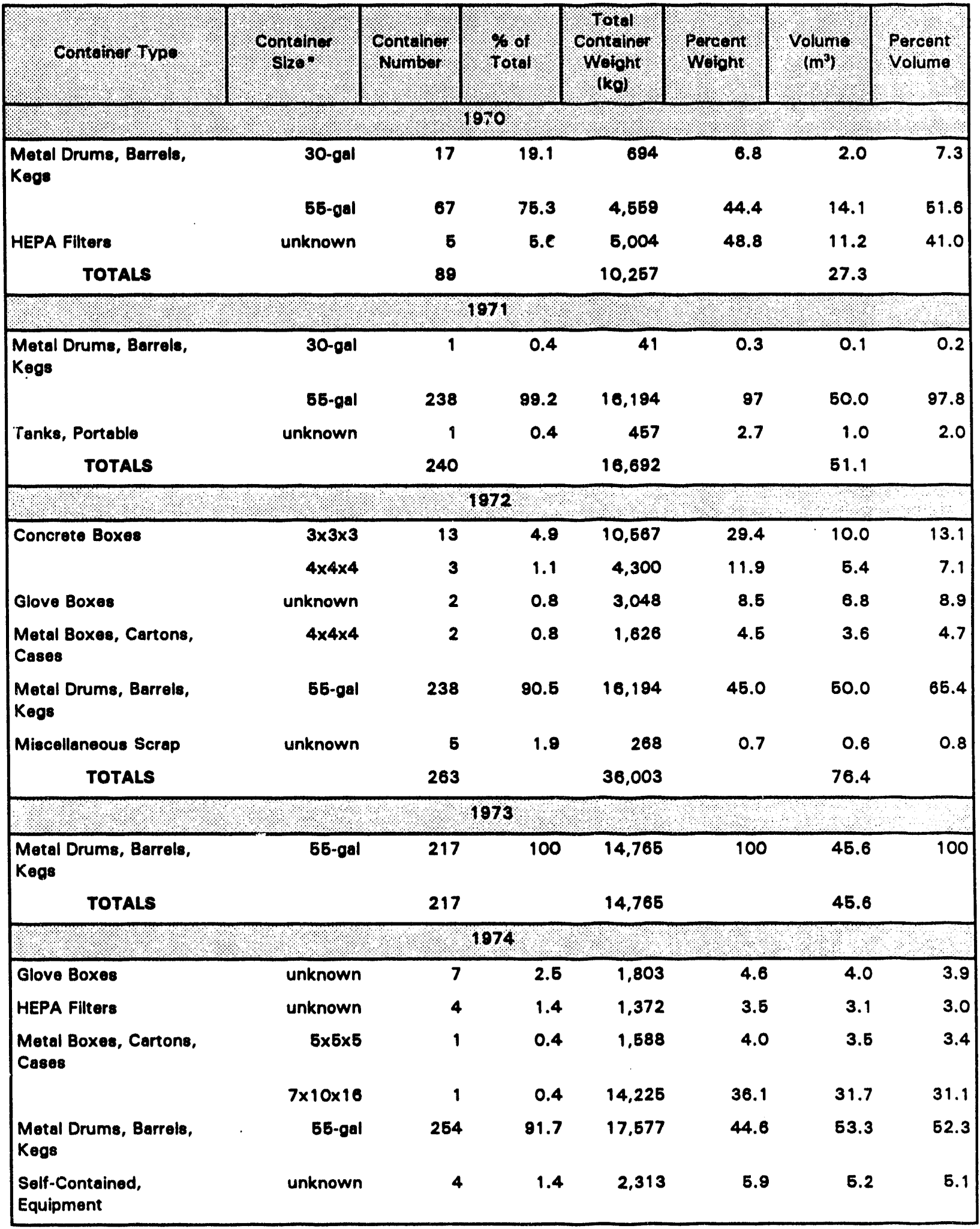


Table 5-3. TRU Waste Generated at Building 325 Westinghouse Hanford by Container Type and Year. (4 sheets)

\begin{tabular}{|c|c|c|c|c|c|c|c|}
\hline ( & Contong & Continn & 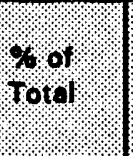 & 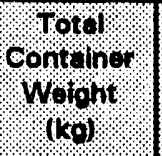 & Woront & Volumo & Porcent \\
\hline Tanks, Portable & unk. wn & 6 & 2.2 & 505 & 1.3 & 1.1 & 1.1 \\
\hline TOTALS & & 277 & & 38,383 & & 101.9 & \\
\hline \multicolumn{8}{|c|}{1976} \\
\hline $\begin{array}{l}\text { Mietal Boxes, Cartons, } \\
\text { Cases }\end{array}$ & $1,250 \mathrm{ft}^{3}$ & 1 & 0.4 & 16,876 & 44.9 & 35.4 & 39.2 \\
\hline $\begin{array}{l}\text { Motal Drums, Barreis, } \\
\text { Kegs }\end{array}$ & 55-gal & 218 & 97.3 & 15,445 & 43.6 & 45.8 & 50.7 \\
\hline Wooden Boxes, Certons, & $4 \times 4 \times 4$ & 6 & 2.2 & 4,064 & 11.6 & 9.1 & 10.1 \\
\hline TOTALS & & 224 & & 35,385 & & 90.3 & \\
\hline \multicolumn{8}{|c|}{1976} \\
\hline Metal Boxes, Cartons, & $4 \times 4 \times 5$ & 1 & 0.6 & 1,016 & 7.0 & 2.3 & 5.5 \\
\hline & $4 \times 5 \times 9$ & 1 & 0.6 & 2,286 & 15.8 & 5.1 & 12.2 \\
\hline \multirow{2}{*}{$\begin{array}{l}\text { Metal Drums, Barrels, } \\
\text { Kegs }\end{array}$} & 56-gal & 162 & 98.2 & 11,022 & 76.2 & 34.0 & 81.3 \\
\hline & 110-gal & 1 & 0.6 & 136 & 0.9 & 0.4 & 1.0 \\
\hline TOTALS & & 165 & & 14,480 & & 41.8 & \\
\hline \multicolumn{8}{|c|}{1877} \\
\hline Concrete Boxes & $3 \times 3 \times 3$ & 1 & 0.7 & 796 & 4.9 & 0.8 & 1.8 \\
\hline $\begin{array}{l}\text { Concrete Cylinders, } \\
\text { Casks }\end{array}$ & $7 \times 12$ & 1 & 0.7 & 5,869 & 36.3 & 13.1 & 30.3 \\
\hline Metal Drums, Barrels, & 55-gal & 140 & 98.6 & 9,521 & 58.8 & 29.4 & 67.9 \\
\hline TOTALS & & 142 & & 16,186 & & 43.3 & \\
\hline \multicolumn{8}{|c|}{1978} \\
\hline Concrete Boxes & $3 \times 3 \times 3$ & 9 & 2.8 & 7,691 & 14.7 & 6.3 & 8.4 \\
\hline $\begin{array}{l}\text { Metal Drums, Barrels, } \\
\text { Kegs }\end{array}$ & 55-gal & 307 & 96.9 & 42,781 & 81.5 & 64.5 & 85.6 \\
\hline Wooden Boxes, Cartons, & $3.17 \times 5 \times 10.3$ & 1 & 0.3 & 1,996 & 3.8 & 4.6 & 6.1 \\
\hline TOTALS & & 317 & & 62,468 & & 75.4 & \\
\hline \multicolumn{8}{|c|}{1979} \\
\hline Metal Drums, Barrels, & 56-gal & 437 & 100 & 29,733 & 100 & 91.8 & 100 \\
\hline TOTALS & & 437 & & 29,733 & & 91.8 & \\
\hline
\end{tabular}




\section{WHC-EP-0696}

Table 5-3. TRU Waste Generated at Building 325 Westinghouse Hanford by Container Type and Year. (4 sheets)

\begin{tabular}{|c|c|c|c|c|c|c|c|}
\hline contaner typo & contolno & containg & $\%$ ot & $\begin{array}{l}\text { Tontalnat } \\
\text { woroht } \\
\text { (ko) }\end{array}$ & $\begin{array}{l}\text { Percents } \\
\text { Woloht }\end{array}$ & $\mathrm{volum}$ & $\begin{array}{l}\text { Percent } \\
\text { Volume }\end{array}$ \\
\hline \multicolumn{8}{|c|}{1880} \\
\hline $\begin{array}{l}\text { Metal Boxes, Cartona, } \\
\text { Cases }\end{array}$ & $4.2 \times 6.2 \times 15.6$ & 1 & 1.1 & 2,496 & 28.7 & 11.5 & 37.6 \\
\hline Motal Drums, Berrols, & 65-gal & 91 & 98.9 & 6,192 & 71.3 & 19.1 & 62.4 \\
\hline TOTALS & & 92 & - & 8,687 & & 30.6 & \\
\hline \multicolumn{8}{|c|}{1081} \\
\hline \multicolumn{8}{|c|}{1982} \\
\hline $\begin{array}{l}\text { Motal Drums, Barrels, } \\
\text { Kegs }\end{array}$ & $\because$ 55-gal & 26 & 92.9 & 1.786 & 36.9 & 6.5 & 36.4 \\
\hline Metal Boxes, Cartons, & $\because 12 \times 6 \times 7$ & 2 & 7.1 & 3,049 & 63.1 & 9.6 & 63.6 \\
\hline TOTALS & & 28 & & 4,836 & & 15.1 & \\
\hline \multicolumn{8}{|c|}{1983} \\
\hline & & \multicolumn{2}{|c|}{1984} & & & & \\
\hline Metal Drums, Barrele, & $\because$ 55-gal & 6 & 100 & 405 & 100 & 1.3 & 100 \\
\hline TOTALS & & 6 & & 405 & & 1.3 & \\
\hline \multicolumn{8}{|c|}{1986} \\
\hline $\begin{array}{l}\text { Metal Boxes, Cartons, } \\
\text { Cases }\end{array}$ & $4 \times 6 \times 10$ & 1 & 11.1 & 2,042 & 76.2 & 7.1 & 80.7 \\
\hline Metal Drums, Barrels, & $\because 56-g a l$ & 8 & 88.8 & 639 & 23.8 & 1.7 & 19.3 \\
\hline TOTALS & & 9 & & 2,881 & & 8.8 & \\
\hline \multicolumn{8}{|c|}{1980} \\
\hline \multicolumn{8}{|c|}{1087} \\
\hline Motel Drums, Barrels, & - 5b-gal & 5 & 100 & 186 & 100 & 1.1 & 100 \\
\hline TOTALS & & 5 & & 186 & & 1.1 & \\
\hline \multicolumn{8}{|c|}{1888} \\
\hline \multicolumn{8}{|c|}{1988} \\
\hline
\end{tabular}


$\succ \varepsilon-9$

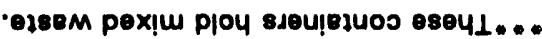

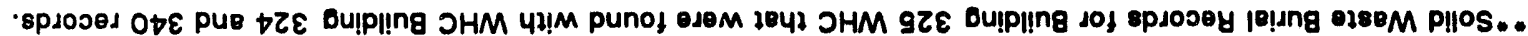

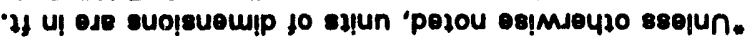

\begin{tabular}{|c|c|c|c|c|c|c|c|}
\hline \multicolumn{8}{|c|}{8001} \\
\hline \multicolumn{8}{|c|}{1801} \\
\hline \multicolumn{8}{|c|}{0681} \\
\hline ounjos & puplol & 140101 & \%1010 & $10 \%$ & ropunos & $\$ \% 10 \% 5$ & \%or \\
\hline
\end{tabular}

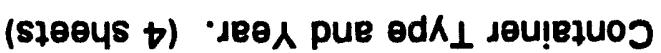

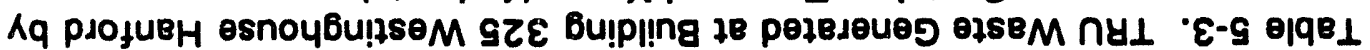


Table 5-4. Non-TRU Waste Generated at Building 325 Westinghouse Hanford by Container Type and Year. (10 sheets)

\begin{tabular}{|c|c|c|c|c|c|c|c|}
\hline contingr trop & contonn & Continbor & $\%$ \%ort: & $\begin{array}{l}\text { Totrol } \\
\text { conganor } \\
\text { wort }\end{array}$ & $\begin{array}{l}\text { Porcent } \\
\text { Woont }\end{array}$ & Volume & Peroent? \\
\hline \multicolumn{8}{|c|}{1070} \\
\hline $\begin{array}{l}\text { Burlap, Cloth, Paper, or } \\
\text { Plastic Bags }\end{array}$ & unknown & 1 & 0.2 & 889 & 2.0 & 2.0 & 1.3 \\
\hline Concrete Boxes & $2 \times 3 \times 6$ & 2 & 0.3 & 3919 & 9.0 & 2.0 & 1.3 \\
\hline $\begin{array}{l}\text { Fiberboard/Plastic Boxes, } \\
\text { Cartons, Cases }\end{array}$ & unknown & 603 & 91.6 & 7658 & 17.6 & 76.8 & 48.7 \\
\hline HEPA Filter & unknown & 10 & 1.5 & 1669 & 3.6 & 3.5 & 2.2 \\
\hline $\begin{array}{l}\text { Metal Boxes, Cartons, } \\
\text { Cases }\end{array}$ & $4 \times 5 \times 6$ & 6 & 0.9 & 4293 & 9.8 & 9.6 & 6.1 \\
\hline $\begin{array}{l}\text { Metal Drums, Barrels, } \\
\text { Kogs }\end{array}$ & 65-gol & 10 & 1.6 & 680 & 1.6 & 2.1 & 1.3 \\
\hline Miscelleneous Scrap & unknown & 10 & 1.5 & 3295 & 7.6 & 14.1 & 8.9 \\
\hline $\begin{array}{l}\text { Self-Contained, } \\
\text { Equipment }\end{array}$ & unknown & 2 & 0.3 & 1867 & 4.3 & 4.2 & 2.7 \\
\hline $\begin{array}{l}\text { Trucks, Flatbeds, } \\
\text { Compactor, Loadlugger }\end{array}$ & unknown & 3 & 0.5 & 610 & 1.4 & 1.4 & 0.9 \\
\hline \multirow{4}{*}{$\begin{array}{l}\text { Wooden Boxes, Cartons, } \\
\text { Ceses }\end{array}$} & $4 \times 4 \times 7$ & 3 & 0.6 & 4283 & 9.8 & 9.6 & 6.1 \\
\hline & $4 \times 4 \times 9$ & 2 & 0.3 & 3668 & 8.4 & 8.2 & 6.2 \\
\hline & $4 \times 5 \times 7$ & 5 & 0.8 & 8890 & 20.4 & 19.8 & 12.6 \\
\hline & $4 \times 5 \times 8$ & 1 & 0.2 & 2033 & 4.7 & 4.6 & 2.9 \\
\hline TOTALS & & 658 & & 43654 & & 157.8 & \\
\hline \multicolumn{8}{|c|}{1971} \\
\hline $\begin{array}{l}\text { Burlap, Cloth, Poper, or } \\
\text { Plastic Bags }\end{array}$ & unknown & 6 & 0.5 & 44 & 0.2 & 1.5 & 0.8 \\
\hline \multirow{3}{*}{$\begin{array}{l}\text { Fiberboard/Plastic Boxes, } \\
\text { Cartons, Cases }\end{array}$} & $2 \mathrm{ft}^{3}$ & 34 & 2.8 & 185 & 0.7 & 2.1 & 1.2 \\
\hline & - unknown & 18 & 1.5 & 228 & 0.8 & 2.3 & 1.3 \\
\hline & unknown & 1128 & 81.1 & 14437 & 51.2 & 143.9 & 79.2 \\
\hline Glove Boxes & unknown & 2 & 0.2 & 830 & 2.9 & 1.9 & 1.1 \\
\hline \multirow{2}{*}{$\begin{array}{l}\text { Metal Drums, Barrels, } \\
\text { Kegs }\end{array}$} & $\cdots$ 65-gal & 3 & 0.2 & 204 & 0.7 & 0.6 & 0.3 \\
\hline & 55-gal & 32 & 2.6 & 2177 & 7.7 & 6.7 & 3.7 \\
\hline Miscellaneous Scrap & unknown & 4 & 0.3 & 2659 & 9.1 & 5.7 & 3.1 \\
\hline Self-Contained Equipment & unknown & 6 & 0.5 & 787 & 2.8 & 1.8 & 1.0 \\
\hline
\end{tabular}




\section{WHC-EP-0696}

Table 5-4. Non-TRU Waste Generated at Building 325 Westinghouse Hanford by Container Type and Year. (10 sheets)

\begin{tabular}{|c|c|c|c|c|c|c|c|}
\hline \% & $100 \%$ & \%ontor & $\%$ & coroting. & pront? & romp & poroptr? \\
\hline Tanks, Portable & unknown & 1 & 0.1 & 635 & 2.3 & 1.4 & 0.8 \\
\hline $\begin{array}{l}\text { Trucks, Flatbeds, } \\
\text { Compactor, Loadhugger }\end{array}$ & unknown & 4 & 0.3 & 6147 & 21.8 & 13.7 & 7.5 \\
\hline TOTALS & & 1238 & & 28234 & & 181.6 & \\
\hline & & & 172 & & & ২ঃ: & \\
\hline $\begin{array}{l}\text { Burlap, Cloth, Paper, or } \\
\text { Plastic Bags }\end{array}$ & unknown & 29 & 2.2 & 2286 & 3.5 & 7.1 & 2.8 \\
\hline Concrete Boxee & unknown & 1 & 0.1 & 1887 & 2.8 & 1.8 & 0.7 \\
\hline \multirow{2}{*}{$\begin{array}{l}\text { Fiberboard/Plastic Boxes, } \\
\text { Cartons, Cases }\end{array}$} & $2 \mathrm{ft}^{3}$ & 3 & 0.2 & 22 & 0.1 & 0.1 & 0.1 \\
\hline & unknown & 1174 & 89.0 & 16885 & 26.1 & 150.0 & 68.8 \\
\hline $\begin{array}{l}\text { Metal Drums, Barrels. } \\
\text { Kege }\end{array}$ & 66-gal & 68 & 4.4 & 4168 & 6.4 & 12.3 & 4.8 \\
\hline \multirow[t]{2}{*}{ Miscellaneous Scrap } & $\cdots$ unknown & 10 & 0.8 & 2722 & 4.2 & 0.3 & 0.1 \\
\hline & unknown & 17 & 1.3 & 216 & 0.3 & 1.8 & 0.7 \\
\hline Self-Contained Equipment & unknown & 13 & 1.0 & 16460 & 25.4 & 36.7 & 14.4 \\
\hline $\begin{array}{l}\text { Truck, Flatbeds, } \\
\text { Compector, Loadlugger }\end{array}$ & unknown & 13 & 1.0 & 18543 & 28.6 & 41.3 & 16.2 \\
\hline Wouden Boxes, Cartons, & $4 \times 4 \times 8$ & 1 & 0.1 & 1626 & 2.6 & 3.6 & 1.4 \\
\hline TOTALS & & 1318 & & 64803 & & 265.1 & \\
\hline \multicolumn{8}{|c|}{1913} \\
\hline Dump Truck & unknown & 1 & 0.1 & 1905 & 2.5 & 1.7 & 0.5 \\
\hline $\begin{array}{l}\text { Fiberboard/Plastic Boxes, } \\
\text { Cartons, Cases }\end{array}$ & unknown & 1548 & 91.8 & 18673 & 26.1 & 198.0 & 62.9 \\
\hline Glove Boxes & unknown & 1 & 0.1 & 787 & 1.0 & 1.8 & 0.6 \\
\hline HEPA Filtere & unknown & 1 & 0.1 & 127 & 0.2 & 0.3 & 0.1 \\
\hline \multirow{2}{*}{$\begin{array}{l}\text { Metal Drums, Barrele, } \\
\text { Kegs }\end{array}$} & 30-gol & 4 & 0.2 & 177 & 0.2 & 0.5 & 0.2 \\
\hline & 55-gal & 93 & 5.5 & 11189 & 14.8 & 19.8 & 6.3 \\
\hline Miscellaneous Scrap & unknown & 18 & 1.1 & 14918 & 19.8 & 33.3 & 10.6 \\
\hline Self-Contained Equipment & unknown & 4 & 0.2 & 610 & 0.8 & 1.5 & 0.6 \\
\hline Tanks, Portable & unknown & 1 & 0.1 & 2169 & 2.9 & 4.9 & 1.6 \\
\hline
\end{tabular}


Table 5-4. Non-TRU Waste Generated at Building 325 Westinghouse Hanford by Container Type and Year. (10 sheets)

\begin{tabular}{|c|c|c|c|c|c|c|c|}
\hline $8010 \% 1 \%$ & 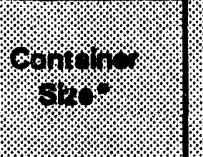 & ronkn. & \%1010 & gromoln & woph & 10.010\%? & rophnt \\
\hline $\begin{array}{l}\text { Trucke, Flatbeds, } \\
\text { Compector, Loadkoger }\end{array}$ & unknown & 14 & 0.8 & 23794 & 31.6 & 53.0 & 16.8 \\
\hline TOTALS & & 1688 & & 76339 & & 314.8 & \\
\hline & & & 18 & & & & \\
\hline $\begin{array}{l}\text { Burlap, Cloth, Paper, or } \\
\text { Plastic Bage }\end{array}$ & unknown & 3 & 0.2 & 426 & 0.8 & 1.0 & 0.4 \\
\hline $\begin{array}{l}\text { Fiberboard/Plastic Boxes, } \\
\text { Cartons, Cases }\end{array}$ & unknown & 1568 & 92.0 & 19902 & 36.9 & 199.7 & 72.4 \\
\hline Glove Boxes & unknown & 2 & 0.1 & 1054 & 1.8 & 2.3 & 0.8 \\
\hline \multirow{2}{*}{$\begin{array}{l}\text { Motal Drums, Barrels, } \\
\text { Kege }\end{array}$} & 30-gal & 23 & 1.3 & 838 & 1.7 & 2.8 & 1.0 \\
\hline & 55-gal & 67 & 3.8 & 8378 & 16.1 & 14.2 & 6.1 \\
\hline Miscellaneous Scrap & unknown & 20 & 1.2 & 5720 & 10.3 & 13.4 & 4.9 \\
\hline Self-Contained Equipment & unknown & 1 & 0.1 & 748 & 1.3 & 1.7 & 0.6 \\
\hline Trucks, Flatbeds, & unknown & 20 & 1.2 & 18346 & 33.1 & 40.9 & 14.8 \\
\hline TOTALS & & 1702 & & 66513 & & 276.0 & \\
\hline & & & 978 & & & & \\
\hline $\begin{array}{l}\text { Burlap, Cloth, Papor, or } \\
\text { Plastic Bags }\end{array}$ & unknown & 36 & 1.6 & 6760 & 3.4 & 13.4 & 2.3 \\
\hline \multirow[t]{2}{*}{ Concrete Boxes } & $3 \times 3 \times 3$ & 7 & 0.3 & 6676 & 3.3 & 5.3 & 0.9 \\
\hline & $4 \times 4 \times 4$ & 1 & $<0.1$ & 1887 & 1.1 & 1.8 & 0.3 \\
\hline \multirow{2}{*}{$\begin{array}{l}\text { Fiberboard/Plastic Boxes, } \\
\text { Cartons, Cases }\end{array}$} & $2 \mathrm{ft}^{3}$ & 6 & 0.3 & 41 & $<0.1$ & 0.8 & 0.1 \\
\hline & unknown & 1967 & 86.0 & 24882 & 14.7 & 250.7 & 43.4 \\
\hline $\begin{array}{l}\text { Fiberboard/Plastic Drums, } \\
\text { Barrels, Kegs }\end{array}$ & unknown & 1 & $<0.1$ & 672 & 0.3 & 1.3 & 0.2 \\
\hline Glove Boxes & unknown & 1 & ○ 0.1 & 12347 & 7.3 & 27.5 & 4.8 \\
\hline HEPA Filters & unknown & 37 & 1.6 & 2169 & 1.3 & 6.9 & 1.2 \\
\hline Metal Cylinder, Casks & unknown & 12 & 0.5 & 7876 & 4.6 & 17.6 & 3.0 \\
\hline $\begin{array}{l}\text { Metal Drums, Barrels, } \\
\text { Kegs }\end{array}$ & 65-gal & 143 & 6.3 & 15935 & 9.4 & 30.4 & 5.3 \\
\hline Miscellaneous Scrap & unknown & 37 & 1.6 & 10422 & 6.1 & 23.1 & 4.0 \\
\hline Self-Contained Equipment & unknown & 6 & 0.3 & 6332 & 3.1 & 13.1 & 2.3 \\
\hline Tanks, Portable & unknown & 7 & 0.3 & 2185 & 1.3 & 4.9 & 0.9 \\
\hline
\end{tabular}


Table 5-4. Non-TRU Waste Generated at Building 325 Westinghouse Hanford by Container Type and Year. (10 sheets)

\begin{tabular}{|c|c|c|c|c|c|c|c|}
\hline (2) & 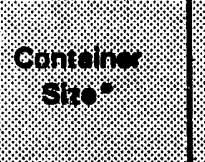 & worno & $18 \%$ & conolo & rop & భ. & Yoroons \\
\hline $\begin{array}{l}\text { Trucks, Flatbeds, } \\
\text { Compector, Losdhoger }\end{array}$ & unknown & 8 & 0.3 & 24181 & 14.3 & 53.9 & 9.3 \\
\hline \multirow{11}{*}{$\begin{array}{l}\text { Wooden Boxes, Cartons, } \\
\text { Cases }\end{array}$} & $2 \times 2 \times 7$ & 1 & $<0.1$ & 6400 & 3.8 & 14.3 & 2.5 \\
\hline & $2 \times 4 \times 4$ & 1 & $<0.1$ & 181 & 0.1 & 0.8 & 0.2 \\
\hline & $3.3 \times 3.3 \times 3.3$ & 1 & $<0.1$ & 470 & 0.3 & 1.1 & 0.2 \\
\hline & $4 \times 4 \times 6$ & 1 & $<0.1$ & 1016 & 0.6 & 2.3 & 0.4 \\
\hline & $4 \times 4 \times 8$ & 8 & 0.4 & 14631 & 8.6 & 32.6 & 5.6 \\
\hline & $4 \times 4 \times 12$ & 1 & $<0.1$ & 181 & 0.1 & 6.4 & 0.8 \\
\hline & $4 \times 8 \times 10$ & 1 & $<0.1$ & 4084 & 2.4 & 9.1 & 1.6 \\
\hline & $4.5 \times 5.6 \times 24$ & 1 & $<0.1$ & 7667 & 4.5 & 17.1 & 3.0 \\
\hline & $4.6 \times 5.6 \times 8.7$ & 1 & $<0.1$ & 2792 & 1.7 & 6.2 & 1.1 \\
\hline & $5 \times 11 \times 12$ & 1 & $<0.1$ & 4082 & 2.4 & 18.7 & 3.2 \\
\hline & $5 \times 6 \times 28$ & 1 & $<0.1$ & 8890 & 6.2 & 19.8 & 3.4 \\
\hline TOTALS & & 2288 & & 169818 & & 678.2 & \\
\hline & & & 76 & & & & $\%$ \\
\hline $\begin{array}{l}\text { Burlap, Cloth, Paper or } \\
\text { Plastic Bags }\end{array}$ & unknown & 13 & 0.5 & 768 & 0.4 & 1.7 & 0.3 \\
\hline \multirow[t]{2}{*}{ Concrete Boxes } & $3 \times 3 \times 3$ & 8 & 0.3 & 6368 & 3.1 & 6.1 & 1.0 \\
\hline & unknown & 1 & $<0.1$ & 737 & 0.4 & 0.7 & 0.1 \\
\hline $\begin{array}{l}\text { Fiberboard/Plastic Boxes, } \\
\text { Cartons, Cases }\end{array}$ & unknown & 2274 & 85.1 & 28583 & 14.0 & 287.0 & 47.7 \\
\hline HEPA Filters & unknown & 80 & 3.4 & 8470 & 4.2 & 17.8 & 3.0 \\
\hline \multirow{4}{*}{$\begin{array}{l}\text { Metal Boxes, Cartons, } \\
\text { Cases }\end{array}$} & $2 \times 3 \times 4$ & 9 & $<0.1$ & 305 & 0.1 & 0.7 & 0.1 \\
\hline & $3 \times 3 \times 4$ & 2 & 0.1 & 914 & 0.5 & 2.0 & 0.3 \\
\hline & $4 \times 4 \times 6$ & 1 & $<0.1$ & 1219 & 0.6 & 2.7 & 0.5 \\
\hline & $4 \times 5 \times 6$ & 2 & 0.1 & 127 & 0.1 & 0.3 & 0.1 \\
\hline \multirow{4}{*}{$\begin{array}{l}\text { Motal Drums, Barrols, } \\
\text { Kegs }\end{array}$} & $110-\mathrm{gal}$ & 8 & 0.3 & 1502 & 0.7 & 3.4 & 0.6 \\
\hline & $30-081$ & 1 & $<0.1$ & 41 & $<0.01$ & 0.1 & $<0.1$ \\
\hline & - " 55-gal & 25 & 0.9 & 9072 & 4.5 & 5.3 & 0.9 \\
\hline & 65-gal & 134 & 5.0 & 13035 & 6.4 & 28.5 & 4.7 \\
\hline
\end{tabular}


Table 5-4. Non-TRU Waste Generated at Building 325 Westinghouse Hanford by Container Type and Year. (10 sheets)

\begin{tabular}{|c|c|c|c|c|c|c|c|}
\hline ช & 6.0.1. & 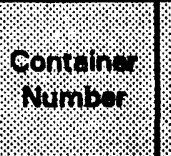 & ro, & conornots? & propto & $\begin{array}{l}\text { wolno } \\
\text { wn }\end{array}$ & polums \\
\hline Miscellanoous Scrap & unknown & 33 & 1.2 & 20327 & 10.0 & 45.3 & 7.5 \\
\hline Solf-Conteined Equipment & unknown & 41 & 1.5 & 2889 & 1.4 & 8.4 & 1.1 \\
\hline Tanke Portable & unknown & 3 & 0.1 & 20297 & 10.0 & 46.3 & 7.5 \\
\hline $\begin{array}{l}\text { Trucks, Flatbeds, } \\
\text { Compector, Loadlugoer }\end{array}$ & unknown & 18 & 0.7 & $\mathbf{5 0 8 5 9}$ & 24.8 & 86.9 & 14.5 \\
\hline \multirow{7}{*}{$\begin{array}{l}\text { Wooden Boxee, Cartons, } \\
\text { Cases }\end{array}$} & $2 \times 3 \times 4$ & 1 & $<0.1$ & 305 & 0.1 & 0.7 & 0.1 \\
\hline & $3 \times 3 \times 3$ & 1 & $<0.1$ & 343 & 0.2 & 0.8 & 0.1 \\
\hline & $3 \times 3 \times 4$ & 1 & $<0.1$ & 467 & 0.2 & 1.0 & 0.2 \\
\hline & $4 \times 4 \times 6$ & 1 & $<0.1$ & 12193 & 6.0 & 2.7 & 0.5 \\
\hline & $4 \times 4 \times 8$ & 12 & 0.6 & 19606 & 9.6 & 43.5 & 7.2 \\
\hline & $4 \times 6 \times 10$ & 1 & $<0.1$ & 3048 & 1.5 & 6.8 & 1.1 \\
\hline & $4 \times 6 \times 8$ & 1 & $<0.1$ & 2438 & 1.2 & 6.4 & 0.9 \\
\hline TOTALS & & 2673 & & 203613 & & 601.1 & \\
\hline & & & $\%$ & & & & $\$$ \\
\hline $\begin{array}{l}\text { Burlap, Cloth, Paper or } \\
\text { Plastic Bags }\end{array}$ & unknown & 13 & 0.5 & 3925 & 2.8 & 9.0 & 1.5 \\
\hline \multirow{3}{*}{$\begin{array}{l}\text { Fiberboard/Plastic Boxes, } \\
\text { Cartons, Cases }\end{array}$} & $2 \mathrm{ft}^{3}$ & 36 & 1.4 & 456 & 0.3 & 4.4 & 0.7 \\
\hline & $\cdots$ unknown & $\mathbf{5 5}$ & 2.2 & 699 & 0.5 & 7.0 & 1.2 \\
\hline & unknown & 2031 & 80.1 & 235 & 0.2 & 260.5 & 44.5 \\
\hline $\begin{array}{l}\text { Fiberboard/Plastic Drums, } \\
\text { Barrels, Kege }\end{array}$ & unknown & 1 & $<0.1$ & 38 & $<0.1$ & 0.1 & $<0.1$ \\
\hline HEPA Filters & unknown & 69 & 2.7 & 1170 & 0.8 & 9.4 & 1.6 \\
\hline \multirow{4}{*}{$\begin{array}{l}\text { Motal Boxes, Cartons, } \\
\text { Cases }\end{array}$} & $108 \mathrm{ft}^{\mathrm{s}}$ & 1 & $<0.1$ & 1372 & 1.0 & 3.1 & 0.5 \\
\hline & $2 \times 2 \times 3$ & 8 & 0.4 & 1300 & 0.9 & 2.9 & 0.5 \\
\hline & $4 \times 5 \times 9$ & 16 & 0.6 & 2784 & 2.0 & 6.2 & 1.1 \\
\hline & unknown & 2 & 0.1 & 188 & 0.1 & 0.4 & 0.1 \\
\hline \multirow[t]{2}{*}{ Metal Cylinder, Casks } & $\cdots$ unknown & 2 & 0.1 & 1218 & 0.9 & 2.7 & 0.5 \\
\hline & unknown & 2 & 0.1 & 81 & 0.1 & 0.2 & $<0.1$ \\
\hline \multirow{2}{*}{$\begin{array}{l}\text { Metal Drums, Barrels, } \\
\text { Kege }\end{array}$} & 110-gal & 2 & 0.1 & 272 & 0.2 & 0.9 & 0.1 \\
\hline & 30-gal & 8 & 0.4 & 348 & 0.3 & 2.0 & 0.3 \\
\hline
\end{tabular}


Table 5-4. Non-TRU Waste Generated at Building 325 Westinghouse Hanford by Container Type and Year. (10 sheets)

\begin{tabular}{|c|c|c|c|c|c|c|c|}
\hline 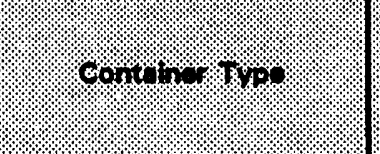 & 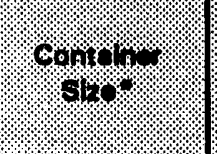 & $6 \%$ & $10 \%$ & 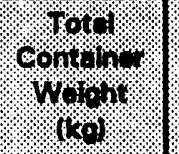 & propts & 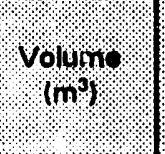 & roorons \\
\hline & 5-gal & 2 & 0.1 & 48 & $<0.1$ & 0.1 & $<0.1$ \\
\hline & $\bullet \bullet$ 56-oal & 7 & 0.3 & 826 & 0.6 & 1.6 & 0.3 \\
\hline & 56-gal & 110 & 4.3 & 18782 & 13.3 & 23.4 & 4.0 \\
\hline Miscellaneous Scrap & unknown & 57 & 2.3 & 6809 & 4.9 & 24.9 & 4.3 \\
\hline \multirow[t]{2}{*}{ Self-Contained Equipment } & $\cdots$ unknown & 12 & 0.6 & 5183 & 3.7 & 11.5 & 2.0 \\
\hline & unknown & 18 & 0.7 & 6230 & 4.4 & 13.9 & 2.4 \\
\hline \multirow[t]{2}{*}{ Tanks, Portable } & $\cdots$ unknown & 1 & $<0.1$ & 800 & 0.6 & 1.8 & 0.3 \\
\hline & unknown & 1 & $<0.1$ & 9525 & 6.7 & 21.2 & 3.6 \\
\hline $\begin{array}{l}\text { Trucke, Flatbeds, } \\
\text { Compactor, Loadlugger }\end{array}$ & unknown & 23 & 0.9 & 23305 & 16.5 & 53.7 & 9.2 \\
\hline \multirow{14}{*}{$\begin{array}{l}\text { Wooden Boxes, Cartons, } \\
\text { Cases }\end{array}$} & $0.7 \times 0.7 \times 6$ & 1 & $<0.1$ & 68 & 0.1 & 0.2 & $<0.1$ \\
\hline & $2 \times 2 \times 2$ & 1 & $<0.1$ & 227 & 0.2 & 0.2 & $<0.1$ \\
\hline & $2 \times 2 \times 3$ & 6 & 0.2 & 699 & 0.5 & 1.6 & 0.3 \\
\hline & $2 \times 2 \times 6$ & 1 & $<0.1$ & 203 & 0.1 & 0.5 & 0.1 \\
\hline & $2 \times 4 \times 4$ & 1 & $<0.1$ & 406 & 0.3 & 0.9 & 0.1 \\
\hline & $\cdots 3 \times 3 \times 3$ & 2 & 0.1 & 394 & 0.3 & 0.9 & 0.1 \\
\hline & $3 \times 3 \times 3$ & 9 & 0.4 & 2172 & 1.5 & 4.8 & 0.8 \\
\hline & $3 \times 3 \times 4$ & 1 & $<0.1$ & 467 & 0.3 & 1.0 & 0.2 \\
\hline & $\cdots 4 \times 4 \times 4$ & 2 & 0.1 & 1603 & 1.1 & 3.6 & 0.6 \\
\hline & $4 \times 4 \times 4$ & 6 & 0.2 & 4876 & 3.5 & 10.9 & 1.9 \\
\hline & $\cdots 4 \times 4 \times 6$ & 2 & 0.1 & 2439 & 1.7 & 5.4 & 0.8 \\
\hline & $\cdots 4 \times 4 \times 8$ & 8 & 0.3 & 13004 & 8.2 & 28.0 & 4.9 \\
\hline & $4 \times 4 \times 8$ & 17 & 0.7 & 27010 & 19.1 & 61.6 & 10.5 \\
\hline & $\cdots 4 \times 6 \times 8$ & 1 & $<0.1$ & 1829 & 1.3 & 4.1 & 0.7 \\
\hline TOTALS & & 2536 & & 141093 & & 585.5 & \\
\hline & & \multicolumn{2}{|c|}{1078} & ঝ & 18 & 18 & \\
\hline $\begin{array}{l}\text { Burlap, Cloth, Paper or } \\
\text { Plastic Bage }\end{array}$ & unknown & 7 & 0.2 & 280 & 0.2 & 0.9 & 0.1 \\
\hline Concrete Boxes & $3 \times 3 \times 3$ & 3 & 0.1 & 2241 & 1.3 & 2.2 & 0.3 \\
\hline $\begin{array}{l}\text { Concrete Cylinders, } \\
\text { Casks }\end{array}$ & $7 \times 12$ & 2 & 0.1 & 1746 & 1.0 & 9.9 & 1.1 \\
\hline
\end{tabular}


Table 5-4. Non-TRU Waste Generated at Building 325 Westinghouse Hanford by Container Tye and Year. (10 sheets)

\begin{tabular}{|c|c|c|c|c|c|c|c|}
\hline \%on. & $60 \%$ & ${ }^{2}$ & $10 \%$ & 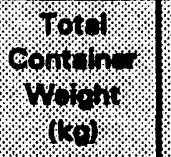 & $10 \% 0 \%$. & volnt? & rophts \\
\hline $\begin{array}{l}\text { Fiberboard/Plastic Boxes, } \\
\text { Cartons, Cases }\end{array}$ & unknown & $\begin{array}{c}2910 \\
.\end{array}$ & 85.2 & 40888 & 24.1 & 372.6 & 42.6 \\
\hline HEPA Filtere & unknown & 182 & $\mathbf{6 . 3}$ & 7024 & 4.1 & 22.5 & 2.8 \\
\hline $\begin{array}{l}\text { Motal Boxes, Cartons, } \\
\text { Cases }\end{array}$ & $5 \times 5 \times 7$ & 1 & $<0.1$ & 770 & 0.5 & 4.8 & 0.5 \\
\hline \multirow{6}{*}{$\begin{array}{l}\text { Motal Drume, Berrels, } \\
\text { Koge }\end{array}$} & $110-001$ & 1 & $<0.1$ & 464 & 0.3 & 0.4 & 0.1 \\
\hline & 30-gal & 3 & 0.1 & 180 & 0.1 & 0.4 & 0.1 \\
\hline & 6-gal & 3 & 0.1 & 68 & $<0.1$ & 0.2 & $<0.1$ \\
\hline & 1-gal & 2 & 0.1 & 27 & $<0.1$ & 0.01 & $<0.1$ \\
\hline & $\cdots$ 65-gal & 6 & 0.2 & 1520 & 0.8 & 1.3 & 0.1 \\
\hline & 56-gol & 159 & 4.6 & 20450 & 12.1 & 33.8 & 3.9 \\
\hline Miscellaneous Scrap & unknown & 52 & 1.5 & 14776 & 8.7 & 70.1 & 8.0 \\
\hline Self-Contained Equipment & unknown & 17 & 0.6 & 4684 & 2.7 & 62.2 & 7.1 \\
\hline $\begin{array}{l}\text { Trucks, Flatbeds, } \\
\text { Compactor, Loadlugger }\end{array}$ & unknown & 16 & 0.6 & 28683 & 16.8 & .121 .6 & 13.9 \\
\hline \multirow{13}{*}{$\begin{array}{l}\text { Wooden Boxes, Cartons, } \\
\text { Cases }\end{array}$} & $1.7 \times 1.8 \times 4.8$ & 1 & $<0.1$ & 158 & 0.1 & 0.4 & 0.1 \\
\hline & $2 \times 2 \times 4$ & 1 & $<0.1$ & 158 & 0.1 & 0.5 & 0.1 \\
\hline & $3 \times 4 \times 6$ & 1 & $<0.1$ & 762 & 0.6 & 1.7 & 0.2 \\
\hline & $3 \times 4 \times 8$ & 1 & $<0.1$ & 218 & 0.1 & 2.7 & 0.3 \\
\hline & $3 \times 5 \times 5$ & 2 & 0.1 & 748 & 0.4 & 4.3 & 0.6 \\
\hline & $4 \times 4 \times 4$ & 1 & $<0.1$ & 813 & 0.6 & 1.8 & 0.2 \\
\hline & $4 \times 4 \times 8$ & 25 & 0.7 & 36480 & 20.8 & 97.9 & 11.2 \\
\hline & $4 \times 6 \times 6$ & 1 & $<0.1$ & 653 & 0.4 & 4.1 & 0.5 \\
\hline & $4 \times 8 \times 14$ & 1 & $<0.1$ & 1016 & 0.6 & 12.7 & 1.6 \\
\hline & $6 \times 5 \times 8$ & 1 & $<0.1$ & 2540 & 1.5 & 6.7 & 0.7 \\
\hline & $6 \times 8 \times 12$ & 1 & $<0.1$ & 1308 & 0.8 & 16.3 & 1.9 \\
\hline & $8 \times 8 \times 12$ & 1 & $<0.1$ & 1688 & 0.9 & 21.8 & 2.5 \\
\hline & unknown & 17 & 0.5 & 371 & 0.2 & 3.4 & 0.4 \\
\hline TOTALS & & 3427 & & 169534 & & 876.1 & \\
\hline
\end{tabular}


Table 5-4. Non-TRU Waste Generated at Building 325 Westinghouse Hanford by Container Type and Year. (10 sheets)

\begin{tabular}{|c|c|c|c|c|c|c|c|}
\hline W & ?. & \%onnol. & $80 \%$ & 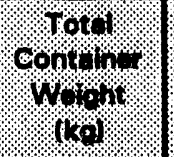 & woron & Volume & $\begin{array}{l}\text { Porcont } \\
\text { Volume }\end{array}$ \\
\hline \multicolumn{8}{|c|}{$18 \%$} \\
\hline $\begin{array}{l}\text { Burlap, Cloth, Paper or } \\
\text { Plastic Bage }\end{array}$ & unknown & 12 & 0.4 & 161 & 0.1 & 0.4 & 0.1 \\
\hline Concrete Boxes & $3 \times 3 \times 3$ & 7 & 0.2 & 7439 & 4.7 & 4.7 & 0.6 \\
\hline \multirow{3}{*}{$\begin{array}{l}\text { Fiberboard/Plastic Boxes, } \\
\text { Certons, Cases }\end{array}$} & $2 \mathrm{ft}^{3}$ & 20 & 0.7 & 85 & 0.1 & 1.0 & 0.1 \\
\hline & $\cdots$ unknown & 14 & 0.6 & 178 & 0.1 & 1.8 & 0.2 \\
\hline & unknown & 2397 & 82.3 & 30834 & 18.3 & 305.6 & 41.2 \\
\hline $\begin{array}{l}\text { Fiberboard/Plastic Drums, } \\
\text { Barrels, Kege }\end{array}$ & unknown & 1 & $<0.1$ & 41 & $<0.1$ & 0.2 & $<0.1$ \\
\hline Glove Boxes & unknown & 1 & $<0.1$ & 186 & 0.1 & 0.9 & 0.1 \\
\hline \multirow[t]{2}{*}{ HEPA Filters } & $\cdots$ unknown & 10 & 0.3 & 782 & 0.6 & 1.4 & 0.2 \\
\hline & unknown & 218 & 7.6 & 6188 & 3.9 & 26.4 & 3.6 \\
\hline $\begin{array}{l}\text { Motal Boxes, Cartons, } \\
\text { Ceses }\end{array}$ & $6 \times 8 \times 12$ & 1 & $<0.1$ & 2041 & 1.3 & 16.3 & 2.2 \\
\hline \multirow{3}{*}{$\begin{array}{l}\text { Motal Drums, Barrels, } \\
\text { Kege }\end{array}$} & 30-gal & 2 & 0.1 & 77 & 0.1 & 0.3 & $<0.1$ \\
\hline & 56-0al & 113 & 3.8 & 13628 & 8.6 & 24.0 & 3.2 \\
\hline & 5-gal & 2 & 0.1 & 46 & $<0.1$ & 0.1 & $<0.1$ \\
\hline Miecellaneous Scrap & unknown & 31 & 1.1 & 9032 & 5.7 & 22.2 & 3.0 \\
\hline Self-Contained Equipment & unknown & 19 & 0.7 & 1879 & 1.2 & 3.8 & 0.5 \\
\hline Tanks, Portable & unknown & 2 & 0.1 & 16 & $<0.1$ & 0.1 & $<0.1$ \\
\hline $\begin{array}{l}\text { Trucks, Flatbeds, } \\
\text { Compactor, Losdlugger }\end{array}$ & unknown & 28 & 1.0 & 46369 & 28.6 & 181.7 & 24.6 \\
\hline \multirow{8}{*}{$\begin{array}{l}\text { Wooden Boxes, Cartons, } \\
\text { Cases }\end{array}$} & $2 \times 2 \times 8$ & 1 & $<0.1$ & 406 & 0.3 & 0.9 & 0.1 \\
\hline & $3 \times 4 \times 4$ & 1 & $<0.1$ & 622 & 0.4 & 1.4 & 0.2 \\
\hline & $3 \times 4 \times 8$ & 1 & $<0.1$ & 363 & 0.2 & 2.7 & 0.4 \\
\hline & $4 \times 4 \times 12$ & 1 & $<0.1$ & 1048 & 0.7 & $\mathbf{6 . 4}$ & 0.7 \\
\hline & $4 \times 4 \times 8$ & 24 & 0.8 & 29374 & 18.6 & 87.0 & 11.7 \\
\hline & $4 \times 5 \times 7$ & 1 & $<0.1$ & 544 & 0.3 & 4.0 & 0.5 \\
\hline & $6 \times 8 \times 14$ & 2 & 0.1 & 6243 & 3.8 & 38.1 & 6.1 \\
\hline & $6 \times 8 \times 8$ & 1 & $<0.1$ & 2080 & 1.3 & 10.8 & 1.5 \\
\hline TOTALS & & 2812 & & 168448 & & 741.3 & \\
\hline
\end{tabular}


Table 5-4. Non-TRU Waste Generated at Building 325 Westinghouse Hanford by Container Type and Year. (10 sheets)

\begin{tabular}{|c|c|c|c|c|c|c|c|}
\hline 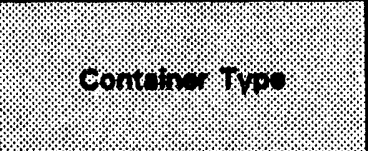 & $1000 \%$ & \%onp. & 1801 & 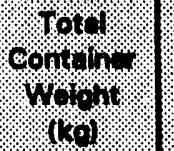 & mpn & volun & roprofs? \\
\hline \multicolumn{8}{|c|}{1080} \\
\hline $\begin{array}{l}\text { Fiberboard/Plastic Boxes, } \\
\text { Cartons, Cases }\end{array}$ & unknown & 211 & B4.6 & 8468 & 11.4 & 40.8 & 30.6 \\
\hline HEPA Filtere & unknown & 84 & 14.0 & 918 & 1.0 & 7.1 & 5.3 \\
\hline $\begin{array}{l}\text { Motal Boxes, Cartons, } \\
\text { Cases }\end{array}$ & unknown & 1 & 0.3 & 162 & 0.3 & 0.3 & 0.2 \\
\hline \multirow{2}{*}{$\begin{array}{l}\text { Motal Drums, Barrels, } \\
\text { Kage }\end{array}$} & 56-gal & 74 & 18.1 & 16562 & 32.4 & 15.7 & 11.7 \\
\hline & $\bullet$ 55-gal & 31 & 8.0 & 2108 & 4.4 & 6.6 & 4.9 \\
\hline Self-Contained Equipment & unknown & 1 & 0.3 & 544 & 1.1 & 1.6 & 1.2 \\
\hline \multirow{2}{*}{$\begin{array}{l}\text { Wooden Boxus, Cartons, } \\
\text { Ceses }\end{array}$} & $4 \times 4 \times 8$ & 13 & 3.4 & 10187 & 21.2 & 47.1 & 25.2 \\
\hline & -16x4x4 & 2 & 0.6 & 13118 & 27.3 & 14.6 & 10.9 \\
\hline TOTALS & & 387 & & 48080 & & 133.8 & \\
\hline \multicolumn{8}{|c|}{1881} \\
\hline Dump Truck & unknown & 1 & 8.3 & 2204 & 22.2 & 1.6 & 3.8 \\
\hline \multirow{3}{*}{$\begin{array}{l}\text { Wooden Boxes, Cartons, } \\
\text { Cases }\end{array}$} & $\because 4 \times 4 \times 8$ & 3 & 26.0 & 1363 & 13.7 & 10.8 & 27.6 \\
\hline & $4 \times 4 \times 8$ & 6 & 50.0 & 5443 & 54.9 & 21.7 & 64.7 \\
\hline & $\because 5.6 \times 6 \times 3$ & 2 & 16.7 & 908 & 9.2 & 5.6 & 14.1 \\
\hline TOTALS & & 12 & & 9818 & & 39.7 & \\
\hline \multicolumn{8}{|c|}{$198 \%$} \\
\hline \multicolumn{8}{|c|}{1083} \\
\hline $\begin{array}{l}\text { Wooden Boxes, Cartons, } \\
\text { Cases }\end{array}$ & $\because 8 \times 4 \times 4$ & 11 & 78.6 & 13301 & 83.0 & 42.1 & 97.7 \\
\hline \multirow[t]{2}{*}{ HEPA Concrate Boxes } & $2 \times 2 \times 3$ & 3 & 21.4 & 2729 & 17.0 & 1.0 & 2.3 \\
\hline & & 14 & & 16030 & & 43.1 & \\
\hline \multicolumn{8}{|c|}{1089} \\
\hline \multicolumn{8}{|c|}{1086} \\
\hline
\end{tabular}




\section{WHC-EP-0696}

Table 5-4. Non-TRU Waste Generated at Building 325 Westinghouse Hanford by Container Type and Year. (10 sheets)

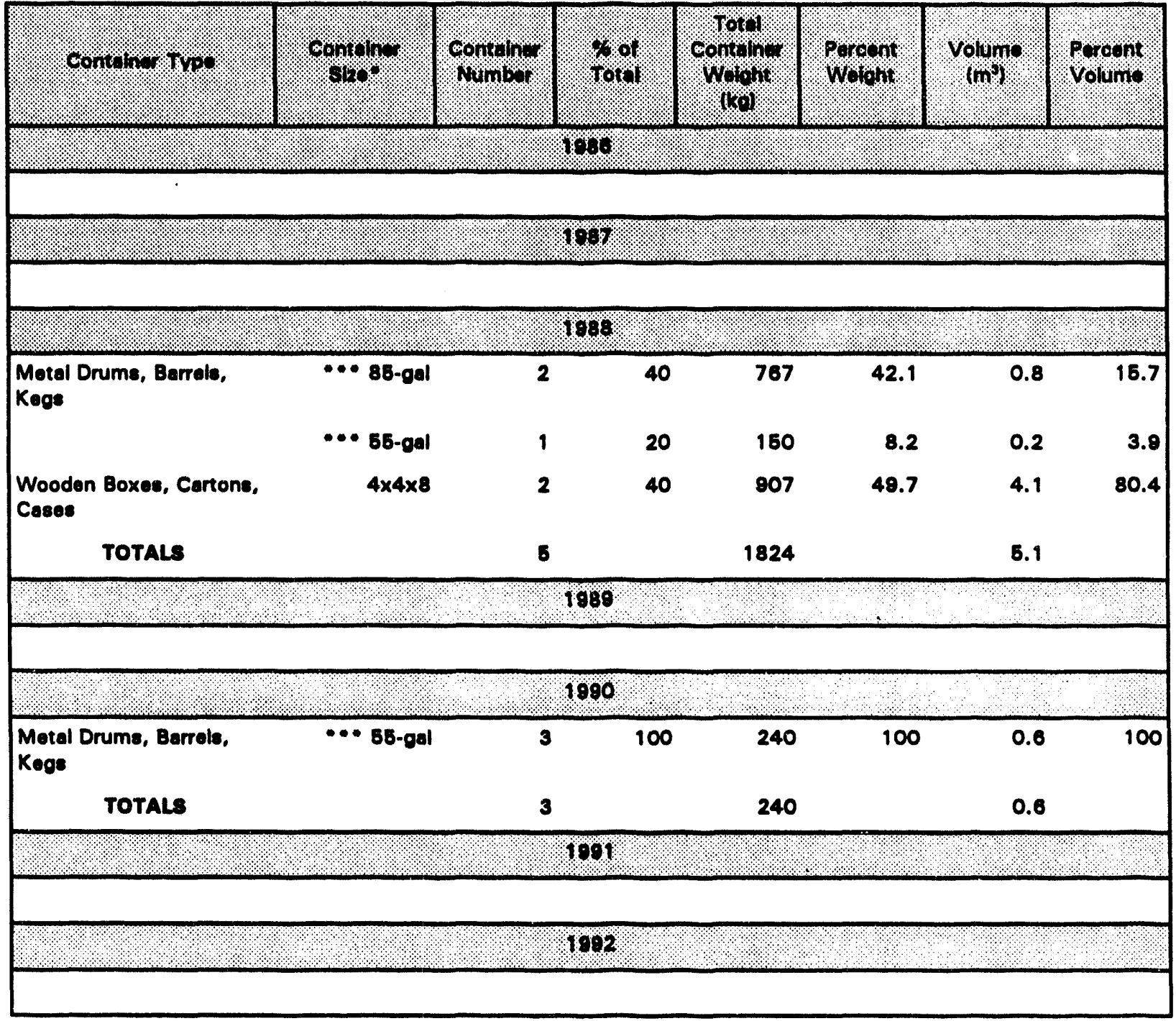

- Uniess otherwise noted, units of dimensions are in $\mathrm{ft}$.

- Solid Waste Burial Records for Building 325 WHC that were found with WHC Building 324 and 340 records.

*- These containers hold mixed wasto. 
Table 5-5. Building 325 Westinghouse Hanford Waste Summary Data for 55-Gallon Drums.

\begin{tabular}{|c|c|c|c|c|c|c|c|c|c|c|c|c|c|c|c|c|c|c|c|c|c|c|c|}
\hline $55 \mathrm{dm}$ & 100 & 107 & 1072 & 1078 & $107 \%$ & 1978 & 1976 & 1877 & 1078 & $107 \%$ & 1000 & 1981 & 1892 & 1083 & $18 \%$ & $10 \%$ & $106 \%$ & $1 \%$ & 10 & 10.9 & $10 \%$ & 101 & $1 \%$ \\
\hline \multicolumn{24}{|l|}{$0 \%=\%$} \\
\hline TRU Count & 67 & 238 & 238 & 217 & 254 & 218 & 162 & 140 & 307 & 437 & 91 & 0 & $20^{\circ}$ & 0 & $6^{*}$ & $\mathbf{8}^{\bullet}$ & 0 & $5^{\bullet}$ & 0 & 0 & 0 & 0 & 0 \\
\hline TRU Wt. (kal & 4650 & 16184 & 16184 & 14766 & 17577 & 15445 & 11022 & 8521 & 42781 & 20733 & 6182 & 0 & 1786 & 0 & $405^{\circ}$ & $630^{\circ}$ & 0 & $180^{\circ}$ & 0 & $\mathbf{0}$ & $\mathbf{0}$ & 0 & 0 \\
\hline TRU Vol. $\left(m^{3}\right)$ & 14.1 & 60.0 & 60.0 & 45.6 & 53.3 & 45.8 & 34.0 & 28.4 & 64.5 & 91.8 & 19.1 & 0 & $5.6^{\circ}$ & $\mathbf{0}$ & $1.3^{\circ}$ & $1.7 \bullet$ & 0 & $1.1^{\bullet}$ & 0 & 0 & 0 & 0 & $\mathbf{0}$ \\
\hline IRU un count & 0 & 0 & 0 & 0 & 0 & 0 & 0 & 0 & 0 & 0 & 0 & 0 & 0 & 0 & o & 0 & 0 & 0 & 0 & 0 & 0 & $\mathbf{0}$ & o \\
\hline Non-TFu Count & 10 & 36 & 68 & 93 & 67 & 143 & 160 & 117 & 165 & 113 & $\begin{array}{c}31 \cdot 1 \\
74\end{array}$ & 0 & 0 & $\mathbf{0}$ & 0 & $\mathbf{0}$ & 0 & 0 & 1 & 0 & 3 & 0 & 0 \\
\hline Non-ThU Wh. & 600 & 2301 & 4168 & 11180 & 6378 & 15936 & 22107 & 19000 & 21970 & 13028 & $\begin{array}{l}2108 \% 1 \\
15662\end{array}$ & 0 & 0 & 0 & 0 & $\mathbf{0}$ & 0 & $\mathbf{0}$ & 150 & 0 & 240 & 0 & 0 \\
\hline Mon-TAU Vol. (m') & 2.1 & 7.3 & 12.3 & 19.8 & 14.2 & 30.4 & 73.8 & 24.9 & 36.1 & 24.0 & $\begin{array}{l}6.5 \% \\
15.7\end{array}$ & 0 & 0 & $\mathbf{0}$ & 0 & 0 & 0 & 0 & 0.2 & 0 & 0.6 & 0 & 0 \\
\hline $\begin{array}{l}\text { Non-Tru MN } \\
\text { Count }\end{array}$ & 0 & 3 & 0 & 0 & 0 & 0 & 25 & 7 & 6 & $\mathbf{0}$ & $\begin{array}{l}0 \\
.\end{array}$ & 0 & 0 & 0 & 0 & $\mathbf{0}$ & 0 & 0 & 1 & 0 & 3 & $\mathbf{0}$ & 0 \\
\hline \multicolumn{24}{|l|}{$P n=m-1$} \\
\hline \% TRU Count & $87 \%$ & $87 \%$ & $60 \%$ & $70 \%$ & $79 \%$ & $60 \%$ & $60 \%$ & $54 \%$ & $65 \%$ & $79 \%$ & $46 \%$ & & $100 \%$ & & $100 \%$ & $100 \%$ & & $100 \%$ & $0 \%$ & & $0 \%$ & & \\
\hline XNon-TRU Count & $13 \%$ & $13 x$ & $20 \%$ & $30 \%$ & $21 \%$ & $40 x$ & $50 \%$ & $46 \%$ & $36 \%$ & $21 \%$ & $\begin{array}{l}16 \% \cdot 1 \\
30 \%\end{array}$ & & $0 x$ & & $0 x$ & $0 \%$ & & $0 x$ & $\begin{array}{c}100 \\
x\end{array}$ & & $\begin{array}{c}100 \\
\times\end{array}$ & & \\
\hline WX TRU & $87 \%$ & $87 \%$ & $80 \%$ & $57 \%$ & $68 \%$ & $49 \%$ & $33 x$ & $33 \%$ & $66 \%$ & $6 x$ & $26 \%$ & & $100 \%$ & & $100 \%$ & $100 \%$ & & $100 \%$ & $0 \%$ & & $0 x$ & & \\
\hline Wr\% Non-TFW & $13 \%$ & $13 x$ & $20 \%$ & $43 \%$ & $32 \%$ & $51 \%$ & $67 \%$ & $67 \%$ & $34 \%$ & $31 \%$ & $\begin{array}{l}9 \% \% \\
65 \%\end{array}$ & & $0 \%$ & & $0 \%$ & $0 x$ & & $O x$ & $\begin{array}{c}100 \\
\%\end{array}$ & & $\begin{array}{c}100 \\
\times\end{array}$ & & \\
\hline Vol\% TFU & $87 \%$ & $87 \%$ & $80 \%$ & $70 \%$ & $79 \%$ & $60 \%$ & $32 \%$ & $54 \%$ & $66 \%$ & $79 \%$ & $46 \%$ & & $100 \%$ & & $100 \%$ & $100 \%$ & & $100 \%$ & $0 \%$ & & $0 \%$ & & \\
\hline Vot\% Non-TRU & $13 \%$ & $13 \%$ & $20 \%$ & $30 \%$ & $21 \%$ & $40 \%$ & $68 \%$ & $46 \%$ & $36 \%$ & $21 \%$ & $\begin{array}{c}16 \% \cdot 1 \\
38 \%\end{array}$ & & $0 \%$ & & $.0 \%$ & $0 x$ & & $0 \%$ & $\stackrel{100}{x}$ & & $\begin{array}{c}100 \\
x\end{array}$ & & \\
\hline $\begin{array}{l}\text { Ximw Nan-TRU } \\
\text { by Count }\end{array}$ & & $9 \%$ & & & & & $16 \%$ & $6 \%$ & $4 \%$ & & & & & & & & & & $\stackrel{100}{*}$ & & $\begin{array}{c}100 \\
x\end{array}$ & & \\
\hline
\end{tabular}

-Solid Weste Burial Reconds that were found under WHC Bullding 324 and 340 rocords. 
Table 5-6. Building 325 Westinghouse Hanford Waste Summary Data for Containers Other Than 55-Gallon Drums.

\begin{tabular}{|c|c|c|c|c|c|c|c|c|c|c|c|c|c|c|c|c|c|c|c|c|c|c|c|}
\hline $\mathrm{Ond}_{\mathrm{Con}} \mathrm{O}$ & 1000 & $101 \%$ & 1ond & 1973 & 1074 & 1075 & 1076 & 1977 & 1078 & 1070 & 1090 & $1001 \%$ & 1002 & $100 \%$ & $109 \%$ & 1006 & $10 \%$ & $1 \%$ & 100 & $8 \%$ & $\%$ & 101 & 101 \\
\hline \multicolumn{24}{|l|}{ Dithomon } \\
\hline TRU Count & 22 & 2 & 25 & 0 & 23 & 6 & 3 & 2 & 10 & 0 & 1 & 0 & $2^{\circ}$ & $\mathbf{0}$ & 0 & 1 & 0 & 0 & 0 & 0 & 0 & 0 & 0 \\
\hline TrU Wh. (kal) & 5608 & 498 & 18000 & 0 & 21806 & 19940 & 3438 & 6665 & 9687 & 0 & 2496 & 0 & $3040^{\circ}$ & 0 & 0 & 2042 & 0 & 0 & 0 & 0 & 0 & $\mathbf{0}$ & 0 \\
\hline TRU Vol. $\left(m^{2}\right)$ & 13.2 & 1.1 & 26.4 & 0 & 48.6 & 44.6 & 7.8 & 13.9 & 10.9 & 0 & 11.6 & 0 & $9.6^{\circ}$ & 0 & o & 7.1 & 0 & 0 & o & 0 & 0 & 0 & 0 \\
\hline TRU MN Count & 0 & 0 & 0 & $\mathbf{0}$ & $\mathbf{0}$ & 0 & 0 & 0 & $\mathbf{0}$ & 0 & $\mathbf{0}$ & 0 & o & 0 & 0 & 0 & o & $\mathbf{0}$ & 0 & 0 & 0 & 0 & $\mathbf{0}$ \\
\hline Non-TRU Count & 648 & 1203 & 1281 & 1693 & 1635 & 2146 & 2514 & 2418 & 3262 & 2790 & $\begin{array}{l}2 \% \\
200\end{array}$ & $\begin{array}{c}5 \% \\
7\end{array}$ & 0 & $14^{\circ}$ & 0 & 0 & 0 & 0 & 4 & 0 & 0 & 0 & 0 \\
\hline Non-TRU Wt. kal & 42074 & 25063 & 100047 & 64160 & 47136 & 153683 & 181608 & 121486 & 147664 & 144820 & $\begin{array}{c}13110 \% \\
17270\end{array}$ & $\begin{array}{c}2272 \% \\
7647\end{array}$ & o & 10050 & 0 & 0 & 0 & 0 & 1674 & 0 & 0 & 0 & 0 \\
\hline Non-TRU Vod. $\left(m^{3}\right)$ & 165.7 & 174.3 & 242.8 & 205.0 & 261.8 & 547.8 & 567.3 & 560.6 & 841.0 & 717.3 & $\begin{array}{c}14.6 \% \\
97.0\end{array}$ & $\begin{array}{c}16.6 \% 1 \\
23.2\end{array}$ & 0 & 43.1० & 0 & 0 & 0 & 0 & 4.0 & 0 & 0 & 0 & 0 \\
\hline $\begin{array}{l}\text { Non-TRU anw } \\
\text { Coumt }\end{array}$ & 0 & 18 & 10 & 0 & 0 & $\mathbf{0}$ & o & 65 & $\mathbf{0}$ & 24 & 0 . & - & o & 0 & 0 & 0 & 0 & 0 & 2 & 0 & o & o & 0 \\
\hline \multicolumn{24}{|l|}{ 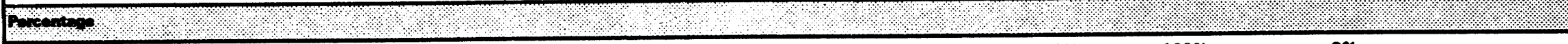 } \\
\hline \$TRU Count & $\mathbf{3 x}$ & $<1 \%$ & $2 \%$ & ox & $1 \%$ & $<1 \%$ & $<1 \%$ & $<1 x$ & $<1 \%$ & $0 x$ & $<1 \%$ & 0 & $100 \%$ & $0 x^{*}$ & & $100 x$ & & & ox & & & & \\
\hline KNon-ThU Count & 87\% & $100 \%$ & $98 \%$ & $100 x$ & $90 \%$ & $100 \%$ & $100 \%$ & $100 \%$ & $100 \%$ & $100 \%$ & $\begin{array}{l}<1 \% \% \\
100 \%\end{array}$ & $\begin{array}{l}42 \% \cdot \% \\
68 \%\end{array}$ & $0 x \cdot$ & $100 \%$ & & $0 x$ & & & $100 \%$ & & & & \\
\hline$w *$ TRU & $12 \%$ & $2 *$ & $25 \%$ & ox & $32 \%$ & $11 \%$ & $2 \%$ & $5 \%$ & $6 \%$ & $\mathbf{0 x}$ & $7 \%$ & $0 x$ & $100 \%$ & ox- & & $100 x$ & & & ox & & & & \\
\hline WtX Non-TRU & $68 \%$ & $88 \%$ & $75 \%$ & $100 \%$ & $68 \%$ & $89 \%$ & $98 \%$ & $95 \%$ & $94 \%$ & $100 \%$ & $\begin{array}{l}40 \% \cdot 1 \\
53 \%\end{array}$ & $\begin{array}{c}23 \% \cdot 1 \\
77 x\end{array}$ & $\mathbf{0 x}$ & $100 \%$ & & ox & & & $100 \%$ & & & & \\
\hline Vol\% TRU & $8 \%$ & $1 \%$ & $10 \%$ & ox & $16 \%$ & $\mathbf{8 \%}$ & $1 \%$ & $2 \%$ & $1 \%$ & ox & $\mathbf{9 x}$ & $0 \%$ & $100 \%$ & ox日 & & $100 \%$ & & & $0 \%$ & & & & \\
\hline Vol\% Non-TAU & $92 \%$ & $99 \%$ & $80 \%$ & $100 \%$ & $84 \%$ & $92 \%$ & $99 \%$ & $98 \%$ & $99 \%$ & $100 \%$ & $\begin{array}{l}12 \% \cdot 1 \\
78 \%\end{array}$ & $\begin{array}{l}42 \% \cdot 1 \\
58 \%\end{array}$ & $0 \%$ & $100 \%$ & & ox & & & $100 x$ & & & & \\
\hline \%MW Non-TRU & & $1 \%$ & $1 \%$ & & & & & $4 *$ & & $1 \%$ & & & & & & & & & $50 \%$ & & & & \\
\hline
\end{tabular}

-Solid Waste Burial Plocords thet were found under WHC Builing 324 and 340 records. 
Table 5-7. Building 325 Westinghouse Hanford TRU Waste in 55-Gallon Drums: Drum Count by Storage Location.

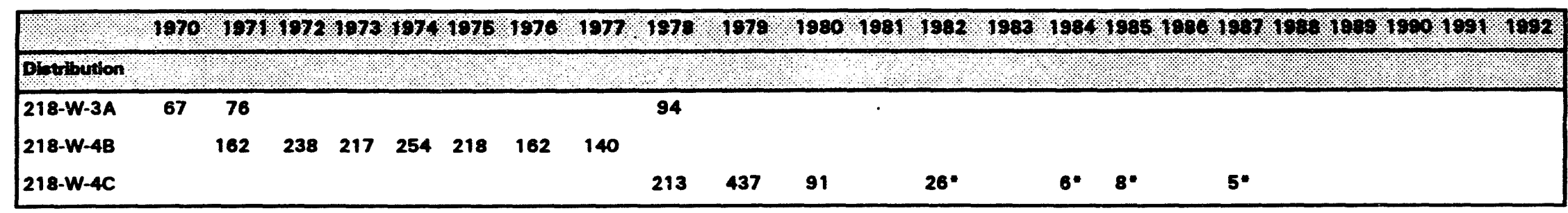

"Solid Waste Burial Records that werb found under WHC Building 324 and 340 records.

Table 5-8. Building 325 Westinghouse Hanford TRU Waste in Containers Other Than 55-Gallon Drums: Container Count by Storage Location.

\begin{tabular}{|c|c|c|c|c|c|c|c|c|c|c|c|c|c|c|c|c|c|c|c|c|c|}
\hline$\$$ & 1970 & 187 & $1972 \% 1873$ & 1874 & 1976 & 1879 & 1977 & 10781879 & 1980 & 1081 & 1982 & 1083 & 1884 & 1085 & 1880 & 1881 & $4 \%$ & $19 \%$ & $10 \%$ & 1991 & 1808 \\
\hline Distributon & & & & & & & & & & & & & & & & & & & & 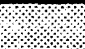 & \\
\hline 218-W-3A & 22 & 2 & & 2 & 6 & & & 7 & & & & & & & & & & & & & \\
\hline $218-W-4 B$ & & & 25 & 21 & & 3 & 2 & 3 & & & & & & & & & & & & & \\
\hline $218-W-4 C$ & & & & & & & & & 1 & & $2 *$ & & & 1 & & & & & & & \\
\hline
\end{tabular}

"Solid Waste Buriel Rocords that were found under WHC Building 324 and 340 records.

Table 5-9. Distribution and Percentage for Building 325 Westinghouse Hanford of TRU 55-Gallon Drum Physical Contents by Storage Location - 218-W-3A.

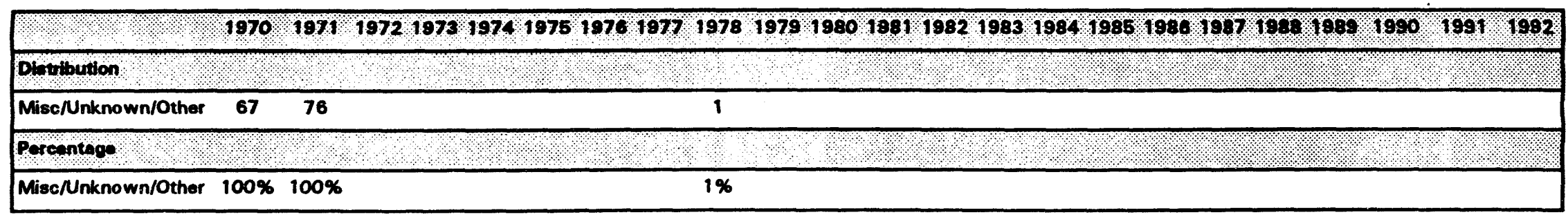


WHC-EP-0696

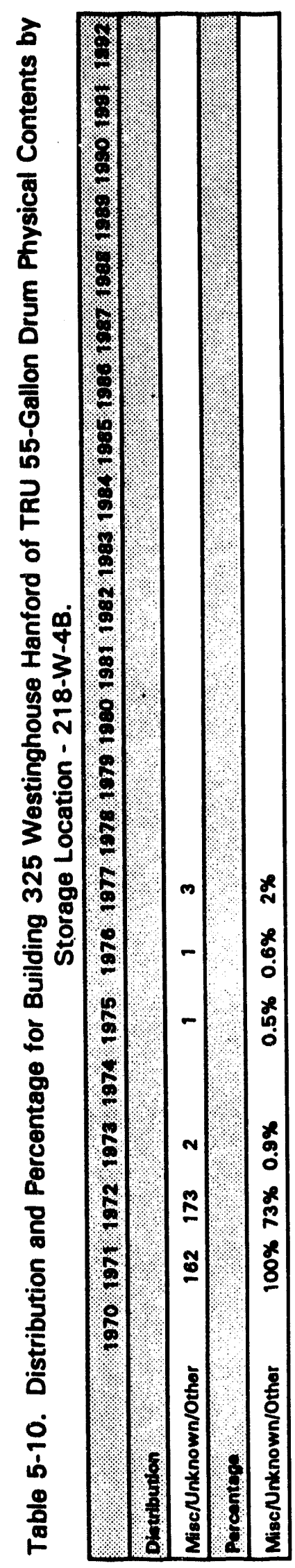


Table 5-11. Distribution and Percentage for Building 325 Westinghouse Hanford of TRU 55-Gallon Drums Physical Contents by Storage Location - 218-W-4C. (2 sheets)

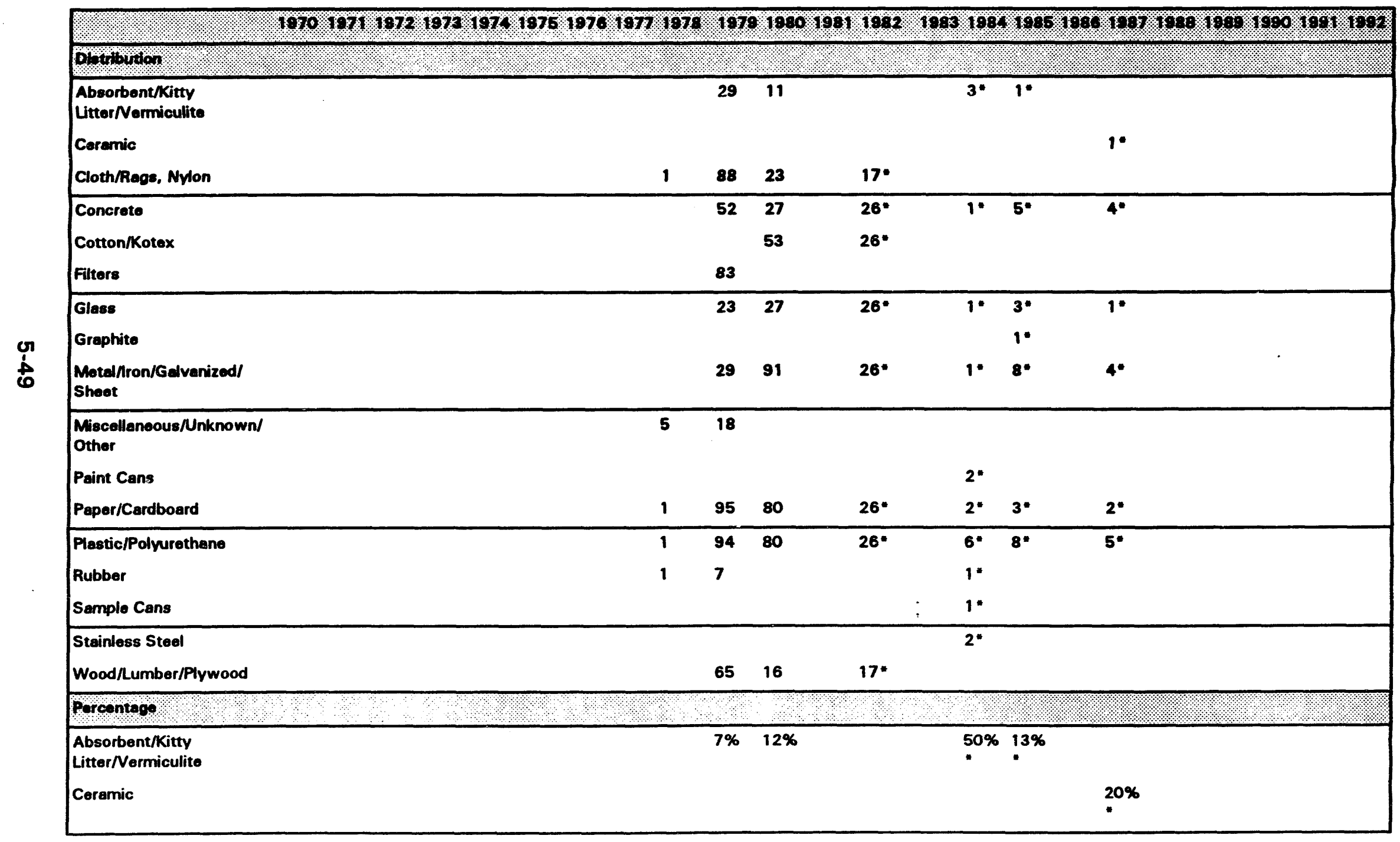


Table 5-11. Distribution and Percentage for Building 325 Westinghouse Hanford of TRU 55-Gallon Drums Physical Contents by Storage Location - 218-W-4C. (2 sheets)

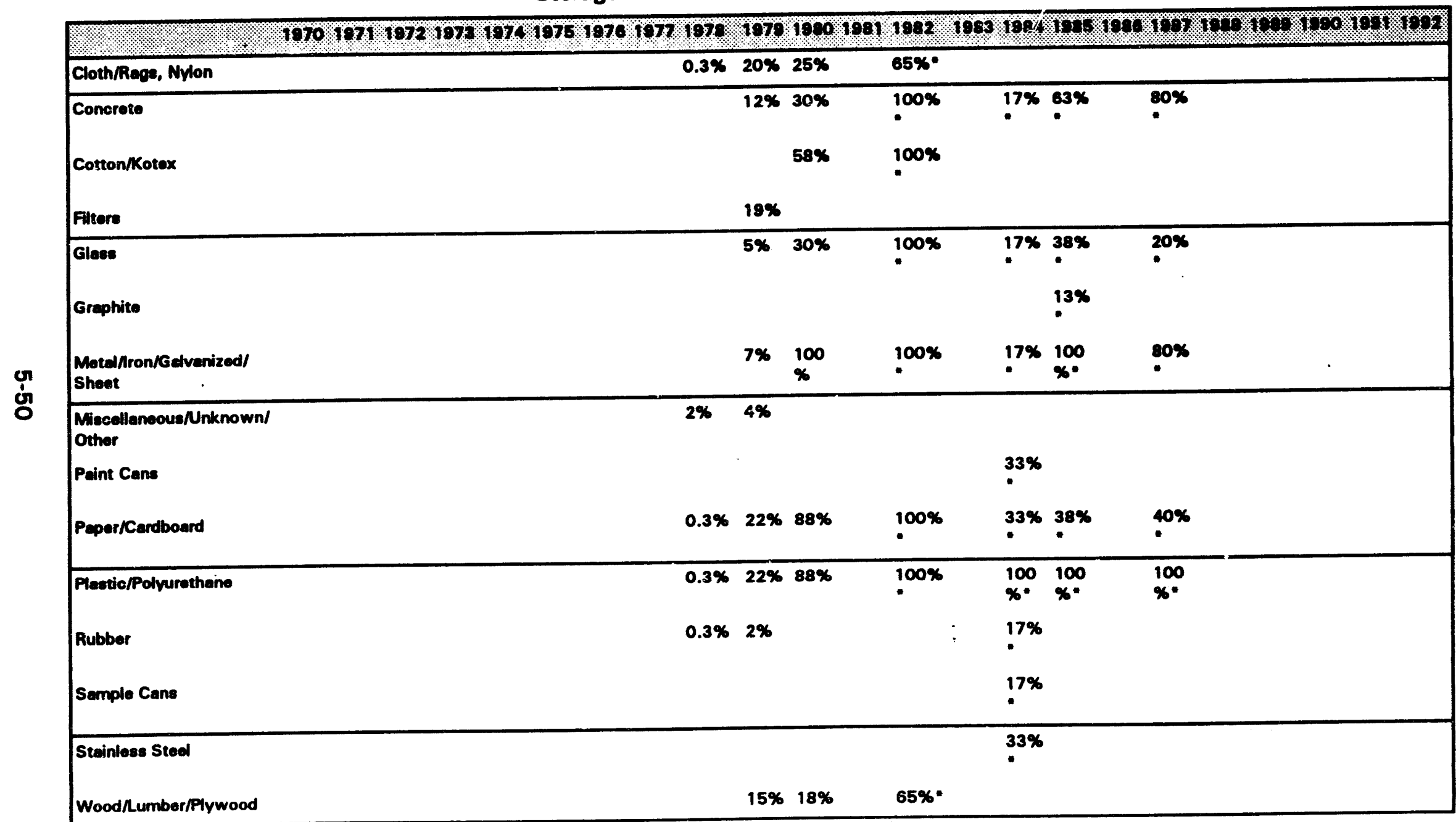


Table 5-12. Distribution and Percentage for Bıelding 325 Westinghouse Henford of TRU Containers Other Than 55-Gallon Drums Physical Contents by Storage Location - 218-W-3A.

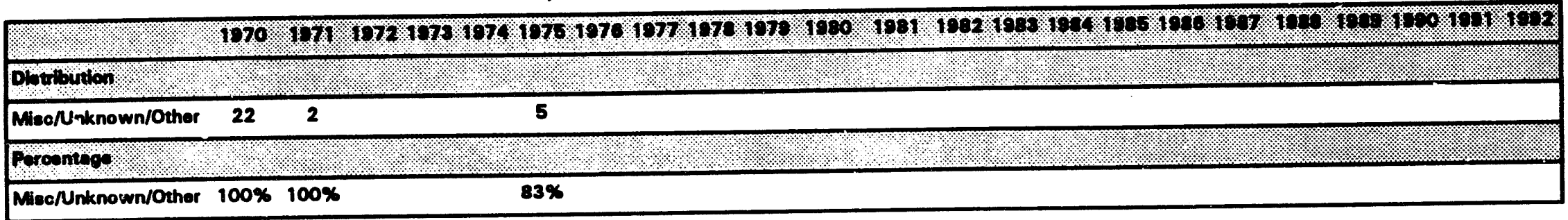

Table 5-13. Distribution and Percentage for Building 325 Westinghouse Hanford of TRU Containers Other Than 55-Gallon Drums Physical Contents by Storage Location - 218-W-4B.

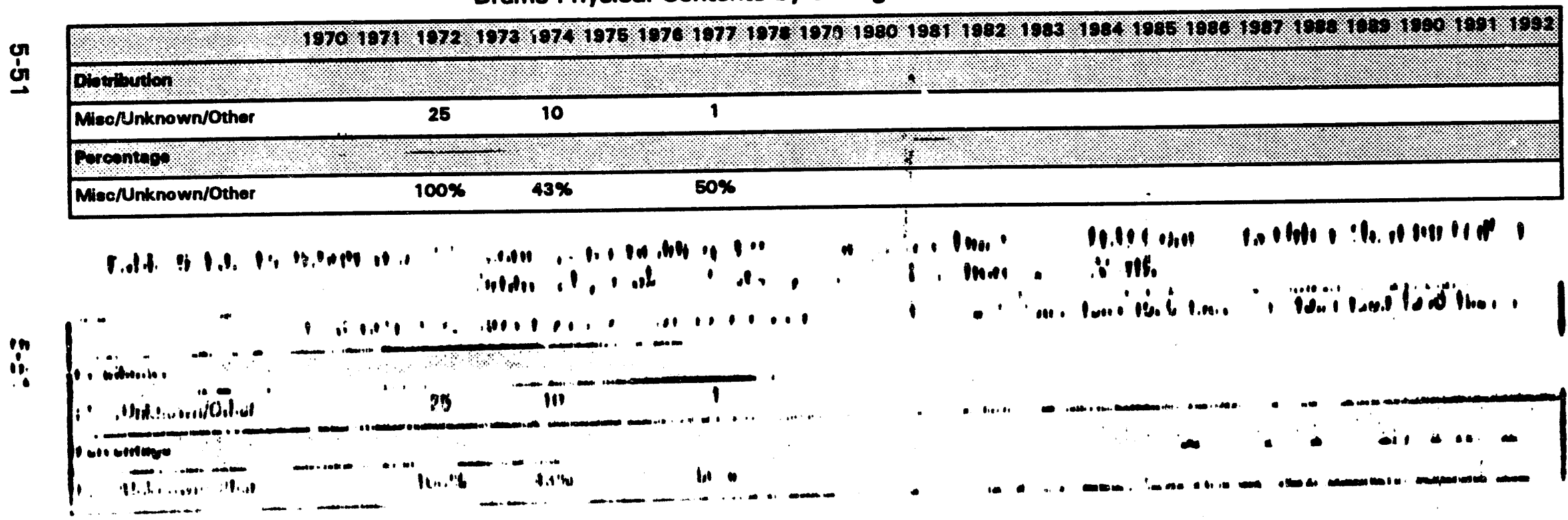


Table 5-14. Distribution and Percentage for Building 325 Westinghouse Hanford of TRU Containers Other Than 55-Gallon Drums Physical Contents by Storage Location - 218-W-4C.

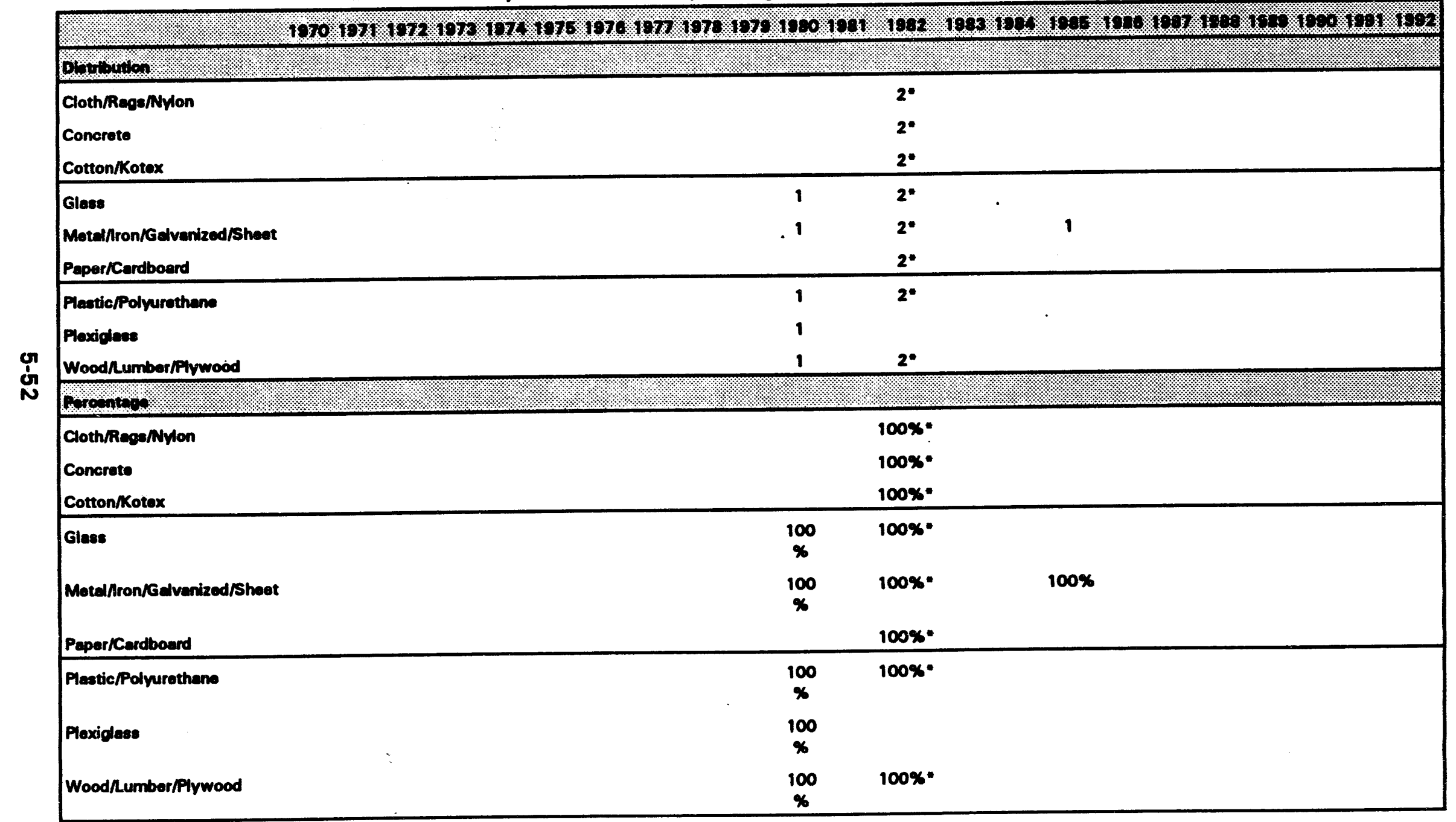


Table 5-15. Building 325 Westinghouse Hanford TRU Waste in 55-Gallon Drums: Total Grams TRU by Storage Location.

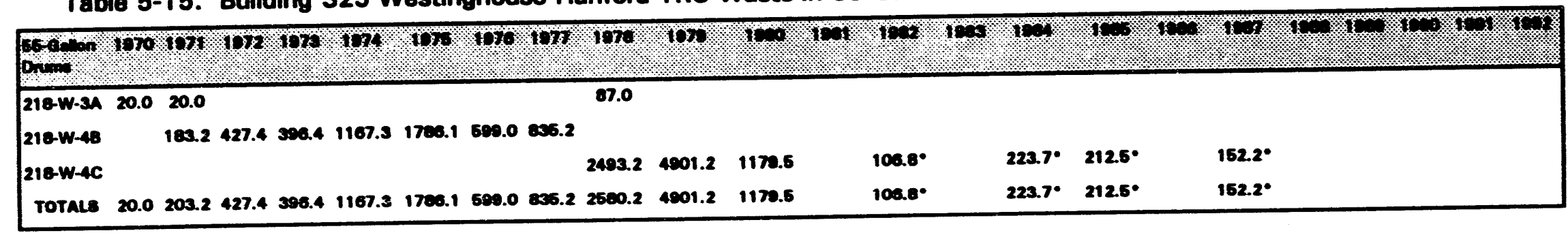

Table 5-16. Building 325 Westinghouse Hanford TRU Waste in Containers Other than 55-Gallon Drums: Total Grams by Storage Location.

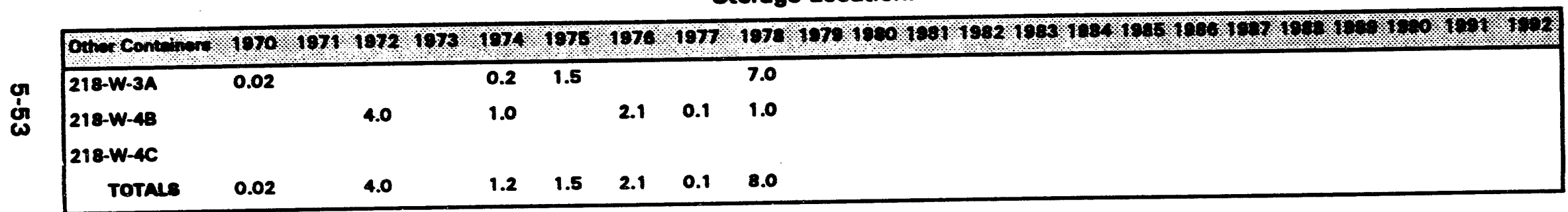


Table 5-17. Isotopes in 55-Gallon Drums for Westinghouse Hanford Building 325 (1970-1992).

\begin{tabular}{|c|c|c|c|}
\hline 100092 & Yror 16 & Gernuty (ด) & Reoctation \\
\hline${ }^{241} \mathrm{Am}$ & $433 Y$ & 0.6 & $a, r$ \\
\hline${ }^{246} \mathrm{Cm}$ & $8.5 \times 10^{3} \mathrm{Y}$ & 2.0 & $a, r$ \\
\hline${ }^{237} \mathrm{~Np}$ & $2.14 \times 10^{\circ} \mathrm{Y}$ & 20.7 & $a, v$ \\
\hline${ }^{230} \mathrm{Pu}$ & $87.74 Y$ & 1.3 & $a, r$ \\
\hline 232Th & $1.41 \times 10^{10} \mathrm{Y}$ & 33973 & $a, v$ \\
\hline $233 \mathrm{U}$ & $1.592 \times 10^{5} Y$ & 12.0 & $a, v$ \\
\hline Pu total & 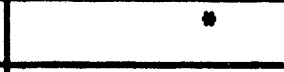 & 14059.09 & $"$ \\
\hline U depleted & 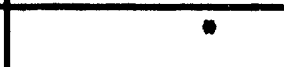 & 225019.14 & 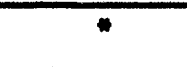 \\
\hline$U$ enriched & 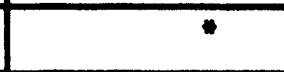 & 19009.19 & 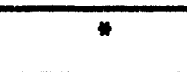 \\
\hline$U$ natural & - & 561016.18 & 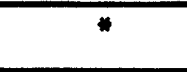 \\
\hline
\end{tabular}

$Y=$ Year

" = Varies according to isotope.

Table 5-18. Isotopes in Other Containers for Westinghouse Hanford Building 325

(1970-1992).

\begin{tabular}{|c|c|c|c|}
\hline 1001000 & Yhate & OHantio 10 & Radiation \\
\hline Pu total & $*$ & $16.96 g$ & 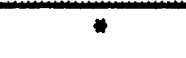 \\
\hline$U$ enriched & 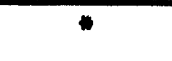 & $35.00 \mathrm{~g}$ & $*$ \\
\hline$U$ natural & - & $32.00 \mathrm{~g}$ & 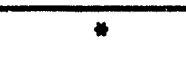 \\
\hline
\end{tabular}


Table 5-19. Building 325: Chemicals Used at Westinghouse Hanford Company Devolopment Laboratory (1970-1976). (3 sheets)

\begin{tabular}{|c|c|c|c|}
\hline 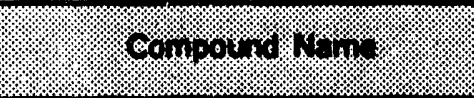 & rong & 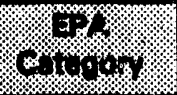 & 901 alfirmotion. \\
\hline Acetic Acid & $\mathrm{CH}_{3} \mathrm{CO}_{2} \mathrm{H}$ & D & corrosive \\
\hline Acetone & $\mathrm{CH}_{3} \mathrm{C}_{2} \mathrm{OH}_{3}$ & D & flammable liquid \\
\hline Aluminum & Al & D & flammable solid \\
\hline Ammonia (Anhydrous) & $\mathrm{NH}_{3}$ & B & \\
\hline Ammonium Oxalate & $\left(\mathrm{NH}_{4}\right)_{2} \mathrm{C}_{2} \mathrm{O}_{4} \mathrm{H}_{2} \mathrm{O}$ & & OAM-A' \\
\hline Barium Nitrate & $\mathrm{Ba}\left(\mathrm{NO}_{3}\right)_{2}$ & C & oxidizer \\
\hline Beryllium & Be & (1 $\begin{array}{c}X \\
\text { Dust) }\end{array}$ & Poison B \\
\hline Boric Acid & $\mathrm{H}_{3} \mathrm{BO}_{3}$ & $\mathbf{B}$ & \\
\hline Calcium Carbonate & $\mathrm{CaCO}_{3}$ & & \\
\hline Calcium Chloride & $\mathrm{CaCl}_{2}$ & D & \\
\hline Calcium Nitrate & $\mathrm{Ca}\left(\mathrm{NO}_{3}\right)_{4}$ & & oxidizer \\
\hline Ceric Nitrate & $\mathrm{Ce}\left(\mathrm{NO}_{3}\right)_{4}$ & & \\
\hline Cerous Nitrate & $\mathrm{Co}\left(\mathrm{NO}_{3}\right)_{3}$ & D & \\
\hline Cesium & Cs & & \\
\hline Chromic Acid & $\mathrm{H}_{2} \mathrm{CrO}_{4}$ & C & \\
\hline Citric Acid & $\mathrm{C}_{3} \mathrm{H}_{4} \mathrm{OH}(\mathrm{COOH})_{3}$ & & \\
\hline Diatomateous Earth & $\mathrm{SiO}_{2}$ & & \\
\hline Ethanol & $\mathrm{CH}_{3} \mathrm{CH}_{2} \mathrm{OH}$ & & combustible flammable liquid \\
\hline Ferric Ammonium Sulfate & $\mathrm{Fe}_{3} \mathrm{NH}_{4}\left(\mathrm{SO}_{4}\right)_{2}$ & & \\
\hline Ferric Nitrate & $\mathrm{Fe}\left(\mathrm{NO}_{3}\right)_{3} \cdot 6 \mathrm{H}_{2} \mathrm{O}$ & C & oxidizer \\
\hline Ferric Sulfate & $\mathrm{Fe}_{2}\left(\mathrm{SO}_{4}\right)_{3}$ & & \\
\hline Hydrazine & $\mathrm{H}_{2} \mathrm{NNH}_{2} \mathrm{H}_{2} \mathrm{O}$ & B & flammable liquid, poison/corrosive \\
\hline Hydrochloric Acid & $\mathrm{HCl}$ & D & nonflammable gas, corrosive \\
\hline lodine & $I_{2}$ & & \\
\hline Iron & $\mathbf{F e}$ & & \\
\hline Isopropyl Alcohol & $\mathrm{C}_{3} \mathrm{H}_{7} \mathrm{OH}$ & & Flammable liquid \\
\hline Lanthanum-Neodynium Nitrate & $\mathrm{La}\left(\mathrm{NO}_{3}\right)_{3}(\mathrm{Nd})\left(\mathrm{NO}_{3}\right)_{3}$ & & \\
\hline Lead Nitrate & $\mathrm{Pb}\left(\mathrm{NO}_{3}\right)_{2}$ & & oxidizer \\
\hline Magnesium Nitrate & $\mathrm{Mg}\left(\mathrm{NO}_{3}\right)_{2}$ & & \\
\hline Mercuric Nitrate & $\mathrm{Ho}\left(\mathrm{NO}_{3}\right)_{2} \mathrm{H}_{2} \mathrm{O}$ & A & oxidizer/poison \\
\hline Mathanol & $\mathrm{CH}_{3} \mathrm{OH}$ & D & flammable liquid \\
\hline Mineral Oil & Light Hydrocarbons & & \\
\hline
\end{tabular}




\begin{tabular}{|c|c|c|c|}
\hline & & 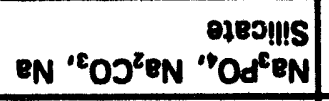 & e(1408solog) OPLL \\
\hline & & "(EN $\left.N^{2} O^{2} H^{2} O\right)^{1} H^{2} \partial^{2} N$ & 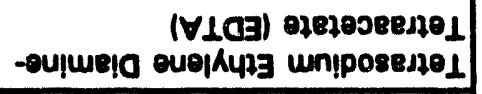 \\
\hline \multirow[t]{8}{*}{ DẠsod100 } & 0 & $\cos ^{2} \mathrm{H}$ & 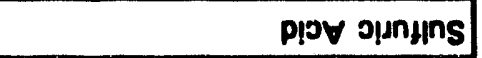 \\
\hline & & $" O^{x H^{2}}$ & Jeons \\
\hline & & icoN)/S & oledin un!̣uodzs \\
\hline & & IOINOS & joots ssopulets \\
\hline & & $\cos ^{2} \mathrm{EN}$ & oleyns un!pos \\
\hline & $\mathbf{a}$ & ${ }^{2} \mathrm{O}: 5 \mathrm{O}^{2} \mathrm{eN}$ & ofeou!s wn!pos \\
\hline & & 'OdeN & oleydsoud un!pos \\
\hline & & $\mathrm{O}_{\mathrm{S}} \mathrm{J}_{\mathrm{B}}^{\mathrm{BN}}$ & ofepexo un!pos \\
\hline Jez!p!x:0| & 5 & 'ONEN & จน!H!N wn!pos \\
\hline \multirow[t]{3}{*}{. Jez!p|xo } & $\mathbf{a}$ & EONEN & oIEAIN Un!pos \\
\hline & & IEN & op!pol un!pos \\
\hline & $\mathbf{8}$ & $1008 \mathrm{~N}$ & DluoHYOOdAH un!pos \\
\hline \multirow[t]{3}{*}{ OA!s01000 } & 0 & HOQN & op!xospAH wn!pos \\
\hline & & $\mathrm{O}^{2} \mathrm{H}^{2} \mathrm{O}^{2} \mathrm{~J} \mathrm{~J}^{2} \mathrm{BN}$ & olewos4ग! un!pos \\
\hline & $\mathbf{a}$ & $\mathrm{OJJ}^{2} \mathrm{BN}$ & oleuoquej un!pos \\
\hline \multirow[t]{2}{*}{ 28-wบo } & & $\mathrm{O}^{2} \mathrm{H}^{\prime} \mathrm{OSHEN}$ & oxeuns!g unipos \\
\hline & & 'OSHEN & oleuoqueoig unipos \\
\hline sez|p|xo & $x$ & 'ONOV & OZRJIIN JOAIIS \\
\hline sozı̣p!xo & $\mathbf{a}$ & rouwy & oleueourused unisselod \\
\hline \multirow[t]{4}{*}{ ¿V-WHO } & & $\cos ^{2} x$ & orewosuगg unissezod \\
\hline & $\mathbf{a}$ & $\cos ^{2} x$ & oleuoquej unissezod \\
\hline & & 'OJHX्र & oreuoquejg unissetod \\
\hline & & spunoduos unịples & zuowes pueppod \\
\hline Onisossos & & $0^{2} d$ & oplxoyued snojoydsoud \\
\hline \multirow[t]{3}{*}{ OApsousos } & $\mathbf{a}$ & ${ }^{\prime} O d^{4} H$ & PlOY opoydsoud \\
\hline & & 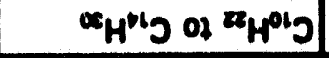 & UoquesospAH UlHeded jeusoN \\
\hline & & 'ONH & P!OY snoגมn \\
\hline \multirow[t]{2}{*}{ 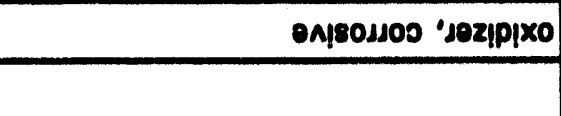 } & 3 & EONH & PIOY D!MIIN \\
\hline & & ow & unuepgAyow \\
\hline 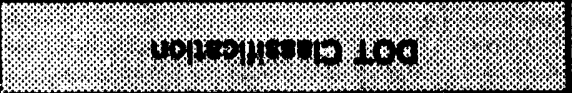 & \%(2) & $3^{3}+1.91$ & 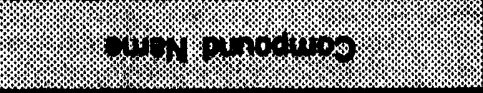 \\
\hline
\end{tabular}

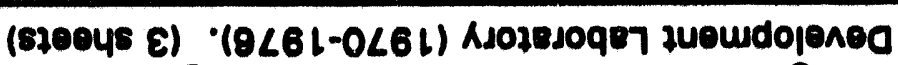

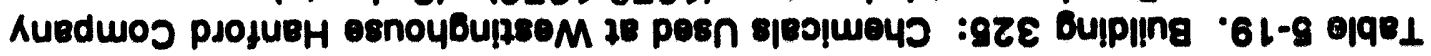




\section{WHC-EP-0696}

Table 5-19. Building 325: Chemicals Used at Weatinghouse Hanford Company Development Laboratory (1970-1976). (3 sheets)

\begin{tabular}{|c|c|c|c|}
\hline 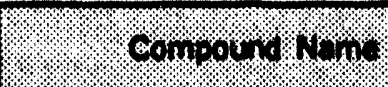 & roming & 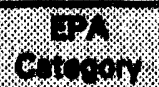 & Dor clinulicotion \\
\hline Tributyl Phosphate & $\left(\mathrm{C}_{4} \mathrm{H}_{2}\right)_{5} \mathrm{PO}_{4}$ & D & \\
\hline Turco $45020^{4}$ & $\begin{array}{l}\mathrm{KOH}_{1} \mathrm{~K}_{2} \mathrm{CrO}_{40} \\
\mathrm{KMnO}_{4}\end{array}$ & & \\
\hline Turco $4518^{4}$ & $\begin{array}{l}\mathrm{HO}_{2} \mathrm{CCO}_{22} \mathrm{H}_{2} \mathrm{H}_{2} \mathrm{O} \\
\text { Sodlum Dodeoyl } \\
\text { Benene Sultonate }\end{array}$ & & \\
\hline Urea & $\mathrm{CO}\left(\mathrm{NH}_{2}\right)_{2}$ & & \\
\hline Vanadium & v & & \\
\hline Zinc Nitrate & $\mathrm{Zn}\left(\mathrm{NO}_{3}\right)_{2}$ & & \\
\hline Zirconium & $\mathbf{Z r}$ & & \\
\hline
\end{tabular}

'Other regulated material with an anesthetic, irritating, noxious, toxic or other properties than can cause discomfort to persons in the event of leakage.

${ }^{2}$ Other regulated material capable of causing significant damage from leakage.

TTradename - Proctor and Gamble.

^Tradename - Turco Products, Inc. 
Table 6-20. Bullding 328: Chemicals Used at Analytical Laboratory (1983-1976). (2 sheots)

\begin{tabular}{|c|c|c|c|}
\hline 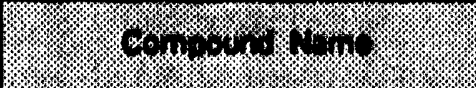 & $10 \%$ & 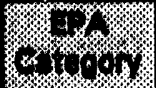 & 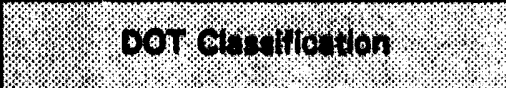 \\
\hline Acetona & $\mathrm{CH}_{2} \mathrm{C}_{2} \mathrm{OH}_{3}$ & $\mathbf{D}$ & flammable liquid \\
\hline Aluminum Nitrate Nonahydrate & $\mathrm{Al}\left(\mathrm{NO} \mathrm{O}_{\mathrm{g}} \mathrm{OH}_{2} \mathrm{O}\right.$ & $\mathbf{D}$ & oxidizer \\
\hline Ammonia (Anhydrous) & $\mathrm{NH}_{3}$ & $\mathbf{B}$ & \\
\hline Ammonium Hydroxide & $\mathrm{NH}_{4} \mathrm{OH}$ & $\mathbf{C}$ & corrosive, OAM-A' \\
\hline Ammonium Oxalate & $\left(\mathrm{NH}_{4}\right)_{2} \mathrm{C}_{2} \mathrm{O}_{4} \cdot \mathrm{H}_{2} \mathrm{O}$ & & OAM-A' \\
\hline Bromocresol Purple & $\mathrm{C}_{7} \mathrm{H}_{0} \mathrm{OHBr}$ & & \\
\hline Calcium Carbonate & $\mathrm{CaCO}_{3}$ & & \\
\hline Calcium Chloride & $\mathrm{CaCl}_{2}$ & D & \\
\hline Carbon Tetrachloride & $\mathrm{CCl}_{4}$ & $\mathbf{D}$ & ORM-A' \\
\hline Ceric Sulfate & $\mathrm{Ce}\left(\mathrm{SO}_{4}\right)_{2}$ & $\mathbf{C}$ & \\
\hline Cesium Nitrate & $\mathrm{CaNO}_{3}$ & $D$ & \\
\hline Ethanol & $\mathrm{C}_{2} \mathrm{H}_{6} \mathrm{OH}$ & & combustible, flammable liquid \\
\hline Ferrous Ammonium Sulfate & $\mathrm{Fe}\left(\mathrm{NH}_{4}\right)_{2}\left(\mathrm{SO}_{4}\right)_{2} \cdot 6 \mathrm{H}_{2} \mathrm{O}$ & & \\
\hline Hydrazine & $\mathrm{H}_{2} \mathrm{NNH}_{2} \mathrm{H}_{2} \mathrm{O}$ & $\mathbf{B}$ & flammable liquid, poison/corrosive \\
\hline Hydrochloric Acid & $\mathrm{HCl}$ & D & non-flammable gas, corrosive \\
\hline Hydrofluoric Acid & HF & $\mathbf{B}$ & corrosive \\
\hline Hydrogen Paroxide & $\mathrm{H}_{2} \mathrm{O}_{2}$ & $\mathbf{B}$ & oxidizer \\
\hline Hydroiodic Acid & HI & & \\
\hline Hydroxylamine Hydrochloride & $\mathrm{NH}_{2} \mathrm{OH}+\mathrm{HCl}$ & & \\
\hline lodine & $I_{2}$ & & \\
\hline Iron & Fe & & \\
\hline Kerosene & $\mathrm{C}_{10} \mathrm{H}_{22}$ to $\mathrm{C}_{10} \mathrm{H}_{34}$ & & combustible \\
\hline Magnesium Oxide & MgO & & \\
\hline Mercury & $\mathrm{Hg}$ & $\mathbf{x}$ & ORM-B ${ }^{2}$ \\
\hline Mercuric Nitrate & $\mathrm{Hg}\left(\mathrm{NO}_{3}\right)_{2} \mathrm{H}_{2} \mathrm{O}$ & A & oxidizer/poison \\
\hline Methanol & $\mathrm{CH}_{3} \mathrm{OH}$ & $\mathbf{D}$ & flammable liquid \\
\hline Methyl Ethyl Ketone & $\mathrm{CH}_{3} \mathrm{COC}_{2} \mathrm{H}_{5}$ & $\mathbf{D}$ & flammable liquid \\
\hline Methyl Isobutyl Ketone & $\mathrm{CH}_{3} \mathrm{COC}_{4} \mathrm{H}_{2}$ & D & \\
\hline Methyl Lactic Acid & $\mathrm{CH}_{3} \mathrm{CH}(\mathrm{OH}) \mathrm{CO}_{2} \mathrm{CH}_{3}$ & & \\
\hline Nitric Acid & $\mathrm{HNO}_{3}$ & $\mathbf{C}$ & oxidizer, corrosive \\
\hline Nitrous Acid & $\mathrm{HNO}_{2}$ & & \\
\hline Oxalic Acid & $\mathrm{HO}_{2} \mathrm{CCO}_{2} \mathrm{H}_{2} \mathrm{H}_{2} \mathrm{O}$ & & \\
\hline
\end{tabular}


Table 5-20. Bullding 325: Chemicals Used at Analytical Laboratory (1963-1976). (2 sheots)

\begin{tabular}{|c|c|c|c|}
\hline 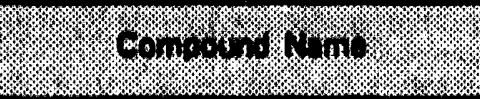 & $6 \%$ & 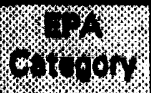 & $60 \%$ erpitionon \\
\hline Perchloric Acid & $\mathrm{HClO}_{4}$ & & \\
\hline Perlodic Acld & 104 & & . \\
\hline Phosphoric Acid & $\mathrm{H}_{9} \mathrm{PO}_{4}$ & $\mathbf{D}$ & corrosive \\
\hline Potesslum Hydroxide & KOH & $\mathbf{C}$ & corrosive \\
\hline Potassium lodate & $\mathrm{KIO}$ & & \\
\hline Potasalum Parmanganate & $\mathrm{KMnO}_{4}$ & $\mathbf{D}$ & oxidizer \\
\hline Silver Nitrate & AgNO, & $x$ & oxidizer \\
\hline Sodlum Carbonate & $\mathrm{Na}_{2} \mathrm{CO}_{3}$ & $\mathbf{D}$ & \\
\hline Sodium Hydroxide & NAOH & $\mathbf{C}$ & corrosive \\
\hline Stainieas Steel & Fonicr & & \\
\hline Strontium Nitrate & Sr $\left(\mathrm{NO}_{2} \mathrm{l}_{2}\right.$ & & \\
\hline Sulfuric Acid & $\mathrm{H}_{2} \mathrm{SO}_{4}$ & $\mathbf{C}$ & corrosive \\
\hline $\begin{array}{l}\text { Tetrasodium Ethylene Diamino- } \\
\text { Tetraacetate (EDTA) }\end{array}$ & $\mathrm{N}_{2} \mathrm{C}_{2} \mathrm{H}_{4}\left(\mathrm{C}_{2} \mathrm{H}_{2} \mathrm{O}_{2} \mathrm{Nal}_{4}\right.$ & & \\
\hline Tetraphenyl Boron & $\left(\mathrm{C}_{0} \mathrm{H}_{8}\right)_{4} \mathrm{~B}$ & & \\
\hline Thenoyltrifluoroacetone & $(\mathrm{CH})_{3} \mathrm{SCOCH}_{2} \mathrm{COCF}_{3}$ & & \\
\hline Tide (Detergent) & $\begin{array}{l}\text { Sodium allicate } \\
\text { compounds }\end{array}$ & & \\
\hline Tributyl Phosphate & $\left(\mathrm{C}_{4} \mathrm{H}_{2}\right)_{3} \mathrm{PO}_{4}$ & $\mathbf{D}$ & \\
\hline Trichloroethane & $\mathrm{CH}_{3} \mathrm{CCl}_{3}$ & & ORM-A' \\
\hline Tri-iso-octylamine & $\left(\left(\mathrm{CH}_{3}\right)_{2} \mathrm{CH}\left(\mathrm{CH}_{2}\right)_{3}\right]_{3} \mathrm{~N}$ & D & \\
\hline Tri-n-octylamine & {$\left[\left(\mathrm{CH}_{3}\right)\left(\mathrm{CH}_{2}\right)_{7}\right]_{3} \mathrm{~N}$} & & \\
\hline Turco Alkaline (Rust Remover) & NaOH, Kerosene & & \\
\hline Turco Deseal Zit $2^{4}$ & $\begin{array}{l}\text { Mothylene Chloride, } \\
\text { Acetic Acid }\end{array}$ & & \\
\hline Turco Plaudit4 & $\begin{array}{l}\text { No Hazardous } \\
\text { Compounds }\end{array}$ & & \\
\hline Vanadium Pentoxide & $\mathrm{V}_{2} \mathrm{O}_{8}$ & C & \\
\hline Xylene & $\mathrm{C}_{0} \mathrm{H}_{4}\left(\mathrm{CH}_{3}\right)_{2}$ & C & flammable liquid \\
\hline Zirconyl Nitrate & $2 \mathrm{OO}\left(\mathrm{NO}_{3}\right)_{2}$ & & \\
\hline
\end{tabular}

'Other regulated material with an anesthetic, irritating, noxious, toxic or other properties than can cause discomfort to persons in the event of leakage.

${ }^{2}$ Other regulated material capable of causing significant damage from leakage.

TTradename - Proctor and Gamble.

‘Tradename - Turco Products, Inc. 
Table 5-21. Summary of Information from Solid Waste Storage Disposel Records (SWSDR) for TRU Wastes with Hazandous Contents from Building 325 Westinghouse Hanford. (2 sheets)

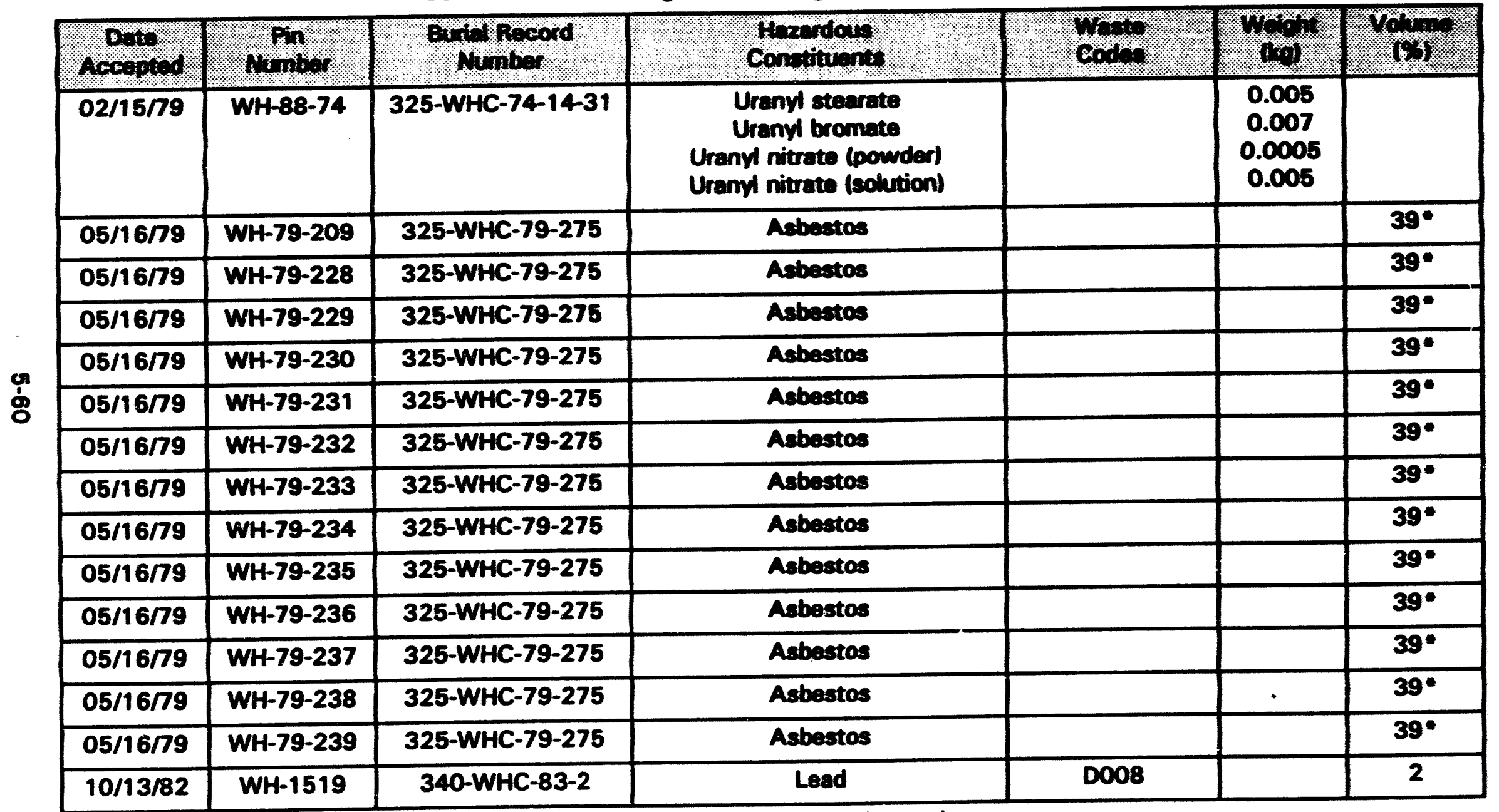

- Volume percents represent entire shipment, rather than individual containers. 
Table 5-22. Summary of Information from Solid Waste Storage Disposal Records (SWSDR) for Low Lovel Wastes with Hazardous Contents from Building 325 Westinghouse Hanford.

\begin{tabular}{|c|c|c|c|c|c|c|}
\hline $\begin{array}{l}\text { Date } \\
\text { Accepted }\end{array}$ & nombor & $\begin{array}{l}\text { Eurill Record } \\
\text { Number }\end{array}$ & Herendous & Wows & (10\%) & 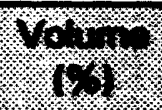 \\
\hline $07 / 31 / 71$ & & WAD-35-71-029 & Lead (brick) & & & \\
\hline $04 / 07 / 72$ & & WHC-325-23-72-013 & Lead (brick) & & 2721 & \\
\hline $10 / 05 / 76$ & & 325-WHC-760098 & Lead & & & \\
\hline $05 / 10 / 77$ & & 325-WHC-77-054 & Sodium & & & \\
\hline $08 / 31 / 77$ & WHC-77-163 & 325-WHC-77-099 & Lead & & & \\
\hline $08 / 31 / 77$ & WHC-77-211 & 325-WHC-77-099 & Lead & & & \\
\hline $10 / 05 / 77$ & WHC-77-272 & 325-WHC-77-112 & Lead & & & \\
\hline $10 / 05 / 77$ & WHC-77-273 & 325-WHC-77-112 & Lead & & $\cdot$ & \\
\hline $02 / 13 / 78$ & WH-78-128 & 325-WHC-78-015 & Lead (pig) & & & \\
\hline $06 / 05 / 79$ & & 325-WHC-79-75 & Asbestos & & & 4 \\
\hline $08 / 15 / 88$ & $325 W-88-0003$ & 325-WHC-89-01 & $\begin{array}{l}\text { Ammonium fluoride } \\
\text { Potessium hydroxide } \\
\text { Ammonium nitrate } \\
\text { Ammonium floro-zirconate }\end{array}$ & & $\begin{array}{c}3.0 \\
18.0 \\
3.0 \\
7.5\end{array}$ & $\begin{array}{r}2 \\
12 \\
2 \\
5\end{array}$ \\
\hline
\end{tabular}




\section{WHC-EP-0696}

This page intentionally left blank. 
WHC-EP-0696

\subsection{CHARACTERIZATION OF RETRIEVABLY STORED SOLID WASTE GENERATED AT BUILDING 325 BY PNL}

The information found in this section is based primarily on data from the Solid Waste Information and Tracking System (SWITS) database. This database, which incorporated the older R-SWIMS database, is used to track information on radioactive and other wastes stored or disposed at the Hanford Site. Transuranic solid waste packages have been tracked since 1970, however, changes in the requirements and regulations governing radioactive wastes have left their mark in the quantity and quality of the data contained in this database. Caveats are included in the text to alert the reader to changes that may affect the interpretation of the data provided.

Solid Waste Burial Records (SWBR) are the source for the data in SWITS and were used as a secondary source of information in this section. Between 1970 and 1980, the 325 Building was used as a collection point for all waste in the 300 Area. Although waste originated from other buildings, the SWITS database recorded the originating source as Building 325. Review of the original SWBRs indicates a different 300 Area facility as the originating source for the waste. Tables 6-1 and 6-2 show the breakdown of waste designated as being produced by PNL at Building 325, but originating at other facilities.

Since 1980, solid waste originating from PNL at Building 325 was sent to Buildings 327 or $\mathbf{3 4 0}$ for interim storage and assay. Subsequently, these waste containers were recorded in SWITS as originating from Buildings 327 or 340 rather than from Building 325. Review of the SWBRs indicates the 325 Building as the originating source of the waste. Data taken from the burial records are incorporated in the tables and figures in this section and are clearly labeled.

The bulk of the data provided in this section is limited to information about the TRU waste that was generated at Building 325 by PNL; however, some general information on the non-TRU waste is included for completeness. The term non-TRU waste is used instead of LLW because a small percentage of the unsegregated waste is designated only as not TRU. Tables 6-3 and 6-4 present TRU and non-TRU waste generation summaries by container type and year. As the initial retrieval efforts and WRAP 1 will focus on 55-gal drums, these containers are considered separately from all other container types. Therefore, the term "other containers" in this report will refer to all container types except 55-gal drums. The term "drum" refers to 55-gal drums only, unless specified otherwise.

The original SWITS data that form the basis for most of the tables and figures in this section can be found in Appendix B. Each computer run is preceded by the query used to generate the data.

Because of the large number of figures and tables in this section, they are placed at the end of the chapter. 


\subsection{SUMMARY OF BUILDING 325 WASTE GENERATION BY PNL}

\subsubsection{Waste Stored in 55-Callon Sted Drums}

Table 6-5 contains waste summary data for 55-gal drums of radioactive waste generated at Building 325 by PNL between 1970 and 1992. The upper portion of this table presents the number, total weight, and total volume of TRU waste drums. The number of TRU mixed waste drums is also indicated. The same information for non-TRU waste follows. At the bottom of the table are the relative percentages of TRU and non-TRU wastes by container number, weight, and volume. Figures 6-1 through 6-6 present this information graphically.

6.1.1.1 Number of Drums. Between 1970 and 1992, 3,786 55-gal drums of radioactive waste were generated by PNL at Building 325. This total includes 2,069 drums of non-TRU and 1,717 drums of TRU waste. The total number of radioactive mixed waste drums is 46, which includes 43 non-TRU MW drums and 3 TRU MW drums. Figure 6-1 provides a graph of the number of 55-gal drums of TRU and non-TRU generated by PNL at Building 325 by year. The major features of this graph are directly related to historical events that took place at Building 325.

The Commercial Alpha Waste program was developed in the early 1970's to study and implement an alpha waste reduction plan. Six tasks provided objectives for the treatment and disposal of alpha waste through waste segregation, waste product criterion and evaluation, and waste container criterion and evaluation. Results from the program as seen in Figure 6-1 reveal a general decline in TRU waste from 1973 to 1978. At the same time, the number of non-TRU containers generally increased. This is depicted more clearly in Figure 6-2, which shows the relative percentages of TRU and non-TRU by year.

Note that, according to the database, 1988 was the only year that TRU waste was not generated; non-TRU waste filled 92 drums in that year.

6.1.1.2 Weight. In the period between 1970 and 1992 approximately $121,744 \mathrm{~kg}$ of TRU waste and $251,801 \mathrm{~kg}$ of non-TRU waste generated by PNL at Building 325 were stored in 55-gallon drums. These numbers are estimates since container weights were not required for individual containers prior to 1977. During the R-SWIMS data reentry program in the mid1980's, all pre-1977 containers were assigned standard weights. All 55-gal drums, for instance, were assigned a value of $68 \mathrm{~kg}(150 \mathrm{lb})$. Since 1979, the average weight of all 325 drums generated by PNL has been $102 \mathrm{~kg}$. However, while the average weight of TRU drums from that period was $79 \mathrm{~kg}$, the average woight of non-TRU drums was $117 \mathrm{~kg}$, making these drums over $48 \%$ heavier than the TRU drums. In addition, the average weight per drum of TRU waste increased yearly between 1985 and 1992.

\begin{tabular}{|c|c|c|c|c|c|c|c|c|}
\hline $\begin{array}{c}\text { Average Weight } \\
\text { (kg) }\end{array}$ & 1985 & 1986 & 1987 & 1988 & 1989 & 1990 & 1991 & 1992 \\
\cline { 2 - 9 } & 74 & N/A & 88 & N/A & 90 & 95 & 102 & 133 \\
\hline
\end{tabular}


Figure 6-3 presents the total weight of both TRU and non-TRU radioactive wastes stored annually in 55-gal drums. The relative weight percentages for TRU and non-TRU drums generated annually by PNL at Building 325 are shown in Figure 6-4.

The large TRU peak in 1979 is probably due to the removal of a 15 ton crane. At that time, it was replaced with a 30 ton crane.

Two trends worth noting both started in the 1980's. First, according to the database, no non-TRU waste was generated between 1981 and 1987. Secondly, non-TRU waste started at a high in 1988 with 92 containers and generally declined over the next four years.

6.1.1.3 Volume. The total volume of waste generated by PNL at Building 325 and stored in 55-gal drums is $790 \mathrm{~m}^{3}$. of which $361 \mathrm{~m}^{3}$ is TRU and $429 \mathrm{~m}^{3}$ is non-TRU. Annual waste generation information is shown in Figure 6-5; the volume percentages of radioactive waste stored in 55-gal drums are provided in Figure 6-6. Since all 55-gal drums have a volume of $0.21 \mathrm{~m}^{3}$, the patterns shown in Figures 6-5 and 6-6 are the same as those described for Figures 6-1 and 6-2 (drum number), respectively. They are included here so that comparisons with later figures can be made (Figures 6-11 and 6-12).

\subsubsection{Waste Stored in Containers Other Than 55-Gallon Steel Drums}

Radioactive solid wastes have been stored or disposed of in a wide variety of containers other than 55-gal steel drums. Other container types used for solid waste generated at Building 325 by PNL include the following:

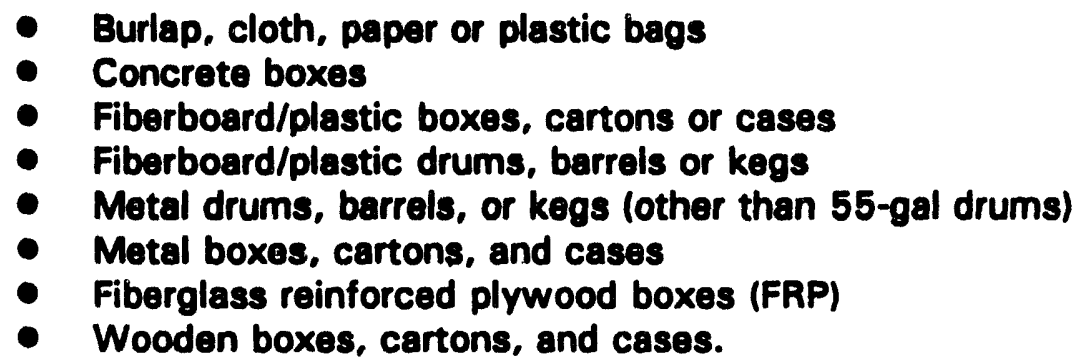

Some items were disposed of or stored without additional packaging, such as dump trucks; gloveboxes; HEPA filters; miscellaneous scrap; trucks, flatbeds, compactors, loadluggers; self-contained equipment; portable tanks, and metal cylinder casks. These items are also included in the "Other Container" category.

Table 6-6 summarizes the waste data for containers of radioactive waste other than 55-gal drums that were generated at Building 325 by PNL between 1970 and 1992. The upper portion of this table indicates the number, total weight and total volume of TRU waste stored in other containers. The number of other containers of TRU mixed waste also is provided. The same information for non-TRU waste follows. At the bottom of this table are the relative percentages of TRU and non-TRU wastes by container number, weight, and volume. Figures 6-7 through 6-12 present these data graphically. 
WHC-EP-0696

6.1.2.1 Number of Other Containers. Between 1972 and 1992, 49,588 other containers of radioactive waste were generated at Building 325 by PNL. Of these containers, 379 hold TRU waste, while 49,209 contain non-TRU waste. For reference, during the same period of time a total of 3,786 55-gallon drums of radioactive solid waste were generated at Building 325 by PNL. Figure 6-7 is a graph of the number of other containers of TRU and non-TRU waste generated at Building 325 by PNL annually.

The largest number of other containers containing TRU waste was generated in 1970 with $\mathbf{3 6 1}$ containers. This is the result of an inordinately large number of fiberboard/plastic boxes, cartons, and cases. The vast majority of other containers contain non-TRU waste. In the years from 1970 to 1992, over $99 \%$ of the waste is non-TRU, with fiberboard/plastic boxes, cartons, and cases accounting for over $96 \%$ of the total.

Figure 6-8 presents the relative percentages of TRU and non-TRU containers other than 55-gallon drums. One thing is apparent from this figure: Almost all of the waste in other containers is non-TRU.

The probable explanation for the large TRU peak in 1970 is the decontamination and repair of a major exhaust stack. Additionally, 41 HEPA filters were disposed of as TRU waste.

6.1.2.2 Weight. In the period between 1970 and 1992, approximately $1,309,081 \mathrm{~kg}$ of radioactive solid waste was generated at 325 by PNL and placed in containers other than 55-gal drums. This total includes approximately $42,204 \mathrm{~kg}$ of TRU and $1,266,877 \mathrm{~kg}$ of nonTRU contaminated material. These weights are approximations because individual container weights were not required to be recorded for individual containers prior to 1977. During an update of the R-SWIMS database in the mid-1980's, all waste containers without recorded weights were assigned standard values. For example, all 2 cubic foot boxes were given a standard weight of $5.5 \mathrm{~kg}(12 \mathrm{lb})$ and 5 gallon lard cans were assigned a standard weight of $22.7 \mathrm{~kg}(50 \mathrm{lb})$.

Figure 6-9 depicts the weight of TRU and non-TRU waste stored in containers other than 55-gallon drums on an annual basis. The most notable features of this graph are the TRU waste peaks in 1970, 1974, 1978, 1982, and 1992. The large weight of waste disposed of in these years is the result of the shipment of several large containers to retrievable storage including thres concrete boxes in 1974, one portable tank in 1978, one $6 \mathrm{ft} \times 6 \mathrm{ft} \times 7 \mathrm{ft}$ metal box, carton, or case in 1982, and one $5.6 \mathrm{ft} \times 6.5 \mathrm{ft} \times 9.3 \mathrm{ft}$ metal box, carton, or case in 1992. The WHC-MR-0388 report (Gerber 1992) also indicated that some contaminated soil wes also removed from around the radioactive liquid waste system line 1970. These six containers account for over $73 \%$ of the TRU waste weight in other containers buried between 1970 and 1992. Fiberboard/plastic boxes, cartons, and cases account for $50 \%$ of the non-TRU waste weight. The removal of trucks, flatbeds, compactors, loadluggers in 1970 and dump trucks in 1974 contributed to the TRU waste peaks in 1970 and 1974.

Figure 6-10 shows the percent contribution of TRU and non-TRU waste to the total annual weight of waste packaged in other containers. Because of the practic s of storing TRU waste in drums when possible, proportionately less of the other container waste is TRU. 
6.1.2.3 Volume. The volume of waste generated at Building 225 by PNL and packaged in containers other than 55-gal drums during the period from 1970 through 1992 is $8,090 \mathrm{~m}^{3}$. Transuranic wastes account for $103 \mathrm{~m}^{3}$ of the total volume; non-TRU wastes account for $7,987 \mathrm{~m}^{3}$. Figure 6-11 shows annual volumes of TRU and non-TRU wastes generated at Building 325 by PNL and packaged in containers other than 55-gal drums. Figure 6-12 shows the relative contribution of TRU and non-TRU wastes to the total volume of waste in other containers for each year.

\subsubsection{Summary of Waste Ceneration Rates at 325 Between 1970 and 1992 by PNL}

6.1.3.1 Number of Waste Containers. Between 1970 and 1992, there were 53,374 containers of radioactive solid waste generated at Building 325 by PNL. Fifty-five gallon drums account for 3,786 of the total number of containers. Containers of transuranics comprise only $4 \%$ of the total container numbers.

Figure 6-13 shows the total numbers of both TRU and non-TRU waste containers generated on an annual basis during this period. The difference in the amount of waste characterized as TRU and non-TRU from 1970 to 1992 is most evident in this graph. As mentioned previously, the Commercial Alpha Waste Program helped to reduce the amount of alpha waste progressively throughout the 1970's. In addition, changes in the DOE (formerly AEC) regulations and disposal practices directly affected the proportion of the waste determined to be TRU and non-TRU. A significant portion of the waste stored as TRU prior to 1982 may be redesignated as LLW upon characterization at WRAP.

6.1.3.2 Total Weight. Approximately $1,682,626 \mathrm{~kg}$ of radioactive solid waste was generated at Building 325 by PNL between 1970 and 1992. Waste packaged in 55-gal drums accounts for about $22 \%$ of the total weight of waste from Building 325 , with $78 \%$ of the total weight comprised of waste packaged in some other type of waste container.

Waste designated as TRU makes up $10 \%$ of the total weight while waste designated as non-TRU makes up the remainder. Figure 6-14 provides a graph of the total weight of TRU and non-TRU waste generated at 325 by PNL on a yearly basis between 1970 and 1992 . This graph shows that as the number of containers declined in later years, the weight of waste also declined but not in the same proportion.

The majority of the weight, $74 \%$, of TRU waste is contained in $55-g a l$ drums followed by concrete boxes with $20,448 \mathrm{~kg}$ or $12 \%$ of the TRU waste weight.

6.1.3.3 Total Volume. In the period between 1970 and $1992,8880 \mathrm{~m}^{3}$ of radioactive solid wastes were generated at Building 325 by PNL. Only $\mathbf{9 \%}$ of this volume was comprised of 55-gal drums; the remaining $91 \%$ of the volume was made up of other container types. Transuranic wastes account for $5 \%$ of the waste volume, while non-TRU wastes make up the remaining 95\%. Figure 6-15 shows the total volume of TRU and non-TRU waste generated at Building 325 by PNL on a yearly basis. 


\subsection{WASTE CONTANERS}

\subsubsection{TRU Waste Containors}

Table 6-3 provides a summary of the TRU waste generated annually at Building 325 by PNL from 1970 to 1992 sorted by container type. The greatest number of containers are 55-gal drums, 1,717 by count; however, there are also significant numbers of fiberboard/ plastic boxes, cartons, and cases in 1970. The percentages for all container types and the weight and volume of these containers are shown for each year in this table.

\subsubsection{Non-TRU Waste Containors}

Table 6-4 provides a summary of the non-TRU waste generated by PNL Building 325 from 1970 to 1992 by container type. Fiberboard/plastic boxes, cartons, and cases were the most common containers used accounting for more than $92 \%$ of the total non-TRU waste count. Significant numbers of 55-gal drums - metal drums, barrels, and kegs were also generated. The greatest weight of non-TRU waste, $40 \%$, is comprised of waste in fiberboard/plastic boxes, cartons, and cases.

\subsection{TRU WASTE STORACE LOCATIONS}

Table 6-7 provides the storage locations for TRU waste packaged in 55-gallon drums by year. Storage locations for TRU waste packaged in other containers are shown in Table 6-8. In general, $69 \%$, of the drums and $93 \%$ of other containers generated at Building 325 by PrJL are stored at burial ground 218-W-4B. A summary of Building 325 waste generated by PNL by storage location follows:

- 218-W-3A - Burial Ground 218-W-3A consists of 14 earthen-bottom, gravel filled trenches, with waste emplaced from May 1970 to April 1988. Ninety-five drums and other containers from Building 325 by PNL were disposed here in the years between 1970 and 1975; they contain no hazardous constituents.

- 218-W-4B - Burial Ground 218-W-4B, consisting of 14 trenches and 5 caissons, accepted retrievable waste through September of 1978. One thousand-one hundred and ninety-two drums and other containers from Building 325 by PNL were buried here from 1970 to 1978.

- 218-W-4C - Burial Ground 218-W-4C consists of six trenches with the first waste emplaced in March 1978. Three-hundred thirty eight 55-gal drum TRU waste was sent to this area from 1978 to 1987 with one other container emplaced in 1982.

- 224T - 224T is an above-ground storage structure known as the Transuranic Storage and Assay Facility (TRUSAF). TRU waste in 55-gal drums from Building 325 by PNL were stored here from 1987 and 1992. Nine of the total 113 drums contained one or more of the following hazardous constituents: asbestos, mercury, lead, and nitric acid. 
- 2403-WA - Storage location 2403-WA is part of the Central Waste Complex. Only five 55-gal drums of TRU waste were sent here for storage between 1991 and 1992.

- 2402-WE - Storage Location 2402-WE is part of the Central Waste Complex. One metal box, carton, or case measuring $5.6 \mathrm{ft} \times 6.5 \mathrm{ft} \times 9.3 \mathrm{ft}$ was sent here for storage in 1992.

\subsection{PHYSICAL CONTENTS OF TRU WASTE CONTAINERS}

The physical contents of TRU 55-gal drums are shown in Tables 6-9 through 6-13. There is a separate table for each storage location. The top portion of each table indicates the number of drums for which a given component is listed; the bottom part of the table indicates the percentage of the total drums from Building 325 by PNL in that burial location that contain the listed constituent. The physical contents for TRU waste containers other than 55-gal drums can be found in Tables 6-14 through 6-17.

One of the most interesting components is in storage location 218-W-4C. In 1980, animal waste is recorded as present in $47 \%$ of the TRU drums that were listed as being shipped from PNL Building 325. It should be noted that this waste was not from any animal research taking place at PNL Building 325. Rats were known to be used in Building 331 for biological studies. In fact, during a recent review of the SWBRs for one record indicated "lots of rats" were present. These animal carcasses were generally packed in lime.

It should be noted that prior to 1978, physical contents were not required to be listed on the burial records. A great many of the contents on early records are listed only as "Miscellaneous".

During an interview with 325 Building personnel, it was indicated that there might be a potential problem with unvented aerosol cans. Aerosol cans of Windex' and WD-40 ${ }^{2}$ were disposed of and sent for burial from the 325 Building.

\subsection{RADIOLOGICAL DESCRIPTION OF BUILDING 325 SOLID WASTE BY PNL}

Table 6-18 shows the number of grams of transuranics contained in waste stored in 55-gal drums each year by storage location; Table 6-19 provides the same information for containers other than 55-gal drums. Figure 6-16 combines the information on these tables in a graph that shows the total amount of transuranics present in waste packages from PNL Building 325 by year.

\footnotetext{
${ }^{1}$ Trademark of Drackett Company.

${ }^{2}$ Trademark of WD-40 Company.
} 
WHC-EP-0696

\subsection{RADIOACTIVE ISOTOPES PRESENT IN BUILDINO 326 SOLID WASTE BY PNL.}

Tables 6-20 and 6-21 contain information on radioactive isotopes found in 55-gal drums and other containers, respectively.

The following isotopes are all referred to specifically in the Building 325 Safety Analysis Reports: ${ }^{131} \mathrm{I},{ }^{0} \mathrm{Sr},{ }^{00} \mathrm{Sr},{ }^{103} \mathrm{Ru}$, and ${ }^{236} \mathrm{U}$. Although these isotopes used in PNL processes and laboratories may or may not be present in the solid waste stream, they are mentioned here for completeness.

\section{7 hazARDOUS CONSTITUENTS OF SOLID WASTE OENERATED AT 325 BY PNL}

This section provides a review of the data on the hazardous components known or suspected to be in the solid waste stream from Building 325 by PNL. Information was gathered from several sources, including: the Solid Waste Information Tracking System, Solid Waste Burial Records, personnel interviews, an internal report examining hazardous components of solid waste, Inventory of Chemicals Used at Hanford Site Production Plants and Support Operations (KJem 1990), and Unstable and Reactive Chemicals in Transuranic Retrievable Waste at the Hanford Site (Reddinger 1992).

\subsubsection{Chemical Invemtories}

Table 6-22 provides a list of chemicals used at 325 by the PNL Development and Production Laboratory from 1955 to 1976. In addition, Table 5-20, discussed previously in Section 5.7.1, provides a list of chemicals used at the 325 Building Analytical Laboratory from 1953 to 1976. These lists were developed by Klem (1990) to support waste characterization efforts for the single-shell tanks. Though these lists characterize chemicals used in the Analytical Laboratory, they do not specify which of these chemicals were used by PNL and/or Westinghouse Hanford.

The final two columns in both of the tables cited above contain information on the toxicity and hazards associated with each of the chemicals listed. The first of these columns lists the EPA toxicity category, which was determined using the Registry of Toxic Effects of Chemical Substances Quarterly Issue (NIOSH 1992) and the EPA "Spill Table" (EPA 1989). In cases where the two sources differed, the more recent source, the Registry of Toxic Effects, was used. Category $X$ marks the most toxic chemicals, followed by Categories $A$, B, C, and D, listed in order of decreasing toxicity. The EPA "Spill Table" is incorporated by reference into the Washington State Administrative Code, Chapter 173-303, Dangerous Waste Regulations. The Registry of Toxic Effects is a quarterly update to the EPA "Spill Tab/e." The final column lists the U.S. Department of Transportation (DOT) designation found in the DOT Hazardous Materials Table (49 CFR 172.101). 


\subsubsection{8olld Wasto Information and Traoking System (8WIT8)}

The information about the hazardous constituents of the solld waste generated by PNL at Building 325 can be found in Tables 6-23 and 6-24. According to the SWITS database, only 9 drums from Building 325 PNL contain mixed waste. The greatest percent of mixed waste was found in 1992 when 13\% of all 58-gal drums generated at Building 325 by PNL contained mixed waste.

The hazardous constituent field in the SWITS database also was searched for containers from Building 325 genorated by PNL. Table 6-25 liats the hazardous constituents of TRU 55-gal drums sorted by storage location and year. In addition, the percentage of PNL drums in the burial location that contain each constituent is indicated. There were no records found that listed PNL TRU containers other than 55-gal drums with mixed waste.

\subsubsection{Burial Records}

Table 6-25 contains a summary of the information obtained from a review of the PNL Building 325 burial records for all TRU mixed waste containers. Table 6-27 is a summary of the low-level mixed waste containers. The presence of asbestos-containing waste material was included in the summary tables despite the fact that it is not considered to be mixed waste. Lead shielding is also not considered to be waste at the current time.

\subsubsection{Intorviows with 328 Bulling Porsonnel}

From the information gained in interviews with 325 Building personnel, a list of known or suspected components in the solid waste stream has been compiled. This list of components was discussed above and can be found in Section 5.7.4. As this list applies to the entire 325 Building Analytical Laboratory, it is expected that these constituents may be part of the PNL waste stream as well the Westinghouse Hanford waste stream. 
Figure 6-1. Bb-Gallon Drum Waste Count Generated at Building 325 by PNL Annually.
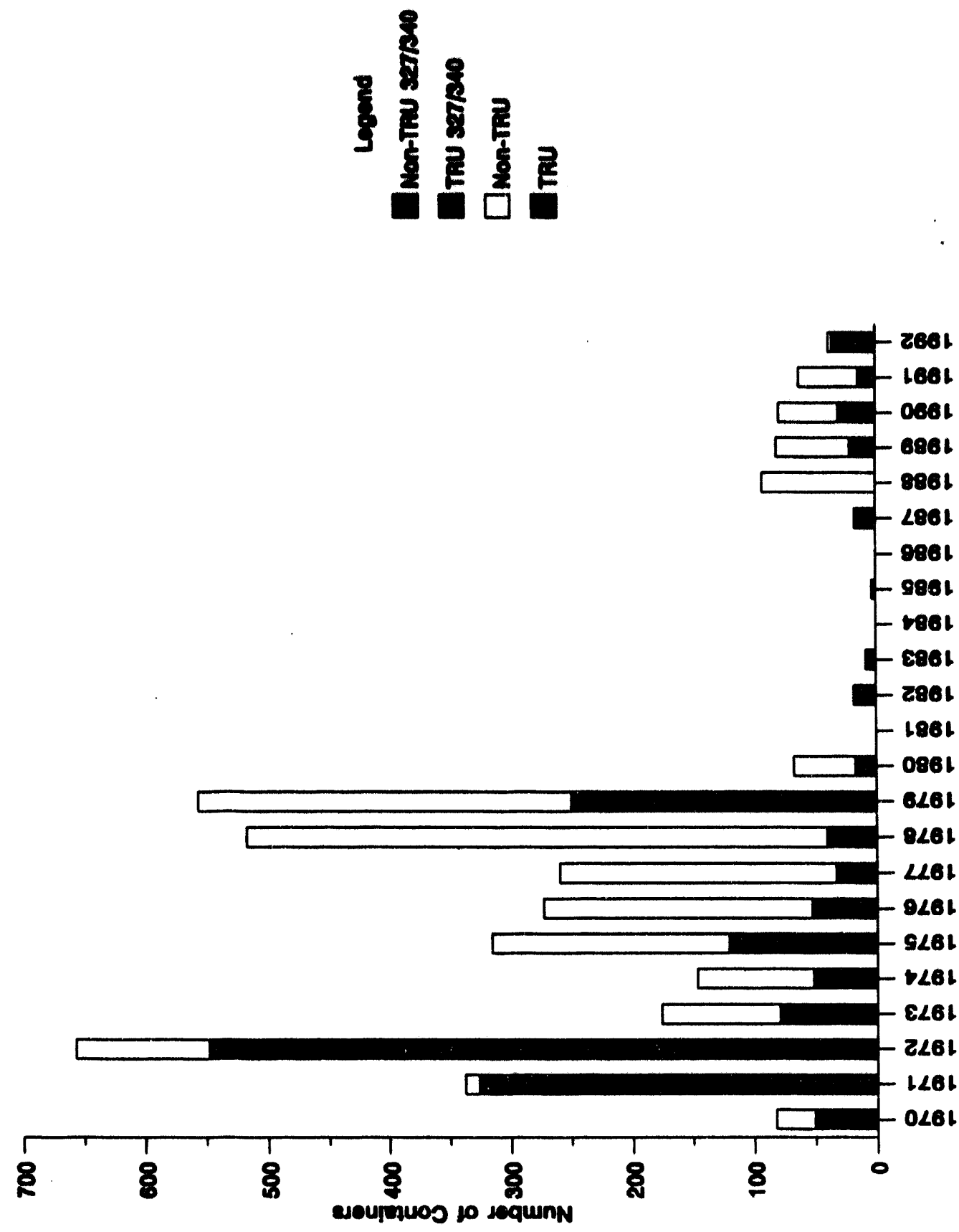
WHC-EP-0696

Figure 6-2. Percentege of Bullding 325 TRU/Non-TAU 86-Gallon Drums by PNL.
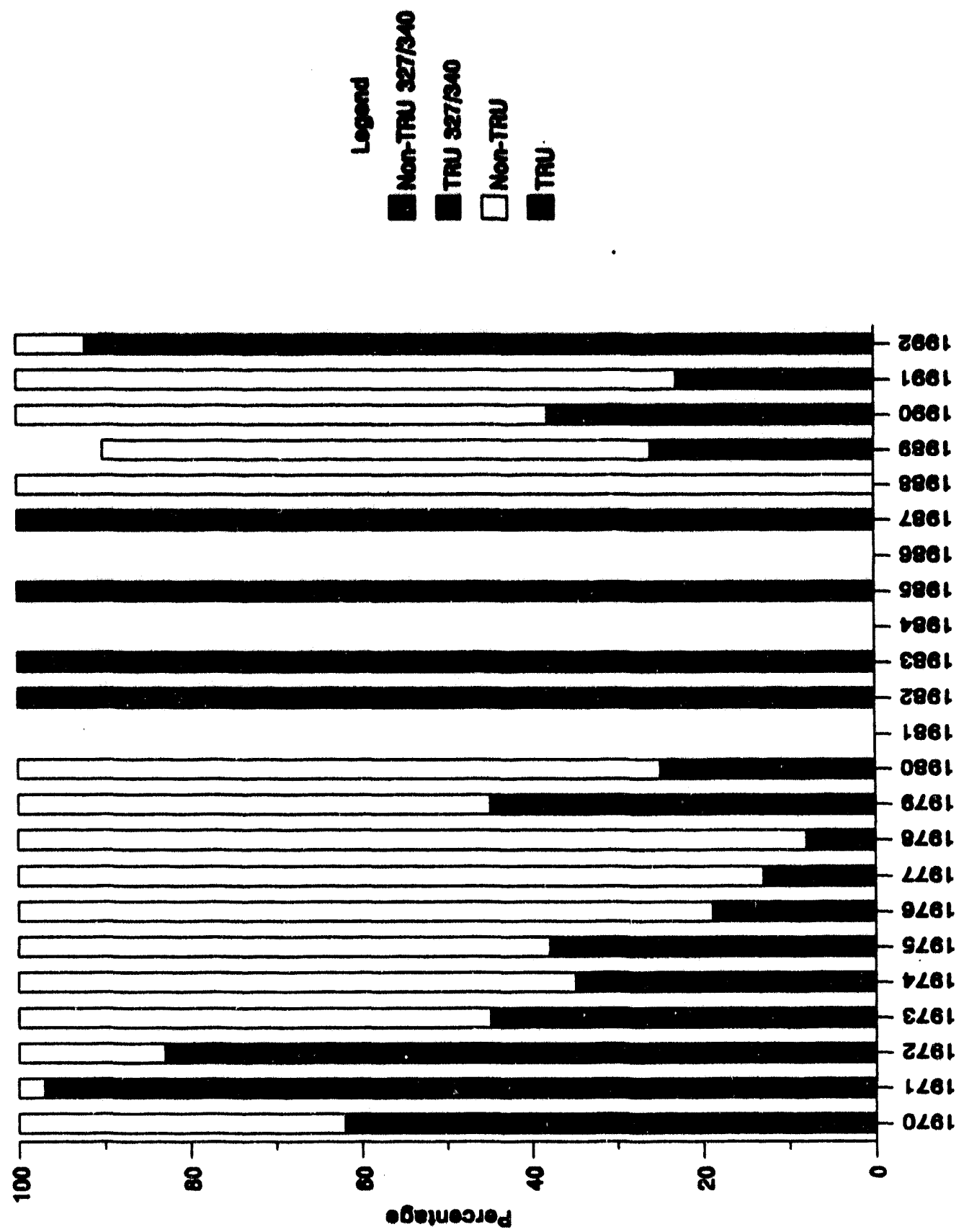
Figure 6-3. Woight of 58-Gallon Drum Waste Cenorated at Bullding 328 by PNL Annually.
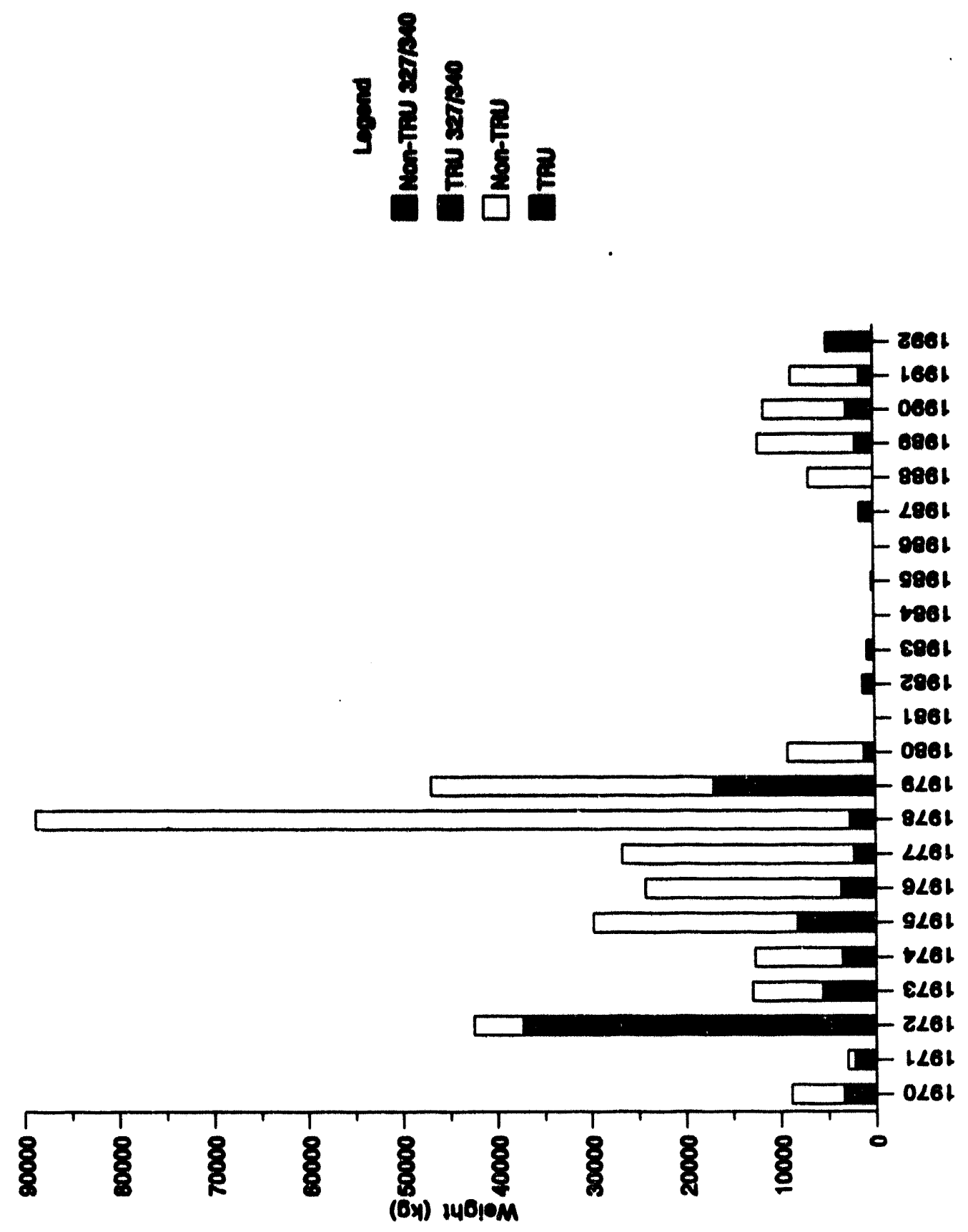
WHC-EP-0696

Figure 6-4. Woight Porcentege of Building 325 85-Gallon Drums by PNL.
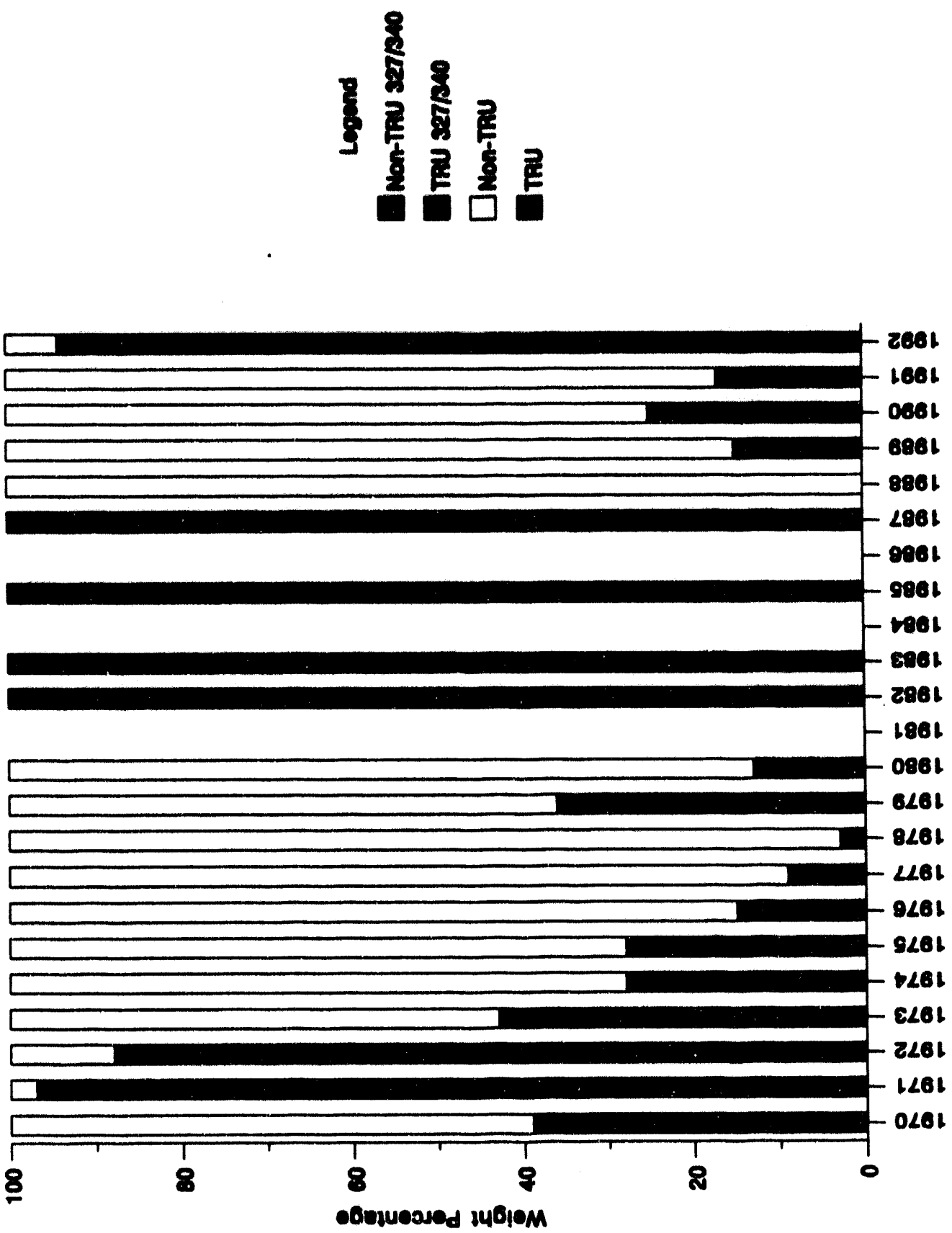


\section{WHC-EP-0696}

Figure 6-5. Volume of 55-Callon Drum Waste Generated at Building 325 by PNL Annually.
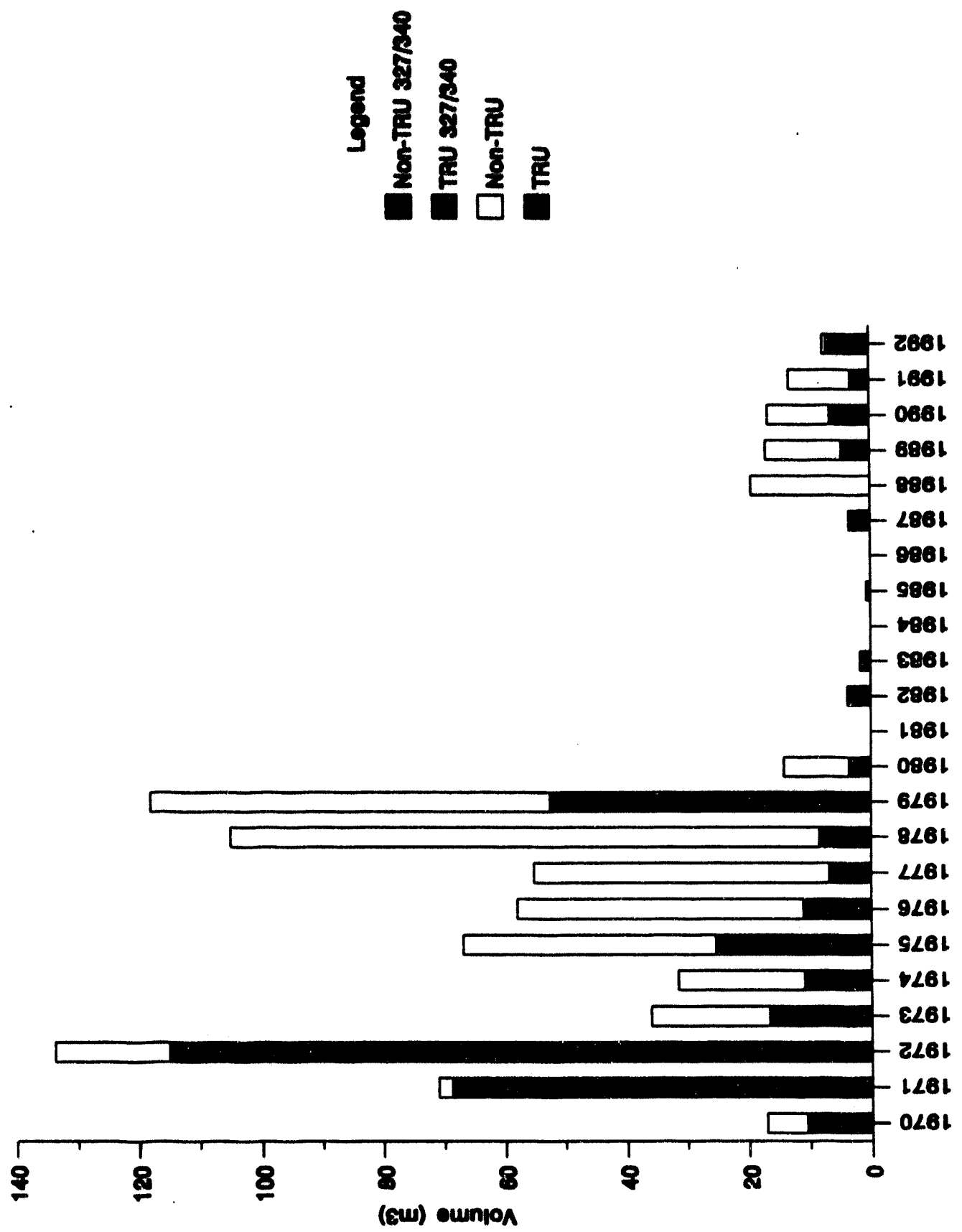
है
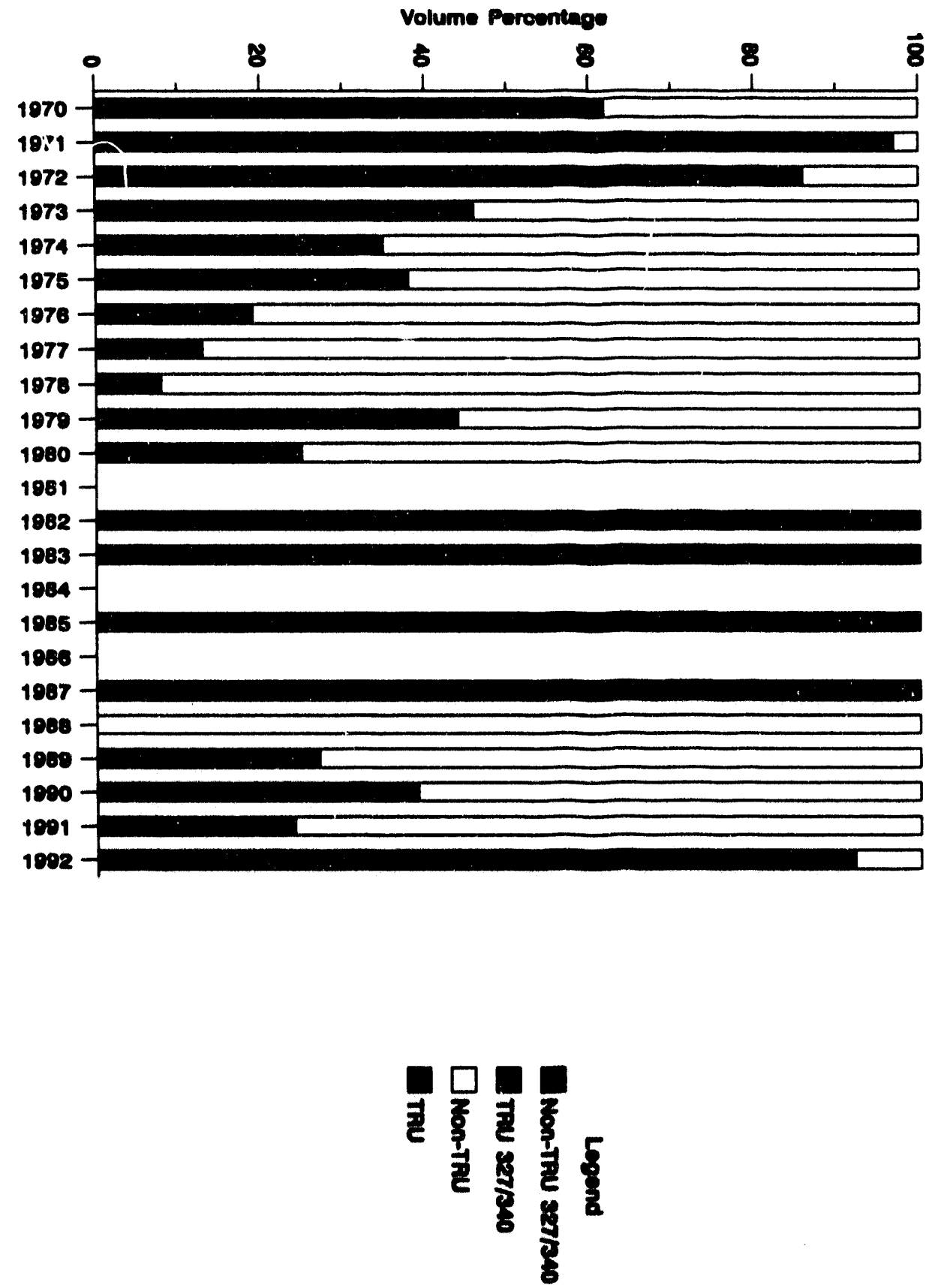

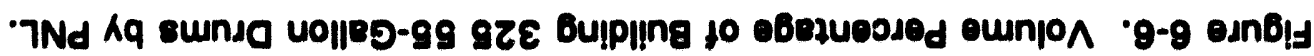


Figure 6-7. Other Container Waste Count Generated at Building 325 by PNL Annually.
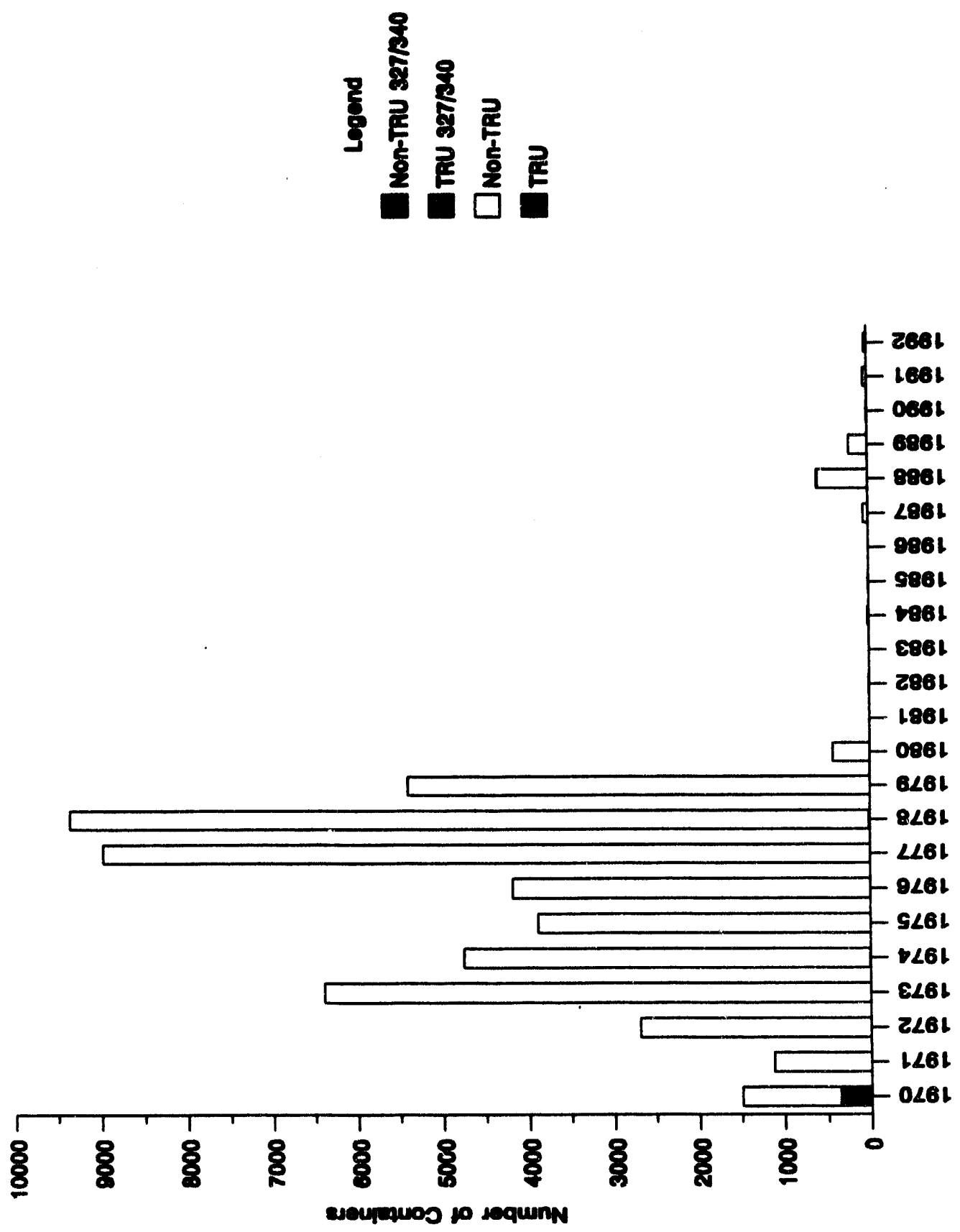
WHC-EP-0696

Figure 6-8. Percentage of Building 325 TRU/Non-TRU Other Containers by PNL.
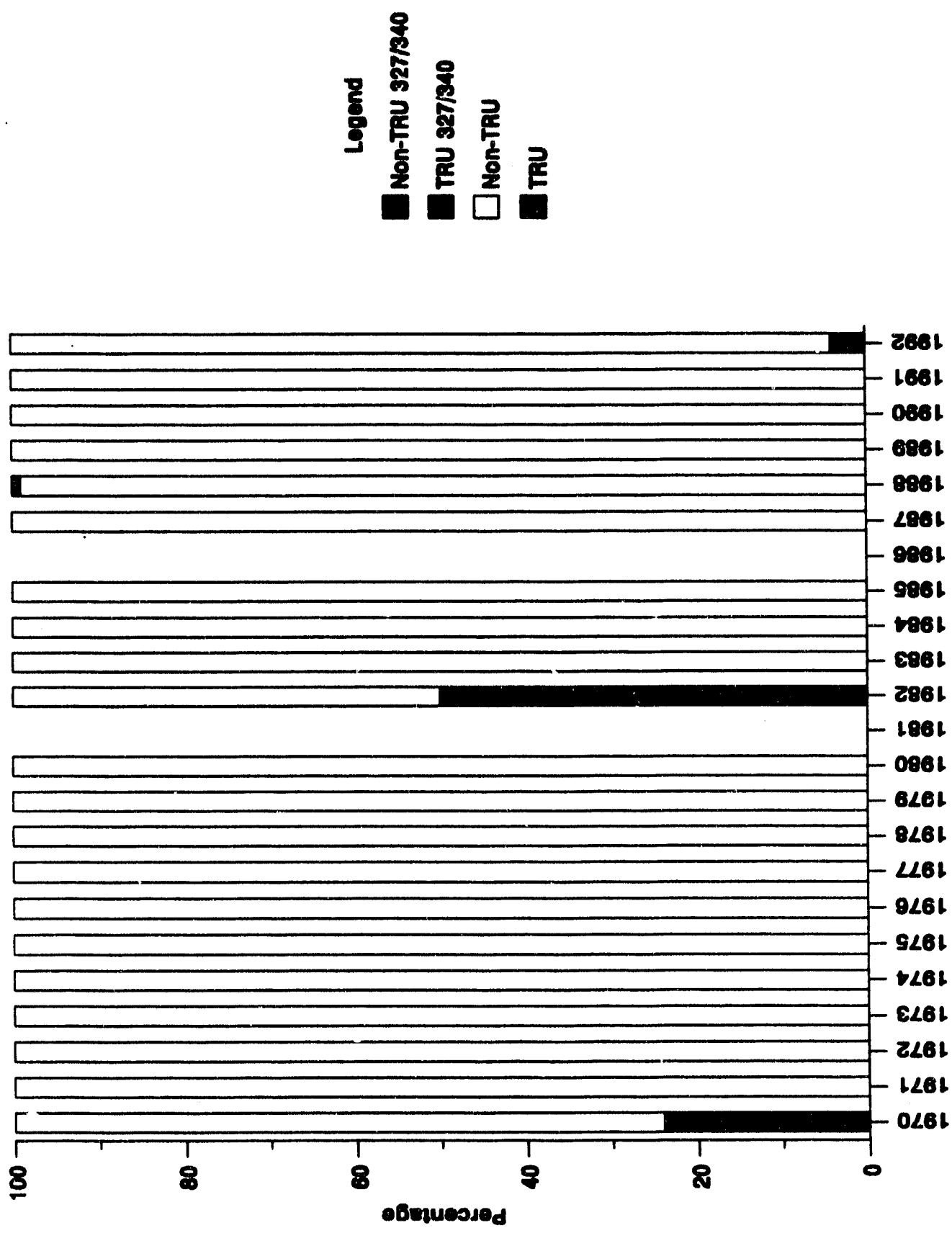


\section{WHC-EP-0696}

Figure 6-9. Weight of Other Containers Generated at Building 325 by PNL Annually.
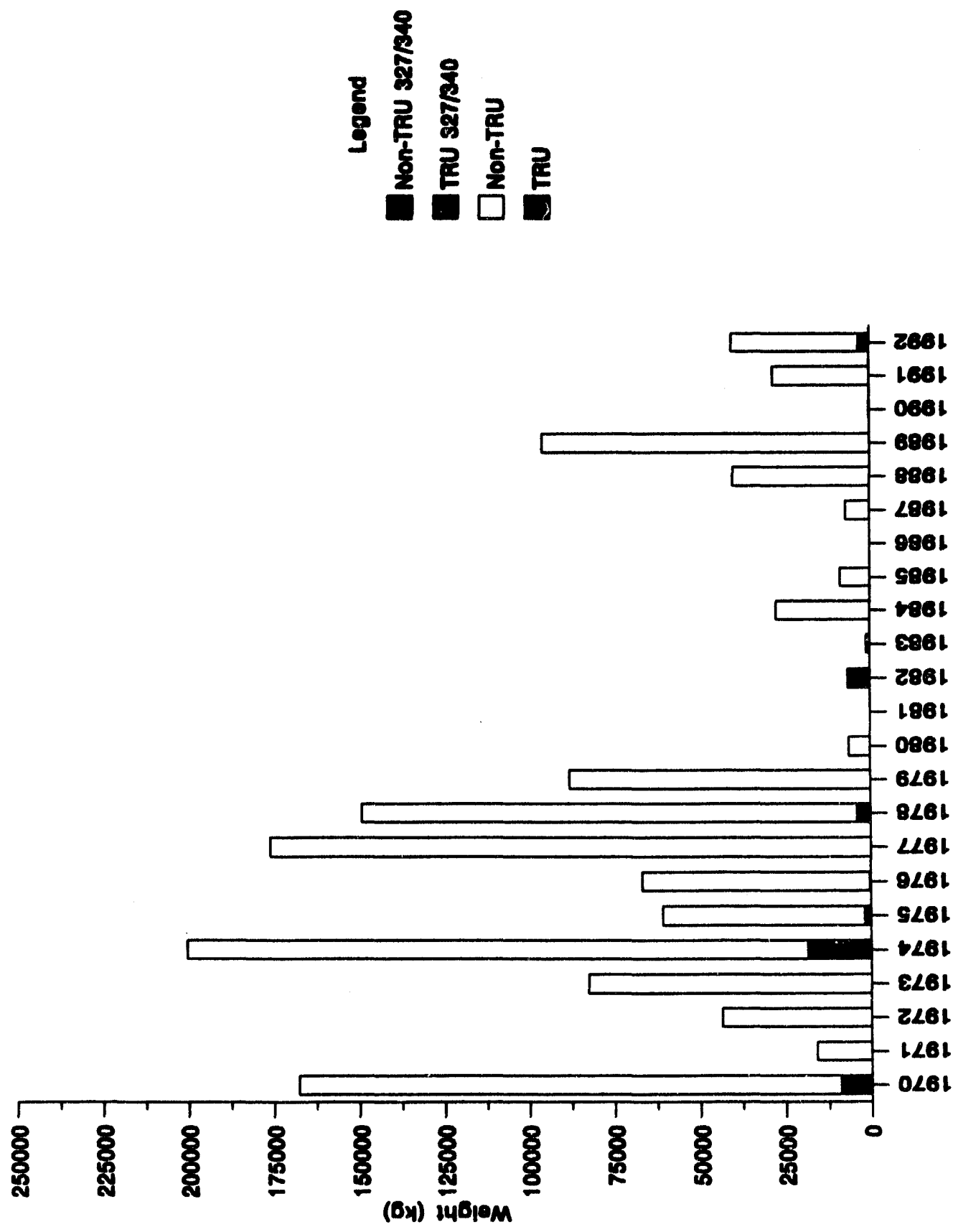


\section{WHC-EP-0696}

Figure 6-10. Weight Percentage of Other Containers Generated at Building $\mathbf{3 2 5}$ by PNL Annually.
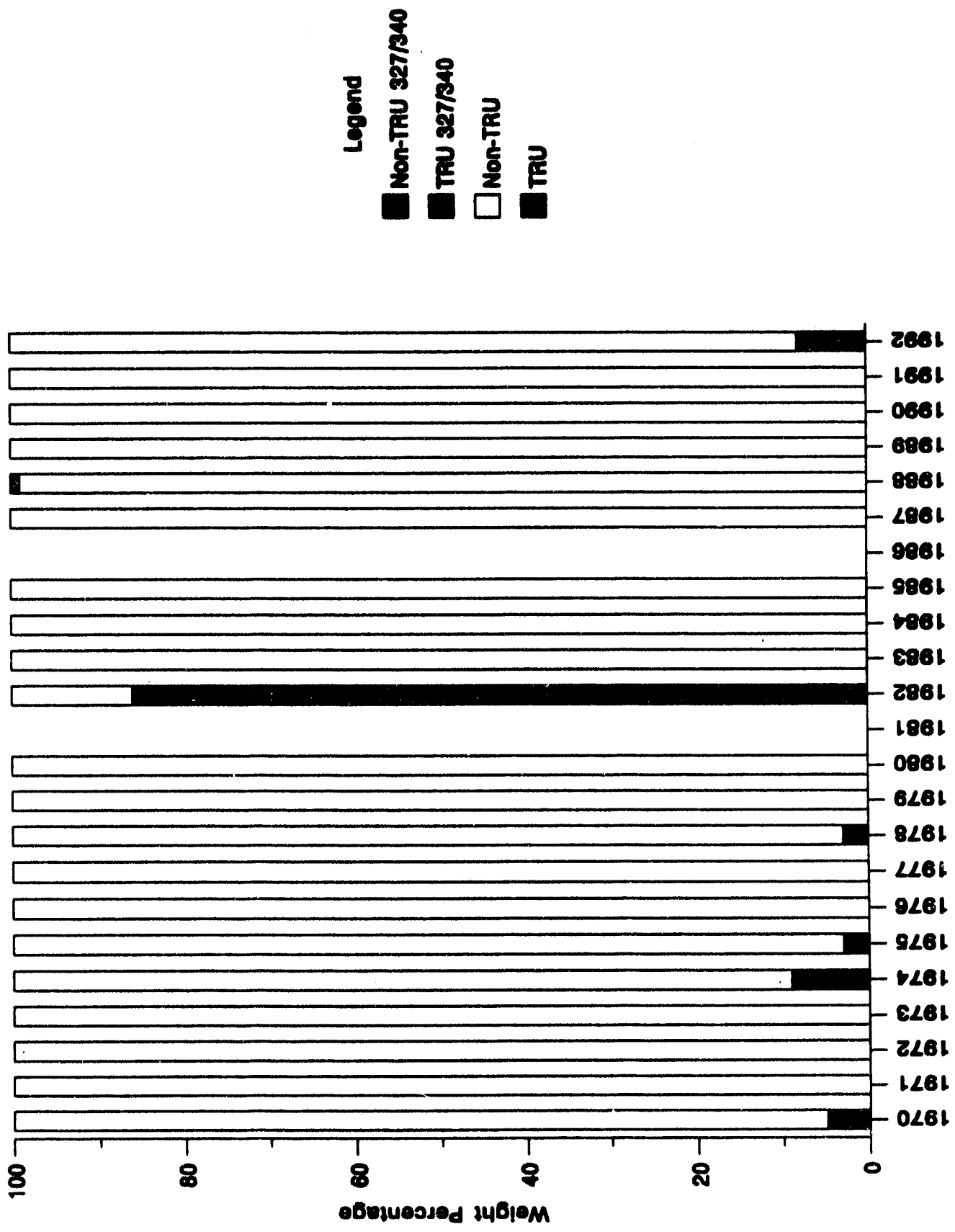


\section{WHC-EP-0696}

Figure 6-11. Volume of Other Containers Generated at Building 325 by PNL Annually.
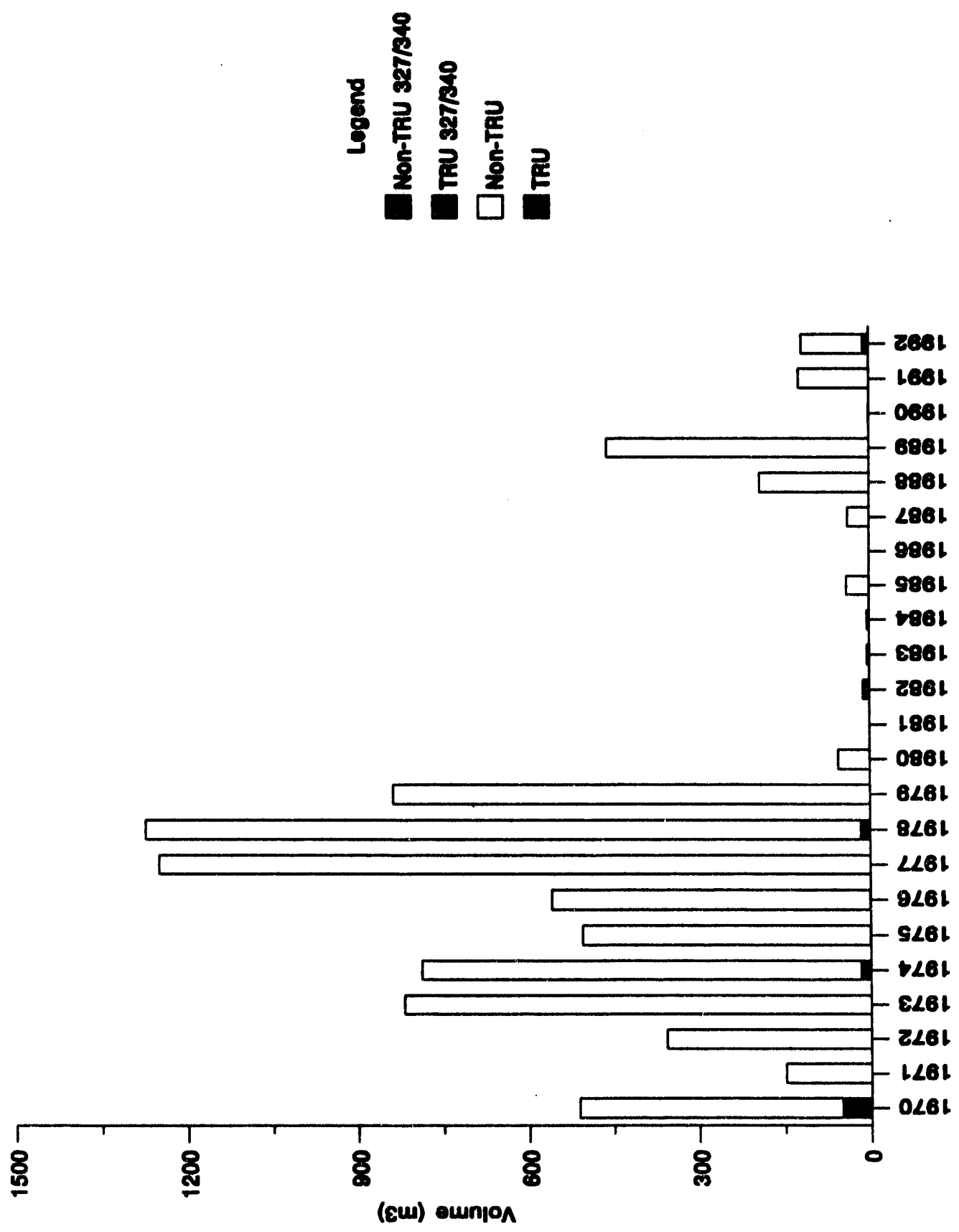


\section{WHC-EP-0696}

Figure 6-12. Volume Percentage of Other Containers Generated at Building $\mathbf{3 2 5}$ by PNL Annually.
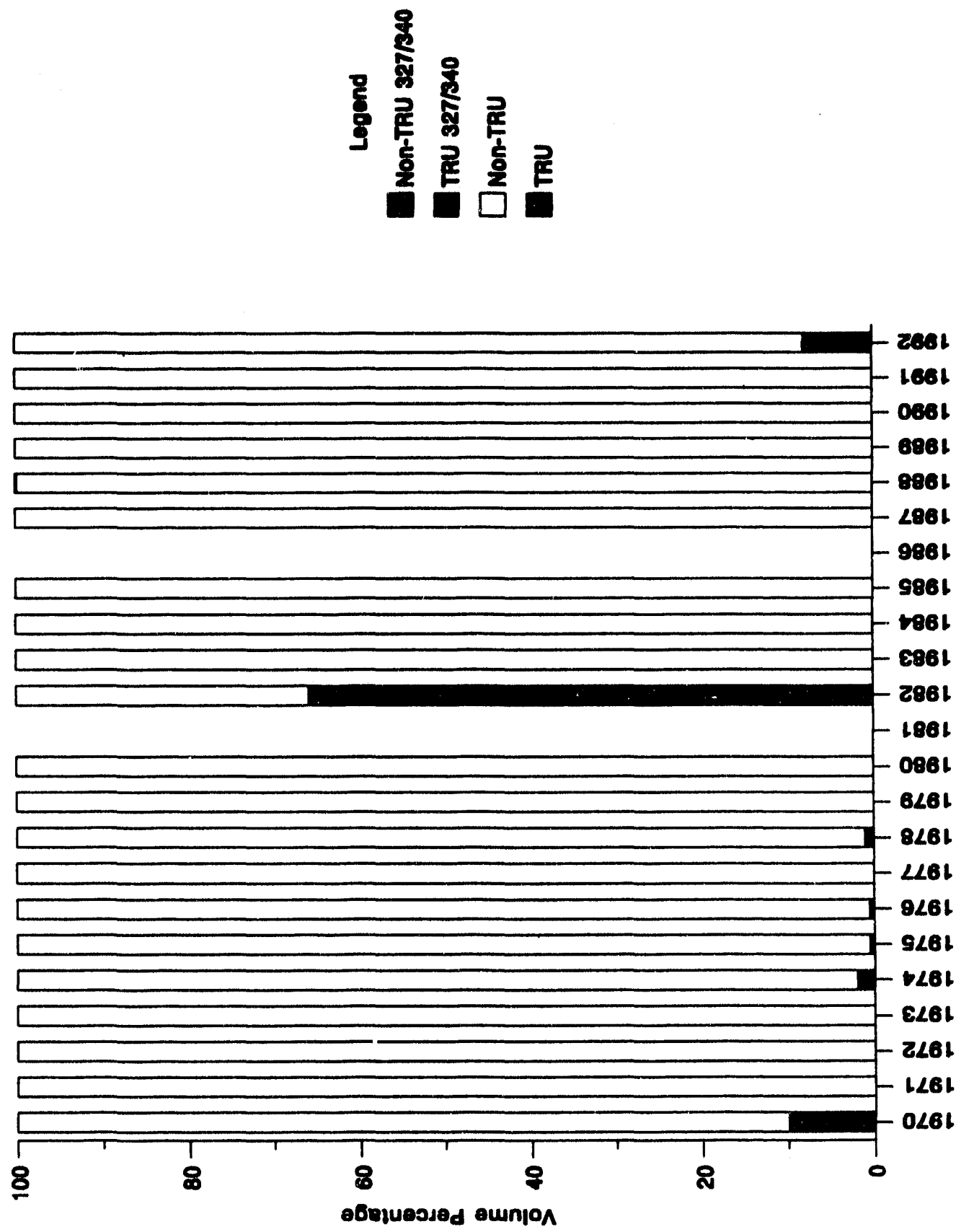

$\frac{8}{4}$ 

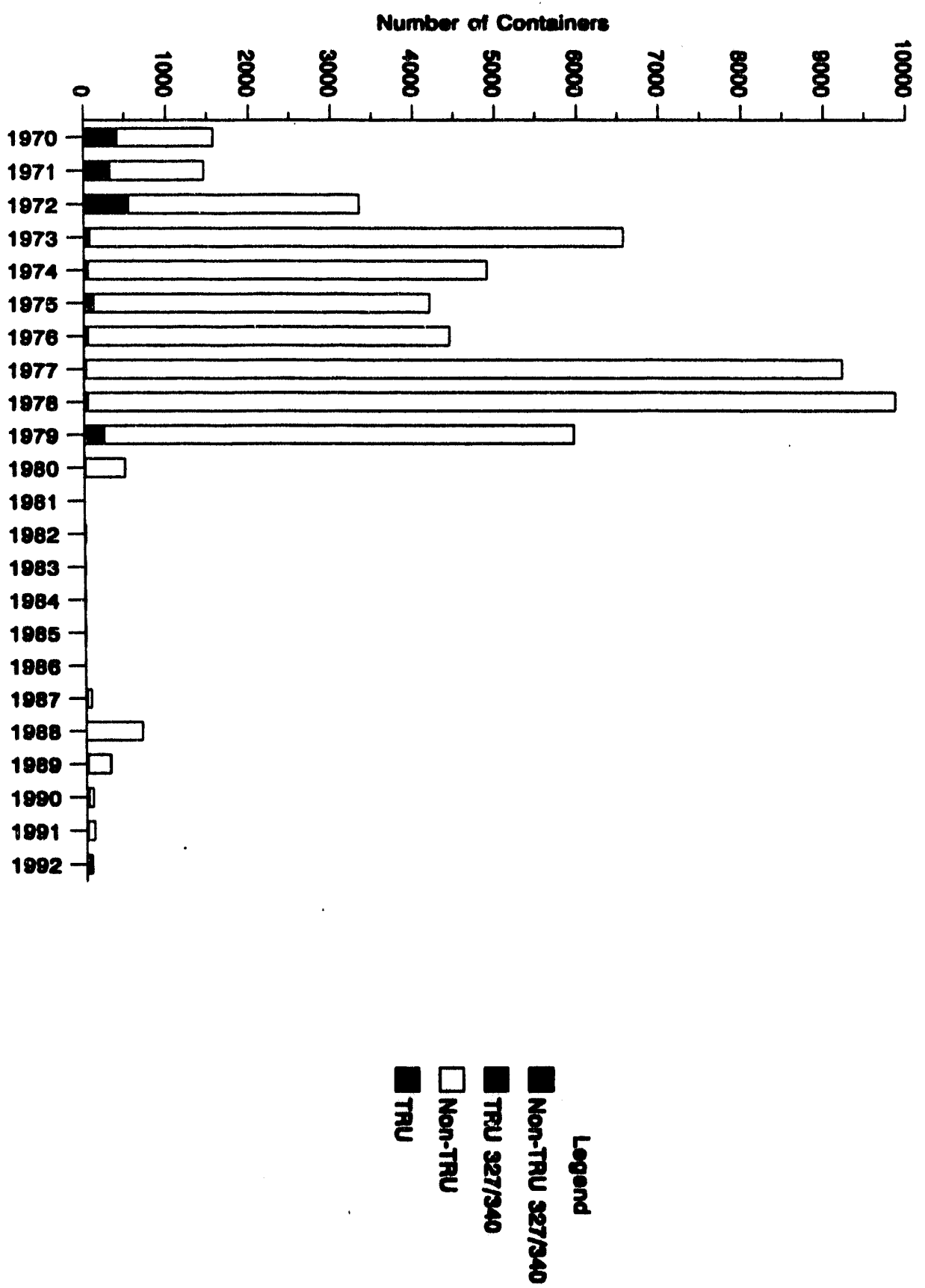

- Allenuuళ 7Nd Aq gZE Bu!pı!ng to

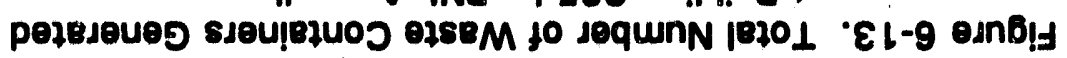




\section{$\varepsilon 乙-9$}

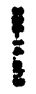
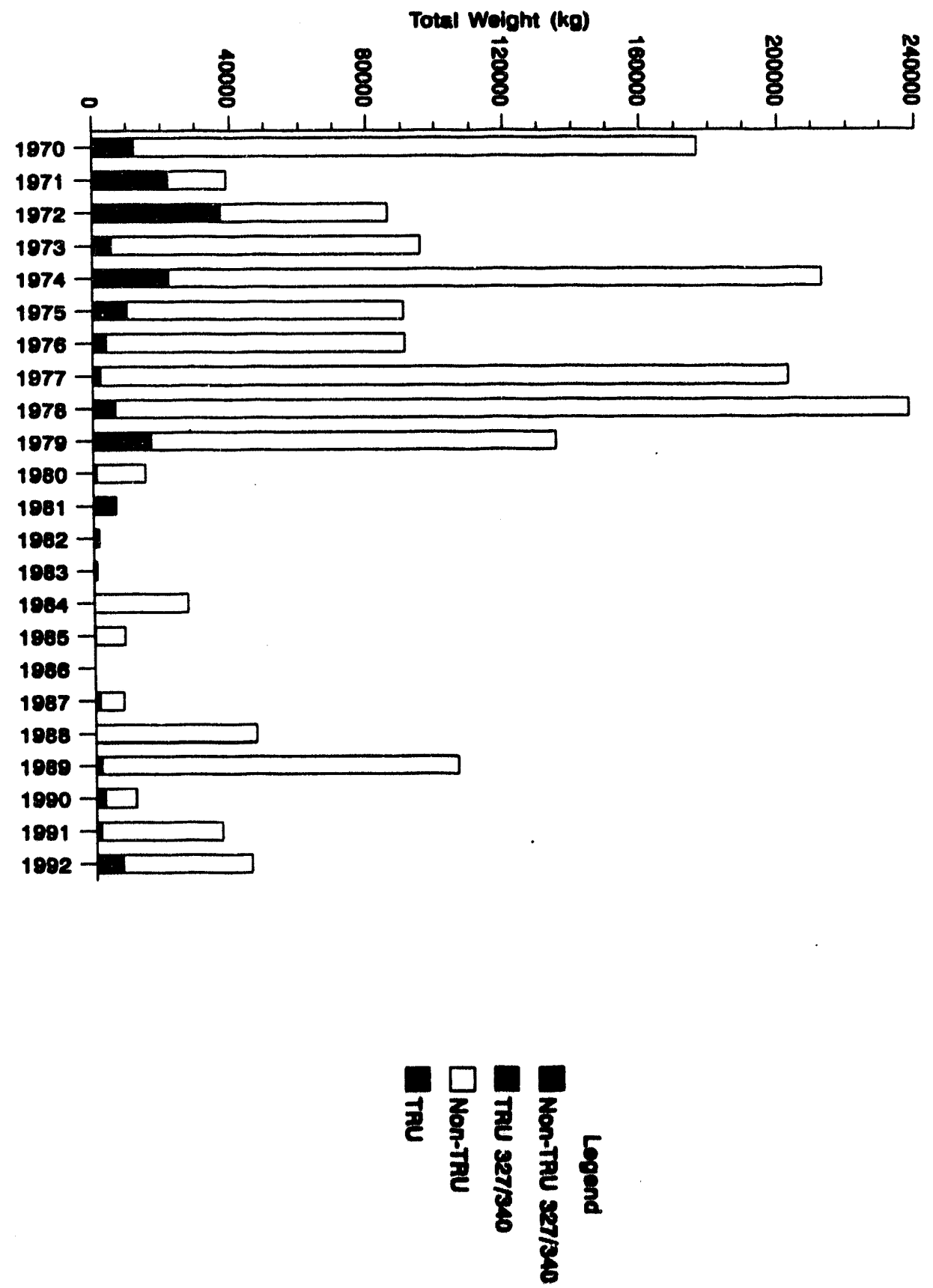

- Allenuu $\forall$ 7Nd Aq 9ZE Bulp|!ng

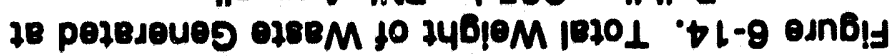


$\downarrow Z-9$

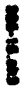

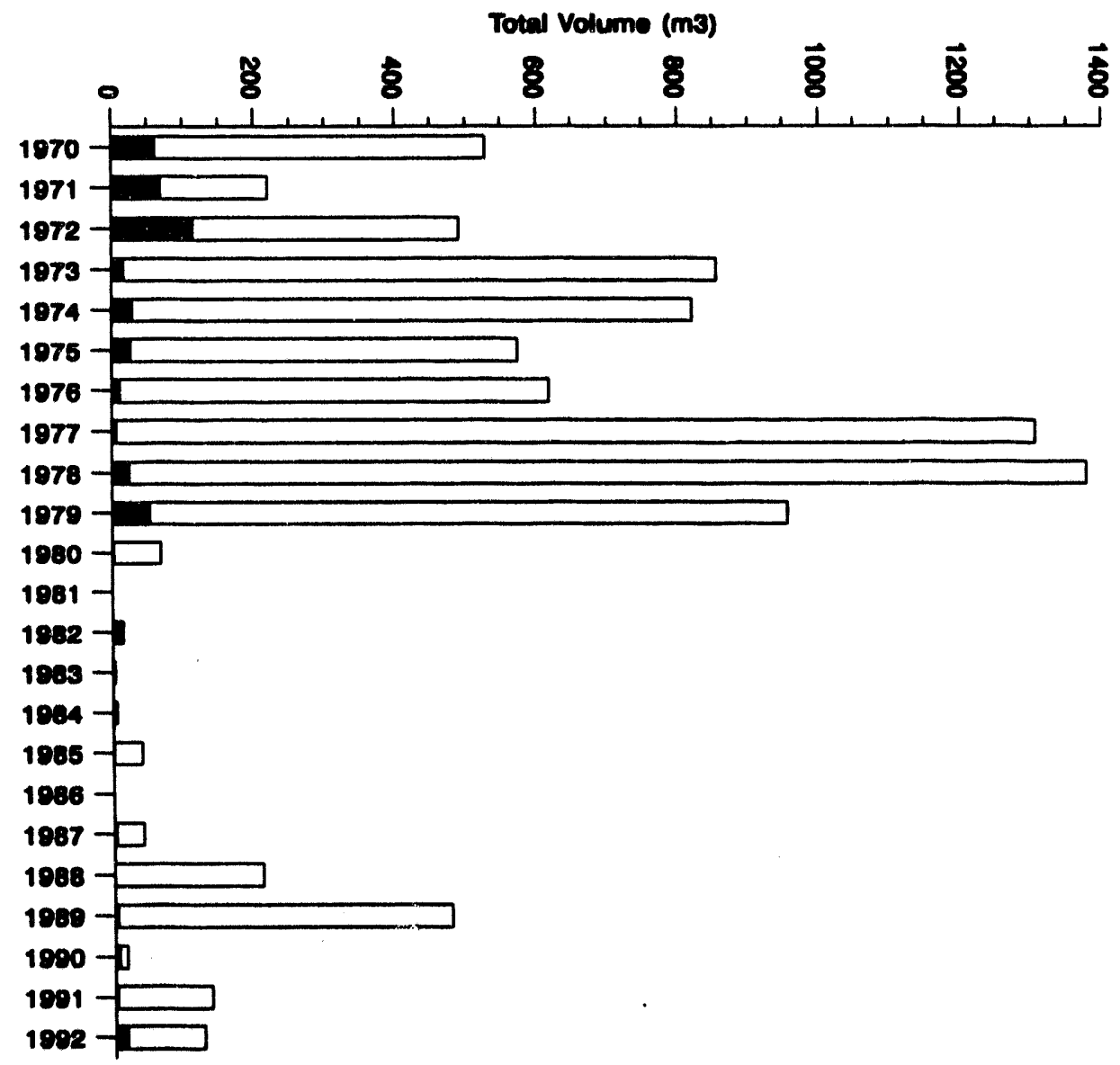

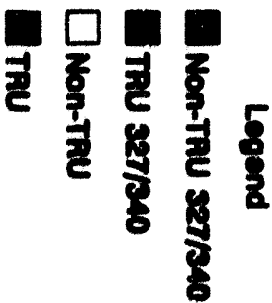

- Allenuu 7 Nd Kq 928 Bu!p|!ng

te pejesoues ezseM to ounjon 10701 ' 91 -9 edn6!d

\section{0-dヨ-JHM}




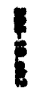
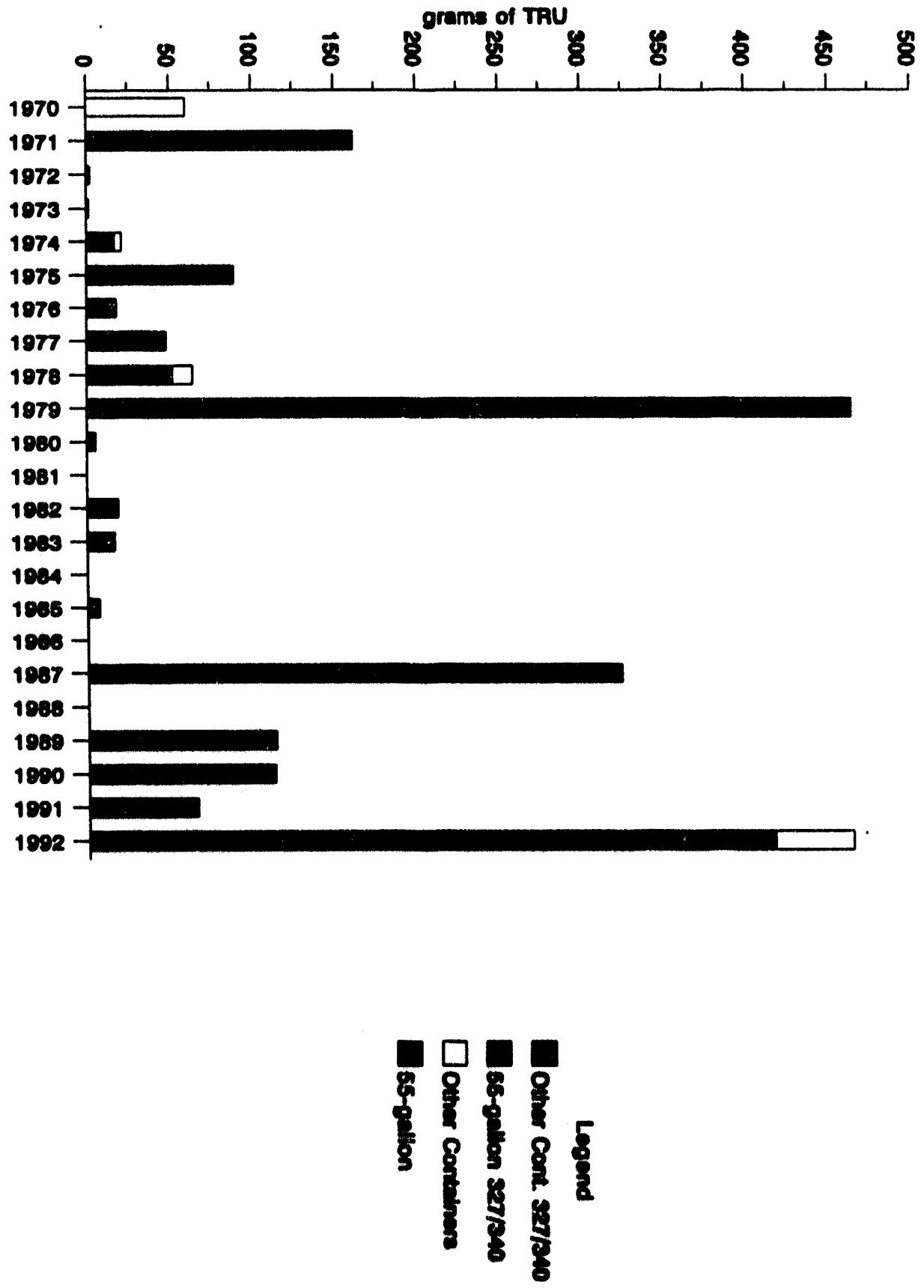

- Allenuu to pozesouog ezsem ny 10 swejo '81-9 ednoly 
Table 6-1. TRU Wastes Attributed to PNL Building 325 but Generated at Other Facilities.

\begin{tabular}{|c|c|c|c|c|c|c|c|}
\hline Year & Source & $\begin{array}{l}\text { Container } \\
\text { Number }\end{array}$ & $\begin{array}{c}\text { Percent of } \\
\text { Total }\end{array}$ & $\begin{array}{l}\text { Woight } \\
\text { (kg) }\end{array}$ & $\begin{array}{l}\text { Weight } \\
\text { Percent }\end{array}$ & $\begin{array}{c}\text { Volume } \\
\left(m^{2}\right)\end{array}$ & $\begin{array}{l}\text { Vohmo } \\
\text { Percent }\end{array}$ \\
\hline \multirow[t]{3}{*}{1970} & 308 & 7 Others & $2 \%$ & 286 & $3 \%$ & 0.8 & $2 \%$ \\
\hline & 308 & 6 Drums & $12 \%$ & 408 & $12 \%$ & 1.2 & $11 \%$ \\
\hline & Total: & 6onors & $\begin{array}{l}2 \% \\
12 \%\end{array}$ & $\frac{286}{108}$ & 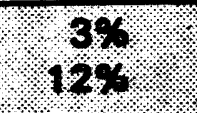 & $7_{1}^{1}$ & $\sqrt{2}$ \\
\hline \multirow[t]{2}{*}{1974} & 325B & 3 Others & $100 \%$ & 18634 & $100 \%$ & 17.9 & $100 \%$ \\
\hline & Toral & 3 others. & $100 \%$ & $1868 \%$ & $100 \%$ & $4 \%$ & 13.6 \\
\hline \multirow[t]{2}{*}{1976} & 3708 & 1 Other & $100 \%$ & 305 & $100 \%$ & 0.7 & $100 \%$ \\
\hline & Toral & 184, & $100 \%$ & 305. & $1005 \%$ & 8 & $10 \%$ \\
\hline \multirow[t]{3}{*}{1979} & $303 C$ & 48 Drums & $19 \%$ & 1360 & $8 \%$ & 10.0 & $19 \%$ \\
\hline & 324 & 36 Drums & $14 \%$ & 1814 & $11 \%$ & 7.6 & $14 \%$ \\
\hline & Toral & 84 Broms & $34 \%$ & 3114 & 1084 & to & $4 \%$ \\
\hline
\end{tabular}


Table 6-2. Low-Level Wastes Attributed to PNL Building 325 but Generated at Other Facilities. (2 sheets)

\begin{tabular}{|c|c|c|c|c|c|c|c|}
\hline Year & Source & $\begin{array}{l}\text { Container } \\
\text { Number }\end{array}$ & $\begin{array}{c}\text { Percent of } \\
\text { Total }\end{array}$ & $\begin{array}{l}\text { Woight } \\
\text { (kg) }\end{array}$ & $\begin{array}{l}\text { Weight } \\
\text { Percent }\end{array}$ & $\begin{array}{c}\text { Volume } \\
\left(m^{3}\right)\end{array}$ & $\begin{array}{l}\text { Volume } \\
\text { Percent }\end{array}$ \\
\hline \multirow[t]{4}{*}{1970} & 327 & 2 Others & $<1 \%$ & 366 & $<1 \%$ & 0.8 & $<1 \%$ \\
\hline & 340 & 20 Others & $2 \%$ & 6843 & $4 \%$ & 16.8 & $4 \%$ \\
\hline & 340 & 24 Drums & $77 \%$ & 4899 & $91 \%$ & 5.1 & $78 \%$ \\
\hline & Total & $\begin{array}{l}22 \text { Others } \\
24 \text { Drums. }\end{array}$ & $2 \%$ & $\begin{array}{l}7209 \\
4899\end{array}$ & $\begin{array}{r}5 \% \\
91 \%\end{array}$ & 1786 & $\% \%$ \\
\hline \multirow[t]{2}{*}{1972} & 331 & 96 Others & $4 \%$ & 1219 & $3 \%$ & 12.1 & $3 \%$ \\
\hline & Torel & 96 Others & $4 \%$ & 1219 & $3 \%$ & 18 & $6 \%$ \\
\hline \multirow[t]{3}{*}{1973} & 340 & 11 Others & $<1 \%$ & 794 & $1 \%$ & 2.8 & $<1 \%$ \\
\hline & 3706 & 33 Others & $1 \%$ & 419 & $1 \%$ & 5.1 & $1 \%$ \\
\hline & Total & 44 Others & $1 \%$ & 1213 & 19 & 79 & $18 \%$ \\
\hline \multirow[t]{4}{*}{1974} & 306 & 2 Others & $<1 \%$ & 5265 & $3 \%$ & 11.6 & $2 \%$ \\
\hline & 309 & 30 Others & $1 \%$ & 17450 & $10 \%$ & 38.4 & $5 \%$ \\
\hline & 309 & 7 Drums & $7 \%$ & 658 & $7 \%$ & 1.5 & $7 \%$ \\
\hline & Total & $\begin{array}{l}32 \text { Others } \\
7 \text { Drums }\end{array}$ & $1 \%$ & $\begin{array}{r}22715 \\
658\end{array}$ & $\frac{13 \%}{7 \%}$ & 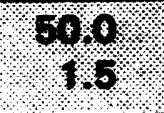 & $\frac{68 \%}{7 \%}$ \\
\hline \multirow[t]{2}{*}{1977} & 306 & 6 Drums & $3 \%$ & 805 & $3 \%$ & 1.3 & $3 \%$ \\
\hline & Total & 6 Drums. & $3 \%$ & 805 & $3 \%$ & $4: 3$. & 383 \\
\hline \multirow[t]{2}{*}{1978} & 306 & 19 Others & $<1 \%$ & 2721 & $2 \%$ & 63.8 & $5 \%$ \\
\hline & 324 & 63 Drums & $13 \%$ & 3266 & $4 \%$ & 13.1 & $14 \%$ \\
\hline
\end{tabular}


Table 6-2. Low-Level Wastes Attributed to PNL Building 325 but Generated at Other Facilities. 12 sheets)

\begin{tabular}{|c|c|c|c|c|c|c|c|}
\hline Year & Source & $\begin{array}{l}\text { Container } \\
\text { Number }\end{array}$ & $\begin{array}{c}\text { Percent of } \\
\text { Total }\end{array}$ & $\begin{array}{l}\text { Weight } \\
\text { (kg) }\end{array}$ & $\begin{array}{l}\text { Woight } \\
\text { Percent }\end{array}$ & $\begin{array}{l}\text { Volume } \\
\left(m^{3}\right)\end{array}$ & $\begin{array}{l}\text { Volume } \\
\text { Percent }\end{array}$ \\
\hline & totel & $\begin{array}{l}19 \text { others } \\
68 \text { Drums. }\end{array}$ & 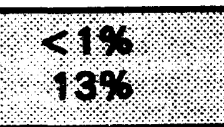 & $\begin{array}{l}2921 \\
3266\end{array}$ & $\begin{array}{l}2 \% \\
6 \%\end{array}$ & $\frac{6 x 8}{48}$ & 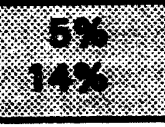 \\
\hline \multirow[t]{8}{*}{1979} & 306 & 7 Others & $<1 \%$ & 9044 & $10 \%$ & 22.0 & $3 \%$ \\
\hline & 331 & 23 Others & $<1 \%$ & 292 & $<1 \%$ & 2.9 & $<1 \%$ \\
\hline & 327 & 9 Others & $<1 \%$ & 114 & $<1 \%$ & 1.1 & $<1 \%$ \\
\hline & 329 & 6 Others & $<1 \%$ & 76 & $<1 \%$ & 0.8 & $<1 \%$ \\
\hline & 340 & 4 Others & $<1 \%$ & 51 & $<1 \%$ & 0.5 & $<1 \%$ \\
\hline & 3720 & 2 Others & $<1 \%$ & 25 & $<1 \%$ & 0.3 & $<1 \%$ \\
\hline & 3745 & 2 Others & $<1 \%$ & 25 & $<1 \%$ & 0.3 & $<1 \%$ \\
\hline & Torel: & 58 Others & $1 \%$ & 9627 & 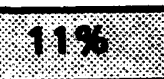 & 79 & $x^{3}$ \\
\hline \multirow[t]{2}{*}{1980} & 306 & 21 Drums & $42 \%$ & 5410 & $67 \%$ & 4.4 & $42 \%$ \\
\hline & Totel & 21 Drums & $42 \%$ & 6410 & 6783 & $\%$ & $9 \%$ \\
\hline \multirow[t]{2}{*}{1985} & $325 A$ & 4 Others & $80 \%$ & 8269 & $95 \%$ & 35.4 & $89 \%$ \\
\hline & Total. & A Others: & $80 \% \%$ & 8269 & 958 & 3.64. & $99 \%$ \\
\hline
\end{tabular}




\section{WHC-EP-0698}

Table 6-3. TRU Waste Generated at Building 325 by PNL by Contairier Type and Year. (4 sheots)

\begin{tabular}{|c|c|c|c|c|c|c|c|}
\hline 3.1. & \%1, & $\% \%$ & $1 \%$ & \%, & \% & +ron & romp \\
\hline \multicolumn{8}{|c|}{$1 \%$} \\
\hline $\begin{array}{l}\text { Burlep, Cloth, Pepar, or } \\
\text { Plactio Bags }\end{array}$ & unknown & 4 & 1.0 & 1242 & 10.0 & 2.8 & 4.2 \\
\hline $\begin{array}{l}\text { Fiberboend/Fiactic Boxes, } \\
\text { Centon, or Ceses }\end{array}$ & unknown & 318 & 70.8 & 4430 & 36.9 & 40.4 & 68.6 \\
\hline \multirow[t]{3}{*}{ HEPA Fitem } & unknown & 8 & 1.0 & 833 & 4.3 & 1.2 & 1.0 \\
\hline & 8-gal & 4 & 1.0 & 264 & 2.1 & 0.6 & 0.1 \\
\hline & 30-gal & 20 & 7.0 & 1184 & 9.6 & 3.4 & 6.6 \\
\hline $\begin{array}{l}\text { Motal Drums, Berrob, } \\
\text { Kegs }\end{array}$ & 85-gal & 81 & 12.4 & 3443 & 27.8 & 10.6 & 17.2 \\
\hline \multirow{3}{*}{$\begin{array}{c}\text { Trucke, Flatbeds, } \\
\text { Compactor, Loadlugoer } \\
\text { Totales }\end{array}$} & unknown & 1 & 0.2 & 1270 & 10.3 & 2.8 & 4.6 \\
\hline & & 412 & & 12382 & & 61.8 & \\
\hline & & & $11 \%$ & & & & \\
\hline \multirow[t]{3}{*}{$\begin{array}{l}\text { Motal Drums, Berrols, } \\
\text { Kege }\end{array}$} & 8b-gal & 327 & 100 & 22240 & 100 & 68.7 & 100 \\
\hline & & 327 & & 22240 & & 68.7 & \\
\hline & & & 112 & & & & \\
\hline \multirow{3}{*}{$\begin{array}{l}\text { Motal Drums, Berreb, } \\
\text { Koge }\end{array}$} & Bb-gal & 848 & 100 & 37286 & 100 & 115.1 & 100 \\
\hline & & 548 & & 37286 & & 118.1 & \\
\hline & & & 1018 & & & 1 & \\
\hline $\begin{array}{l}\text { Motal Drums, Barroks, } \\
\text { Koos }\end{array}$ & 68-gal & 78 & 100 & E834 & 100 & 16.8 & 100 \\
\hline TOTALS & & 70 & & 5834 & & 16.6 & \\
\hline & & & $\% 8$ & 1 & & & \\
\hline \multirow[t]{2}{*}{ Concrote Boxes } & $4 \times 4 \times 8$ & 1 & 1.8 & 3774 & 17.0 & 3.6 & 12.5 \\
\hline & $4 \times 7 \times 9$ & 2 & 3.6 & 14860 & 67.0 & 14.3 & 49.7 \\
\hline $\begin{array}{l}\text { Motal Drums, Barrels, } \\
\text { Kogs }\end{array}$ & 65-gal & 62 & 84.6 & 3638 & 16.0 & 10.9 & 37.8 \\
\hline TOTAL & & 58 & & 22172 & & 28.8 & \\
\hline
\end{tabular}


Table 6-3. TRU Waste Generated at Building 325 by PNL by Container Type and Year. (4 sheets)

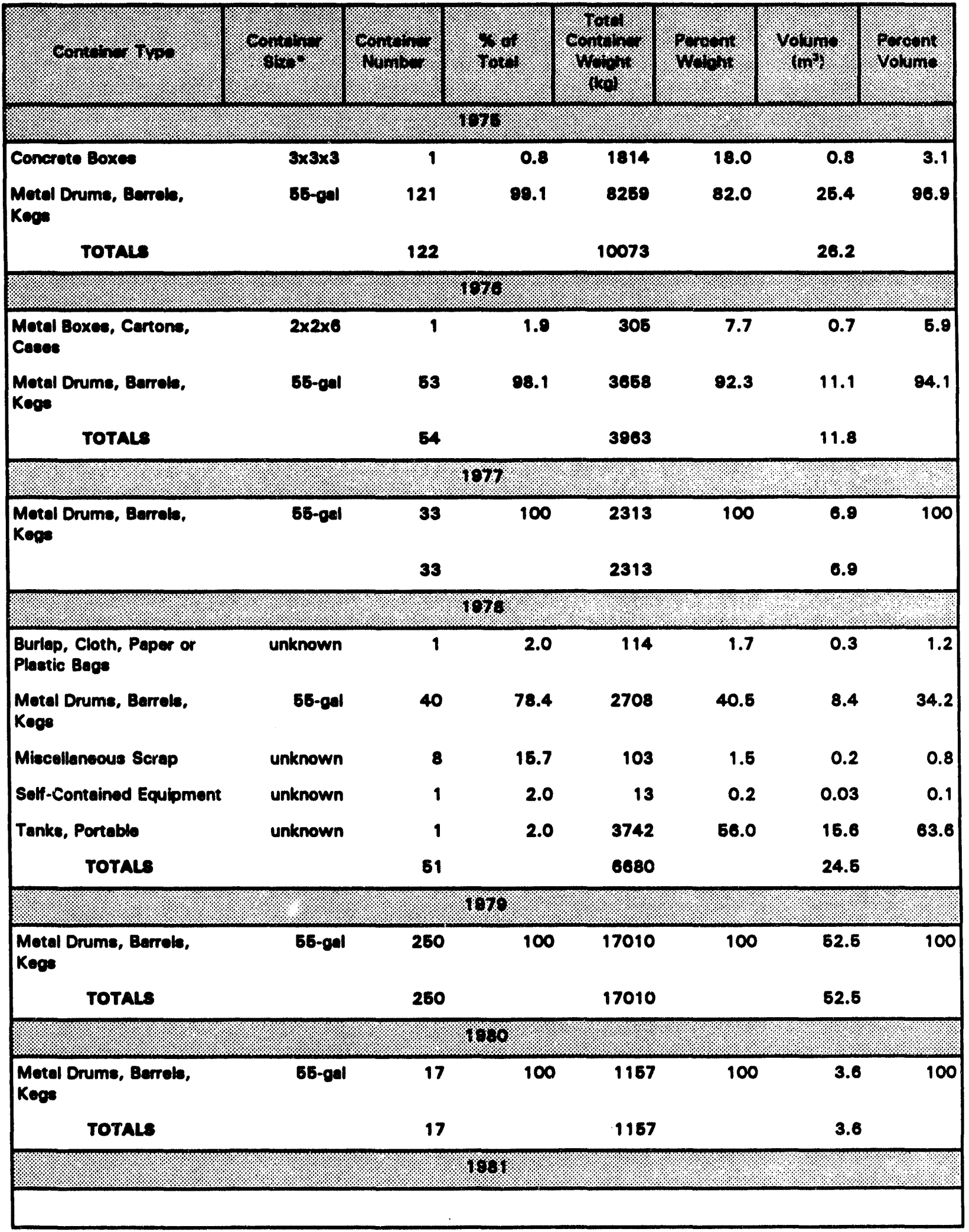




\section{WHC-EP-0696}

Table 6-3. TRU Waste Generated at Building 325 by PNL by Container Type and Year. (4 sheets)

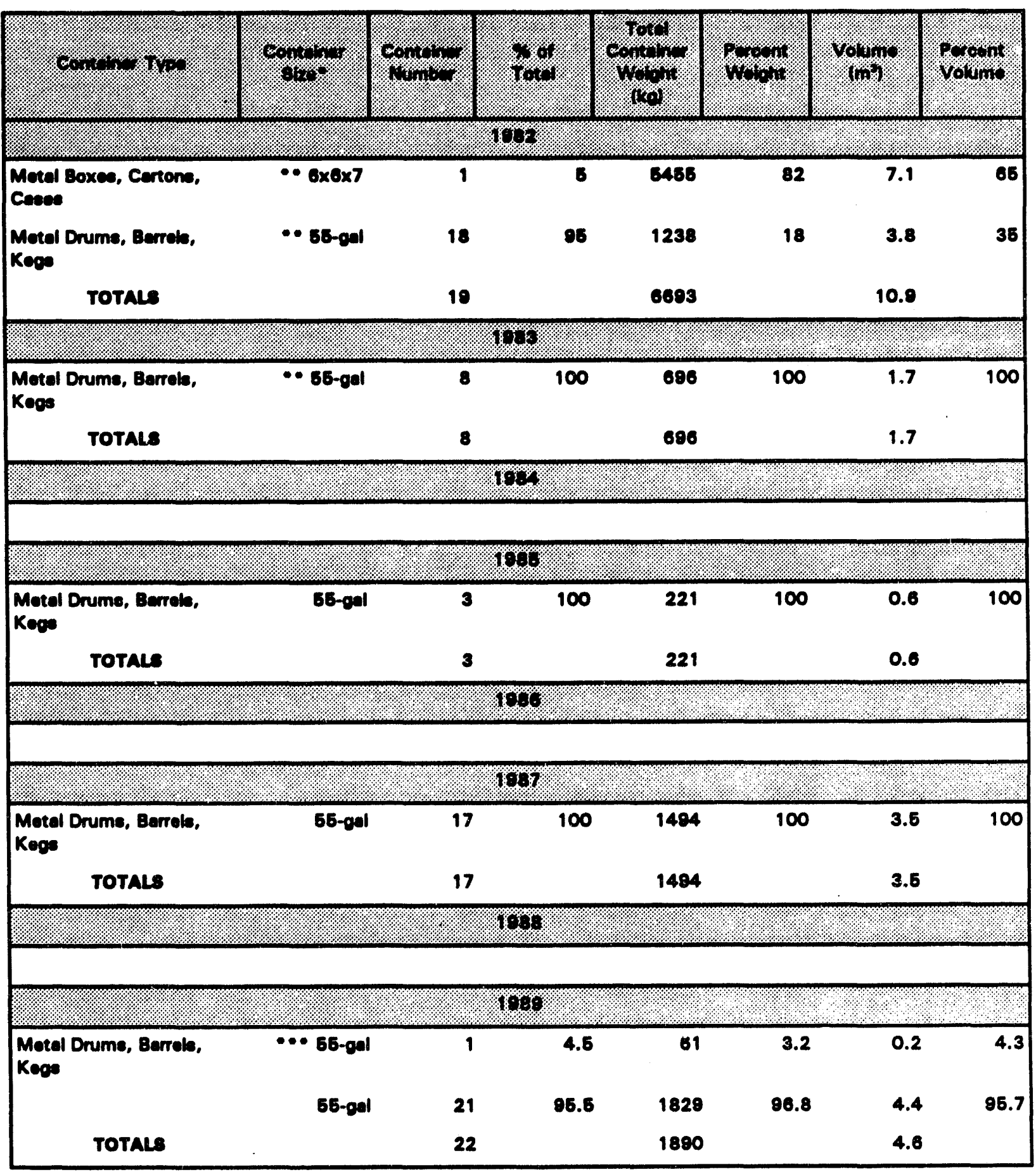




\section{WHC-EP-0696}

Table 6-3. TRU Waste Generated at Building 325 by PNL by Container Type and Year.

(4 sheets)

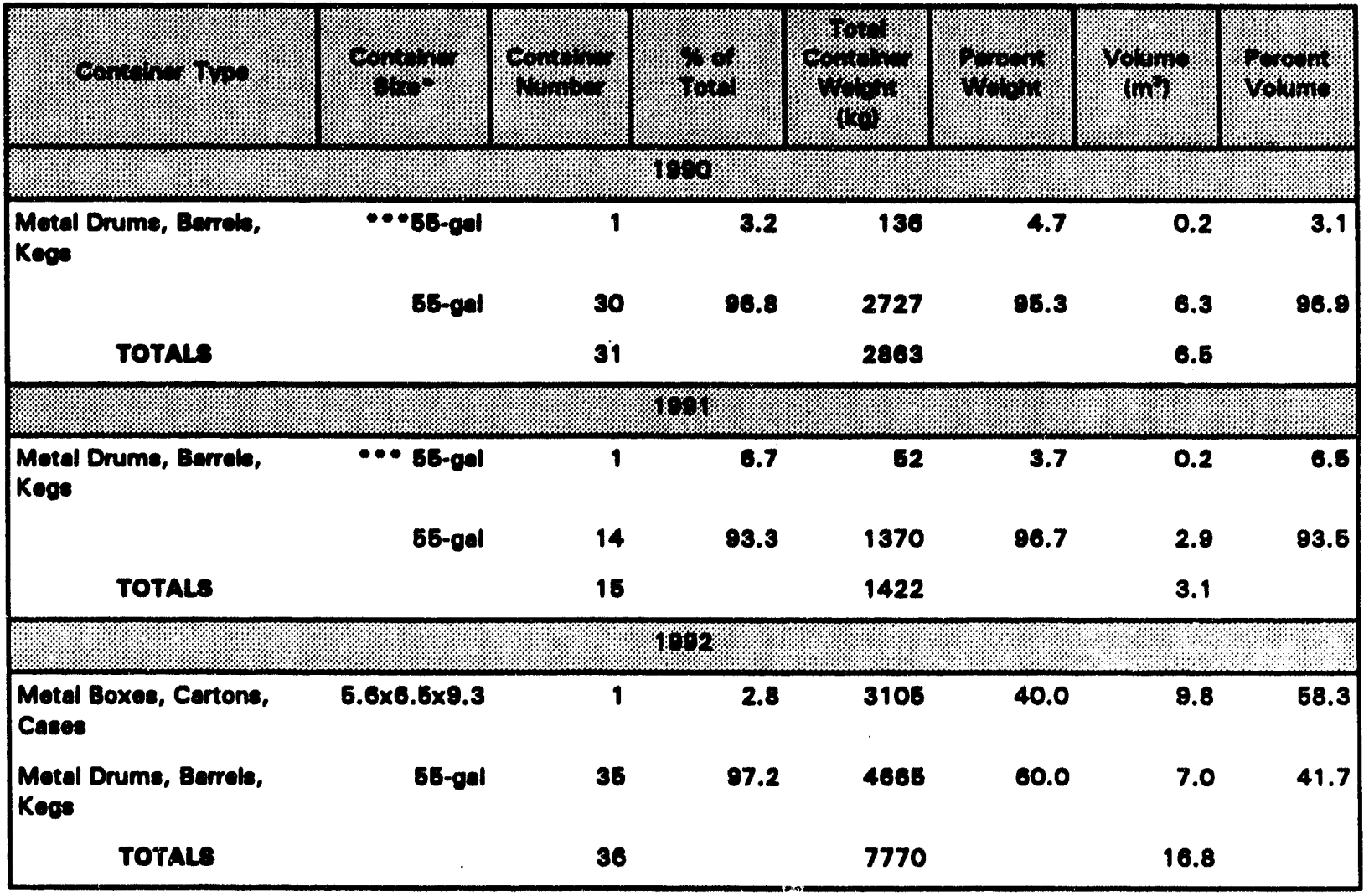

- Unlese otherwibe noted, unite of dimensione ere in ft.

- "Solid Weste Burial Recorde for PNL Bullding 326 that were found under PNL Bulldinge 327 and 340 records. - "Contalnere hold mbxed waete. 


\section{WHC-EP-0696}

Table 6-4. Non-TRU Waste Generated at Building 325 by PNL by Container Type and Year. (9 sheets)

\begin{tabular}{|c|c|c|c|c|c|c|c|}
\hline 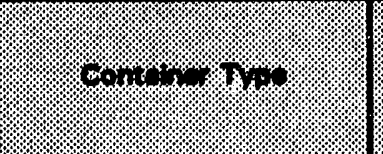 & 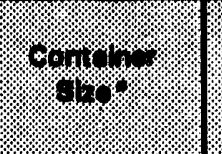 & $\%+1 \%$ & $4 \%$ & $\frac{6}{6 \%}$ & $1 \%$ & 1.0101\% & 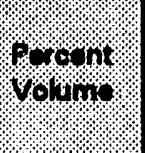 \\
\hline \multicolumn{8}{|c|}{$18 \%$} \\
\hline \multirow{2}{*}{$\begin{array}{l}\text { Flberboerd/Plactic Boxee, } \\
\text { Cartone, Cases }\end{array}$} & $\cdots$ unknown & 7 & 0.8 & 80 & 0.1 & 0.8 & 0.2 \\
\hline & unknown & 1071 & 02.3 & 13754 & 8.4 & 136.2 & 29.2 \\
\hline Glove Boxes & unknown & 2 & 0.2 & 826 & 0.6 & 1.8 & 0.4 \\
\hline HEPA Filtere & unknown & 2 & 0.2 & 386 & 0.2 & 0.8 & 0.2 \\
\hline \multirow{3}{*}{$\begin{array}{l}\text { Metal Drums, Barrals, } \\
\text { Kege }\end{array}$} & $30-981$ & $\boldsymbol{8}$ & 0.5 & 245 & 0.1 & 0.7 & 0.2 \\
\hline & $\bullet \bullet$ 86-gal & 1 & 0.1 & 68 & $<0.1$ & 0.2 & $<0.1$ \\
\hline & B8-gal & 30 & 2.6 & $\mathbf{6 3 3 0}$ & 3.3 & 6.3 & 1.3 \\
\hline Mbcollaneous Scrap & unknown & 22 & 1.0 & 70381 & 42.8 & 156.8 & 33.6 \\
\hline Self-Contained Equipment & unknown & 10 & 0.0 & 26868 & 16.4 & 69.8 & 12.8 \\
\hline $\begin{array}{l}\text { Trucks, Flatbeds, } \\
\text { Compectore, Loaduggers }\end{array}$ & unknown & 4 & 0.3 & 40007 & 24.4 & 89.2 & 19.1 \\
\hline \multirow{2}{*}{$\begin{array}{l}\text { Wooden Boxes, Cartons, } \\
\text { Cases }\end{array}$} & $3 \times 3 \times 4$ & 3 & 0.3 & 1828 & 1.1 & 4.1 & 0.9 \\
\hline & $4 \times 5 \times 6$ & 3 & 0.3 & 4446 & 2.7 & 9.9 & 2.1 \\
\hline TOTALS & & 1161 & & 104186 & & 466.9 & \\
\hline \multicolumn{8}{|c|}{$18 \%$} \\
\hline $\begin{array}{l}\text { Fiberboard/Plastic Boxes, } \\
\text { Cartons, Cases }\end{array}$ & unknown & 1116 & 98.7 & 14174 & 86.0 & 142.2 & 94.0 \\
\hline $\begin{array}{l}\text { Motal Drums, Barrols, } \\
\text { Kegs }\end{array}$ & 56-gal & 11 & 1.0 & 748 & 4.6 & 2.3 & 1.6 \\
\hline Wooden Boxes, Cartons, & unknown & 4 & 0.4 & 1760 & 10.6 & 6.7 & 4.4 \\
\hline TOTALS & & 1131 & & 16682 & & 151.2 & \\
\hline \multicolumn{8}{|c|}{$1 \% \%$} \\
\hline $\begin{array}{l}\text { Fiberboard/Plastic Boxes, } \\
\text { Cartons, Cases }\end{array}$ & unknown & 2624 & 93.8 & 33436 & 68.6 & 333.7 & 88.8 \\
\hline $\begin{array}{l}\text { Metal Drums, Barrels. } \\
\text { Kege }\end{array}$ & 65-gal & 100 & 3.8 & $\mathbf{6 2 1 8}$ & 10.7 & 18.7 & 6.0 \\
\hline $\begin{array}{l}\text { Self-Contained, } \\
\text { Equipment }\end{array}$ & unknown & 1 & $<0.1$ & 38 & 0.1 & 1.0 & 0.3 \\
\hline Tanks, Portable & unknown & 3 & 0.1 & 228 & 0.5 & 0.6 & 0.1 \\
\hline $\begin{array}{l}\text { Trucks, Flatbede, } \\
\text { Compactor, Loadlugger }\end{array}$ & unknown & 2 & 0.1 & 3601 & 7.4 & 8.0 & 2.1 \\
\hline $\begin{array}{l}\text { Wooden Boxes, Cartons, } \\
\text { Cases }\end{array}$ & $4 \times 4 \times 8$ & 2 & 0.1 & 3251 & 6.7 & 7.3 & 1.8 \\
\hline
\end{tabular}


WHC-EP-0696

Table 6-4. Non-TRU Waste Generated at Building 325 by PNL by Container Type and Year. (9 shoets)

\begin{tabular}{|c|c|c|c|c|c|c|c|}
\hline (4. & \%... & wor. & \% & \% & $\% \%$ & 1.m\% & Yo.no \\
\hline $\begin{array}{l}\text { Woodan Boxes, Cartons, } \\
\text { Cases (cont) }\end{array}$ & unknown & $\mathbf{8 6}$ & 2.0 & 2891 & 6.1 & 6.7 & 1.8 \\
\hline Totals & & 2797 & & 48784 & & 378.9 & \\
\hline \multicolumn{8}{|c|}{$1 \% 8$} \\
\hline $\begin{array}{l}\text { Burlep, Cloth, Peper or } \\
\text { Plestic Bags }\end{array}$ & unknown & 12 & 0.2 & 182 & 0.2 & 3.0 & 0.4 \\
\hline $\begin{array}{l}\text { Fiberboerd/Piactic Boxee, } \\
\text { Cartons, Caces }\end{array}$ & unknown & 6374 & 88.2 & 80088 & 80.0 & 812.2 & 96.0 \\
\hline $\begin{array}{l}\text { Motal Boxes, Certons, } \\
\text { Cases }\end{array}$ & $2 \times 2 \times 3$ & 1 & $<0.1$ & 182 & 0.2 & 0.3 & $<0.1$ \\
\hline \multirow{2}{*}{$\begin{array}{l}\text { Motal Drums, Berrols, } \\
\text { Koge }\end{array}$} & 30-gal & 1 & $<0.1$ & 41 & 0.1 & 0.1 & $<0.1$ \\
\hline & 55-gal & 97 & 1.6 & 7360 & 8.2 & 19.3 & 2.3 \\
\hline Self-Contained Equipment & unknown & 2 & $<0.1$ & 616 & 0.7 & 1.4 & 0.2 \\
\hline $\begin{array}{l}\text { Truaks, Flatbeds, } \\
\text { Compactor, Loadugoer }\end{array}$ & unknown & 1 & $<0.1$ & 667 & 0.7 & 1.6 & 0.2 \\
\hline TOTALs & & 6488 & & 89044 & & 837.8 & \\
\hline \multicolumn{8}{|c|}{$\% \%$} \\
\hline Dump Truok & unknown & 18 & 0.3 & 76421 & 40.0 & 73.4 & 9.3 \\
\hline $\begin{array}{l}\text { Fiberboard/Pleatic Boxes, } \\
\text { Cartone, Caces }\end{array}$ & unknown & 4672 & 96.4 & 60448 & 31.2 & 595.5 & 76.2 \\
\hline \multirow{2}{*}{$\begin{array}{l}\text { Metal Drums, Barruls, } \\
\text { Kege }\end{array}$} & $30-0.1$ & 9 & 0.2 & 387 & 0.2 & 1.1 & 0.1 \\
\hline & 55-gal & 85 & 2.0 & 9189 & 4.8 & 20.6 & 2.6 \\
\hline Miscellaneous Scrap & unknown & 3 & 0.1 & 1284 & 0.7 & 2.8 & 0.3 \\
\hline Self-Contained Equipment & unknown & 1 & $<0.1$ & 64 & $<0.1$ & 0.1 & $<0.1$ \\
\hline $\begin{array}{l}\text { Trucks, Flatbeds, } \\
\text { Compactor, Loadhoger }\end{array}$ & unknown & 2 & $<0.1$ & 8264 & 2.8 & 11.7 & 1.5 \\
\hline \multirow{8}{*}{$\begin{array}{l}\text { Wooden Boxes, Cartons, } \\
\text { Cases }\end{array}$} & $2 \times 2 \times 8$ & 1 & $<0.1$ & 408 & 0.2 & 0.8 & 0.1 \\
\hline & $2 \times 2 \times 9$ & 1 & $<0.1$ & 467 & 0.2 & 1.0 & 0.1 \\
\hline & $2 \times 4 \times 5$ & 1 & $<0.1$ & 608 & 0.3 & 1.1 & 0.1 \\
\hline & $2 \times 4 \times 8$ & 13 & 0.3 & 10565 & $\mathbf{6 . 6}$ & 23.6 & 3.0 \\
\hline & $4 \times 4 \times 4$ & 14 & 0.3 & 11381 & 6.0 & 25.4 & 3.2 \\
\hline & $4 \times 4 \times 6$ & 1 & $<0.1$ & 1016 & 0.5 & 2.3 & 0.3 \\
\hline & $4 \times 4 \times 8$ & 4 & 0.1 & 8504 & 3.4 & 14.5 & 1.8 \\
\hline & $5 \times 6 \times 7$ & 1 & $<0.1$ & 2607 & 1.4 & 6.0 & 0.8 \\
\hline
\end{tabular}




\section{WHC-EP-0696}

Table 6-4. Non-TRU Waste Generated at Building 325 by PNL by Container Type and Year. (9 sheets)

\begin{tabular}{|c|c|c|c|c|c|c|c|}
\hline \%. & $1 \%$ & \%n\% & 108 & $\frac{6}{6}$ & $4 \%+1 \%$ & rop & $10.01 \%$ \\
\hline $\begin{array}{l}\text { Wooden Boxes, Cartons, } \\
\text { Coees (cont) }\end{array}$ & unknown & 14 & 0.3 & 8307 & 2.8 & 11.8 & 1.6 \\
\hline TOTALS & & 4848 & & 180828 & & 791.8 & \\
\hline & & & 18 & & & & \\
\hline $\begin{array}{l}\text { Burlap, Cloth, Paper or } \\
\text { Plaotic Bacs }\end{array}$ & unknown & 7 & 0.2 & 781 & 1.0 & 1.7 & 0.3 \\
\hline Concrote Boxee & $3 \times 3 \times 3$ & 1 & $<0.1$ & 1814 & 2.3 & 0.8 & 0.2 \\
\hline $\begin{array}{l}\text { Fiberbourd/Plastic Boxes, } \\
\text { Cartons, Cases }\end{array}$ & unknown & 3801 & 93.2 & 48282 & 68.8 & 484.2 & 88.6 \\
\hline HEPA Fittore & unknown & 23 & 0.6 & 978 & 1.2 & 2.2 & 0.4 \\
\hline $\begin{array}{l}\text { Metal Boxes, Cartons, } \\
\text { Cases }\end{array}$ & unknown & 7 & 0.2 & 800 & 1.0 & 1.8 & 0.3 \\
\hline \multirow{2}{*}{$\begin{array}{l}\text { Motel Drume, Barrols, } \\
\text { Kege }\end{array}$} & 30-gol & 8 & 0.2 & 826 & 0.7 & 1.2 & 0.2 \\
\hline & 56-gal & 198 & 4.8 & 21491 & 26.7 & 41.4 & 7.6 \\
\hline Miscellaneous Scrap & unknown & 21 & 0.6 & 2760 & 3.4 & 6.2 & 1.1 \\
\hline $\begin{array}{l}\text { Self-Contained, } \\
\text { Equipment }\end{array}$ & unknown & 16 & 0.4 & 1864 & 2.3 & 4.4 & 0.8 \\
\hline $\begin{array}{l}\text { Trucke, Flatbede, } \\
\text { Compactor, Loadlugger }\end{array}$ & unknown & 1 & $<0.1$ & 1270 & 1.6 & 2.8 & 0.6 \\
\hline TOTALs & & 4080 & & 80545 & & 546.7 & \\
\hline & & \multicolumn{2}{|c|}{$107 \%$} & & & ২ & ४ \\
\hline $\begin{array}{l}\text { Fiberboard/Plastic Boxes, } \\
\text { Cartons, Cases }\end{array}$ & unknown & 4141 & 84.3 & 53050 & 60.8 & 528.1 & 87.3 \\
\hline HEPA Filtere & unknown & 10 & 0.2 & 1716 & 2.0 & 3.8 & 0.6 \\
\hline $\begin{array}{l}\text { Metal Boxes, Cartons, } \\
\text { Casese }\end{array}$ & unknown & 3 & 0.1 & 381 & 0.4 & 0.8 & 0.1 \\
\hline Metal Cylindor, Caoks & unknown & 1 & $<0.1$ & 254 & 0.3 & 0.6 & 0.1 \\
\hline \multirow{2}{*}{$\begin{array}{l}\text { Metal Drums, Barrels, } \\
\text { Kegs }\end{array}$} & 30-gal & 3 & 0.1 & 122 & 0.1 & 0.4 & 0.1 \\
\hline & 65-gal & 220 & 5.0 & 20857 & 23.7 & 46.8 & 7.7 \\
\hline Miscellaneous Scrap & unknown & 2 & 0.1 & 13 & $<0.1$ & 0.03 & $<0.1$ \\
\hline $\begin{array}{l}\text { Solf-Contained, } \\
\text { Equipment }\end{array}$ & unknown & 6 & 0.1 & 689 & 0.7 & 1.3 & 0.2 \\
\hline \multirow{2}{*}{$\begin{array}{l}\text { Trucks, Flatbeds, } \\
\text { Compactor, Loadlugger }\end{array}$} & unknown & 2 & 0.1 & 3378 & 3.9 & 7.6 & 1.2 \\
\hline & $1 \times 2 \times 3$ & 1 & $<0.1$ & 76 & 0.1 & 0.2 & $<0.1$ \\
\hline
\end{tabular}


Table 6-4. Non-TRU Waste Generated at Building 325 by PNL by Container Type and Year. (9 sheets)

\begin{tabular}{|c|c|c|c|c|c|c|c|}
\hline 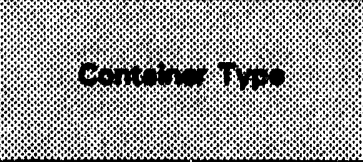 & 1.0\%1\%. & \%on & 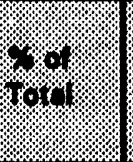 & \%or. & $40 \%$ & rom & rogno \\
\hline \multirow{3}{*}{$\begin{array}{l}\text { Wooden Boxen, Cartons, } \\
\text { Coeces }\end{array}$} & $4 \times 4 \times 4$ & 1 & $<0.1$ & 813 & 0.8 & 1.8 & 0.3 \\
\hline & $4 \times 4 \times 8$ & 2 & 0.1 & 3281 & 3.7 & 7.3 & 1.2 \\
\hline & $6 \times 6 \times 6$ & 1 & $<0.1$ & 2743 & 3.1 & 6.1 & 1.0 \\
\hline Totals & & 4303 & & 87042 & & 604.8 & \\
\hline \multicolumn{8}{|c|}{$18 \%$} \\
\hline $\begin{array}{l}\text { Burlap, Cloth, Paper or } \\
\text { Plastio Bags }\end{array}$ & unknown & 86 & 0.8 & 408 & 2.2 & 10.5 & 0.8 \\
\hline \multirow{2}{*}{$\begin{array}{l}\text { Fiberboard/Plastic Boxes, } \\
\text { Cartons, Cases }\end{array}$} & $2 \mathrm{ft} .^{3}$ & 101. & 1.1 & 702 & 0.3 & 3.1 & 0.2 \\
\hline & unknown & 8483 & 92.3 & 109287 & 64.6 & 1082.4 & 83.4 \\
\hline HEPA Filtere & unknown & 67 & 0.7 & 3834 & 1.9 & 11.2 & 0.9 \\
\hline \multirow{2}{*}{$\begin{array}{l}\text { Metal Boxes, Cartons, } \\
\text { Cases }\end{array}$} & $4 \times 4 \times 5$ & 1 & $<0.1$ & 1018 & 0.5 & 2.3 & 0.2 \\
\hline & $80.8 \mathrm{ft}^{3}$ & 13 & 0.1 & 13372 & 6.7 & 29.8 & 2.3 \\
\hline \multirow{4}{*}{$\begin{array}{l}\text { Motel Drums, Barrele, } \\
\text { Kege }\end{array}$} & 2-oal & $\mathbf{B}$ & 0.1 & 91 & 0.1 & 0.1 & $<0.1$ \\
\hline & 6.gal & 78 & 0.8 & 2416 & 1.2 & 6.6 & 0.4 \\
\hline & 30-gal & 4 & $<0.1$ & 163 & 0.1 & 0.7 & 0.1 \\
\hline & 65-gal & 227 & 2.6 & 24405 & 12.2 & 48.3 & 3.7 \\
\hline \multirow[t]{2}{*}{ Miscellaneoue Scrap } & $\cdots$ unknown & 1 & $<0.1$ & 236 & 0.1 & 0.2 & $<0.1$ \\
\hline & unknown & 44 & 0.5 & 2005 & 1.0 & 7.3 & 0.6 \\
\hline $\begin{array}{l}\text { Solf-Contained, } \\
\text { Equipment }\end{array}$ & unknown & 8 & 0.1 & 1070 & 0.6 & 2.5 & 0.2 \\
\hline Tanks, Portable & unknown & 1 & $<0.1$ & 2515 & 1.3 & 6.6 & 0.4 \\
\hline \multirow{10}{*}{$\begin{array}{l}\text { Wooden Boxes, Cartons, } \\
\text { Cases }\end{array}$} & $0.7 \times 1 \times 1.2$ & 11 & 0.1 & 200 & 0.1 & 0.2 & $<0.1$ \\
\hline & $\because 1 \times 4 \times 4$ & 1 & $<0.1$ & 363 & 0.2 & 0.5 & $<0.1$ \\
\hline & $1 \times 4 \times 4$ & 1 & $<0.1$ & 305 & 0.2 & 0.7 & 0.1 \\
\hline & $1.5 \times 1.5 \times 0.7$ & 7 & 0.1 & 13 & $<0.1$ & 0.3 & $<0.1$ \\
\hline & $2 \times 2 \times 2$ & 1 & $<0.1$ & 140 & 0.1 & 0.3 & $<0.1$ \\
\hline & $2 \times 2 \times 4$ & 2 & $<0.1$ & 408 & 0.2 & 0.9 & 0.1 \\
\hline & $3 \times 3 \times 3$ & 13 & 0.1 & 2015 & 1.0 & 7.0 & 0.6 \\
\hline & $3 \times 3 \times 4$ & 1 & $<0.1$ & 467 & 0.2 & 1.0 & 0.1 \\
\hline & $3 \times 6 \times 16$ & 1 & $<0.1$ & 3658 & 1.8 & 8.2 & 0.6 \\
\hline & $4 \times 0.4 \times 0.8$ & 2 & $<0.1$ & 762 & 0.4 & 1.7 & 0.1 \\
\hline
\end{tabular}




\section{WHC-EP-0696}

Table 6-4. Non-TRU Waste Generated at Building 325 by PNL by Container Type and Year. (9 sheets)

\begin{tabular}{|c|c|c|c|c|c|c|c|}
\hline - & \%, & $600 \%$ & $1 \%$ & $10 \%$ & $4 \% \%$ & $\% 10 \%$ & nom? \\
\hline \multirow{10}{*}{$\begin{array}{l}\text { Wooden Boxes, Certons, } \\
\text { Cases (cont) }\end{array}$} & $4 \times 1.8 \times 1.6$ & 1 & $<0.1$ & 114 & 0.1 & 0.3 & $<0.1$ \\
\hline & $\cdots 4 \times 4 \times 4$ & 1 & $<0.1$ & 1481 & 0.7 & 1.8 & 0.1 \\
\hline & $4 \times 4 \times 4$ & 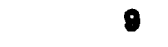 & 0.1 & 6184 & 3.1 & 18.3 & 1.3 \\
\hline & $4 \times 4 \times 6$ & 1 & $<0.1$ & 1210 & 0.8 & 2.7 & 0.2 \\
\hline & $4 \times 4 \times 7$ & 7 & 0.1 & 2642 & 1.3 & 20.6 & 1.6 \\
\hline & $4 \times 4 \times 8$ & 3 & $<0.1$ & 4876 & 2.4 & 10.8 & 0.8 \\
\hline & $4 \times 7 \times 8$ & 1 & $<0.1$ & 2848 & 1.4 & 6.3 & 0.5 \\
\hline & $4.6 \times 6.6 \times 8.7$ & 1 & $<0.1$ & 828 & 0.4 & 1.8 & 0.1 \\
\hline & $5 \times 5 \times 8$ & 1 & $<0.1$ & 8897 & 2.8 & 5.7 & 0.4 \\
\hline & unknown & 8 & 0.1 & 611 & 0.3 & 1.7 & 0.1 \\
\hline TOTALS & & 9189 & & 200681 & & 1288.4 & \\
\hline & & & 70 & & 0 & & २ \\
\hline \multirow{2}{*}{$\begin{array}{l}\text { Burlap, Cloth, Paper or } \\
\text { Plaatic Bags }\end{array}$} & $\cdots$ unknown & 1 & $<0.1$ & 18 & $<0.1$ & 0.03 & $<0.1$ \\
\hline & unknown & 84 & 1.0 & 2711 . & 1.2 & 13.3 & 1.0 \\
\hline \multirow{3}{*}{$\begin{array}{l}\text { Fiberboard/Plestic Boxes, } \\
\text { Cartons, Cases }\end{array}$} & $2 \mathrm{ft}^{3}$ & 112 & 1.1 & 1311 & 0.6 & 4.0 & 0.3 \\
\hline & $\cdots$ unknown & 88 & 0.6 & 921 & 0.4 & 7.4 & 0.6 \\
\hline & unknown & 8735 & 89.0 & 118363 & 51.2 & 1111.0 & 82.1 \\
\hline $\begin{array}{l}\text { Fiberboard/Plastic Drums, } \\
\text { Berrals, Kege }\end{array}$ & unknown & 1 & $<0.1$ & 41 & $<0.1$ & 0.2 & $<0.1$ \\
\hline Glove Boxes & unknown & 4 & $<0.1$ & 782 & 0.3 & 22.8 & 1.7 \\
\hline HEPA Filtero & unknown & 143 & 1.6 & 6126 & 2.2 & 20.0 & 1.5 \\
\hline $\begin{array}{l}\text { Metal Boxes, Cartons, } \\
\text { Cases }\end{array}$ & unknown & 1 & $<0.1$ & 810 & 0.4 & 8.2 & 0.6 \\
\hline \multirow{4}{*}{$\begin{array}{l}\text { Motal Drums, Barrols, } \\
\text { Kegs }\end{array}$} & 8-ool & 70 & 0.7 & 1208 & 0.6 & 4.5 & 0.3 \\
\hline & 30-gal & 14 & 0.1 & 840 & 0.4 & 1.7 & 0.1 \\
\hline & $\cdots$ 56-gal & 1 & $<0.1$ & 464 & 0.2 & 0.2 & $<0.1$ \\
\hline & 55-gal & 476 & 4.8 & 86813 & 37.0 & 96.4 & 7.1 \\
\hline Miscellaneoue Scrap & unknown & 67 & 0.7 & 2610 & 1.1 & 37.2 & 2.7 \\
\hline $\begin{array}{l}\text { Self-Contained, } \\
\text { Equipment }\end{array}$ & unknown & 26 & 0.3 & 1642 & 0.7 & 4.2 & 0.3 \\
\hline \multirow{2}{*}{$\begin{array}{l}\text { Wooden Boxes, Cartons, } \\
\text { Cases }\end{array}$} & $2 \times 2 \times 4$ & 1 & $<0.1$ & 608 & 0.2 & 1.1 & 0.1 \\
\hline & $2 \times 4 \times 4$ & 1 & $<0.1$ & 408 & 0.2 & 0.9 & 0.1 \\
\hline
\end{tabular}


Table 6-4. Non-TRU Waste Generated at Building 325 by PNL by Container Type and Year. (9 sheets)

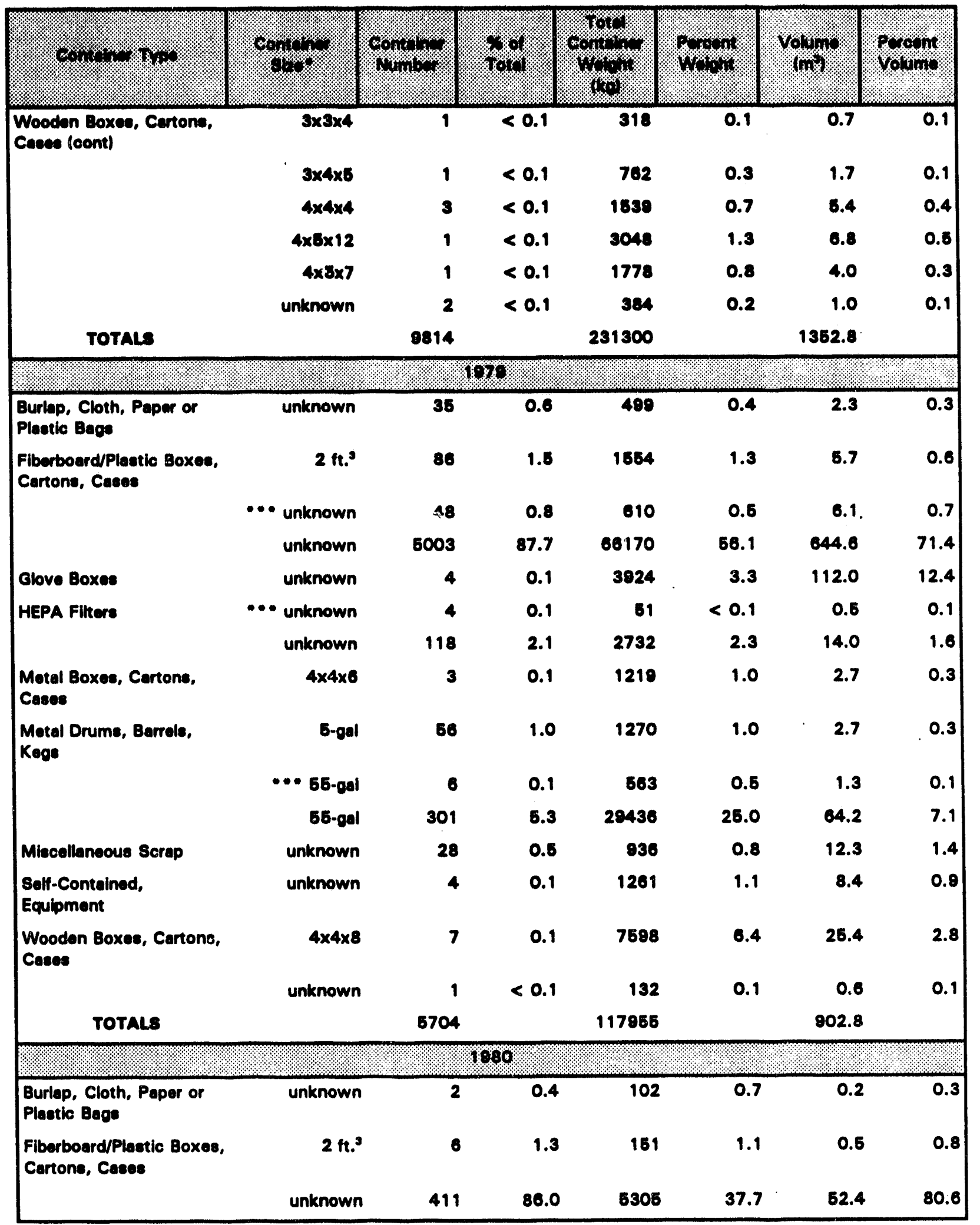




\section{WHC-EP-0696}

Table 6-4. Non-TRU Waste Generated at Bullding 325 by PNL by Container Type and Year. (9 sheots)

\begin{tabular}{|c|c|c|c|c|c|c|c|}
\hline 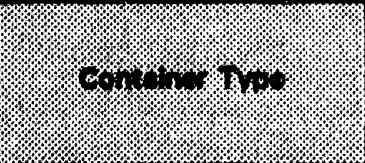 & opor. & 6.8\% & $8 \%$ & $\frac{10}{60}$ & $86 \%$ & $6010 \%$ & 1\%, \\
\hline HEPA Filtere & unknown & 8 & 1.7 & 481 & 3.4 & 1.3 & 2.0 \\
\hline $\begin{array}{l}\text { Motel Drums, Berrole, } \\
\text { Koge }\end{array}$ & B8-gal & 80 & 10.8 & 8020 & 87.0 & 10.6 & 16.3 \\
\hline Mhoollaneous sorep & unknown & 1 & 0.2 & 22 & 0.2 & 0.04 & 0.1 \\
\hline TOTALS & & 478 & & 14000 & & 68.0 & \\
\hline \multicolumn{8}{|c|}{$1 \%$} \\
\hline \multicolumn{8}{|c|}{$1 \%$} \\
\hline Wooden Boxes, Cartone, & $\because 8 \times 4 \times 4$ & 1 & 100.0 & 800 & 100.0 & 3.6 & 100.0 \\
\hline TOTALS & & 1 & & 808 & & 3.6 & \\
\hline \multicolumn{8}{|c|}{108} \\
\hline Fiberglase Roinforced & $\because 8 \times 4 \times 4$ & 1 & 100.0 & 900 & 100.0 & 3.6 & 100.0 \\
\hline TOTALS & & 1 & & 808 & & 3.6 & \\
\hline \multicolumn{8}{|c|}{$18 \%$} \\
\hline Tanks, Porteble & unknown & 10 & 100.0 & 27216 & 100.0 & 4.0 & 100.0 \\
\hline TOTALs & & 10 & & 27216 & & 4.0 & \\
\hline \multicolumn{8}{|c|}{$101 \%$} \\
\hline Glove Boxee . & unknown & 1 & 20.0 & 431 & 6.0 & 3.7 & 9.3 \\
\hline Motal Boxes, Cartons, & unknown & 4 & 80.0 & 8289 & 98.0 & 35.9 & 90.7 \\
\hline TOTALS & & $\mathbf{6}$ & & 8700 & & 39.6 & \\
\hline \multicolumn{8}{|c|}{180} \\
\hline \multicolumn{8}{|c|}{$108 \%$} \\
\hline Miacellaneoue Scrap & unknown & 46 & 92.0 & 3276 & 47.4 & 21.6 & 67.0 \\
\hline Wooden Boxes, Cartons, & $4 \times 5 \times 8$ & 4 & 8.0 & 3629 & 62.6 & 16.2 & 43.0 \\
\hline TOTALs & . & 60 & & 6805 & & 37.7 & \\
\hline \multicolumn{8}{|c|}{1088} \\
\hline $\begin{array}{l}\text { Fiberboard/Plastic Boxes, } \\
\text { Cartons, Cases }\end{array}$ & unknown & 314 & 48.7 & 5284 & 11.3 & 40.0 & 18.1 \\
\hline HEPA Filtere & unknown & 76 & 11.1 & 7278 & 16.6 & 38.1 & 18.2 \\
\hline $\begin{array}{l}\text { Metal Boxes, Cortons, } \\
\text { Cases }\end{array}$ & $2 \times 3 \times 4$ & 18 & 2.6 & 2041 & 4.4 & 10.2 & 4.9 \\
\hline
\end{tabular}


Table 6-4. Non-TRU Waste Generated at Building 328 by PNL by Container Type and Year. (9 sheots)

\begin{tabular}{|c|c|c|c|c|c|c|c|}
\hline $6 \%$ & \%. & \%+1, & $10 \%$ & א. & कम & +14, & \%का \\
\hline $\begin{array}{l}\text { Motal Boxes, Cartona, } \\
\text { Caces (oomt) }\end{array}$ & $2.8 \times 2.8 \times 4$ & 18 & 2.8 & 2041 & 4.4 & 10.2 & 4.0 \\
\hline \multirow{3}{*}{$\begin{array}{l}\text { Motal Drume, Berrela, } \\
\text { Kege }\end{array}$} & $\bullet \bullet \bullet$ 8E-gal & 3 & 0.4 & 211 & 0.8 & 0.6 & 0.3 \\
\hline & B8-all & 80 & 13.0 & 6803 & 14.0 & 18.7 & 8.8 \\
\hline & $\bullet$ B-gal & 8 & 1.2 & 90 & 0.2 & 0.2 & 0.1 \\
\hline Mbeollaneous serep & unknown & 146 & 21.3 & 9818 & 20.6 & 28.8 & 14.2 \\
\hline $\begin{array}{l}\text { Wooden Boxes, Cartons, } \\
\text { Cases }\end{array}$ & $4 \times 4 \times 8$ & 18 & 2.2 & 13008 & 28.1 & 81.8 & 28.6 \\
\hline TOTALS & & 687 & & 40746 & & 209.6 & \\
\hline \multicolumn{8}{|c|}{$1 \%$} \\
\hline $\begin{array}{l}\text { Fiberboard/Plastic Boxea, } \\
\text { Cartone, Cases }\end{array}$ & unknown & 78 & 27.8 & 1239 & 1.2 & 10.6 & 2.2 \\
\hline HEPA Filtere & unknown & 31 & 11.4 & 1280 & 1.2 & 9.8 & 2.1 \\
\hline \multirow{2}{*}{$\begin{array}{l}\text { Motal Druma, Berrole; } \\
\text { Kege }\end{array}$} & $\bullet \bullet$ BE-gal & 24 & 8.8 & 7138 & 6.8 & 6.0 & 1.1 \\
\hline & E8-gal & 38 & 12.8 & 3183 & 3.1 & 7.3 & 1.6 \\
\hline \multirow{2}{*}{$\begin{array}{l}\text { Wooden Boxes, Certons, } \\
\text { Cases }\end{array}$} & $4 \times 4 \times 8$ & 108 & 30.6 & 91036 & 87.7 & 439.6 & 93.1 \\
\hline & & 273 & & 103888 & & 472.2 & \\
\hline \multicolumn{8}{|c|}{$\%$} \\
\hline \multirow{4}{*}{$\begin{array}{l}\text { Motal Drums, Barrels, } \\
\text { Kege }\end{array}$} & $\cdots 8-g a l$ & 3 & 8.6 & 68 & 0.7 & 0.1 & 1.0 \\
\hline & $\leadsto 30-\mathrm{gal}$ & 3 & $\mathbf{5 . 8}$ & 105 & 1.1 & 0.4 & 3.8 \\
\hline & $\bullet \bullet$ 56.gal & 4 & 7.4 & 1364 & 15.4 & 0.8 & 7.6 \\
\hline & 56-gal & 44 & 81.6 & 7336 & 82.7 & 9.2 & 87.6 \\
\hline TOTALS & & 54 & & 8870 & & 10.6 & \\
\hline \multicolumn{8}{|c|}{$10 \%$} \\
\hline \multirow{4}{*}{$\begin{array}{l}\text { Motal Drums, Barrols, } \\
\text { Kegs }\end{array}$} & $\cdots$ 8-gal & 2 & 2.4 & 28 & 0.1 & 0.04 & $<0.1$ \\
\hline & $\because$ 20-gal & 3 & 3.6 & 80 & 0.3 & 0.2 & 0.2 \\
\hline & $\bullet 30-g a l$ & 1 & 1.2 & 39 & 0.1 & 0.1 & 0.1 \\
\hline & $\bullet \bullet$ 5E-cal & 2 & 2.4 & 341 & 1.0 & 0.4 & 0.3 \\
\hline $\begin{array}{l}\text { Motal Drume, Barrels, } \\
\text { Kege }\end{array}$ & 58-gal & 46 & 54.8 & 6841 & 19.4 & 9.6 & 7.2 \\
\hline
\end{tabular}


WHC-EP-0696

Table 6-4. Non-TRU Waste Generated at Building 328 by PNL by Container Type and Year. (9 sheets)

\begin{tabular}{|c|c|c|c|c|c|c|c|}
\hline 2.1. & $8 \%$ & \%ong & $10 \%$ & $\frac{1}{4}$ & \%xp & +1mp & roonor. \\
\hline $\begin{array}{l}\text { Wooden Boxes, Certons, } \\
\text { Cesese }\end{array}$ & $4 \times 4 \times 8$ & 30 & 36.7 & 27932 & 79.2 & 122.4 & 92.2 \\
\hline Totals & & 84 & & 38208 & & 132.7 & \\
\hline \multicolumn{8}{|c|}{$1 \%$} \\
\hline \multirow{2}{*}{$\begin{array}{l}\text { Motel Drume, Berrale, } \\
\text { Kegs }\end{array}$} & $\bullet \bullet-86.0 a l$ & 2 & 6.8 & 174 & 0.8 & 0.4 & 0.4 \\
\hline & 85-gal & 1 & 3.3 & 90 & 0.3 & 0.2 & 0.2 \\
\hline $\begin{array}{l}\text { Wooden Boxes, Cartons, } \\
\text { Cascis }\end{array}$ & $4 \times 4 \times 8$ & 27 & 80.0 & 37138 & 90.2 & 108.0 & 90.5 \\
\hline TOTALS & & 30 & & 37409 & & 108.6 & \\
\hline
\end{tabular}

- Unlese otherwise noted, units of dimensions are in ft.

- Solid Weate Burlal Records for PNL Bullding 325 that were found under PNL Buildinge 327 and 340 records.

- Containers hold mixed waste. 
Table 6-5. Building 325 Waste Summary Data for 55-Gallon Drums by PNL.

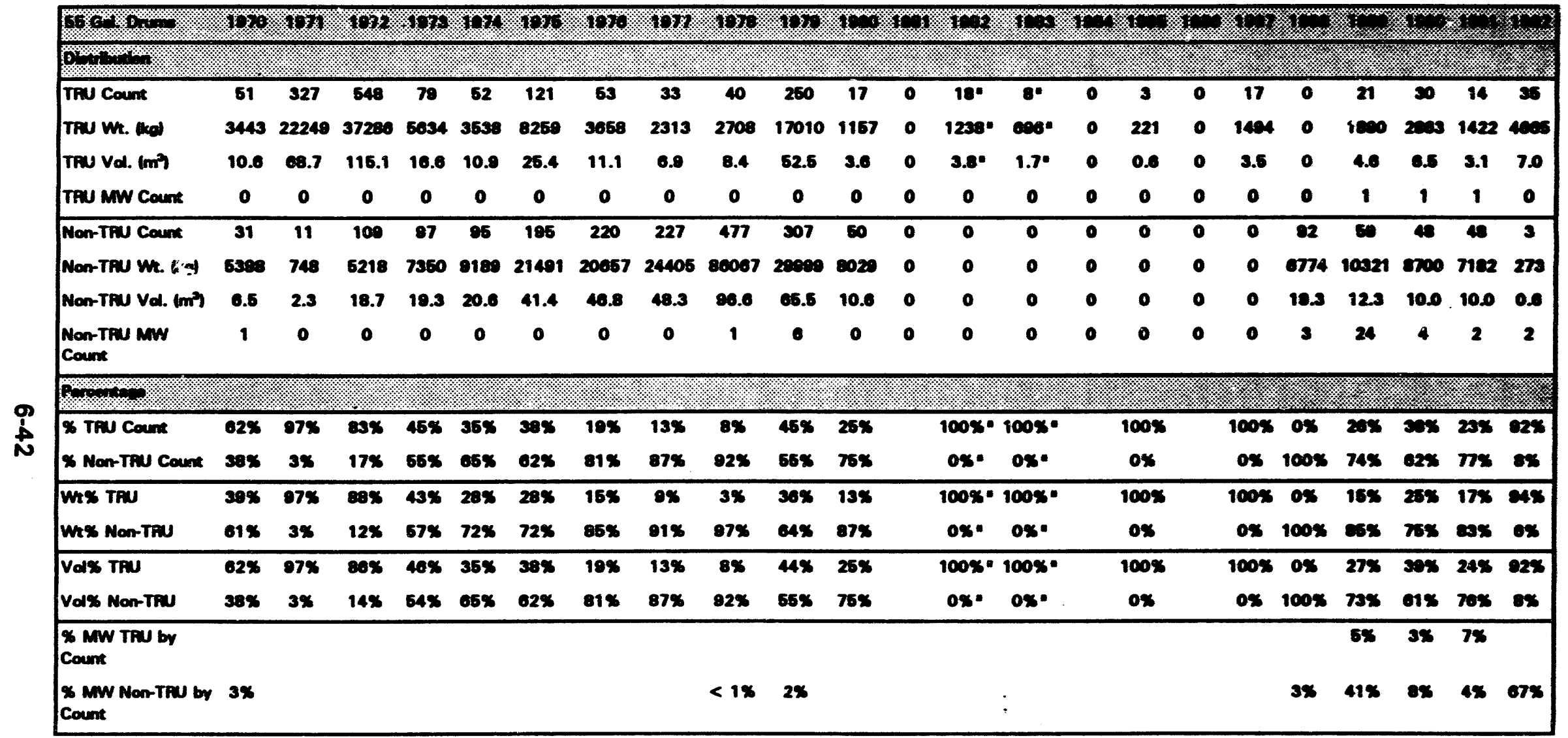

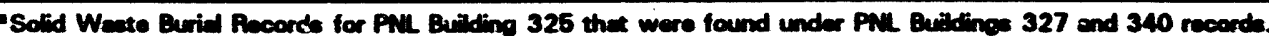


Table 6-6. Building 325 Waste Summary Data for Waste Containers Other Than 55-Gallon Druma by PNL.

\begin{tabular}{|c|c|c|c|c|c|c|c|c|c|c|c|c|c|c|c|c|c|c|c|c|c|c|c|}
\hline $6+1 \%$ & $110 \%$ & 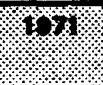 & $11^{4}$ & $101 \%$ & 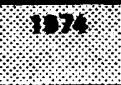 & $10 \%$ & $10 \%$ & $10 \%$ & $11 \%$ & $11 \%$ & $10 \%$ & $4 x^{3}$ & $10 \%$ & $\%$ & ${ }^{10}$ & 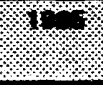 & $\sqrt{1}$ & . & . & . & I & . & 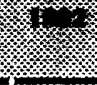 \\
\hline \multicolumn{24}{|l|}{$6=1$} \\
\hline TPu Count & 361 & 0 & 0 & 0 & 3 & 1 & 1 & 0 & 11 & 0 & 0 & 0 & $1 \bullet$ & 0 & 0 & 0 & 0 & 0 & 0 & 0 & 0 & 0 & 1 \\
\hline TRU Wt. (ka) & 8910 & 0 & 0 & 0 & 18634 & 1814 & 300 & 0 & 3072 & 0 & 0 & 0 & $5456^{\circ}$ & 0 & 0 & 0 & 0 & 0 & 0 & 0 & 0 & 0 & 9106 \\
\hline $\operatorname{Tm}_{\left(\mathrm{m}^{\prime}\right)}$ Vol. & 51.0 & 0 & 0 & 0 & 17.9 & 0.8 & 0.7 & 0 & 16.1 & 0 & 0 & 0 & $7.1^{\circ}$ & 0 & 0 & $\mathbf{0}$ & 0 & 0 & 0 & 0 & 0 & 0 & 2.8 \\
\hline $\begin{array}{l}\text { TFu mW } \\
\text { Count }\end{array}$ & 0 & 0 & 0 & 0 & $\mathbf{0}$ & 0 & 0 & 0 & 0 & 0 & $\mathbf{0}$ & 0 & 0 & 0 & 0 & 0 & 0 & 0 & $\mathbf{0}$ & 0 & 0 & 0 & 0 \\
\hline $\begin{array}{l}\text { Non-TRU } \\
\text { Count }\end{array}$ & 1130 & 1120 & 2088 & 6391 & 4753 & 3806 & 4173 & 8962 & 2337 & 5307 & 428 & 0 & $1 \bullet$ & $1 \bullet$ & 10 & 5 & 0 & 60 & $8 \% 1587$ & 214 & - & 30 & 27 \\
\hline $\begin{array}{l}\text { Non-TRU Wh } \\
\text { Nkel }\end{array}$ & 158788 & 16934 & 43540 & 82694 & 181630 & 59054 & 66386 & 178160 & 145233 & 87868 & 0001 & 0 & $\infty 00^{\circ}$ & $\cos$ & 27216 & 8700 & 0 & 6005 & (2001 & 93634 & 170 & 28000 & 37130 \\
\hline $\begin{array}{l}\text { Non-Tru } \\
\text { Vol. }\left(m^{2}\right)\end{array}$ & 460.4 & 148.0 & 357.2 & 818.5 & 771.2 & 505.3 & 658.0 & 1250.1 & 1256.2 & 037.3 & 54.4 & 0 & $3.6^{\circ}$ & 3.6 & 4.0 & 30.6 & 0 & 37.7 & $\begin{array}{l}0.2 \% \\
180.1\end{array}$ & 450.8 & 0.5 & 122.7 & 100 \\
\hline $\begin{array}{l}\text { Non-Tru } \\
\text { MN Count }\end{array}$ & 7 & $\mathbf{0}$ & 0 & 0 & 0 & 0 & 0 & 10 & 50 & 62 & - & 0 & 0 & $\mathbf{0}$ & 0 & 0 & 0 & 0 & 0 & 0 & - & - & 0 \\
\hline \multicolumn{24}{|l|}{$8+1+0$} \\
\hline XTfu Count & $24 \%$ & $0 \%$ & $0 \%$ & $0 \%$ & $<1 \%$ & $<1 \%$ & $<1 \%$ & $0 x$ & $<1 \%$ & $0 \%$ & $0 x$ & & $50 \%$ & $0 x$ & ox & $0 x$ & & $0 x$ & $0 x$ & $0 x$ & ox & $0 x$ & 48 \\
\hline $\begin{array}{l}\text { XNlon-TRU } \\
\text { Count }\end{array}$ & $70 \%$ & $100 \%$ & $100 \%$ & $100 \%$ & $100 \%$ & $100 \%$ & $100 \%$ & $100 \%$ & $100 \%$ & $100 \%$ & $100 \%$ & & $50 \%$ & $100 x$ & $100 \%$ & $100 x$ & & $100 \%$ & $\begin{array}{l}1 \times \% 1 \\
00 \%\end{array}$ & $100 x$ & $100 x$ & $100 x$ & $\operatorname{sex}$ \\
\hline Wr\% TRU & $5 \%$ & $0 \%$ & $0 x$ & $0 \%$ & $9 x$ & $3 x$ & $<1 x$ & ox & $3 x$ & $0 x$ & $0 \%$ & & $\infty x$ & $0 x$ & $0 x$ & $0 x$ & & ox & $0 x$ & $0 x$ & $o x$ & $0 x$ & $a x$ \\
\hline $\min _{\text {TRU NON- }}$ & $95 \%$ & $100 x$ & $100 \%$ & $100 \%$ & $81 \%$ & $97 \%$ & $100 \%$ & $100 \%$ & $97 \%$ & $100 \%$ & $100 \%$ & & $14 \%$ & $100 \%$ & $100 \%$ & $100 x$ & & $100 \%$ & $\begin{array}{l}0.2 \% \% \\
100 \%\end{array}$ & $100 x$ & $100 \%$ & $100 x$ & $22 \%$ \\
\hline Val\% TRU & $10 \%$ & $0 \%$ & $0 x$ & $0 \%$ & $2 \%$ & $<1 \%$ & $<1 \%$ & $0 x$ & $1 \%$ & $0 \%$ & ox & & $66 \%$ & $0 \%$ & $0 \%$ & $0 x$ & & $0 x$ & $0 x$ & $0 \%$ & ox & $0 x$ & $8 x$ \\
\hline $\begin{array}{l}\text { Vol\% Non- } \\
\text { TRU }\end{array}$ & $80 \%$ & $100 x$ & $100 \%$ & $100 \%$ & $98 \%$ & $100 \%$ & $100 \%$ & $100 \%$ & $90 \%$ & $100 \%$ & $100 \%$ & & $34 \%$ & $100 \%$ & $100 \%$ & $100 \%$ & & $100 \%$ & $\begin{array}{l}0.1 \times 11 \\
100 \%\end{array}$ & $100 \%$ & $100 \%$. & $.100 \%$ & $92 \%$ \\
\hline $\begin{array}{l}\text { XMW Non- } \\
\text { TRU by } \\
\text { Count }\end{array}$ & $1 \%$ & & & & & & & $<1 \%$ & $1 \%$ & $1 \%$ & & & & & & & & & & & $100 \%$ & $17 \%$ & \\
\hline
\end{tabular}


Table 6-7. Building 325 TRU Waste in 55-Gallon Drums by PNL: Drum Count by Storage Location.

\begin{tabular}{|c|c|c|c|c|c|c|c|c|c|c|c|c|c|c|c|c|c|c|c|c|c|c|c|}
\hline & 19 & $10 \%$ & $10 \%$ & $10 \%$ & 1101 & 1983 & $101 \%$ & 107 & 1079 & $107 \%$ & $10 \%$ & $10 \%$ & $104 \%$ & 1003 & $10 \%$ & 4013 & $101 \%$ & $101 \%$ & 14 & $1 / 4$ & 1.4 & $14 \%$ & $1 \%$ \\
\hline $04 \%=1 \%$ & & & & & & & & & & & & & & & & & & & & & & & \\
\hline $218-W-3 A$ & 35 & 22 & & 14 & & 1 & & & & & & & & & & & & & & & & & \\
\hline $218-W-4 B$ & 16 & 305 & 548 & 65 & 52 & 120 & 53 & 33 & & & & & & & & & & & & & - & & \\
\hline $218-W-4 C$ & & & & & & & & & 40 & 250 & 17 & & $18 "$ & 8* & & 3 & & 2 & & & & & \\
\hline $224 T$ & & & & & & & & & & & & & & & & & & 15 & & 22 & 31 & 13 & 32 \\
\hline 2403-WA & & & & & & & & & & & & & & & & & & & & & & 2 & 3 \\
\hline
\end{tabular}

"Solid Weate Buriel Rocorde for PNL Building 325 that wore found under PANL Buildinge 327 and 340 records.

Table 6-8. Building 325 TRU Waste in Containers Other Than 55-Gallon Drums by PNL: Container Count by Storage Location.

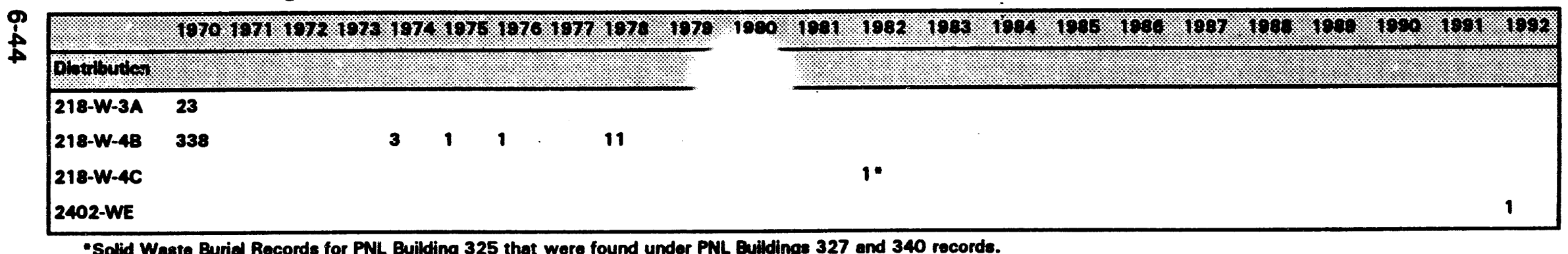

-Solid Westo Buriel Records for PNL Building 325 that were found under PNL Buildings 327 and 340 records. 
Table 6-9. Distribution and Percentage of TRU 55-Gallon Drum Physical Contents by Storage Location - 218-W-3A.

\begin{tabular}{|c|c|c|c|c|c|}
\hline & 1070 & $1971=$ & $107 \% 1078197 \% 1976$ & 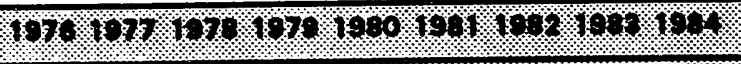 & 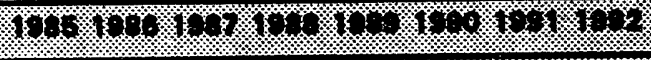 \\
\hline \multicolumn{6}{|l|}{$0 \% \%+1 \%$} \\
\hline Animal Waste & & & 7 & ; & \\
\hline Mise/Unknown/Other & 35 & 22 & 7 & & \\
\hline \multicolumn{6}{|l|}{ Promines. } \\
\hline Animel Weste & & & $50 \%$ & & \\
\hline Misc/Unknown/Other & $100 \%$ & $100 \%$ & $100 \%$ & & \\
\hline
\end{tabular}

Table 6-10. Distribution and Percentage of TRU 55-Gallon Drum Physical Contents by Storage Location - 218-W-4B.

\begin{tabular}{|c|c|c|c|c|c|c|c|c|c|}
\hline & 1020 & 197 & 1072 & 1973 & 1972 & 1976 & 1978 & 1077 & 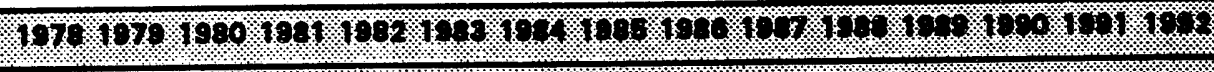 \\
\hline Dormition & & $x$ & & & a. & & & & F \\
\hline \multicolumn{10}{|c|}{$\begin{array}{l}\text { Dirtsoil/Dintomoceove } 8 \\
\text { Earth }\end{array}$} \\
\hline Misc/Unknown/Other & 8 & 305 & 548 & 65 & 52 & 120 & 53 & 33 & \\
\hline \multicolumn{10}{|c|}{ 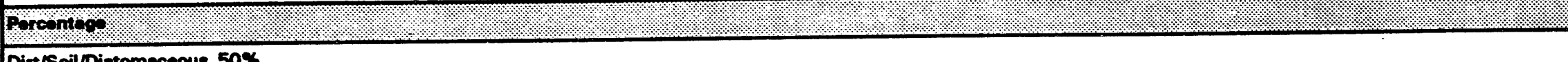 } \\
\hline $\begin{array}{l}\text { Dirt/Soil/Dintomacoous } \\
\text { Eerth }\end{array}$ & $50 \%$ & & & & & & & & \\
\hline Misc/Unknown/Other & $50 \%$ & $100 \%$ & $100 \%$ & $100 \%$ & $100 \%$ & $100 \%$ & $100 \%$ & $100 \%$ & \\
\hline
\end{tabular}


Table 6-11. Distribution and Percentage of TRU 55-Gallon Drum Physical Contents by Storage Location - 218-W-4C. (2 sheets)

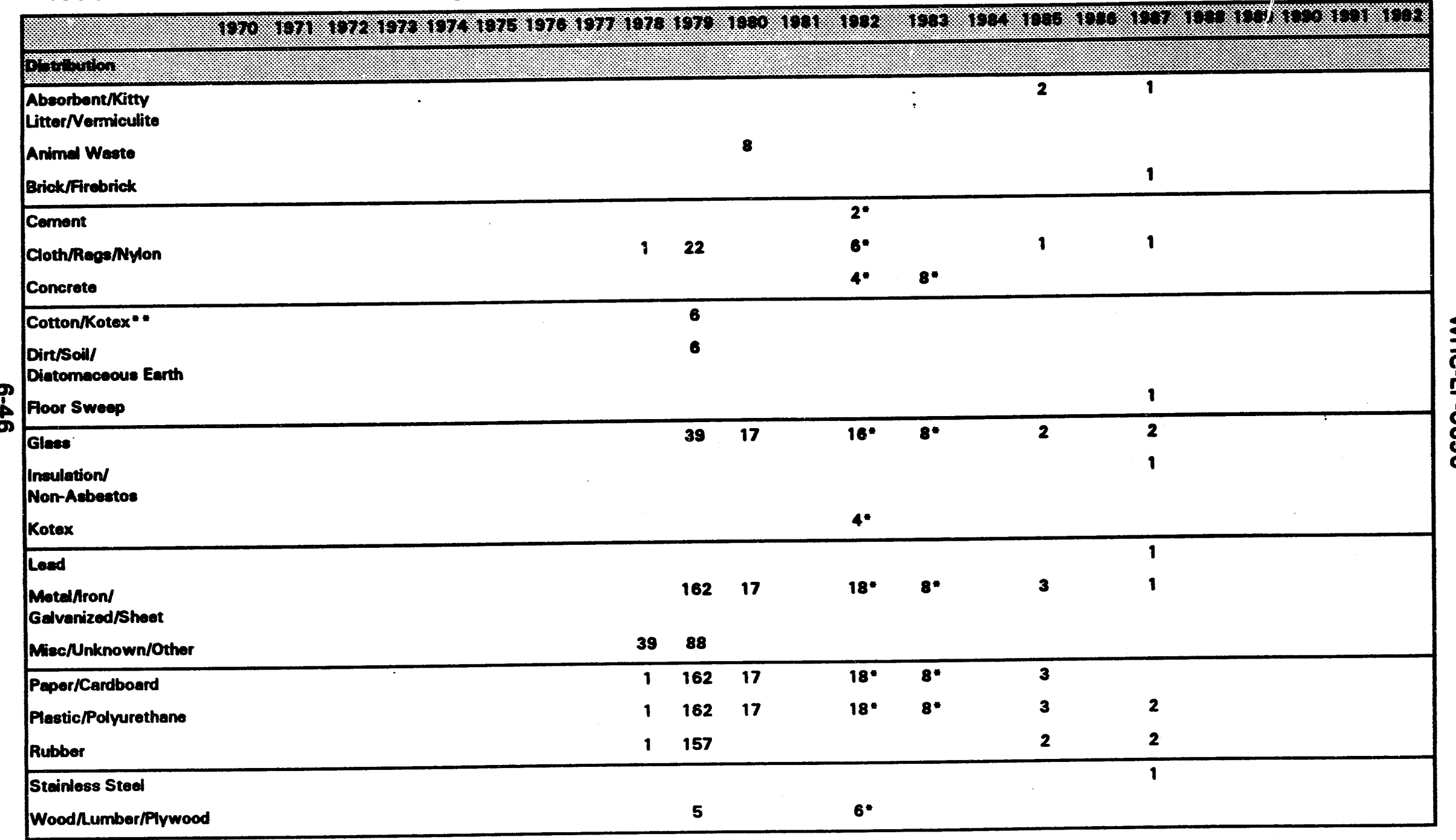


Table 6-11. Distribution and Percentage of TRU 55-Gallon Drum Physical Contents by Storage Location - 218-W-4C. (2 sheets)

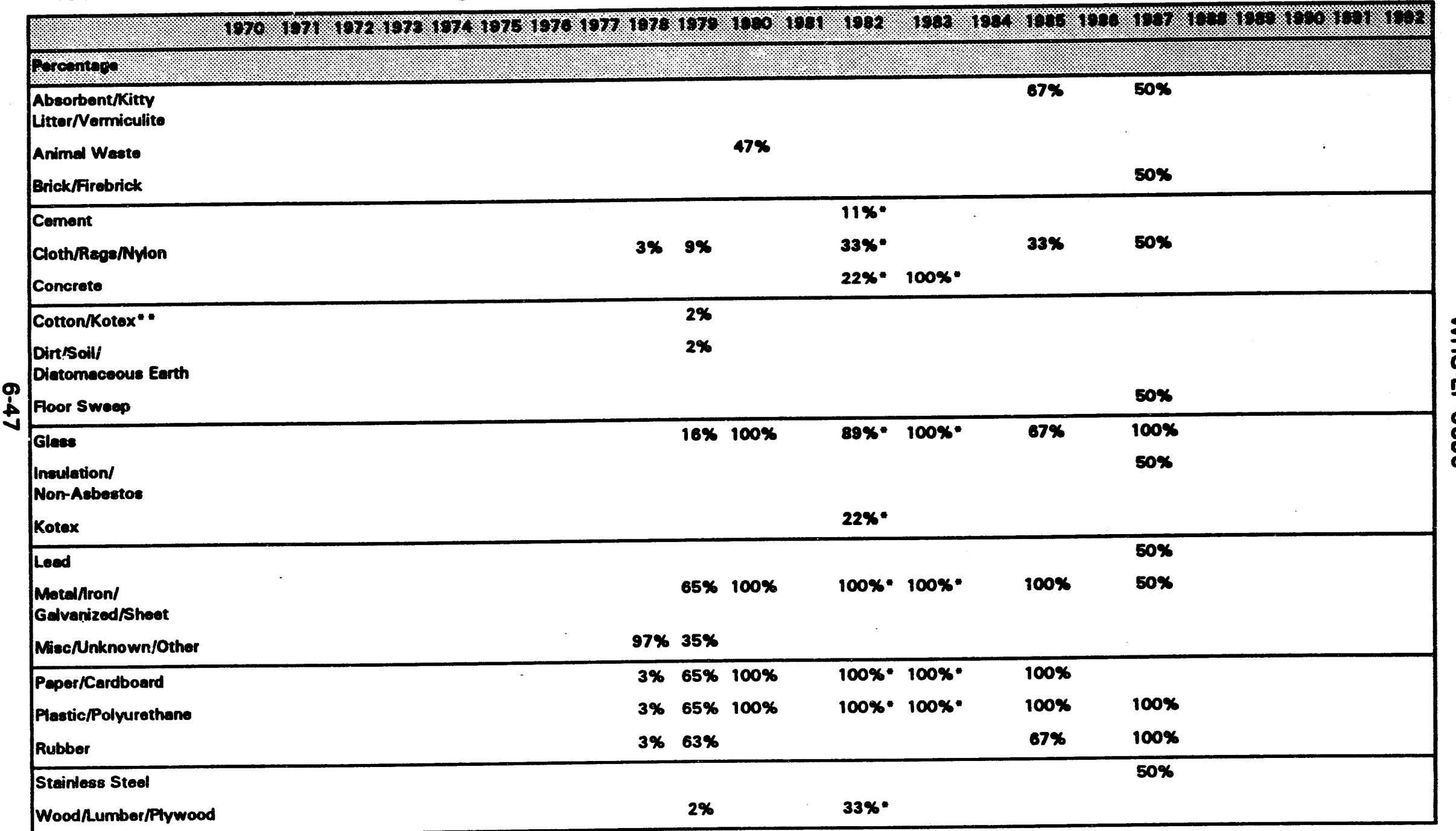

-Solid Waste Buriel Records for PNL Building 325 that were found under PNL Buildings 327 and 340 records.

- Tredemark of 
Table 6-12. Distribution and Percentage of TRU 55-Gallon Drum Physical Contents by Storage Location - 224T. (3 sheets)

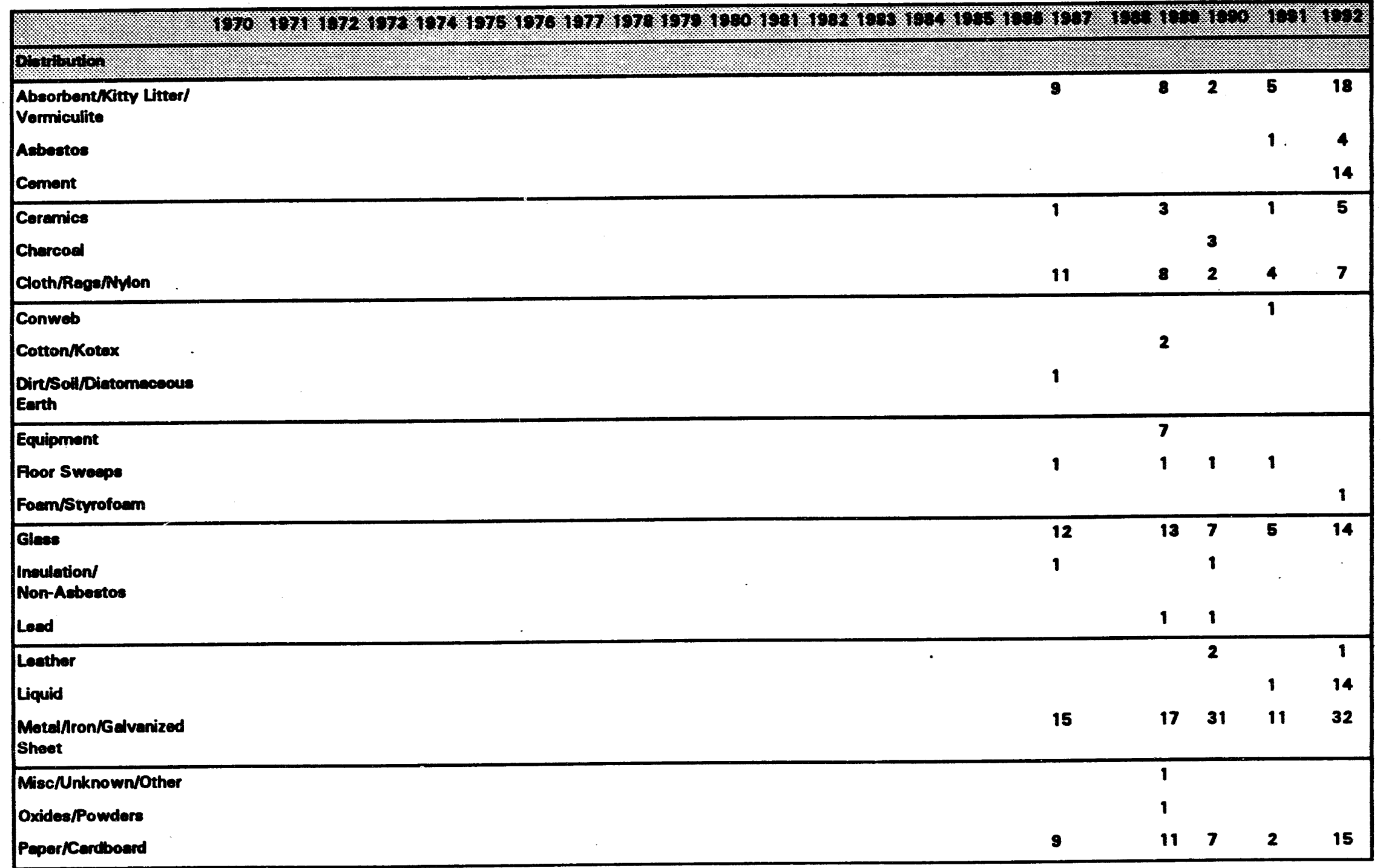


Table 6-12. Distribution and Percentage of TRU 55-Gallon Drum Physical Contents by Storage Location - 224T. (3 sheets)

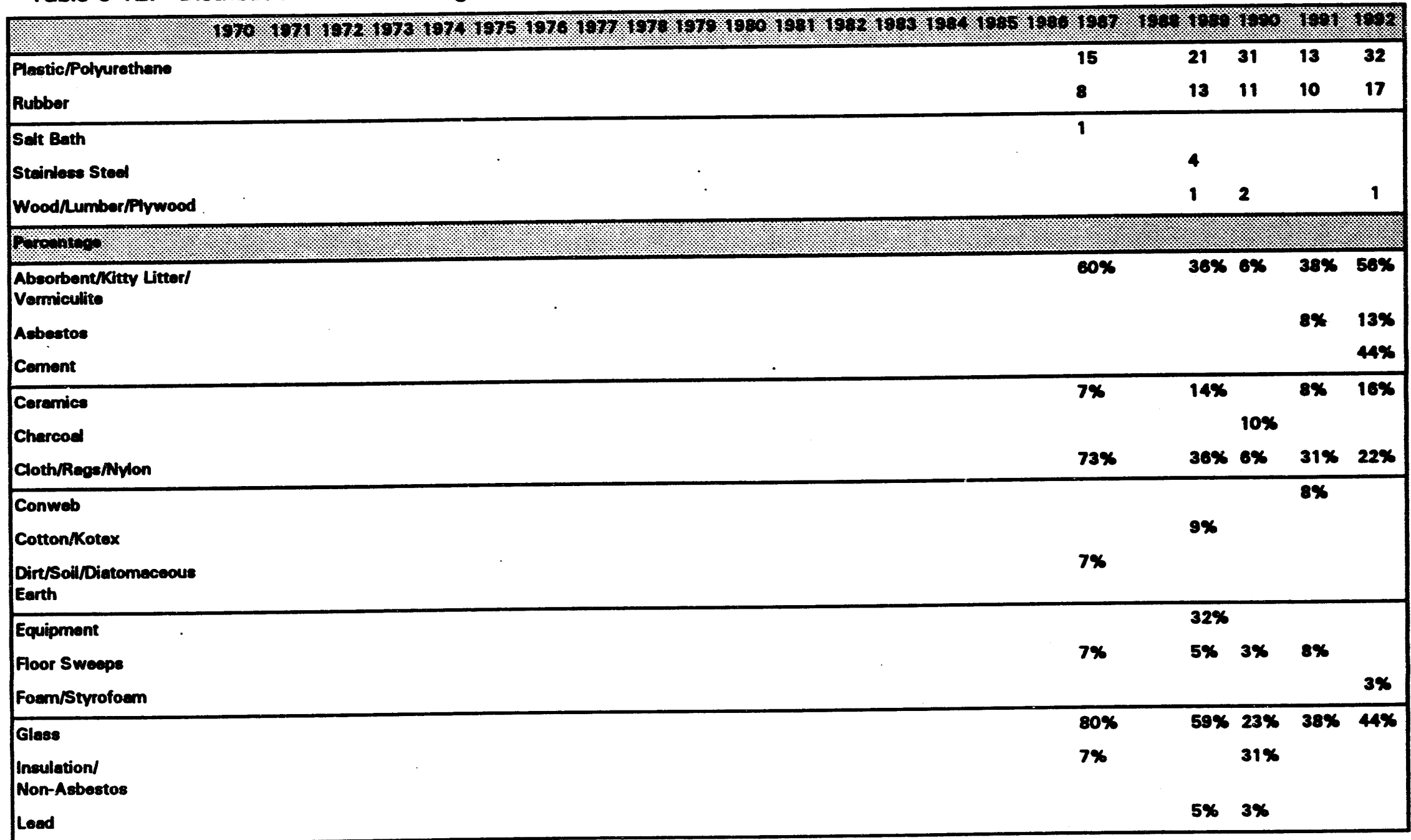


Table 6-12. Distribution and Percentage of TRU 55-Gallon Drum Physical Contents by Storage Location - 224T. (3 sheets)

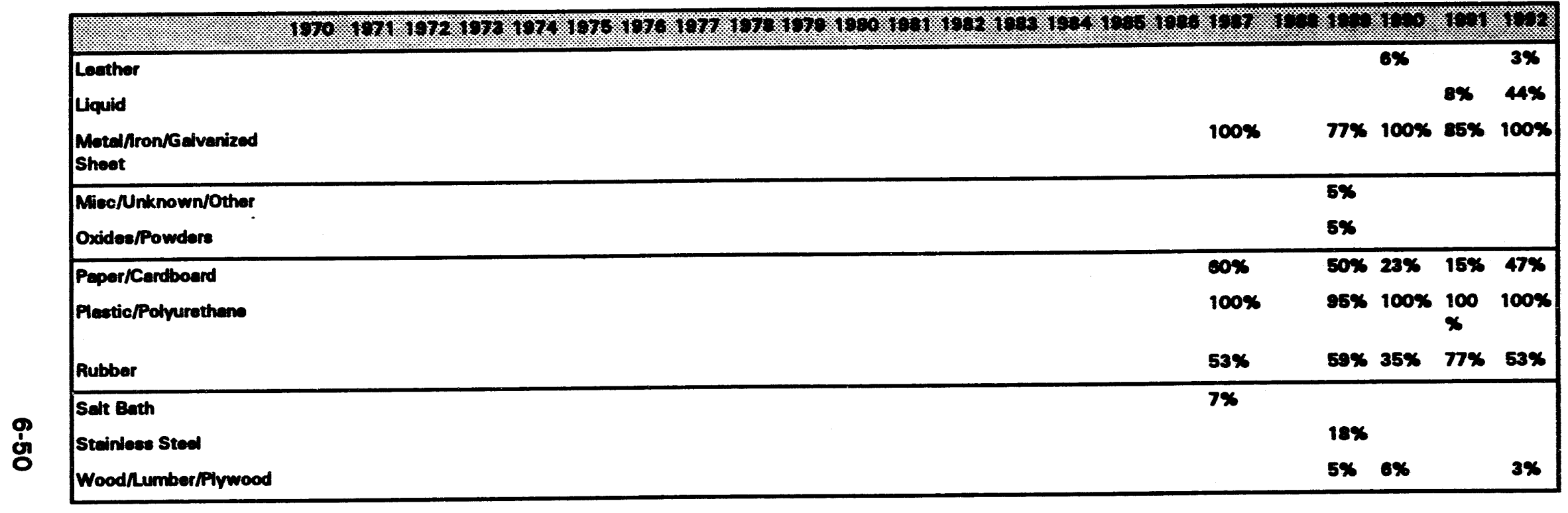


Table 6-13. Distribution and Percentage of TRU 55-Gallon Drum Physical Contents by Storage Location - 2403-WA.

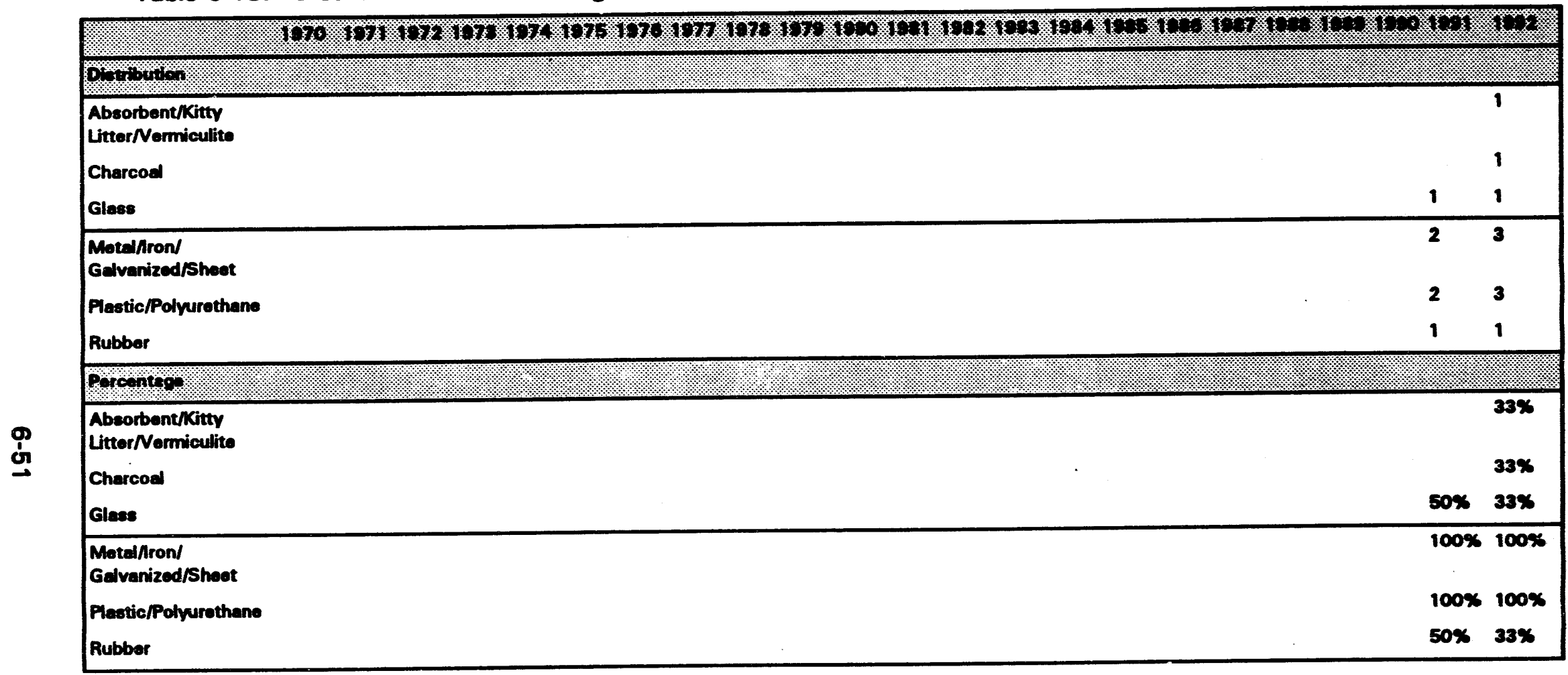


Table 6-14. Distribution and Percentage of TRU Containers Other Than 55-Gallon Drum Physical Contents by Storage Location - 218-W-3A.

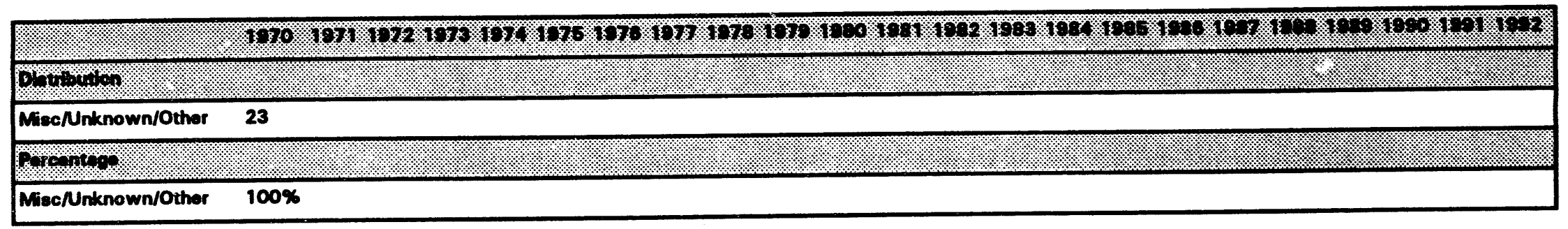

Table 6-15. Distribution and Percentage of TRU Containers Other Than 55-Gallon Drum Physical Contents by Storage Location - 218-W-4B.

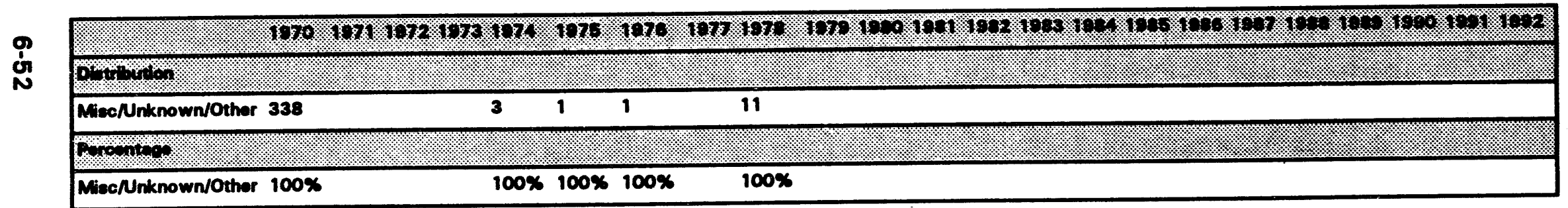


Table 6-16. Distribution and Percentage of TRU Containers Other Than 55-Gallon Drum Physical Contents by Storage Location - 218-W-4C.

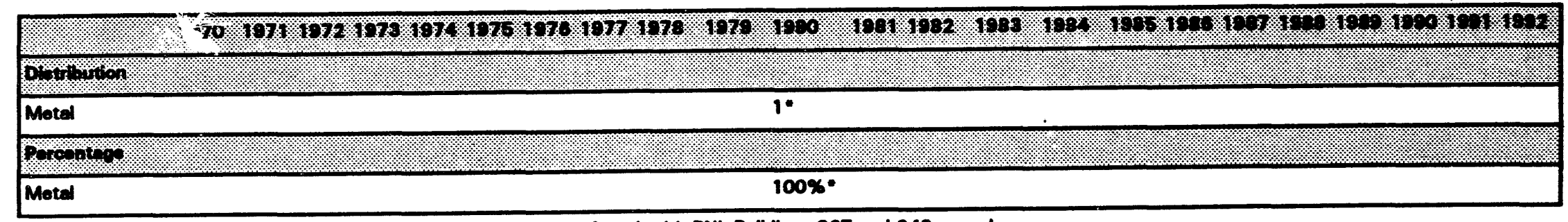

"Solid Weate Buriel Records for PNL Building 325 that were found with PNL Buildings 327 and 340 records. 
WHC-EP-0696

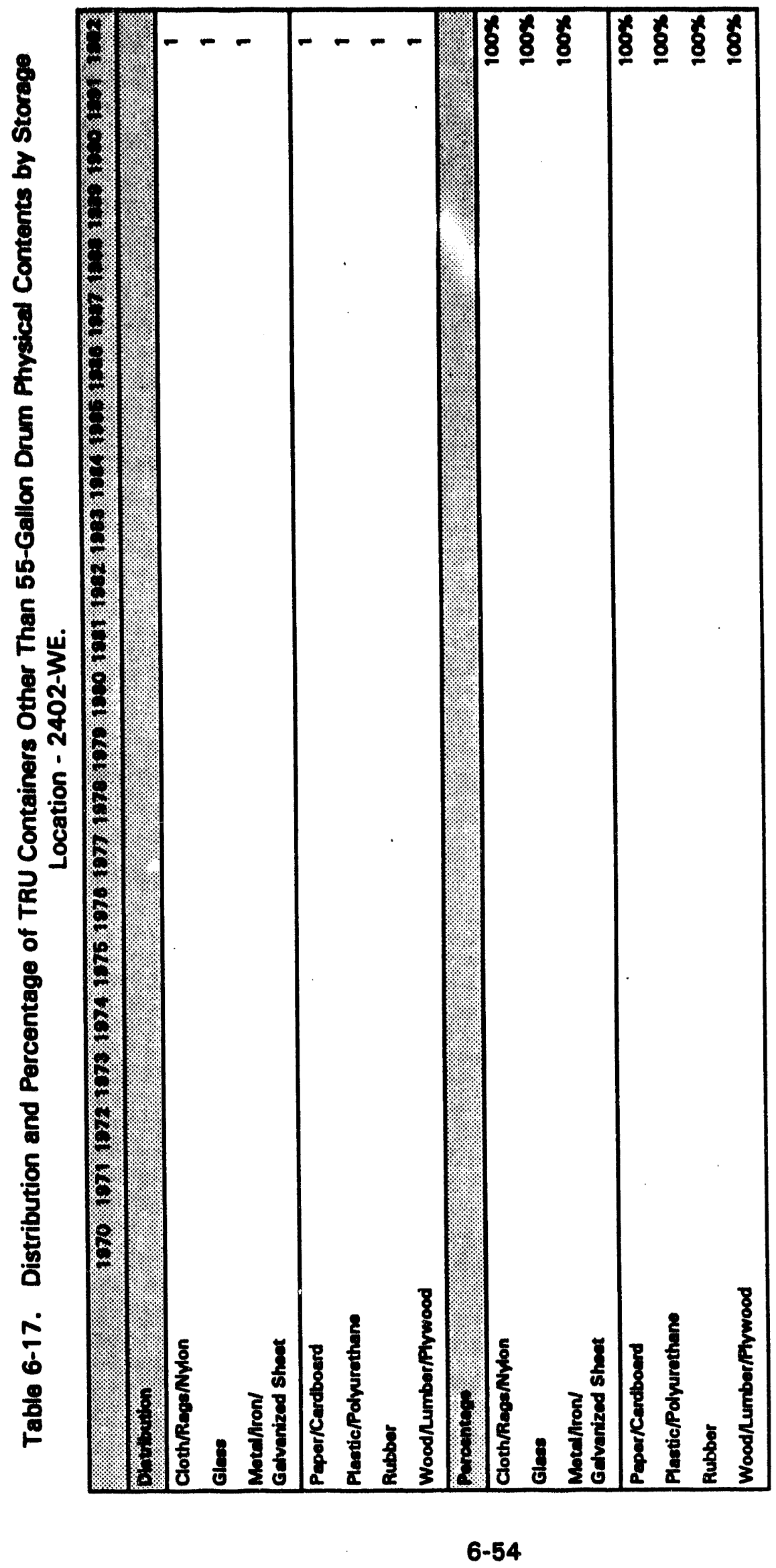


Table 6-18. Building 325 TRU Waste by PNL in 55-Gallon Drums: Total Grams TRU by Storage Location.

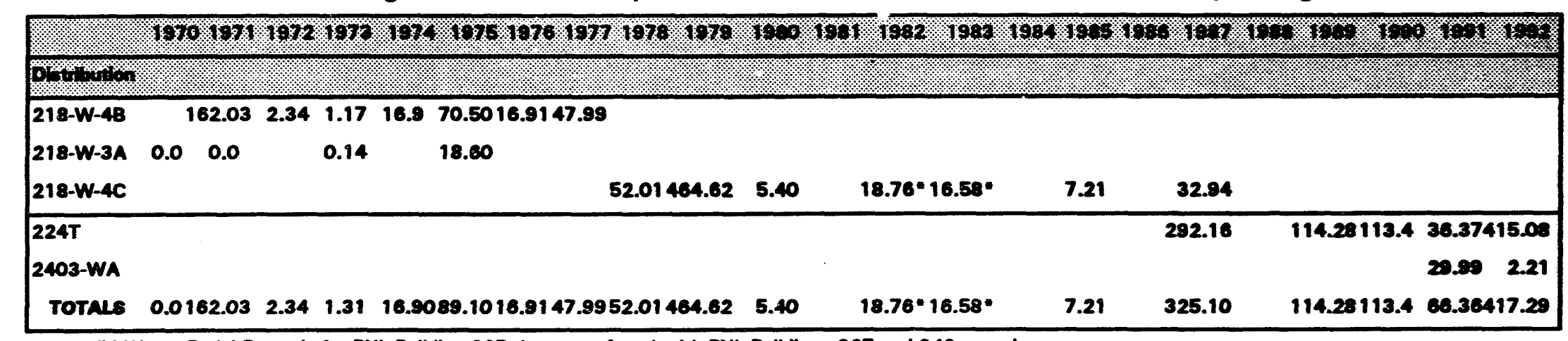

"Solid Weate Buriel Rocords for PNL Building 325 that wore found with PNL Buildinge 327 and 340 records.

Table 6-19. Building 325 TRU Waste by PNL in Containers Other Than 55-Gallon Drums: Total Grams TRU by Storage Location.

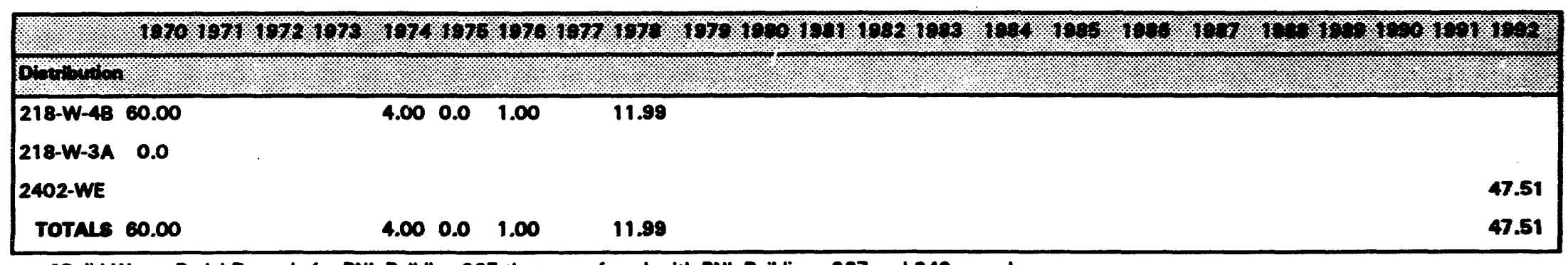

"Solid Waste Burial Records for PNL Building 325 that were found with PNL Buildings 327 and 340 records. 


\section{WHC-EP-0896}

Table 6-20. Isotopes in 55-Gallon Drums.

\begin{tabular}{|c|c|c|c|}
\hline 15 & 400 & 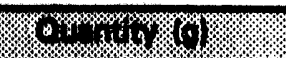 & $8 \% 000$ \\
\hline${ }^{21} \mathrm{Am}$ & $433 Y$ & 21.807523 & $a, r$ \\
\hline${ }^{m} \mathrm{Am}$ & $7.37 \times 10^{9} \mathrm{Y}$ & 0.0510001 & $\overline{a, v}$ \\
\hline 1578 & $2.851 M$ & 0.07401 & $r$ \\
\hline${ }^{140} \mathrm{C}_{0}$ & 2840 & 0.000037 & $\beta, r$ \\
\hline${ }^{2 x} \mathrm{Cm}$ & $28.8 Y$ & 0.001368 & $0, v$ \\
\hline${ }^{m} \mathrm{Cm}$ & $18.11 Y$ & 1.378693 & $0, r$ \\
\hline${ }^{m} \mathrm{Cm}$ & $8.5 \times 10^{9} y$ & 49.980000 & $a, y$ \\
\hline${ }^{\infty} \mathrm{Co}_{0}$ & $5.271 Y$ & $7.33 \times 10^{-4}$ & $\overline{B, y}$ \\
\hline${ }^{19} \mathrm{Cs}$ & $2.062 Y$ & 0.000476 & B, $r$ \\
\hline${ }^{137} \mathrm{Cs}$ & $30.17 Y$ & 0.0741 & A, $r$ \\
\hline${ }^{152} \mathrm{Eu}$ & $13 Y$ & $1.9 \times 10^{4}$ & B. $r$ \\
\hline TSEU & $8.5 Y$ & $6.47 \times 10^{-4}$ & B, $r$ \\
\hline SMn & 3120 & $=$ & $r$ \\
\hline${ }^{5} \mathrm{Nb}$ & 35.00 & $2 \times 10^{-6}$ & $\beta, \gamma$ \\
\hline $237 \mathrm{~Np}$ & $2.14 \times 10^{6} Y$ & 6.013921 & $a, r$ \\
\hline 19Ppm & $2.6234 Y$ & 427.23 & $\beta, \gamma$ \\
\hline ESPu & $87.74 Y$ & 0.480396 & $a, r$ \\
\hline rapu & $2.41 \times 10^{6} Y$ & 306.0857 & $a, v$ \\
\hline${ }^{200} \mathrm{Pu}$ & $6.57 \times 10^{3} Y$ & 36.8253 & $a, r$ \\
\hline${ }^{241} \mathrm{Pu}$ & $14.4 Y$ & 3.165925 & $\beta, \alpha, \gamma$ \\
\hline${ }^{2+2} \mathrm{Pu}$ & $3.76 \times 10^{6} \mathrm{Y}$ & 0.852415 & $a, r$ \\
\hline${ }^{100} \mathrm{Ru}$ & 3670 & $7.704 \times 10^{-3} \mathrm{~g}$ & $\beta$ \\
\hline${ }^{100} \mathrm{Ah}$ & 29.85 & $=$ & $\bar{B}, \gamma$ \\
\hline${ }^{125} \mathrm{Sb}$ & $2.7 Y$ & 0.000155 & $\beta, r$ \\
\hline 120Te & 580 & 0.000155 & $r$ \\
\hline 220Th & $1.913 \mathrm{Y}$ & $5.38 \times 10^{-4}$ & $0, y$ \\
\hline 232Th & $1.41 \times 10^{10} \mathrm{\gamma}$ & 24444 & $0, r$ \\
\hline${ }^{50} \mathrm{Zr}$ & 64.00 & $2 \times 10^{-6}$ & $\overline{\beta, a}$ \\
\hline Pu total & $\cdot$ & 1477.77 & " \\
\hline U depleted & - & 334653.81 & - \\
\hline$U$ enriched & $\div$ & 10027.49 &. \\
\hline U natural & - & 409169.27 & . \\
\hline
\end{tabular}

$Y=$ Years

D = Days

$S=$ Seconds

$H=$ Hours

M = Minutes. 
WHC-EP-0696

Table 6-21. Isotopes in Other Containers than 55-Gallon Drums for 1970 - 1992.

\begin{tabular}{|c|c|c|c|}
\hline 10000 & भII & gremento & Frodiation \\
\hline${ }^{241} \mathrm{Am}$ & $433 Y$ & 2.2 & $a, r$ \\
\hline${ }^{147} \mathrm{Pm}$ & $2.6234 Y$ & 8572.5 & $\boldsymbol{\beta}$ \\
\hline${ }^{250} \mathrm{Pu}$ & $87.74 Y$ & 0.064 & $a, r$ \\
\hline${ }^{230} \mathrm{Pu}$ & $2.41 \times 10^{4} Y$ & 36.8 & $a, v$ \\
\hline${ }^{240} \mathrm{Pu}$ & $6.57 \times 10^{3} Y$ & 7.31 & $a, r$ \\
\hline${ }^{241} \mathrm{Pu}$ & $14.4 Y$ & 1.14 & $\bar{\beta}$ \\
\hline${ }^{242} \mathrm{Pu}$ & $3.76 \times 10^{6} Y$ & 0.338 & $a, r$ \\
\hline $233 \mathrm{U}$ & $1.592 \times 10^{6} \mathrm{Y}$ & 55.0 & $a, r$ \\
\hline Pu total & 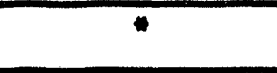 & 41.14 & 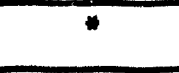 \\
\hline U depleted & 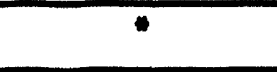 & 11432.98 & 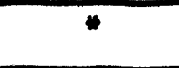 \\
\hline$U$ enriched & . & 175.00 & 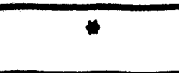 \\
\hline U natural & " & 1.00 & " \\
\hline
\end{tabular}

$Y=$ Years. 


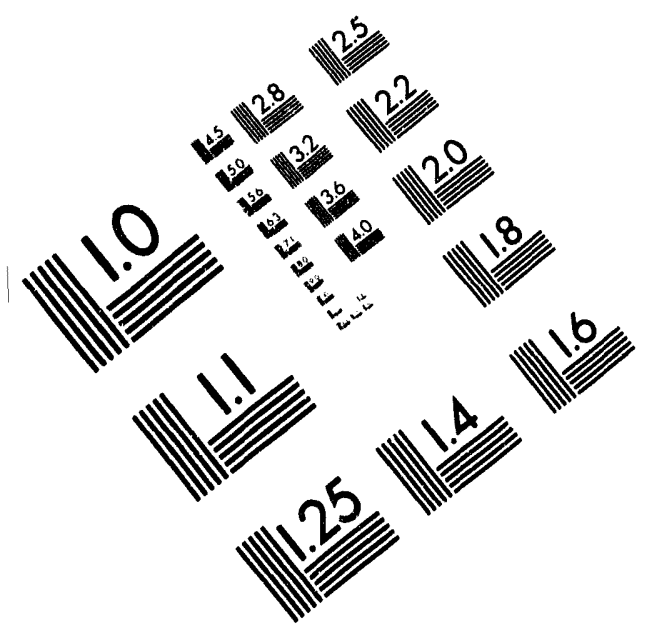

•JNI • $\exists$ כ

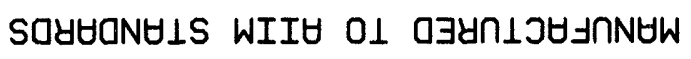
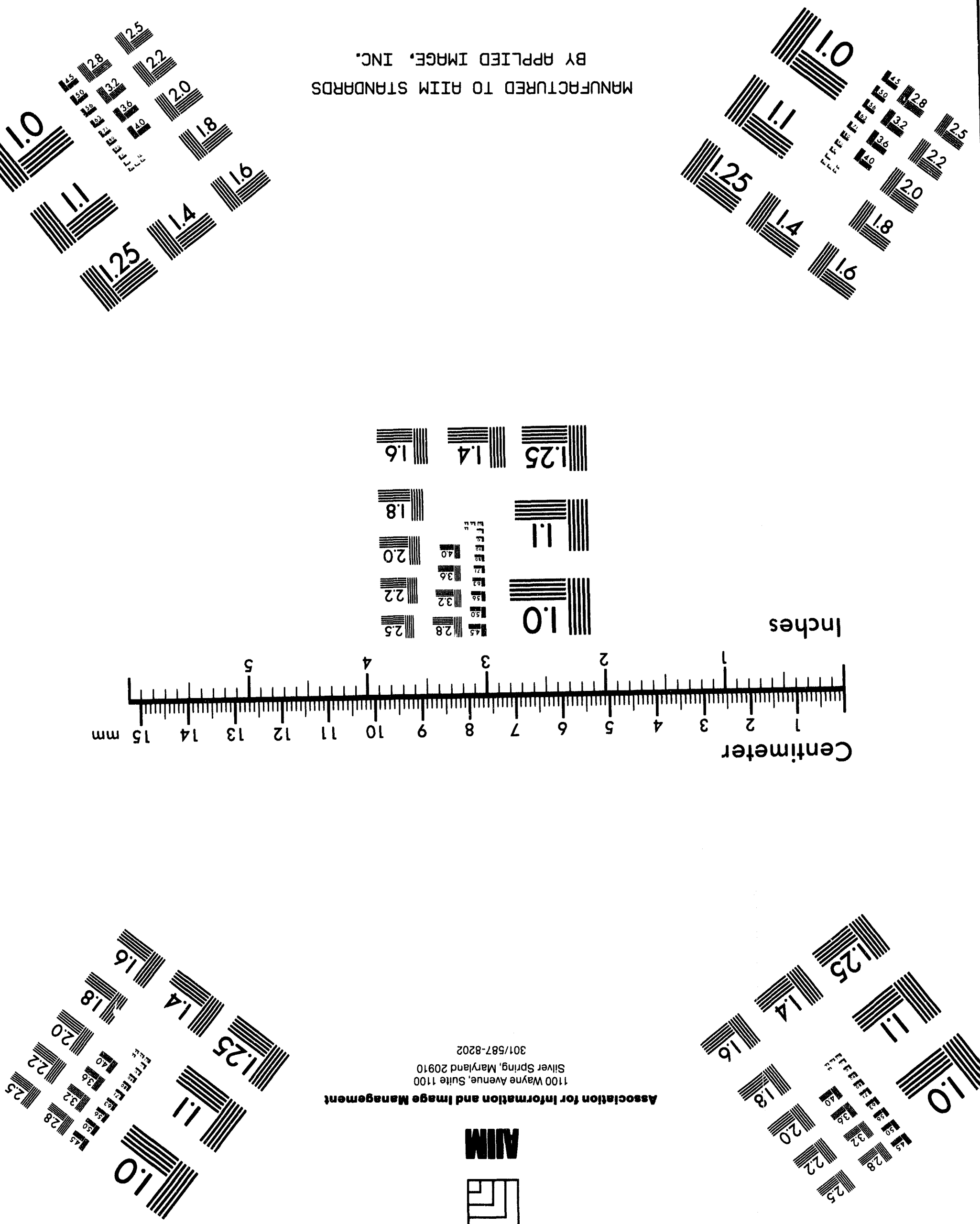

20Z8- $\angle 89 / 10 \varepsilon$

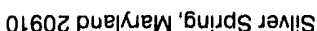

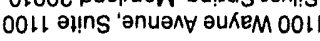

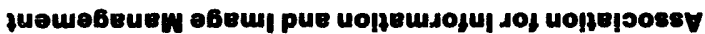
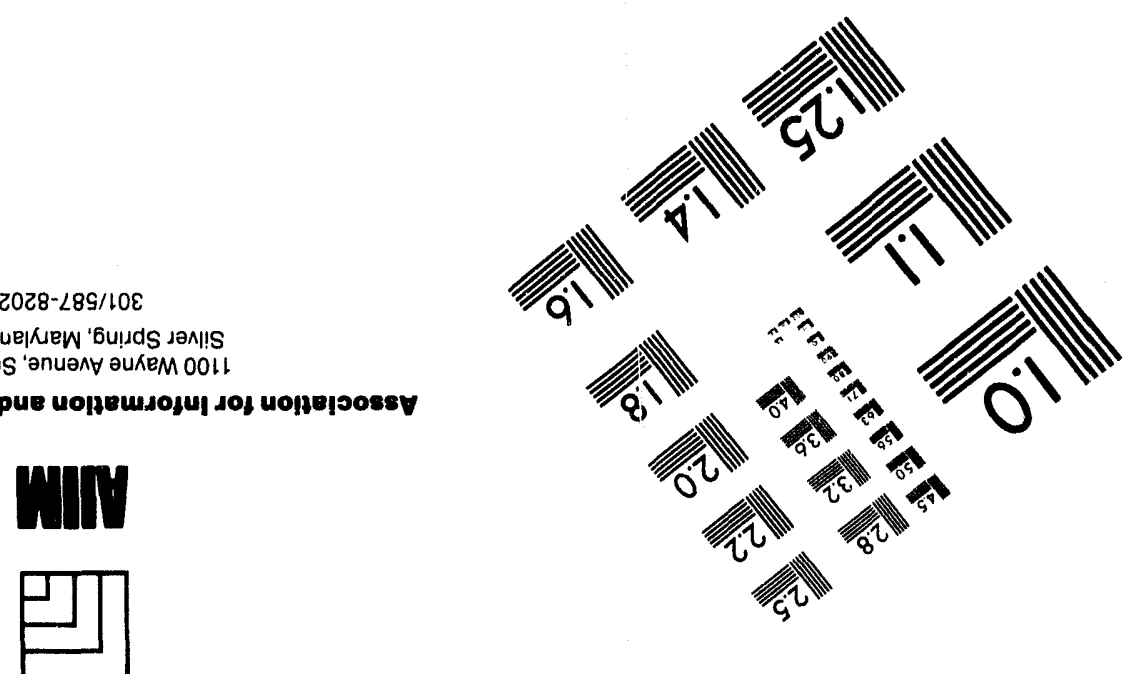

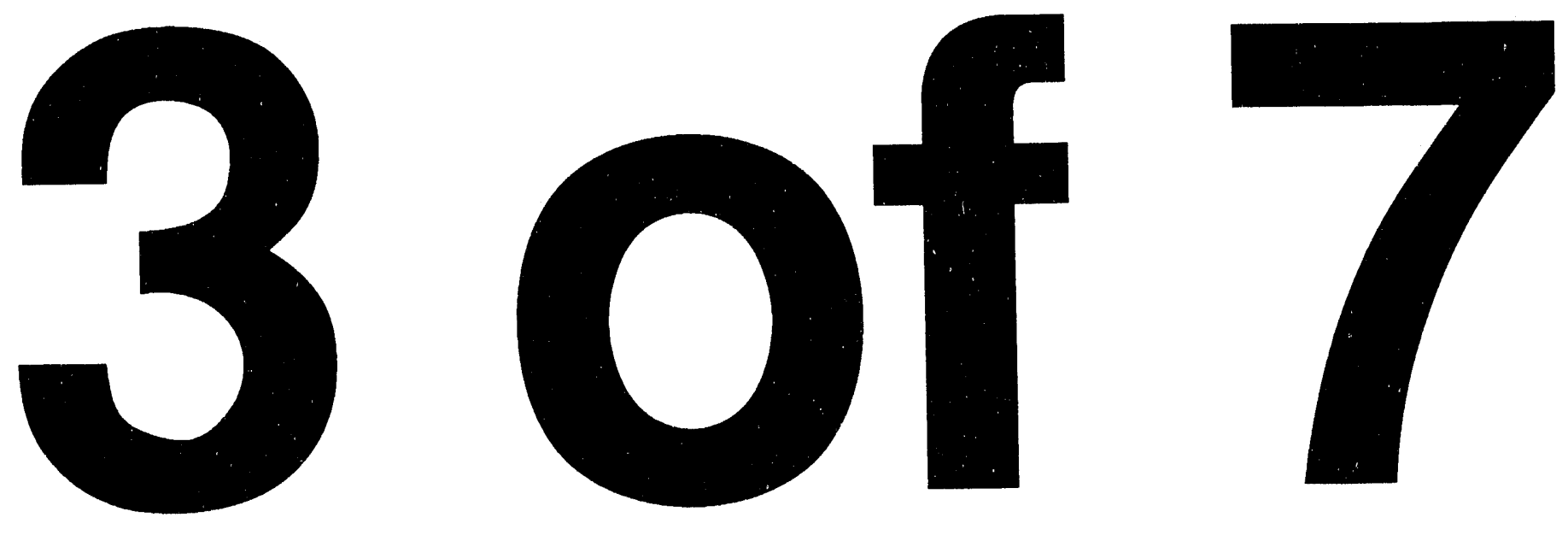
Table 6-22. Chemicals Used at Building 325: Pacific Northwest Laboratory Development and Production Laboratory (1955-1976). (2 sheets)

\begin{tabular}{|c|c|c|c|}
\hline 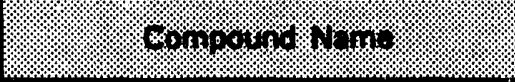 & $40+4 \%$ & 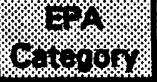 & 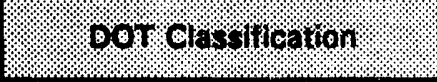 \\
\hline Aluminum Nitrate Nonahydrate & $\mathrm{Al}\left(\mathrm{NO}_{3}\right)_{3} \cdot 9 \mathrm{H}_{2} \mathrm{O}$ & D & oxidizer \\
\hline Acetone & $\mathrm{CH}_{3} \mathrm{C}_{2} \mathrm{OH}_{3}$ & D & flammable liquid \\
\hline Ammonium Hydroxide & $\mathrm{NH}_{4} \mathrm{OH}$ & C & corrosive, OAM-A \\
\hline Carbon Tetrachloride & $\mathrm{CCl}_{4}$ & D & ORM-A' \\
\hline Cesium Nitrate & $\mathrm{CsNO}_{3}$ & D & \\
\hline Copper Nitrate & $\mathrm{Cu}\left(\mathrm{NO}_{3}\right)_{2}$ & D & oxidizer \\
\hline Dowex $50^{3}$ (1X Resin) & $\begin{array}{l}\text { Sulfonate Polystyrene Divinyl } \\
\text { Benzene }\end{array}$ & & \\
\hline Ethanol & $\mathrm{C}_{2} \mathrm{H}_{6} \mathrm{OH}$ & & combustible flammable liquid \\
\hline Ferrous Sulfate & $\mathrm{Fe}\left(\mathrm{SO}_{3} \mathrm{NH}_{2}\right)_{2}$ & & \\
\hline Hydrazine & $\mathrm{H}_{2} \mathrm{HHN}_{2} \cdot \mathrm{H}_{2} \mathrm{O}$ & B & $\begin{array}{l}\text { flammable liquid, } \\
\text { Poison/Corrosive }\end{array}$ \\
\hline Hydrochloric Acid & $\mathrm{HCl}$ & $\mathbf{D}$ & $\begin{array}{l}\text { Non-Flammable gas, } \\
\text { Corrosive }\end{array}$ \\
\hline Hydrofluoric Acid & HF & B & corrosive \\
\hline Hydroxylamine Nitrate & $\mathrm{NH}_{2} \mathrm{OHHNO}_{3}$ & & \\
\hline Lanthanum Nitrate & $\mathrm{La}\left(\mathrm{NO}_{3}\right)_{3}$ & $\mathbf{D}$ & \\
\hline Methanol & $\mathrm{CH}_{3} \mathrm{OH}$ & $\mathbf{D}$ & flammable liquid \\
\hline Mercury & Ho & $x$ & ORM-B2 \\
\hline Nitric Acid & $\mathrm{HNO}_{3}$ & C & oxidizer, corrosive \\
\hline Nitrilotriacetic Acid (NTA) & $\mathrm{N}\left(\mathrm{CH}_{2} \mathrm{COOH}_{3}\right.$ & D & \\
\hline Normal Paraffin Hydrocarbon & $\mathrm{C}_{10} \mathrm{H}_{22}$ to $\mathrm{C}_{14} \mathrm{H}_{30}$ & & \\
\hline Oxalic Acid & $\mathrm{HO}_{2} \mathrm{CCO}_{2} \mathrm{H}_{2} \mathrm{H}_{2} \mathrm{O}$ & & \\
\hline $\begin{array}{l}\text { Pentasodium Diethylene Triamine } \\
\text { Pentaacetate (DTPA) }\end{array}$ & $\mathrm{N}_{3}\left(\mathrm{CH}_{2}\right)_{4}\left(\mathrm{COONa}_{5}\right.$ & & \\
\hline Potassium Permanganate & $\mathrm{KMnO}_{4}$ & D & oxidizer \\
\hline Platinum & $\mathbf{P t}$ & & \\
\hline Sodium Dichromate & $\mathrm{Na}_{2} \mathrm{Cr}_{2} \mathrm{O}_{7} \cdot 2 \mathrm{H}_{2} \mathrm{O}$ & B & oxidizer \\
\hline Sodium Fluoride & NaF. & C & ORM-B2, corrosive \\
\hline Sodium Hydroxide & $\mathrm{NaOH}$ & C & corrosive \\
\hline Sodium Nitrate & $\mathrm{NaNO}_{3}$ & D & oxidizer \\
\hline Sodium Phosphate & $\mathrm{Na}_{3} \mathrm{PO}_{4}$ & & \\
\hline Sodium Sulfate & $\mathrm{Na}_{2} \mathrm{SO}_{4}$ & & \\
\hline Strontium Nitrate & $\mathrm{Sr}\left(\mathrm{NO}_{3}\right)_{2}$ & & \\
\hline
\end{tabular}


WHC-EP-0696

Table 6-22. Chemicals Used at Building 325: Pacific Northwest Laboratory Development and Production Laboratory (1955-1976). (2 sheets)

\begin{tabular}{|c|c|c|c|}
\hline $60 n 10041 \% 1410$ & 80.101018 & $8010 \%$ & 00196125itication \\
\hline Sulfuric Acid & $\mathrm{H}_{2} \mathrm{SO}_{4}$ & C & corrosive \\
\hline $\begin{array}{l}\text { Tetrasodium Ethylene Diamine } \\
\text { Tetraacetate (EDTA) }\end{array}$ & $\mathrm{N}_{2} \mathrm{C}_{3} \mathrm{H}_{4}\left(\mathrm{C}_{2} \mathrm{H}_{2} \mathrm{O}_{2} \mathrm{Na}_{4}\right.$ & & \\
\hline Tributyl Phosphate & $\left(\mathrm{C}_{4} \mathrm{H}_{6}\right)_{3} \mathrm{PO}_{4}$ & c) & \\
\hline $\begin{array}{l}\text { Trisodium Hydroxyethyl Ethylene- } \\
\text { Diamine Triacetate (HEDTA) }\end{array}$ & $\mathrm{N}_{2} \mathrm{C}_{2} \mathrm{H}_{4}\left(\mathrm{C}_{2} \mathrm{H}_{2} \mathrm{O}_{2} \mathrm{Na}_{3}\left(\mathrm{C}_{2} \mathrm{H}_{4} \mathrm{OH}\right)\right.$ & & \\
\hline Turco (Fabrifilm) ${ }^{4}$ & $\begin{array}{l}\text { Toluene, Butanol, } \\
\text { Isopropanol, Acetone }\end{array}$ & & \\
\hline Turco $4502 \mathrm{D}^{4}$ & $\mathrm{KOH}_{1} \mathrm{~K}_{2} \mathrm{CrO}_{4}, \mathrm{KMnO}_{4}$ & & \\
\hline Turco $4518^{4}$ & $\begin{array}{l}\mathrm{HO}_{2} \mathrm{CCO}_{2} \mathrm{H}_{2} \mathrm{H}_{2} \mathrm{O}, \text { Sodium } \\
\text { Dodecyl Benzene Sulfonate }\end{array}$ & & \\
\hline Yttrium Nitrate & $\mathrm{Y}\left(\mathrm{NO}_{3}\right)_{3}$ & & \\
\hline Zinc Nitrate & $\mathrm{Zn}\left(\mathrm{NO}_{3}\right)_{2}$ & & \\
\hline
\end{tabular}

'Other regulated material with an anesthetic, irritating, noxious, toxic or other properties that can cause discomfort to persons in the event of leakage.

${ }^{2}$ Other regulated material capable of causing significant damage from leakage.

${ }^{3}$ Tradename - Dow Chemical.

¿Tradename - Turco Products, Inc. 
Table 6-23. Building 325 TRU 55-Gallon Drums Containing Hazardous Constituents by PNL: Drum Count by Storage Location.

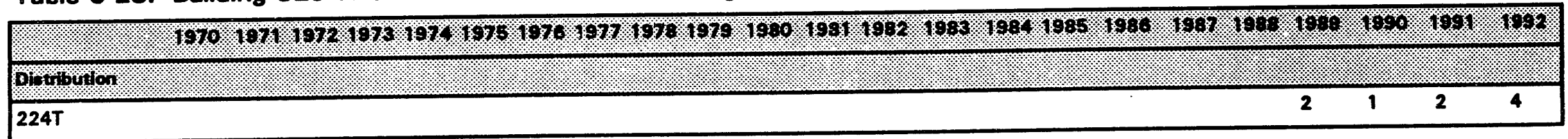

Table 6-24. Number and Percentage of Building 325 TRU 55-Gallon Drums Containing Hazardous Constituents by Storage Location - 224T.

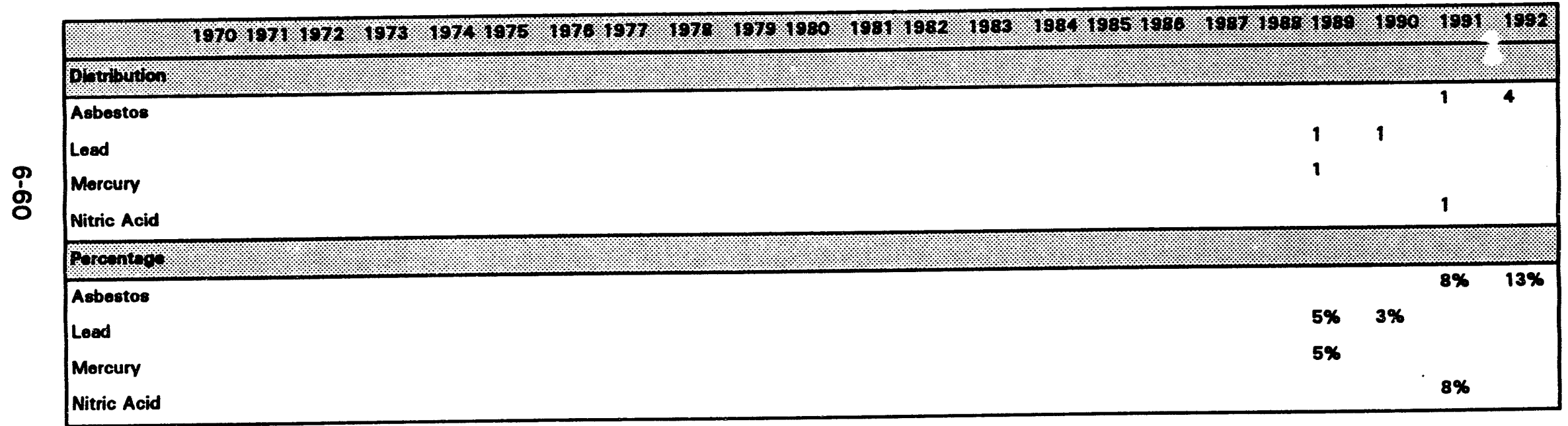

Table 6-25. Summary of Information from Solid Waste Storage Disposal Records (SWSDR) for TRU Wastes with Hazardous Contents from PNL at Building 325.

\begin{tabular}{|c|c|c|c|c|c|c|}
\hline Accepted & Nomber & Burial Recoro & oonstituents & $\begin{array}{l}\text { waste } \\
\text { oodes }\end{array}$ & Weght & Wolono \\
\hline $02 / 13 / 87$ & PNL-186004 & 325-PNL-87-2 & Lead (bricks) & D008 & 34.1 & 3 \\
\hline
\end{tabular}


Table 6-26. Summary of Information from Solid Waste Storage Disposal Records (SWSDR) for LLW Wastes with Hazardous Contents from PNL at Building 325. (2 sheets)

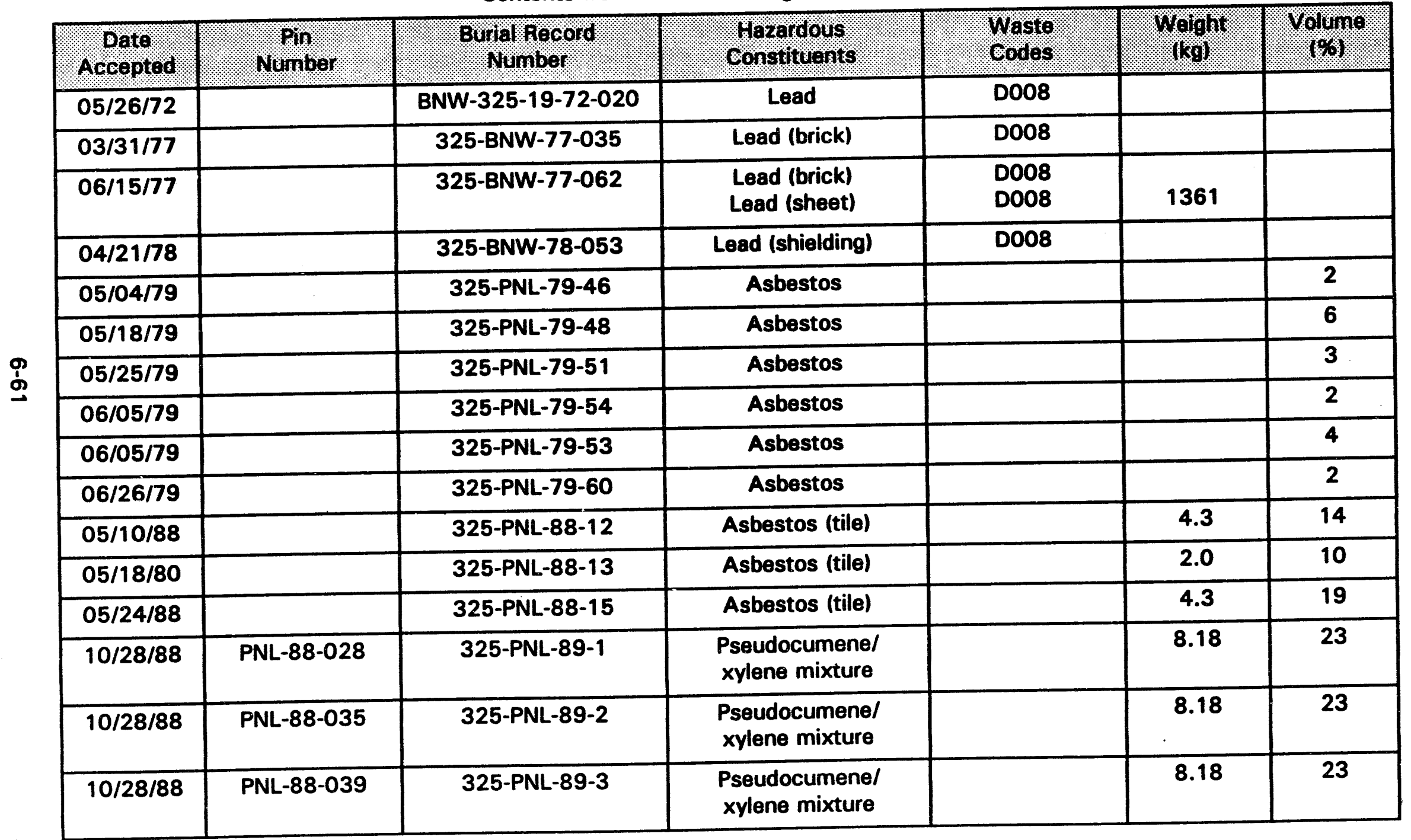


Table 6-26. Summary of Information from Solid Waste Storage Disposal Records (SWSDR) for LLW Wastes with Hazardous Contents from PNL at Building 325. (2 sheets)

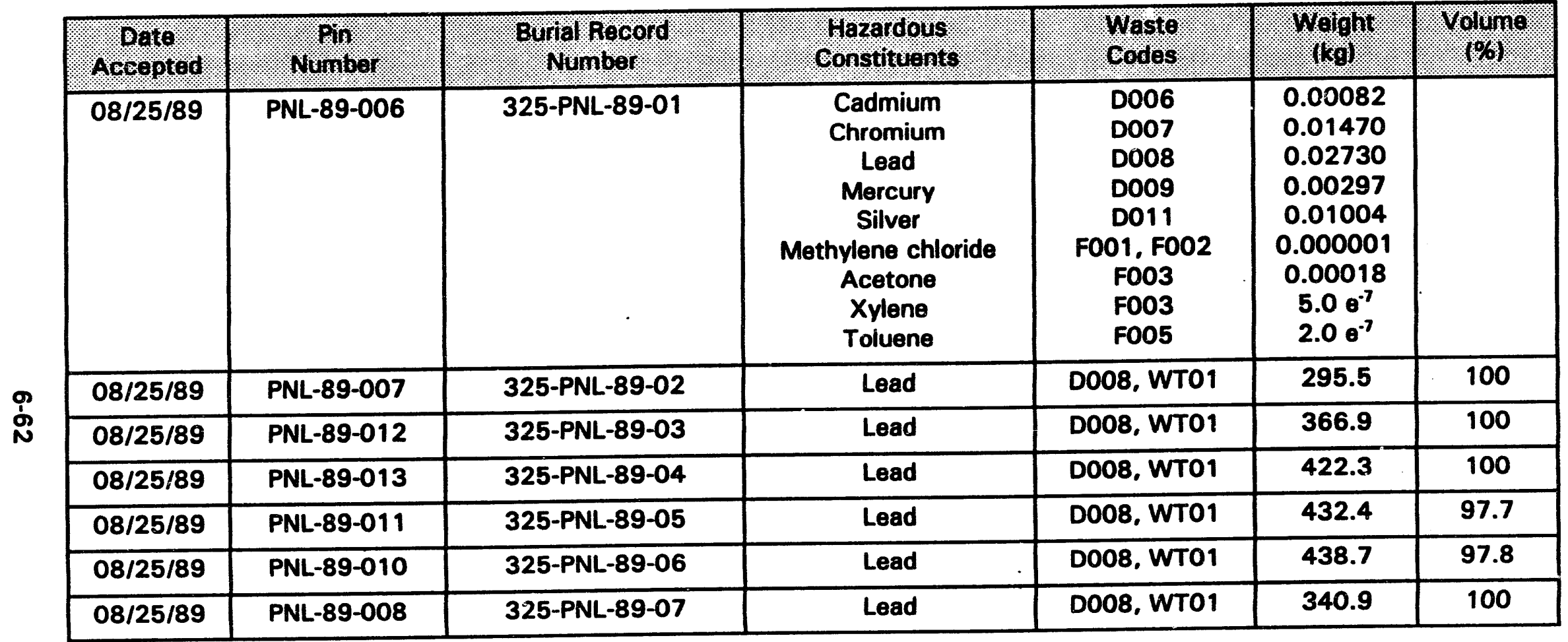




\section{WHC-EP-0696}

\subsection{CHARACTERIZATION OF RETRIEVABLY STORED SOLID WASTE GENERATED BY 325A HOT CELLS ANNEX}

The information found in this section is based primarily on data from the Solid Waste Information and Tracking System (SWITS) database. This database, which incorporated the Jlder R-SWIMS database, is used to track information on radioactive and other wastes stored or disposed at the Hanford Site. Transuranic solid waste packages have been tracked since 1970, however, changes in the requirements and regulations governing radioactive wastes have left their mark in the quantity and quality of the data contained in this database. Caveats are included in the text to alert the reader to changes that may affect the interpretation of the data provided.

The bulk of the data provided is limited to information about the TRU waste that was generated at 325A, however, some general information on the non-TRU waste is included for completeness. The term non-TRU waste is used instead of LLW because a small percentage of the unsegregated waste is designated only as not TRU. Tables 7-1 and 7-2 present TRU and non-TRU waste generation summaries by container type and year. Since the initial retrieval efforts and WRAP 1 will focus on 55-gal drums, these containers are considered separately from all other container types. Therefore, the term "other containers" in this report will refer to all container types except 55-gal drums. The term "drum" refers to 55-gal drums only, unless specified otherwise.

The 325A Hot Cells Annex is composed of three cells - A, B, and C. During the past 25 years, the 325A Hot Cells Annex has been operated by PNL. This is waste that was not included in the previous section on PNL Building 325. Appendix C contains the original SWITS queries and computer runs for PNL 325A. Because of the large number of figures and tables, they are placed at the end of the chapter.

\subsection{SUMMARY OF 325A WASTE GENERATION}

\subsubsection{Waste Stored in 55-Gallon Steel Drums}

Table 7-3 contains waste summary data for 55-gal drums of radioactive waste generated at the 325A Annex between 1970 and 1992. The upper portion of this table presents the number, total weight, and total volume of TRU waste drums. The number of TRU mixed waste drums are also indicated. The same information for non-TRU waste follows. At the bottom of the table are the relative percentages of TRU and non-TRU wastes by container number, weight, and volume. Figures 7-1 through 7-6 present this information graphically.

7.1.1.1 Number of Drums. Between 1970 and 1979, fifty-four 55-gal drums of radioactive waste were generated at the 325A Annex. This total includes 39 drums of non-TRU and 15 drums of TRU waste. The total number of radioactive mixed waste drums is 15 , all of which are non-TRU. Figure 7-1 provides a graph of the number of 55-gal drums of TRU and non-TRU wastes generated at the 325A Annex by PNL annually. The major features of this graph are directly related to historical events that took place at the $325 \mathrm{~A}$ Annex. 


\section{WHC-EP-0696}

The Nuclear Waste Vitrification Project (NWVP) peaked in 1978-1979. The large nonTRU container count in 1978 is probably a result of the processing of light water reactor fuel in cells $A$ and $C$. This operation as well as modifications to $A$ and $C$ cells, were part of NWVP and generally contributed to the waste.

Other NWVP support operations in B and C cells between 1977 and 1979 contributed to the TRU and non-TRU waste generation. The B cell was involved with high-level waste behavior studies and light reactor fuel dissolution testing. The C cell's support of the FFTF by solvent degradation testing as well as molten salt electrode portion experiments probably contributed to the waste generated at 325A Hot Cell Annex (Gerber 1992).

Figure 7-2 presents the relative percentages for TRU and non-TRU drums generated annually at the 325A Annex.

7.1.1.2 Weight. In the period between 1970 and 1979 approximately 4,195 $\mathrm{kg}$ of TRU waste and 8,591 $\mathrm{kg}$ of non-TRU waste generated at the 325A Annex were stored in 55-gal drums. These numbers are estimates since container weights were not required for individual containers prior to 1977. During the R-SWIMS data reentry program in the mid1980's, all pre-1977 containers were assigned standard weights. All 55-gal drums, for instance, were assigned a value of $68 \mathrm{~kg}$ (150 lbs). However, while the average weight of TRU drums from that period was $280 \mathrm{~kg}$, the average weight of non-TRU drums was $296 \mathrm{~kg}$, making these drums more than $16 \mathrm{~kg}$ heavier than the TRU drums.

Figure 7-3 presents the total weight of both TRU and non-TRU radioactive wastes stored annually in 55-gal drums. The relative weight percentages for TRU and non-TRU drums generated annualiy at the 325A Annex are shown in Figure 7-4.

7.1.1.3 Volume. The total volume of waste generated at the 325A Annex and stored in 55-gal drums between 1970 and 1992 is $11 \mathrm{~m}^{3}$, of which $3 \mathrm{~m}^{3}$ is TRU and $8 \mathrm{~m}^{3}$ is non-TRU. Annual waste generation information is shown in Figure 7-5; the volume percentages of radioactive waste stored in 55-gal drums are provided in Figure 7-6. Since all 55-gal drums have a volume of $0.21 \mathrm{~m}^{3}$, the patterns shown in Figures 7-5 and 7-6 are the same as those described for Figures $7-1$ and 7-2 (drum number), respectively. They are included here so that comparisons with later figures (7-11 and 7-12) can be made.

\subsubsection{Waste Stored in Containers Other Than 55-Gallon Steel Drums}

Radioactive solid wastes have been stored or disposed of in a wide variety of containers other than 55-gal steel drums. Other container types used for solid waste generated at the 325A Annex include the following:

- Burlap, cloth, paper or plastic bags

- Metal drums, barrels, or kegs (other than 55-gal drums)

- Wooden boxes, cartons, and cases

- Metal cylinders, casks. 
Some iterns were disposed of or stored without additional packaging, such as portable tanks; miscellaneous scrap; trucks, flatbeds, compactors, loadluggers; and self-contained equipment. These items are also included in the "Other Container" category.

Table 7-4 summarizes the waste data for containers of radioactive waste other than 55-gal drums that were generated at the 325A Annex between 1970 and 1992. The upper portion of this table indicates the number, to:al weight, and total volume of TRU waste stored in other containers. The number of other containers of TRU mixed waste is also provided. The same information for non-TRU waste follows. At the bottom of this table are the relative percentages of TRU and non-TRU wastes by container number, weight and volume. Figures 7-7 through 7-12 present these data graphically.

7.1.2.1 Number of Other Containers. Between 1970 and 1992, 341 other containers of radioactive waste were generated at 325A. Of these containers, 108 hold TRU waste, while 233 contain non-TRU waste. For reference, during the same period of time a total of fiftyfour 55-gal drums of radioactive solid waste were generated at the 325A Annex. Figure 7-7 is a graph of the number of other containers of TRU and non-TRU waste generated at 325A by year. The largest number of other containers containing TRU waste was generated in 1971 with 38 containers. This is the result of an inordinately large number of 5-gal metal drums. Over $68 \%$ of the containers other than 55 -gal drums generated at $325 \mathrm{~A}$ contain nonTRU waste. The peaks in the number of non-TRU other contains in 1978 and 1979 probably result from NWVP support.

Figure 7-8 presents the relative percentages of TRU and non-TRU containers other than 55-gal drums generated at $325 \mathrm{~A}$ by year. One thing apparent from this figure is that after 1975 almost all of the waste in other containers was non-TRU.

7.1.2.2 Weight. In the period between 1970 and 1992, approximately $121,113 \mathrm{~kg}$ of radioactive solid waste was generated at the 325A Annex and placed in containers other than $55-\mathrm{gal}$ drums. This total includes approximately $31,517 \mathrm{~kg}$ of TRU and $89,596 \mathrm{~kg}$ of nonTRU contaminated material. These weights are approximations because individual container weights were not required to be recorded for individual containers prior to 1977. During an update of the R-SWIMS database in the mid-1980's, all waste containers without recorded weights were assigned standard values. For example, all $2 \mathrm{ft}^{3}$ boxes were given a standard weight of $5.5 \mathrm{~kg}(12 \mathrm{lb})$ and $5-$ gal lard cans were assigned a standard weight of $22.7 \mathrm{~kg}$ (50 lb).

Figure 7-9 depicts the weight of TRU and non-TRU waste stored in containers other than 55-gal drums on an annual basis. The most notable feature of this graph is the TRU waste peak in 1975. The large weight of waste disposed of in this year is probably due to the shipment of 11 metal cylinders or casks to retrievable storage. Figure 7-10 shows the percent contribution of TRU and non-TRU waste to the total annual weight of waste packaged in other containers.

7.1.2.3 Volume. The volume of waste generated at the 325A Annex and packaged in containers other than 55-gal drums during the period from 1970 through 1992 is $305 \mathrm{~m}^{3}$. Transuranic wastes account for $69 \mathrm{~m}^{3}$ of the total volume; non-TRU wastes account for $236 \mathrm{~m}^{3}$. Figure 7-11 shows annual volumes of TRU and non-TRU wastes generated by the 


\section{WHC-EP-0696}

325A Annex and packaged in containers other than 55-gal drums. Figure 7-12 shows the relative contribution of TRU and non-TRU wastes to the total volume of waste in other containers for each year.

\subsubsection{Summary of Waste Generation Rates at the 325A Annex Between 1970 and 1992}

7.1.3.1 Number of Waste Containers. Between 1970 and 1992 there were 395 containers of radioactive solid waste generated at the 325A Annex. Fifty-five gallon drums account for $14 \%$ of the total number of containers. Containers of transuranics comprise $31 \%$ of the total container numbers.

Figure 7-13 shows the total numbers of both TRU and non-TRU waste containers generated on an annual basis during this period. The difference in the amount of waste characterized as TRU and non-TRU from 1970 to 1992 is most evident in this graph. Changes in the DOE (formerly AEC) regulations and the 325A Annex disposal practices directly affected the proportion of the waste determined to be TRU and non-TRU. A significant portion of the waste stored as TRU prior to 1982 may be redesignated as LLW upon characterization at WRAP.

Notice that no waste was generated in 1974 unlike the early 1970's. The Hot Cells Isotope campaign, which included a ${ }^{238}$ Pu recovery program, may account for the early 1970's waste. Large peaks in 1978-1979 are probably due to NWVP support.

The NWVP program was put on standby in 1980. During the rest of the 1980's, waste generated probably resulted from some of the several 325A Annex operations such as:

- Material characterization leach test of waste glass nuclear fuels

- Post irradiation examination of Boron shield from N Reactor

- Characterization of neutral cladding removal waste

- Method development to convert cladding to non-TRU waste

- Characterization of double-shell tank slurry

- Fuel iodine analysis

- Fuel uranium dissolution methods for N Reactor

- Recovery testing of Sr using antimonic acid.

Memos dated from the early 1990's indicate a hot cell cleanout and HWVP radioactive testing. This may account for the waste in 1992.

7.1.3.2 Total Weight. Approximately $133,899 \mathrm{~kg}$ of radioactive solid waste was generated at the 325A Annex between 1970 and 1992. Waste packaged in 55-gal drums accounts for about $10 \%$ of the total weight of waste from the 325A Annex, with $90 \%$ of the total weight comprised of waste packaged in some other type of waste container.

Waste designated as TRU makes up $27 \%$ of the total weight while waste designated as non-TRU makes up the remainder. Figure 7-14 provides a graph of the total weight of TRU and non-TRU waste generated at the 325A Annex on a yearly basis between 1970 and 1992. 
The large TRU weight peaks in 1975 and 1976 probably resulted from extended renovations to the $325 \mathrm{~A}$ Annex prior to 1975 , as well as a variety of operations in the 325A Annex during 1975. In 1975, A cell was involved in the separation and purification of fission products as well as ${ }^{147} \mathrm{Pm}$ production. Radiochemical studies were performed in $\mathrm{B}$ cell while C cell was used for separation and purification of ${ }^{238} \mathrm{Pu}$ and ${ }^{237} \mathrm{~Np}$ from fuel elements. All of these operations contributed to TRU waste peaks in 1975-1976.

7.1.3.3 Total Volume. In the period between 1970 and $1992,316 \mathrm{~m}^{3}$ of radioactive solid wastes were generated at the 325A Annex. Only $4 \%$ of this volume is comprised of 55-gal drums; the remaining $96 \%$ of the volume is made up of other container types. Transuranic wastes account for $23 \%$ of the waste volume, while non-TRU wastes make up the remaining $77 \%$. Figure $7-15$ shows the total volume of TRU and non-TRU waste generated at the 325A Annex on a yearly basis. The most notable feature of this graph is the peak in 1975. It is interesting to note that metal cylinders or casks account for $100 \%$ of the TRU waste volume for 1975. For the Non-TRU portion of the 1975 peak, metal cylinders or casks account for $50 \%$.

\subsection{WASTE CONTAINERS}

\subsubsection{TRU Waste Containers}

Table 7-1 provides a summary of the TRU waste generated annually from 1970 to 1992 by the 325A Annex sorted by container type. The greatest number of containers are 5-gal metal drums, barrels, or kegs, $75 \%$ by count. Eight percent of the TRU waste is contained in 11 metal cylinders or casks of unknown container size, followed by $11 \%$ of the weight in 55-gal drums. The percentages for all container types and the weight and volume of these containers are shown for each year in this table.

\subsubsection{Non-TRU Waste Containers}

Table 7-2 provides a summary of the non-TRU waste generated by the 325A Annex from 1970 to 1992 by container type. Miscellaneous scrap was the most common type of waste generated. The greatest weight of non-TRU waste, $40 \%$, is comprised of waste in wooden boxes, cartons, or cases although there are also large amounts of waste weight from trucks, flatbeds, compactors, or loadluggers.

\subsection{TRU WASTE STORAGE LOCATIONS}

Table 7-5 provides the storage locations for TRU waste packaged in 55-gal drums by year. In general, the bulk of the drums generated in one year are stored primarily in one 
facility. Storage locations for TRU waste packaged in other containers are shown in

Table 7-6. A summary of the 325A Annex waste by storage location follows:

- 218-W-3A - Burial Ground 218-W-3A consists of 14 earthen-bottom, gravel filled trenches with waste emplaced from May 1970 to April 1988. Drums and other containers from the 325A Annex were disposed here between 1975 and 1976 containing no hazardous constituents.

- 218-W-4B - Burial Ground 218-W-4B, consisting of 14 trenches, accepted retrievable waste through September of 1978. Drum and other container waste from the 325A Annex was buried here from 1970 to 1983.

- 218-W-4C - Burial Ground 218-W-4C consists of six trenches with the first waste emplaced in March 1978. Drum waste from the 325A Annex was sent to this area in 1979.

\subsection{PHYSICAL CONTENTS OF TRU WASTE CONTAINERS}

The physical contents of 55-gal drums are shown in Tables 7-7 through 7-8. There is a separate table for each storage location. The top portion of each table indicates the number of drums for which a given component is listed; the bottom part of the table indicates the percentage of the total drums from the 325A Annex in that burial location that contain the listed constituent. The physical contents for TRU waste containers other than 55-gal drums can be found in Tables 7-9 through 7-10.

Interviews with 325 Building personnel indicate possible unvented aerosols cans may be among the non-TRU waste containers. Aerosol cans may have contained Windex ${ }^{\mathrm{m}}$ and WD-40".

It should he noted that prior to 1978, physical contents were not required to be listed on the burial records. A great many of the contents on early records are listed only as "Miscellaneous."

\subsection{RADIOLOGICAL DESCRIPTION OF THE 325A ANNEX SOLID WASTE}

Table 7-11 shows the number of grams of transuranics contained in waste stored in 55-gal drums each year by storage location; Table 7-12 provides the same information for containers other than 55-gal drums. Figure 7-16 combines the information on these tables in a graph that shows the total amount of transuranics present in waste packages from the 325A Annex by year. It should be noted that not all SWITS records for TRU Waste containers list the number of grams of TRU, particularly in the early 1970's. 
WHC-EP-0696

\subsection{RADIOACTIVE ISOTOPES PRESENT IN THE 325A ANNEX SOLID WASTE}

Information on radioactive isotopes was obtained from the SWITS database and from the 325A Annex Safety Analysis Report (Anderson and Hanthorn 1975). Isotopes known to be in the waste stream are presented in Table 7-13 for 55-gal drums and Table 7-14 for other containers. The present isotopes listed in the safety analysis report as being used in the 325A Annex proc jsses and laboratories may or may not be in the solid waste stream. The following are isotopes suspected to be present in the 325A solid waste stream: ${ }^{241} \mathrm{Am}$. ${ }^{243} \mathrm{Am},{ }^{144} \mathrm{Ce}, \mathrm{Cm},{ }^{131} \mathrm{I},{ }^{237} \mathrm{~Np},{ }^{147} \mathrm{Pm},{ }^{230} \mathrm{Pu}$, and ${ }^{230} \mathrm{Pu}$.

\section{7 haZARDOUS CONS ITTUENTS OF SOLID WASTE GENERATED BY 325A HOT CELLS ANNEX}

This section provides a review of the data on the hazardous components known or suspected to be in the solid waste stream from Westinghouse Hanford and PNL Westinghouse Hanford 325A Annex Hot Cells. Information was gathered from several sources, including: the Solid Waste Information Tracking System, Solid Waste Burial Records, personnel interviews, an internal report examining hazardous components of solid waste, Inventory of Chemicals Used at Hanford Site Production Plants and Support Operations (Klem 1990), and Unstable and Reactive Chemicals in Transuranic Retrievable Waste at the Hanford Site (Reddinger 1992).

\subsubsection{Chemical Inventories}

Table 5-20, discussed previously in Section 5.7.1, provides a list of chemicals used at the 325 Building Analytical Laboratory from 1953 to 1976. This list was developed by Klem (1990) to support waste characterization efforts for the single shell tanks. Though this list characterizes chemicals used in the Analytical Laboratory, it does not specify, if any, which of these chemicals originated in the 325A Hot Cells Annex.

\subsubsection{Solid Waste Information and Tracking System (SWITS)}

The hazardous constituent field in the SWITS database was searched for all containers from PNL 325 Hot Cells that contained radioactive mixed waste. This search indicated that no mixed waste containers from the 325A Hot Cells Annex were sent to the solid waste burial grounds.

\subsubsection{Burial Records}

A review of microfilmed burial records revealed hazardous constituents that were sent to the solid waste burial grounds from the Westinghouse Hanford and PNL 325A Hot Cells Annex. Table 7-15 contains a summary of the PNL 325A Annex TRU mixed weste containers and their hazardous contents. The PNL 325A low-level mixed waste containers are summarized in Table 7-16. 


\subsubsection{Interviows with 325 Bullding Personnel}

From the information gained in interviews with 325 Building personnel, a list of known or suspected components in the solid waste stream has been compiled. This list of components can be found in Section 5.7.4. As this list applies to the entire 325 Building Analytical Laboratory, it is expected that some of the constituents may be present in the Hot Cells waste stream as well. 


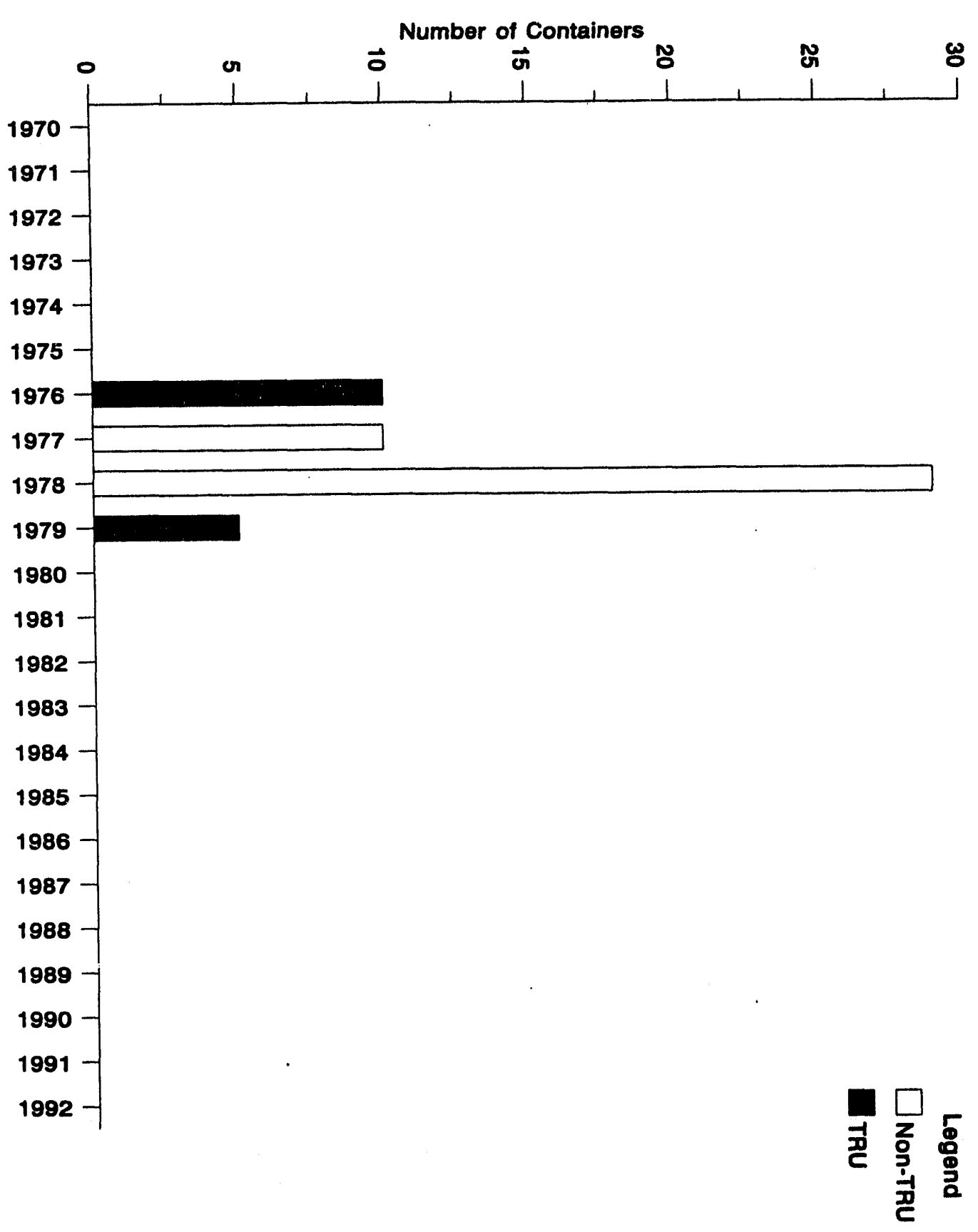

-A॥enuu $\forall$ xөuu $\forall \forall 92 \varepsilon$

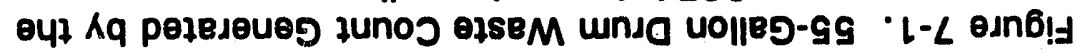


OL-L

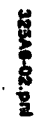

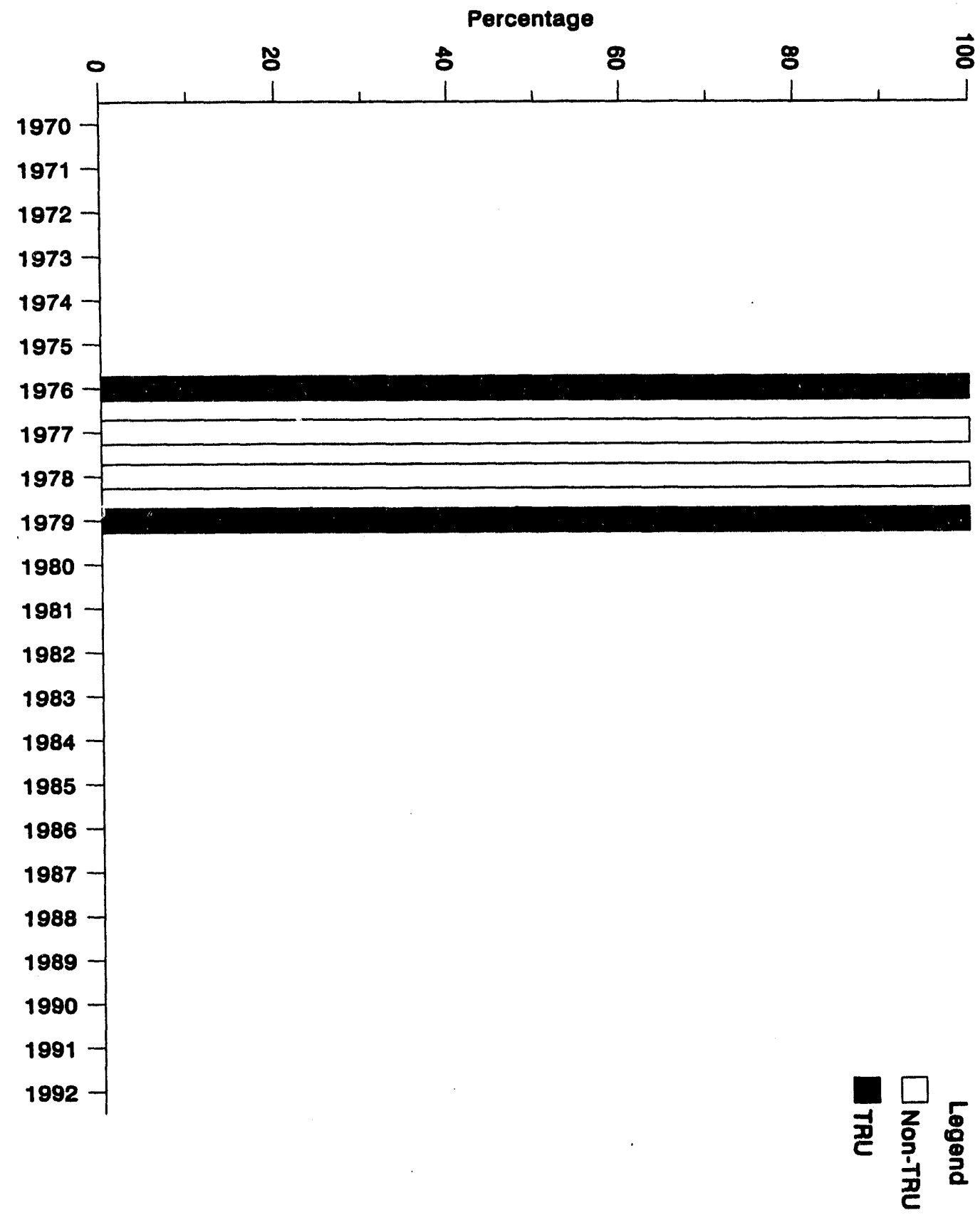

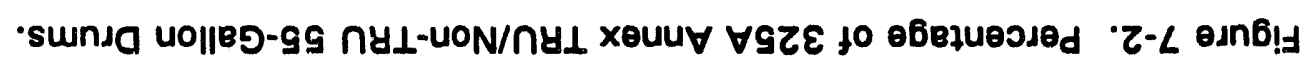


$L L-L$

葛

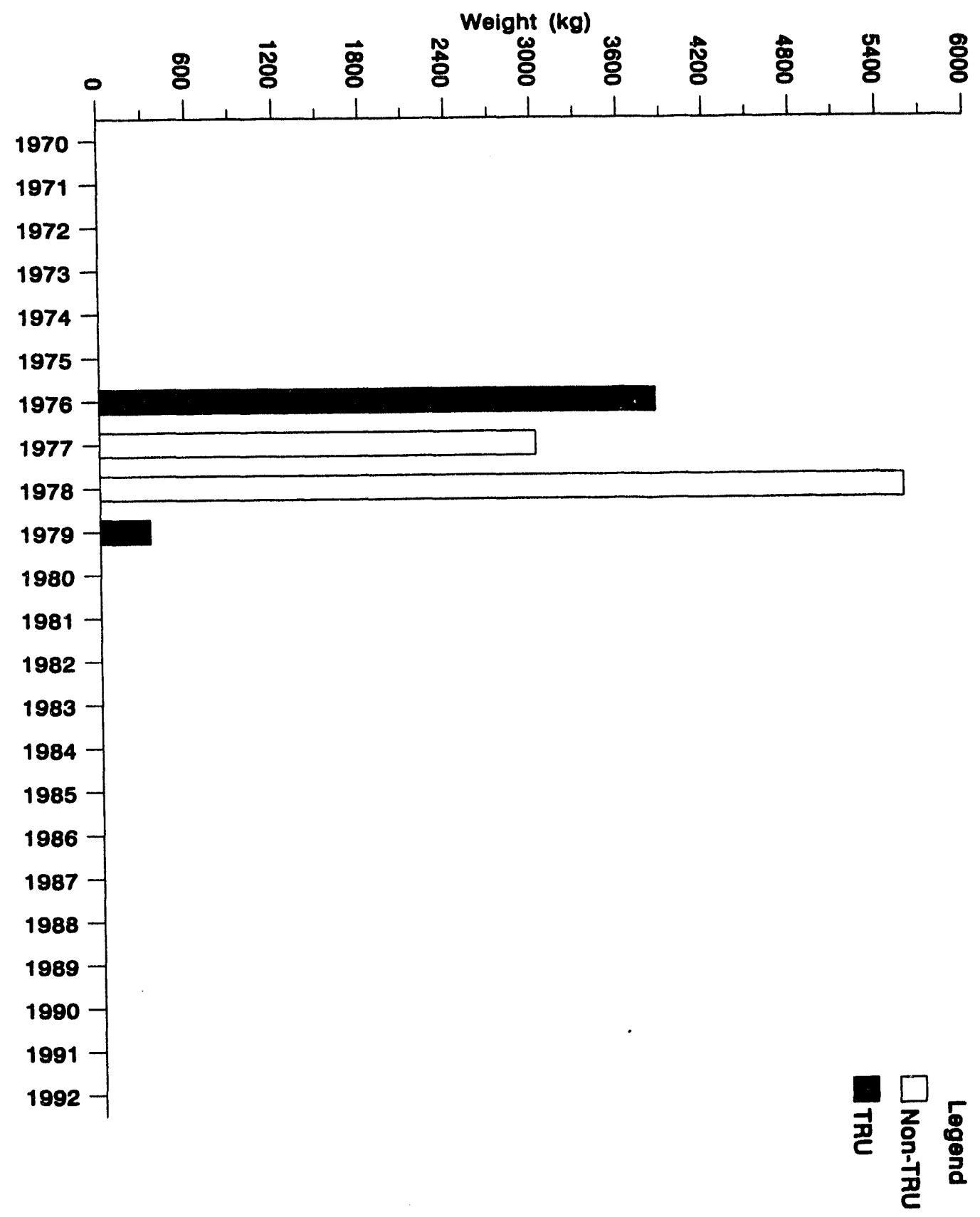

$\cdot$ -

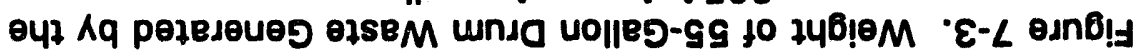


$\frac{8}{8}$

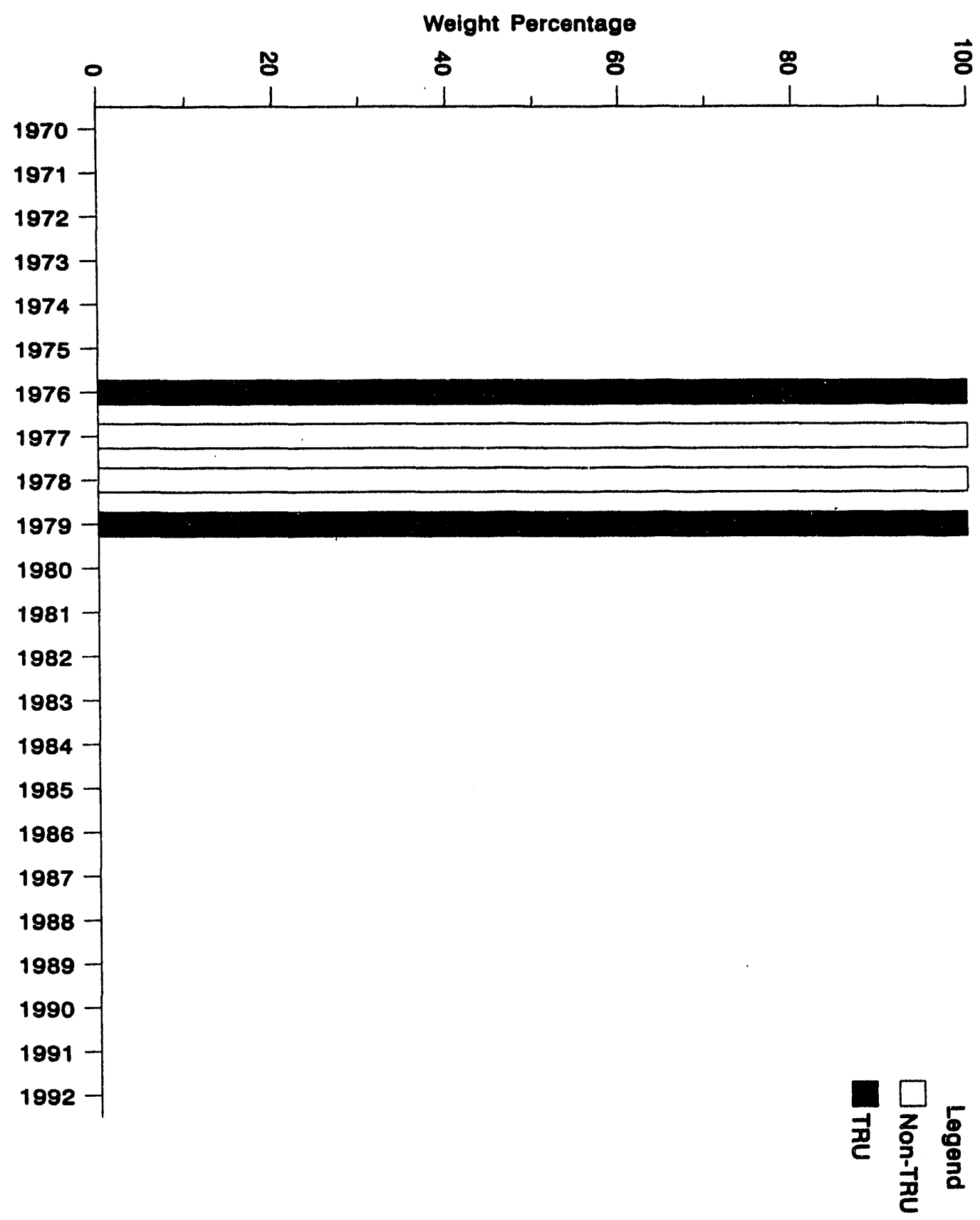

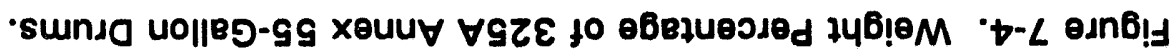


$\varepsilon\llcorner-L$

8
8
2

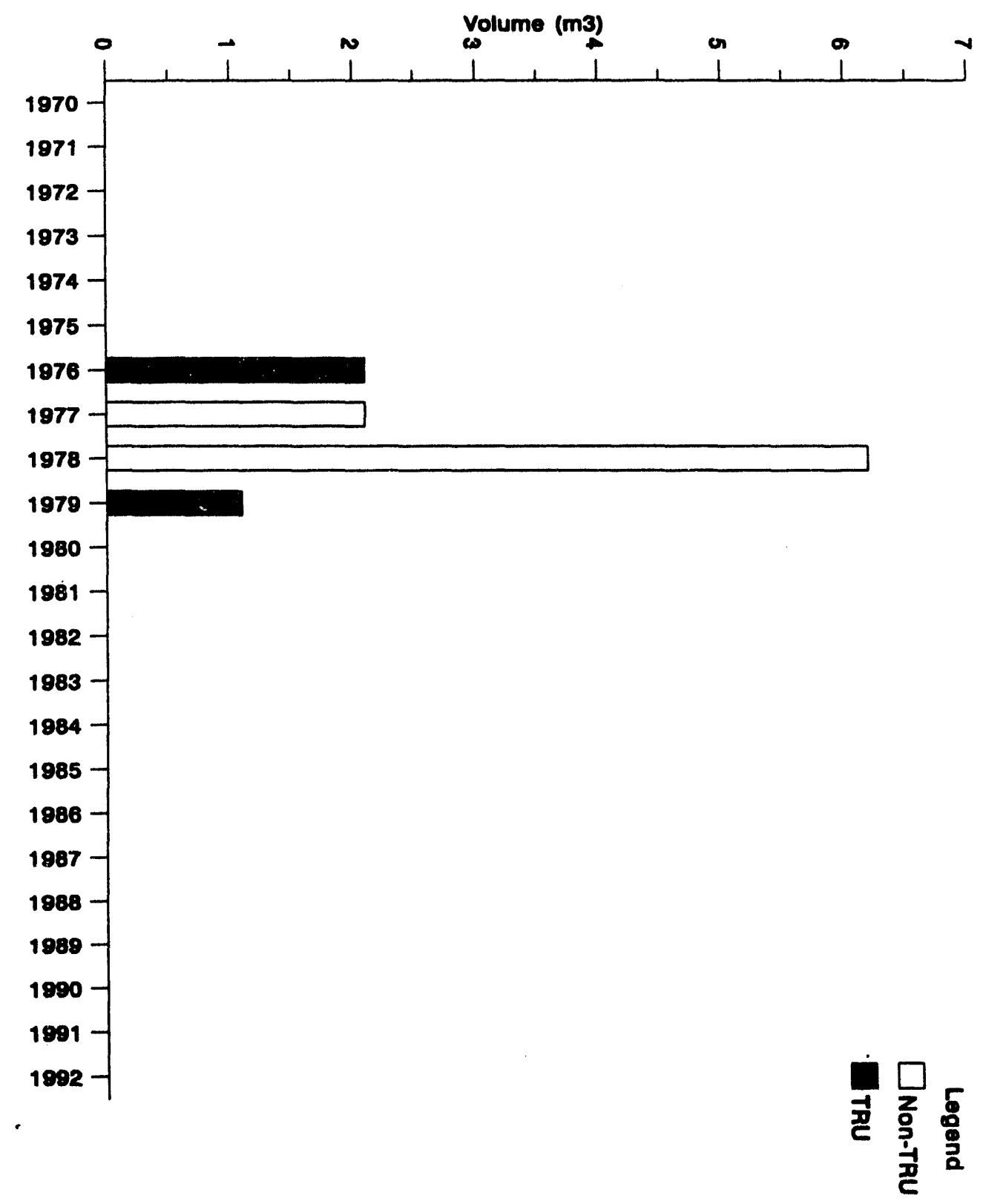

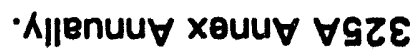

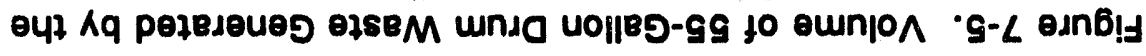


$\rightarrow l-L$

8
8
8

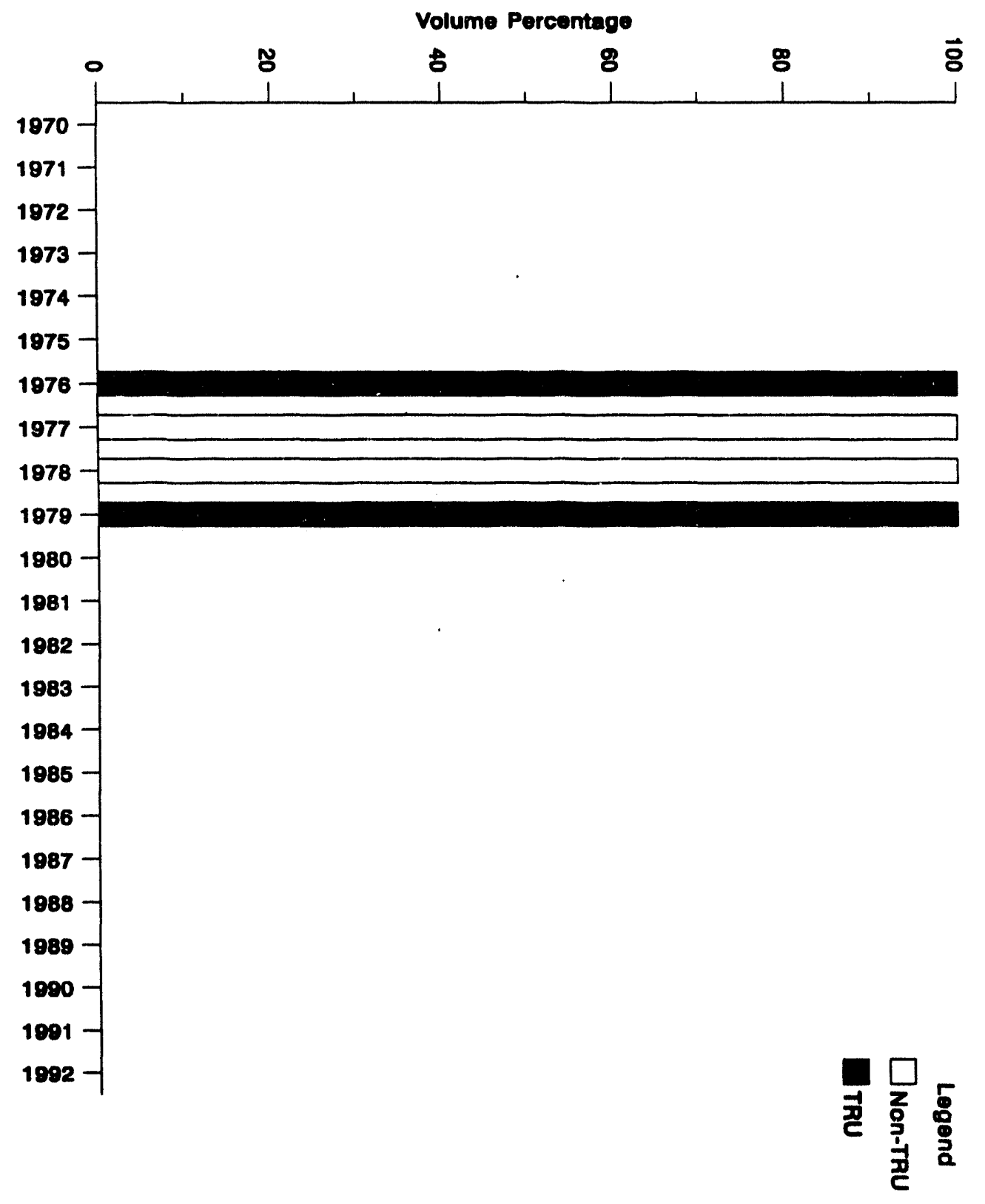

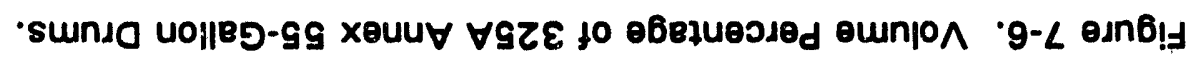




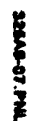

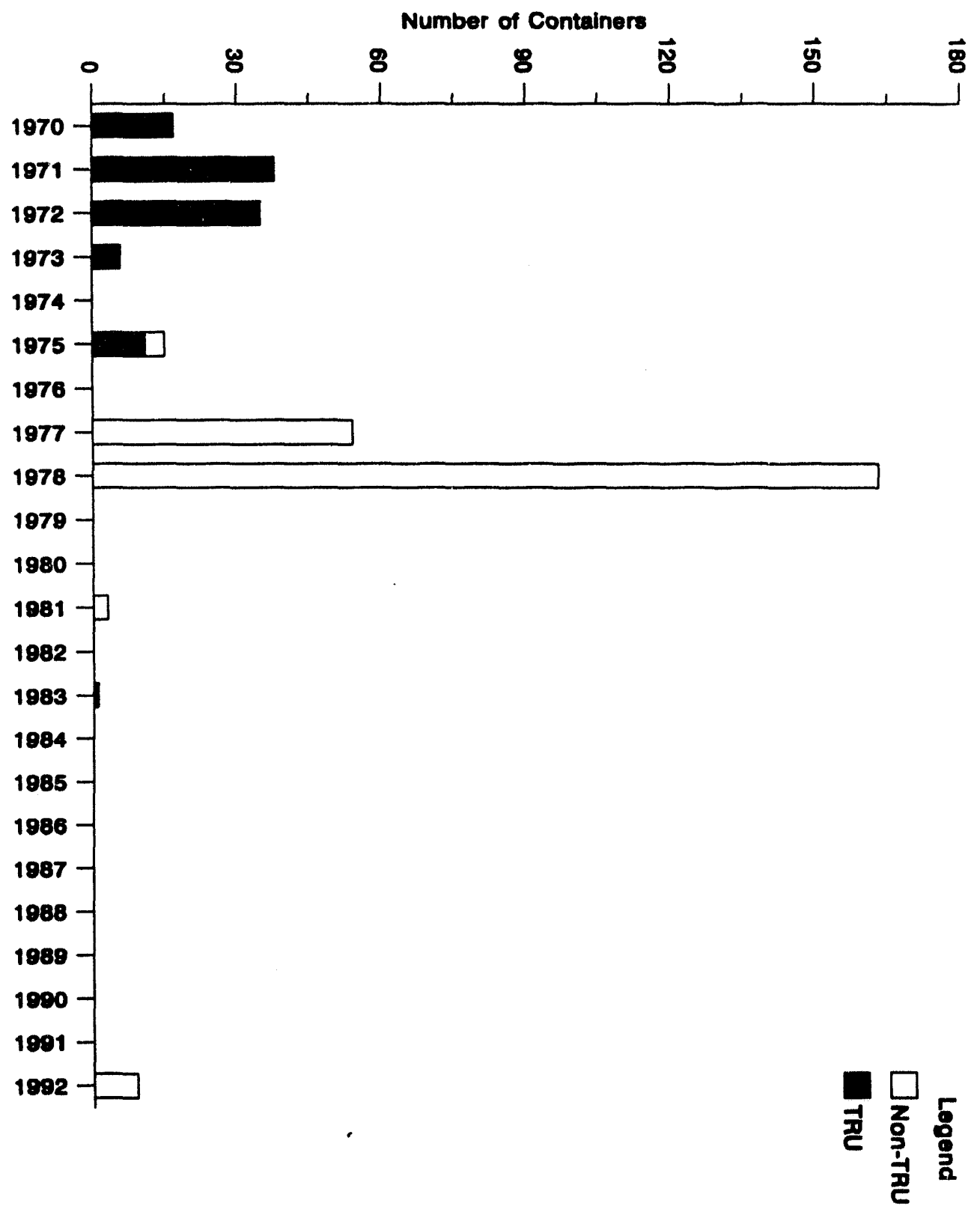

- ᄉ॥enuu $\forall$ Xөuu $\forall \forall 9 Z \varepsilon$

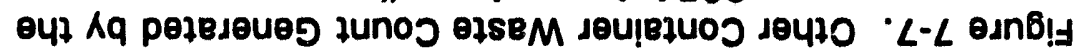


WHC-EP-0696

Figure 7-8. Percentage of 325A Annex TRU/Non-TRU Other Containers.
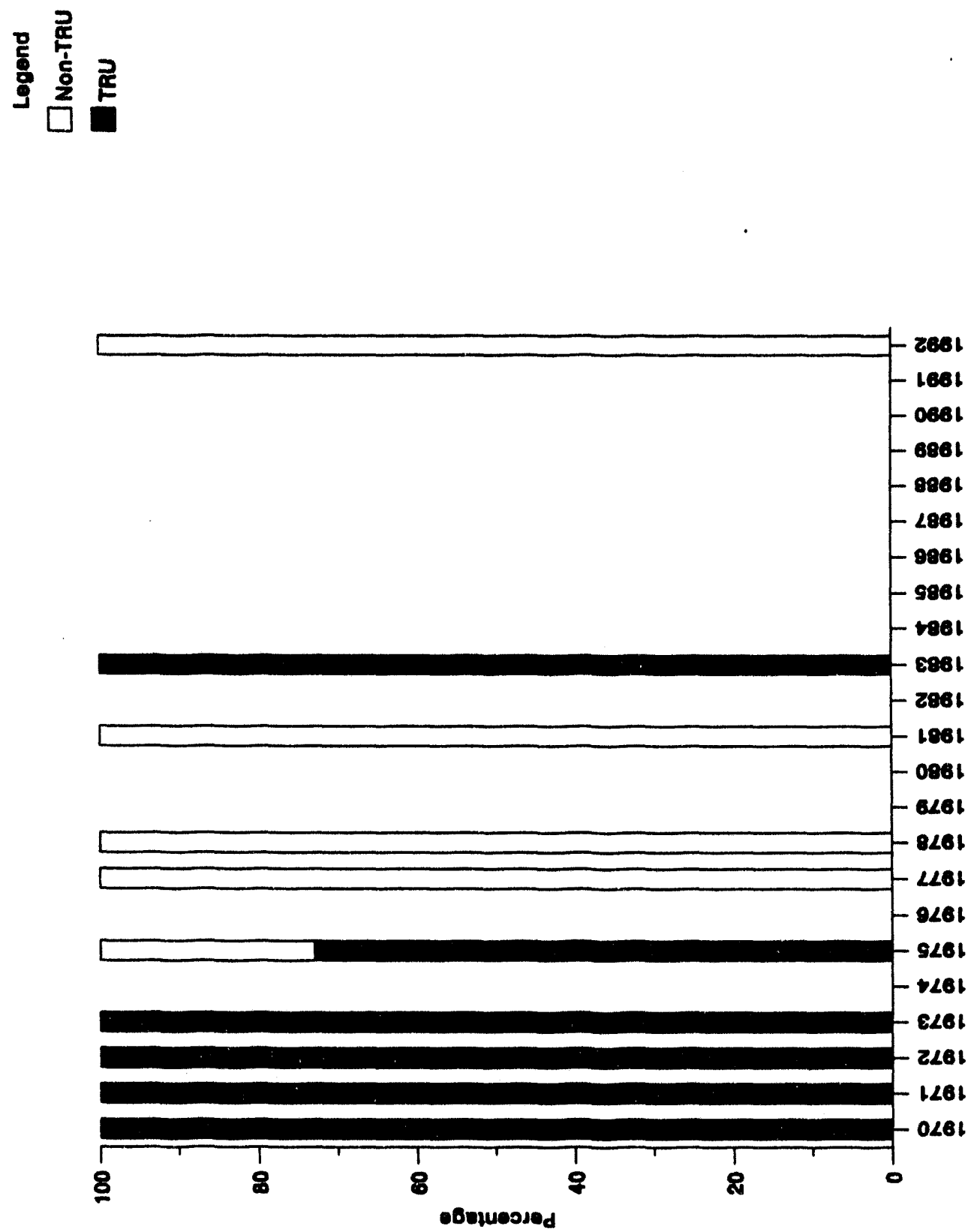

8 


\section{WHC-EP-0696}

Figure 7-9. Weight of Other Containers Generated by the 325A Annex Annually.

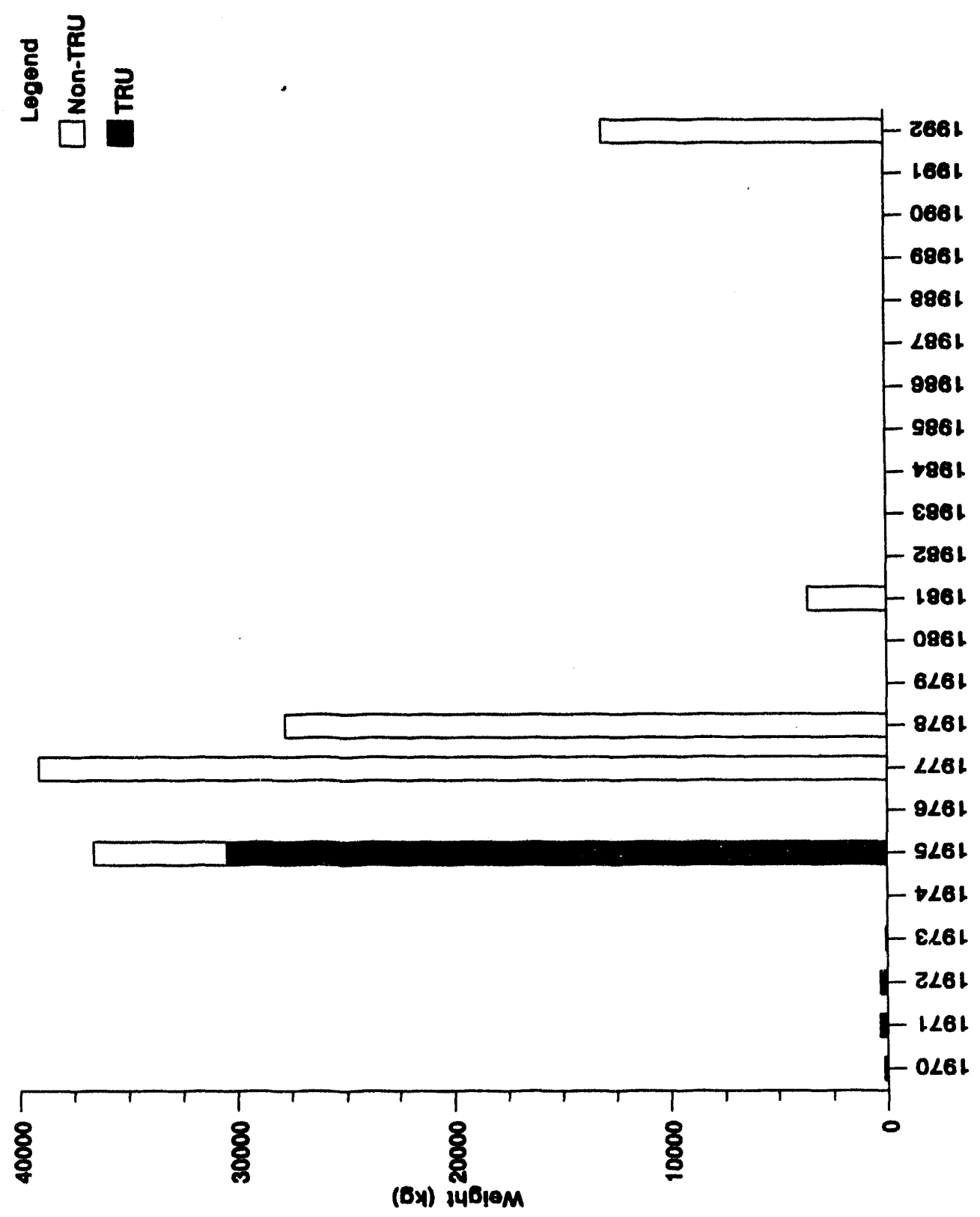




\section{$8 l-L$}

8

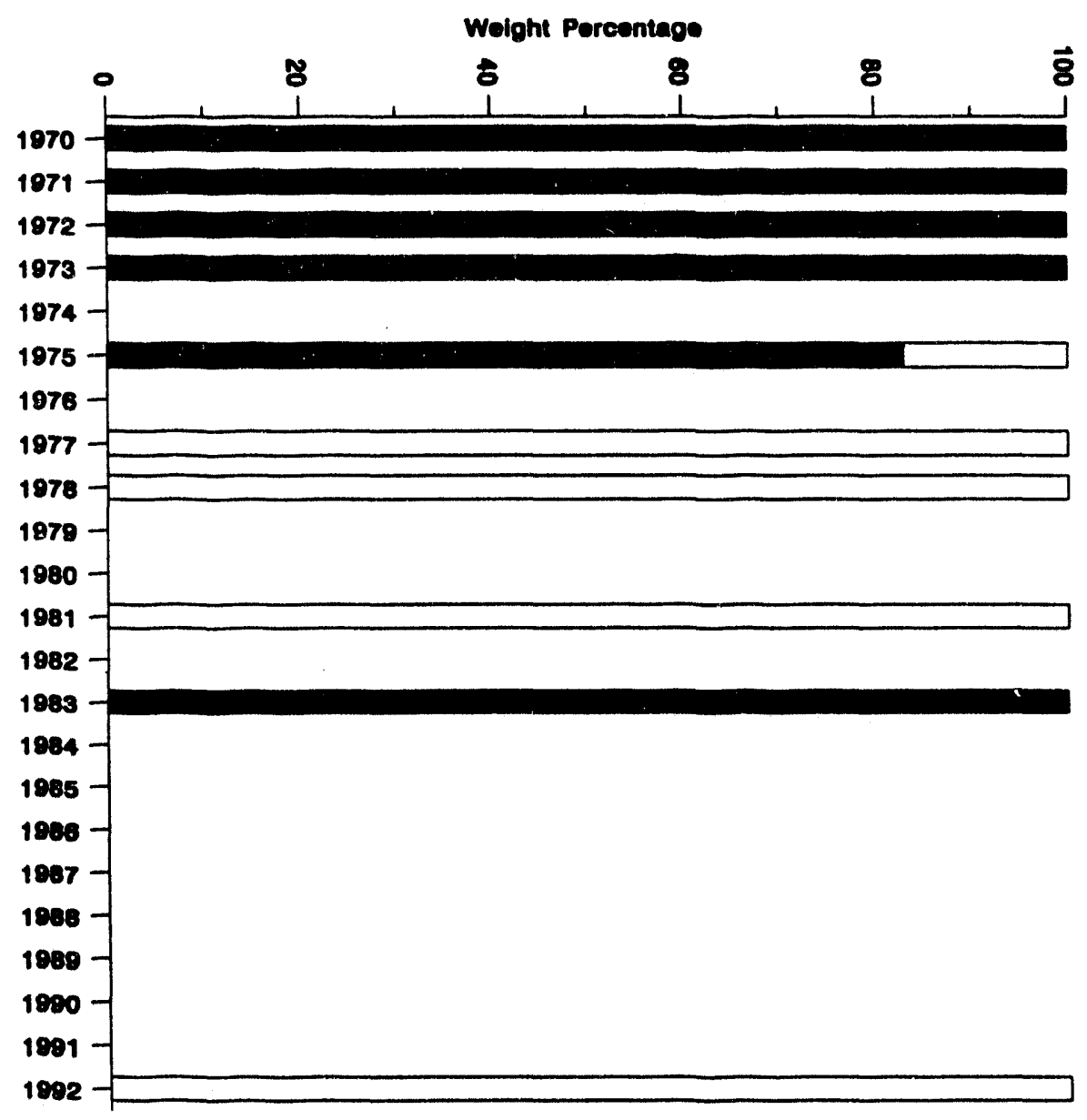

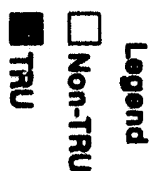

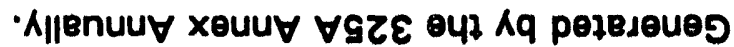

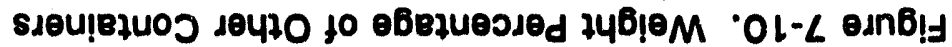


Figure 7-11. Volume of Other Containers Generated by the 325A Annex Annually.

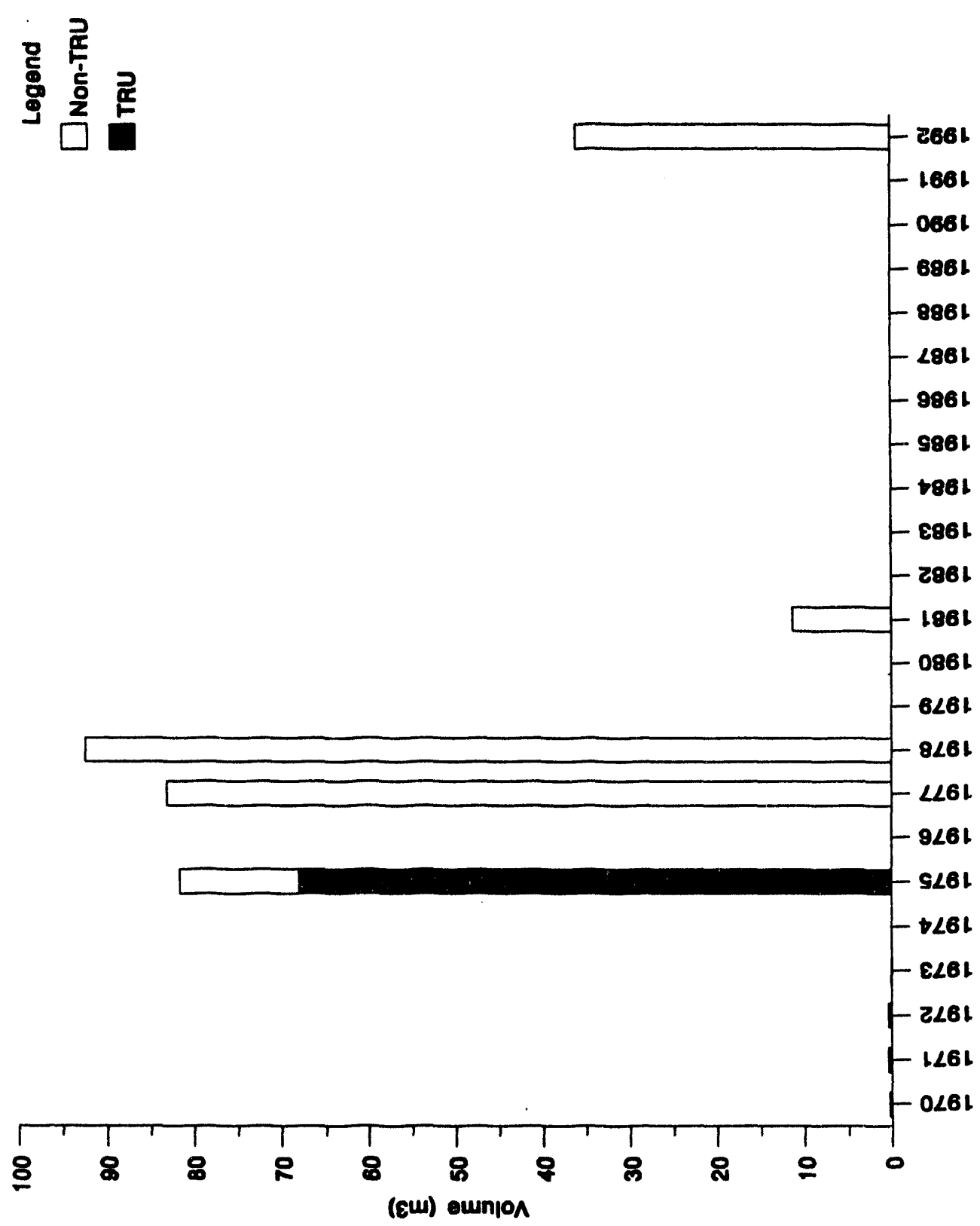




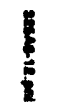

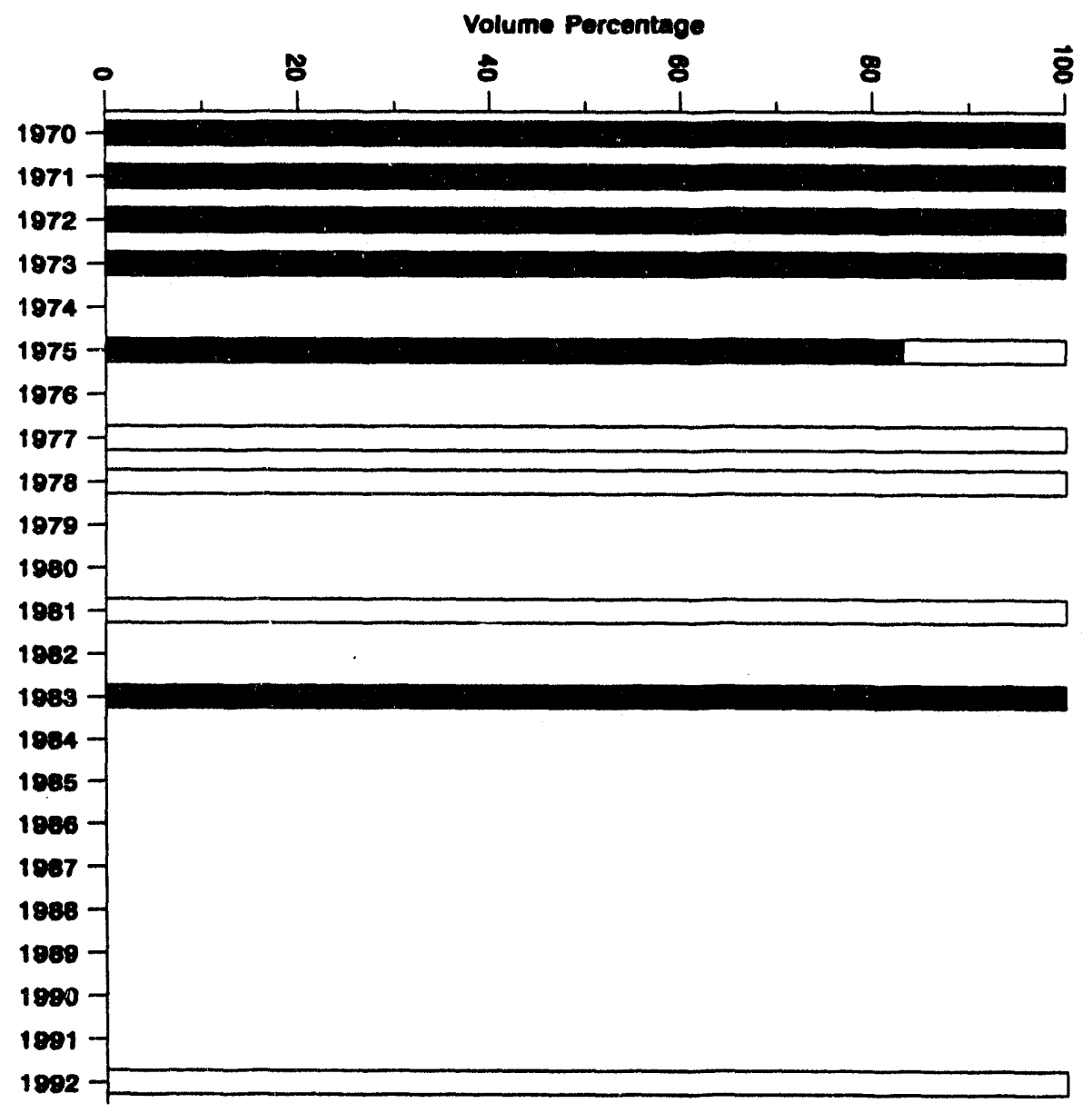

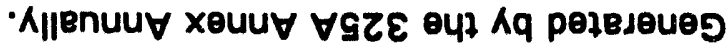

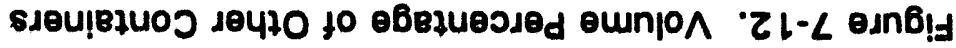


$1 z-L$

$$
8
$$

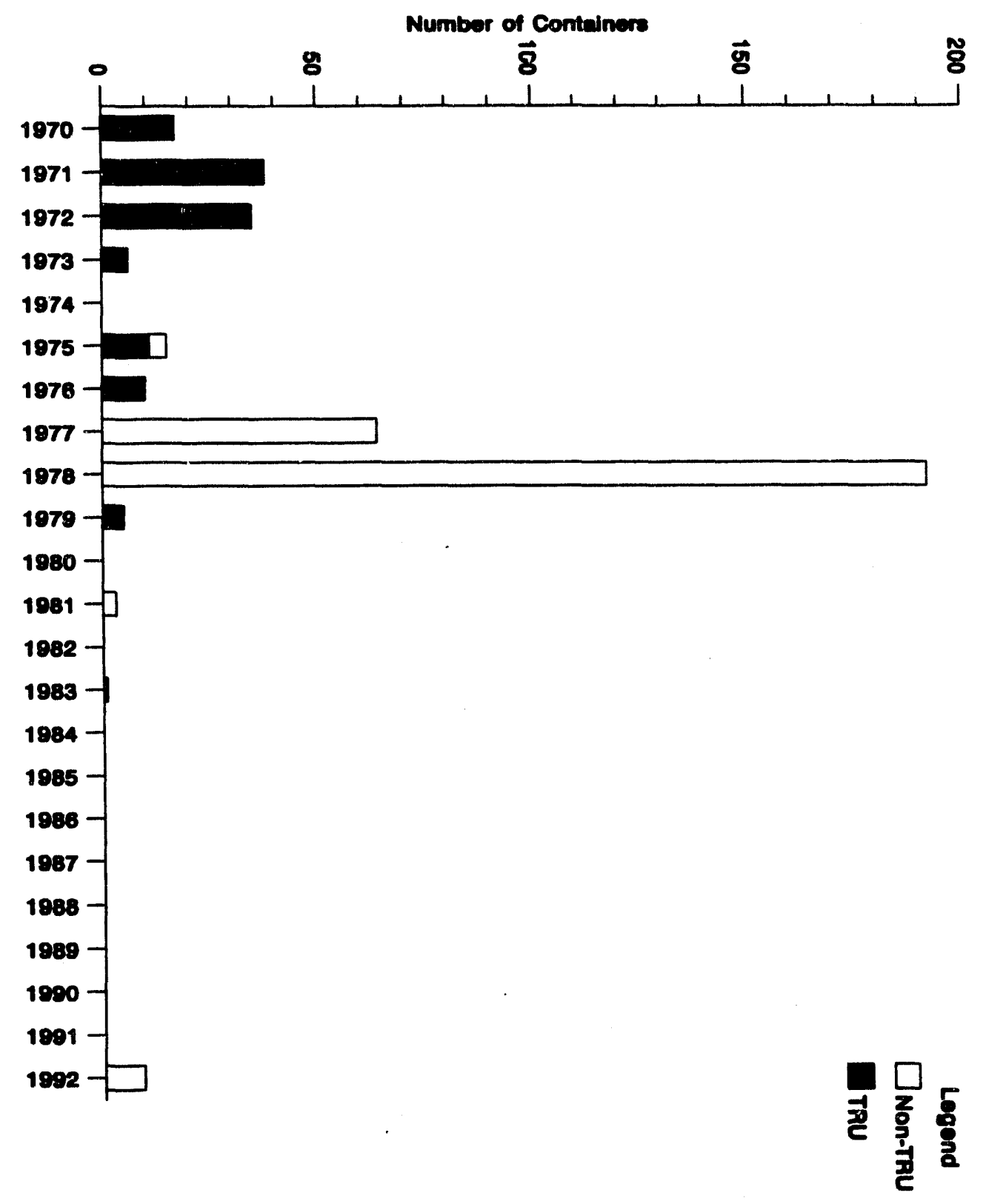

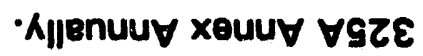

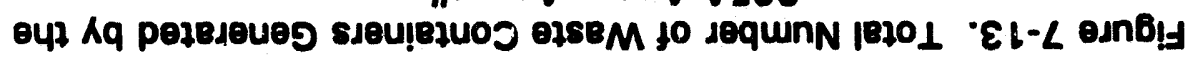


$2 z-L$

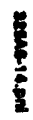

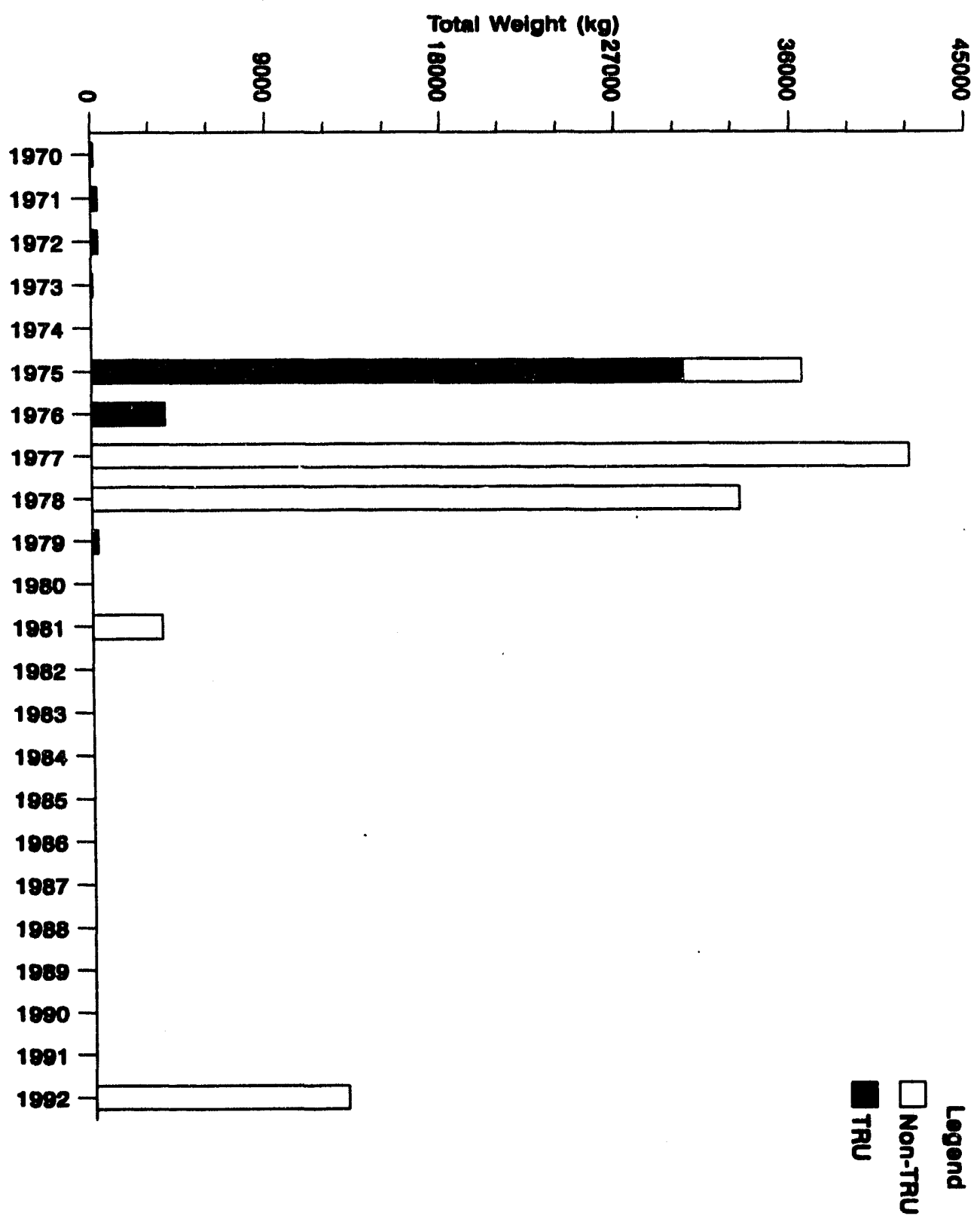

- Aाןenuu $\forall$ xeuu $\forall \forall 92 \varepsilon$

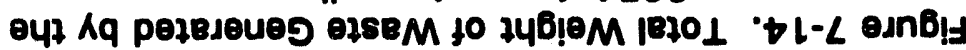

9690-dJ-JHM 
Figure 7-15. Total Volume of Waste Generated by the 325A Annex Annually.

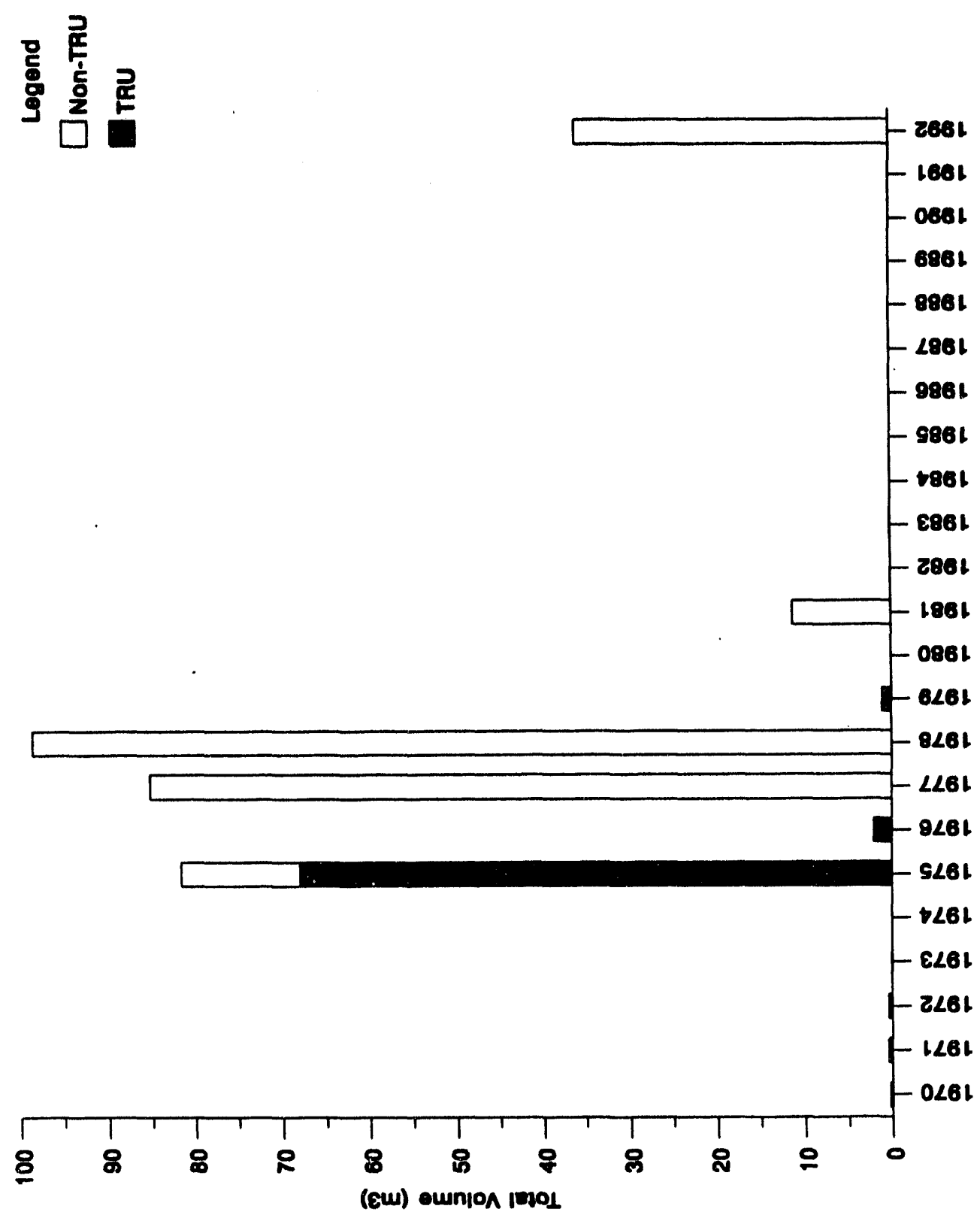




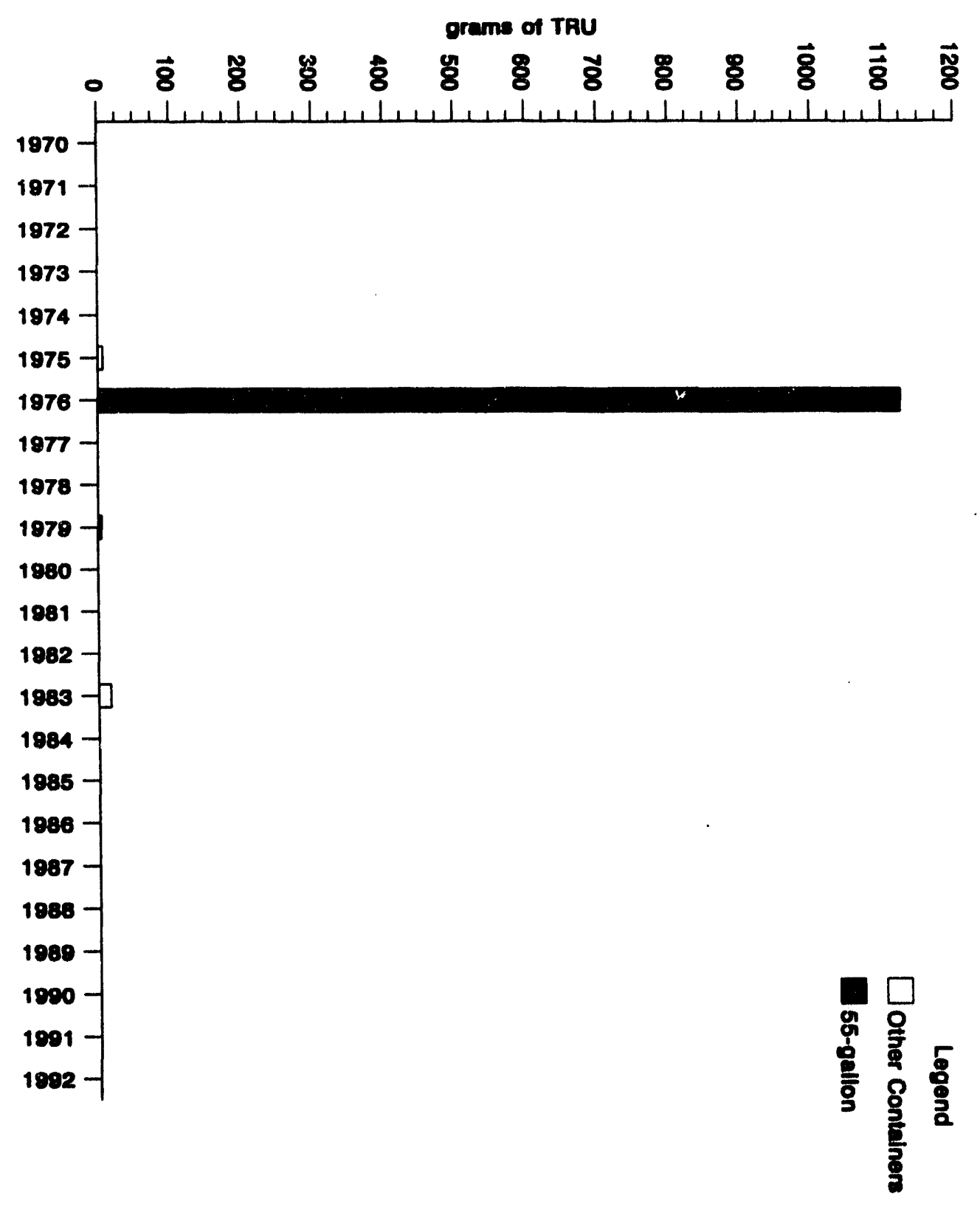


Table 7-1. TRU Waste Generated at the 325A Annex by Container Type and Year. (2 sheets)

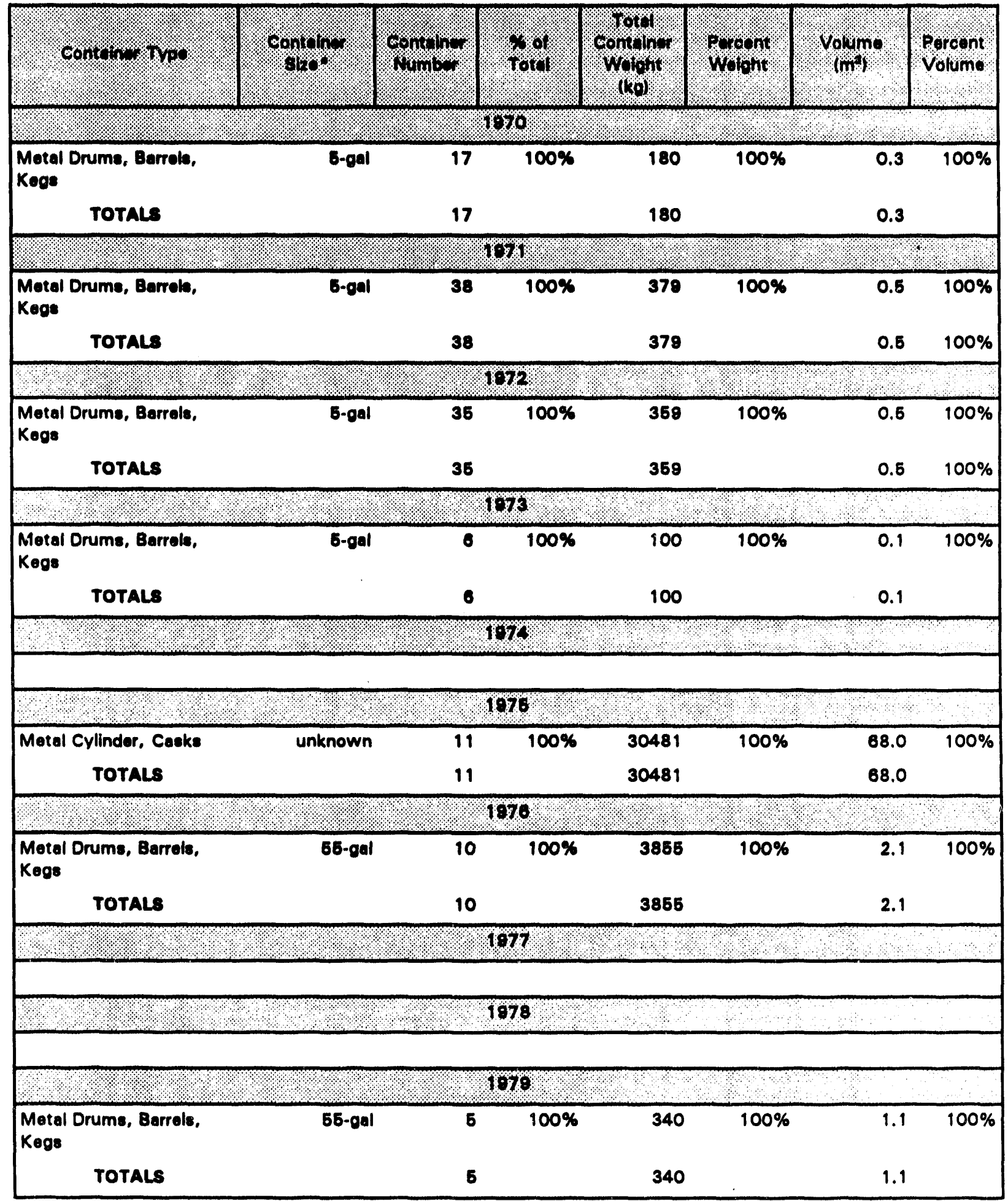


WHC-EP-0696

Table 7-1. TRU Waste Generated at the 325A Annex by Container Type and Year. (2 sheets)

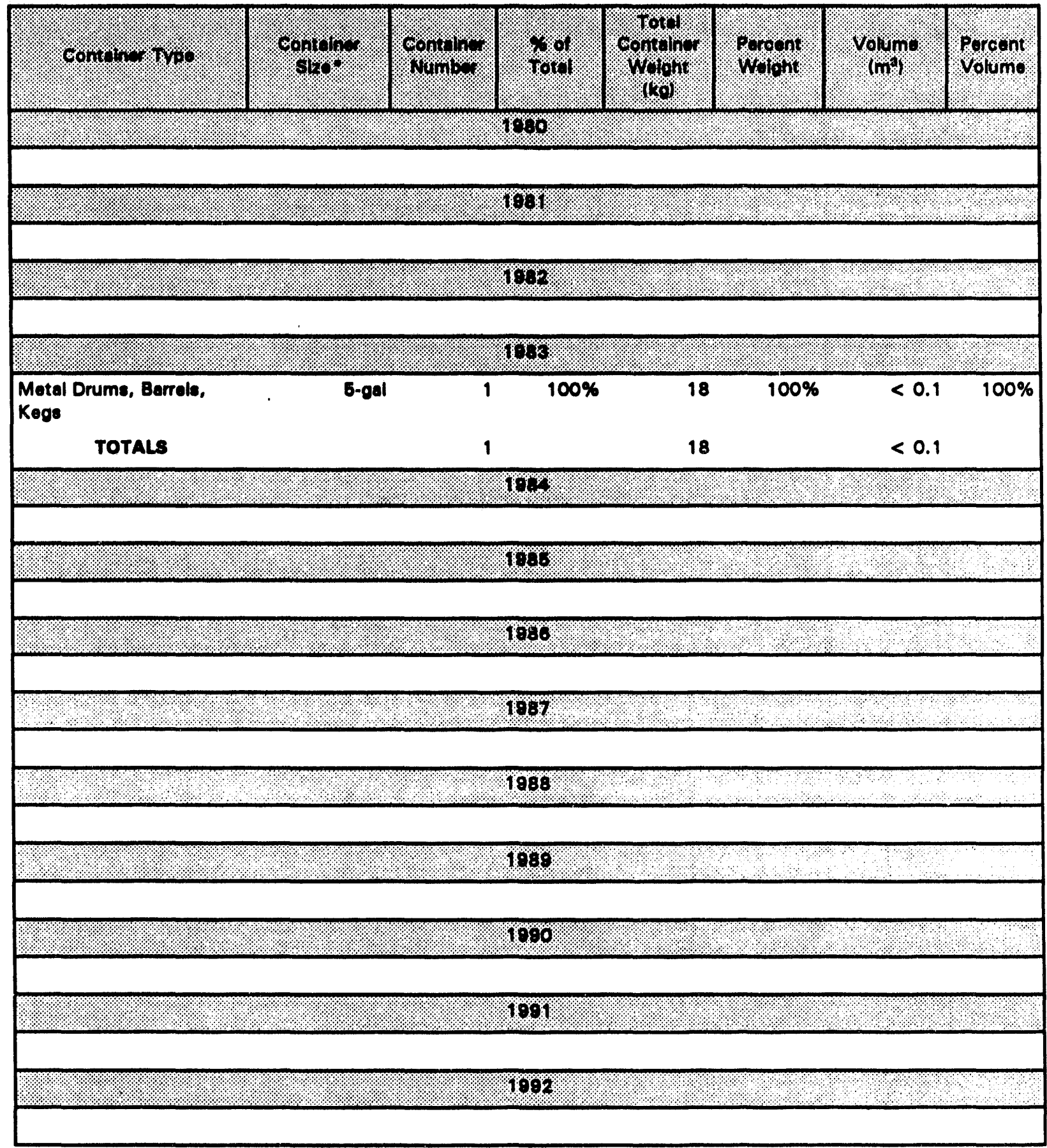

-Unlese otherwise noted, units of dimensions are in $\mathrm{ft}$.

- These containers hold mixed waste. 
WHC-EP-0696

Table 7-2. Non-TRU Waste Generated at the 325A Annex by Container Type and Year. (3 sheets)

\begin{tabular}{|c|c|c|c|c|c|c|c|}
\hline reon & oonton. & o.m. & 1010 & conoring & wornts & volum & $\begin{array}{l}\text { Porcent: } \\
\text { volume }\end{array}$ \\
\hline \multicolumn{8}{|c|}{1020} \\
\hline \multicolumn{8}{|c|}{1021} \\
\hline \multicolumn{8}{|c|}{1012} \\
\hline \multicolumn{8}{|c|}{$18 \%$} \\
\hline \multicolumn{8}{|c|}{1010} \\
\hline \multicolumn{8}{|c|}{1076} \\
\hline Matal Cylinder, Cacks & unknown & 2 & $50.0 \%$ & 6475 & $89.8 \%$ & 12.2 & $89.7 \%$ \\
\hline Miscellaneous Scrap & unknown & 2 & $60.0 \%$ & 622 & $10.2 \%$ & 1.4 & $10.3 \%$ \\
\hline TOTAL8 & & 4 & & 6097 & & 13.6 & \\
\hline \multicolumn{8}{|c|}{1076} \\
\hline \multicolumn{8}{|c|}{1071} \\
\hline $\begin{array}{l}\text { Burlap, Cloth, Paper, or } \\
\text { Plastic Bags }\end{array}$ & unknown & 28 & $40.6 \%$ & 1445 & $10.6 \%$ & 9.9 & $11.6 \%$ \\
\hline \multirow{2}{*}{$\begin{array}{l}\text { Metal Drums, Barrels, } \\
\text { Kegs }\end{array}$} & 110-gal & 1 & $1.6 \%$ & 188 & $0.4 \%$ & 0.4 & $0.5 \%$ \\
\hline & 65.gal & 10 & $15.6 \%$ & 3016 & $7.2 \%$ & 2.1 & $2.6 \%$ \\
\hline Miscellaneous Scrap & unknown & 1 & $1.6 \%$ & 38 & $0.1 \%$ & 0.9 & $1.1 \%$ \\
\hline Tanke, Portable & unknown & 6 & $8.4 \%$ & 7780 & $18.4 \%$ & 17.3 & $20.3 \%$ \\
\hline \multirow{3}{*}{$\begin{array}{l}\text { Wooden, Boxes, Cartona, } \\
\text { Cases }\end{array}$} & $3 \times 4 \times 6$ & 1 & $1.6 \%$ & 914 & $2.2 \%$ & 2.0 & $2.3 \%$ \\
\hline & $4 \times 4 \times 4$ & 9 & $14.1 \%$ & 7316 & $17.4 \%$ & 16.3 & $19.1 \%$ \\
\hline & $4 \times 4 \times 8$ & 10 & $15.6 \%$ & 18407 & $43.7 \%$ & 36.3 & $42.6 \%$ \\
\hline TOTALS & & 64 & & 42083 & & 85.2 & \\
\hline
\end{tabular}




\section{WHC-EP-0696}

Table 7-2. Non-TRU Waste Generated at the 325A Annex by Container Type and Year.

(3 sheets)

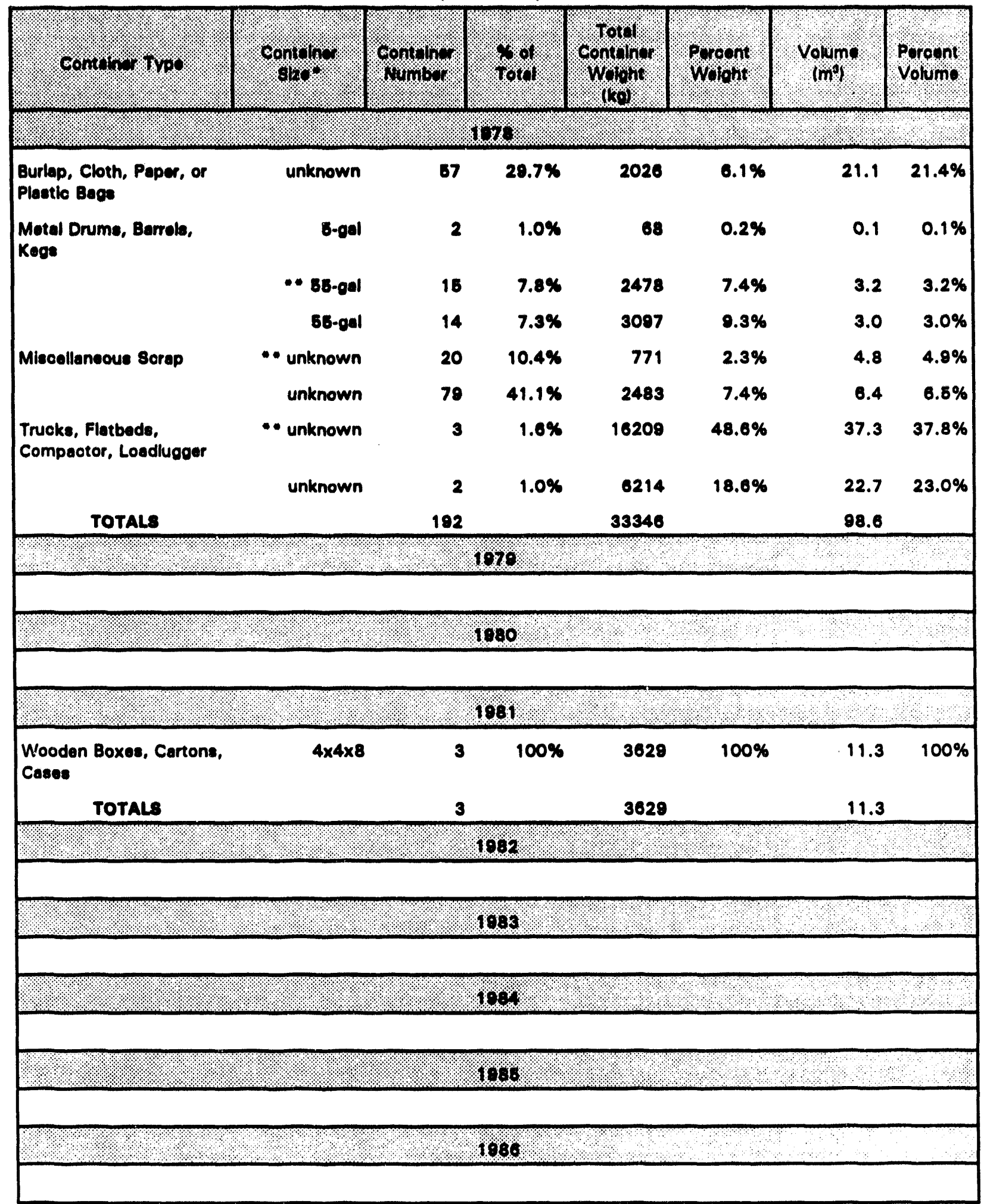


WHC-EP-0696

Table 7-2. Non-TRU Waste Generated at the 325A Annex by Container Type and Year. (3 sheots)

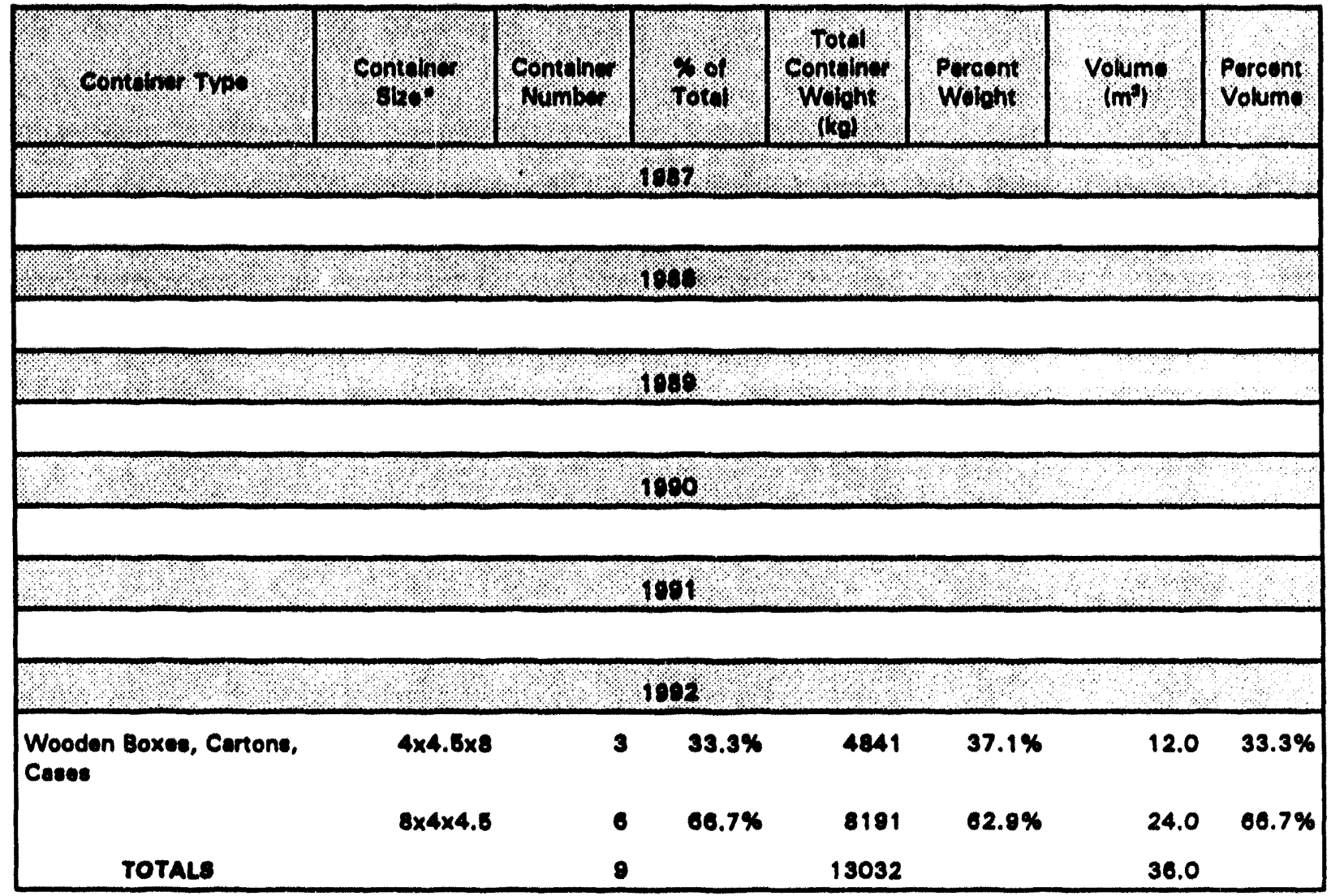

- Unlese otherwise noted, unite of dimenaiona are in ft.

- These containere hold mixed waste. 
Table 7-3. The 325A Annex Waste Summary Data for 55-Gallon Drums.

\begin{tabular}{|c|c|c|c|c|c|c|c|c|c|c|c|c|c|c|c|c|c|c|c|c|c|c|c|}
\hline 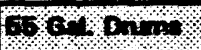 & 1010 & $101 \%$ & 121 & 1096 & 1021 & 1876 & 1976 & 1872 & rope & 1978 & 1890 & 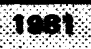 & 1802 & rest & 1eos & 1808 & roos & $10 \%$ & 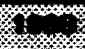 & x & 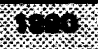 & K & 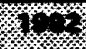 \\
\hline \multicolumn{24}{|l|}{ 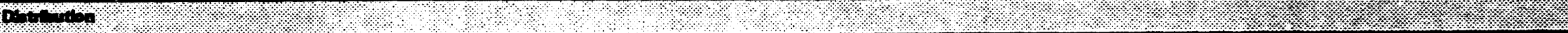 } \\
\hline TRU Count & 0 & 0 & o & 0 & 0 & 0 & 10 & $\mathbf{0}$ & 0 & 5 & 0 & $\mathbf{0}$ & 0 & o & $\mathbf{0}$ & $\mathbf{0}$ & $\mathbf{0}$ & 0 & $\mathbf{0}$ & 0 & $\mathbf{0}$ & 0 & o \\
\hline TRU Wt. (kg) & o & 0 & $\mathbf{0}$ & $\mathbf{0}$ & $\mathbf{0}$ & 0 & 3655 & 0 & 0 & 340 & o & o & 0 & $\mathbf{0}$ & 0 & $\mathbf{0}$ & 0 & $\mathbf{0}$ & $\mathbf{0}$ & $\mathbf{0}$ & o & o & $\mathbf{0}$ \\
\hline TRU Vol. $\left(\mathrm{m}^{2}\right)$ & o & 0 & 0 & o & 0 & 0 & 2.1 & 0 & 0 & 1.1 & 0 & $\mathbf{0}$ & - & 0 & 0 & 0 & 0 & 0 & $\mathbf{0}$ & $\mathbf{0}$ & 0 & $\mathbf{0}$ & $\mathbf{0}$ \\
\hline $\begin{array}{l}\text { TRu ma } \\
\text { Count }\end{array}$ & 0 & 0 & $\mathbf{0}$ & 0 & 0 & 0 & 0 & $\mathbf{0}$ & $\mathbf{0}$ & o & 0 & o & 0 & 0 & 0 & - & - & 0 & 0 & - & o & o & $\mathbf{0}$ \\
\hline $\begin{array}{l}\text { Non-TRU } \\
\text { Court }\end{array}$ & $\mathbf{0}$ & $\mathbf{0}$ & 0 & $\mathbf{0}$ & $\mathbf{0}$ & o & 0 & 10 & 20 & 0 & o & $\mathbf{0}$ & 0 & 0 & 0 & 0 & 0 & 0 & 0 & $\mathbf{0}$ & $\mathbf{0}$ & 0 & 0 \\
\hline Non-TRU Wh. & 0 & 0 & $\mathbf{0}$ & 0 & 0 & o & 0 & 3010 & 6675 & o & 0 & $\mathbf{0}$ & 0 & 0 & 0 & o & 0 & o & 0 & $\mathbf{0}$ & 0 & 0 & $\mathbf{0}$ \\
\hline $\begin{array}{l}\text { Non-TRU Vol. } \\
\left(m^{2}\right)\end{array}$ & 0 & $\mathbf{0}$ & $\mathbf{0}$ & $\mathbf{0}$ & 0 & $\mathbf{0}$ & 0 & 2.1 & 0.2 & 0 & - & 0 & $\mathbf{0}$ & $\mathbf{0}$ & 0 & $\mathbf{0}$ & $\mathbf{0}$ & $\mathbf{0}$ & $\mathbf{0}$ & 0 & 0 & $\mathbf{0}$ & $\mathbf{0}$ \\
\hline $\begin{array}{l}\text { Non-TRU MAN } \\
\text { Count }\end{array}$ & $\mathbf{0}$ & 0 & $\mathbf{0}$ & 0 & 0 & 0 & 0 & 0 & 16 & o & 0 & $\mathbf{0}$ & $\mathbf{0}$ & 0 & 0 & $\mathbf{0}$ & 0 & $\mathbf{0}$ & 0 & 0 & 0 & 0 & $\mathbf{0}$ \\
\hline \multicolumn{24}{|l|}{ 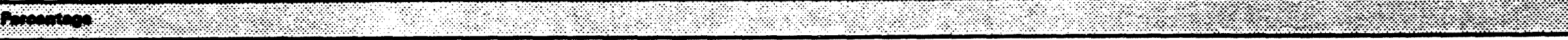 } \\
\hline XTRU Courst & & & & & & & $100 \%$ & $0 \%$ & $0 x$ & $100 x$ & & & & & & & & & & & & & \\
\hline $\begin{array}{l}\text { Esion-TAU } \\
\text { Court }\end{array}$ & & & & & & & ox & $100 \%$ & $100 x$ & $0 x$ & & & & & & & & & & & & & \\
\hline WE TRU & & & & & & & $100 \%$ & $0 x$ & $0 x$ & $100 \%$ & & & & & & & & & & & & & \\
\hline Whx Mon-TRU & & & & & & & $0 \%$ & $100 \%$ & $100 x$ & ox & & & & & & & & & & & & & \\
\hline Vol\% Tfu & & & & & & & $100 x$ & $0 \%$ & $0 x$ & $100 \%$ & & & & & & & & & & & & & \\
\hline Vols Alon-TRU & & & & & & & $0 x$ & $100 x$ & $100 x$ & $0 \times$ & & & & & & & & & & & & & \\
\hline $\begin{array}{l}\text { XMW Non- } \\
\text { TRU by Count }\end{array}$ & & & & & & & & & $52 \%$ & & & & & & & & & & & & & & \\
\hline
\end{tabular}


Table 7-4. The 325A Annex Waste Summary Data for Waste Containers Other Than 55-Gallon Drums.

\begin{tabular}{|c|c|c|c|c|c|c|c|c|c|c|c|c|c|c|c|c|c|c|c|c|c|c|c|}
\hline $\mathrm{CH} / \mathrm{Cl}=$ & 1070 & 107. & 1072 & 107\% & $10 \%$ & 1008 & 1070 & 107 & 1070 & 107\%: & $m$ & 1001 & $\operatorname{lom}$ & 100 & $10 \%$ & 10 & 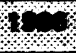 & $1 \%$ & -1 & 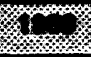 & w. & 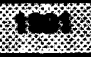 & $x$ \\
\hline $0 \%$ & & I & (3) & (1: & (1) & . & 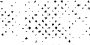 & so & 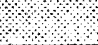 & 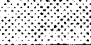 & & 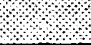 & & & $\%$ & (:) & (1) & (m) & (1) & 2 & & : & 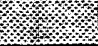 \\
\hline TreU count & 17 & 38 & 36 & 6 & 0 & 11 & 0 & 0 & 0 & 0 & o & 0 & 0 & 1 & 0 & 0 & 0 & 0 & 0 & 0 & 0 & - & 0 \\
\hline Tru we & 100 & 370 & 369 & 100 & 0 & 30401 & 0 & o & o & 0 & 0 & 0 & - & 18 & 0 & 0 & 0 & 0 & 0 & 0 & - & 0 & - \\
\hline TRU Vol. (min) & 0.3 & 0.5 & 0.5 & 0.1 & 0 & 68.0 & 0 & 0 & o & 0 & 0 & 0 & o & $<0.1$ & 0 & 0 & 0 & 0 & - & - & - & - & - \\
\hline The ImW Count & o & 0 & 0 & 0 & 0 & 0 & o & o & - & - & - & 0 & 0 & - & o & 0 & 0 & 0 & - & 0 & 0 & - & - \\
\hline Nore-TRU Count & 0 & 0 & 0 & 0 & 0 & 4 & 0 & 54 & 103 & 0 & 0 & 3 & - & 0 & o & - & 0 & 0 & - & 0 & 0 & - & - \\
\hline Non-the we wat & - & o & - & 0 & o & $\infty 007$ & 0 & 30007 & $27 m 1$ & 0 & - & 3020 & o & 0 & 0 & 0 & 0 & o & 0 & - & - & - & 13002 \\
\hline Mom-TRU Vol. (mn') & - & 0 & 0 & 0 & 0 & 13.6 & 0 & 83.1 & 92.4 & 0 & 0 & 11.3 & 0 & 0 & - & 0 & o & o & - & - & 0 & - & 300 \\
\hline $\begin{array}{l}\text { Non-TFu unv } \\
\text { Count }\end{array}$ & 0 & o & 0 & 0 & o & 0 & 0 & 0 & 23 & - & - & 0 & - & - & - & 0 & 0 & 0 & - & o & - & - & - \\
\hline rasion & & & & (?: & & 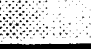 & & s & \% & . & 19 & 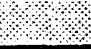 & 3. & ( & & & & 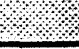 & ( & 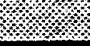 & & & I \\
\hline$x \operatorname{Tru}$ conse & $100 x$ & $100 x$ & $100 \%$ & $100 \%$ & & $73 \%$ & & $0 x$ & ox & & & ox & & $100 \%$ & & & & & & & & & $\infty x$ \\
\hline XNlor-TEU Count & $0 \%$ & $0 x$ & $0 x$ & $0 \%$ & & $27 \%$ & & $100 x$ & $100 \%$ & & & $100 \%$ & & ox & & & & & & & & & $100 x$ \\
\hline$m \times \mathrm{ThU}$ & $100 \%$ & $100 x$ & $100 \%$ & $100 \%$ & & $00 \%$ & & ox & ox & & & ox & & $100 \%$ & & & & & & & & & $\infty x$ \\
\hline WTK Man-TRU & $0 x$ & $0 x$ & $0 x$ & $0 \%$ & & $17 \%$ & & $100 \%$ & $100 \%$ & & & $100 \%$ & & $0 x$ & & & & & & & & & $100 \%$ \\
\hline Vol* TFU & $100 \%$ & $100 \%$ & $100 \%$ & $100 \%$ & & 83\% & & ox & $0 \%$ & & & ox & & $100 \%$ & & & & & & & & & ox \\
\hline Valx Mon-TRU & $0 x$ & $0 x$ & ox & $0 \%$ & & $17 \%$ & & $100 \%$ & $100 \%$ & & & $100 x$ & & $0 x$ & & & & & & & & & $100 x$ \\
\hline $\begin{array}{l}\text { Xanw Mon-TRU } \\
\text { by Coume }\end{array}$ & & & & & & & & & $14 \%$ & & & & & & & & & & & & & & \\
\hline
\end{tabular}


Table 7-5. The 325A Annex TRU Waste in 55-Gallon Drums: Drum Count by Storage Location.

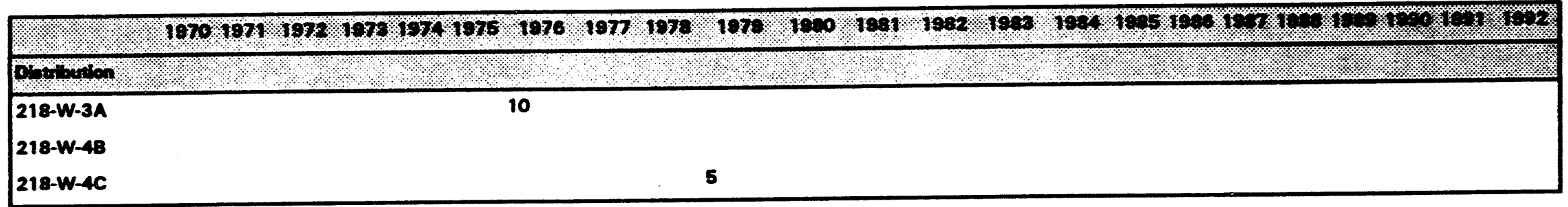

Table 7-6. The 325A Annex TRU Waste in Containers Other Than 55-Gallon Drums: Container Count by Storage Location.

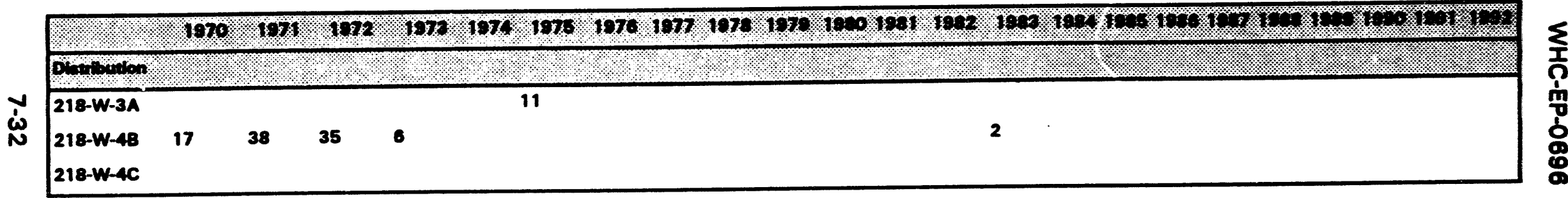

Table 7-7. Distribution and Percentage of TRU 55-Gallon Drum Physical Contents by Storage Location - 218-W-3A.

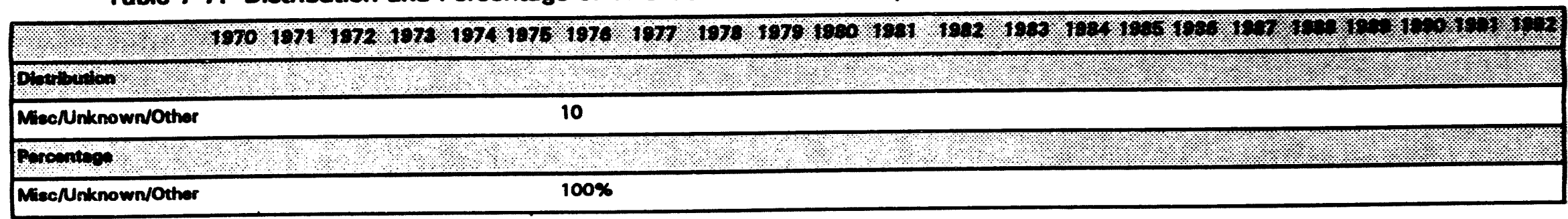


Table 7-8. Distribution and Percentage of TRU 55-Gallon Drum Physical Contents by Storage Location - 218-W-4C.

\begin{tabular}{|c|c|c|c|c|c|c|c|c|c|}
\hline 4. & 1970 1971 $19721973 / 974197519761977 \quad 1978$ & 1979.1080 & 1981 & 1982 & 1989 & 198\%. & 19081090 & $19 \%$ & $1=10=10 \% 1 \% 1 \%$ \\
\hline Dintibution. & ২ & , & & & & $1 .:$ & & & $=0$ \\
\hline Misc/Unknown/Othor & & 5 & & & & & & & \\
\hline Proconteos: & & & & & & & & & \\
\hline Misc/Nnknown/Other & & $100 \%$ & & & & & & & \\
\hline
\end{tabular}

Table 7-9. Distribution and Percentage of TRU Containers Other than 55-Gallon Drums Physical Contents by Storage Location - 218-W-3A.

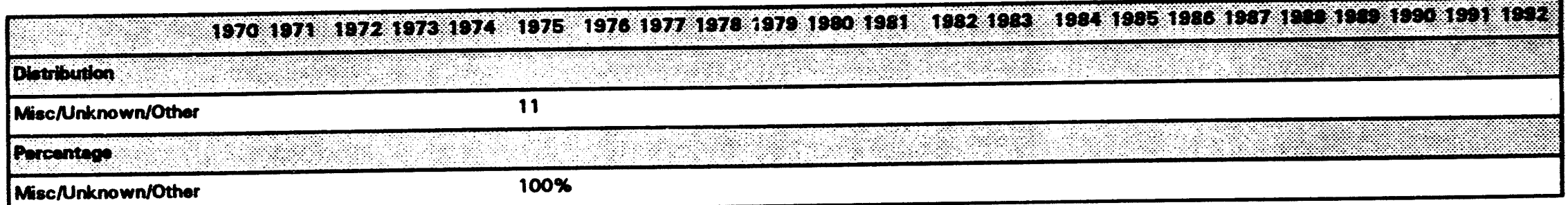


Table 7-10. Distribution and Percentage of TRU Containers Other than 55-Gallon Drums Physical Contents by Storage Location - 218-W-4B.

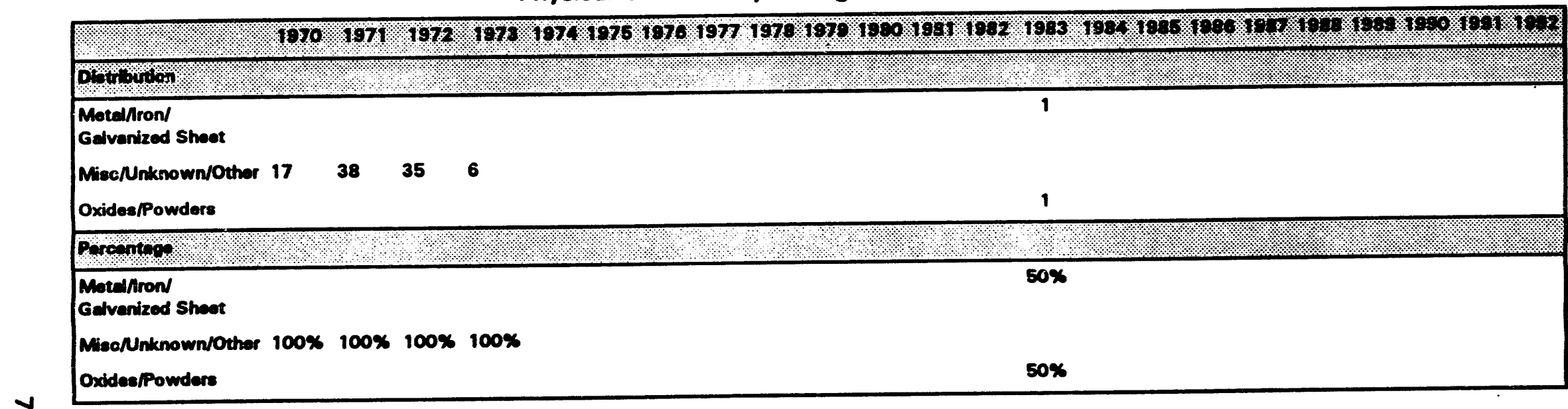

Table 7-11. The 325A Annex TRU Waste in 55-Gallon: Total Grams TRU by Storage Location.

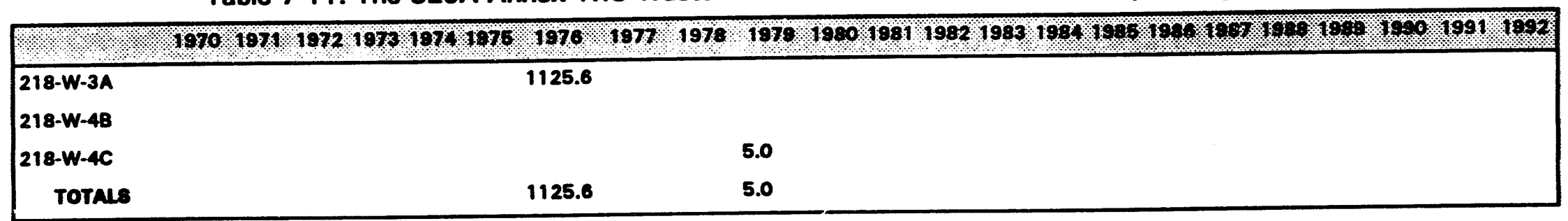


Table 7-12. The 325A Annex TRU Waste in Containers Other than 55-Gallon Drums: Total Grams TRU by Storage Location.

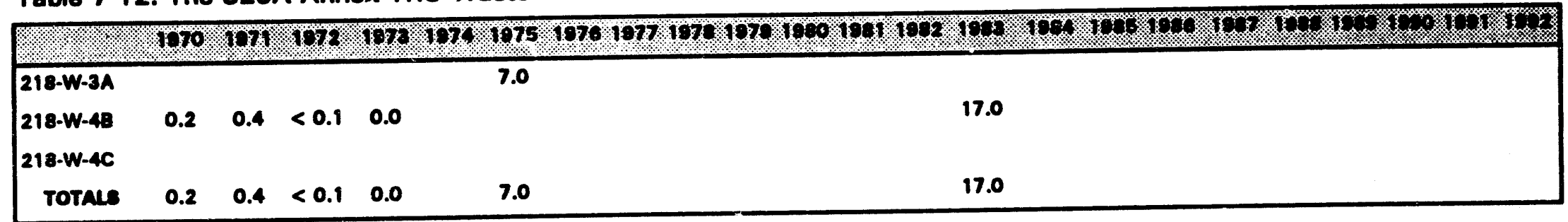


Table 7-13. Isotopes in 55-Gallon Drums for the 325A Annex for 1970-1992.

\begin{tabular}{|c|c|c|c|}
\hline (3.1.0. & $7 x_{3} ; 1$ & Q.3.1. & $5 \%, 1,10 \%$ \\
\hline${ }^{232} \mathrm{Th}$ & $1.41 \times 10^{10} \mathrm{Y}$ & 22600 & $a, r$ \\
\hline $233 u$ & $1.592 \times 10^{6} Y$ & 4.0 & $a, r$ \\
\hline Pu total & 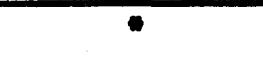 & 1126.0 & $*$ \\
\hline
\end{tabular}

$Y=$ Years

* = Varies according to isotope.

Table 7-14. Isotopes in Other Containers for the 325A Annex for 1970-1992.

\begin{tabular}{|c|c|c|c|}
\hline $1042 \%$ & 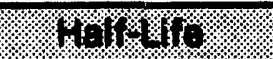 & $01811 \%(1)$ & 680131309 \\
\hline${ }^{137} \mathrm{Ba}$ & $2.551 M$ & 30.087 & $r$ \\
\hline${ }^{137} \mathrm{Cs}$ & $30.17 Y$ & 30.087 & $\beta, \gamma$ \\
\hline 129 & $1.6 \times 10^{7} Y$ & 0.5 & $\beta, \gamma$ \\
\hline${ }^{237} \mathrm{~Np}$ & $2.14 \times 10^{6} Y$ & 0.011 & $a, r$ \\
\hline${ }^{100} \mathrm{Rh}$ & $130 \mathrm{M}$ & 1.01 & $\beta, \gamma$ \\
\hline${ }^{106} R u$ & 3670 & 1.01 & $\beta$ \\
\hline${ }^{\circ 0} \mathrm{Sr}$ & $28.8 Y$ & 8200 & $\beta$ \\
\hline 232Th & $1.41 . \times 10^{10} \mathrm{Y}$ & 30.0 & $a, r$ \\
\hline${ }^{233} \mathrm{U}$ & $1.592 \times 10^{5} Y$ & 17.0 & $a, r$ \\
\hline soY & $49.7 M$ & 30.0 & $a$ \\
\hline Pu total & 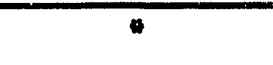 & 7.5948 & $*$ \\
\hline
\end{tabular}

$Y=$ Years

$D=$ Days

$M=$ Minutes

- = Varies according to isotope. 
Table 7-15. Summary of Information from Sol id Waste Burial Records (SWBR) for TRU Wastes with Hazardous Contents from 325A Hot Cell Annex by PNL.

\begin{tabular}{|c|c|c|c|c|c|c|}
\hline Accepted. & (1. & Burlal Recard' : : & $\begin{array}{l}\text { Hazarous } \\
\text { const tuients }\end{array}$ & $\begin{array}{l}\text { yestes } \\
\text { codes }\end{array}$ & mol & 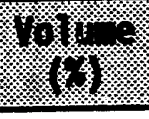 \\
\hline $08 / 11 / 70$ & $325-70-008$ & & Resin & & & \\
\hline $08 / 12 / 70$ & $325-70-209$ & & Resin & & & \\
\hline $10 / 16 / 70$ & $325-70--216-038$ & & Resin & & & \\
\hline $11 / 01 / 72$ & $325-73-011$ & 325-A-BNW-72-025 & Resin & & & \\
\hline $02 / 14 / 73$ & $325-73-025$ & 325-A-BNW-73-008 & Resin & & & \\
\hline $02 / 14 / 73$ & $325-73-026$ & 325-A-BNW-73-009 & Resin & & & \\
\hline $02 / 15 / 73$ & $325-73-027$ & 325-A-BNH-73-010 & Resin & & & \\
\hline $02 / 15 / 73$ & $325-73-028$ & 325-A-BNH-73-011 & Resin & & & \\
\hline
\end{tabular}


Table 7-16. Summary of Information from Solid Waste Burial Records (SWBR) for Low-Level Wastes with Hazardous Contents from 325A Hot Cell Annex by PNL. (3 sheets)

\begin{tabular}{|c|c|c|c|c|c|c|}
\hline iceepled & 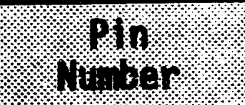 & Burglifl Record: & gonsuritivents: & Modres. & $4+4)^{2}$ & $(9)=2$ \\
\hline $04 / 07 / 70$ & $325-70-010$ & & Resin & & & \\
\hline $04 / 05 / 71$ & $325-71-336$ & $B N W-325-A-71-016$ & Resin & & & \\
\hline $04 / 07 / 71$ & $325-71-340$ & BNW-325-A-71-020 & Resin & & & \\
\hline $04 / 08 / 71$ & $325-71-343$ & BNW-325-A-71-023 & Resin & & & \\
\hline $12 / 07 / 71$ & $325-72-014$ & BNH-325-A-71-049 & Resin & & & \\
\hline $04 / 20 / 72$ & & BNH-325-A-72-022-006 & Resin & & & \\
\hline $01 / 29 / 73$ & $325-73-023$ & 325-A-BNH-73-006 & Resin & & & \\
\hline $01 / 31 / 73$ & $325-73-024$ & 325-A-BNH-73-007 & Resin & & & \\
\hline $02 / 20 / 73$ & $325-73-029$ & 325-A-BNW-43-012 & Resin & & & \\
\hline $03 / 15 / 73$ & $325-73-037$ & $325-A-B N H-73-020$ & Resin & & & \\
\hline $07 / 09 / 73$ & $325-74-005$ & 325-A-BNW-73-034 & Resin & & & \\
\hline $07 / 09 / 73$ & $325-74-006$ & 325-A-BNW-73-035 & Resin & & & \\
\hline $07 / 10 / 73$ & $325-74-007$ & 325-A-BNW-73-036 & Resin & & & \\
\hline $07 / 24 / 73$ & $325-74-016$ & 325-A-BNH-73-045 & Resin & & & \\
\hline $09 / 17 / 73$ & $325-74-017$ & 325-A-BNW-73-048 & Resin & & & \\
\hline $10 / 09 / 73$ & $325-74-024$ & 325-A-BNW-73-054 & Resin & & & \\
\hline $02 / 25 / 74$ & $325-74-034$ & $325-A-B N W-74-001$ & Resin & & & \\
\hline $03 / 06 / 74$ & $325-74-039$ & 325-A-BNW-74-006 & Resin & & & \\
\hline
\end{tabular}


Table 7-16. Summary of Information from Solid Waste Burial Records (SWBR) for Low-Level Wastes with Hazardous Contents from 325A Hot Cell Annex by PML. (3 sheets)

\begin{tabular}{|c|c|c|c|c|c|c|}
\hline $\begin{array}{l}\text { Oater } \\
\text { iccepted }\end{array}$ & $\begin{array}{l}\text { Pin } \\
\text { romber }\end{array}$ & $\begin{array}{l}\text { Burfial Recond } \\
\text { Number }\end{array}$ & $\begin{array}{l}\text { Hazardous } \\
\text { constituents }\end{array}$ & $\begin{array}{l}\text { Haste: } \\
\text { codes: }\end{array}$ & 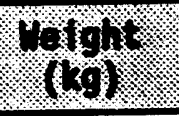 & 46 \\
\hline $05 / 16 / 74$ & $325-74-050$ & 325-A-BNW-74-017 & Resin & & & \\
\hline $05 / 17 / 74$ & $325-74-051$ & $325-A-B N M-74-018$ & Resin & & & \\
\hline $05 / 22 / 74$ & $325-74-057$ & 325-A-BMN-74-024 & Resin & & & \\
\hline $07 / 18 / 74$ & $325-75-013$ & 325-A-BNW-74-042 & Resin & & & \\
\hline $02 / 27 / 75$ & $325-75-48$ & 325-A-BNH-75-015 & Resin & & & \\
\hline $03 / 05 / 75$ & $325-75-049$ & 325-A-BMH-75-016 & Resin & & & \\
\hline $03 / 05 / 75$ & $325-75-050$ & 325-A-BNU-75-017 & Resin & & & \\
\hline $03 / 07 / 75$ & $325-75-054$ & 325-A-BNH-75-021 & Resin & & & \\
\hline $03 / 10 / 75$ & $325-75-056$ & 325-A-BNH-75-023 & Resin & & & \\
\hline $03 / 11 / 75$ & $325-75-058$ & 325-A-BNW-75-025 & Resin & & & \\
\hline $05 / 07 / 75$ & $325-75-71$ & 325-A-BNU-75-031 & Resin & & & \\
\hline $05 / 07 / 75$ & $325-75-72$ & 325-A-BNU-75-032 & Resin & & & \\
\hline $06 / 20 / 74$ & 325-75-93. & 325-A-BNU-75-052 & Resin & & & \\
\hline $06 / 23 / 75$ & $325-75-94$ & 325-A-BNW-75-053 & Resin & & & \\
\hline $06 / 23 / 75$ & $325-75-95$ & 325-A-BNW-75-054 & Resin & & & \\
\hline $04 / 02 / 76$ & $325-76-050$ & 325-A-BNU-76-028 & Resin & & & \\
\hline $04 / 02 / 76$ & $325-76-051$ & $325-A-B N H-76-029$ & Resin & & & \\
\hline $01 / 17 / 77$ & $325-77-018$ & $325-A-B N W-77-001$ & Resin & & & \\
\hline
\end{tabular}


Table 7-16. Summary of Information from Solid Waste Burial Records (SWBR) for Low-Level Wastes with Hazardous Contents from 325A Hot Cell Annex by PNL. (3 sheets)

\begin{tabular}{|c|c|c|c|c|c|c|}
\hline Ocrepted & Plilo. & Burtal Record & 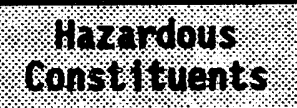 & godes & (1) & 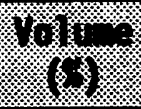 \\
\hline $01 / 17 / 77$ & $325-77-019$ & $325-A-B N W-77-002$ & Resin & & & \\
\hline $01 / 19 / 77$ & $325-77-020$ & $325-A-B N W-77-003$ & Resin & & & \\
\hline $01 / 26 / 77$ & $325-77-024$ & 325-A-BNW-77-004 & Resin & & & \\
\hline $01 / 21 / 77$ & $325-77-021$ & 325-A-BNW-77-003A & Resin & & & \\
\hline $01 / 21 / 77$ & $325-77-022$ & 325-A-BNH-77-003B & Resin & & & \\
\hline $06 / 15 / 77$ & $325-77-029$ & 325-BNW-77-062 & $\begin{array}{l}\text { Lead(sheets) } \\
\text { Lead (bricks) }\end{array}$ & & 1361 & \\
\hline $08 / 24 / 77$ & $325-77-030$ & 325-A-BNW-77-003 & Lead & & & \\
\hline $05 / 25 / 78$ & $325-78-004$ & 325-A-BNW-78-004 & Lead (scraps) & & & \\
\hline $08 / 11 / 78$ & $325-78-006$ & 325-A-BNW-78-06 & Lead & & & \\
\hline $10 / 10 / 78$ & $325-78-009$ & 325-A-PNL-78-09 & Welding lead & & & \\
\hline $05 / 25 / 79$ & $325-79-012$ & 325-A-PNL-79-11 & Resin & & & \\
\hline $05 / 30 / 79$ & $325-79-013$ & 325-A-PNL-79-12 & Resin & & & \\
\hline $06 / 20 / 79$ & $325-79-025$ & 325-A-PNL-79-24 & Resin & & & \\
\hline
\end{tabular}




\subsection{CHARACTERIZATION OF RETRIEVABLY STORED SOLID WASTE GENERATED BY $323 B$ HOT CELLS ANNEX}

The information found in this section is based primarily on data from the Solid Waste Information and Tracking System (SWITS) database. This database, which incorporated the older R-SWIMS database, is used to track information on radioactive and other wastes stored or disposed at the Hanford Site. Transuranic solid waste packages have been tracked since 1970. Changes in the requirements and regulations governing radioactive wastes have left their mark in the quantity and quality of the data contained in this database. Caveats are included in the text to alert the reader to changes that may affect the interpretation of the data provided.

The 325B west wing, with its six interconnecting hot cells, has been operated by both PNL and Westinghouse Hanford during the last 30 years. Custody was transferred from PNL to Westinghouse Hanford in 1970, and Westinghouse Hanford operated 325B until 1987 when custody of the facility was returned to PNL. Tables 8-1 through 8-5 present a summary of the TRU waste generated by Westinghouse Hanford between 1970 and 1985. Table 8-6 presents the TRU waste generation summary for PNL at the 325B Annex.

Appendix D contains the original SWITS queries and computer runs for the Westinghouse Hanford 325B Annex. Appendix E contains the original SWITS queries and computer runs for PNL 325B Annex. Since the only records retrieved for the PNL 325B Annex are from 1970, Westinghouse Hanford 325B data was used to generate a majority of the tables and figures in this section. Because of the large number of figures and tables, they are placed at the end of the chapter.

\subsection{SUMMARY OF 325B WASTE GENERATION}

A summary of the waste generated at the 325B Annex by Westinghouse Hanford can be found in Table 8-1. This is waste that was not included in Section 5.0 and 6.0, Westinghouse Hanford and PNL Building 325 waste, but was entered into the database as 325B Annex waste. This table is organized by container type and year.

\subsubsection{Waste Stored in 55-Gallon Steel Drums}

According to the SWITS database, no TRU waste was generated at the 325B Annex by Westinghouse Hanford or PNL and stored in 55-gal drums.

\subsubsection{Waste Stored in Containers Other Than 55-Gallon Steel Drums}

The only container type used to contain the solid waste generated at the 325B Annex was the 1-gal metal drum. Some items were disposed of and stored without additional packaging such as self-contained equipment. These items also are included in the "Other Container" category. 
Table 8-2 summarizes the waste data for containers of radioactive waste other than 55-gal drums for Westinghouse Hanford that were generated at the 325B Annex between 1970 and 1992. This table indicates the number, total weight and total volume of TRU waste stored in other containers. Figures 8-1 through 8-3 present these data graphically.

8.1.2.1 Number of Other Contalners. Between 1970 and 1985, 355 other containers of radioactive waste were generated at the 325B Annex by Westinghouse Hanford. These containers all contain TRU waste. Figure 8-1 is a graph of the number of other containers of TRU waste generated at the 325B Annex by Westinghouse Hanford annually. The largest number of other containers containing TRU waste was generated in 1971 with 80 containers. This is the result of an inordinately large number of 1-gal metal drums, barrels, or kegs. In 1970, twenty-five 1-gal containers of TRU waste was generated at the 325B Annex by PNL.

According to interviews with 325B personnel, the 325B Hot Cells Annex was mainly an analytical facility performing two operations using spent fuel. One operation was spent fuel burnup analyses which involved determining how long fuel had been in the reactor and how much fuel had been used. The second operation was hydrothermal testing. In this operation, spent fuel was received from the 324 Building, loaded into autoclaves, reacted, down loaded and disposed of or dried and disposed of. The hydrothermal testing work was part of the Basalt Waste Isolation Project during the mid-1980's. These two operations should account for a majority of waste generated at the 325B Annex.

8.1.2.2 Weight. In the period betwieen 1970 and 1985, approximately $916 \mathrm{~kg}$ of radioactive solid waste was generated at the 325B Annex by Westinghouse Hanford and placed in containers other than 55-gal drums. Figure 8-2 depicts the weight of TRU stored in containers other than 55-gal drums on an annual basis. In 1970, an additional $75 \mathrm{~kg}$ of TRU waste was generated at the 325B Annex by PNL.

The weight totals are approximations because individual container weights were not required prior to 1977. During an update of the R-SWIMS database in the mid-1980's, all waste containers without recorded weights were assigned standard values. For 325B Hot Cells Annex data, 1-gal cans appear to have been given a standard weight of $2.6 \mathrm{~kg}$ for 1970 through 1972. The heaviest weight for a 1 -gal can was recorded in 1977 as $4.3 \mathrm{~kg}$ compared to the average weight per container of $2.5 \mathrm{~kg}$ for 1970 to 1992.

8.1.2.3 Volume. The volume of waste generated at the 325B Annex by Westinghouse Hanford and packaged in containers other than 55-gal drums during the period from 1970 through 1985 is less than $2 \mathrm{~m}^{3}$. Transuranic wastes account for all of the total volume. Figure 8-3 shows annual volumes of TRU waste generated by the 325B Annex and packaged in containers other than 55-gal drums. In 1970, $0.1 \mathrm{~m}^{3}$ of TRU waste was generated at the 325B Annex by PNL.

The volume of TRU waste generated at 325B follows a pattern similar to the number of containers of TRU waste generated at 325B. 


\subsection{WASTE CONTANIERS}

\section{2:1 TRU Waste Containars}

Table 8-1 provides a summary of the TRU waste generated annually at the 325B Annex by Westinghouse Hanford sorted by container type. The greatest number of containers are 1-gal metal cans, $91 \%$ by count for both PNL and Westinghouse Hanford.

In December 1975, the 325B facility had just resumed operations after extensive modifications. This could explain the lack of data for 1974 and 1975. The typical mid1970's operations included analytical analysis of irradiated mixed oxides fuels and fuel cladding samples such as follows:

- Analytical woighing

- Sample dissolution

- Sample dilution

- Aliquoting

- Emission spectrographic analysis

- Titrimetric analysis

- Solvent extraction

- Ion exchange separations.

Fuel waste from the burnup analysis was solidified and disposed of as a salt cake in 1-gal buckets. Fuel waste from hydrothermal testing was disposed of in a powder form. Personnel from the 325B Annex indicated that there is a backlog of hydrothermal testing waste that was never disposed of.

\subsubsection{Non-TRU Waste Containors}

According to the SWITS database, the 325B Annex generated no non-TRU waste either under PNL or Westinghouse Hanford from 1970 to 1992.

\subsection{TRU WASTE STORAGE LOCATIONS}

Table 8-3 provides the storage location for TRU waste packaged in 1-gal containers by year. All the 3258 Annex waste by Westinghouse Hanford or PNL is stored in the caissons in burial ground 218-W-4B.

\subsection{PHYSICAL CONTENTS OF TRU WASTE CONTAINERS}

The physical contents of the 1-gal containers are shown in Tables 8-4. The top portion of this table indicates the number of containers for which a given component is listed; the bottom part of the table indicates the percentage of the total drums generated at the 
328B Annex in that burial location that contain the listed constituent. Some of the more interesting physical contents include fual pins or rods in 1983, graphite in 1980, and rubber in 1983 and 1984.

It should be noted that prior to 1978, phyaical contents were not required to be listed on the burial records. A great many of the contents on early records are liated only as "Miscellanoous."

\subsection{RADIOLOGICAL DESCRIPTION OF 3285 8OLID WASTE}

Table 8-5 shows the number of grams of transuranics contained in waste stored in 1-gal containers cans each year by Westinghouse Hanford. Figure 8-4 shows the total amount of transuranics present in waste peckages from the 325B Annex by Westinghouse Hanford. It should be noted that not all SWITS records for TRU Waste containers list the number of grams of TRU, particularly in the early 1970's.

\subsection{RADIOACTIVE I8OTOPES PRESENT IN $328 B$ SOLID WASTE}

Information on radioactive isotopes was obtained from the SWITS database and from the 325B Safety Analysis Report (SAR) Supplement (Anderson and Hanthorn 1975). Isotopes known to be in the waste stream are presented in Table 8-7.

\subsection{HAZARDOUS CONSTITUENTS OF SOLID WASTE GENERATED BY $328 B$ HOT CELLS ANNEX}

This section provides a review of the data on the hazardous components known or suspected to be in the solid waste stream from Westinghouse Hanford and PNL 325B Annex Hot Cells. Information was gathered from several sources, which include the SWITS database, SWBRs, personnel interviews, an internal report examining hazardous components of solid waste, Inventory of Chemicals Used at Hanford Site Production Plants and Support Operations (Klem 1990), and Unstable and Reactive Chemicals in Transuranic Retrievable Waste at the Hanford Site (Reddinger 1992).

\subsubsection{Chemical Inventories}

Table 5-22, discussed previously in Section 5.7.1, provides a list of chemicals used at the 325 Building Analytical Laboratory from 1953 to 1976. This list was developed by Klem (1990) to support waste characterization efforts for the single shell tanks. Though this list characterizes chemicals used in the Analytical Laboratory, it does not specify which of these chemicals may have been used in the 325B Hot Cells Annex. 


\subsubsection{8olld Waste Information and Traoking Syotom (SWITs)}

The hazardous constituent field in the SWITS databese was searched for all containers from Westinghouso Hanford and PNL $328 B$ Hot Cells Annax that contained radioactive mixed waste. This search indicated that no mixed waste containers from the 328B Hot Cells Annex were sent to the caissons.

\subsubsection{Build Records}

The SWBR or SWSDR for a given container is the source of waste container information abstracted for the SWITS database. Often these records will have more detalled information on the hazardous components of waste containers than is found in SWITS. Additional data can also be found on the supplementary forms often attached to the SWBR of SWSDR. These supplementary documents include Uniform Hazardous Waste Manifests, Contents Inventory Sheets, NRC 741 Forms, and Storege/Disposal Approval Records (SDARS).

A thorough search of these microfilmed records verifies the results of the SWITS database search, which indicated that no mixed waste containers from the Westinghouse Hanford and PNL 328B Hot Cells Annex were sent to the solid waste burial grounds.

\subsubsection{Interviows with 328 Bulling Parsonnel}

From the information gained in interviews with 325 Building personnel, a list of known or suspected hazardous components in the solid waste stream has been compiled. This list of components can be found in Section 5.7.4. As this list applies to the entire 325 Building Analytical Laboratory, it is expected that some of the constituents may be part of the Hot Cell waste stream from the $325 B$ Annex as well. 
Flgure 8-1. Other Conteiner Waste Count Generated by the Westinghouse Hanford 325B Annex Annually.

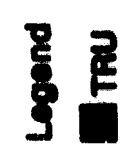

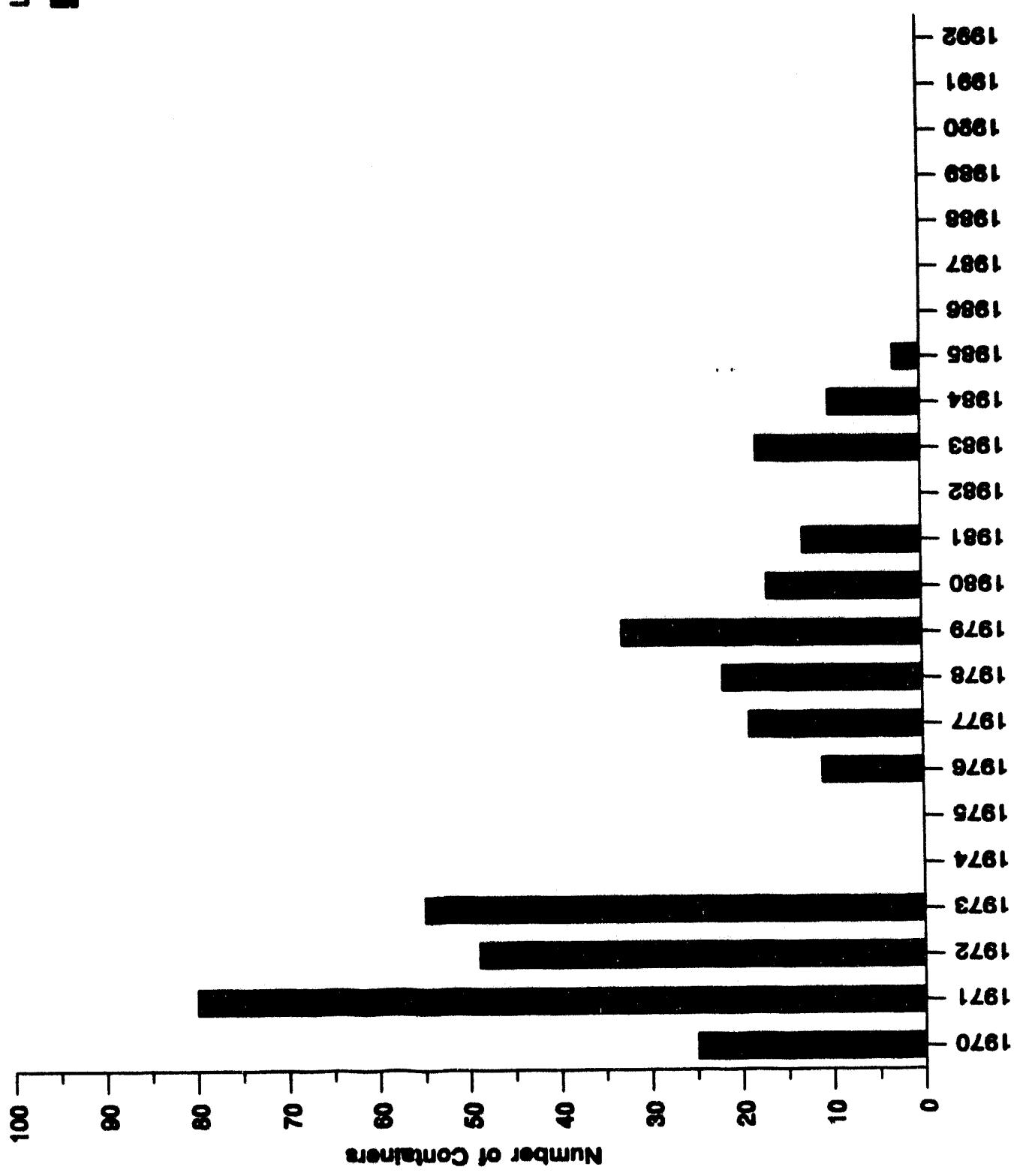


Figure 8-2. Weight of Other Containers Generated by the Westinghouse Hanford 325B Annex Annually.

홍로

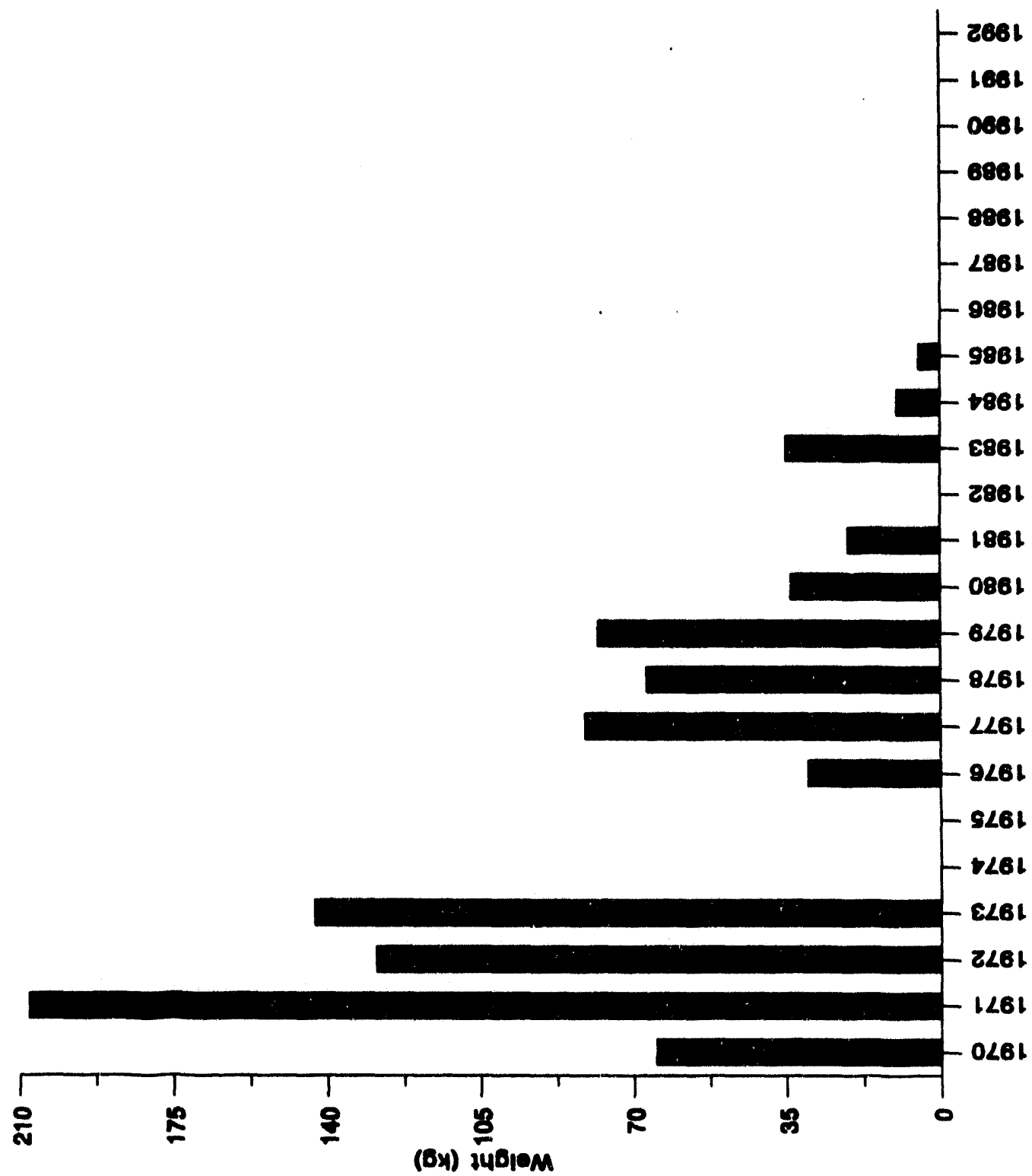




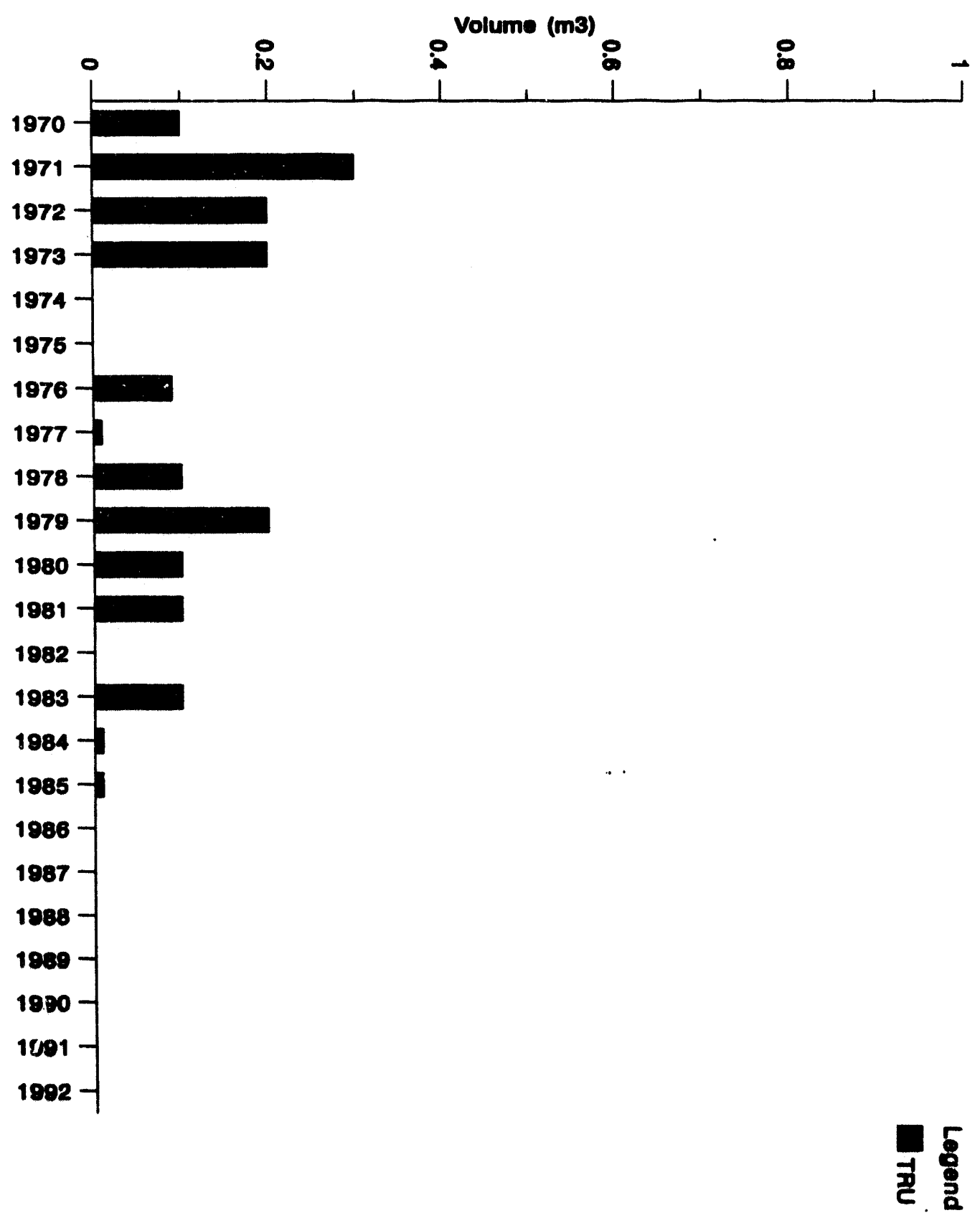

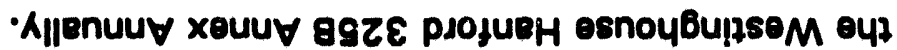

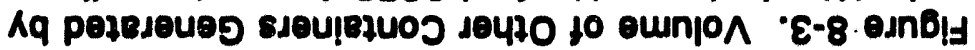


Figure 8-4. Grams of TRU Waste Generated by the Westinghouse Hanford 325B Annex Annually.

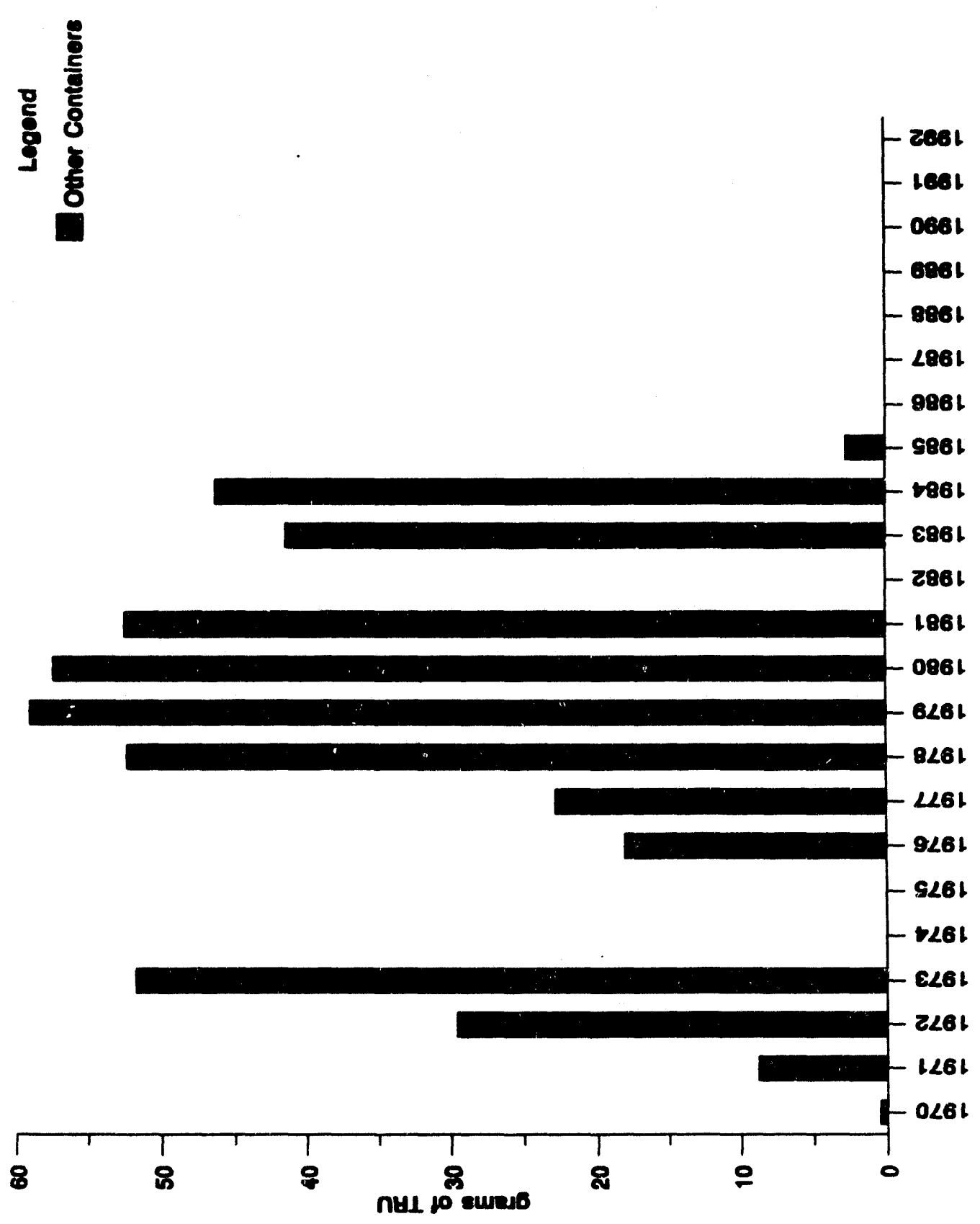




\section{WHC-EP-0696}

Table 8-1. TRU Waste Generated at the Westinghouse Hanford 325B Annex by Container Type and Year. (3 sheets)

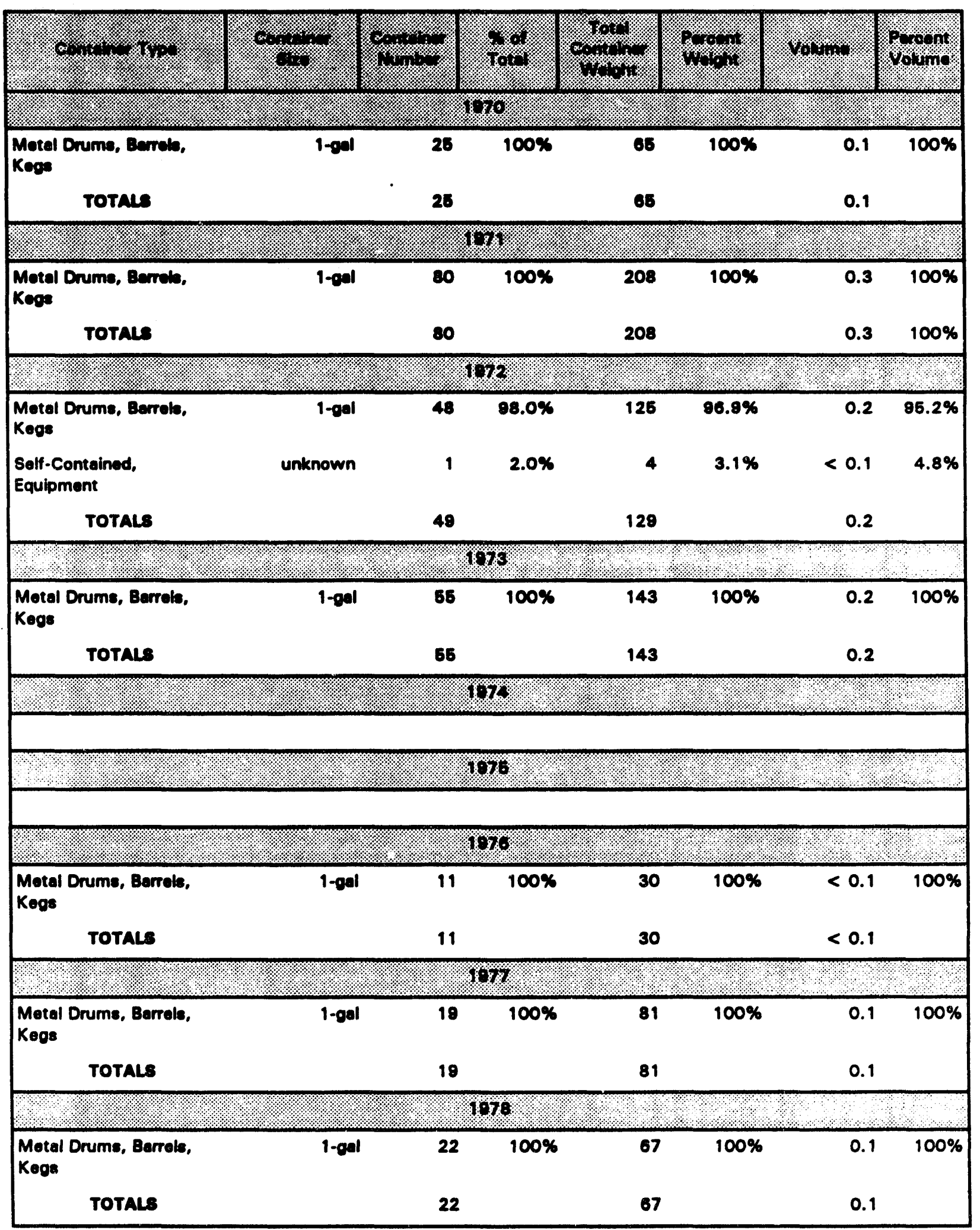




\section{WHC-EP-0696}

Table 8-1. TRU Waste Generated at the Westinghouse Hanford 325B Annex by Container Type and Year. (3 sheets)

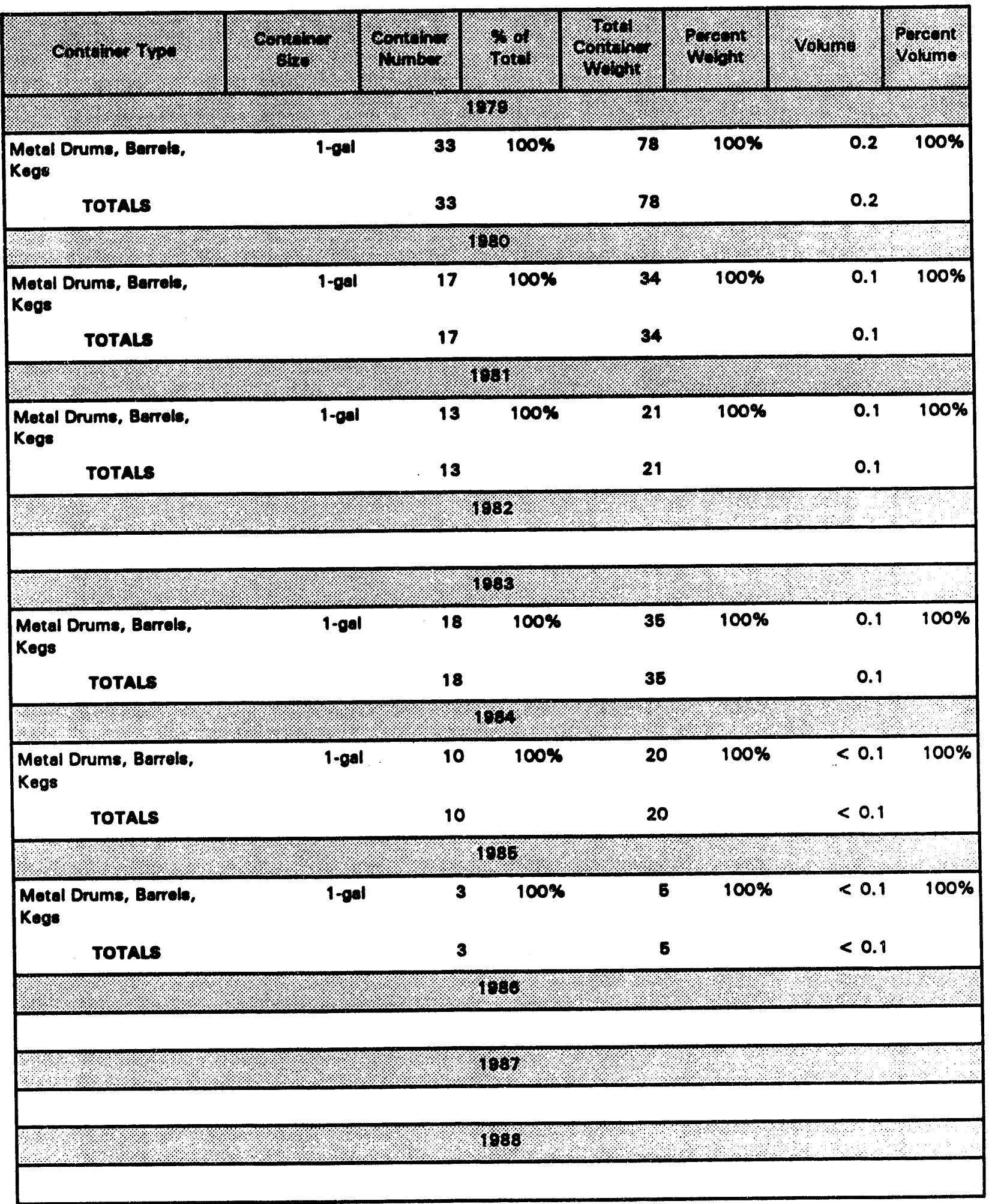


$2 l-8$

1013

1.1 .1

$\times 1.13$

\section{8}

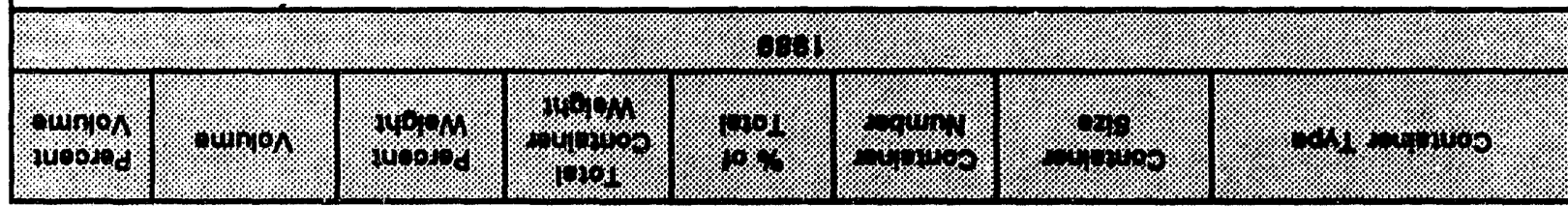

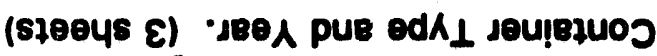

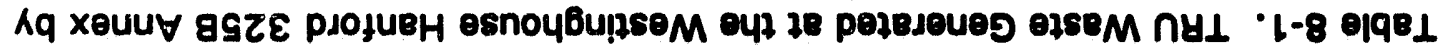


Table 8-2. The 325B Annex Westinghouse Hanford Waste Summary Data for Waste Containers Other Than 55-Gallon Drums.

\begin{tabular}{|c|c|c|c|c|c|c|c|c|c|c|c|c|c|c|c|c|c|c|c|c|c|c|c|}
\hline $0+1,00+1+101$ & 1870 & 107 & $12 \%$ & 1078 & 101 & 1076 . & 1170 & $10 \%$ & 100 & $0 \%$ & 1000 & 1001 & $100 \%$ & $100 \%$ & $108 \%$ & $16=$ & 10 & I & 1 & 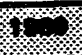 & (1. & $10 \%$ & W \\
\hline \multicolumn{24}{|c|}{$0+300-1 \% 4 \%$} \\
\hline TRU Count & 25 & 80 & 49 & 65 & 0 & 0 & 11 & 19 & 22 & 33 & 17 & 13 & 0 & 18 & 10 & 3 & 0 & 0 & 0 & 0 & 0 & 0 & 0 \\
\hline TRU Wt. (kg) & 65 & 200 & 129 & 143 & 0 & 0 & 30 & 81 & 67 & 78 & 34 & 21 & 0 & 36 & 20 & 5 & 0 & $\mathbf{0}$ & $\mathbf{0}$ & $\mathbf{0}$ & o & 0 & 0 \\
\hline TRU Vol. (m?) & 0.1 & 0.3 & 0.2 & 0.2 & 0 & 0 & $<0.1$ & 0.1 & 0.1 & 0.2 & 0.1 & 0.8 & 0 & 0.1 & $<0.1$ & $<0.1$ & 0 & 0 & 0 & $\mathbf{0}$ & 0 & 0 & 0 \\
\hline IFU MNW Count & 0 & 0 & 0 & 0 & 0 & 0 & 0 & 0 & 0 & 0 & 0 & 0 & 0 & 0 & $\mathbf{0}$ & 0 & $\mathbf{0}$ & 0 & o & $\mathbf{0}$ & 0 & 0 & o \\
\hline Non-TRU Count & $\mathbf{0}$ & 0 & 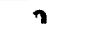 & 0 & 0 & 0 & 0 & 0 & $\mathbf{0}$ & 0 & 0 & 0 & 0 & 0 & 0 & 0 & 0 & 0 & $\mathbf{0}$ & 0 & 0 & 0 & 0 \\
\hline Non-Tru Wh. & 0 & 0 & 0 & 0 & 0 & 0 & 0 & 0 & 0 & 0 & 0 & 0 & 0 & 0 & 0 & 0 & 0 & 0 & 0 & 0 & 0 & 0 & 0 \\
\hline Non-TRU Vol. & 0 & 0 & 0 & 0 & 0 & $\mathbf{0}$ & 0 & 0 & $\mathbf{0}$ & 0 & 0 & $\mathbf{0}$ & 0 & 0 & 0 & 0 & 0 & 0 & $\mathbf{0}$ & $\mathbf{0}$ & 0 & 0 & 0 \\
\hline Non-TRU MW & & & $\mathbf{0}$ & 0 & 0 & $\mathbf{0}$ & $\mathbf{0}$ & $\mathbf{0}$ & 0 & - & & & o & o & $\mathbf{0}$ & o & - & 0 & o & o & 0 & $\mathbf{0}$ & 0 \\
\hline $\begin{array}{l}\text { Non-TFu MN } \\
\text { Count }\end{array}$ & $\mathbf{0}$ & 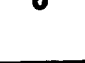 & 0 & 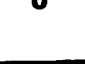 & 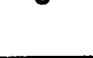 & & & & & & & & & & & & & & & & & & \\
\hline \multicolumn{24}{|l|}{ Count } \\
\hline \multicolumn{24}{|l|}{ XTRU Count } \\
\hline \multicolumn{24}{|l|}{$\begin{array}{l}\text { XNon-TFU } \\
\text { Count }\end{array}$} \\
\hline \multicolumn{24}{|l|}{$W t \times$ TPU } \\
\hline \multicolumn{24}{|l|}{ Wh\% Non-TPU } \\
\hline \multicolumn{24}{|l|}{ Vol\% TRU } \\
\hline \multicolumn{24}{|l|}{$\begin{array}{l}\text { Vol\% TFU } \\
\text { Vol\% Non-TRU }\end{array}$} \\
\hline $\begin{array}{l}\text { TMW Non-TRU } \\
\text { by Couns }\end{array}$ & & & & & & & & & & & & & & & & & & & & & & & \\
\hline
\end{tabular}


Table 8-3. The 325B Annex Westinghouse Hanford TRU Waste in Containers Other Than 55-Gallon Drums: Container Count by Storage Location.

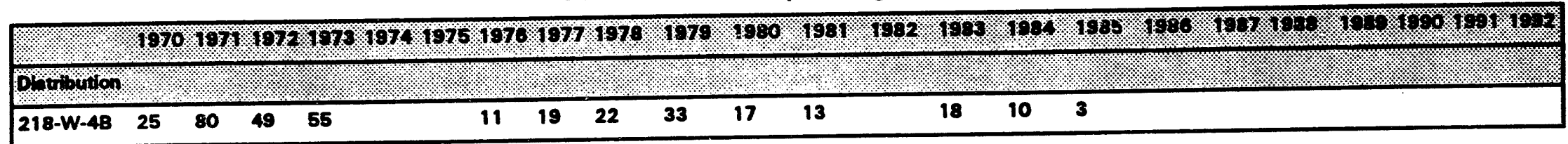


Table 8-4. Distribution and Percentage of TRU Containers Other than 55-Gallon Drum Physical Contents by Storage Location - 218-W-4B.

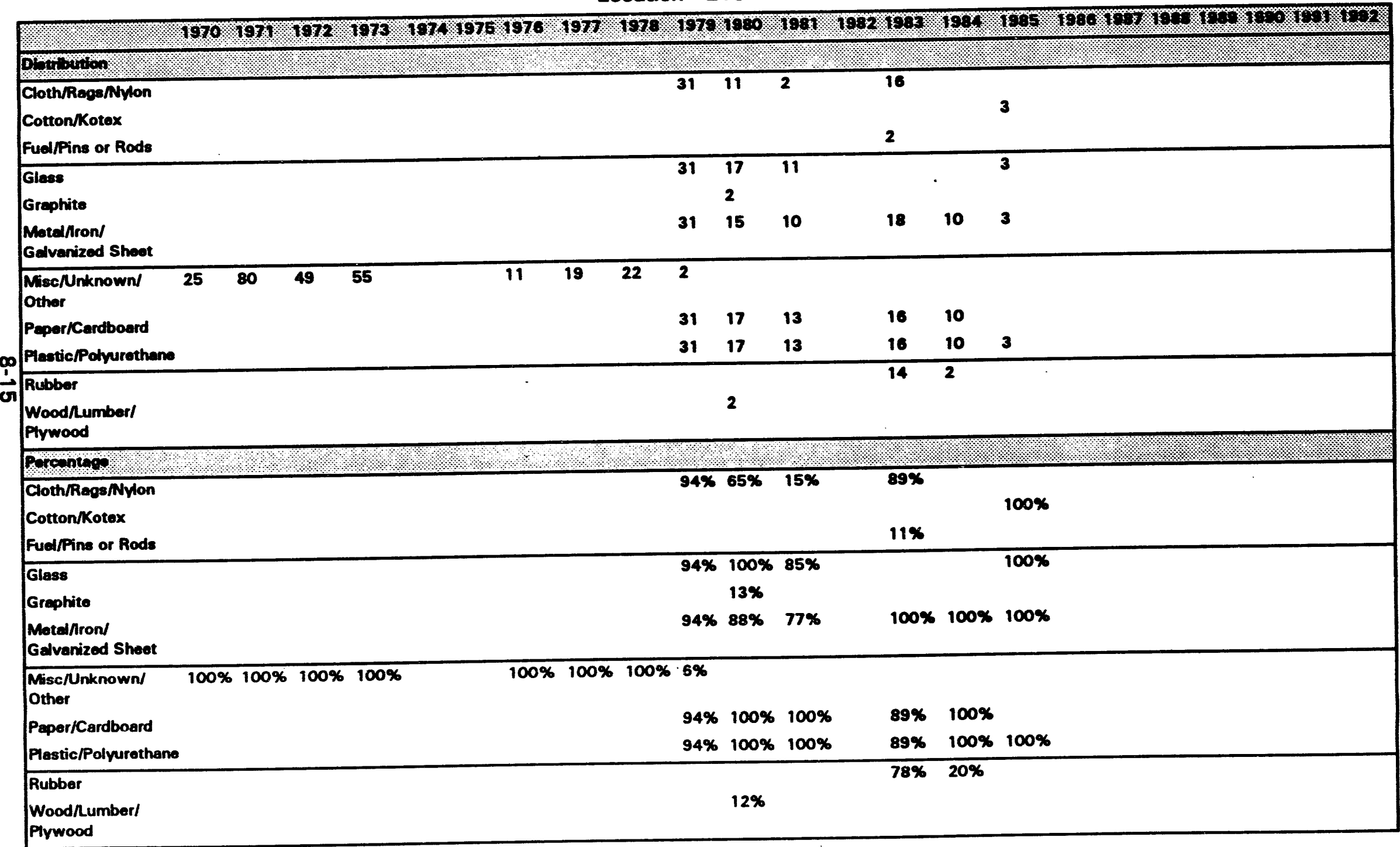


Table 8-5. The 325B Annex Westinghouse Hanford TRU Waste in Containers Other Than 55-Gallon Drums: Total Grams TRU by Storage Location.

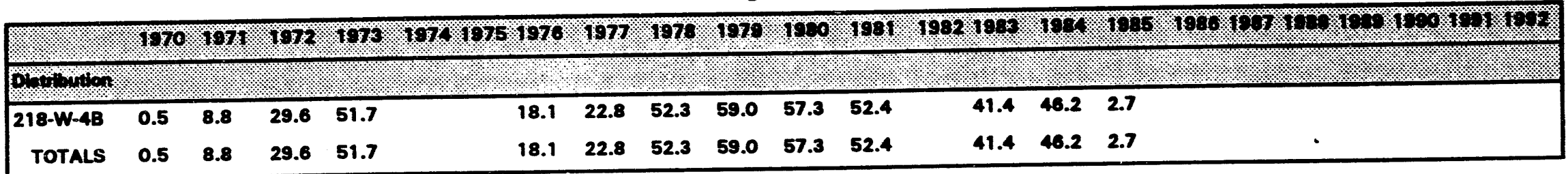


Table 8-6. Characterization of TRU Waste from the PNL 325B Annex in 1 Gallon

Containers Stored at 218-W-4B.

\begin{tabular}{|l|c|}
\hline & \\
\hline TRU Containers & 25 \\
\hline TRU Wt. $(\mathrm{kg})$ & 75 \\
\hline TRU Volume $\left(\mathrm{m}^{3}\right)$ & 0.1 \\
\hline TRU Grams & 0.01 \\
\hline
\end{tabular}

Table 8-7. Isotopes in Other Containers for the 325B Annex from 1970 to 1992.

\begin{tabular}{|c|c|c|c|}
\hline $19010 \%$ & Halfigrs. & oronory (o) & Radiation \\
\hline${ }^{241} \mathrm{Cm}$ & $433 Y$ & 0.2 & $a, r$ \\
\hline${ }^{237} \mathrm{~Np}$ & $2.14 \times 10^{\circ} \mathrm{Y}$ & 0.93 & $a, r$ \\
\hline${ }^{232} \mathrm{Th}$ & $1.41 . \times 10^{10} Y$ & 183.41 & $a, r$ \\
\hline${ }^{233} \mathrm{U}$ & $1.592 \times 10^{6} Y$ & 3.64 & $a, r$ \\
\hline Pu total & $*$ & $0.005 / 438.0431$ & - \\
\hline U depleted & 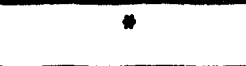 & $0 / 1373.74$ & 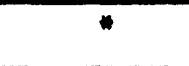 \\
\hline$U$ enriched & 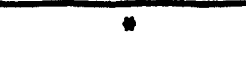 & $5.0 / 4001.44$ & * \\
\hline U natural & 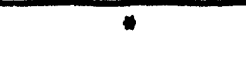 & $0 / 163.42$ & 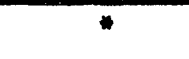 \\
\hline
\end{tabular}

PNLMestinghouse Hanford.

- = Varies according to isotopes

$Y=$ Years. 
WHC-EP-0696

This page intentionally left blank. 


\section{WHC-EP-0898}

\subsection{AEFERENCE8}

AEC, 1970, Immediate Action Directive 0811-21, U.S. Atomic Energy Commission, Washington, D.C.

AEC, 1973, Atomic Energy Commission Manual, Chapter 0511, "Radloactive Waste Management," U.S. Atomic Energy Commiesion, Washington, D.C.

Anderson, B. C., J. D. Anderson, J. A. Domitor, D. R. Duncan, L. A. Fort, D. C. McCann, and S. J. Stone, 1991, Contect-Handled Transuranic Waste Characterization Based on Existing Records, WHC-EP-0225, Revision 1, Westinghouse Hanford Company, Richland, Washington.

Anderson, H. J., and H. E. Hanthorn, 1975, Safoty Analysis Repoit for the 325 Radiochemistry Building. TC-299, Revision 1, Hanford Engineering Development Laboratory, Westinghouse Hanford Company, Richland, Washington.

Anderson, H. J., and H. E. Hanthorn, 1977, Safoty Analysis Report for the 325 Radiochemistry Building. TC-299, Revision 2, Hanford Engineering Development Laboratory, Westinghouse Hanford Company, Richland, Washington.

DOE, 1980, Radioactive Waste Management, DOE Order 5820.2A, U.S. Department of Energy, Washington, D.C.

DOE, 1982, Management of Transuranic Material, DOE Order 5820.1, U.S. Department of Energy, Washington, D.C.

DOE, 1987, Radioactive Waste: Byproducts Material Final Rule, 10 CFR 962, Federal Register, 52 FR 159397-1592, U.S. Department of Energy, Washington, D.C.

DOE, 1988, Radioactive Waste Management, DOE Order 5820.2A, U.S. Department of Energy, Washington, D.C.

DOT, 1989, Transportation, Title 49, Code of Federal Regulations, Parts 100 to 199 , U.S. Department of Transportation. Washington, D.C.

Duncan, D. R., D. A. Burbank, B. C. Anderson, and J. A. Demiter, Application of Service Examinations to Transuranic Waste Container Integrity at the Hanford Site, WHC-SA-1450FP, Westinghouse Hanford Company, Richland, Washington, October 1992.

Ecology, 1989, Dangerous Waste Regulations, Chapter 173-303, Washington Administrative Code, Washington State Department of Ecology, Olympia, Washington.

EPA, 1976, Resource Conservation and Recovery Act, Public Law 94-580, 90 Stat. 2003, 42 USC 6901 et seq.

EPA, 1989, Spill Table, 40 CFR 302.4, Environmental Protection Agency, Washington, D.C. 


\section{WHC-EP-0696}

EPA, 1990, Licensing Requirements for Land Disposal of Radioactive Waste. Title 10, Code of Federal Regulations, Part 61, U.S. Environmental Protection Agency, Washington, D.C.

EPA, 1991, Interim Status Standards for Owners and Operators of Hazardous Waste Treatment, Storage, and Disposal Facillities, Title 40, Code of Federal Regulations, Part 265, U.S. Environmental Protection Agency, Washington, D.C.

Gerber, M. S., 1992, Past Practices Tochnical Characterization Study - 300 Area - Hanford Site. WHC-MR-0388, Westinghouse Hanford Company, Richland, Washington.

HEDL, 1975a, Analytical Chemistry Laboratory Manual, MG-28, Volume 2: Analytical Mothods for Mixed Uranium-Plutonium Oxide Fuel, Hanford Engineering Developmental Laboratory, Weatinghouse Hanford Company, Richland, Washington.

HEDL, 1975b, Analytical Chemistry Laboratory Manual, MG-28, Volume 3: Analytical Mothods for Boron Carbide Absorbers, Hanford Engineoring Developmental Laboratory, Westinghouse Hanford Company, Richland, Washington.

Klem, M. J., 1990, Inventory of Chemicals Used at Hanford Site Production Plants and Support Operations (1944-1980), WHC-ED-0172, Revision 1, Westinghouse Hanford Company, Rlchland, Washington.

McCormack, C. G., 1969, 325 Building Safoty Analysis Report, BNWL-CC-1913, BattelleNorthwest, Richland, Washington. Please note that this document is not publicly available due to its age.

NIOSH, 1992, Registry of Toxic Effects of Chemical Substances Quarterly Issue, July 1992 Microfiche edition, National Institute for Occupational Safety and Health, Cincinnati, Ohio.

Reddinger, R.W., 1992, Unstable and Reactive Chemicals in TRU Retrievable Waste at the Hanford Site - A Review of Available Date, WHC-EP-0603, Westinghouse Hanford Company, Richiand, Washington.

U.S. Department of Interior, 1973, Fire and Explosion Hazard Evaluation - Waste Storage Facility - AEC Hanford Operations - Richland, Washington, United States Department of Interior, Mining Enforcement and Safety Administration. Health and Safety Technical Support, Washington, D.C.

WHC, 1981, Waste Marragement Manual, MG-17, Rev. 17, Hanford Engineering Development Laboratory, Westinghouse Hanford Company, Richland, Washington.

Willis, N.P., and Triner, 1991, Hanford Site Radioactive Solid Waste Acceptance Criteria, WHC-EP-0063-3, Westinghouse Hanford Company, Richland, Washington.

WIPP, 1989, TRU Waste Acceptance Criteria for the Waste Isolation Pilot Plant, WIPP-DOE-069, Revision 3, Westinghouse Electric Corporation, Carlsbad, New Mexico. 
WHC-EP-0696

\begin{abstract}
APPENDIX A
DATA ON BUILDINO 325 WASTE GENERATION BY WESTINGHOUSE HANFORD FROM THE SOLID WASTE INFORMATION TRACKINO SYSTEM
\end{abstract}


The information found in this appendix is from the Solid Waste Information and Tracking Syetem (SWITS) database. This database incorporates the older R-SWIMS database and is used to track information on radioactive and other wastes stored or disposed at the Hanford Site.

Each SWITS data run in this appendix is preceded by the query used to generate the data. A brief explanation of the run and any additional information needed to understand the data presented is also included.

The bulk of the date provided is limited to information about TRU waste generated at Building 325 by Westinghouse Hanford; however, some general information on the nonTRU waste is included for comploteness. The term non-TRU is used, instead of LLW, because a small percentage of the waste has been designated only as not TRU. It is believed that most, if not all, of the non-TRU waste is LLW.

The data runs in this appendix are further segregated by waste container type. Since initial retrieval efforts and WRAP 1 will focus on 55-gal drums, these container types are considered separately.

Some general information about SWITS database and the codes used follows. Please note the disclaimer found on the next page. 


\section{WHC-EP-0696}

DON'T SAY IT -- Write It $l$

Date:

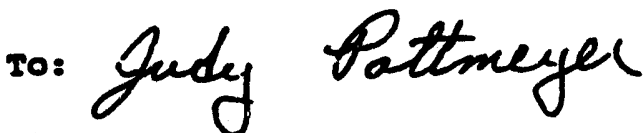

Froms SolId Waste Engineering Data Management Group 113-11 6-4394/6-4020

Re: SNITS DATA REQUEST

Attached for your information and use is the data which you requested. This data represents best avallable information regarding wastes currently in storage at the Hanford site. I trust the information will be suitable to your needs.

Requests for information from the solid waste Information and Tracking system (SWITS) are normally relatively limited in scope, requesting specific data fields or sumbary data. The responses to these requests undergo review during data collection, summary and response preparation.

The response to this request represents a simple reproduction of the swITS Database. Tranamittal of this information is made with the following disclaimers:

1) The information contained in this transmittal is raw data, and represents information provided to Solid Waste Engineering (SWE) on burial records or other documents. This data has not been validated.

2) The information contained in this transmittal is subject to change without notice. Continual update of swITs information and improvement of the software system make it impossible to ensure consistency of this data with the database after transmittal.

3) This information is current as of $12 / 11 / 22$.

If I can be of further assistance to you, do not hesitate to call me. 
WHC-EP-0696

This page intentionally left blank.

A-4 
WHC-EP-0696

SWIR401

Solid waste Information and Tracking system

$05 / 01 / 92$

$13: 25$

Páge: 1

Primary Waste Type code Table

Primary Waste Type Code

$\mathrm{AR}$
$\mathbf{C H}$
$\mathbf{H P}$
$\mathbf{M}$
$\mathbf{M P}$
$\mathbf{N}$
$\mathbf{R}$
$\mathbf{R}$
$\mathbf{R}$

Primary Waste Iype Description

\section{HAZ, ADIII BOTD}

MORAYORIOY CONYROTID

ENTARDO08

HAZNRDOU WASTL PCB

MIXIT

IIXED PCB

NON-REGULATED

PCB

RADIOACTIVE

RADIOACTIVE PCB

unkNown

\section{Ship}

Time Rad

$-\infty$

89

89

89

29

89

29

29

29
N

N

N

N

$Y$

$\boldsymbol{Y}$

N

N

$Y$

$\mathbf{Y}$

N 
WHC-EP-0696

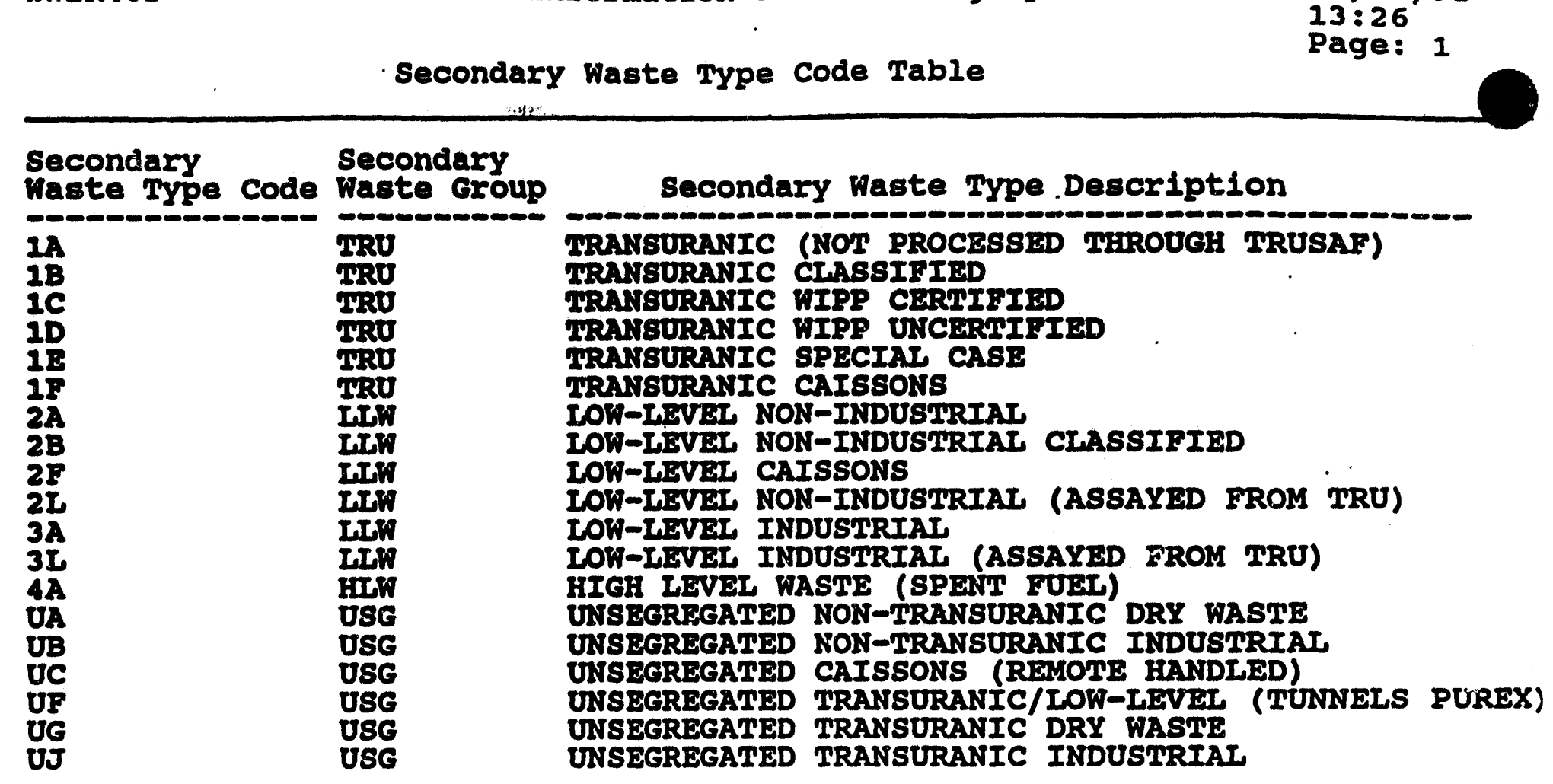


WHC-EP-0696

APPENDIX A.1

CONTAINER NUMBER AND WEIGHT OF TRU WASTE IN 55-CALLON DRUMS BY WASTE TYPE 
This data run gives an overall look at the waste types, container numbers and total weight (in kilograms) of the radioactive wastes generated at Building 325 by Westinghouse Hanford on a yearly basis. These data are for wastes stored in 55-gal drums only. The meanings of primary and secondary waste codes can be found in the introductory materials for this appendix.

The date in the average weight column should be taken cautiously, especially for LLW codes. Concern is warranted because LLW records are still done on a batch basis. This means that one weight datum may be the composite weight for a group of drums. The computer program used simply divided the sum of all weight values by the number of values used to compute the total weight, not the actual number of containers that the value represents.

From 1970 until 1978 individual container weights were not required for TRU drums. During the data re-entry program in the mid-1980's standard weights were assigned for all container types; 55-gal drums were given a standard weight of $68 \mathrm{~kg}$. This is why the average weight/drum for TRU is so consistent during this period. 


\section{WHC-EP-0696}

set linesize 170

pagesize 45

newpage 0

spool pot2. srep

ttitle center ' 325 WHC' -

right 'Page:' format 999 sql.pno skip 1

break on dt skip 1

compute sum of sum (con_gross_wgt) on dt

compute sum of sum(rdet_rswims_count) on dt

compute sum of sum(con_cntr_voI) on dt

col dt

col con_pwtyp_cd

col rdet_swtyp_cd

col sum (ŕdet_rswims_count)

col avg (con_ḡross_wḡt)

col sum (con_gross_wgt)

col sum (con_cntr_vol)

col avg (con_cntr_vol)

col cntyp_descr

col con_size_descr

format a 4

format a4

format a4

format 999999

format 99999.99 heading

format 9999999.99

format 99999.99 heading

format 99999.99 heading

format a 30

format a25

heading "Container Description" heading "Container Size"

select subsEr (to_char(con_tsd_accept_dt, 'YYYY'), 1, 4) dt, cntyp descr, con_size_descr, con_pwtyp_cd, rdet_swtyp_cd, $\operatorname{sum}\left(\bar{r} d e t \_r \bar{s} w i m s \_c o u n t\right)$, sum (con_ōross_wḡt), avg (con_gross_wgt), sum (con_cntr_vol), avg (con_cntr_vol)

from radwaste, contype

where con_tsd_accept_dt between '01-jan-69' and '31-dec-92' and con_srce_cmpny id = 'WHC' and

con_srce_facil_id $=1325^{\prime}$

and con_size_déscr $=155$ GALLON' and con_cntyp_cd = cntyp_cd

group by cntȳp_descr,

con_size_descr,

con_pwtyp̄_cd,

substr (to_char(con_tsd_accept_dt, 'YYYY'), 1, 4),

rdēt_swtyp_cad

;

spool off 


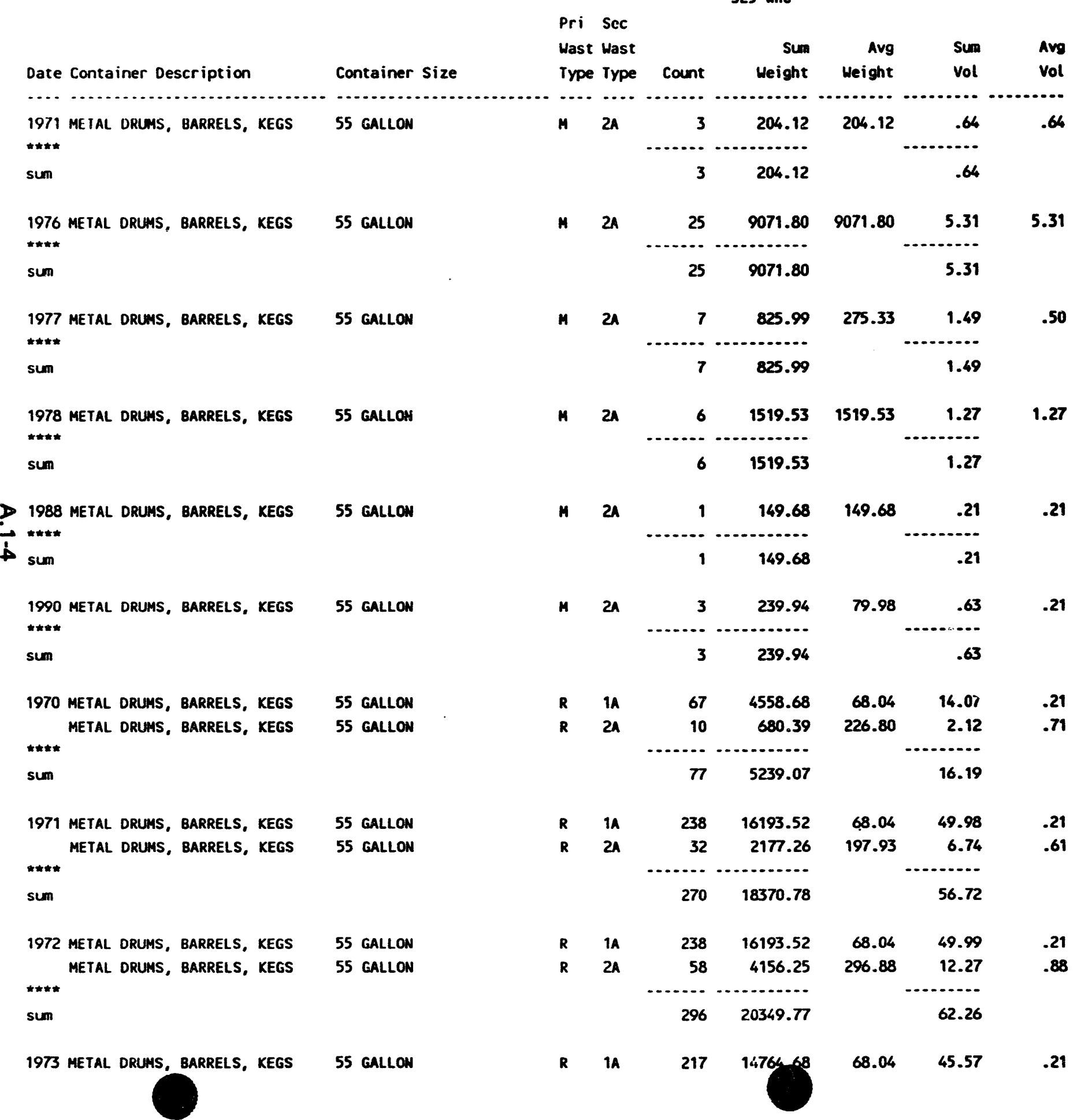




\begin{tabular}{|c|c|c|c|c|c|c|c|c|}
\hline Date Container Description & Container Size & $\begin{array}{l}\text { Pri } \\
\text { Wast } \\
\text { Type }\end{array}$ & $\begin{array}{l}\text { Sec } \\
\text { Wast } \\
\text { Type }\end{array}$ & count & $\begin{array}{r}\text { Sum } \\
\text { Weight }\end{array}$ & $\begin{array}{r}\text { Avg } \\
\text { Weight }\end{array}$ & $\begin{array}{l}\text { Sum } \\
\text { Vol }\end{array}$ & $\begin{array}{l}\text { Avg } \\
\text { vol }\end{array}$ \\
\hline $\begin{array}{l}1973 \text { METAL DRUMS, BARRELS, KEGS } \\
\star \star \star * \star\end{array}$ & 55 GALLON & R & $2 A$ & $\begin{array}{r}93 \\
9 .\end{array}$ & 11189.18 & 508.60 & $\begin{array}{c}19.75 \\
-\ldots . . .\end{array}$ & .90 \\
\hline sum & & & & 310 & 25953.86 & & 65.32 & \\
\hline 1974 METAL DRUMS, BARRELS, KEGS & 55 GALLON & R & 14 & 254 & 17576.99 & 69.20 & 53.34 & .21 \\
\hline $\begin{array}{l}\text { METAL DRUMS, BARRELS, KEGS } \\
\star \star \star \star \star \\
\text { sum }\end{array}$ & 55 GALLON & R & $2 \mathrm{~A}$ & $\begin{array}{r}67 \\
321\end{array}$ & $\begin{array}{r}8378.27 \\
25955.26\end{array}$ & 492.84 & $\begin{array}{c}14.23 \\
67.57\end{array}$ & .84 \\
\hline $\begin{array}{l}1975 \text { METAL ORUMS, BARRELS, KEGS } \\
\text { METAL DRUMS, BARRELS, KEGS } \\
\text { **** } \\
\text { sum }\end{array}$ & $\begin{array}{l}55 \text { GALLON } \\
55 \text { GALLON }\end{array}$ & $\begin{array}{l}\mathbf{R} \\
\mathbf{R}\end{array}$ & $\begin{array}{l}1 A \\
2 A\end{array}$ & $\begin{array}{c}218 \\
143 \\
361\end{array}$ & $\begin{array}{l}15445.05 \\
15935.07 \\
31380.12\end{array}$ & $\begin{array}{r}70.85 \\
408.59\end{array}$ & $\begin{array}{c}45.78 \\
30.37 \\
76 . . \\
76.15\end{array}$ & $\begin{array}{l}.21 \\
.78\end{array}$ \\
\hline $\begin{array}{l}1976 \text { METAL ORLMS, BARRELS, KEGS } \\
\text { METAL DRUMS, BARRELS, KEGS }\end{array}$ & $\begin{array}{l}55 \text { GALLON } \\
55 \text { GALLON }\end{array}$ & $\begin{array}{l}\mathbf{R} \\
\mathbf{R}\end{array}$ & $\begin{array}{l}1 A \\
2 A\end{array}$ & $\begin{array}{l}162 \\
136\end{array}$ & $\begin{array}{l}11022.48 \\
13035.27\end{array}$ & $\begin{array}{r}68.04 \\
449.49\end{array}$ & $\begin{array}{l}34.02 \\
28.45\end{array}$ & $\begin{array}{l}.21 \\
.98\end{array}$ \\
\hline sum & & & & 296 & 24057.75 & & 62.47 & \\
\hline $\begin{array}{l}1977 \text { METAL DRUMS, BARRELS, KEGS } \\
\text { METAL DRUMS, BARRELS, KEGS }\end{array}$ & $\begin{array}{l}55 \text { GALLON } \\
55 \text { GALLON }\end{array}$ & $\begin{array}{l}\mathbf{R} \\
\mathbf{R}\end{array}$ & $\begin{array}{l}1 A \\
2 A\end{array}$ & $\begin{array}{l}140 \\
110\end{array}$ & $\begin{array}{r}9521.06 \\
18782.24\end{array}$ & $\begin{array}{r}68.01 \\
569.16\end{array}$ & $\begin{array}{l}29.40 \\
23.36\end{array}$ & $\begin{array}{l}.21 \\
.71\end{array}$ \\
\hline sum & & & & 250 & 28303.30 & & 52.76 & \\
\hline 1978 METAL DRUMS, BARRELS, KEGS & 55 GALLON & $\begin{array}{l}R \\
0\end{array}$ & 14 & $\begin{array}{l}307 \\
159\end{array}$ & $\begin{array}{l}42781.36 \\
20440.69\end{array}$ & $\begin{array}{l}139.35 \\
454.44\end{array}$ & $\begin{array}{l}64.47 \\
33.76\end{array}$ & .21 \\
\hline $\begin{array}{l}\text { METAL DRUHS, BARRELS, KEGS } \\
\ldots * * * \\
\text { sum }\end{array}$ & SS GALLON & $\mathbf{R}$ & & 466 & $\begin{array}{l}20449.09 \\
63231.05\end{array}$ & & 98.23 & \\
\hline 1979 METAL DRUMS, BARRELS, KEGS & 55 GALLON & R & 14 & 437 & 29733.48 & 68.04 & 91.77 & .21 \\
\hline $\begin{array}{l}\text { METAL DRUMS, BARRELS, KEGS } \\
\text { sum } \\
\text { sum }\end{array}$ & 55 GALLON & $\mathbf{R}$ & $2 \mathrm{~A}$ & $\begin{array}{r}113 \\
550\end{array}$ & $\begin{array}{l}13628.11 \\
43361.59\end{array}$ & 412.97 & $\begin{array}{r}24.00 \\
115.7\end{array}$ & .73 \\
\hline 1980 METAL DRUMS, BARRELS, KEGS & 55 GALLON & $\mathbf{R}$ & $1 \mathrm{~A}$ & 91 & $\begin{array}{r}6191.64 \\
15561.78\end{array}$ & $\begin{array}{r}68.04 \\
2223.11\end{array}$ & $\begin{array}{l}19.11 \\
15.72\end{array}$ & $\begin{array}{r}.21 \\
2.25\end{array}$ \\
\hline $\begin{array}{l}\text { sum } \\
\text { su** METAL DRUHS, BARRELS, KEGS }\end{array}$ & SS GALLON & $\mathrm{k}$ & $2 \mathrm{~A}$ & 165 & 21753.42 & & 34.83 & \\
\hline
\end{tabular}


WHC-EP-0696

This page intentionally left blank.

A.1-6 
WHC-EP-0696

\section{APPENDIX A.2}

BUILDINO 325 WESTINOHOUSE HANFORD TRU WASTE CONTAINERS SORTED BY CONTAINER DESCRIPTION, SIZE, YEAR, AND PRIMARY WASTE TYPE 


\section{WHC-EP-0698}

This subappendix provides information on Building 325 Westinghouse Hanford TRU waste containers, woight, volume, and primary waste type stored at various TRU waste storage facilities on the Hanford Site. The data provides the TRU waste generation from the Building 325 Westinghouse Hanford Facillty by yoar. 
set linesize 170

pagesize 44

newpage 0

spool pot3.srep

ttitle center 'TRU WASTE- 325 WHC' -

right 'Page:' format 999 sql.pno skip 1

break on dt skip 1

compute sum of sum(con_gross_wgt) on dt

compute sum of sum(rdet_rswims_count) on dt

col dt

col con_pwtyp_cd

col rdet_swtyp_de

col sum (con_gross_wgt)

col sum (rdet_rswims_count)

col avg (con_gross_wğt)

col sum (con_cntr. vol)

col avg (con_cntr_vol)

col cntyp descr

col con_size_descr

col con_locn_facil_id

format a4

format a4

heading "Date"

format a4

heading

heading

format 999999.99

format 99999

heading

format 999999.99

format 99999.99 heading

format 99999.99 heading

format a55

format a 45

heading

heading

format' as

heading

"Pri|Wast/Type"

"Sec/Wast/Type"

heading "Welght"

select

cntyp_descr,

consIze descr,

substr (Eo_char(con_tsd_accept_dt, 'YYYY'), 1, 4) dt,

con_pwtyp_cd,

con_locn_facil_id,

$\operatorname{sum}(r d e t$ rswims_count),

sum (con_ōross_wḡt),

avg (con_gross_wgt),

sum (con_cntr_vol),

avg (con_cntr_vol)

from radwaste, contype

where con_tsd_accept_dt between '01-jan-69' and '31-dec-92' and

con_srce_cmpny $1 d=$ 'WHC' and

con_srce_facil_id $=1325^{\prime}$

and rdet_swtyp_cd in ('IA', 'IB','IC', 'IE', 'IF', 'UG', 'UF', 'UJ') and

con_cntyp_cd = cntyp_cd

groüp by centyp_descr,

con_size_descr,

subs̄tr (Eo_char(con_tsd_accept_dt, 'YYYY'), 1, 4),

con_pwtyp_cd,

con_locn_facil_id

spool off 


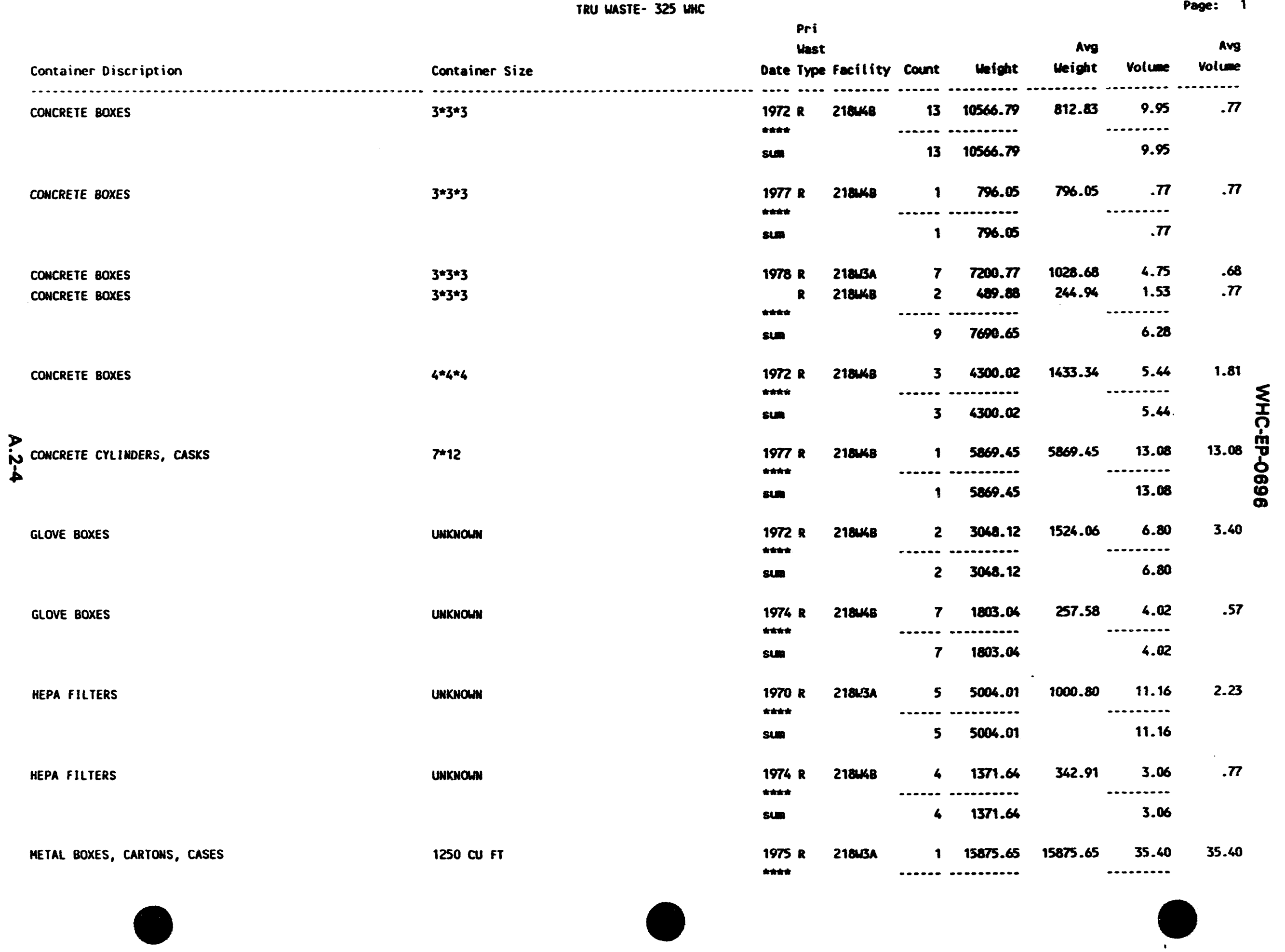


Pri

Wast

Date Type Facility Count Weight

Container size

sum

$1 \quad 15875.65$

Avg Avg

Container Discription

METAL BOXFS, CARTONS, CASES

$4 * 4 * 4$

METAL BOXES, CARTONS, CASES

METAL 8OXES, CARTONS, CASES

$4 * 4 * 5$

$4 * 5 * 9$

$1972 R \quad 218 w 48$
sum

$2 \quad 1625.66$

21625.66

$4 * 6 * 10$

METAL BOXES, CARTONS, CASES

ơ

metal boXes, cartons, cases

$4.2 * 6.2 * 15.6$
$5 * 5 * 5$

$7 * 10 * 16$

190 GaLLON

METAL DRUMS, BARRELS, KEGS

30 GALLON

METAL DRUMS, BARRELS, KEGS

METAL ORUMS, BARRELS, KEgS

METAL DRUMS, BARRELS, KEGS Metal boXes, cartons, cases
metal boXes, cartons, cases

30 GALLON

55 GALLON
$1976 R \quad 218448$

R 218448

sum

\begin{tabular}{rr}
1 & 1016.04 \\
1 & 2286.09 \\
2 & 3302.13 \\
1 & 2042.06 \\
\hline 1 & 2042.06
\end{tabular}

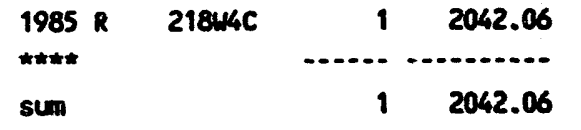

1980 R $21844 C \quad 1 \quad 2494.75$

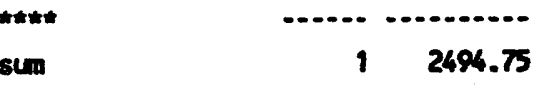

$\begin{array}{rr}1974 R & 21843 A \\ R & 21843 A\end{array}$

sum

1976 R $21844 B$

sum

$1970 R \quad 21843 A$
$\star * \star *$

sum

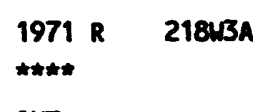

sum

1970 R 21843A

sum

$\begin{array}{rr}1 & 40.32 \\ 1 & 40.02\end{array}$

\begin{tabular}{rr}
67 & 4558.68 \\
\hline 67 & 4558.68
\end{tabular}

Weight Volume volume

35.40

$\begin{array}{rrr}812.83 & 3.62 & 1.81 \\ & 3.62 & \\ & & \\ 1016.04 & 2.27 & 2.27 \\ 2286.09 & 5.10 & 5.10 \\ & \ldots . . . & \\ & 7.36 & \end{array}$

$2042.06 \quad 7.07 \quad 7.07$

11.50

$\begin{array}{llll}1587.57 & 3.54 & 3.54 & 0\end{array}$

$14224.58 \quad 31.72 \quad 31.72$

35.26

$136.08 \quad .42 \quad .42$

.42

$40.82 \quad 1.97 \quad .12$

1.97

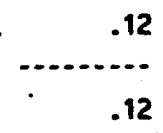

.12

.21

14.07
$2494.75 \quad 11.50 \quad 11.50$

-..........

14.07 
TRU MASTE- 325 WHC

Page: 3

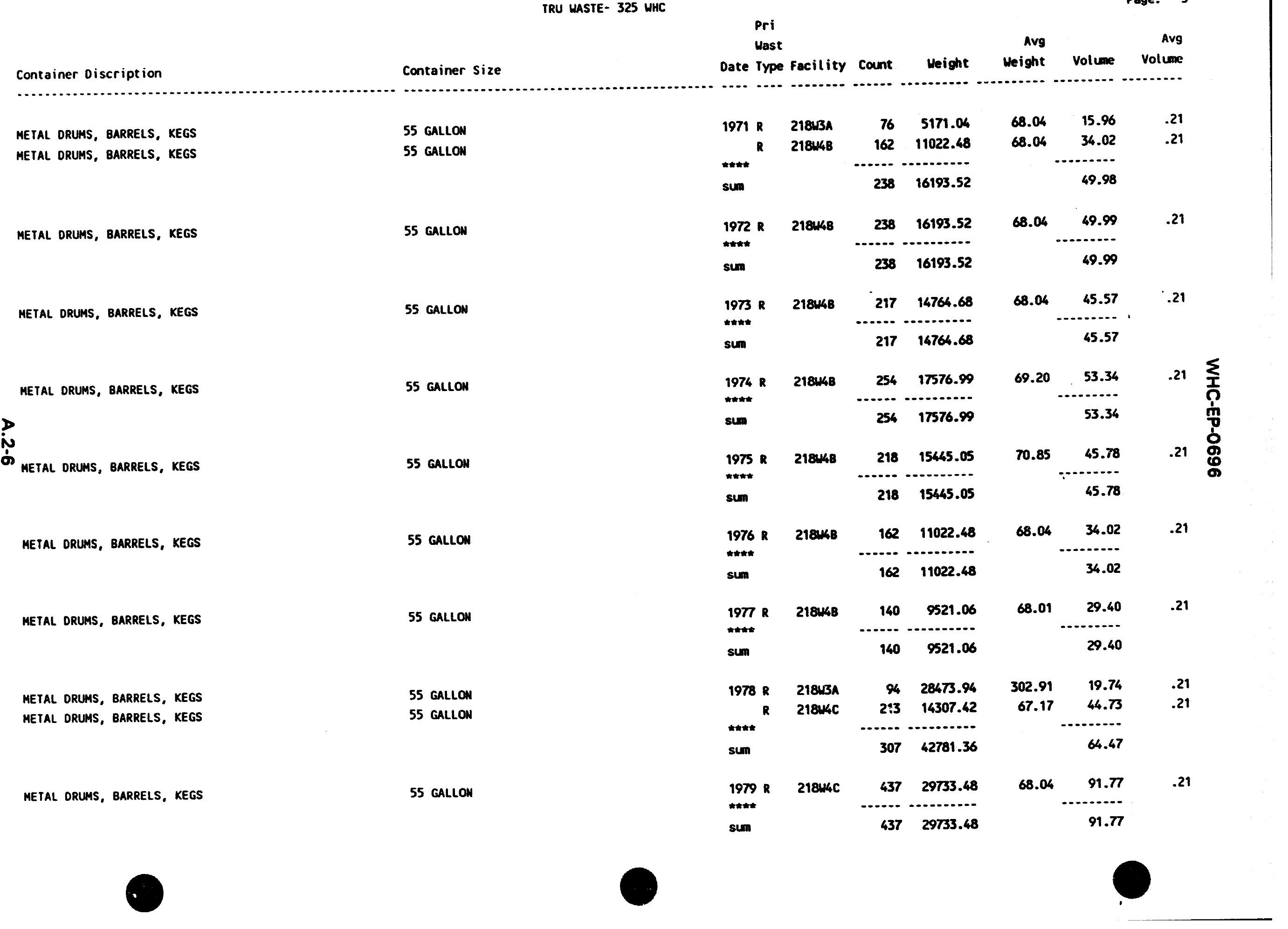




\section{WHC-EP-0696}

This page intentionally left blank.

A.2-8 
WHC-EP-0696

\section{APPENDIX A.3}

BUILDINO 325 WESTINGHOUSE HANFORD NON-TRU WASTE CONTAINERS SORTED BY CONTAINER DESCRIPTION, SIZE, YEAR, AND PRIMARY WASTE TYPE 
This subappendix provides information on Building 325 Westinghouse Hanford nonTRU waste containers, weight, volume, and primary waste type stored at various waste storage facilities on the Hanford Site. The data provides the non-TRU waste generation from the Building 325 Westinghouse Hanford Facility by year. 
set 1 inesize 175

pagesize 45

newpage 0

spool pot3a.srep

ttitle center 'NON-TRU WASTE- 325 WHC' -

right 'Page:l format 999 sql.pno skip 1

break on dt skip 1

compute sum of sum (con_gross_wgt) on dt compute sum of sum(rdet_rswims_count) on dt

col dt

col con_pwtyp_cd

col rdet_swtyp_de

col sum (con_gross_wgt)

col sum (rdet_rswims_count)

col avg (con_gross_wğt)

col sum (con_cntr_vol)

col avg (con_cntr_vol)

col cntyp_descr

col con_size_descr

col con_locn_facil_id

format a4

format a4

format a4

format 999999.99

heading "Date"

heading "Pri|Wast/Type"

heading "Sec/Wast/Type"

format 99999 heading "Count"

format 999999.99

format 99999.99 heading

format 99999.99 heading

format a55

format a 45

heading

heading

format $a 8$

heading "Facility"
heading "Sec|Wast|Code"

heading "Avg|Weight"

"Volume"

"Avg|Volume"

col rdet_swtyp_cd

format a4

select

cntyp_descr,

con_size_descr,

substr (Eo_char(con_tsd_accept_dt, 'YYYY'), 1, 4) dt,

con_pwtyp_cd,

rdet swtyp_cd,

con_Iocn_facil_id,

sum (rdet_rswims_count),

sum (con_gross_wgt),

avg (con_gross_wgt),

sum (con_cntr_vol),

avg (con_cntr_vol)

from radwaste, contype

where con tsd_accept_dt between '01-jan-69' and '31-dec-92' and

con_srce_cmpny _id = 'WHC' and

con_srce_facil_id $=' 325^{\prime}$

and rdet_swtyp_cd in ('2A', '2B', '2L', '3A', '3L', 'UA', 'UB') and

con_cntyp_pd = cntyp_cd

groüp by cntyp_descr,

con size descr,

substr (Eo_char (con_tsd_accept_dt, 'YYYY'), 1, 4) ,

con_pwtyp_cd,

rdet_swtyp $\bar{p} \_d$,

con_Iocn_fācil_id

;

spool off 


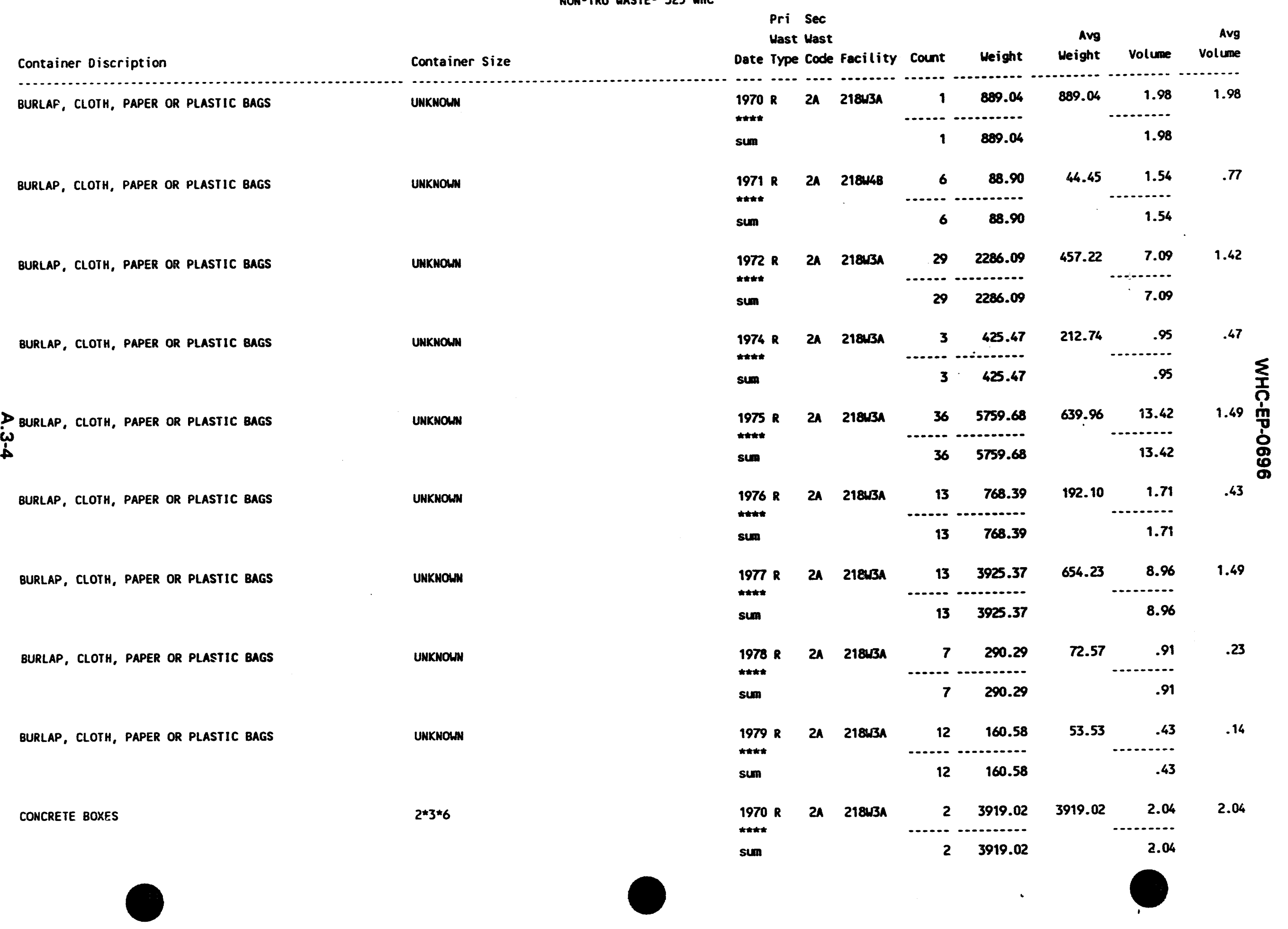




\section{$\sim \quad$ WHC-EP-0696}

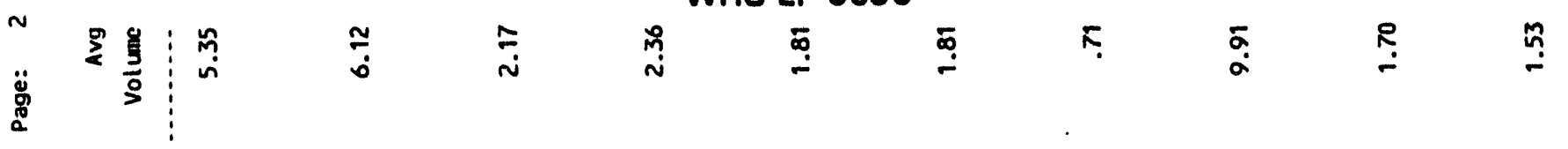

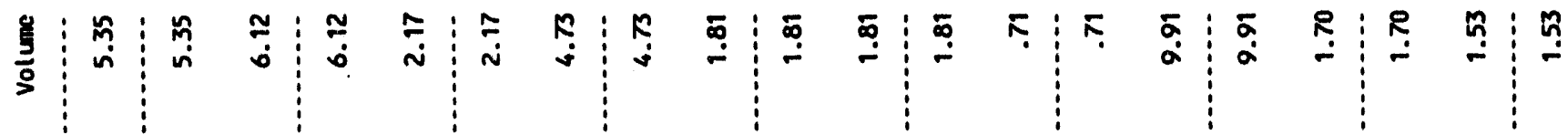

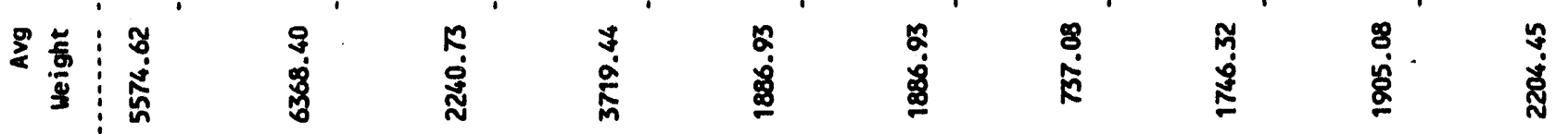

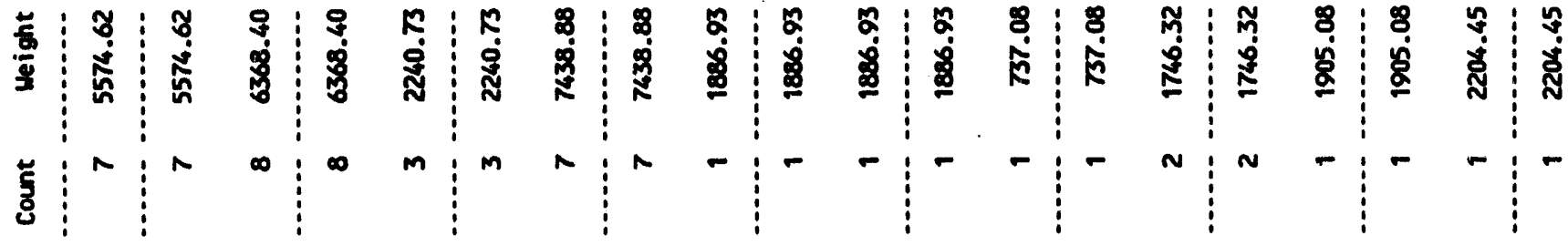

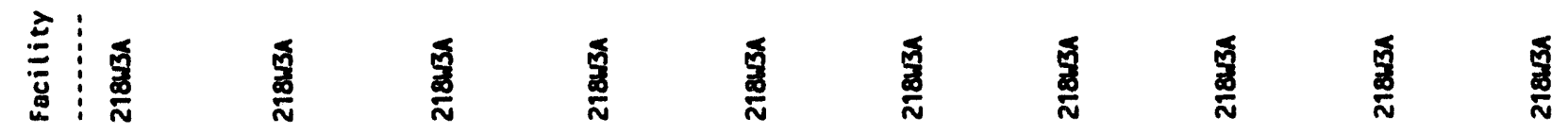

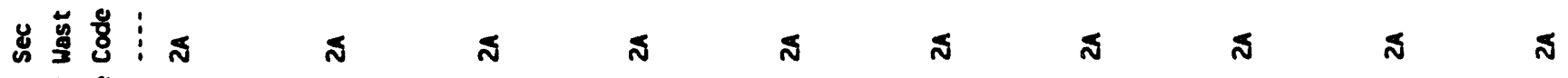

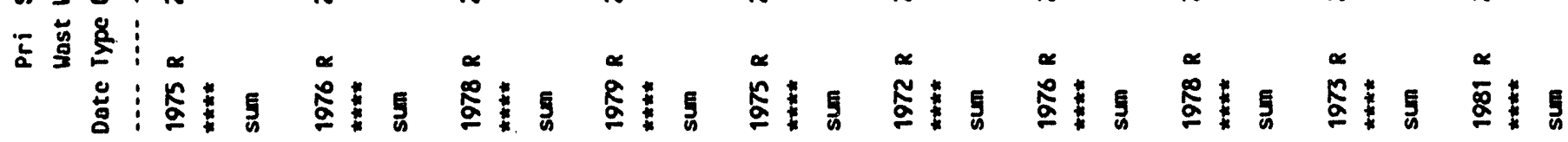

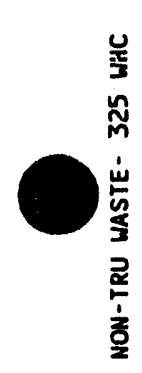

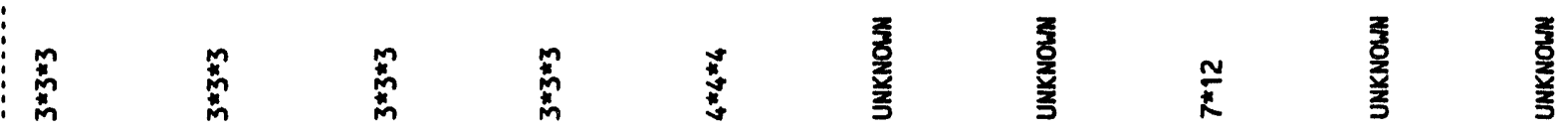
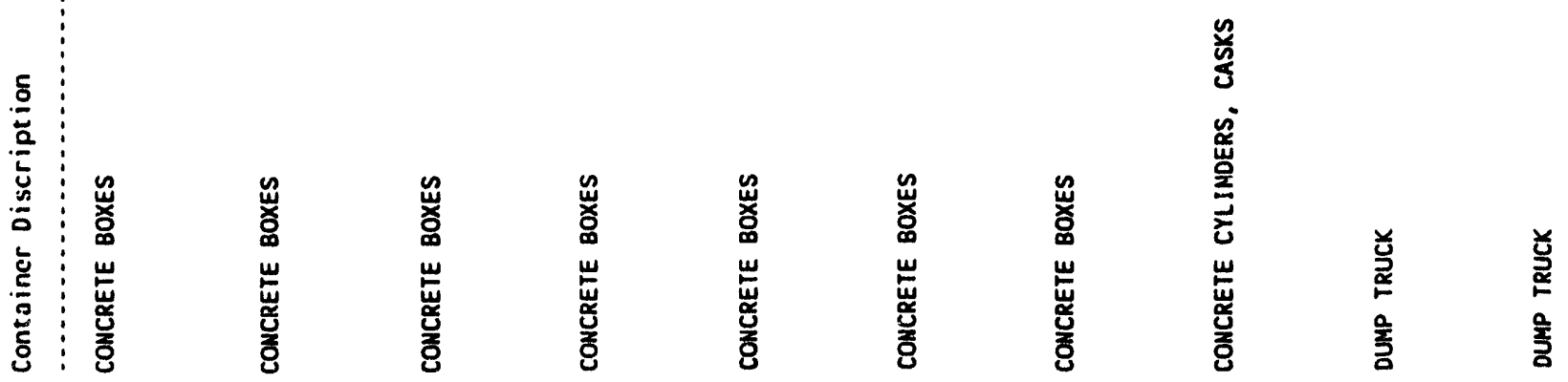


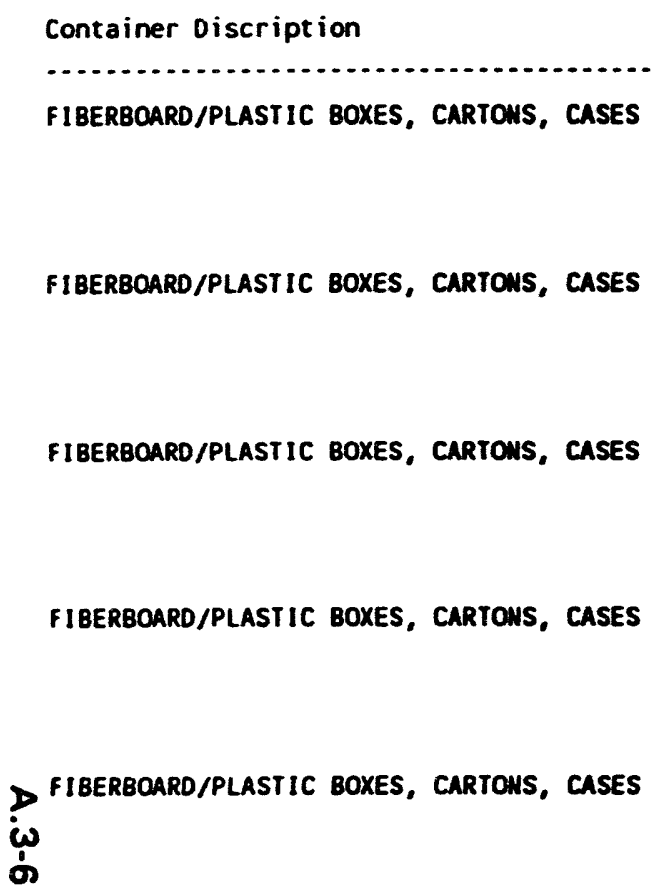

Container Size

UNKNOWM

UNKNOWN

UNKNOWM

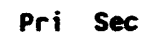

1975

2 a $21813 n$

sum

1977

**แ*

$2 \wedge$

218usA

sum

1979 R $2 A \quad 218433$

1970 tothe

sum
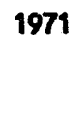

***

sim

1972

tht

sum

$2 a$

218uBA

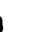

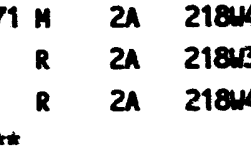

m

1973

*a**

sum

1974 R

$34 \quad 185.06$

$34 \cdot 185.06$

\begin{tabular}{rr}
603 & 7658.39 \\
\hline 603 & 7658.39
\end{tabular}

\section{Avg}

(n)

185.06

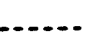

$21.77 \quad .10$

.10

.10

$40.82 \quad .75$

.75

.75

$151.95 \quad 4.36$

4.36

$\begin{array}{rr}31.60 \quad .96 \\ & .9 .\end{array}$

.96

$156.29 \quad 76.84$

76.84

$\begin{array}{lll}228.61 & 2.29 & 2.29\end{array}$

$\begin{array}{lll}300.40 & 69.32 & 3.01\end{array}$

$\begin{array}{lll}358.47 & 74.59 & 3.55\end{array}$

-..........

146.20

$337.71 \quad 149.97 \quad 3.00$

149.97

$\begin{array}{lll}258.86 & 197.95 & 2.60\end{array}$

197.95

$234.14 \quad 199.68$ 
Container Discription

Container Size

FIBERBOARD/PLASTIC BOXES, CARTONS, CASES

FIBERBOARD/PLASTIC BOXES, CARTONS, CASES

FIBERBOARO/PLASTIC BOXES, CARTONS, CASES FIBERBOARD/PLASTIC BOXES, CARTONS, CASES

FIBERBOARD/PLASTIC BOXES, CARTONS, CASES

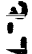

FIBERBOARD/PLASTIC BOXES, CARTONS, CASES FIBERBOARD/PLASTIC BOXES, CARTONS, CASES

FIBERBOARD/PLASTIC BOXES, CARTONS, CASES FIBERBOARO/PLASTIC BOXES, CARTONS, CASES

FIGERBOARD/PLASTIC DRUMS, BARRELS, KEGS

FIGERBOARD/PLASTIC DRUMS, BARRELS, KEGS

FIGERBOARD/PLASTIC DRUMS, BARRELS, KEGS
UNKNOWN

UNKNOWN

UNKNOWN

UNKNOWN

UNKNOWN

UNKNOWII

UNKNOWN

Uaknow

UNKNOWH

UNKMOWM

UNKNOWM

UNKNOWN
Pri Sec

Uast Wast

Date Type Code Facility Count Weight

sum

1975 R $2 A \quad 21843 A$

$1566 \quad 19901.68$

sum

\begin{tabular}{|c|c|c|c|c|}
\hline $1976 R$ & $2 A$ & $21813 A$ & 2274 & 28582.50 \\
\hline & & & 2274 & 28582.50 \\
\hline
\end{tabular}

sin

$\begin{array}{rrr}1977 \text { M } & 2 A & 218 \text { ra } \\ R & 2 A & 21843 A\end{array}$

sum

$1978 R \quad 2 A \quad 21843 A$
Sin:

$\sin$

$\begin{array}{rrr}1979 \text { M } & 2 A & 21843 A \\ R & 2 A & 21803 A\end{array}$

sum

$\begin{array}{ccc}1980 R & 2 A & 21843 A \\ R & 3 A & 21843 A\end{array}$

sum

$55 \quad 698.53$

$2031 \quad 25863.94$

$2086 \quad 26562.47$

$2919 \quad 40887.91$

$2919 \quad 40087.91$

140177

$\begin{array}{rr}14 & 177.81 \\ 2397 & 30633.66\end{array}$

241130811.47

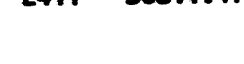

\begin{tabular}{rr}
207 & 2737.87 \\
4 & 2721.54 \\
\hline & 211
\end{tabular}

$211 \quad 5459.41$

1975 R $2 A \quad 21843 A$

sum

\begin{tabular}{cr}
1 & 571.52 \\
\hline 1 & 571.52
\end{tabular}

1977 R $2 A \quad 21843 A \quad 1 \quad 38.10$

แะแ

sum

1979 R $2 A \quad 21843 A$

$\sin$
294

1.27
1.52
1.27

Avg

Aolune volume volume

9.68

$247.35 \quad 250.65 \quad 2.48$

250.65

2.96

286.98

$\begin{array}{lll}349.27 & 7.01 & 3.50\end{array}$

$235.13 \quad 260.51 \quad 2.37$

267.52

$312.12 \quad 372.54$ 372.54

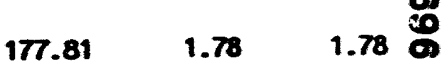

$297.41 \quad 305.45 \quad 2.97$

307.23

$\begin{array}{lll}248.90 & 26.38 & 2.40\end{array}$

$2721.54 \quad 14.50 \quad 14.50$

40.87

1.27

$\begin{array}{lll}38.10 & .09 & .09\end{array}$

$1 \quad 38.10$

.09

40.82

.18
.- .9




\section{Container Discription}

GLOVE BOXES

GLOVE BOXES

GLOVE BOXES

GLOVE BOXES

${ }_{\omega}^{\infty}$

媳 GLOVE BOXES

HEPA FILTERS

MEPA FILTERS

HEPA FILTERS

HEPA FILTERS

HEPA FILTERS
Container Size

UnKNOWN

UNKNOMN

UNKNOWN

UnKMOWH

UNKNOWN

UNKNOWN

UNKNOMN

UNKNOWH

UNKNOWN

UNKNOWM
Pri Sec

Wast Wast

Date Type Code facility Count Weight

Avg

Avg Date ivpe Code Facility Count

1971 R $2 A \quad 21843 A$

$\begin{array}{rr}2 & 829.61 \\ 2 & 829.61\end{array}$

414.8 1.85
1.85

$\begin{array}{lrrrrrr}1973 R & 2 A & 21843 A & 1 & 787.43 & 787.43 & 1.76 \\ \text { sum } & & & \ldots . . & 1 & 787.43 & \end{array}$

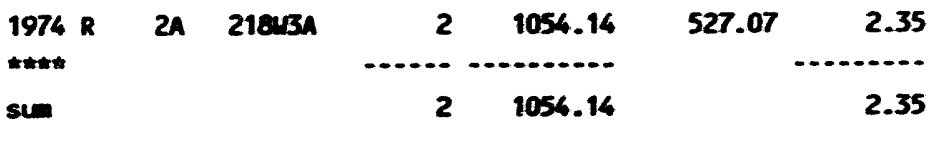

1975 R $2 A \quad 21813 n$

*t*
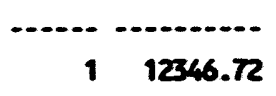

1979 R $2 A \quad 21843$

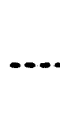

1

sin

$1 \quad 186.43$

1970 R $2 A \quad 21843$ B

101568.5

$10 \quad 1568.51$

sum

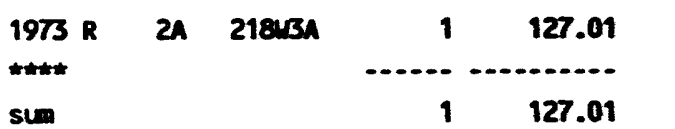

\section{5}

1975

tattat

$2 A \quad 218$ आa

\begin{tabular}{rr}
37 & 2159.09 \\
\hline 37 & 2159.09
\end{tabular}

1976 R 2A 21843A

$90 \quad 8479.4$

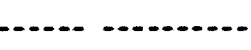

$90 \quad 8479.41$

sum

1977 R

$2 a \quad 21843 n$

\begin{tabular}{rr}
69 & 1169.81 \\
\hline 69 & 1169.81
\end{tabular}

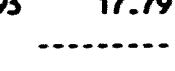

186.43

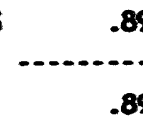

$1568.51 \quad 3.50$ 3.50

$127.01 \quad .28$ .28
.28

$539.77 \quad 6.85$ 6.85

1.71

129.98
1.18

27.52 


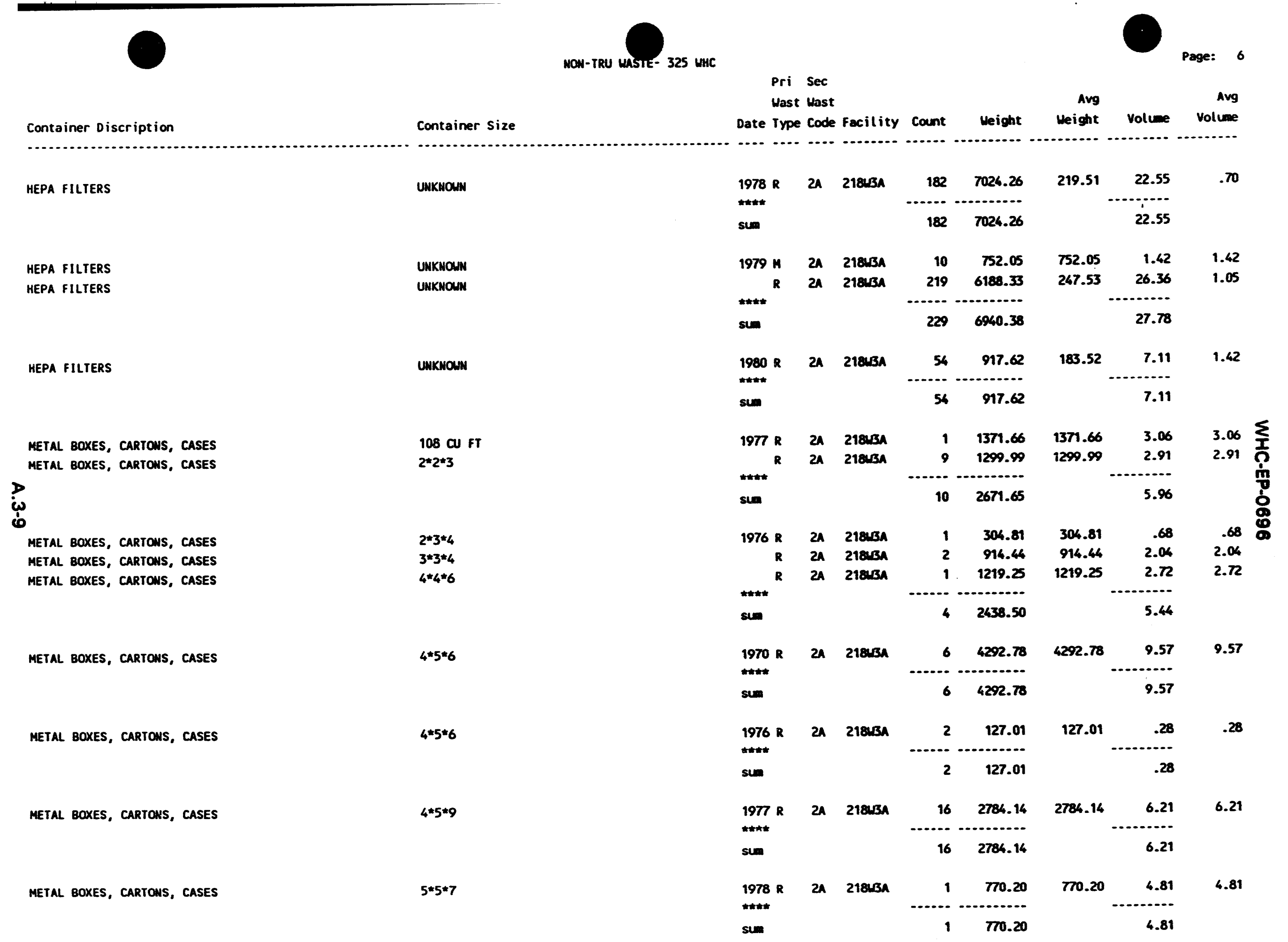




\begin{tabular}{|c|c|c|c|c|c|c|c|c|c|}
\hline Container Discription & Container Size & $\begin{array}{l}\text { Pri } \\
\text { Hast } \\
\text { Date Type }\end{array}$ & $\begin{array}{l}\text { Sec } \\
\text { Wast } \\
\text { code }\end{array}$ & Facility & count & Height & $\begin{array}{r}\text { Avg } \\
\text { Weight }\end{array}$ & Volume & Avo \\
\hline METAL BOXES, CARTONS, CASES & $6 * 8 * 12$ & $\begin{array}{l}1979 \text { R } \\
\text { sum } \\
\text { sum }\end{array}$ & $2 n$ & $21843 n$ & $\begin{array}{r}1 \\
\ldots . . \\
1\end{array}$ & $\begin{array}{c}2041.16 \\
2041.16\end{array}$ & 2041.16 & $\begin{array}{r}16.31 \\
16.31\end{array}$ & 16.31 \\
\hline METAL BOXES, CARTONS, CASES & UMKNOMN & $\begin{array}{l}1977 \text { R } \\
\text { sum }\end{array}$ & $2 n$ & $21843 n$ & $\begin{array}{r}2 \\
2 \\
2\end{array}$ & $\begin{array}{r}187.79 \\
187.79\end{array}$ & 187.79 & $\begin{array}{r}.43 \\
.43\end{array}$ & .43 \\
\hline METAL BOXES, CARTOWS, CASES & UNKMONM & $\begin{array}{l}1980 \mathrm{R} \\
\text { stwt: } \\
\text { sun }\end{array}$ & $2 \AA$ & $21843 A$ & $\begin{array}{c}1 \\
1\end{array}$ & $\begin{array}{r}152.41 \\
152.41\end{array}$ & 152.41 & $\begin{array}{l}.34 \\
.34\end{array}$ & .36 \\
\hline METAL CYLIMDER, CASKS & UNKMOMN & $\begin{array}{l}1975 \text { R } \\
\text { stw" } \\
\text { sum }\end{array}$ & $2 a$ & 218u3a & $\begin{array}{c}12 \\
12\end{array}$ & $\begin{array}{r}7876.14 \\
7876.14\end{array}$ & 3938.07 & $\begin{array}{r}17.56 \\
17.56\end{array}$ & 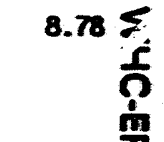 \\
\hline $\begin{array}{l}\text { WetAL CYLINDER, CASKS } \\
\text { metal CYLINDER, CASKS }\end{array}$ & $\begin{array}{l}\text { UnKMOMN } \\
\text { unkNOWM }\end{array}$ & $\begin{array}{l}197 T^{M} \\
\text { Rum } \\
\text { sum }\end{array}$ & $\begin{array}{l}2 A \\
2 A\end{array}$ & $\begin{array}{l}218 \text { ara } \\
218433\end{array}$ & \begin{tabular}{c}
2 \\
2 \\
\hdashline \\
4
\end{tabular} & $\begin{array}{r}1219.25 \\
90.72 \\
1309.97\end{array}$ & $\begin{array}{r}1219.25 \\
45.36\end{array}$ & $\begin{array}{r}2.72 \\
.16 \\
2.87\end{array}$ & $\begin{array}{r}2.728 \\
.00 \\
\end{array}$ \\
\hline METAL DRUMS, BARRELS, KEGS & 1 GALLON & $\begin{array}{l}1978 \text { R } \\
\text { stom } \\
\text { sum }\end{array}$ & 2a & $21843 n$ & $\begin{array}{c}2 \\
2 \\
2\end{array}$ & $\begin{array}{r}27.22 \\
27.22\end{array}$ & 27.22 & $\begin{array}{r}.01 \\
.01\end{array}$ & .01 \\
\hline YETAL drums, BARRELS, KEGS & 110 GALLON & $\begin{array}{l}1976 \text { R } \\
\text { sum }\end{array}$ & $2 A$ & $21843 n$ & $\begin{array}{c}8 \\
8 \\
8\end{array}$ & $\begin{array}{r}1502.30 \\
1502.30\end{array}$ & 751.15 & $\begin{array}{r}3.40 \\
3.40\end{array}$ & 1.70 \\
\hline METAL DRUMS, BARRELS, KEGS & 110 GALLON & $\begin{array}{l}1977 \text { R } \\
\text { sum }\end{array}$ & $2 a$ & 21845a & 2 & $\begin{array}{r}272.15 \\
272.15\end{array}$ & 272.15 & $\begin{array}{l}.85 \\
. . . \\
.85\end{array}$ & .85 \\
\hline METAL ORLMS, BARRELS, KEGS & 110 GALLON & $\begin{array}{l}1978 \text { R } \\
\text { sum }\end{array}$ & $2 a$ & $21843 n$ & $\begin{array}{c}1 \\
1 \\
1\end{array}$ & $\begin{array}{r}453.59 \\
453.59\end{array}$ & 453.59 & $\begin{array}{r}.41 \\
.41\end{array}$ & .41 \\
\hline METAL DRLWS, BARRELS, KEGS & 30 GALLOW & 1973 R & $2 n$ & $21843 A$ & $\begin{array}{r}4 \\
\ldots . . .\end{array}$ & 176.90 & 176.90 & & .46 \\
\hline
\end{tabular}



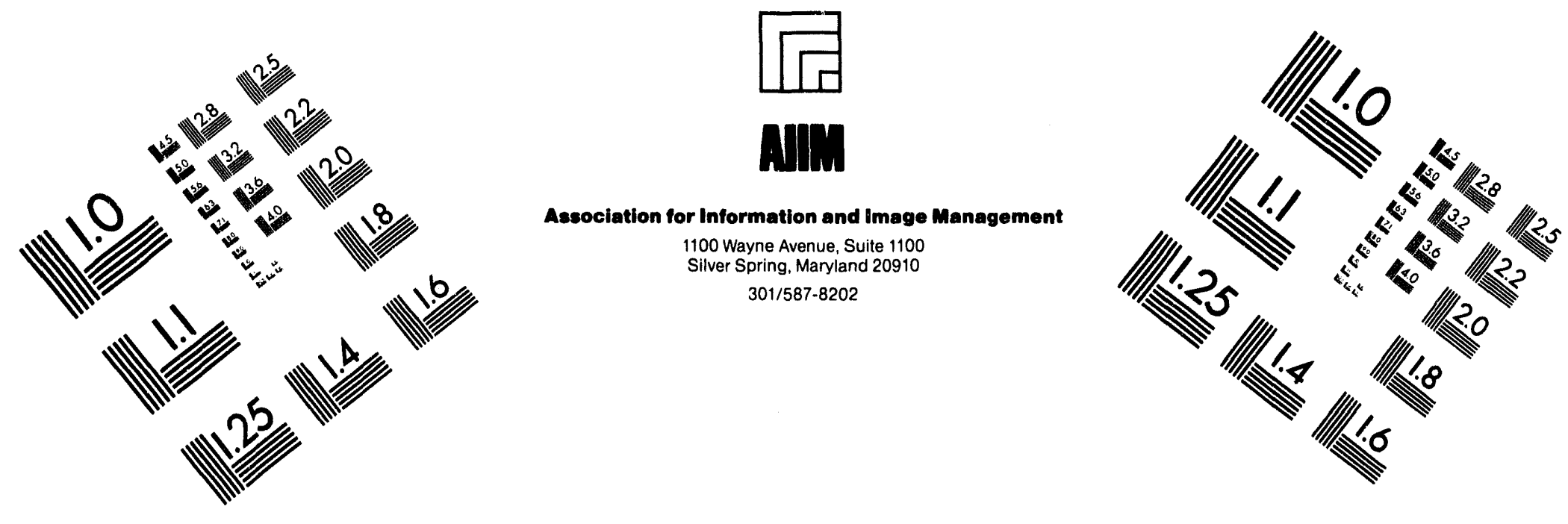

\section{Centimeter}

$\begin{array}{llllllllllllllll}1 & 2 & 3 & 4 & 5 & 6 & 7 & 8 & 9 & 10 & 11 & 12 & 13 & 14 & 15 & \mathrm{~mm}\end{array}$

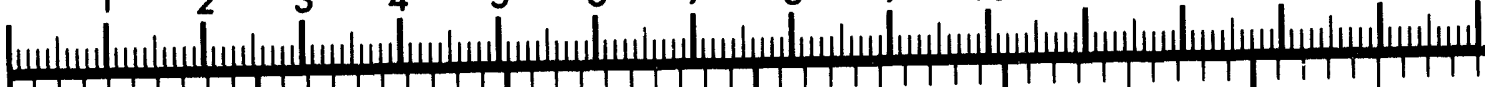

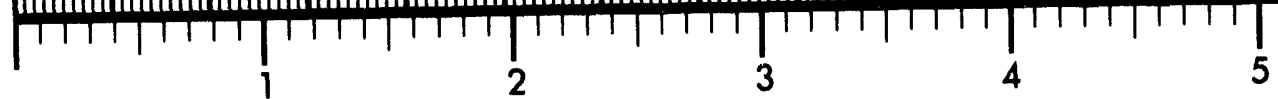
Inches
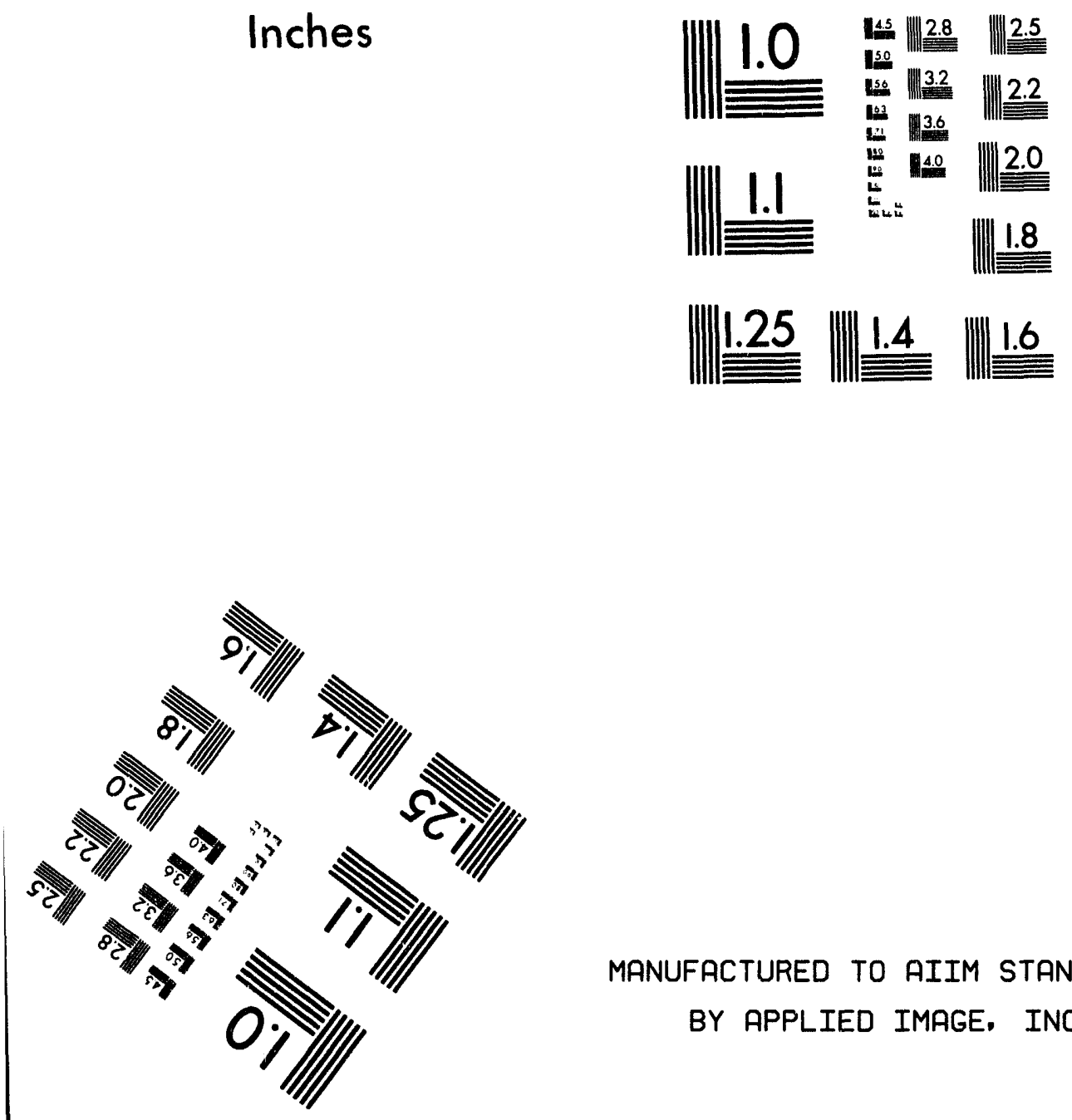

MANUFACTURED TO AIIM STANDARDS

BY APPLIED IMAGE, INC.

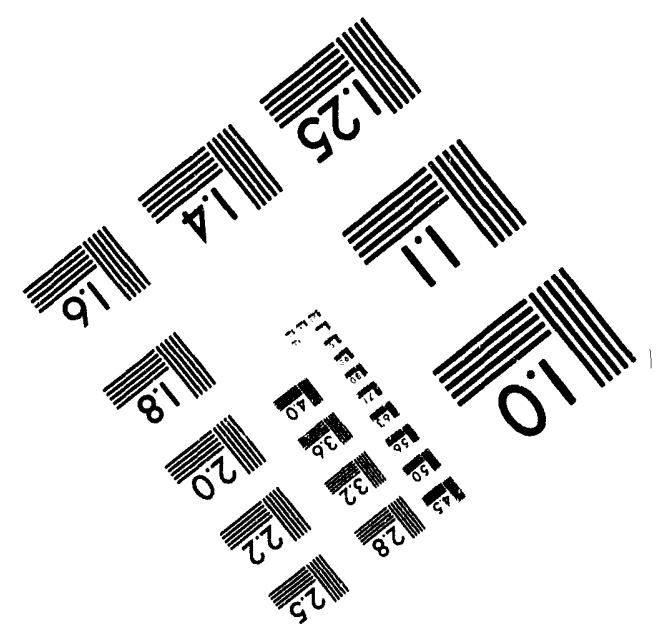



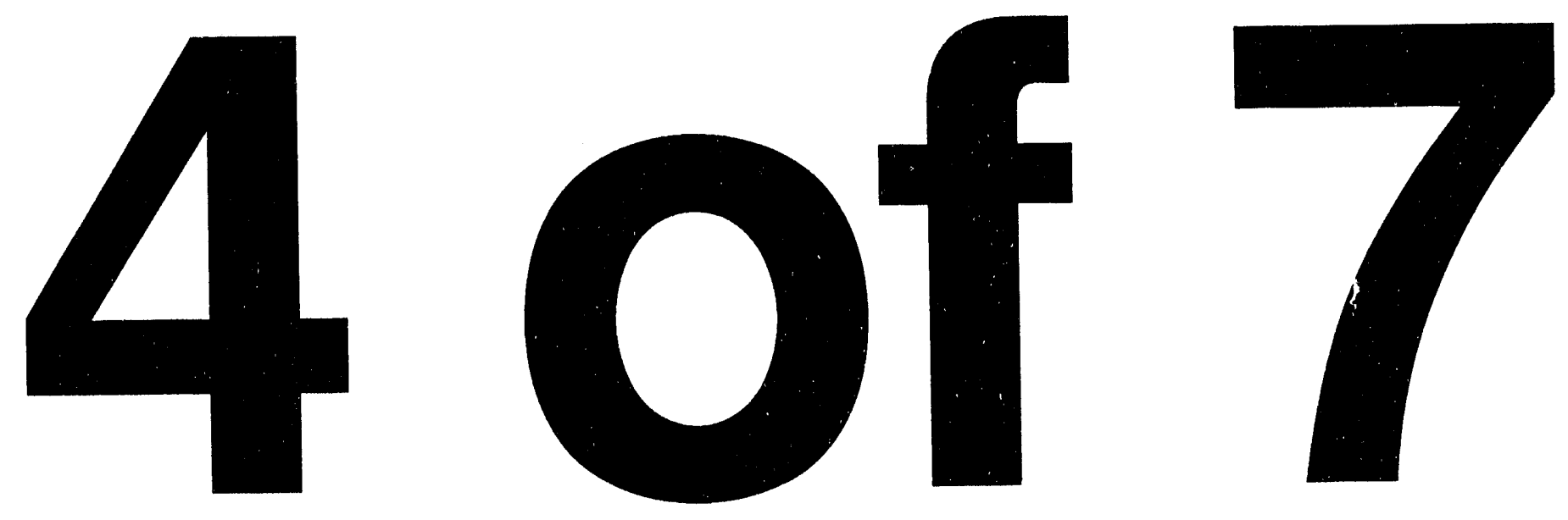


\begin{tabular}{|c|c|c|c|c|c|c|c|c|c|}
\hline Container Discription & Container size & $\begin{array}{r}\text { Pri } \\
\text { Was } \\
\text { Date Typ }\end{array}$ & $\begin{array}{l}\text { Sec } \\
\text { Wast } \\
\text { Code }\end{array}$ & Facility & Count & Height & $\begin{array}{r}\text { Avg } \\
\text { Weight }\end{array}$ & Volume & $\begin{array}{r}\text { Avg } \\
\text { volume }\end{array}$ \\
\hline & & sum & & & 4 & 176.90 & & .46 & \\
\hline METAL ORUMS, BARRELS, KEGS & 30 GALLON & $\begin{array}{l}1974 \text { R } \\
\text { sum }\end{array}$ & $2 \mathrm{~A}$ & 218L3A & $\begin{array}{c}23 \\
2.3\end{array}$ & $\begin{array}{r}938.93 \\
938.93\end{array}$ & 469.47 & $\begin{array}{l}2.75 \\
2.75\end{array}$ & 1.37 \\
\hline METAL DRUMS， BARRELS，KEGS & 30 GALLON & $\begin{array}{l}1976 \text { R } \\
* * * * * \\
\text { sum }\end{array}$ & $2 \mathrm{~A}$ & $21843 A$ & 1 & $\begin{array}{r}40.82 \\
40.82\end{array}$ & 40.82 & $\begin{array}{l}.12 \\
.12\end{array}$ & .12 \\
\hline METAL DRUMS， BARRELS， KEGS & 30 GALLON & $\begin{array}{l}1977 \text { R } \\
\text { ***** } \\
\text { sum }\end{array}$ & $2 \mathrm{~A}$ & 218W3A & 9 & $\begin{array}{l}349.26 \\
349.26\end{array}$ & 116.42 & $\begin{array}{r}2.04 \\
2.04\end{array}$ & .68 \\
\hline $\begin{array}{l}\text { METAL DRUMS, BARRELS, KEGS } \\
\vdots \\
\dot{\nu}\end{array}$ & 30 GALLON & $\begin{array}{l}1978 \text { R } \\
* * * * *\end{array}$ & $2 \mathrm{~A}$ & 21843A & 3 & $\begin{array}{r}190.05 \\
0\end{array}$ & 63.35 & $\begin{array}{r}.38 \\
7\end{array}$ & 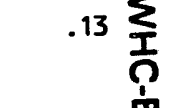 \\
\hline & & sum & & & 2 & 48.08 & & .09 & \\
\hline METAL ORUMS, BARRELS, KEGS & 5 GALLON & $\begin{array}{l}1978 \text { R } \\
\star \star \star \star \star \star\end{array}$ & $2 \mathrm{~A}$ & $218 W 3 A$ & 3 & $\begin{array}{r}68.04 \\
\end{array}$ & 68.04 & .16 & .16 \\
\hline & & sum & & & 3 & 68.04 & & .16 & \\
\hline METAL DRUMS， BARRELS，KEGS & 5 GALLON & $\begin{array}{l}1979 \mathrm{R} \\
\star \star \star \star \star\end{array}$ & $2 \mathrm{~A}$ & $218 W 3 A$ & 2 & $\begin{array}{r}45.36 \\
\end{array}$ & 45.36 & .06 & .06 \\
\hline & & sum & & & 2 & .45 .36 & & .06 & \\
\hline METAL DRUMS， BARRELS，KEGS & 55 GALLON & $\begin{array}{l}1970 \mathrm{R} \\
\star \star \star \star \star\end{array}$ & $2 \mathrm{~A}$ & $218 W 3 A$ & 10 & $\begin{array}{r}680.39 \\
0.39\end{array}$ & 226.80 & $\begin{array}{r}2.12 \\
\end{array}$ & .71 \\
\hline & & sum & & & 10 & 680.39 & & 2.12 & \\
\hline METAL DRUMS, BARRELS, KEGS & 55 GALLON & $1971 \mathrm{M}$ & $2 \mathrm{~A}$ & $218 W 4 B$ & 3 & 204.12 & 204.12 & .64 & .64 \\
\hline METAL DRUMS, BARRELS, KEGS & 55 GALLON & $\mathbf{R}$ & ZA & $218 W 3 A$ & 18 & 1224.71 & 204.12 & 3.78 & .63 \\
\hline
\end{tabular}




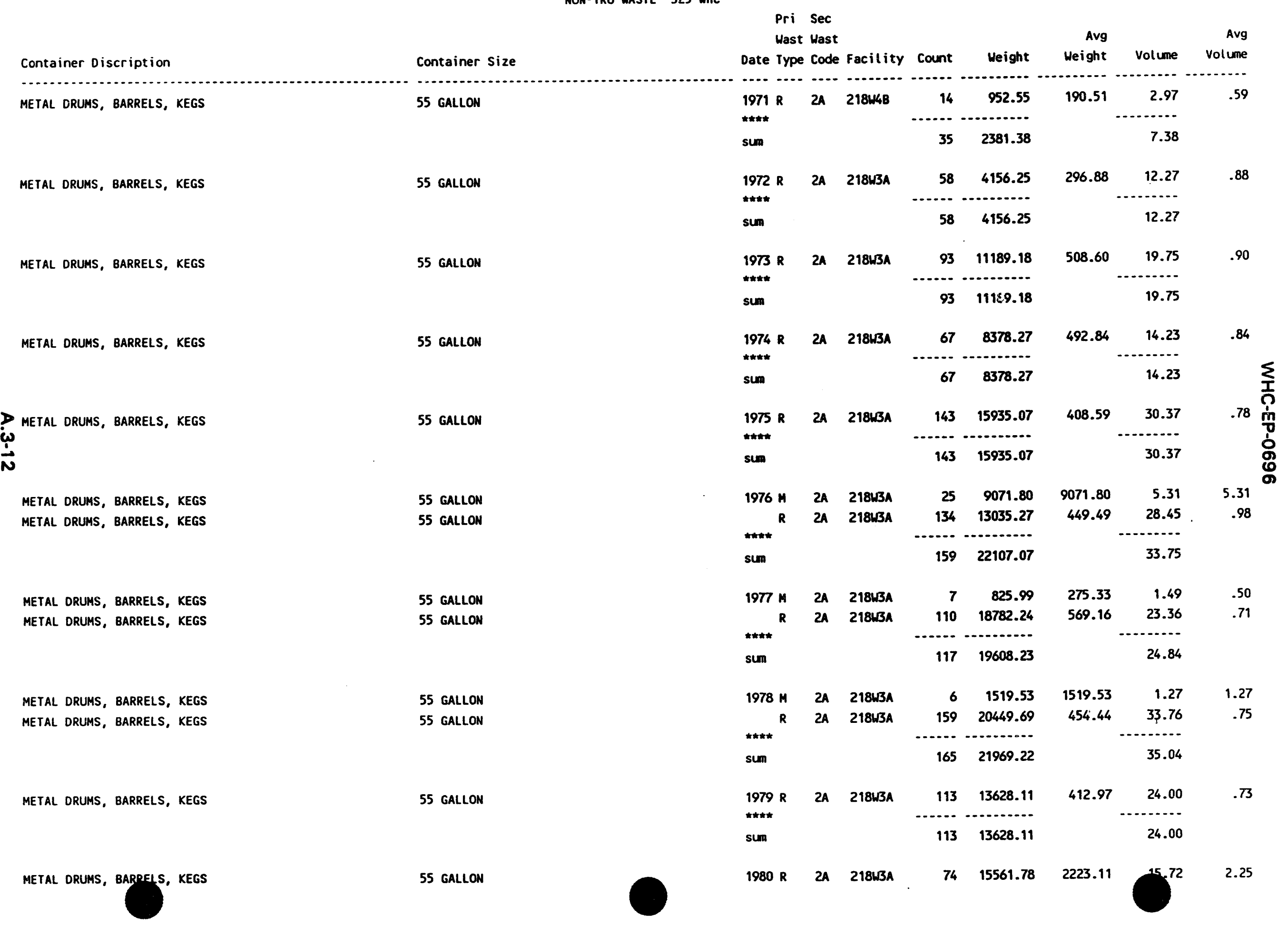


Container Discripition

Container Size

METAL DRUMS, BARRELS, KEGS

METAL DRUMS, BARRELS, KEGS

METAL DRUMS, BARRELS, KEGS

MISCELLANEOUS SCRAP

$\stackrel{\rightarrow}{\Rightarrow}$

MISCELLANEOUS SCRAP

MISCELLANEOUS SCRAP MISCELLANEOUS SCRAP

\section{MISCELLANEOUS SCRAP}

MISCELLANEOUS SCRAP

MISCELLANEOUS SCRAP

55 GALLON

55 GALLON

85 GALLON

UNKNOWN

UNKNOWN

UNKNOWN

UNKNOWN

UNKNOWM

UNKNOWN

UNKNOWN
Pri Sec

Wast Wast

Date Type Code Facility Count Weight Weight volume volume

.....

sum

$74 \quad 15561.78$

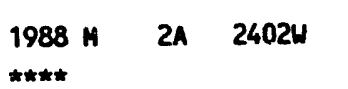

\begin{tabular}{rr}
1 & 149.68 \\
\hline 1 & 149.68
\end{tabular}

sum

1990 M $2 A \quad$ FS6

sum

1988 N $2 A \quad 2402 W C$

1970 R $2 A \quad 218$ A $3 A$

*\#**

sum

1971 R 2A 218W3A

sum

1972 M 2A 218W3A

R $2 A \quad 218 W 3 A$

$\star \star \star \star$

sum

1973 R 2A 218W3A
$\star \star \star \star$
sum

$1974 R \quad 2 A \quad 218 W 3 A$

sum

1975 R 2 A 218W3A

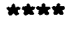

sum

$\begin{array}{rr}3 & 239.94 \\ 3 & 239.94\end{array}$

2766.56

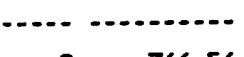

2766.56

\begin{tabular}{rr}
10 & 3294.87 \\
\hline 10 & 3294.87
\end{tabular}

$4 \quad 2559.15$

$4 \quad 2559.15$

\begin{tabular}{rr}
10 & 2721.54 \\
17 & 215.91 \\
\hline 27 & 2937.45
\end{tabular}

215.91

$215.91 \quad 1.91$

2.19

$27 \quad 2937.45$

\begin{tabular}{rr}
18 & 14918.1 \\
\hline 18 & 14918.1
\end{tabular}

$2131.16 \quad 33.26$

.........

33.26

\begin{tabular}{rr}
20 & 5719.77 \\
\hline 20 & 5719.77
\end{tabular}

$817.11 \quad 13.41$

13.41

$801.67 \quad 23.13$
1.78

$3.51 \stackrel{1}{\Omega}$

$\mathbf{1}$
8
1
0
0
0
0
0

$1.43 \stackrel{\%}{\%}$

.28

1.91

4.75

1.92 23.13 


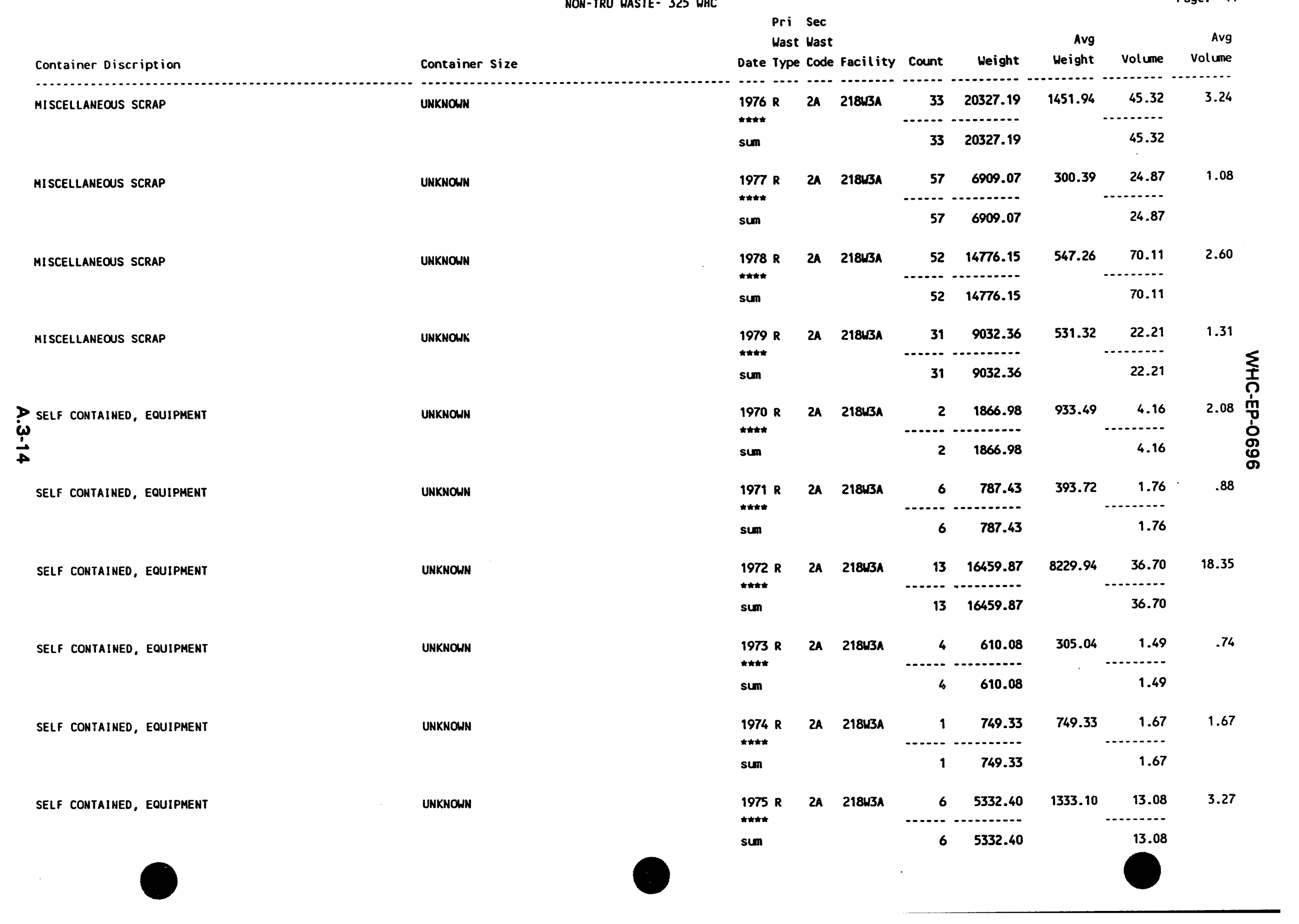




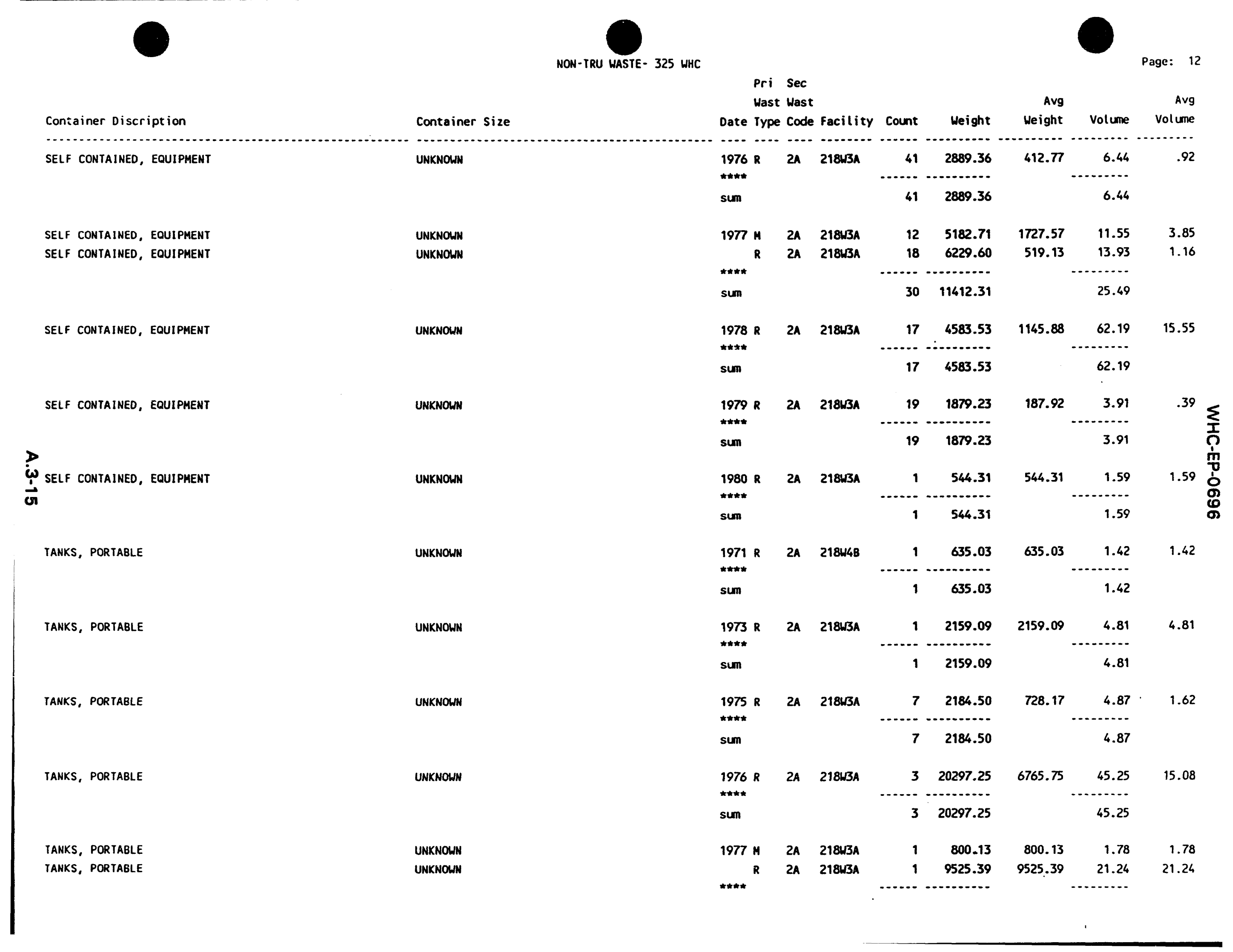




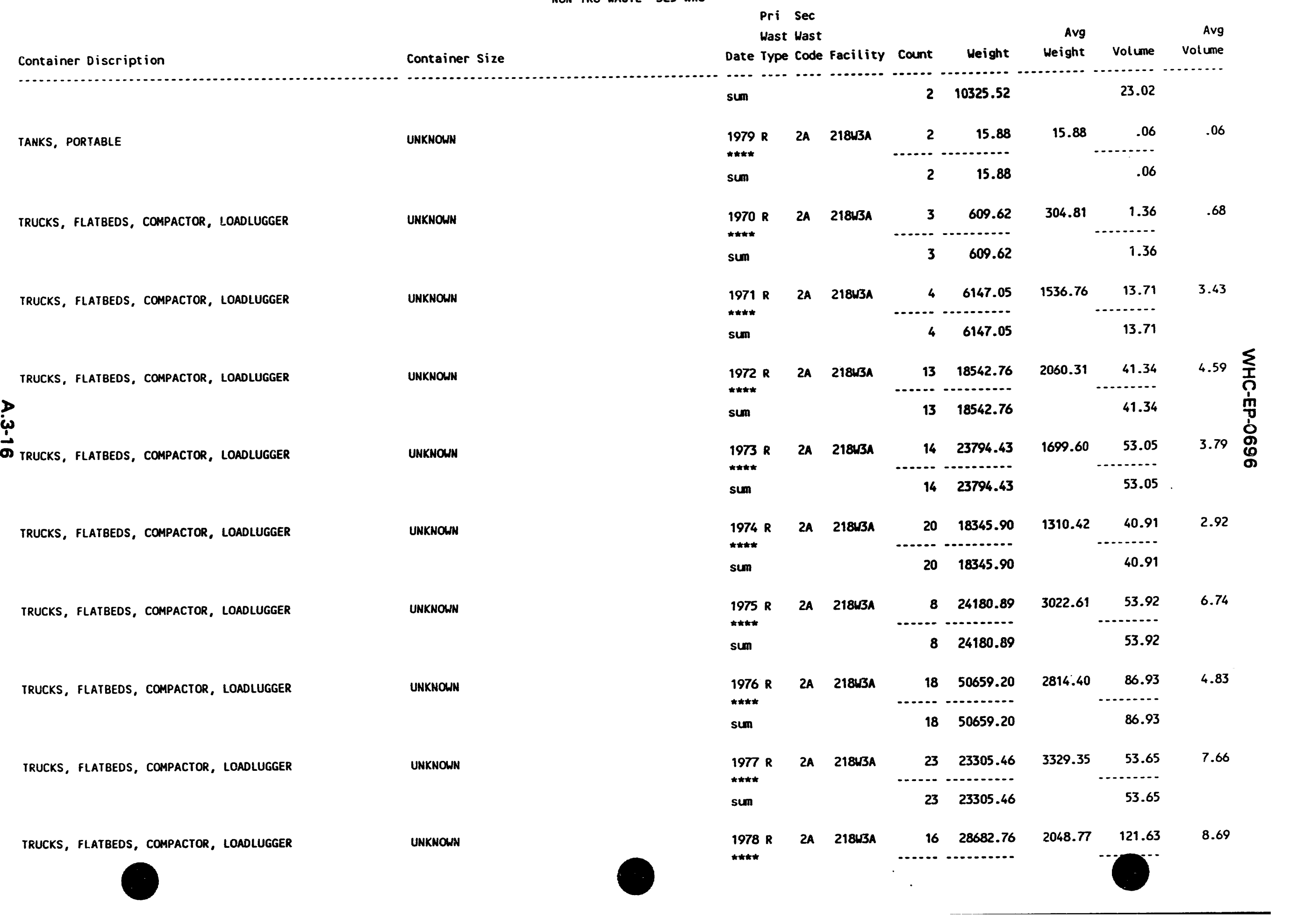




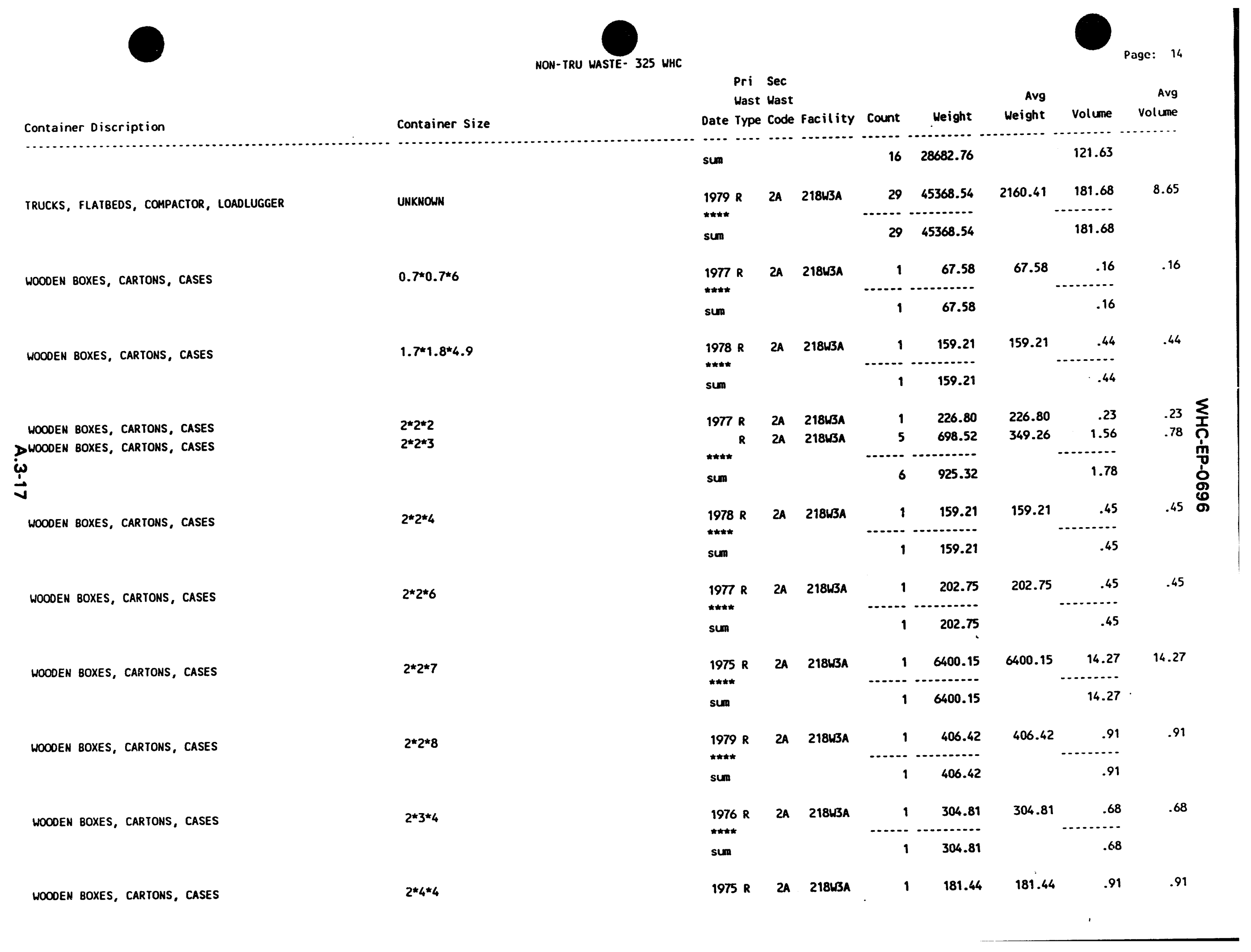




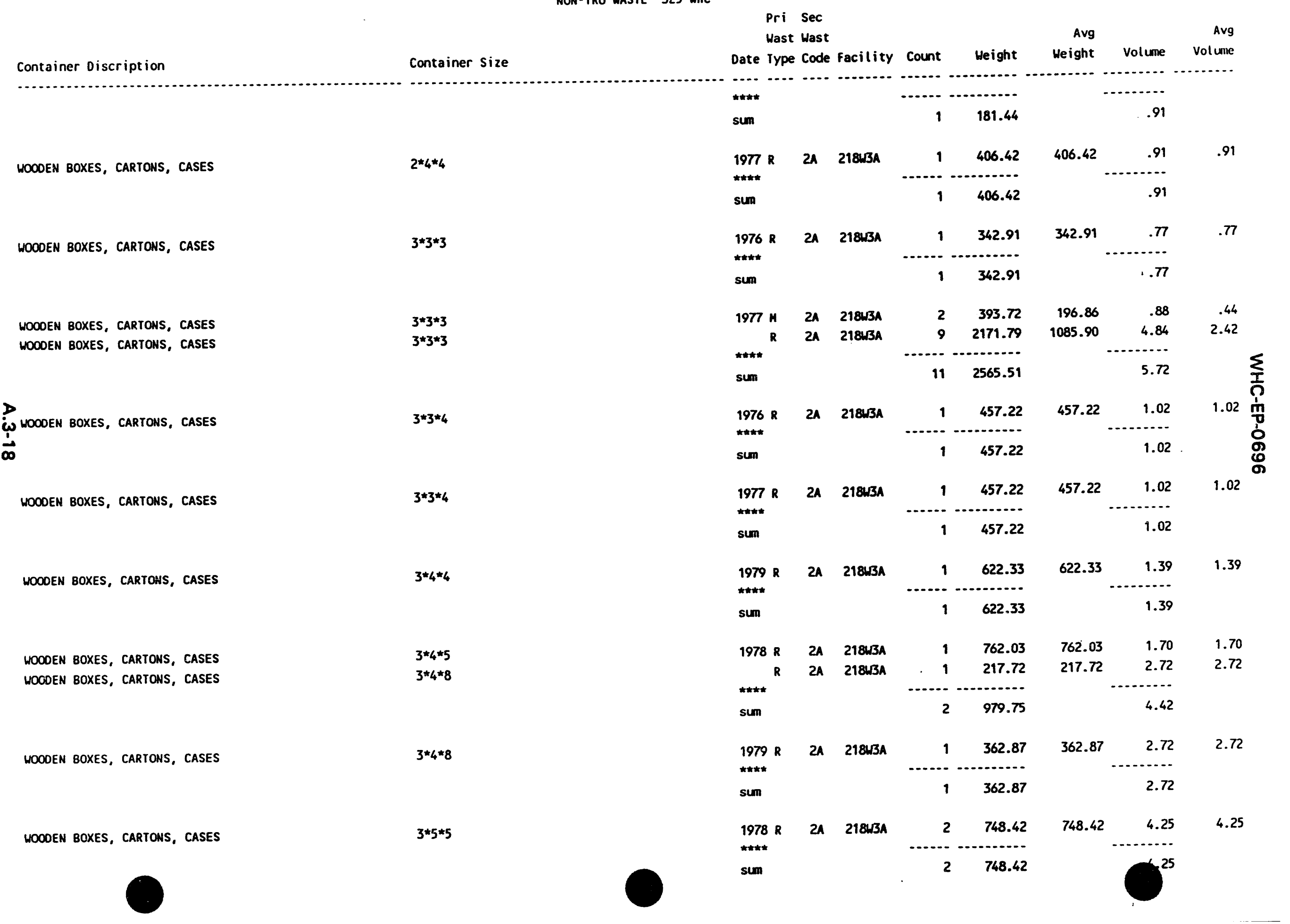




\section{Container Discription}

WOODEN BOXES, CARTONS, CASES WOOOEN BOXES, CARTONS, CASES

WOOOEN BOXES, CARTONS, CASES

HOOOEN BOXES, CARTONS, CASES WOOOEN BOXES, CARTONS, CASES

WOODEN BOXES, CARTONS, CASES ؛

ف

WOOOEN BOXES, CARTONS, CASES

WOOOEN BOXES, CARTONS, CASES

WOODEN BOXES, CARTONS, CASES

WOOOEN BOXES, CARTONS, CASES

WOOOEN BOXES, CARTONS, CASES

WOOOEN BOXES, CARTONS, CASES
Container Size

$3.3 * 3.3 * 3.3$

$4 * 4 * 12$

$4 \star 4 \star 12$

$4 * 4 * 4$

$4 * 4 * 4$

$4 * 4 * 4$

$4 \star 4 \star 5$

$4 * 4 * 6$

$4 * 4 * 6$

$4 \star 4 \star 7$

$4 * 4 * 8$

$4 \star 4 * 8$

Pri Sec

Wast Wast

Date Type Code Facility Count Weight Weight Volume Volume

Count Weight

Avg Volume volume

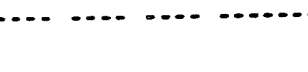

$1975 R \quad 2 A \quad 218 W 3 A$

$R \quad 2 A \quad 21843 A$

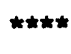

sum

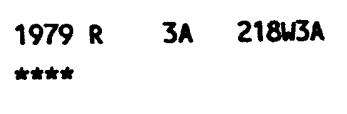

sum

$\begin{array}{rrr}1977 M & 2 A & 21843 A \\ R & 2 A & 218 W 3 A \\ * * * * & & \end{array}$

sum

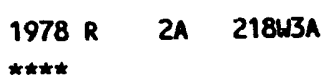

sum

1975 R $2 A \quad 218$ A $3 A$

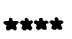

sum

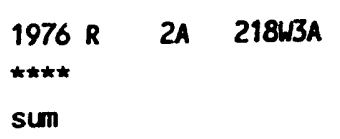

sum

1977 M 2A 218WBA

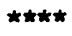

sum

1970 R 2A 218W3A

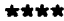

sum

1972 R 2A 218W3A

****

sum

1975 R 2A 21843A

\begin{tabular}{rr}
1 & 470.37 \\
1 & 181.44 \\
\hline 2 & 651.81
\end{tabular}

\begin{tabular}{rr}
1 & 1045.07 \\
\hline 1 & 1045.07
\end{tabular}

$2 \quad 1602.98$

$6 \quad 4876.09$

$\begin{array}{ll}8 & 6479.07\end{array}$

18128

.

$1 \quad 812.83$

$\begin{array}{rr}1 & 1016.04 \\ 1 & 1016.04\end{array}$

$\begin{array}{rrr}470.37 & 1.05 & 1.05 \\ 181.44 & 5.44 & 5.44 \\ & .2 . & \\ & 6.49 & \end{array}$

1045.

5.44
5.44

$801.49 \quad 3.62$

$4876.09 \quad 10.87$

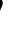$$
\text { ........ }
$$

812.83

812.8

1.81

1016.0

2.27

2.27

\begin{tabular}{rr}
1 & 12192.50 \\
\hline 1 & 12192.50
\end{tabular}

12192.50

2.72

2.72

$\begin{array}{rr}2 & 2438.50 \\ \ldots .2 & \end{array}$

2438.50

2.72

$2 \quad 2438.50$

5.44
...-

5.44

9.57

9.57

$3 \quad 4292.78$

5.44

1.81

10.87

$1.81 \frac{1}{\Omega}$

81

$2.27 \stackrel{0}{\boldsymbol{\sigma}}$

2.72

$1625.67 \quad 3.63$

3.63

$1 \quad 1625.67$

$2438.50 \quad 32.62$ 
Container Discription

Container size

WOCOEN BOXES, CARTONS, CASES

WOOOEN BOXES, CARTONS, CASES WOODEN BOXES, CARTONS, CASES

WOOOEN BOXES, CARTONS, CASES

WOOOEN BOXES, CARTONS, CASES WWOOEN BOXES, CARTONS, CASES

WOODEN BOXES, CARTONS, CASES

WOOOEN BOXES, CAKTONS, CASES

WOOOEN BOXES, CARTONS, CASES

WOOOEH BOXES, CARTONS, CASES

WOOOEN BOXES, CARTONS, CASES

WOOOEN BOXES, CARTONS, CASES

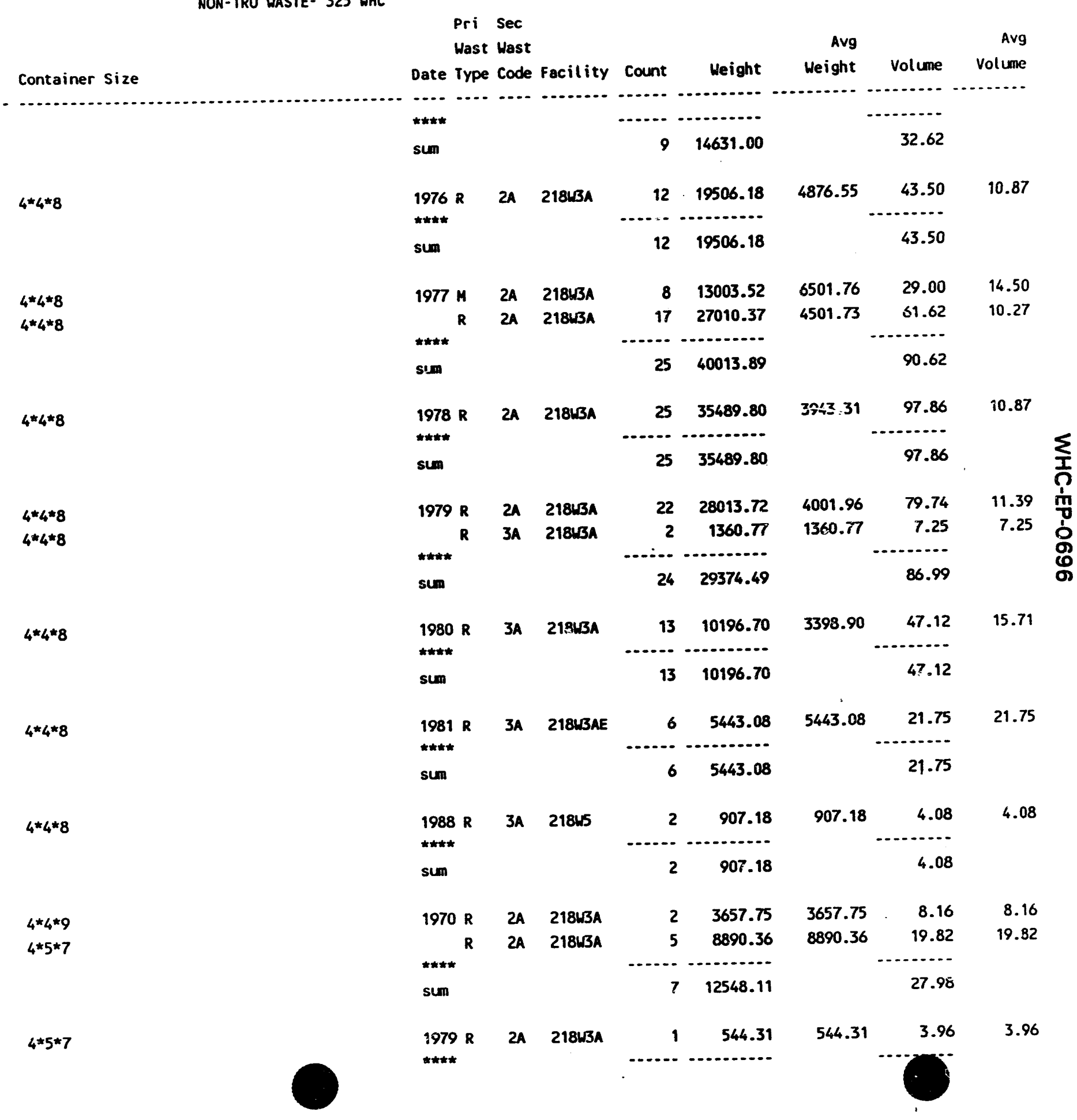




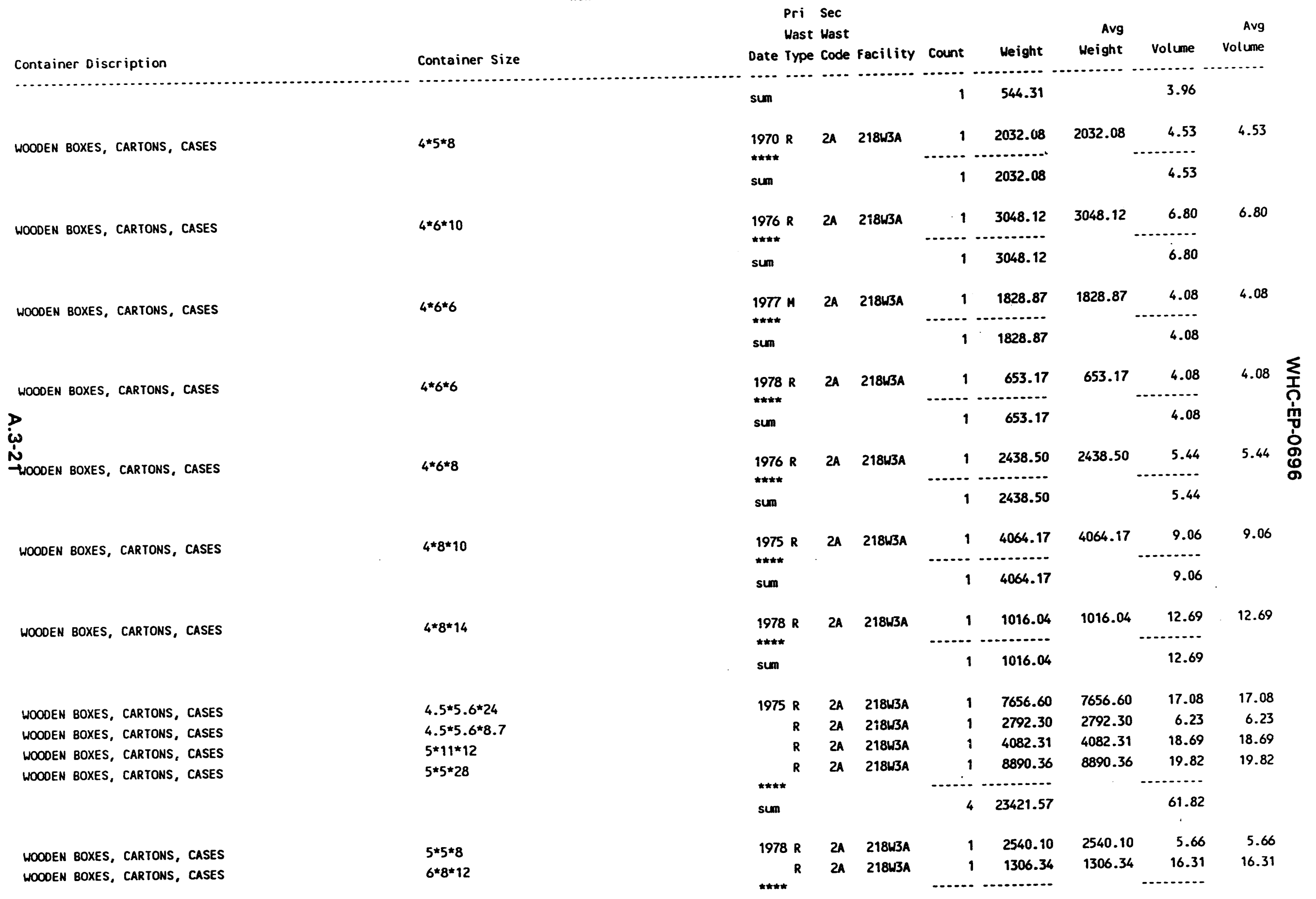




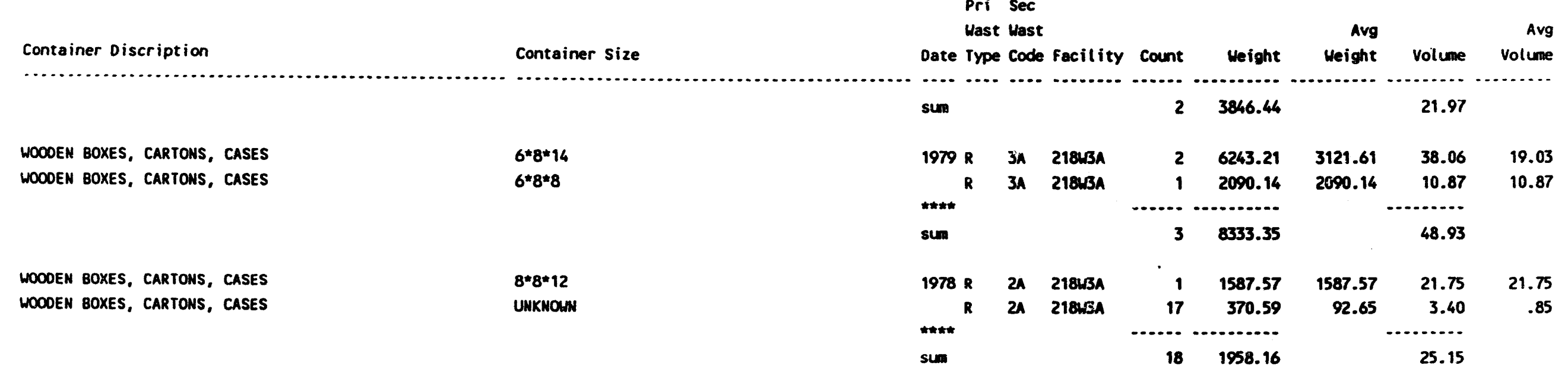

207 rows selected.

$\omega$
$N$
$N$ 
WHC-EP-0696

\section{APPENDIX A.4}

RADIOLOGICAL DATA FOR TRU WASTE CONTAINERS SORTED BY DATE, PRIMARY WASTE TYPE, AND STORAGE FACILITY 


\section{WHC-EP-0696}

This subappendix summarizes the radiological data for all TRU waste containers generated at Building 325 Westinghouse Hanford. Specifically, this computer run provides the total grams of TRU elements in a given container type in a given year. In addition, the average gram loading for a given container type in a given year has been calculated.

In 1991 and 1992 the number of curies (Ci) of alpha radiation was included on the SWBRs. Since the Building 325 Westinghouse Hanford waste generation data is all from 1970 to 1988, these columns contain blank entries. The "Max Dose" column contains the highest value recorded for a given container in a given year, in $\mathrm{mrem} / \mathrm{hr}$.

Additional information about the isotopes present in Building 325 Westinghouse Hanford waste containers can be found in subappendices A.9 and A.10. 
set linesize 170

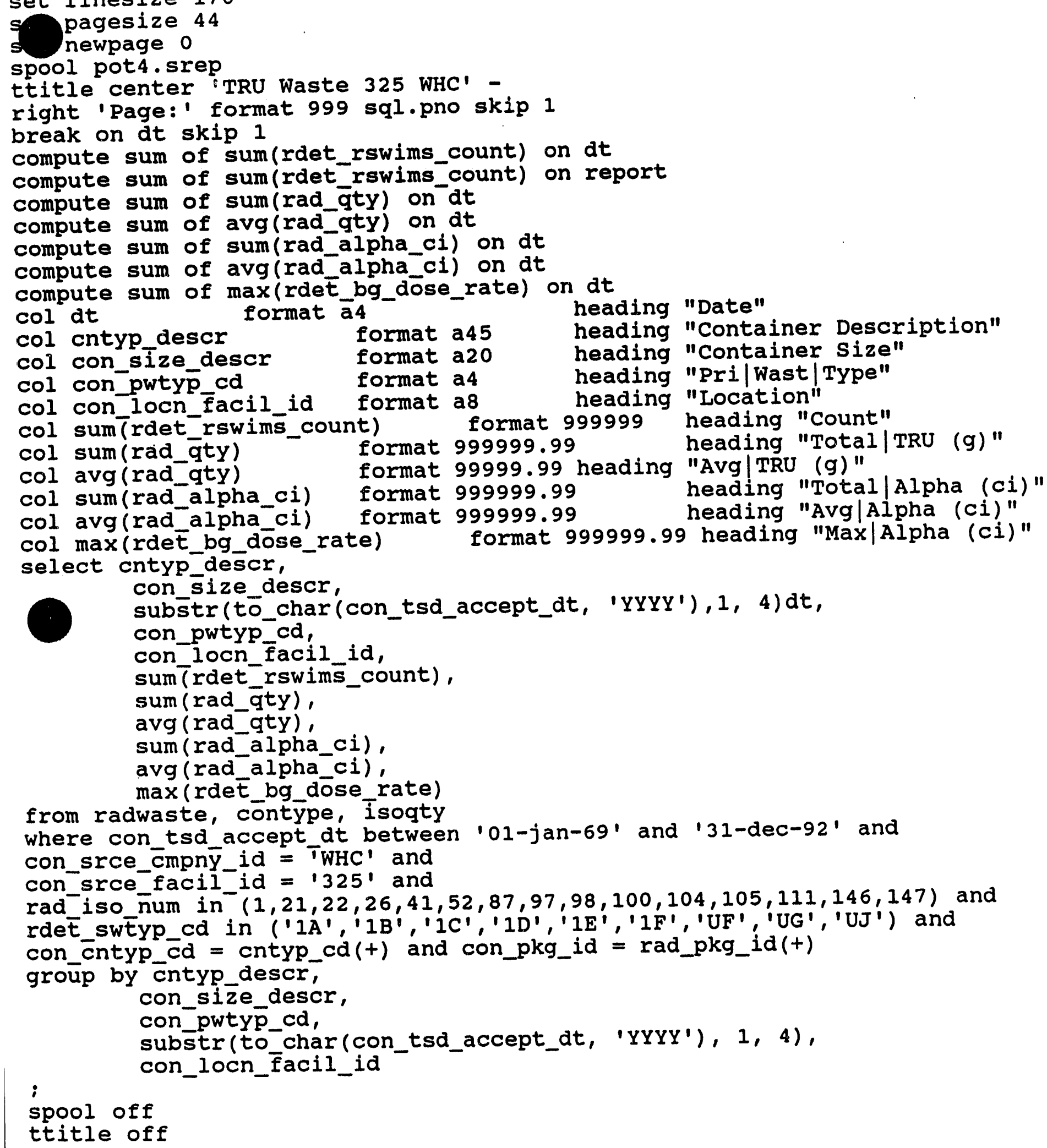

spool off

ttitle off 


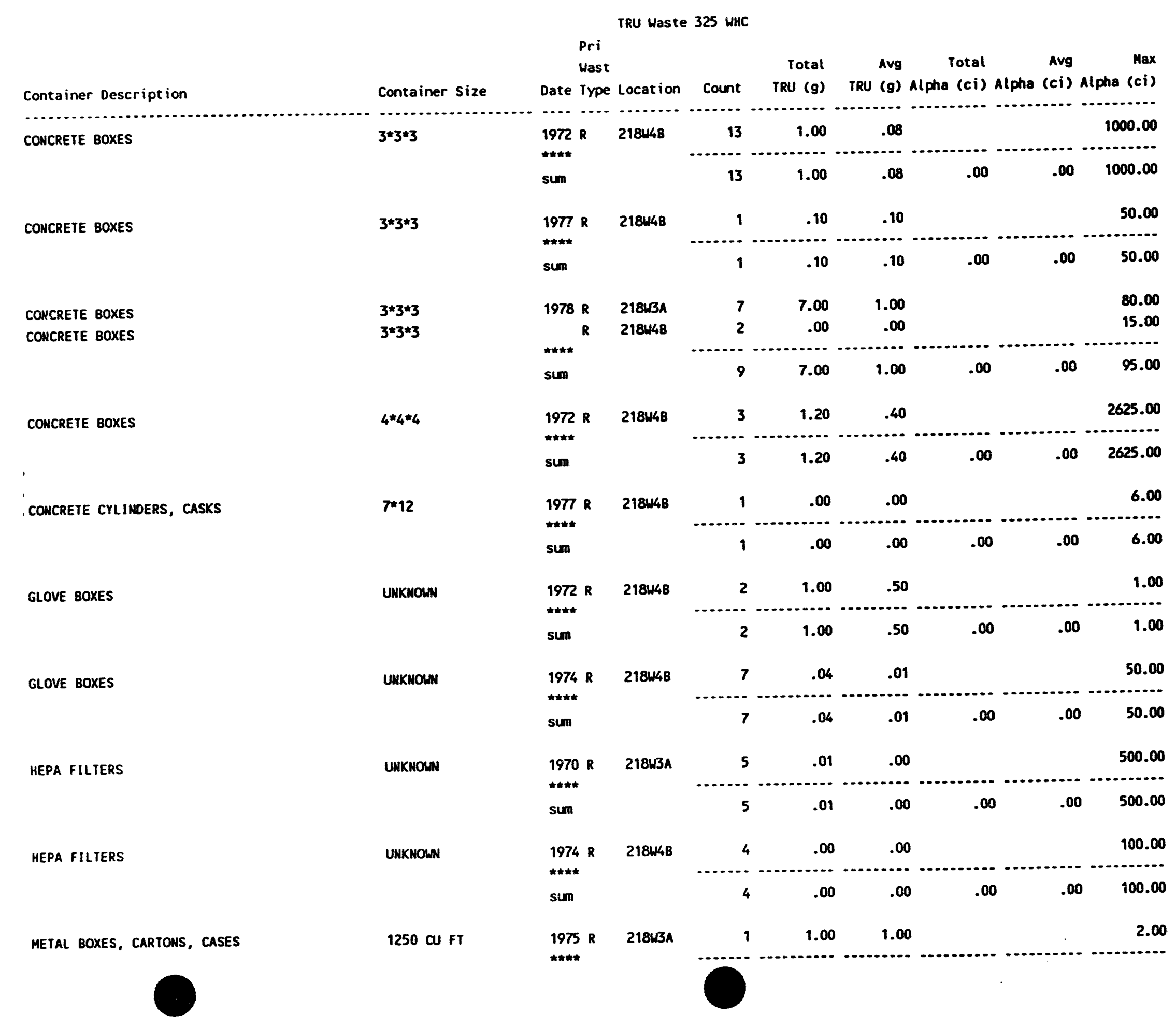




\begin{tabular}{|c|c|c|c|c|c|c|c|c|c|}
\hline & & $\begin{array}{l}\text { Pri } \\
\text { Wast }\end{array}$ & & & Total & Avg & Total & Avg & $\operatorname{Max}$ \\
\hline \multirow[t]{2}{*}{ Container Description } & Container Size & Date Type & Location & Count & TRU (g) & $\operatorname{TRU}(g)$ & Alpha (ci) & Alpha (ci) & Alpha (ci) \\
\hline & & sum & & 1 & 1.00 & 1.00 & .00 & .00 & 2.00 \\
\hline \multirow[t]{2}{*}{ METAL BOXES, CARTONS, CASES } & $4 * 4 * 4$ & $1972 \mathrm{R}$ & 218448 & 2 & .80 & .40 & & & 2625.00 \\
\hline & & sum & & 2 & .80 & .40 & .00 & .00 & 2625.00 \\
\hline METAL BOXES, CARTONS, CASES & $4 * 4 * 5$ & $1976 \mathrm{R}$ & 218448 & 1 & 1.00 & 1.00 & & & 5.00 \\
\hline \multirow[t]{2}{*}{ METAL BOXES, CARTONS, CASES } & $4 * 5 * 9$ & $\mathbf{R}$ & 218148 & 1 & 1.00 & 1.00 & & & 5.00 \\
\hline & & sum & & 2 & 2.00 & 2.00 & .00 & .00 & 10.00 \\
\hline \multirow[t]{2}{*}{ METAL BOXES, CARTOWS, CASES } & $4.2 * 6.2 * 15.6$ & $1980 \mathrm{R}$ & $218 w 4 C$ & 1 & .00 & .00 & & & 6.00 \\
\hline & & sum & & 1 & .00 & .00 & .00 & .00 & 6.00 \\
\hline \multirow{3}{*}{$\begin{array}{l}\text { METAL BOXES, CARTONS, CASES } \\
\text { PMETAL BOXES, CARTONS, CASES } \\
\text { of }\end{array}$} & $5 * 5 * 5$ & 1974 R & 218W3A & 1 & .10 & .10 & & & 5.00 \\
\hline & $7 * 10 * 16$ & $\mathbf{R}$ & $218 W 3 A$ & 1 & .10 & .10 & & & 5.00 \\
\hline & & sum & & 2 & .20 & .20 & .00 & .00 & 10.00 \\
\hline \multirow[t]{2}{*}{ METAL ORUHS, BARRELS, KEGS } & 110 GALLON & $1976 \mathrm{R}$ & 218W4B & 1 & .10 & .10 & & & 25.00 \\
\hline & & sum & & 1 & .10 & .10 & .00 & .00 & 25.00 \\
\hline \multirow[t]{2}{*}{ METAL DRUMS, BARRELS, KEGS } & 30 GALLON & $1970 \mathrm{R}$ & $21843 A$ & 17 & .01 & .00 & & & 10.00 \\
\hline & & 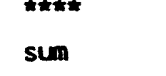 & & 17 & .01 & .00 & .00 & .00 & 10.00 \\
\hline \multirow[t]{2}{*}{ METAL DRUMS, BARRELS, KEGS } & 30 GALLON & $1971 \mathrm{R}$ & $218 W 3 A$ & 1 & .00 & .00 & & & 10.00 \\
\hline & & sum & & 1 & .00 & .00 & .00 & .00 & 10.00 \\
\hline \multirow[t]{2}{*}{ METAL DRUMS, BARRELS, KEGS } & 55 GALLON & $1970 \mathrm{R}$ & $218 W 3 A$ & 67 & 20.01 & .30 & & & 20.00 \\
\hline & & sum & & 67 & 20.01 & .30 & .00 & .00 & 20.00 \\
\hline METAL DRUMS, BARRELS, KEGS & 55 GALLON & $1971 \mathrm{R}$ & $21843 \mathrm{~A}$ & 76 & 20.01 & .26 & & & 50.00 \\
\hline METAL DRUMS, BARRELS, KEGS & 55 GALLON & $\mathbf{R}$ & $218 W 4 B$ & 163 & 183.22 & 1.12 & & & 10.00 \\
\hline
\end{tabular}


Container Description

Metal DRUMS, BarReLs, KEgs

METAL DRUMS, BARRELS, KEGS

METAL DRUMS, BARRELS, KEGS

METAL DRUMS, BARRELS, KEGS

METAL DRUMS, BARRELS, KEGS

METAL DRUMS, BARRELS, KEGS

METAL DRUMS, BARRELS, KEGS METAL DRUMS, BARRELS, KEGS

METAL DRUMS, BARRELS, KEGS

METAL DRUMAS, BARRELS, KEGS
Pri

Wast Iotal Avg Total Avg Max

Container size

Date Type Location Count

TRU (g) TRU (g) Alpha (ci) Alpha (ci) Alpha (ci)

$\begin{array}{lllllll}\text { sum } & 239 & 203.23 & 1.39 & .00 & .00 & 60.00\end{array}$

55 GALLON

$1972 R \quad 218 w 4 B$
$\star \star \star \star$

sum

55 GALLON

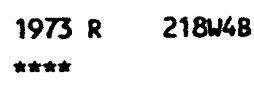

sum

S5 GALLON

$1974 R \quad 218$ R
$\star \star \star \star \star$

sum

55 GALLOM

1975 R $218 W 4 B$
sum

\begin{tabular}{|c|c|c|}
\hline 239 & 427.41 & 1.79 \\
\hline 239 & 427.41 & 1.79 \\
\hline
\end{tabular}

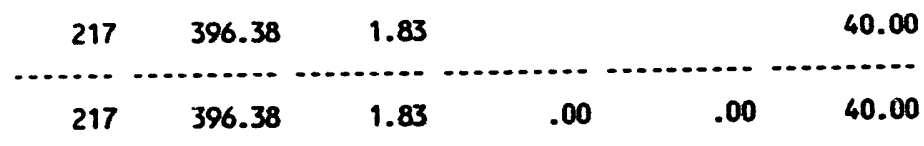

\begin{tabular}{|c|c|c|}
\hline 254 & 1167.35 & 4.60 \\
\hline & 1167.35 & 4.60 \\
\hline
\end{tabular}

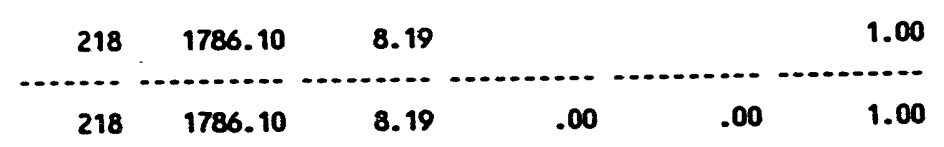

55 GALLON

1976 R $218 w 48$

sum

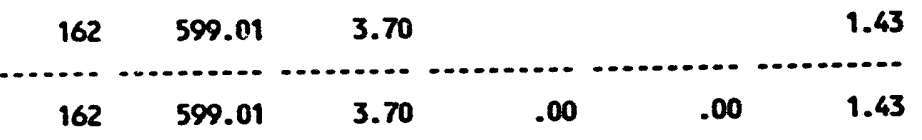

55 GALLON

1977 R 218W4B

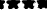

sum

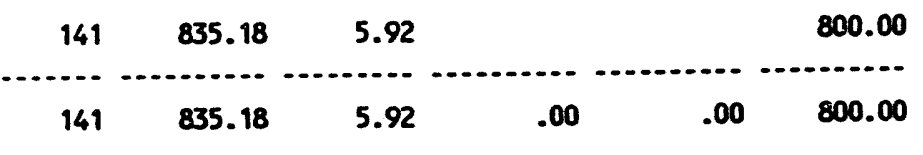

55 GALLON

55 GALLON

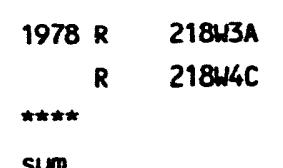

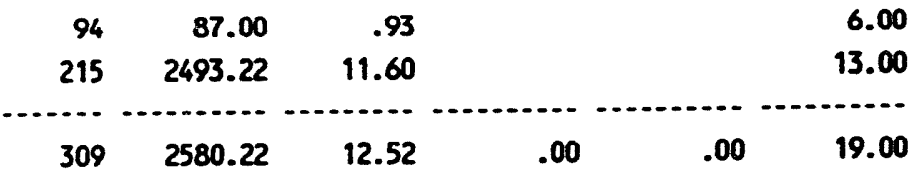

55 GALLON

1979 R $218 W 4 C$
$\star \star \star \star$

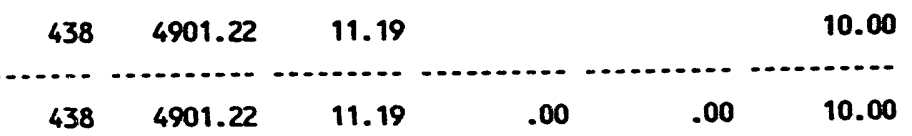

55 GALLON

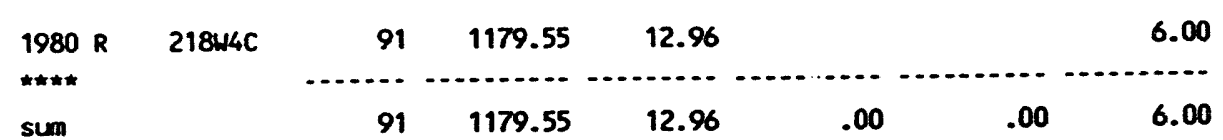




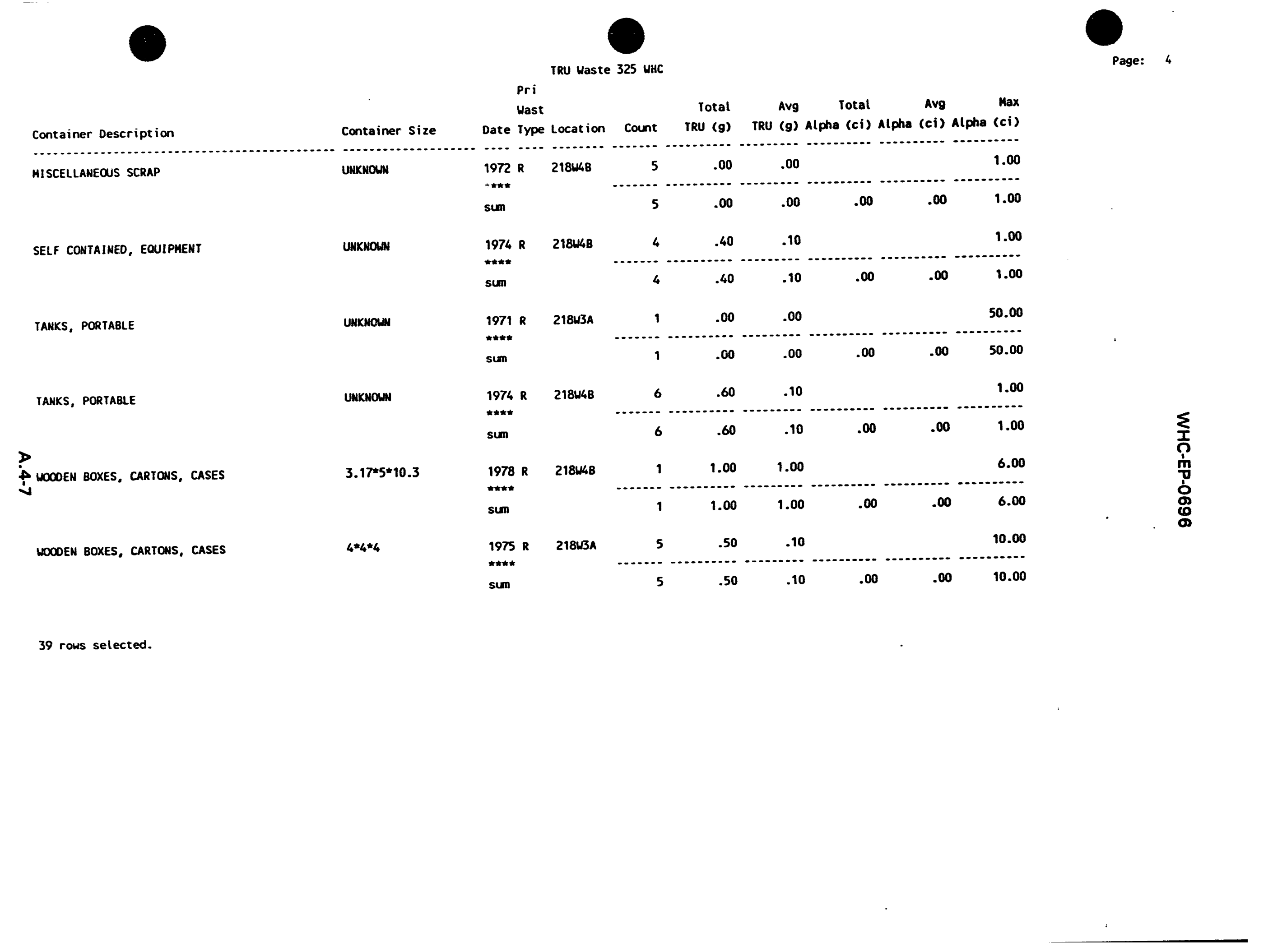


WHC-EP-0696

This page intentionally left blank.

A.4-8 
WHC-EP-0696

APPENDIX A.5

PHYSICAL CONTENTS DESCRIPTION FOR BUILDING 325 WESTINGHOUSE HANFORD 55-GALLON DRUMS CONTAINING TRU WASTE SORTED BY DATE, PRIMARY WASTE TYPE, AND STORAGE FACILITY

A.5-1 
This subappendix describes the physical contents of the 55-gal drums of TRU waste generated by Building 325 Westinghouse Hanford. Prior to 1978, physical contents were not required on the burial records, so a great many of the early records list the contents of the drum as "Miscellaneous."

The printout for this computer run sorts the contents data by storage facility, date and primary waste type. The introduction to Appendix A contains a table of waste codes and their meanings. 


\section{WHC-EP-0696}

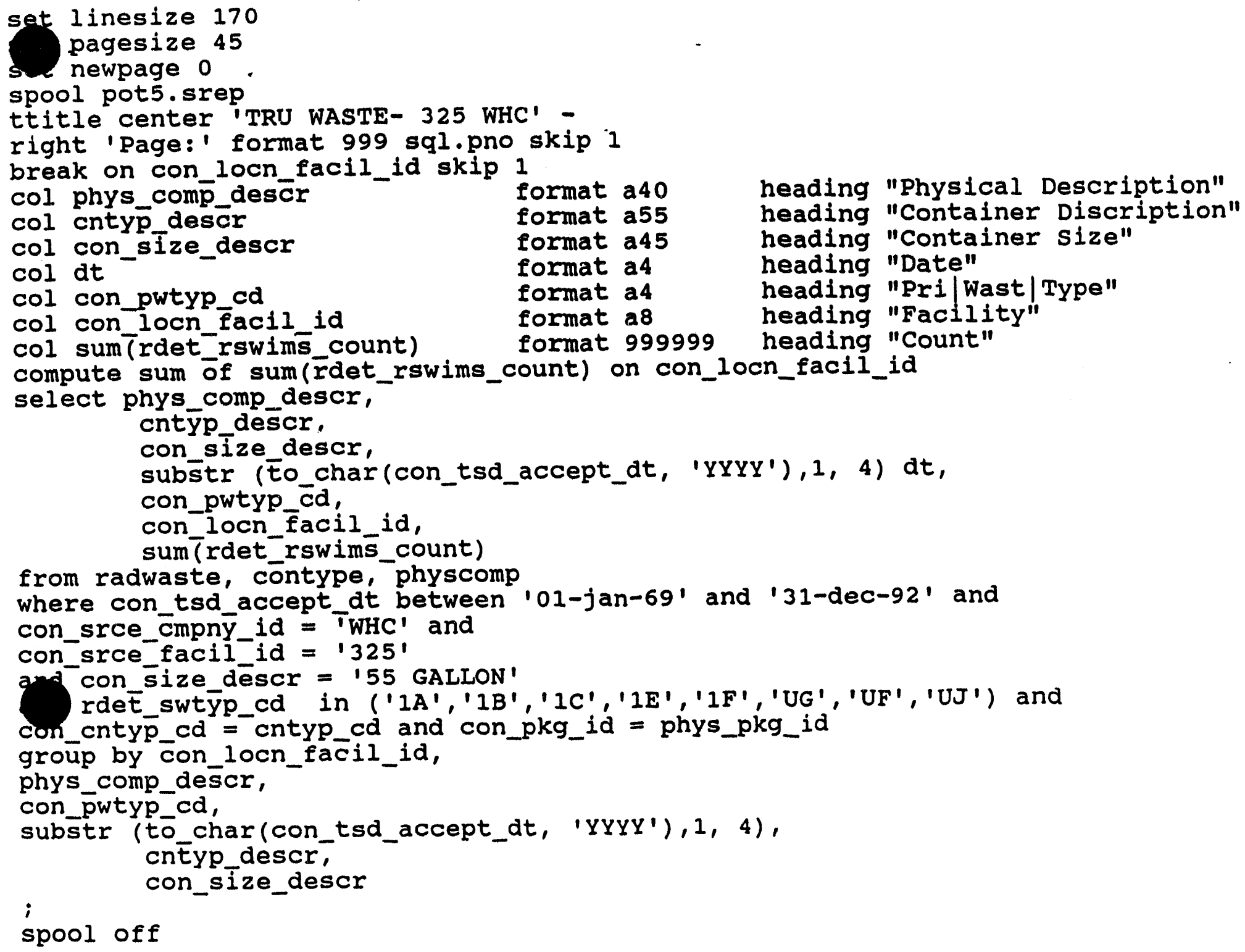

heading "Physical Description" heading "Container Discription" heading "Container size" heading "Date" heading "Pri|Wast|Type" heading "Facility" heading "Count" compute sum of sum(rdet_rswims_count) on con_locn_facil_id

select phys_comp_descr, cntȳp_descr. con_size_descr, substr (Éo_char(con_tsd_accept_dt, 'YYYY'), 1, 4) dt, con pwtyp_cd, conlocn facil_id, sum (rdet_rswims_count)

from radwaste, contype, physcomp

where con_tsd_accept_dt between '01-jan-69' and '31-dec-92' and con_srce_cmpny_id = 'WHC' and con_srce_facil_id $=' 325^{\prime}$

and con_size_descr $=155$ GALLON'

rdeE_swtyp_cd in ('IA', 'IB','IC','IE', 'IF', 'UG', 'UF', 'UJ') and

con_cntyp_cd =-cntyp_cd and con_pkg_id = phys_pkg_id

groūp by con_locn_facil_id,

phys_comp_descr,

con_pwtyp_cd,

substr (to_char(con_tsd_accept_dt, 'YYYY'), 1, 4), cnĒyp_descr, con_size_descr

;

spool off 
TRU WASTE- 325 WHC

Pri

Wast

Physical Description

Container Discription

Container Size

Date Type Facility Count

MISCELLANEOUS/UNKHOUN/OTHER

METAL DRUMS, BARRELS, KEGS

METAL DRUMS, BARRELS, KEGS

55 GALLON

55 GALLON

55 GALLON

MISCELLANEOUS/UNKNOLN/OTHER

METAL DRUMS, BARRELS, KEGS

METAL ORUMS, BARRELS, KEGS
METAL DRUMS, BARRELS, KEGS METAL DRUMS, BARRELS, KEGS METAL DRUMS, BARRELS, KEGS METAL DRUMS, BARRELS, KEGS METAL DRUWS, BARRELS, KEGS METAL DRUMS, BARRELS, KEGS METAL DRUAMS, BARRELS, KEGS METAL DRUMS, BARRELS, KEGS METAL DRUMS, BARRELS, KEGS METAL DRUALS, BARRELS, KEGS METAL DRUMS, BARRELS, KEGS METAL DRUMS, BARRELS, KEGS METAL DRUMS, BARRELS, KEGS METAL DRUMS, BARRELS, KEGS METAL DRUMS, BARRELS, KEGS METAL DRUAHS, BARRELS, KEGS METAL DRUMS, BARRELS, KEGS METAL DRUMS, BARRELS, KEGS METAL DRUMS, BARRELS, KEGS METAL DRUMS, BARRELS, KEGS METAL DRUAS, BARRELS, KEGS METAL DRUMS, BARRELS, KEGS METAL DRUMS, BARRELS, KEGS METAL DRUMS, BARRELS, KEGS METAL DRUMS, BARRELS, KEGS METAL DRUMS, BARRELS, KEGS METAL DRUMS, BARRELS, KEGS METAL DRUMS, BARRELS, KEGS METAL DRUMS, BARRELS, KEGS METAL DRUMS, BARRELS, KEGS

55 GALLON
55 GALLON
55 GALLON
55 GALLON
55 GALLON
55 GALLON

55 GALLON

55 GALLON

55 GALLOH

55 GALLON

55 GALLON

55 GALLON

55 GALLON

55 GALLON

55 GALLON

55 GALLON

55 GALLON

55 GALLON

55 GALLON

55 GALLON

55 GALLON

55 GALLON

55 GALLON

55 GALLON

55 GALLON

55 GALLON

55 GALLON

55 GALLON

55 GALLON

55 GALLON

RUBBER

WOOO/LUMBER/PLYW
WOOO/LUMBER/PLYWOOO

-

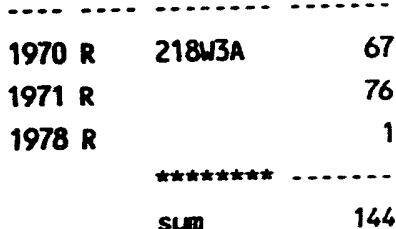

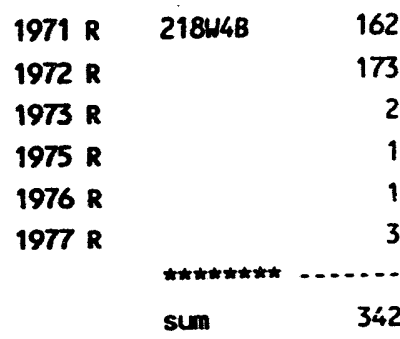

$1979 R$

$1980 \mathrm{R}$

$1979 R$

$1980 R$

$1979 R$

$1980 R$

$1980 R$

$1979 R$

1979 R

$1980 R$

$1979 R$

$1980 R$

$1978 R$

$1979 R$

$1978 R$

1979 R

$1980 R$

$1978 R$

$1979 R$

$1980 R$

$1978 \mathrm{R}$

1979 R

1979 R

$1980 R$

$218 w 4 C$ 
Date rype Facility count

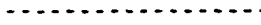

\section{4 rows selected.}




\section{WHC-EP-0696}

This page intentionally left blank.

A.5-6 
WHC-EP-0696

\section{APPENDIX A.6}

PHYSICAL CONTENTS DESCRIPTION FOR BUILDINO 325 WESTINGHOUSE HANFORD TRU WASTE CONTAINERS OTHER THAN 55-GALLON DRUMS

SORTED BY DATE, PRIMARY WASTE TYPE, AND STORAGE FACILITY 
This subappendix describes the physical contents of the TRU waste containers other than 55-gal drums generated at Building 325 Westinghouse Hanford. Prior to 1978, physical contents were not required on the burial records, so a great many of the early records list the contents of the drum as "Miscellaneous."

The printout for this computer run sorts the contents data by storage facility, date and primary waste type. The introduction to Appendix A contains a table of waste codes and their meanings. 
set linesize 170

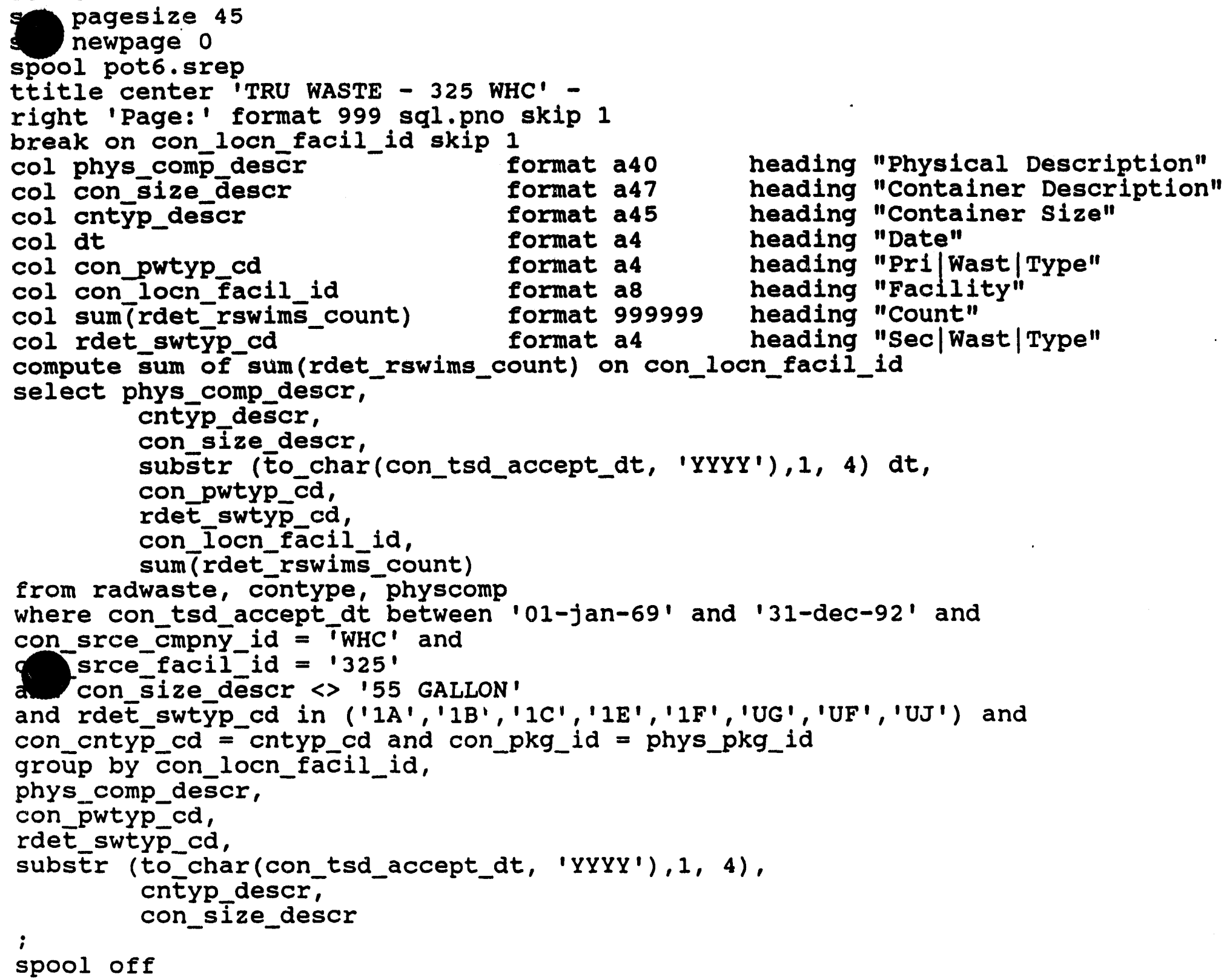




\section{Physical Description}

MISCELLANEOUS/UMKHONH MISCELLANEOUS/UNKNOWM/OTHER MI SCELLLANEOUS/UNKNOWN/OTHER MISCELLLANEOUS/UNKNOWN/OTHER MISCELLANEOUS/UNKNOWM/OTHER

MISCELLANEOUS/UNKNOWM/OTHER MI SCELLLANEOUS/UNKNOWM/OTHER MISCELLANEOUS/UNKNON/OTHER MISCELLANEOUS/UMKNOMM/OTHER MISCELLANEOUS/UNKNONM/OTHER MISCELLANEOUS/UNKNOWN/OTHER MISCELLANEOUS/UMKMOWN/OTHER MISCELLANEOUS/UNKHOWM/OTHER in

\section{GLASS}

METAL/IRON/GALVANIZED/SHEET METAL/IRON/GALVANIZED/SHEET PLASTIC/POLYURATHANE PLEXIGLASS

WOOD/LUMBER/PL YWOOO
Container Size

HEPA FILTERS

METAL DRLMS, BARRELS, KEGS

METAL DRUNS, BARRELS, KEGS

TANKS, PORTABLE

WOCDEN BOXES, CARTONS, CASES

COMCRETE BOXES

CONCRETE BOXES

GLOVE BOXES

METAL BOXES, CARTONS, CASES

MISCELLAMEOUS SCRAP

SELF CONTAIMED, EQUIPMEMT

TAMKS, PORTABLE

CONCRETE BOXES

METAL BOXES, CARTONS, CASES

METAL BOXES, CarTONS, CASES

Metal boXes, cartons, cases

METAL 8OXES, CARTONS, CASES

METAL BOXEs, CARTONS, CASES

METAL BOXEs, CARTONS, CASES
Container Description

UnKMOM⿻

30 Gallow

30 Gallon

Uinknown

$4 * 4 * 4$

$3 * 3 * 3$

$4 * 4 * 4$

Unurionar

$4 * 4 * 4$

UnKMom

unkrom

Unknow

$3 * 3 * 3$

$4.2 * 6.2 * 15.6$

$4.2 * 6.2 * 15.6$

$4 * 6 * 10$

$4.2 * 6.2 * 15.6$

$4.2 * 6.2 * 15.6$

$4.2 * 6.2 * 15.6$
Pri sec

Wast Wast

Date Type Type facility count

1970 R 14 21843a

$1970 R \quad 1 A$

$1971 R \quad 1 A$

$1971 \mathrm{R} 1 \mathrm{~A}$

$1975 \& \quad 14$

$\begin{array}{rr} & 17 \\ & 1 \\ & 1 \\ & 5 \\ \text { sen } & 29\end{array}$

1972 \& IA 218448

1972 R 1A

$1972 R \quad 1 A$

1972 R 1A 2

$1972 R \quad 1 A \quad 5$

$1974 R$ 1A

$1974 R$ IA

$1977 R \quad 1 A$

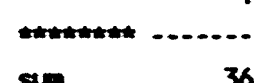

$1960 \mathrm{R} 1 \mathrm{~A}$ 2184AC

$1980 R \quad 1 A$

$1985 R \quad 1 A$

$1980 \mathrm{R} \quad 14$

$1980 \mathrm{R} \quad 1 \mathrm{~A}$

$1980 \& \quad 11$

19 rows selected. 
WHC-EP-0696

\title{
APPENDIX A.7
}

\section{HAZARDOUS CONSTITUENTS OF 55-GALLON} DRUMS CONTAINING TRU WASTE

\author{
A.7-1
}


The presence of hazardous constituents in a radioactive waste drum designates the waste as "Mixed" (primary waste code "M"). In this subappendix the hazardous contents of 55-gal drums generated at Bullding 325 Westinghouse Hanford are sorted by storage facility.

Information concerning the hazardous constituents of waste containers was not required prior to 1986. During the R-SWIMS data reentry program in the mid-1980's an attempt was made to add any available information on the hazardous materials present, however, this information was limited. 


\section{WHC-EP-0696}

set linesize 170

set pagesize 45

newpage 0

I pot7.srep

ttitle center 'TRU waste - 325 wHC ' -

right 'page:' format 999 sql.pno skip 1

break on con_locn_facil_id skip 1

col haz_comp_text

col con_size_descr

format 940

format a 45

col cntȳp_descr

col dt

col con_pwtyp_cd

col con_locn_facil_id

col sum (rdet_rswims_count)

format a 40

format a 4

format a4

format a 8

compute sum or sum(radet_rswims_count) on con_locn_facil_id

select haz_comp_text,

substr (to_char(con_tsd_accept_dt, 'YYYY'), 1, 4) dt,

cntyp_descr,

con_size_descr,

con_pwty $\bar{p} \_c d$,

con_locn_facil_id,

sum (rdet_rswims_count)

from radwaste, contype, chemcomp

where con_tsd_accept_dt between '01-jan-69' and '31-dec-92' and

con_srce_cmpny _id = 'THC' and

con_srce_facil_id = '325'

and con_size_descr $=155$ GALLON'

and rdet_swtȳp_cd in ('IA', 'IB', '1C', '1D', '1E', 'IF', 'UG', 'UF', 'UJ') and

and rdet_swtyp_cd

gulup by con_locn_facil_id,

haz_comp_text,

con_pwtyp_cd,

substr (tō_char(con_tsd_accept_dt, 'YYYY'),1,4),

cnËYp_descr,

con_size_déscr

i

spool of $\mathrm{f}$ 


\section{WHC-EP-0696}

compute sum of sum(rdet_rswims_count) on con_locn_facil_id

select haz comp_text, substr (to_char(con_tsd_accept_dt, 'YYYY'), 1, 4) dt, cntyp descr, con_size_descr, con_pwty $\bar{p} \_c d$, con_locn_facil_id, sum (rdet_rswims_count)

from radwaste, contype, chemcomp

where con_tsd_accept_dt between '01-Jan-69' and '31-dec-92' and con_srce_cmpny_id = 'WHC' and con_srce_facilid $=$ '325'

and con_size_descr $=155$ GALION'

and rdeE_swt $\bar{Y} P_{-} C d$ in $\left({ }^{\prime} 1 A^{\prime},{ }^{\prime} 1 B^{\prime},{ }^{\prime} 1 C^{\prime},{ }^{\prime} 1 D^{\prime},{ }^{\prime} 1 E^{\prime},{ }^{\prime} 1 F^{\prime},{ }^{\prime} U G^{\prime},{ }^{\prime} U F^{\prime},{ }^{\prime} U J^{\prime}\right)$ and con_cntyp_cd - cntyp_cd and con_pkg_id = haz_pkg_id groüp by con_locn_facil_id, haz_comp_texE,

"poE7.sqI" 37 lines, 1226 characters

SQL> Qpot7

no rows selected

SQL>

'VT100: 80 COL" 7 BIT MODE. 
WHC-EP-0696

\author{
APPENDIX A.8 \\ HAZARDOUS CONSTITUENTS OF TRU WASTE CONTAINERS \\ OTHER THAN B8-GALLON DRUMB
}

A.8-1 
The presence of hazardous constituents in a radioactive waste drum designates the waste as "Mixod" (primary waste code "M"). No hazardous constituents are listed in containers other than 85-gal drums generated at Building 325 Westinghouse Hanford; therefore this run did not roveal any hezardous information. 
set linesize 170

pagesize 45

newpage 0

spool pot8.srep

ttitle center 'TRU Waste - 325 WHC' -

right 'Page:' format 999 sql.pno skip 1

break on con_locn_facil_id skip 1

col haz_comp_text

format a40

format a45

heading "Hazardous"

col con_size_descr

format a40

heading "Container Description"

col cntȳp_descr

format a4

heading "Container Size"

col dt

col con pwtyp cd

col con_locn_facil_id

format a4

heading "Date"

format a 8

col sum (rdet_rswims_count)

format 999999

heading "Pr1/Wast|Type"

heading "Facliity"

select haz_comp_tex $\bar{t}$,

heading "Count"

substr (to_char(con_tsd_accept_dt, 'YYYY'),1, 4) dt,

cntyp_descr,

con_size_descr,

con_pwtyp̄_cd,

con_locn facil_id,

rdeE_rswims_coünt

from radwast $\bar{e}$, cont $\bar{y}$ pe, chemcomp

where con tsd_accept dt between '01-jan-69' and '31-dec-92' and

con_srce_cmpny

con_srce_facil_id $=1325^{\prime}$

and con_size_descr $\longleftrightarrow 155$ GALLON'

and rdeE_swtyp_cd in ('IA', 'IB', '1C','ID', 'IE', 'IF', 'UG', 'UF', 'UJ') and

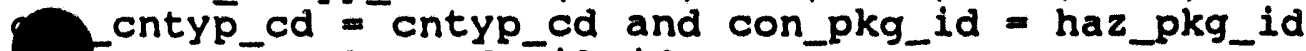

up by con_locn_facil_ia.

haz_comp_text,

con_pwtyp_cd,

cñtyp_descr,

substr (to_chār(con_tsd_accept_dt, 'YYYY'),1,4), coñsize_des̄cr, rdē_rswims_count

;

spool off 
WHC-EP-0696

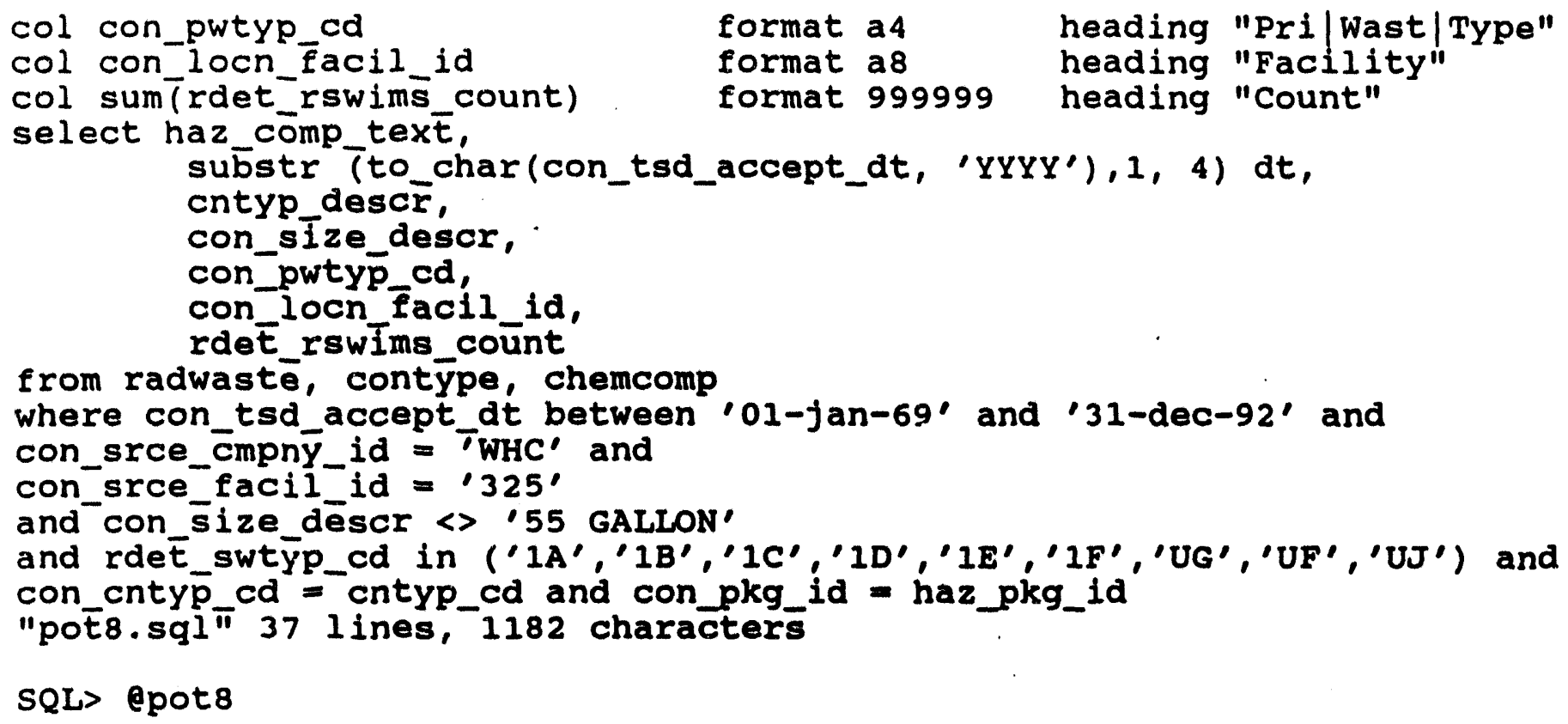


WHC-EP-0696

APPENDIX A.9

ISOTOPES LISTED IN 55-GALLON DAUMS CONTAININO TRU WASTES

A.9-1 
This subappendix contains a listing of the SWITS isotope information for TRU waste from Building 325 Westinghouse Hanford stored in 55-gal drums. The isotope field in SWITS contains several of the following types of information:

- Specific isotope (0.9., Am-241, Np-237, U-233, Pu-238)

- Genoric isotope listings (6.g., Pu, uranium-enriched, uranium-depleted)

- Piutonium-239 fissile gram equivalents (Pu239 FSL GR equiv)

- Total - alpha

- Total bata/gamma

- PE-Ci (plutonium equivalent curies).

This computer run contains the isotope field listing along with the number of TRU waste drums with that listing. The run is sorted by storage facility and year. 


\section{WHC-EP-0696}

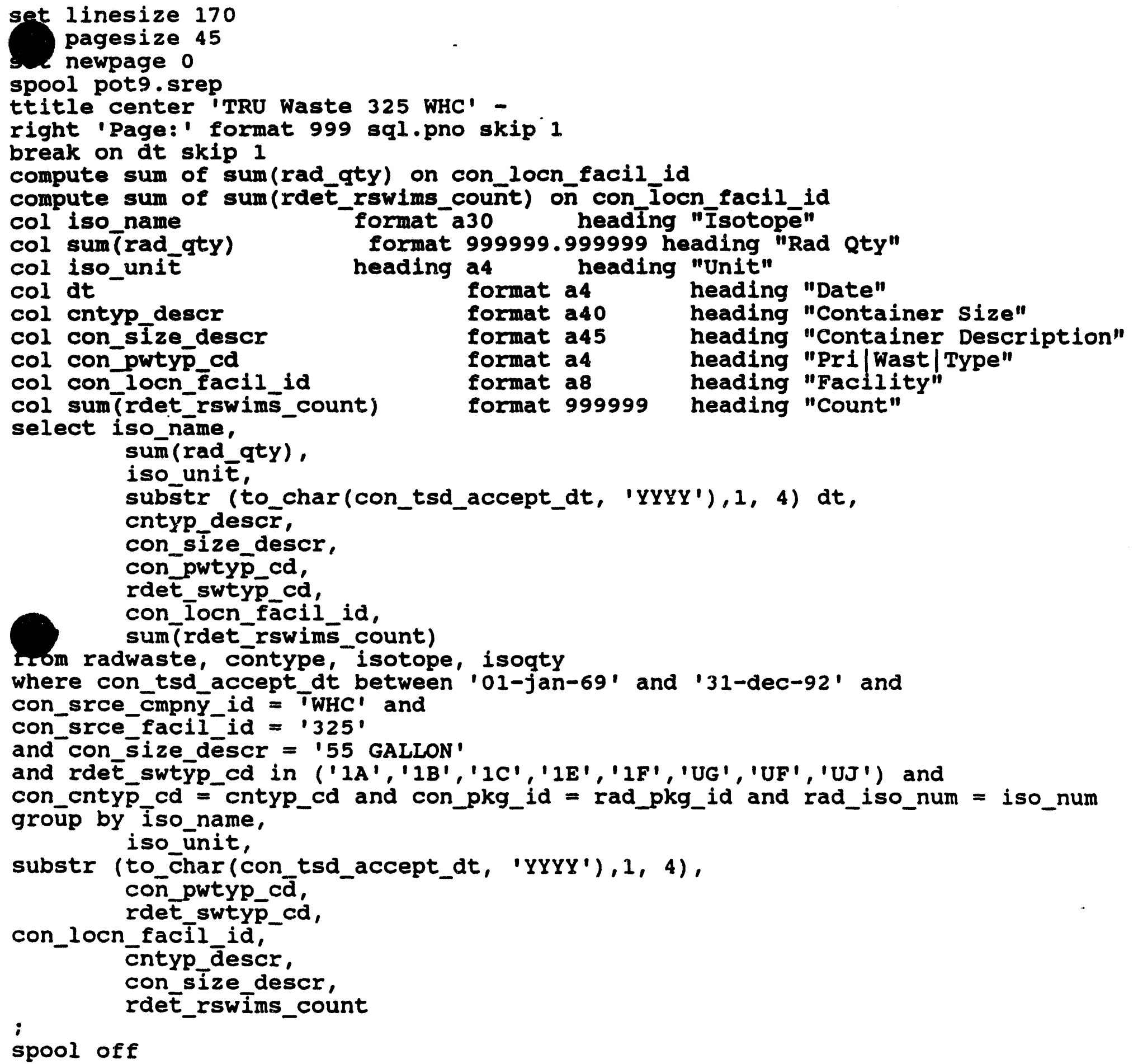




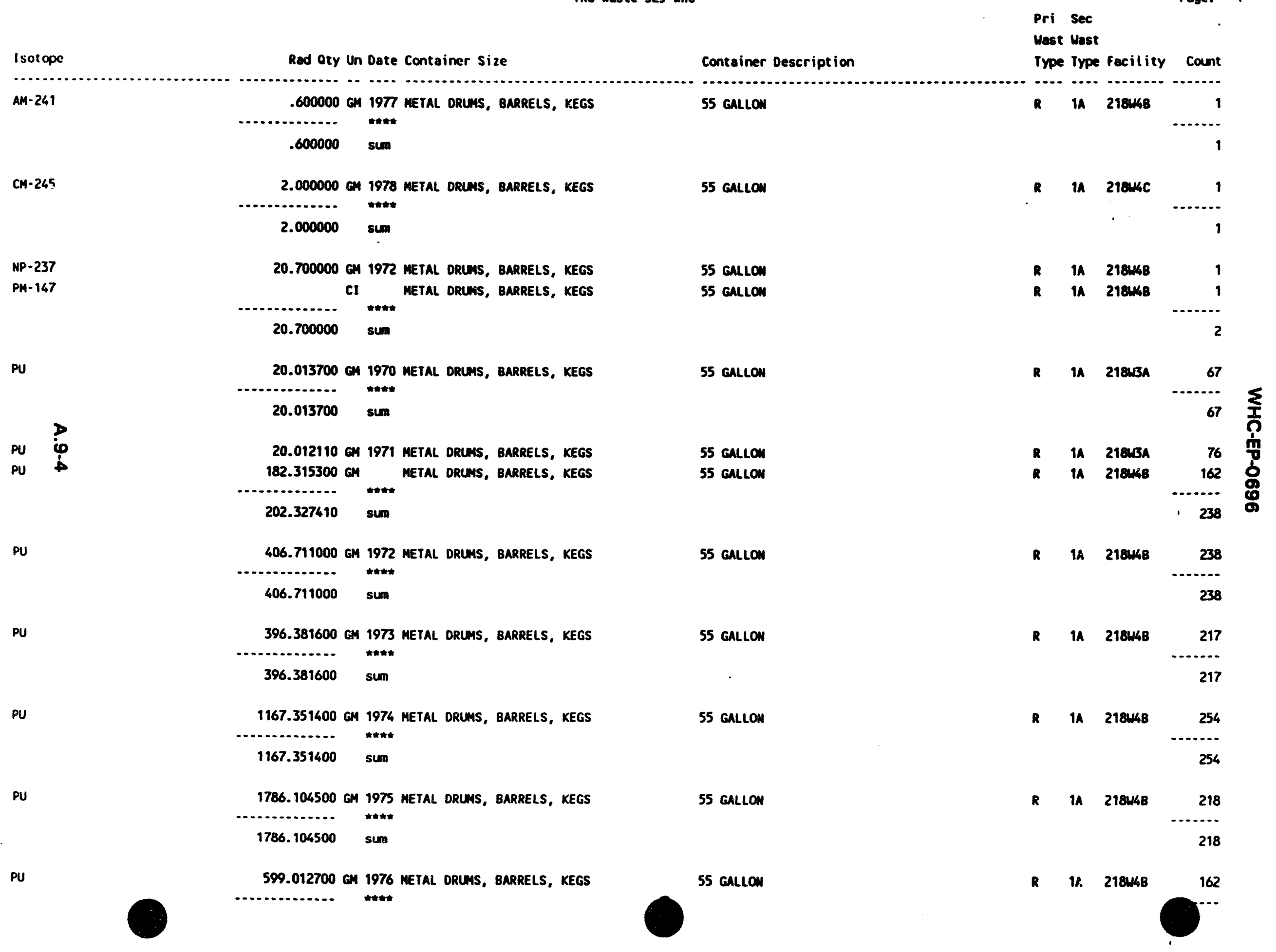


TRU Haste 325 UHC

I sotope

Rad Oty Un Date Container Size

599.012700

sum

PU

834.578100

$\operatorname{sun}$ $2479.222900 \mathrm{CM}$

sum

4900.822700

atot*

$$
\text { ............ Ant }
$$

1179.553200 sum

.900000 sum

.400000 sum $834.600000 \mathrm{GM}$

................

2888.600000 sum \#*\#*

15060.000000 sum
834.578100 GM 1977 METAL DRUMS, BARRELS, KEGS

87.000700 GM 1978 METAL DRUWS, BARRELS, KEGS METAL ORLUA, BARRELS, KEGS

4900.822700 GM 1979 METAL DRUNS, BARRELS, KEGS

1779.553200 GH 1980 METAL DRUNS, BARRELS, KEGS

.900000 GM 1971 METAL DRUMS, BARRELS, KEGS

.400000 GM 1979 METAL DRLHS, BARRELS, KEGS

2054.000000 GM 1971 METAL DRUMS, BARRELS, KEGS METAL DRUAS, BARRELS, KEGS

15060.000000 GM 1972 METAL DRLMS, BARRELS, KEGS

10000.000000 GM 1973 METAL DRUMS, BARRELS, KEGS

10000.000000

Sum
Container Description

Wast Wast

Type Type facility Count

162

55 GALLON

55 GALLON

55 GALLON

55 GALLON

55 GaLLON

55 GALLON

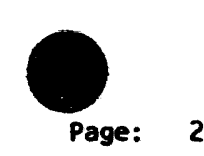

R 11 21814B 140

140

R IA 21843A 94

R $1 R \quad 218$ AC 212

306

R $1 A \quad 218$ 4C 437

437

R 1 1A 2isuac

R 1 A 218448

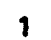

R IA 21844C

1

55 GALLON

55 GALLON

R $1 A \quad 218$ 3A 13

R $1 A \quad 218$ W4B

14

55 GALLON

R IA 21814

10

55 GALLON

.




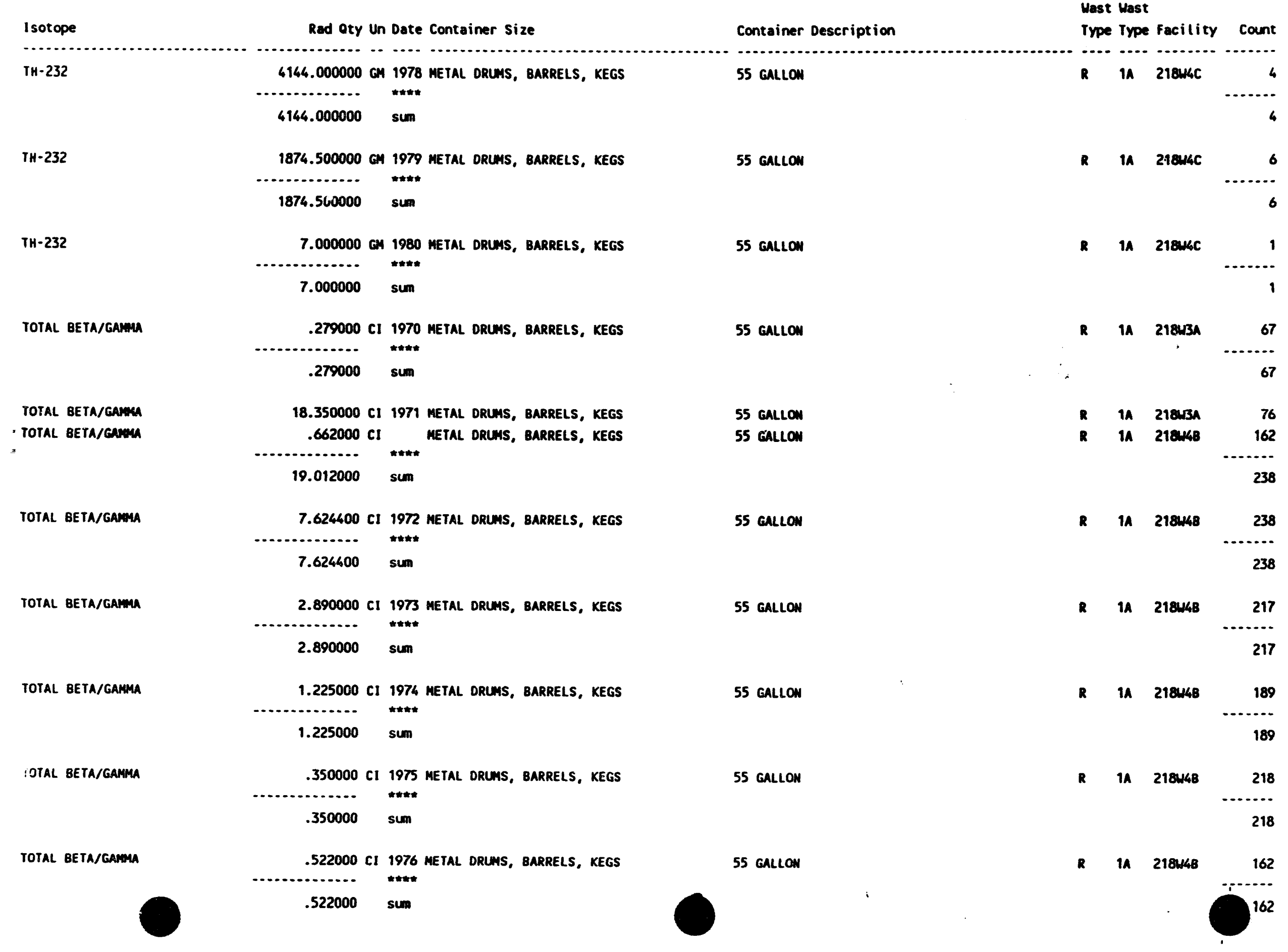


TOTAL BETA/GAMAa

TOTAL BETA/GAMMA TOTAL BETA/GAMMA

TOTAL BETA/gamma

TOTAL GETA/GAMMA

io

$v_{U \cdot 233}$

URANIUM-DEPLETED URANIUM-DEPLETED

URANIUM-DEPLETED

URANIUM-DEPLETED

URANIUM-DEPLETED

URANIUM-DEPLETED
$1.676500 \mathrm{Cl} 1977$ METAL DRUMS, BARRELS, KEGS

55 GALLON

55 GALLON

55 GALLON

4.039000 CI 1978 METAL DRIMS, BARRELS, KEGS $11.408000 \mathrm{C}$ METAL DRUMS, BARRELS, KEGS

15.447000

2.226000 CI 1979 METAL DRUMS, BARRELS, KEGS

2.226000 sum

$10.840000 \mathrm{CI} 1980$ METAL DRUMS, BARRELS, KEGS

55 GALLON

............

10.840000 sum

12.000000 GM 1978 METAL DRUMS, BARRELS, KEGS 12.000000 sum

6938.000000 GM 1971 METAL DRUMS, BARRELS, KEGS $9571.000000 \mathrm{GM}$ METAL DRUMS, BARRELS, KEGS

................

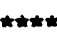

16509.000000 sum

75000.000000 GM 1972 METAL DRUMS, BARRELS, KEGS .............

75000.000000

sum

79.600000 GM 1974 METAL DRUMS, BARRELS, KEGS

55 GALLON

$$
79.600000 \text { sum }
$$

480.870000 GM 1975 METAL DRUMS, BARRELS, KEGS

55 GALLON

$$
480.870000 \text { sum }
$$

4406.000000 GH 1976 METAL DRUMS, BARRELS, KEGS

55 GALLON

55 GALLON

55 GALLON

55 GALLON

55 GALLON
R 1 1A 218468

140

140

R $1 A \quad 218$ 3A $\quad 94$

R IA 218U4C 213

307

R IA 218 MC $\quad 437$

437

R $1 \mathrm{~A} \quad 218 \mathrm{waC} \quad 91$

91

R IA 218:4ac

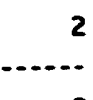

2

R $1 A$ 21843A 1

R 1 IA 218448 B 1

R 1 1A $218448 \quad$ 5

R 1 I 218 48 2

2

R $11 \quad 218448 \quad 39$

39 


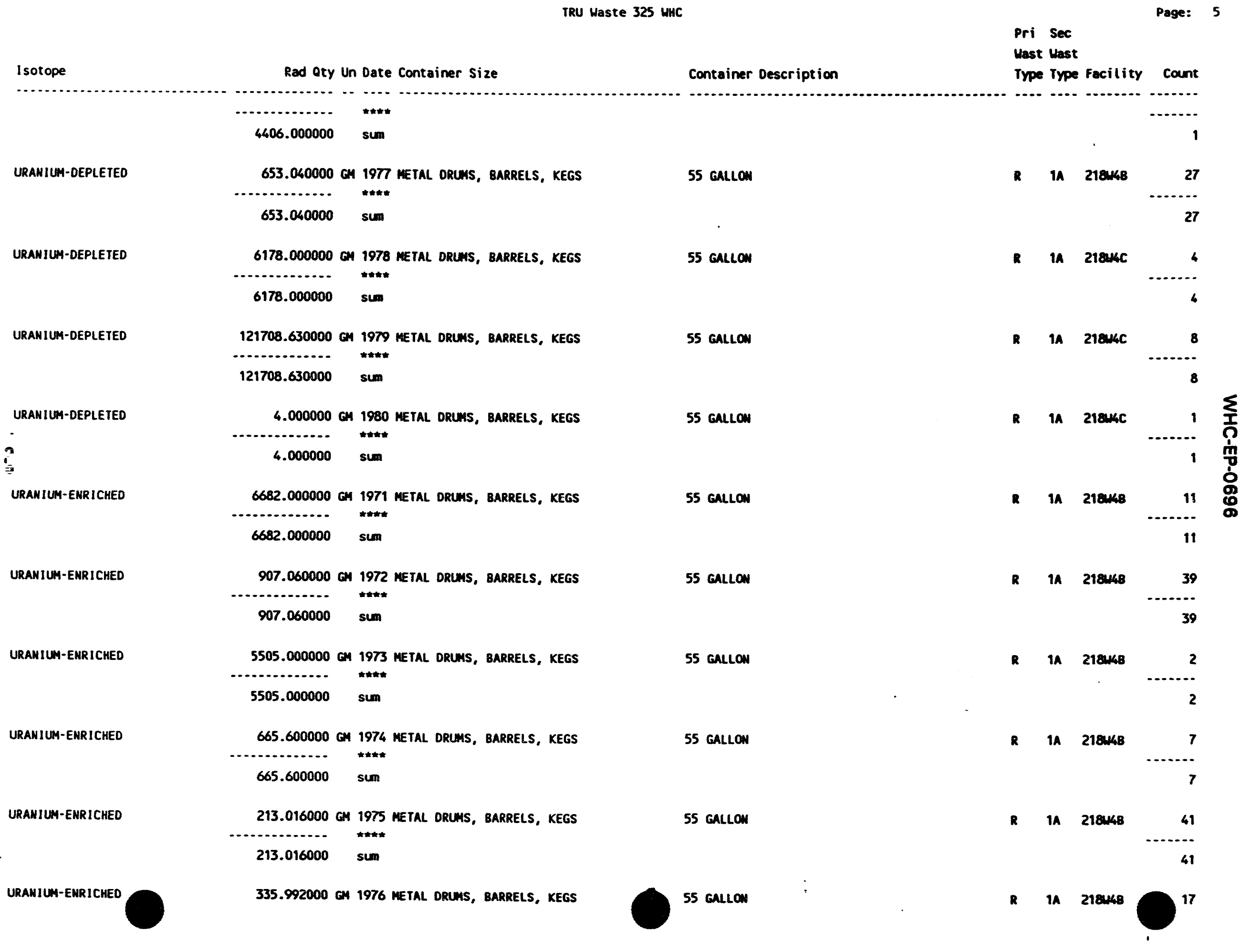


Type Type focility count

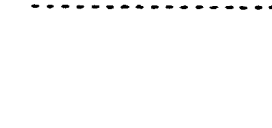

URANIUM-ENRICHED

$$
\text { sum }
$$

Type Type racility

\section{URANIUM-ENRICHED \\ URANIUM-ENRICHED \\ URANIUM-ENRICHED \\ 3 \\ URANIUM-NATURAL}

3 URANIUH-NATURAL

URANIUM-NATURAL

URANIUM-NATURAL

URANIUM-NATURAL

URANIUM-NATURAL
1407.955000 GM 1977 METAL DRUMS, BARRELS, KEGS 1407.955000

1116.950000 on 1978 METAL DRUMS, BARRELS, KEGS ............

1116.950000 sum

1862.680000 GM 1979 METAL DRLMS, BARRELS, KEGS ....

1862.680000 sum

313.000000 GH 1980 METAL DRUMS, BARRELS, KEGS -...............

*t*t

313.000000

sum

148898.000000 GM 1971 METAL ORUNS, BARRELS, KEGS

5814.000000 GI METAL DRUMS, BARRELS, KEGS

$$
\text { -............. }
$$

154712.000000 sum

345513.500000 GM 1972 METAL DRUNS, BARRELS, KEGS

\section{...............}

345513.500000 sum

204.800000 GM 1974 METAL DRUHS, BARRELS, XEGS ...

204.800000 sum

31707.000000 GM 1975 METAL DRUMS, BARRELS, KEGS ...............

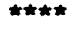

31707.000000 sin

123.047000 GM 1976 METAL DRUMS, BARRELS, KEGS

123.047000 sum

55 GALLON

55 GaLLON

55 GALLOH

55 GALLON

55 gallon

55 GALLON

55 GALLON

55 GALLON

55 GALLON

55 GALLOM

........

218448

40

40

2 in 218urc

218446

48

B 14 218nac

19

19

a 1 2tanac

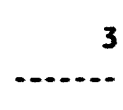

3

R IA 218i3n

12

in 218ura

3

15

R 1 14 218 4kB

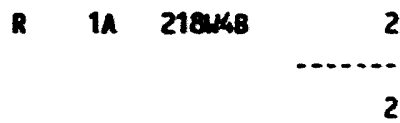

R 1 A 218 4a

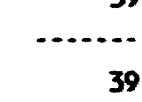




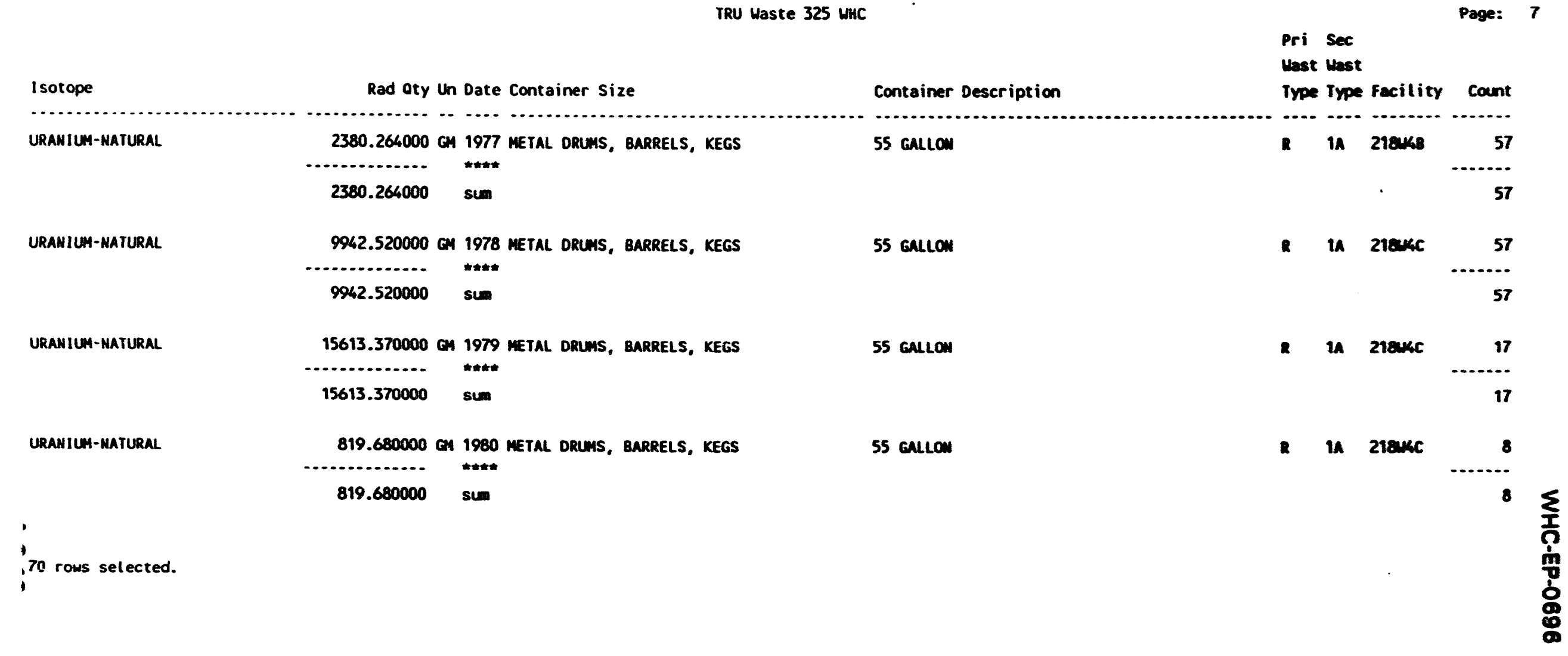


WHC-EP-0896

\author{
APPENDIX A.10 \\ 18OTOPES LISTED IN TRU CONTAINEAS \\ OTHER THAN 8B-GALLON DRUMS
}

A.10-1 
This subappendix contains a liating of the SWITS isotope information for TRU waste from Building 325 Westinghouse Hanford atored in containors other than 85-gal drums. The isotope field in SWITS contains several of the following types of information:

- Specific isotope (0.9., Am-241, Np-237, U-233, Pu-238)

- Generic lsotope listings (0.9., Pu, uranium-enriched, uranium-deploted)

- Piutonium-239 equivalent curies (PE-CI)

- Total-alpho

- Total beta/gamma

- PE-Ci (plutonium equivalent curies)

This computer run contains the isotope field listing along with the number of TRU waste containers with that listing. The run is sorted by storage facility and year. 


\section{WHC-EP-0696}

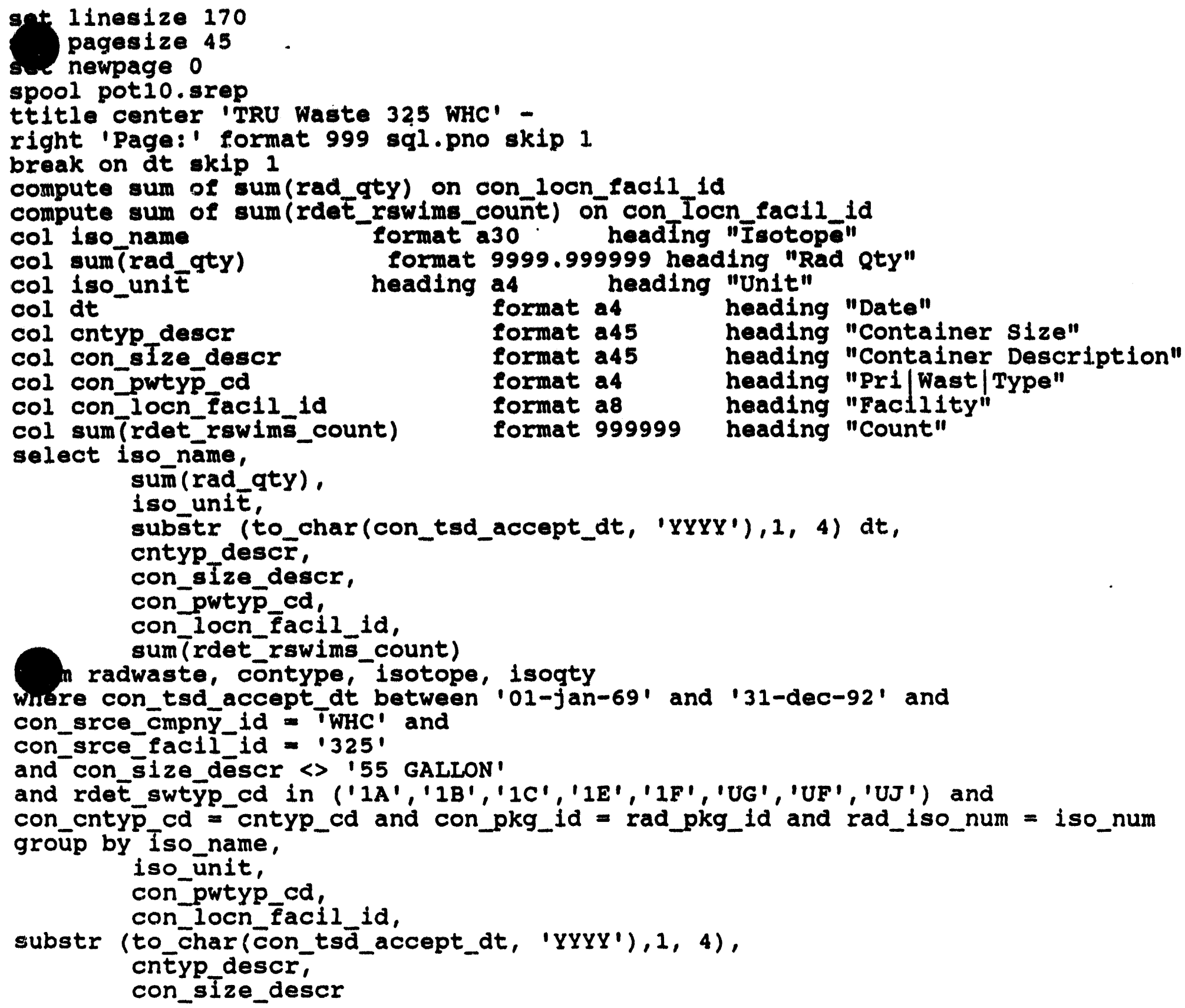

spool off 


\section{Isotope}

PU

PU

PU

PU

PU

PU

PU

$\overrightarrow{8}$

PU

PU

PU

PU

PU

PU

PU

Pu

PU

PU
Rad oty Un Date Container size

Container Description

unst

Type facility count

30 Gallow

METAL ORUMS, BARRELS, KEGS

$.010100 \mathrm{cH}$

mat:

.018500 sin

.000100 GM 1971 METAL DRUUL, BARRELS, KEGS

$.000100 \mathrm{GH}$ TAMKS, PORTABLE

uncuorn

.000200 sua

.100000 GM 1974 METAL bOXES, CARTONS, CASES

.100000 GI METAL BOXES, CARTONS, CASES

............ then

.200000

1.000000 GN 1975 METAL BOXES, CARTOUS, CASES

$.500000 \mathrm{GH}$ WOOOEN BOXES, CARTONS, CASES

$1.500000 \mathrm{sin}$

7.000000 on 1978 CONCRETE BOXES

$3 * 3 * 3$

7.000000 sum

$1.001000 \mathrm{GH} 1972$ CONCRETE BOXES

$1.200000 \mathrm{GH}$ CONCRETE BOXES

$1.000000 \mathrm{GM}$ GLOVE BOXES

.800000 GH METAL BOXES, CARTONS, CASES

$.000500 \mathrm{GH}$

MISCELlanequs SCRap

$4.001500 \mathrm{sum}$

$.040300 \mathrm{GH} 1974$ GLOVE BOXES

$.000400 \mathrm{GH}$ HEPA FILTERS

$.400000 \mathrm{GM}$ SELF CONTAIMED, EQUIPMENT

$.600000 \mathrm{GH}$ tAMKS, PORTABLE

\section{$3 * 3 * 3$}

$4 \div 4=4$

UnKrom

$4 \div 4 \div 4$

UnKNon

unknown

unknow

unknow

unrowoun

$1.040700 \mathrm{sim}$

1.000000 GI 1976 metal 8OXES, CARTOUS, CASES unknow

30 GALLON

1250 CU FT

$4 * 4 * 4$

21843

218:3n

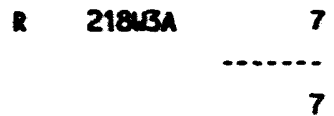

21843A

2181BA

5

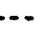

22

218:5s

218usa

1
1
2
2

- 218 ba

218usa

1
1
-2.
2

5
1
8
1
0
8
8

$\begin{array}{rr}218448 & 13 \\ 218448 & 3 \\ 218448 & 2 \\ 218448 & 2 \\ 218448 & 5 \\ & \ldots . . . \\ & 25\end{array}$

21844867

21844864

218448 


Isot
PU
PU
PU
PU
PU
PU
PU

total Beta/gama TOTAL BETA/GAMMA

TOTAL BETA/GAMMA TOTAL BETA/GAMMA

TOTAL BETA/GAMMA TOTAL BETA/GAMMA

TOTAL BETA/GAMMA

TOTAL BETA/GAMMA
Rad oty Un Date Container size

$1.000000 \mathrm{CH} 1976$ METAL BOXES, CARTONS, CASES

$.100000 \mathrm{GH}$

METAL DRUMS, BARRELS, KEGS

2.100000 $\rightarrow+$

sum

$.100000 \mathrm{GM} 1977$ CONCRETE BOXES

$.100000 \mathrm{GH}$
$.000100 \mathrm{GH}$ CONCRETE CYLIMDERS, CASKS
.100100 sin

$.000200 \mathrm{GN} 1978$ CONCRETE BOXES

$1.000000 \mathrm{~cm}$

WOOOEN BOXES, CARTONS, CASES

1.000200

$1.000200 \mathrm{sum}$

.000100 GN 1980 METAL BOXES, CARTOUS, CASES

.000100

$.370000 \mathrm{CI} 1970$ MEPA FILTERS

$.053000 \mathrm{CI}$ METAL DRUHS, BARRELS, KEgS

423000

.005000 Cl 1971 METAL DRUMS, BARRELS, KEGS $3.000000 \mathrm{Cl}$ tANKS, PORTABle

3.005000 sum

.100000 Cl 1974 METAL BOXES, CARTONS, CASES $.100000 \mathrm{Cl}$ metal boXes, cartons, cases ....... twat

.200000 sum

$4 * 4 * 4$

$.100000 \mathrm{Cl} 1975$ WOOOEN BOXES, CARTONS, CASES

100000

$4.860000 \mathrm{Cl} 1978$ CONCRETE BOXES unknoum

30 GALLON

30 Gallon

unknown

$5 * 5 * 5$

$7 * 10 * 16$

Pri

Wast

Type facility Count

4*5*9

110 GALLON

R 218448 , 1

R 21844B $r$. 1

R 218448

R 218448

2

$3 * 3 * 3$

$3.17 * 5 * 10.3$

R 218

218448

R $218 \mathrm{kin}$......

R 218w4 1

r $\quad r . .$.

R $21843 a \quad 5$

R 218uba 17

22

R 21843A 1

R 218u3a

2

R 218u3a

R 21843a $\quad 1$

R 21843A 5

5

$3 * 3 * 3$

R 218:3A 7 


Isotope Rod aty Un Date Container size

Contoiner Description

Pri

Unst

Type focility count

4.860000 sum

$104.000000 \mathrm{Cl} 1972$ CONCRETE BOXES

$63.000000 \mathrm{Cl}$ CONCRETE BOXES

$.002000 \mathrm{Cl}$ GLOVE BOXES

42.000000 CI METAL BOXES, CARTOWS, CASES

$.005000 \mathrm{CI}$ MiscellameOUS SCRAP

209.007000 sum

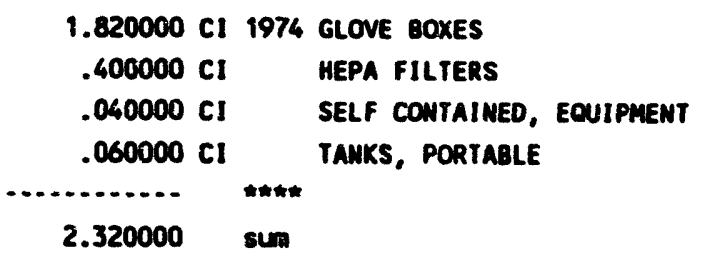

$.005000 \mathrm{Cl} 1976$ METAL BOXEs, CARTONS, CASES

$.005000 \mathrm{Cl}$ METAL BOXES, CARTOWS, CASES

TOTAL BETA/GaMma

TOTAL BETA/gaMMA

TOTAL BETA/GAMMA

TOTAL BETA/GAMMA

TOTAL BETA/GAMMA

TOTAL BETA/GAMMA

URANIUM-ENRI CHED

URANIUM-ENRICHED

URANIUM-MATURAL

URANIUM-MATURAL

*it:

.010000 sum

$.500000 \mathrm{Cl} 1977$ CONCRETE BOXES
$.060000 \mathrm{cl}$ CONCRETE CYLIMDERS, CASKS
$.560000 \mathrm{sum}$
$.300000 \mathrm{cl} 1978$ CONCRETE BOXES
$.060000 \mathrm{cl}$ WOODEN BOXES, CARTONS, CASES
.360000 sum

3*3*3

$7=12$

$3 \star 3 \star 3$

$3.17 * 5 * 10.3$

$4.2 * 6.2 * 15.6$

UNKNOM

UHKnom

UnkMow

unknowy
14.000000 GM 1974 SELF CONTAINED, EQUIPMENT

$21.000000 \mathrm{GH}$ TANKS, ORTABLE

$12.400000 \mathrm{GM}$ SELF CONTAINED, ECUIPMENT

$18.600000 \mathrm{GN}$ tanKS, portable
$3=3 \approx 3$

LuKMOM

$4 * 4 * 4$

Unxmom

UNKMOWN

UMKNOAN

$4 * 4 * 5$

$4 * 5 * 9$

$218 \mathrm{~m}$

R 218048

$\begin{array}{rr}218448 & 13 \\ 218448 & 3 \\ 218448 & 2 \\ 218448 & 2 \\ 218448 & 5 \\ & \ldots . . . \\ & 25\end{array}$

25

$2184 \times 8$

$218 \mathrm{mB}$

a 218468

$218 \times 48$

21

$\frac{3}{2}$
$\frac{1}{8}$
$\frac{8}{8}$
$\frac{8}{0}$ 
Pri

Mast

Container Description

Type facility count

I sotope

Rad aty Un Date Container Size

๓แณแ

66.000000

sum

1.000000 GM 1978 WOOOEN BOXES, CARTONS, CASES *t心
1.000000

URANIUM-NATURAL

sum
$3.17 * 5 * 10.3$
218448

1
Page: 4

55 rows selected. 
WHC-EP-0696

This page intentionally left blank.

A. $10-8$ 
WHC-EP-0696

APPENDIX B

DATA ON BUILDING 325 WASTE GENERATION BY PNL FROM THE SOLID WASTE INFORMATION TRACKING SYSTEM

B-1 


\section{WHC-EP-0696}

The information found in this appendix is from the Solid Waste Information and Tracking System (SWITS) database. This database incorporates the older R-SWIMS database and is used to track information on radioactive and other wastes stored or disposed at the Hanford Site.

Each SWIT'S data run in this appendix is preceded by the query used to generate the data. A brief explanation of the run and any additional information needed to understand the data presented is also included.

The bulk of the data provided is limited to information about TRU waste generated at Building 325 by PNL; however, some general information on the non-TRU waste is included for completeness. The term non-TRU is used, instead of LLW, because a small percentage of the waste has been designated only as not TRU. It is believed that most, if not all, of the non-TRU waste is LLW.

The data runs in this appendix are further segregated by waste container type. Since initial retrieval efforts and WRAP 1 will focus on 55-gal drums, these container types are considered separately.

Some general information about SWITS database and the codes used follows. Please note the disclaimer found on the next page. 


\section{WHC-EP-0696}

DON'T SAY IT -- Write It \&

Dates

To:

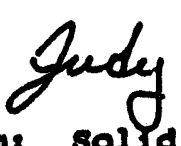

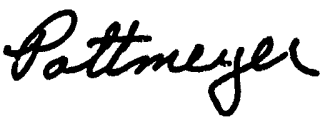

Fromi solld Maste Englneering Data Management Group 113-12 6-4394/6-4020

Res SHITS DATA REQUEST

Attached for your information and ues is the data which you requested. This data represents best avallable information regarding wastes currentiy in storage at the Hanford site. I trunt the Information will be suitable to your neede.

Requeste for Information from the solld waste Information and Tracking syatem (SWITs) are normally relatively limited in scope, requesting speciflc data flelds or summary data. The responses to these requests undergo review during data collection, summary and response preparation.

The response to this request represents a imple reproduction of the swITs Database. Transmittal of this information is made with the following disclaimers:

1) The information contained in this transmittal is raw data, and represents Information provided to Solld waste Englneering (SWE) on burlal records or other documents. This data has not been ralldated.

2) The information contained in this transmittal is subject to change without notice. Continual update of swITS information and improvement of the software system make it impossible to ensure consistency of this data with the database after transmittal.

3) This information is current as of $12 / 11 / 22$.

If I can be of further assistance to you, do not hesitate to call me. 
WHC-EP-0696

This page intentionally left blank. 
WHC-EP-0696

SWIR401

Solid Waste Information and pracking system

$05 / 01 / 92$

$13: 25$

Páge: 1

Primary Waste Type Code Table

Primary Waste Type Code

$-\infty-\infty-\infty-\infty-\infty-\infty$

Af

CII

II

IIP

$\mathbf{M}$

MP

N

$\mathbf{P}$

R

RP

U

Primary Naste Type Description

Ship

Time

Rad

HAg, ADIII HOTD

MORAMORIUK CONHZOTITD

89

magardoos

mazardoUs MASTE PCB

89

89

YIXED

MIXOBD PCB

29

89

NON-REGULATED

PCB

RADIOACTIVE

RADIOACTIVE PCB

29

29

UNRnOWN

29 
WHC-EP-0696

SWIR402

Solid Waste Information and Tracking system

Secondary Waste Type Code Table

$05 / 01 / 92$

$13: 26$

Page: 1

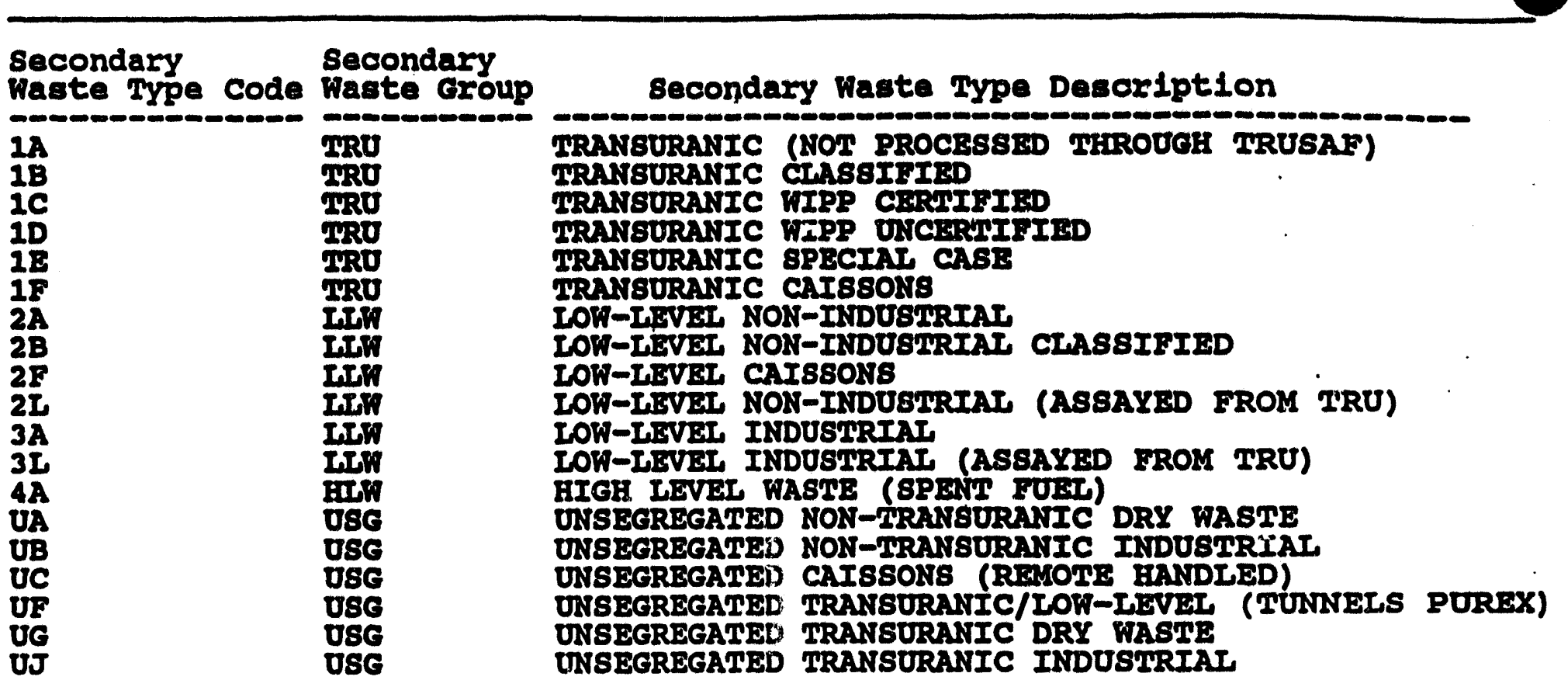


WHC-EP-0896

APPENDIX B.1

CONTAINER NUMBER AND WEIOHT OF TRU WASTE IN 85-CALLON DRUMS BY WASTE TYPE

B.1-1 
This data run gives an overall look at the waste types, container numbers and total woight (in kilograms) of the radioactive wastes generated at Bullding 328 PNL on a yearly basis. These data are for wastes stored in 58-gal drums only. The meanings of primary and secondary waste codes can be found in the introductory materials for this appendix.

The data in the average weight column should be taken cautiously, especially for LLW codes. Concern is warranted because LLW records are still done on a batch basis. This means that one weight datum may be the composite weight for a group of drums. The computer program used simply divided the sum of all woight values by the number of values used to compute the total weight, not the actual number of containers that that value represents.

From 1970 until 1978 individual container weights were not required for TRU drums. During the data reentry program in the mid-1980's standard weights were assigned for all container types; 55-gal drums were given a standard woight of $68 \mathrm{~kg}$. This is why the average weight/drum for TRU is so consistent during this period. 


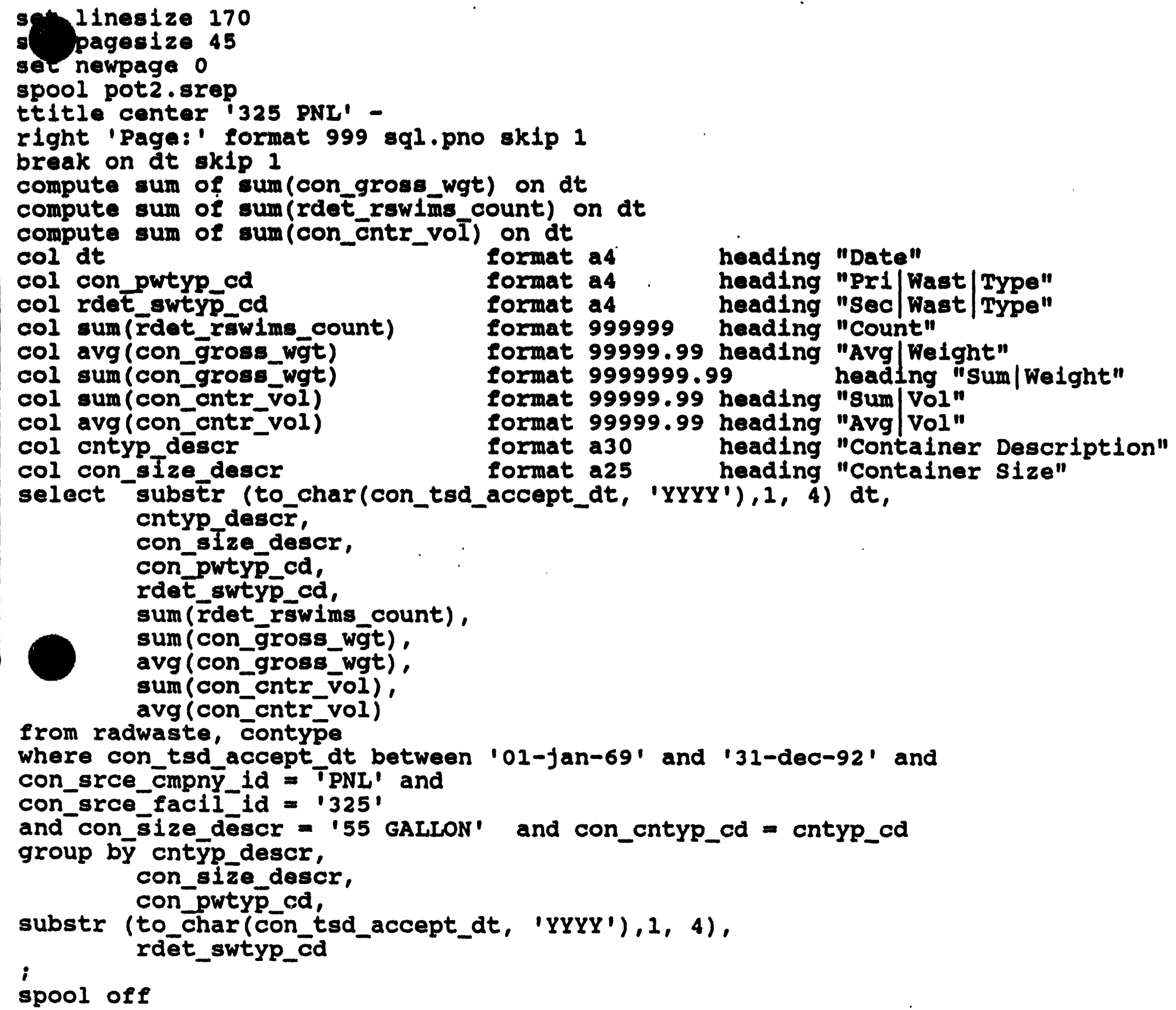




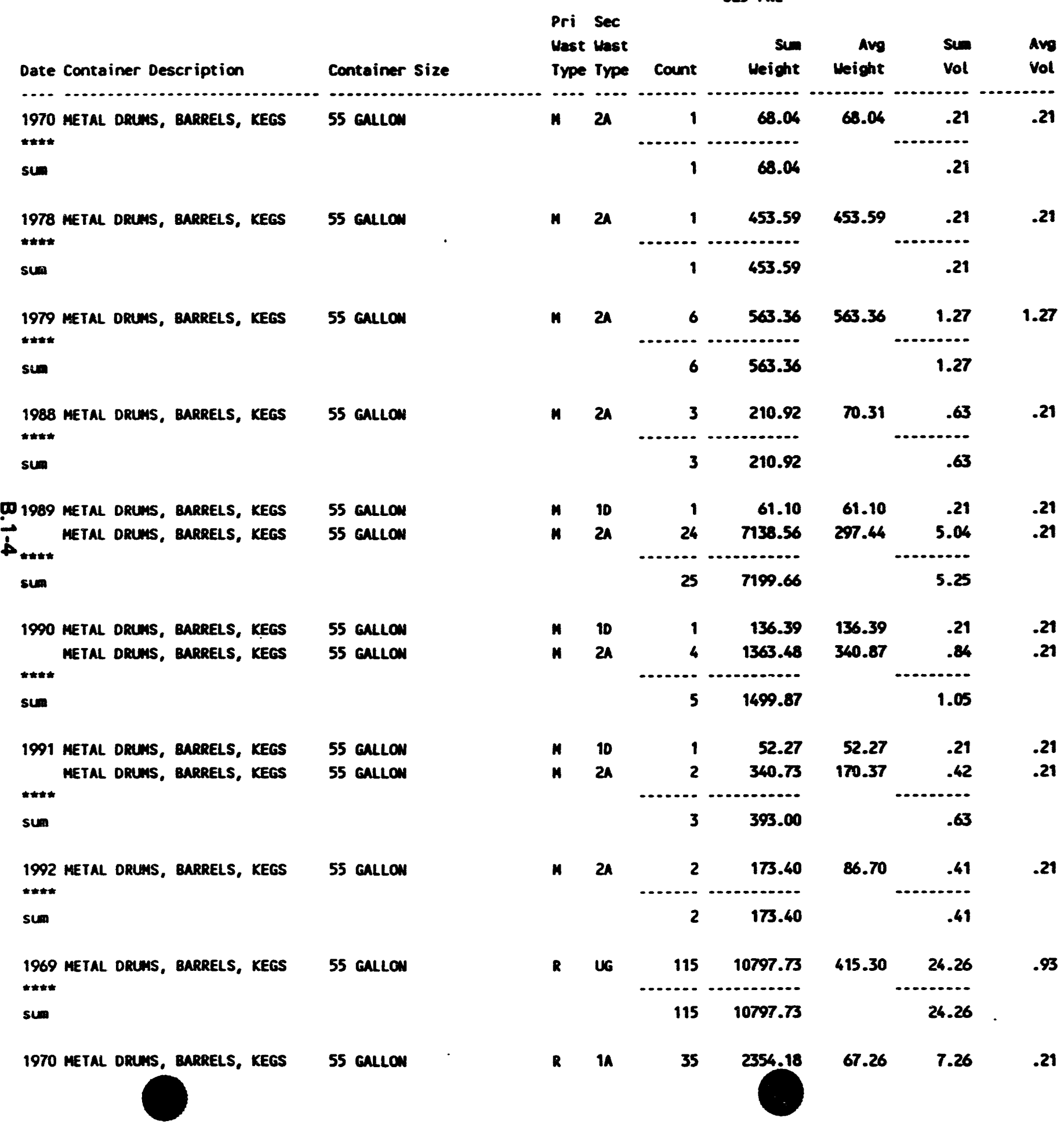




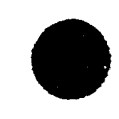

325 PNI

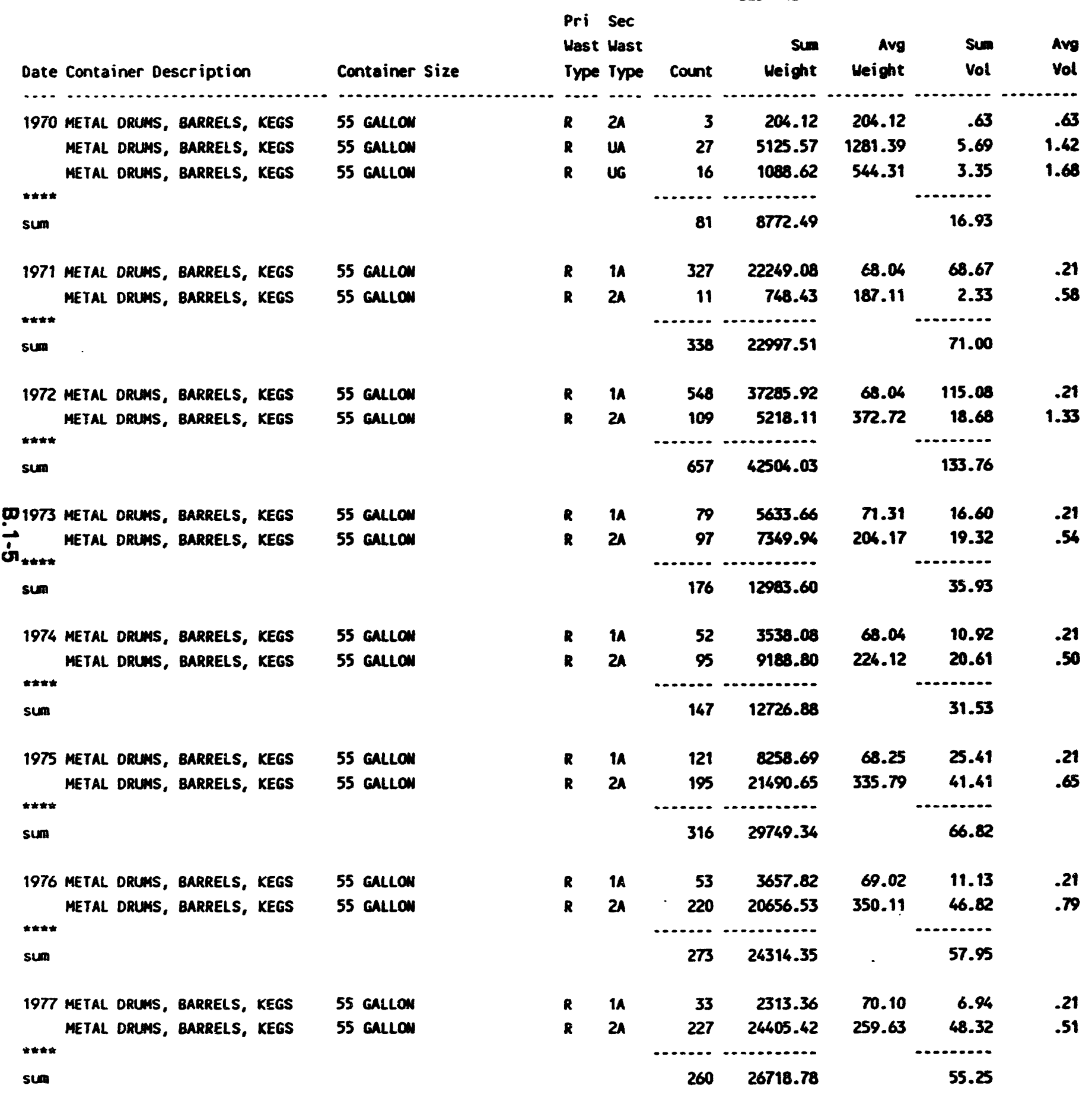


325 PNL

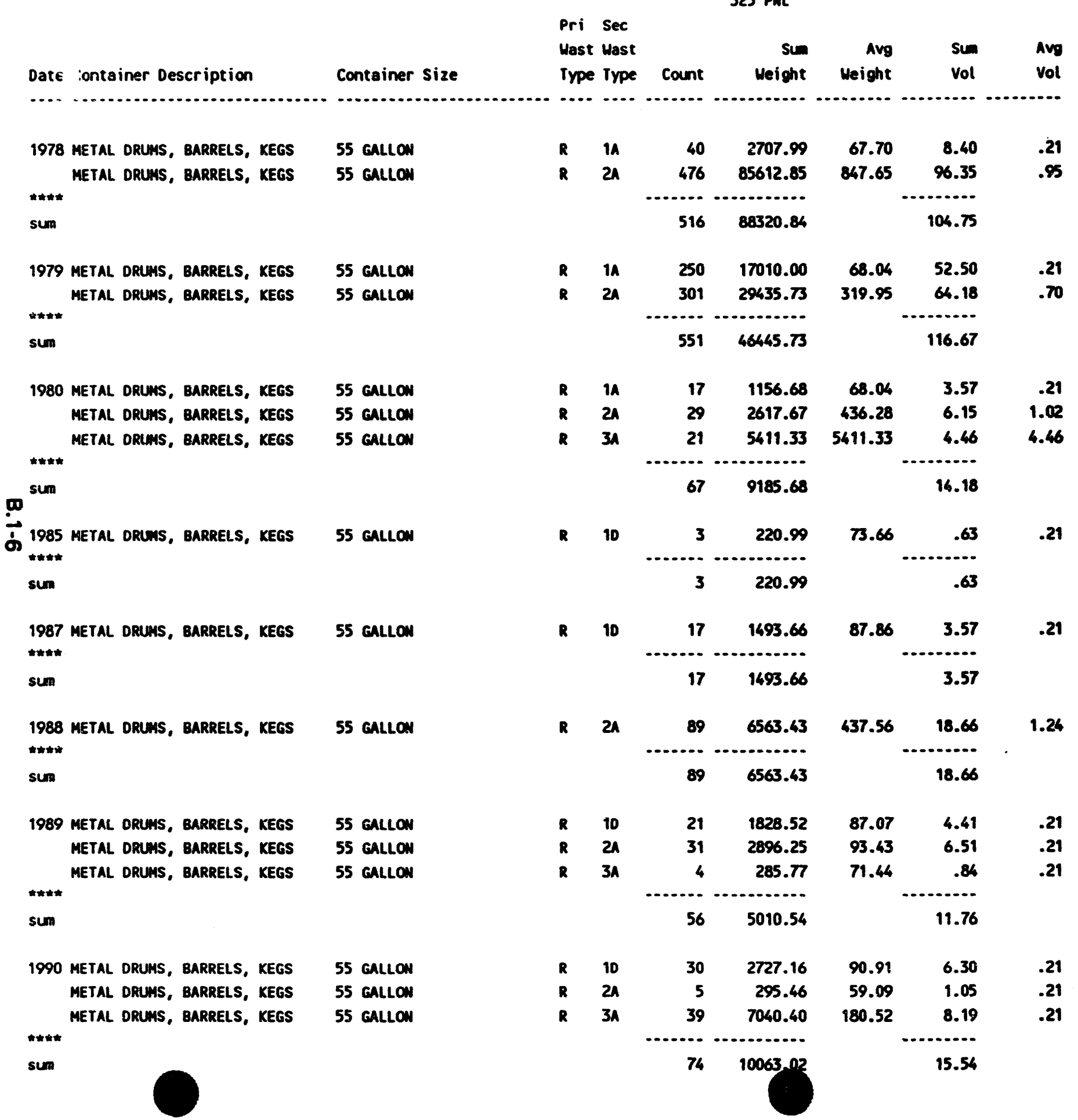


325 PML

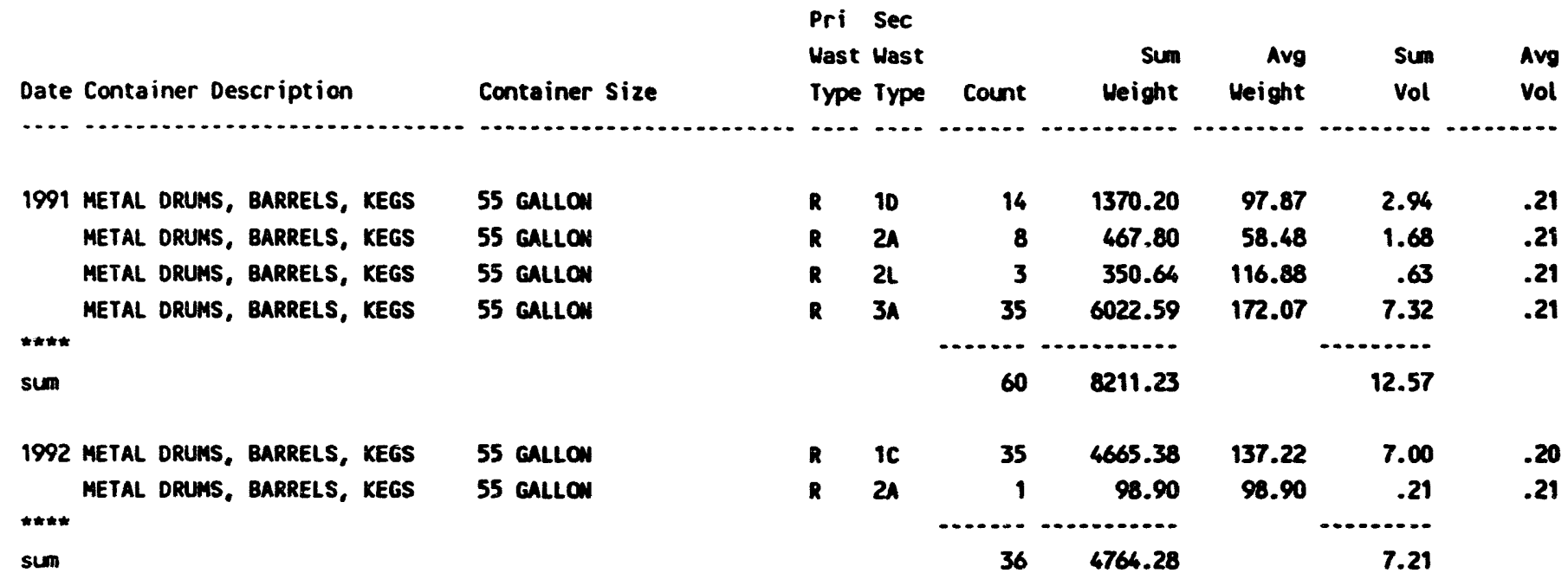

52 rous selected. 
WHC-EP-0696

This page intentionally left blank.

\section{B.1-8}


WHC-EP-0696

APPENDIX B.2

BUILDINO 325 PNL TRU WASTE CONTAINERS SORTED BY CONTAINER DESCRIPTION, SIZE, YEAR, AND PRIMARY WASTE TYPE 


\section{WHC-EP-0696}

This subappendix provides information on Building 325 PNL TRU waste containers, weight, volume, and primary waste type stored at various TRU waste storage facilities on the Hanford Site. The data provides the TRU waste generation from the Building 325 PNL Facility by year. 


\section{WHC-EP-0696}

set linesize 170

pagesize 44

newpage 0

spool pot3.srep

ttitle center 'TRU WASTE - 325 PNL' -

right 'Page:' format 999 sql.pno skip 1

break on dt skip 1

compute sum of sum(con_gross_wgt) on dt

compute sum of sum(rdet_rswims_count) on dt

col dt

col con_pwtyp_cd

col rdet_swtyp_de

col sum (čon_gross_wgt)

col sum (rdet rswims_count)

col avg (con_gross_wgtt)

col sum (con_cntr_vol)

col avg (con_cntr_vol)

col cntyp descr

col con_size_descr

col con_locn_facil_id

format a4

format a4

format a4

format 999999.99

format 99999

format 999999.99

format 99999.99 heading

format 99999.99 heading

format a55

format $a 45$

heading

format as

heading

heading

heading "Date"

heading "Pri|Wast/Type"

heading "Sec|Wast|Type"

heading "Weight"

select

cntyp_descr,

con size descr,

substr (Eo_char (con_tsd_accept_dt, 'YYYY'), 1, 4) dt, con_pwtyp_cd,

con_locn_facil_id,

sum (rdet_rswims_count),

sum (con_gross_wğt),

avg (con_gross_wgt),

sum (con_cntr_vol),

avg (con_cntr_vol)

from radwaste, contyp̄e

where con_tsd_accept_dt between '01-jan-69' and '31-dec-92' and

con_srce_cmpny

con_srce_facil_id = '325'

and rdet_swtyp_cd in ('IA', '1B', 'IC', 'ID', 'IE', 'IF', 'UG', 'UF', 'UJ') and

con_cntyp_cd = cntyp_cd

group by cntyp_descr,

con_size_descr,

substr (Éo_char (con_tsd_accept_dt, 'YYYY'), 1, 4) ,

con pwtyp_cd,

con_locn_facil_id

;

spool off 
Container Discription

Container size

BURLAP, ClOTH, PAPER OR PLASTiC BaGS

BUrlap, Cloth, PaPer OR plastic bags

CONCRETE BOXES

\section{CONCRETE BOXES}

CONCRETE BOXES

$\mathbf{m}$

Nffiberbonro/plastic boxes, cartons, cases

- fiberboard/PLastic boxes, cartons, cases

FIBERBOARD/PLASTIC BOXES, CARTONS, CASES

HEPA FILTERS

HEPA FILTERS

METAL BOXES, CARTONS, CASES

metal boXes, cartons, Cases
unKNOMN

unknows

Unknow

$3 * 3 * 3$

$4 * 4 * 8$

$4 * 7 \times 9$

2 CU FT

unknown

UnKMOMN

unknom

UNKNOWN

$2 \star 2 * 6$

$5.6 * 6.5 * 9.3$
Pri

Wast

Date Type facility count Weight Weight Volune volume

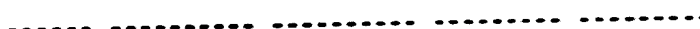

$1970 R \quad 218448$

$4 \quad 1262.38$

$\begin{array}{lll}1242.38 & 2.59 & 2.59\end{array}$

$\rightarrow$ twa

$4 \quad 1242.38$

$\sin$

1978 R $2184 \times 6$

$\begin{array}{rr}1 & 114.30 \\ 1 & 114.30\end{array}$

sum

1975 R $218 \mathrm{me}$
sin

11814.36

$1 \quad 1814.36$

$1974 R \quad 218468$

$137 / 3.87$

\& $218 \mathrm{k}$

214859.60

the

sum

$3 \quad 18633.47$

1969 R 218068

$6 \quad 32.66$

19

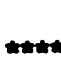

218 we

sun

1970

sum

1969 R 218468

$\sin$

\begin{tabular}{rr}
6 & 32.66 \\
2017 & 26950.51 \\
\hline 2023 & 26903.17
\end{tabular}

1970 R 218 a46

tक⿻上丨

$315 \quad 4636.11$

$315 \quad 436.11$

403.28

40.42

3.67

sum

$27 \quad 1892.37$

4.20

1.40

$\begin{array}{ll}27 & 1892.37\end{array}$

1976 R 2181148

sum

1992 R 2602UE

$8 \quad 533.4$

$8 \cdot 533.42$

266.71

1.19

.59

\begin{tabular}{rr}
1 & 304.81 \\
\hline 1 & 304.81
\end{tabular}

304.81

.68

.68

1.50500 
Container Discription

\section{Container Discription}

METAL DRUNS, BARRELS, KEGS

METAL DRUMS, BARRELS, KEGS METAL DRUMS, BARRELS, KEGS METAL DRUMS, BARRELS, KEGS

METAL DRUMS, BARRELS, KEGS

$\infty$

N Metal dRUAS, Barrels, kegs

Or METAL DRUMS, BARRELS, KEGS

METAL DRUAS, BARRELS, KEGS METAL DRLHS, BARRELS, KEGS

METAL DRUMS, BARRELS, KEGS

METAL DRLiNS, BARRELS, KEGS METAL DRUHS, BARRELS, KEGS

METAL DRUMS, BARRELS, KEGS
Container Size

30 GALLON

30 GALLOU

30 GAlLON

5 GALLON

55 Gallor

55 GALLON

55 Gallon

55 callon

55 GALLON

55 GALLON

55 GALLON

55 GALLON

55 GALLON

Hast

Tye facility count Weight

(t)

sum

1969 R 218448

$\operatorname{sen}$

1970 R 218u3n

R 2180443

R 218468

sin

1969 R 218448

sin

$1970 R \quad 21843$

R 218448

intitit

$\sin$

$1971 R \quad 21813$

$R: 218448$

tht十

sum

1972 R $2180 \times 48$

sum

$\begin{array}{rr}1973 R & 218434 \\ R & 2184448\end{array}$

sum

1974 R 218448

$\sin$

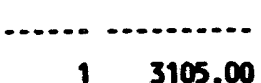

$1 \quad 3105.00$

\begin{tabular}{cr}
5 & 204.12 \\
\hline 5 & 204.12
\end{tabular}

\begin{tabular}{rr}
23 & 938.86 \\
6 & 246.94 \\
4 & 254.01 \\
\hline 33 & 1437.81
\end{tabular}

$\begin{array}{lll}40.82 & 2.67 \quad .12\end{array}$

$264.96 \quad .70 \quad .70$

$\begin{array}{lll}254.01 & .57 & .57\end{array}$

$415.30 \cdot 24.26 \quad .93$

\begin{tabular}{rr}
115 & 10797.75 \\
\hline 115 & 10797.75
\end{tabular}

$35 \quad 2354.18$

\begin{tabular}{rr}
16 & 1008.62 \\
\hline 51 & 3442.80
\end{tabular}

67.26

544.31

7.26

3.35

$51 \quad 3442.80$

$68.04 \quad 4.62 \quad .21$

$68.04 \quad 64.05 \quad .21$

$68.04 \quad 115.08 \quad .21$

\begin{tabular}{rr}
548 & 37285.92 \\
\hline 548 & 37285.92
\end{tabular}

$14 \quad 1211.06$

$65 \quad 4622.60$

$79 \quad 5633.66$

$\begin{array}{lll}86.50 & 2.95 & .21\end{array}$

$68.04 \quad 13.65 \quad .21$

$\begin{array}{lll}68.04 & 10.92 & .21\end{array}$ 


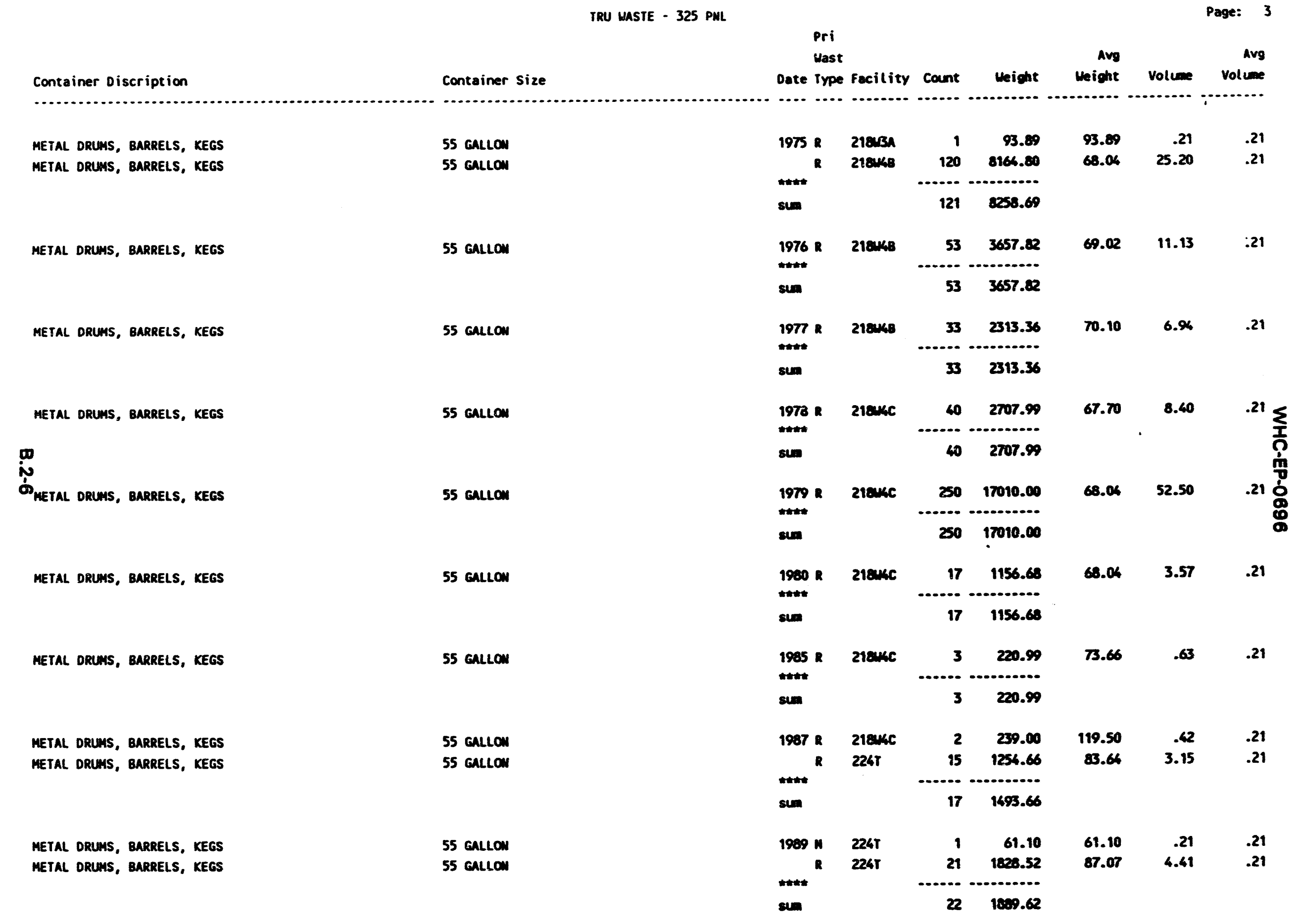




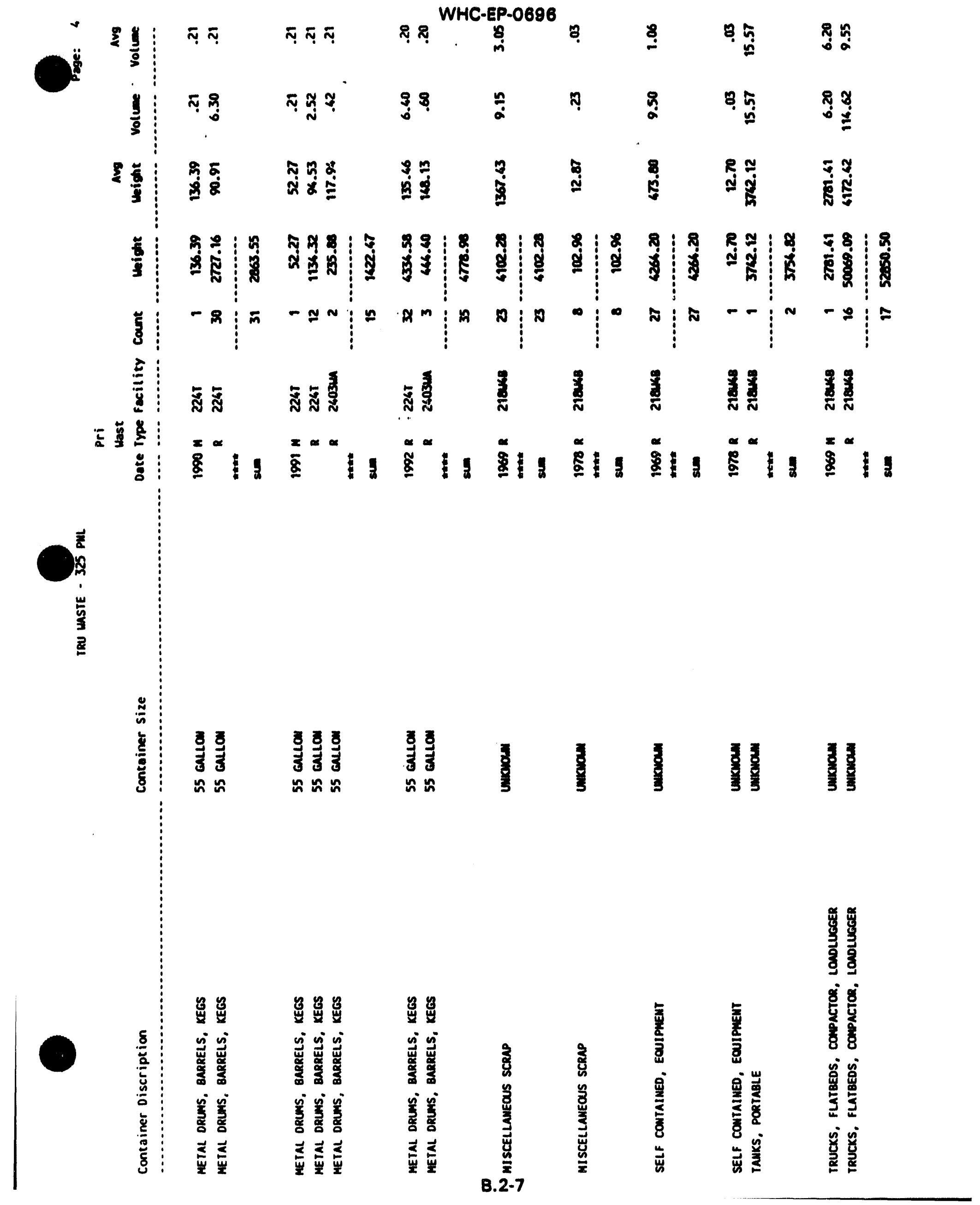




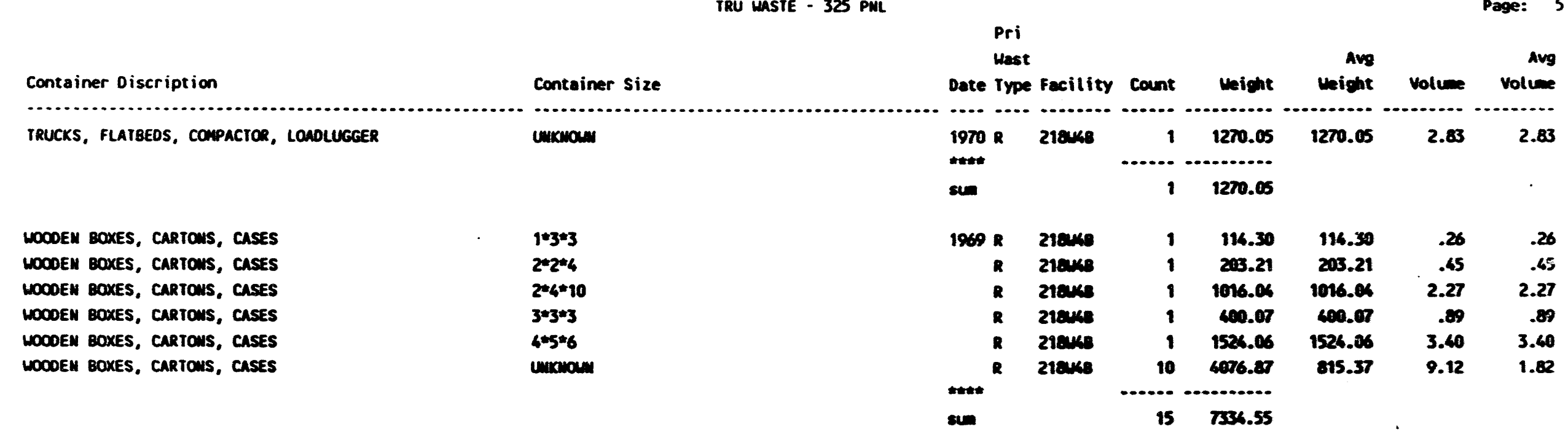

58 rows selected.

w

$\dot{\infty}$ 
WHC-EP-0698

APPENDIX 0.3

\section{BUILOING 325 PNL NON-TRU WASTE CONTAINERS SORTED BY CONTAINEA DESCRIPTION, SIZE, YEAR, \\ AND PRIMARY WASTE TYPE}

B.3-1 
This subappendix provides information on Bullding 325 PNL non-TRU waste containers, woight, volume, and primary waste type stored at various waste storage facilities on the Hanford Site. The data provides the non-TRU waste generation from the Building 325 PNL Facillty by year. 
set linesize 175

pagesize 45

newpage 0

spool pot3a.srep

title center 'NON-TRU WASTE- 325 PNL' -

right 'page:' format 999 sql.pno skip 1

break on dt skip 1

compute sum of sum(con_gross_wgt) on dt

compute sum of sum(rdet_rswims_count) on dt

col dt

col con_pwtyp_cd

col rdet_swtyp_de

col sum(con_gross_wgt)

col sum (rdeE_rswims_count)

col avg (con_ḡross_wğt)

col sum (con_cntr_vol)

col avg (con_cntr_vol)

col cntyp descr

col con_size_descr

col con_locn_facil_id

format a 4

format a4

format a4

format 999999.99

format 99999 heading "Count"

heading "Date"

heading "Pri|Wast/Type"

heading "Sec Wast/TYpe"

format 999999.99

format 99999.99 heading

format 99999.99 heading

format a55

format a45

heading

heading

format a8

heading

format a4

heading

heading "Avg/Weight"

"Volume"

col rdeE_swtỵp_cd

"Avg/Volume"

"Container Discription"

select

cntyp descr,

con_size_descr,

substr (Eo_char(con_tsd_accept_dt, 'YYYY'), 1, 4) dt,

con_pwtyp_cd,

rdet_swtyp_cd,

con Iocn facil id,

sum(rdet_rswims_count),

sum (con_gross_wḡt),

avg (con_gross_wgt),

sum (con_cntr_vol),

avg (con_cntr_vol)

from radwaste, contype

where con_tsd_accept_dt between '01-jan-69' and '31-dec-92' and

con_srce_cmpny _id = 'TPNL' and

con_srce_facil_id $=1325$ '

and rdet_swtyp_cd in ('2A', '2B', '2L', '3A', '3L', 'UA', 'UB') and

con_crityp_cd = cntyp_cd

group by cntyp_descr,

con_size_descr,

substr (Eo_char(con_tsd_accept_dt, 'YYYY'),1, 4),

con_pwtyp_cd,

rdet swty $\bar{p} c d$,

con_Iocn_facil_id

;

spool off 
Container Discription

Container size

BURLAP, ClOTH, PAPER OR PLASTIC baGs

BURLAP, Cloth, PAPER OR PLASTIC bags

BURLAP, CLOTh, PAPER OR PLASTIC BAGS

BURLAP, CLOTH, PAPER OR PLASTIC BAGS

bURlap, cloth, PAPER OR PLASTIC bags

$\infty$

w qurlap, cloth, paper or plastic bags

burlap, cloth, paper or plastic bags

CONCRETE BOXES

DUMP TRUCK

FIBERBOARD/PLASTIC BOXES, CARTONS, CASES

FIBERBOARD/PLASTIC BOXES, CARTOUS, CASES

\section{UnKMOMn}

UNKNOWN

Unknoum

Unknorm

UnKnom

Umknom

UnKnow

$3 * 3 * 3$

UNKNOWN

2 CU FT

2 CU FT
Pri Sec

Wast Hast

Date Type Code facility count Wright

1973 R 24 21813n $12 \quad 152.41$

tht

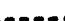

$12 \quad 152.41$

$\operatorname{sen}$
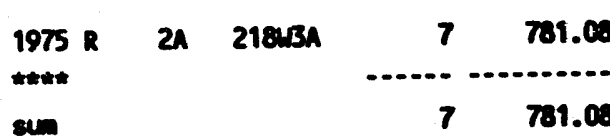

$\sin$

$1977 R \quad 2 A \quad 218$ ara

thtt

sin

$\begin{array}{rrr}1978 n & 2 A & 21833 \\ R & 2 A & 21833\end{array}$

sun

1979 R $2 A \quad 21843$

thitut

sin

1980 R $2 A \quad 218 \pi 3 A$

ד10

sun

$1975: \quad 21 \quad 21813$

1975

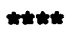

sum

$1974 R \quad 2 A \quad 21843 A$
sum

1977 R

2 21843A

sum

$1978 R \quad 2 A \quad 21843 A$

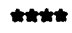

sum

$86-4405.26$

$86 \quad 4405.26$

$35 \quad 498.95$

$35 \quad 498.95$

2101.60

2101.60
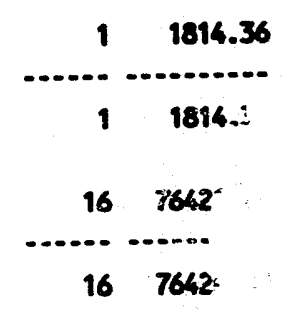

\begin{tabular}{cr}
105 & 701. \\
\hline 101 & 701.72
\end{tabular}

\begin{tabular}{cr}
112 & 1311.33 \\
\hline 112 & 1311.33
\end{tabular}

Avg$$
\begin{array}{lll}
76.21 & 2.97 & 1.49
\end{array}
$$$$
2.97
$$$$
\begin{array}{lll}
260.36 & 1.74 & .58
\end{array}
$$$$
1.74
$$$$
231.86 \quad 10.49 \quad .55
$$

10.49

$\begin{array}{lll}18.14 & .03 & .03\end{array}$

$142.69 \quad 13.30 \quad .70$

13.32

55.

50.80

$.80 \quad .23 \quad .11$

1814.36 .23

.$i T$

4.59

$-3.30 \quad 73.39$

73.39

$7.72 \quad 3.10 \quad .39$

3.10

57.01 
Container Discription Container Size

2 CU FT

FIBERBOARD/PLASTIC BOXES, CARTONS, CASES

FIBERBOARD/PLASTIC BOXES, CARTONS, CASES

FIBERBOARD/PLASTIC BOXES, CARTCWS, CASES FIBERBOARD/PLASTIC BOXES, CARTOUS, CASES FIBERBOARD/PLASTIC BOXES, CARTONS, CASES

FIBERBOARD/PLASTIC BOXES, CARTOUS, CASES FIBERBOARD/PLASTIC BOXES, CarTONS, CASES $\omega$

ì

fiberboaro/PLASTIC bOXES, Cartons, Cases

fIBERBOARD/PLASTIC BOXES, CARTONS, CASES

FIBERBOARD/PLASTIC BOXES, CARTOWS, CASES

FIBERBOARO/PLASTIC BOXES, CARTONS, CASES

FIBERBOARD/PLASTIC BOXES, CARTONS, CASES
2 CU FT

UNKWOW

UAKMOWN

UNKMONH

UMKNOWN

UNKNOWN

UnKMom

UNKNOWN

UNKNOWN

UNKNOWN

UAKNOMN
Pri Sec

Wast Hast

Date Type Code Facility count Weight Weight volume Volume

1980 R $2 A \quad 218013$

$86 \quad 1554.43$

$86 \quad 1554.43$

$\sin$

1970 n $2 A \quad 21843 A$

R $2 A$ 21843a

$R$ in 218ura

tht

sum

$\begin{array}{rrr}1971 R & 2 A & 21843 n \\ R & 2 A & 218448\end{array}$

sum

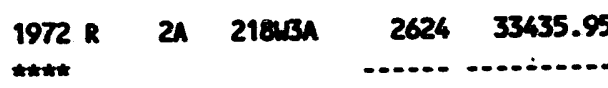

sum

$2624 \cdot 33435.95$

1973

sum

$21 \quad 2183$

$6374 \quad 80965.82$

$6374 \quad 80965.82$

1974

sum

$2 A \quad 218 \% 3 n$

467259448.44

$$
\ldots
$$

467259448.44

1975

t林

$2 A \quad 21843 A$

sum

$3801 \quad 48262.00$

$3801 \quad 48262.00$

1976 R $2 A \quad 21843$

$\operatorname{sun}$

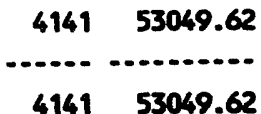

\section{$43.18 \quad 5.73 \quad .16$}

5.73

$37.65 \quad .51 \quad .13$

.51

$\begin{array}{lll}88.90 & .89 & .89^{\circ}\end{array}$

$\begin{array}{lll}132.89 & 92.00 \quad 1.33\end{array}$

$382.04 \quad 44.23 \quad 3.69$

-.........

137.12

$324.77 \quad 68.43$

408.5373 .78

142.21

\begin{tabular}{lll}
522.44 & 333.72 & 5.21 \\
\hline
\end{tabular}

333.72

$710.23 \quad 812.21$

7.12

812.21

$612.87 \quad 595.51$

6.14

595.51

$473.16 \quad 484.22 \quad 4.75$

484.22

$530.50 \quad 528.10$ 


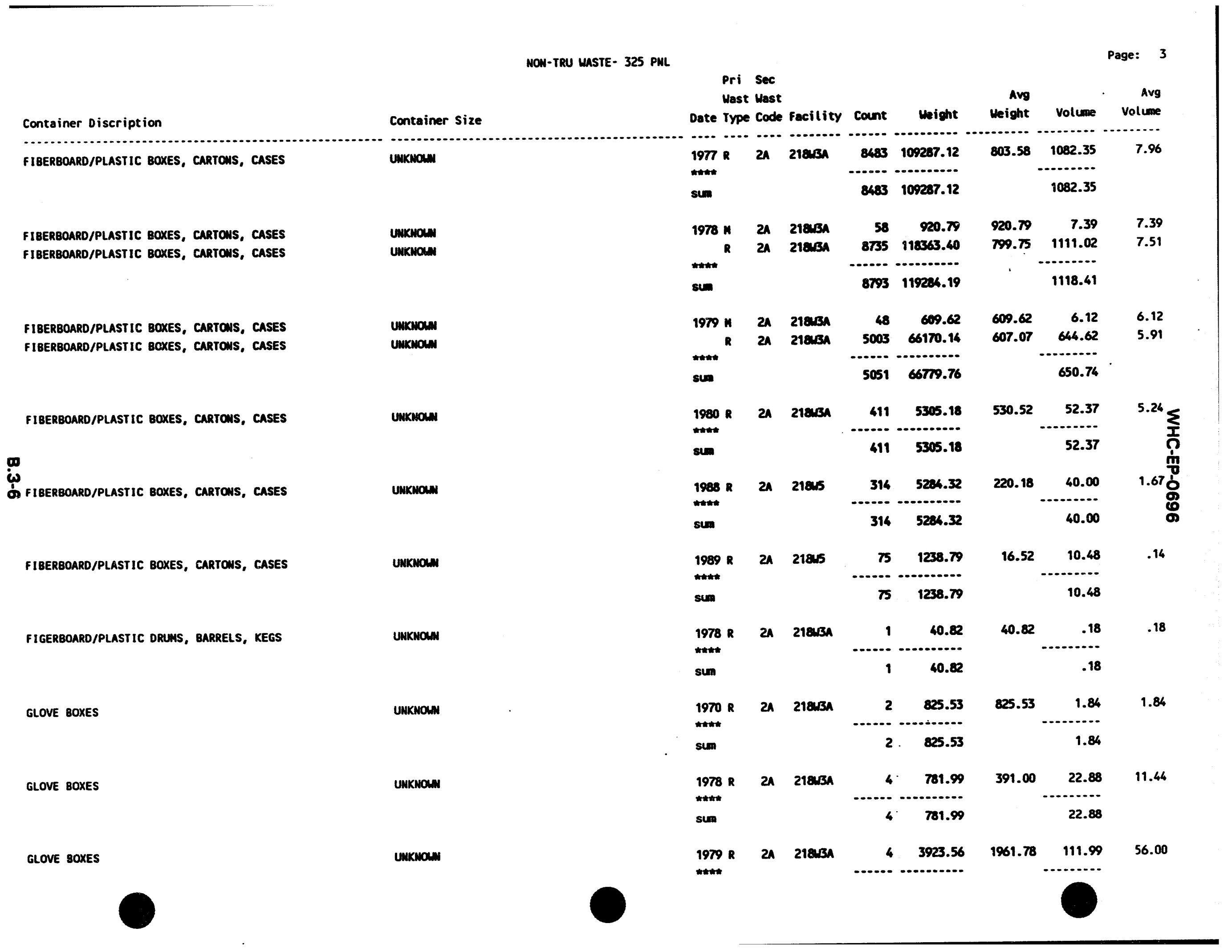




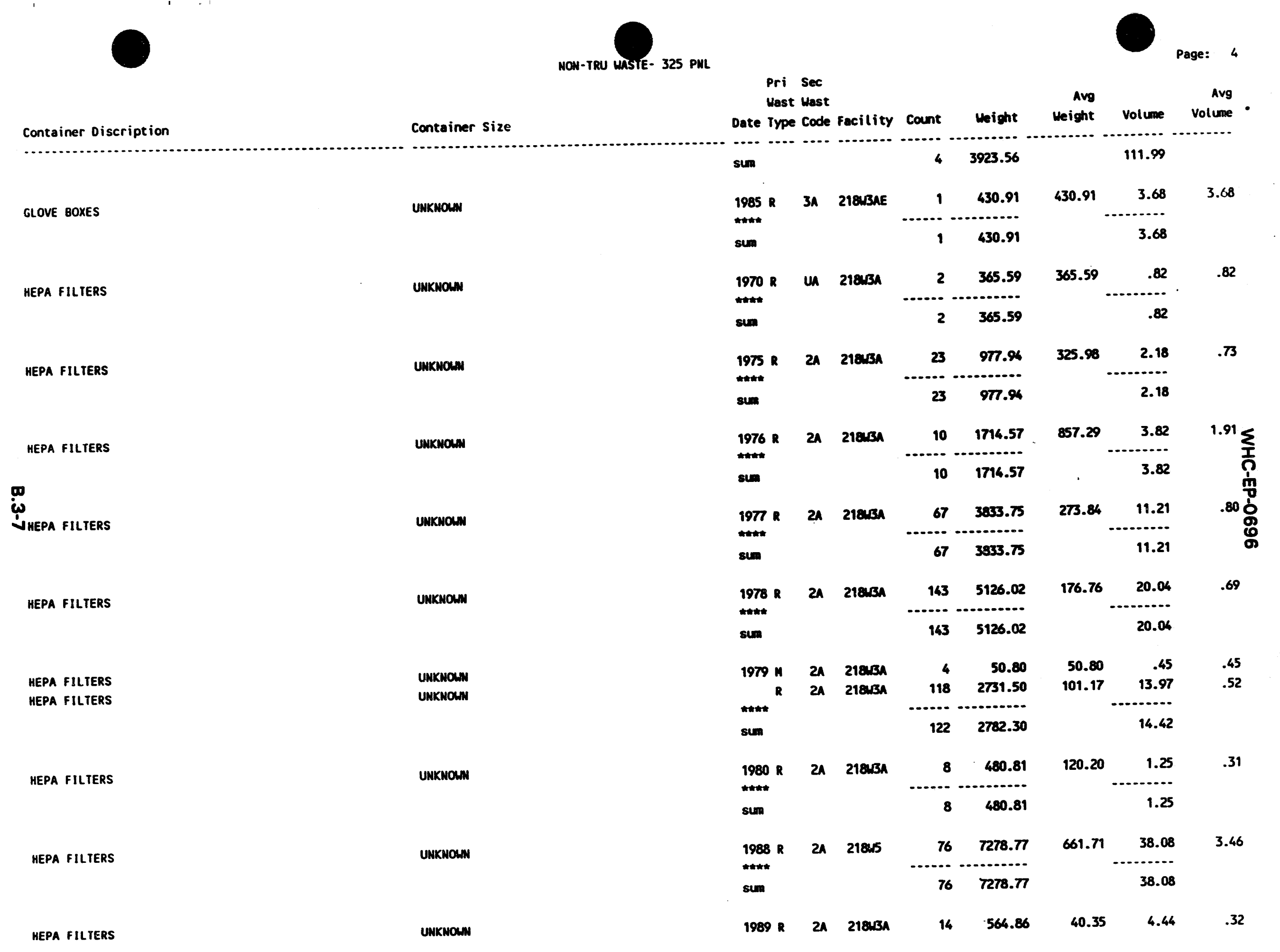




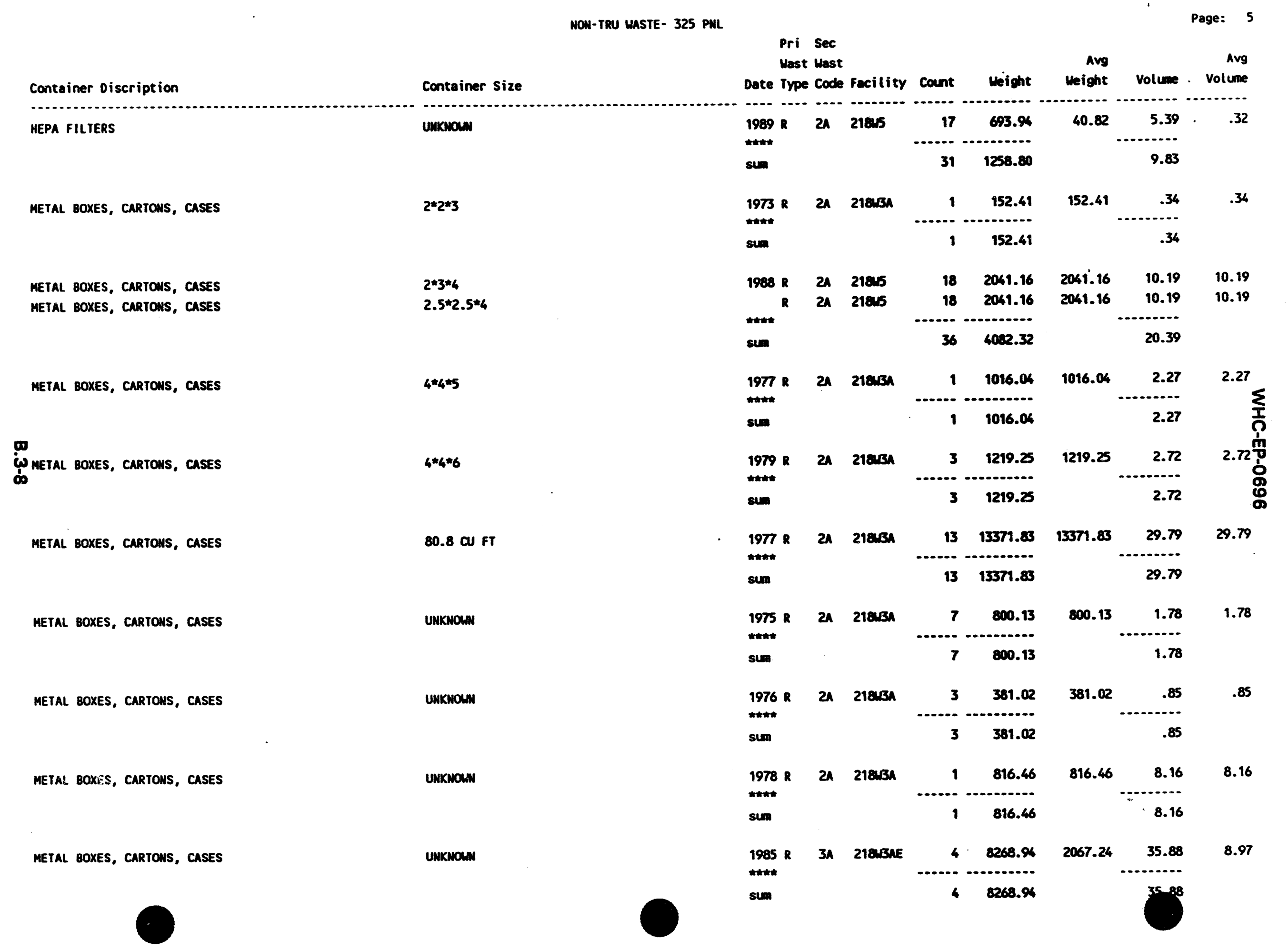




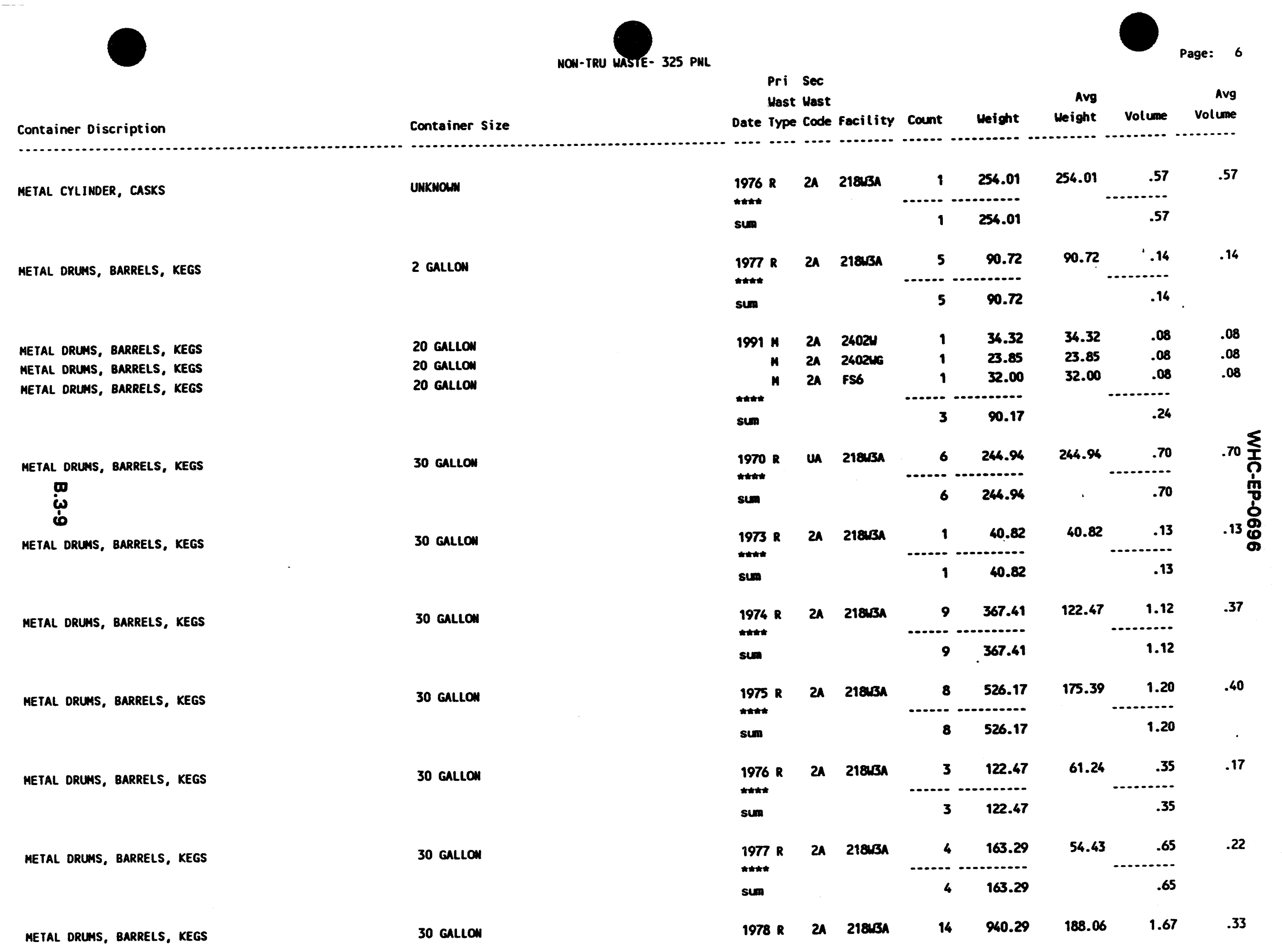




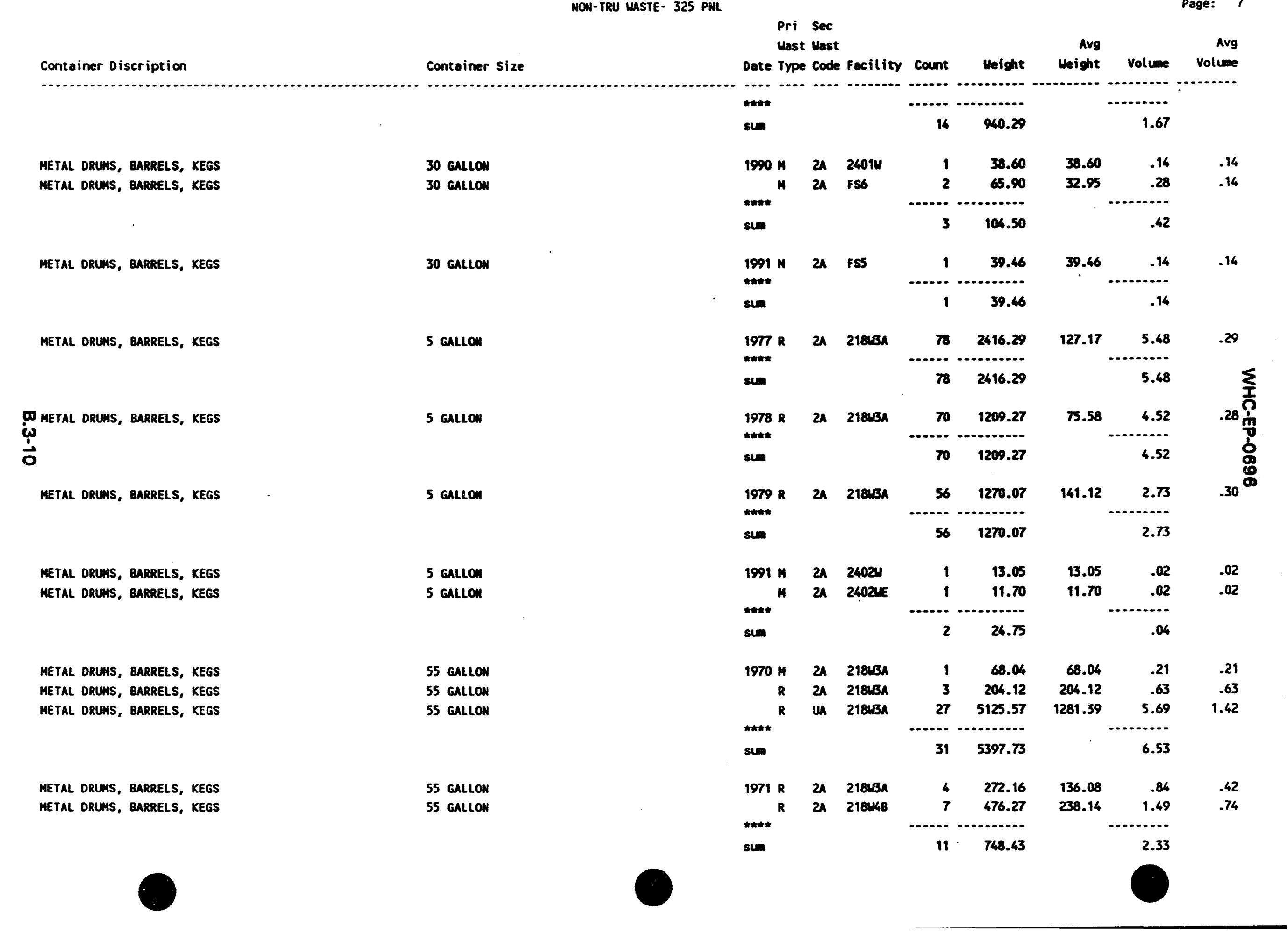




\section{Container Discription}

METAL DRUMS, BARRELS, KEGS

metal dRLUHS, BARRELS, KEGS

metal DRUMS, BarreLS, KegS

METAL DRUMS, BARRELS, KEGS

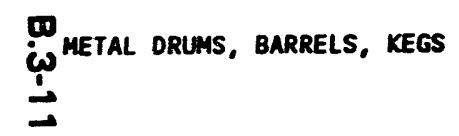

Metal dRUMS, Barrels, KegS

METAL DRUMS, BARRELS, KEGS METAL DRUMS, BARRELS, KEGS

METAL ORUMS, BARRELS, KEGS METAL DRUMS, BARRELS, KEGS

METAL DRUMS, BARRELS, KEGS METAL DRUMS, BARRELS, KEGS

METAL DRUMS, BARRELS, KEGS
Container Size

55 GALLON

55 Gallor

55 GALLON

55 GaLLON

55 GALLON

55 GALLOW

55 GALLOW

55 GALLON

55 GALLON

55 GALLON

55 GALLOW

55 GALLON

55 GALLON
Pri sec

Hast Mast

Date iype code fecility cant Hoight ung volue volume

$\begin{array}{lllll}1972 R & \text { LA } & 21843 A & 109 & 5218.11\end{array}$

***

sum

$109 \quad 5218.11$

372.72

$18.68 \quad 1.33$

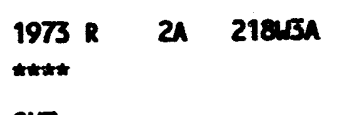

\begin{tabular}{rr}
97 & 7349.94 \\
\hdashline 97 & 7349.94
\end{tabular}

18.68

sun
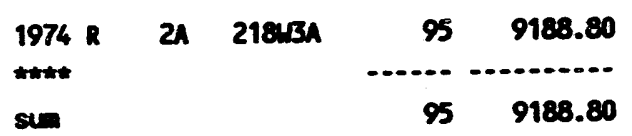

sin

$95 \quad 9188.80$

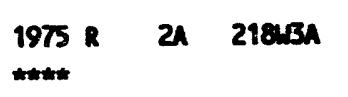

$195 \quad 21490.65$

$195 \quad 21490.65$

sin

$220 \quad 20656.53$

1976 R 2A 21843A

$220 \quad 20656.53$

\section{smang}

1977 R 21 218ura

2270405.42

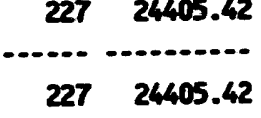

1978 n $24 \quad 21843$

1.53 .59

\begin{tabular}{lr}
$476 \quad 85612.85$ \\
\hline..-
\end{tabular}

47786066.44

sun
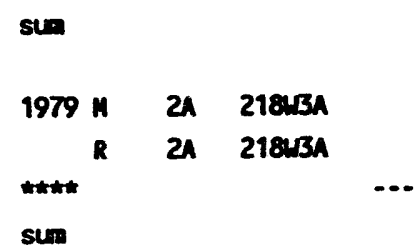

\begin{tabular}{rr}
6 & 563.36 \\
301 & 29435.73 \\
\hline 307 & 29999.09
\end{tabular}

1980 R $2 A \quad 21843 A$

\begin{tabular}{rr}
29 & 2617.67 \\
21 & 5411.33 \\
\hline 50 & 8029.00
\end{tabular}

$224.12 \quad 20.61$

20.61

20.61

$335.79 \quad 41.41$
$-\ldots . .-$

41.41

$350.11 \quad 46.82$

46.82

$259.63 \quad 48.32$

48.32
48.32

$\begin{array}{lll}453.59 & .21 & .21\end{array}$

$847.65 \quad 96.35 \quad .95$

R $3 A \quad 21843 A$

sim

$50 \quad 8029.00$

1988 N 2A 24014

$3 \quad 210.92$

70.31

.21 
Metal dRUMS, BarReLs, KEgS

METAL DRUMS, BARRELS, KEGS METAL DRUMS, BARRELS, KEGS METAL DRUMS, BARRELS, KEGS METAL DRUAS, BARRELS, KEGS METAL DRUAS, BARRELS, KEGS METAL DRUNS, BARRELS, KEGS

METAL DRUAS, BARRELS, KEGS METAL DRUMS, BARRELS, KEGS METAL DRUMS, BARRELS, KEGS W METAL DRUMS, BarRels, Kegs

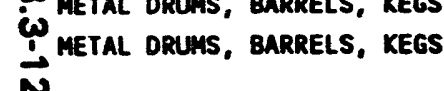

METAL DRUNS, BARRELS, KEGS METAL DRUMS, BARRELS, KEGS METAL DRUMS, BARRELS, XEES METAL DRUMS, BARRELS, KEGS METAL DRUUH, BARRELS, KEGS

METAL DRUMS, BarRels, KEgS METAL ORUMS, BARRELS, KEGS METAL DRUMS, BARRELS, KEGS

METAL DRUMS, BARrELS, KEgS

miscellameOUS SCRAP
55 GALLON

55 Gallon

55 GALLON

55 callow

55 Galloy

55 GalloM

55 GaLLOM

55 Gallon

55 GALLON

55 Gallon

55 Gallon

55 GalloN

55 GALLON

55 GALLON

55 GALLON

55 GALLON

55 GALLON

55 GALLON

55 GALLON

55 GALLON

8 GALLON

Unknom
Date Iype Code facility count Weight Weight Volune Volume

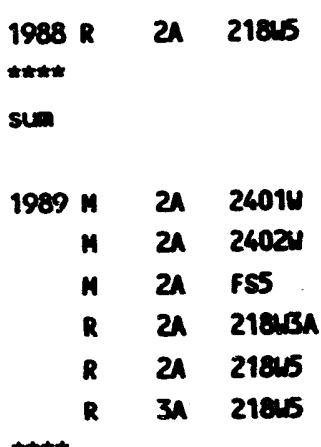

t*t.

$\sin$

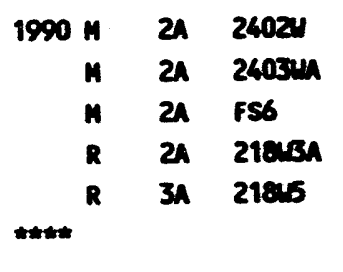

sin

1991 in $2 A$ 2402AE

H $2 A \quad$ FS5

R $2 A$ 21845

R $2 L$ 218MC

R $3 A \quad 2185$

thitut

sum

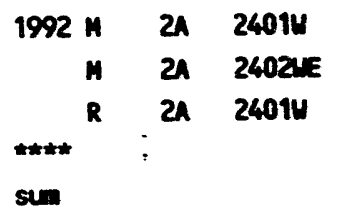

sun

1990 M $21 \quad 240218$
$\sin$

...................

$89 \quad 6563.43$

$92 \quad 6774.55$

437.56

18.66

19.29

\begin{tabular}{rr}
1 & 117.93 \\
22 & 6907.23 \\
1 & 113.40 \\
3 & 200.66 \\
28 & 2687.59 \\
4 & 285.77 \\
\hline & \\
\hline
\end{tabular}

$59 \quad 10320.58$

$\begin{array}{rrr}117.93 & .21 & .21 \\ 313.97 & 4.62 & .21 \\ 113.40 & .21 & .21 \\ 69.55 & .63 & .21 \\ 95.99 & 5.83 & .21 \\ 71.44 & .84 & .21 \\ & \ldots . . . . . . & \end{array}$

12.39

\begin{tabular}{rr}
1 & 490.70 \\
1 & 663.60 \\
2 & 209.10 \\
5 & 295.46 \\
39 & 7040.40 \\
\hline 48 & 8699.34
\end{tabular}

$490.78 \quad .21$

$663.60 \quad .21$

$104.55 \quad .42$

59.09

180.52

1.05
8.19

8.19
-2.0.

10.08

\section{.21}

$.21 \leq$

$.21 \frac{5}{8}$

.21 กิ

$.21 \frac{\mathrm{m}}{\mathrm{v}}$

.08

\begin{tabular}{rr}
1 & 281.73 \\
1 & 59.00 \\
8 & 467.80 \\
3 & 350.64 \\
35 & 6022.59 \\
\hline &..
\end{tabular}

$281.73 \quad .21 \quad .21$

59.00

58.48

116.88

172.07

1.68

.63

7.32

10.05

$48 \quad 7181.76$

$\begin{array}{lll}113.50 & .21 & .21\end{array}$

\begin{tabular}{rr}
1 & 113.50 \\
1 & 59.90 \\
1 & 98.90 \\
\hline 3 & 272.30
\end{tabular}

$59.90 \quad .20 \quad .20$

98.90

.21

.62

21.67

$\begin{array}{rr}3 & 65.00 \\ 3 & 65.00\end{array}$

.09
.09

$1970 R \quad 2 A \quad 2184 B$ $\begin{array}{lll}2 & 6058.28 \quad 3429.14\end{array}$
.21

.21

.21

21

.03 


\section{Container Discription}

MISCELLANEOUS SCRAP

MISCELLAMEOUS SCRAP

MISCELLAMEOUS SCRAP

miscellamequs scrap

miscellamequs scrap Miscellameous SCrap

$\omega$

MISCELLAMEOUS SCRAP

MISCELLAMEOUS SCRAP

miscellaneous scrap

MISCELLAMEOUS SCRAP

MISCELLANEOUS SCRAP
Container size

\section{UnkMONA}

unknow

UnKNOM

Unknown

unxuoun

unkroun

UnKMONM

UNKMOWM

UNKMONN

UnKnow

UnKnOM⿻
Pri Sec

Hast Hast

Date Type Code Facility count Weight

1970 \& un 218u3a $20 \quad 63502.60$

timtert

sum

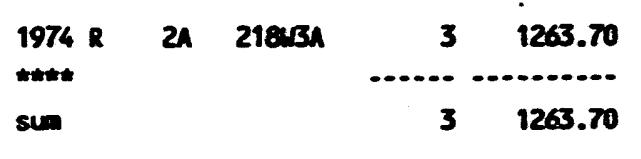

1975

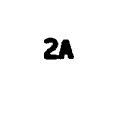

21843

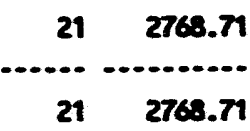

sim

$21 \quad 2768.71$

1976 R 2A 218uBA

$\begin{array}{cr}2 & 12.70 \\ 2 & -12\end{array}$

sum

$2 \quad 12.70$

1977

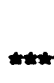

sum

24 218asa

1235.87

44. 2095.12

-....

$45 \quad 2330.99$

$1978 R \quad 2 A \quad 21843$

$67 \quad 2609.94$

$67 \quad 2609.94$

1979 R $2 A \quad 21845$

1979

$28 \quad 035.76$

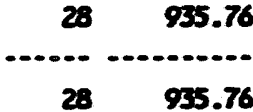

sum

$1980 R \quad 2 A \quad 21813$

1217

$\sin$

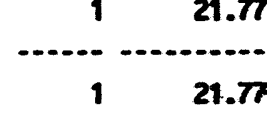

$1987 R \quad 2 A \quad 21845$

tatat

$46 \quad 3275.85$

sen:

$46 \quad 3275.83$

t*t*

$\sin$
$24 \quad 21845$

\begin{tabular}{rr}
146 & 9616.12 \\
\hline 146 & 9616.12
\end{tabular}

Avg

Avg

Weight volume Volume

$\begin{array}{lll}31751.30 & 141.59 & 70.79\end{array}$

$421.23 \quad 2.82 \quad 94$

$r .82$

$692.18 \quad 6.17 \quad 1.54$

6.17

$12.70 \quad .03 \quad .03$

.03

$235.87 \quad .23$

$130.95 \quad 7.26$

-........

7.49

$153.53 \quad 37.18$

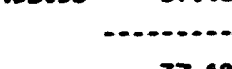

37.18

$\begin{array}{lll}93.58 & 12.32 & 1.23\end{array}$

12.32

$21.77 \quad .04 \quad .04$

.06

$655.17 \quad 21.46$

4.29

21.46 


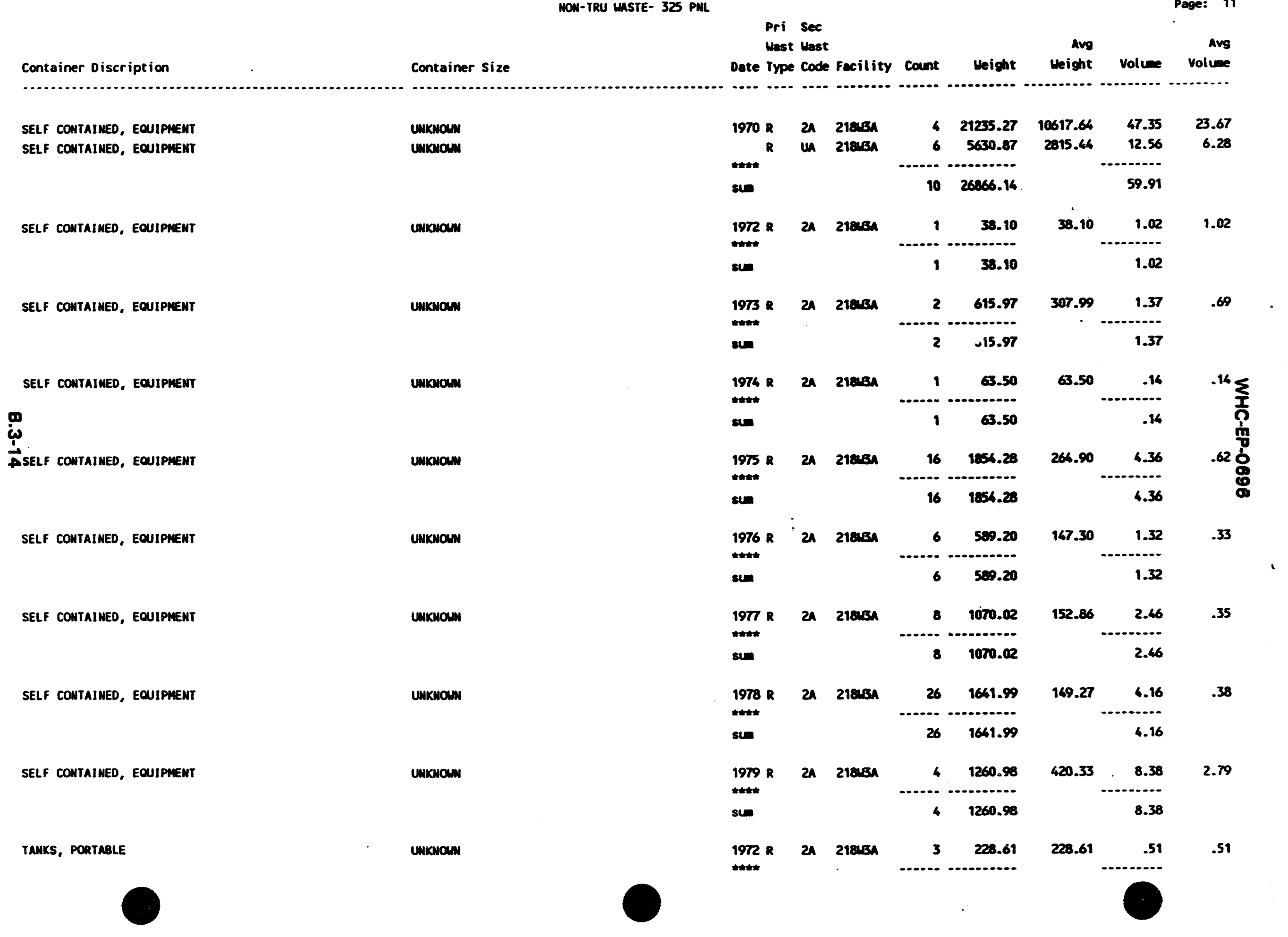


Container Discription

Container Size

TANKS, PORTABLE

TANKS, PORTABLE

TRUCKS, FLATBEDS, COMPACTOR, LOADLUGGER TRUCKS, FLATBEDS, COMPACTOR, LOADLUGGER

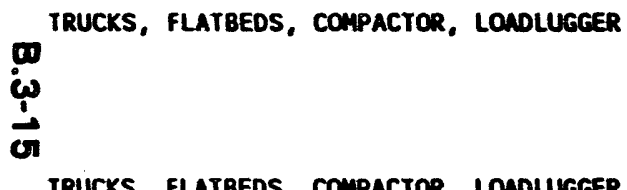

TRUCKS, FLATBEDS, COMPACTOR, LOADLUGGER

TRUCKS, FLATBEDS, COMPACTOR, LOADLUGGER

TRUCKS, FLATBEDS, COMPACTOR, LOADLUGGER

TRUCKS, FLATBEDS, COMPACTOR, LOADLUGGER

WOCOEN BOXES, CARTONS, CASES

WOODEN BOXES, CARTOWS, CASES
UAKNOMH

UNKNOM

UntKMOM

Unkmon

Unknown

UnKMON

Unkmon

UNKNOM

UNKNOWN

$0.7 * 1 * 1.2$

$1 * 2 * 3$
Pri Sec

Wast Wast

Date Type code Facility count Wight

sin

$3 \quad 220.61$

1977 R $2 A$ 21813a

$\begin{array}{rr}1 & 2514.70 \\ 1 & 254.70\end{array}$

$2514.70 \quad 5.61$
$\ldots . r$
$\quad 5.61$

1984 \& 2A 218u3n

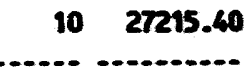

$27215.40 \quad 3.96$

$10 \quad 27215.40$

$\sin$

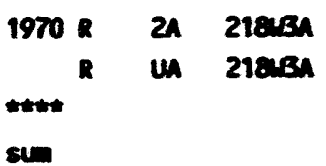

$2 \quad 6550.26$

$2 \quad 35656.38$

$4 \quad 40006.64$

1972 R $2 A$ 218usa

1972

23600.60

$\operatorname{sen}$

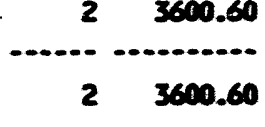

1973 R $2 A \quad 21834$

mats

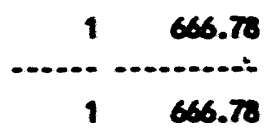

66.78

sem

1974 R $21 \quad 21813$

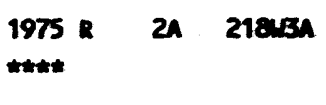

su.

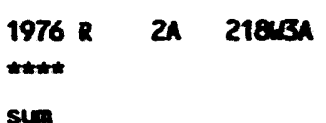

sum

1977 R $2 A \quad 218$ Ba

sum

1976 R $2 A \quad 218$ B

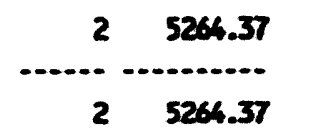

$2632.19 \quad 11.7$

11.74

\begin{tabular}{rr}
1 & 1270.05 \\
\hdashline & 1270.05
\end{tabular}

$\begin{array}{lll}1270.05 & 2.83 & 2.83\end{array}$

2.83

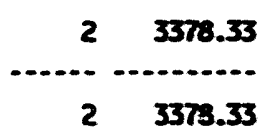

$1689.17 \quad 7.53$

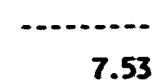

\begin{tabular}{rr}
11 & 199.58 \\
\hline 11 & 199.58
\end{tabular}

199.58

24
$-\ldots . .5$

$76.20 \quad .17$

3.77

5.61

3.96

14.16

37.52

$4.01 \leq$

$1.40 \%$

5.87

.17 


$$
\text { Uast Uast }
$$

Date Type Code facility count whight Weight volume volune

WOOOEN BOXES, CARTOWS, CASES WOODEN BOXES, CARTONS, CASES WOOOEN BOXES, CARTONS, CASES WOOOEN BOXES, CARTONS, CASES WOOOEN BOXES, CARTOUS, CASES

WOODEN BOXES, CARTONS, CASES

WOCOEN BOXES, CARTONS, CASES W WOODEN BOXES, CARTOWS, CASES के

WOOOEN BOXES, CARTONS, CASES

WOODEN BOXES, CARTOWS, CASES WOCOEN BOXES, CARTOUS, CASES

HOOOEN BOXES, CARTONS, CASES WOODEN BOXES, CARTOWS, CASES

WOOOEN BOXES, CARTONS, CASES

WOODEN BOXES, CARTONS, CASES

$1 * 4 * 4$
$1 * 4 * 4$
$1.5 * 1.5 * 0.7$
$2 * 2 * 2$
$2 * 2 * 4$

$2 * 2 * 4$

$2 * 2 * 8$

$2 * 2 * 9$

$2 * 4 * 4$

$2 * 4 * 5$

$2 \star 4 \star 8$

$3 \star 3 \star 3$

$3 * 3 * 4$

$3 \star 3 \star 4$

$3 \star 4 * 4$
....

su

1977 * $2 A \quad 2188$ A

R $2 A \quad 2183 A$

M $2 A$ 218usa

R $2 A$ 218Ba

R $2 A$ 218:3a

thate

sen

1978 R $2 A$ 218usa

su

$\begin{array}{rrr}1974 \& \quad 2 a & 21843 a \\ R & 2 a & 2183 a\end{array}$

sen

$1978 R$ 2A 2185A

$\begin{array}{rrr}1974 R & 2 A & 21843 a \\ R & 2 A & 21843 A\end{array}$

sum

$\begin{array}{rrr}1977 R & 2 A & 21853 \\ k & 2 A & 21843 a\end{array}$

$\sin$

1978 R $2 A \quad 218 \% 34$

tot:

sum

1970 R $2 A \quad 21833$

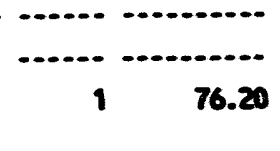

1362.87

304.81

$1 \quad 13.15$

2406.42

-...................

$12 \quad 1226.96$

\begin{tabular}{cr}
1 & 508.02 \\
\hdashline$\ldots .0$
\end{tabular}

\begin{tabular}{rr}
1 & 406.42 \\
1 & 457.22 \\
\hline 2 & 863.64
\end{tabular}

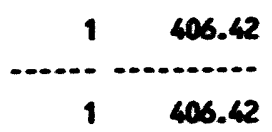

362.87
304.81

13.15

139.71

406.42

$42 \quad .91$

2.65

508.02

1.13
1.13

406.42 .91

457.22

22

1.02

$06.42 \quad .91$

91

$508.02 \quad 1.13$

$3521.67 \quad 23.56$

24.69

$14 \quad 11073.04$

$\begin{array}{rr}13 & 2014.85 \\ 1 & 457.22 \\ \ldots & \ldots . .\end{array}$

402.97

89

457.22

1.02

8.00

$14 \quad 2672.07$

$\begin{array}{rr}1 & 317.51 \\ \ldots & \end{array}$

$\begin{array}{rr}317.51 & .68 \\ & .68\end{array}$

8.00

1317.51

1828.87

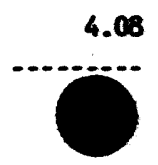




\section{Container Discription}

\section{Container..........................}

MOOOEN BOXES, CARTONS, CASES

WOCOEN BOXES, CARTONS, CASES WOONEN BOXES, CARTOWS, CASES WOOOEN BOXES, CARTONS, CASES

WOODEN BOXES, CARTONS, CASES

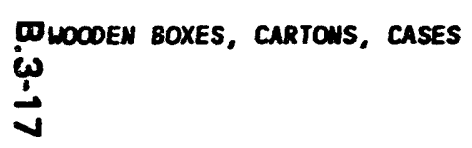

WOCDEN BOXES, CARTOWS, CASES WOODEN BOXES, CARTONS, CASES

WCOOEN BOXES, CARTONS, CASES

WOODEN BOXES, CARTOHS, CASES

WOOOEN BOXES, CARTONS, CASES WOONEN GiXES, CARTONS, CASES

WOONEM BOXES, CARTOUS, CASES
Container Size

$3 * 4 * 5$

3*6*16

$4 * 0.4 * 0.8$

$4 * 1.5 * 1.5$

$4 * 4 \star 4$

$4 * 4 * 4$

$4 * 4 * 4$

$4 * 4 \star 4$

$4 * 4 * 4$

$4 * 4 * 5$

$4 * 4 * 6$

$4 * 4 * 7$

$4 * 4 * 8$
Pri sec

Wast Hast

Date Type Code facility Count Weight

sum

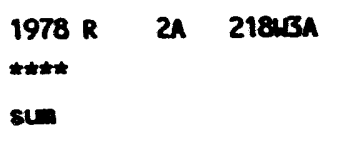

$3 \quad 1828.87$

\begin{tabular}{cr}
1 & 762.03 \\
\hline 1 & 762.03
\end{tabular}

$\begin{array}{rrr}1977 R & 2 A & 21813 A \\ R & 2 A & 21813 A \\ R & 2 A & 21813 A\end{array}$

wtat

sen

$1974 R \quad 2 A \quad 21843 A$

su

1976 R $2 A \quad 218$ sa

$\sin$

$\begin{array}{rrr}1977 M & 2 a & 21843 A \\ R & 2 A & 2184 B A\end{array}$

su

1978 R 2A 21843A

1978

sin

1974 R 2A 21845A

sum

1977 R $2 A \quad 21843 A$

$R \quad 2 A \quad 21843 A$

แか十

sen

1972
$2 \quad 3251.34$

\begin{tabular}{rr}
1 & 3657.75 \\
2 & 762.03 \\
1 & 114.30 \\
\hline 4 & 4534.08
\end{tabular}

$14 \quad 11380.57$

$14 \quad 11380.57$

\begin{tabular}{rr}
1 & 812.83 \\
\hline & 812.83
\end{tabular}

1951.49

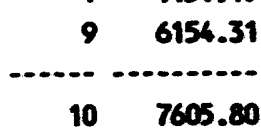

$10 \quad 7605.80$
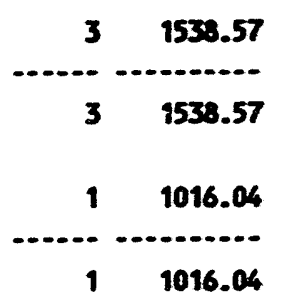

\begin{tabular}{rr}
1 & 1219.25 \\
7 & 2641.71 \\
\hline 8 & 3860.96
\end{tabular}

the

1016.04

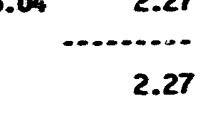

1219.2

2641.71

2.72
20.62
23.33

Avg

Avg

Weight Volune Avg

Weight volume

$\begin{array}{lll}762.03 & 1.70 \quad 1.70\end{array}$

$\begin{array}{lll}3657.75 & 8.16 & 8.16\end{array}$

$\begin{array}{lll}762.03 & 1.70 & 1.70\end{array}$

$\begin{array}{lll}114.30 & .26 & .26\end{array}$

10.11

$\begin{array}{lll}1380.57 & 25.37 \quad 25.37\end{array}$

: 25.37

$812.83-1.81$

1.81
1.81

$1451.49 \quad 1.81$

3.26

18.12

$\begin{array}{lll}769.29 & 5.44 & 2.72\end{array}$

5.44

2.27

2.72

20.62

.33

$1625.67 \quad 7.25$ 

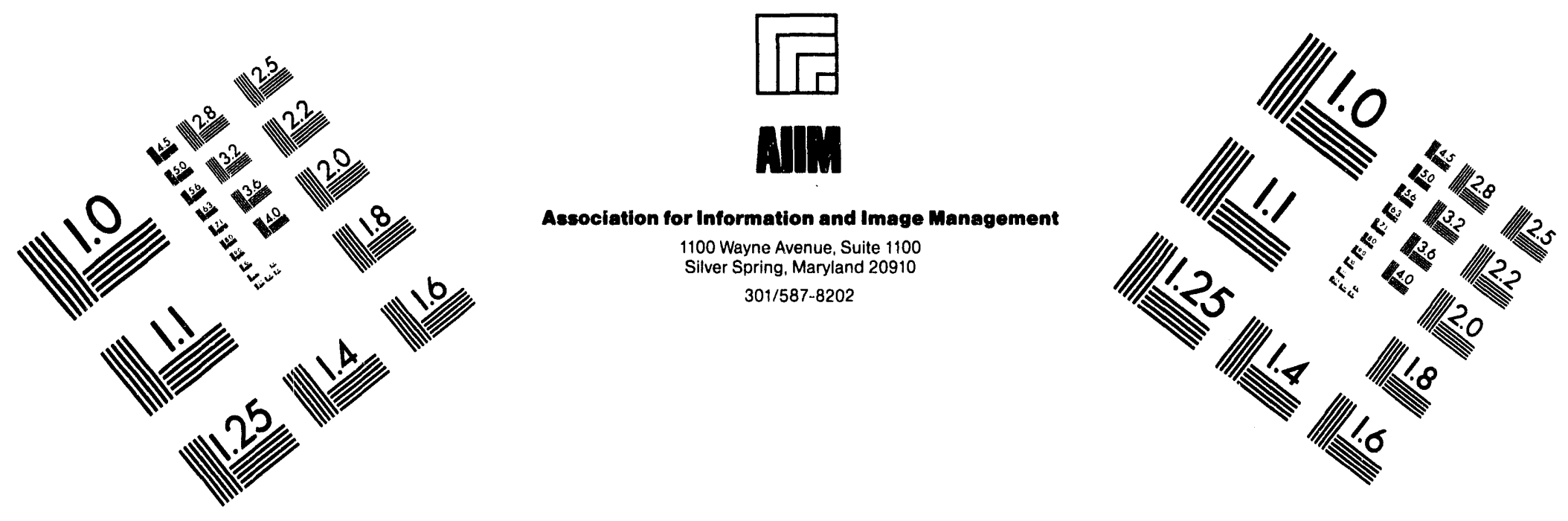

\section{Centimeter}

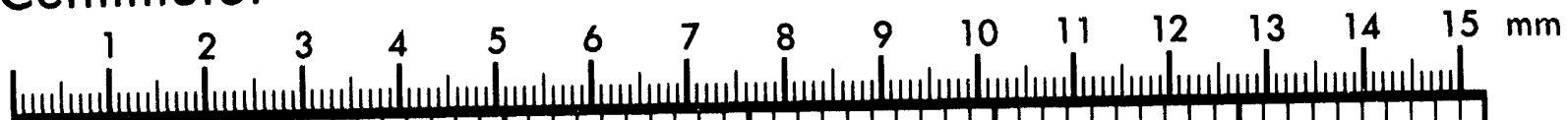

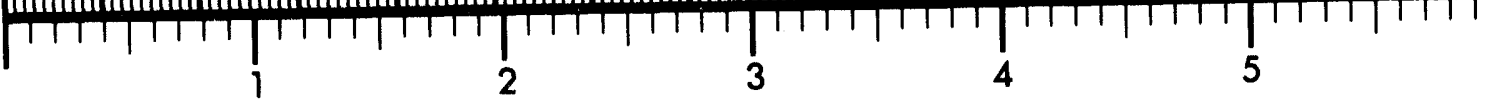
Inches
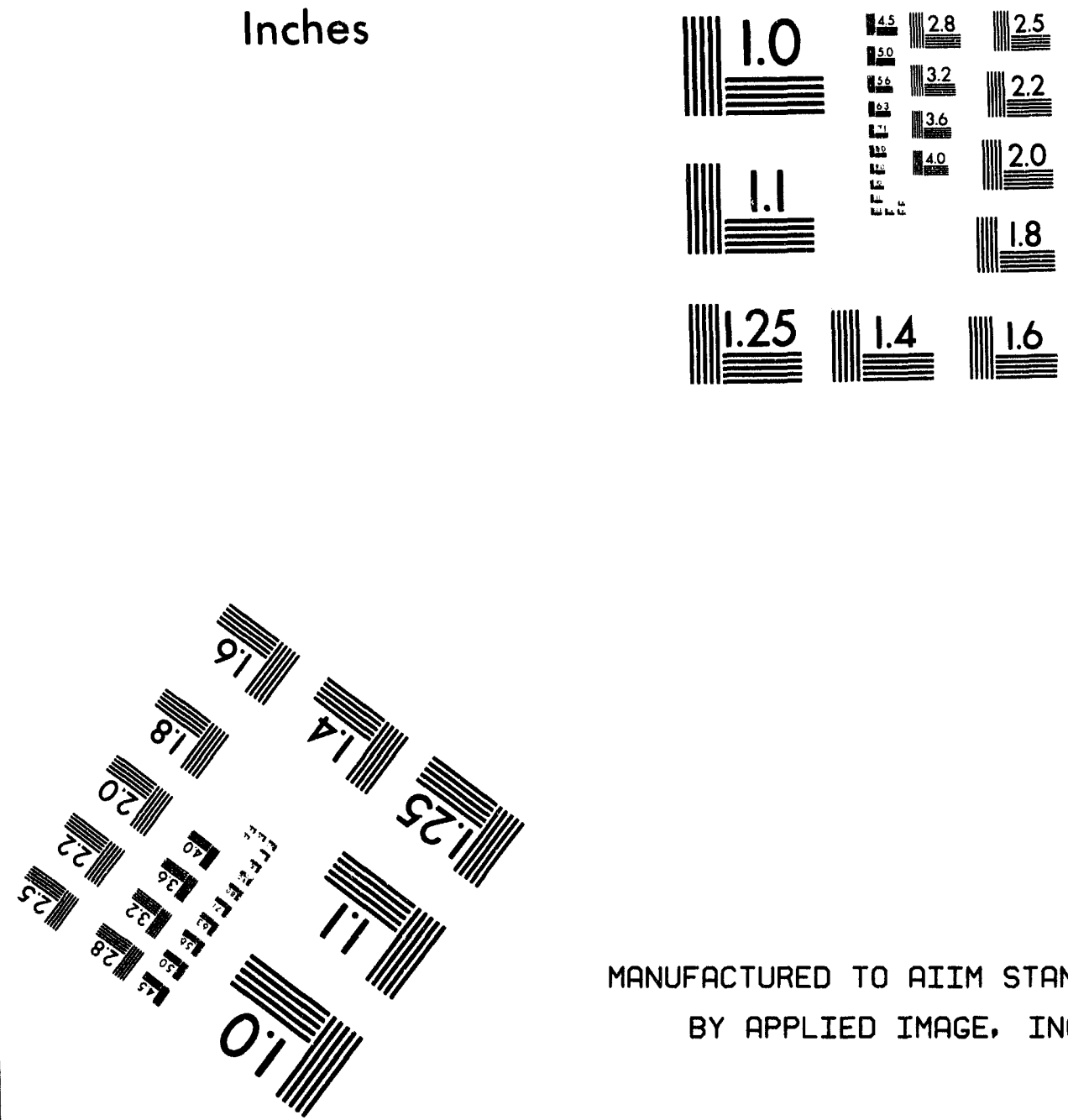

MANUFACTURED TO AIIM STANDARDS

BY APPLIED IMAGE, INC.

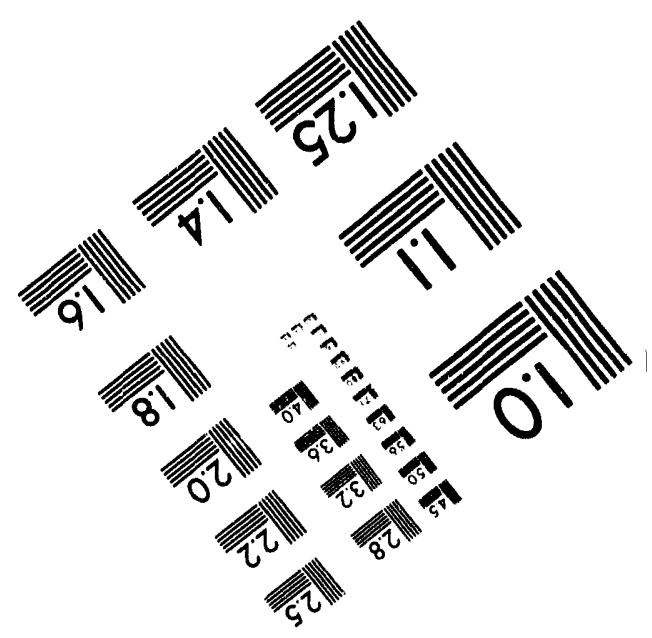



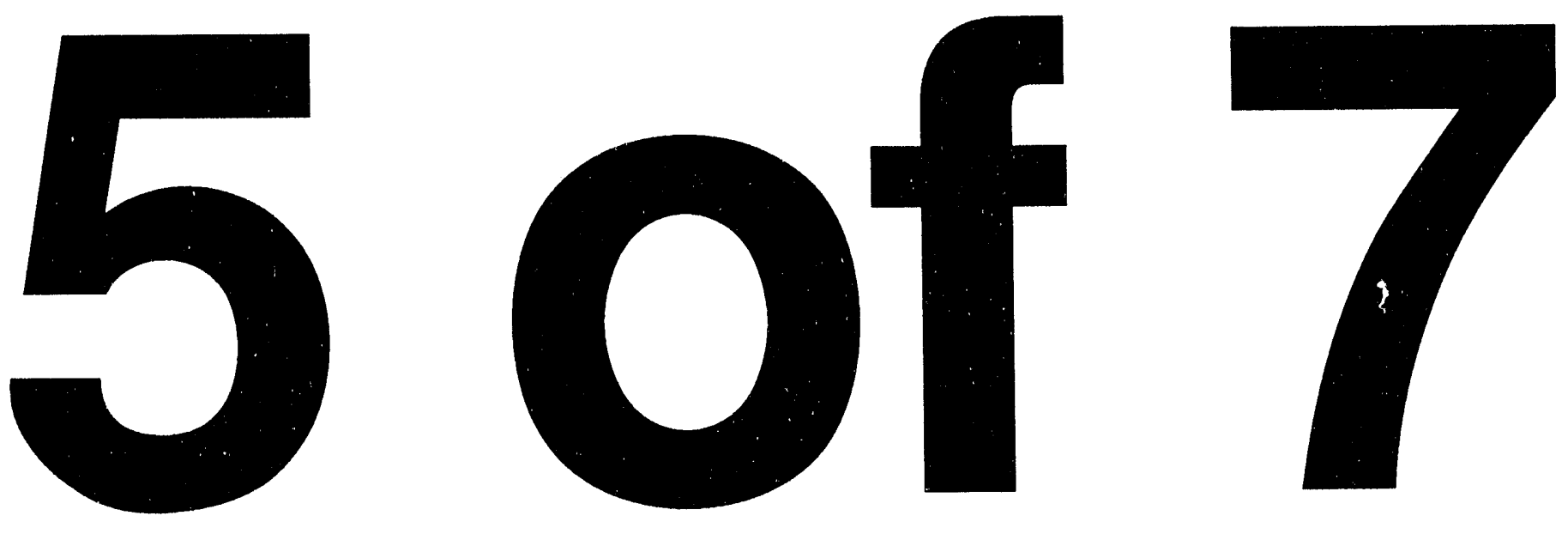


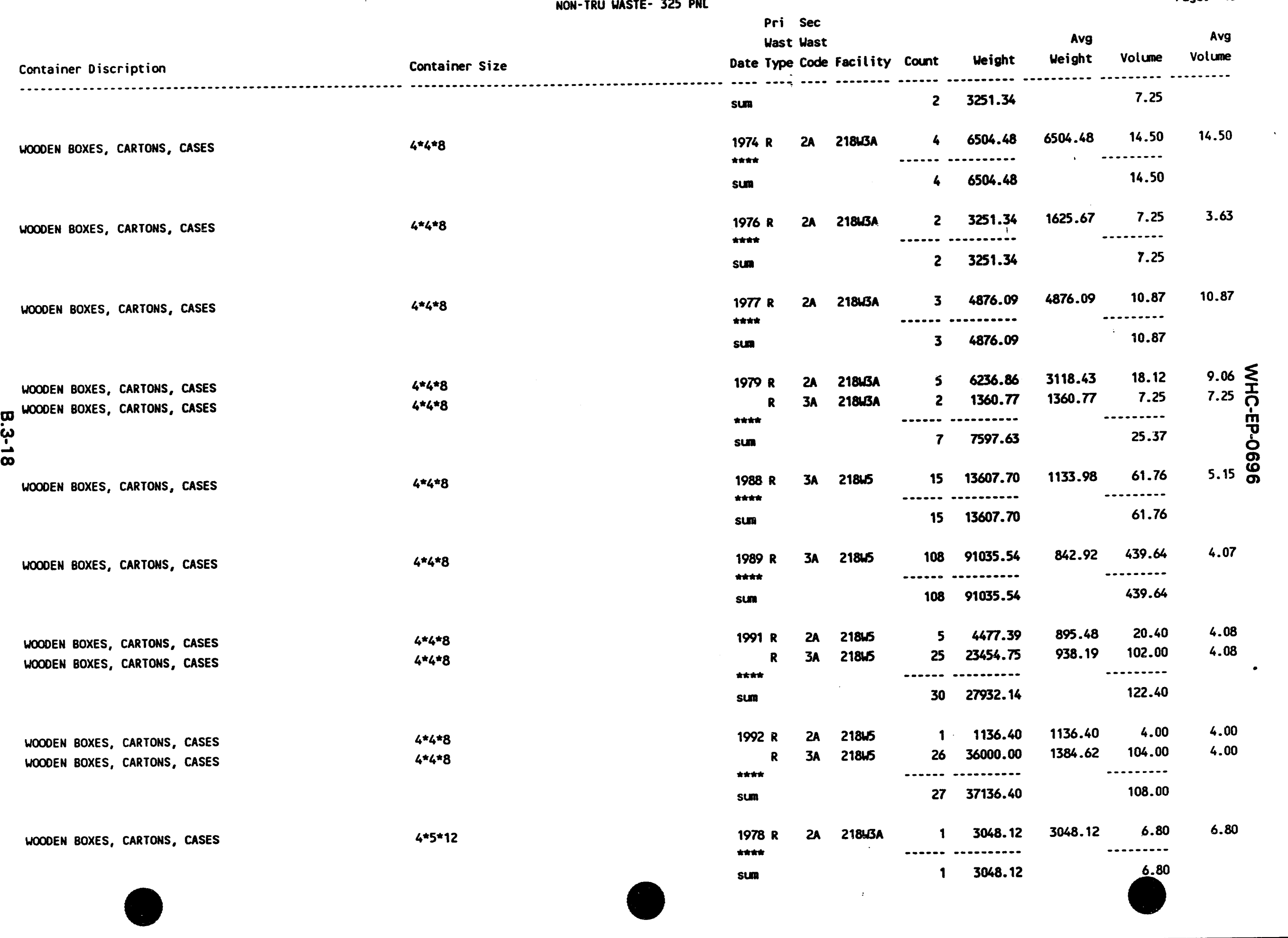




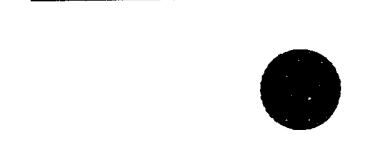

NOW-TRU WASTE- 325 PNL

Page: 16

Container Discription

WOODEN BOXES, CARTONS, CASES

WOOOEN BOXES, CARTONS, CASES

WOOOEN BOXES, CARTONS, CASES

WOOOEN BOXES, CARTONS, CASES WOODEN BOXES, CARTONS, CASES W WOOOEN BOXES, CARIONS, CASES $\dot{\omega}$

WOOOEN BOXES, CARTONS, CASES

WOOOEN BOXES, CARTONS, CASES

HOONEN BOXES, CARTONS, CASES

WOOOEN BOXES, CARTONS, CASES

WOOOEN BOXES, CARTONS, CASES

WOODEN BOXES, CARTONS, CASES
Container size

$4 \star 5 \star 6$

$4 \star 5 \star 7$

$4 \star 5 \star 8$

$4 \star 7 \star 8$

$4.5 * 5.6 * 8.7$

$5 * 5 * 8$

$5 * 6 * 7$

$6 * 6 * 6$

UNKNOWN

UNKNOWN

UNKNOWN

UNKNOWN

Pri Sec

Wast Wast Date Type Code Facility Count Weight

$1970 R \quad 2 A \quad 21843 A$

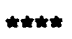

sum

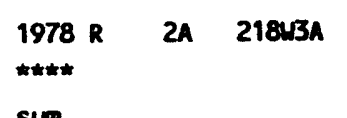

sum

1987 R $3 A \quad 21845$

sum

1977 R $2 A \quad 218433$

R $2 A$ 21843A

R $2 A \quad 21843 a$

stame

sum

1974 R $2 A \quad 21843$ A

sum

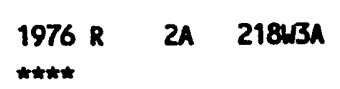

sum

$1971 R \quad 2 A \quad 218448$

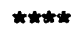

sum

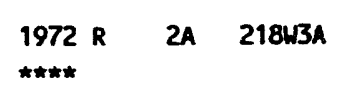

sum

$\underset{\star 1974 R \quad 2 A \quad 21843 A}{ }$

sum

1977 R 2A 21843A
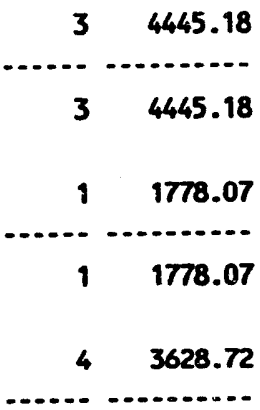

$4 \quad 3628.72$

$\begin{array}{lr}1 & 2844.92 \\ 1 & 825.53\end{array}$

$1 \quad 5896.67$

$3 \quad 9567.12$

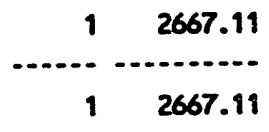

12743.31

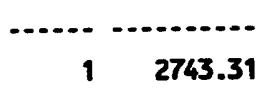

6.12

$4 \quad 1759.93$

$4 \quad 1759.93$

879.97

6.66

6.66

\begin{tabular}{rr}
56 & 2990.97 \\
\hline 56 & 2990.97
\end{tabular}

1495.49

6.67 6.67

\begin{tabular}{rr}
14 & 5307.00 \\
\hline 14 & 5307.00
\end{tabular}

$5307.00 \quad 11.84$

11.84

11.84

$\begin{array}{llll}5 & 610.54 & 203.51 & 1.67\end{array}$
9.91

3.96

4.05

6.34

$1.84 \sum$

5.66 六 
Container Discription

Container size

c........................

WOODEN BOXES, CARTONS, CASES

WOODEN BOXES, CARTONS, CASES

UNKNOWN

UNKNOWN
Pri Sec

Wast Wast

Date Type Code Facility Count Weight

..................

$5 \quad 610.54$

sum

1978 R $2 A \quad 21843 A$

*t*t*

sum

\#แ*

$\sin$

21843

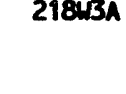

2.384 .20

$\begin{array}{rr}2 & 384.20 \\ 2 & 384.20\end{array}$

192.10

.99
.99

$\begin{array}{rr}1 & 131.99 \\ 1 & 131.99\end{array}$

131.99

.62
$\ldots .62$

Avg

Volume

204 rows selected.

$\boldsymbol{\omega}$
$\dot{\omega}$
$\hat{\alpha}$ 


\section{WHC-EP-0696}

APPENDIX B.4

\section{RADIOLOGICAL DATA FOR TRU WASTE CONTAINERS SORTED BY DATE, PRIMARY WASTE TYPE, AND STORAGE FACILITY}




\section{WHC-EP-0696}

This subappendix summarizes the radiological data for all TRU waste containers generated at Building 325 PNL. Specifically, this computer run provides the total grams of TRU elements in a given container type in a given year. In addition, the average gram loading for a given container type in a given year has been calculated.

In 1991 and 1992 the number of curies (Ci) of alpha radiation was included on the SWBRs. Since the Building 325 PNL waste generation data is all from 1970 to 1988 , these columns contain blank entries. The "Max Dose" column contains the highest value recorded for a given container in a given year, in $\mathrm{mrem} / \mathrm{hr}$.

Additional information about the isotopes present in Building 325 PNL waste containers can be found in subappendices B.9 and B.10. 
WHC-EP-0696

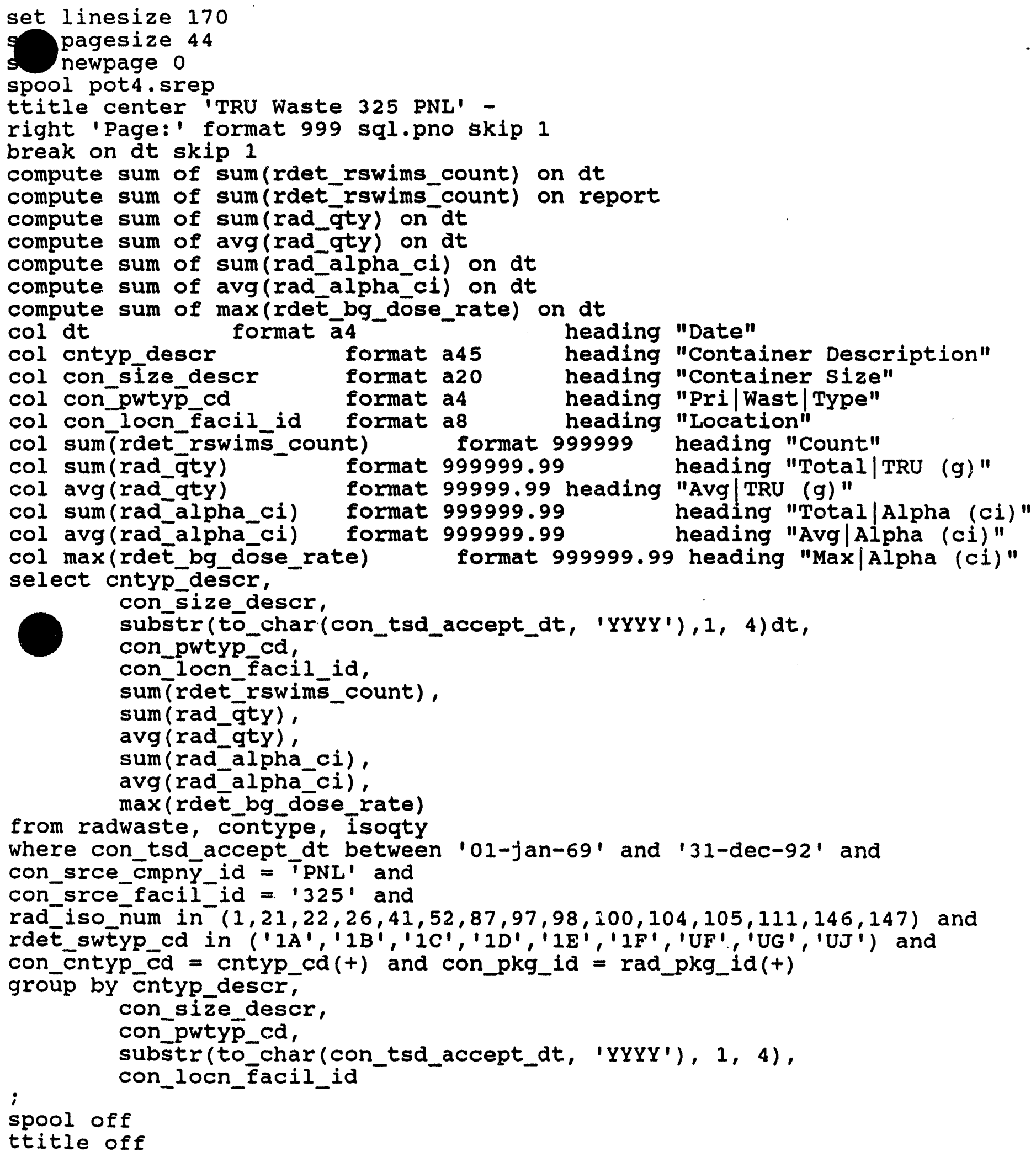


Hast Total Avg Total Avg Max

Container Description

Container Size

Date Type Location Count

1978 R 218448

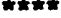

sum

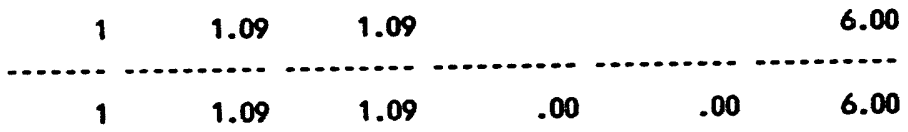

CONCRETE BOXES

$3 * 3 * 3$

1975 R $218 w 4 B$

sum

CONCRETE BOXES

CONCRETE BOXES

FIBERBOARD/PLASTIC BOXES, CARTOWS, CASES

Afiberboard/plastic boxes, cartons, cases

METAL BOXES, CARTONS, CASES

METAL BOXES, CARTONS, CASES

METAL DRUMS, BARRELS, KEGS

METAL DRUMS, BARRELS, KEGS

METAL DRUMS, BARRELS, KEGS
$4 * 4 * 8$

$4 * 7 * 9$

UNKMOWN

UNKNOWN

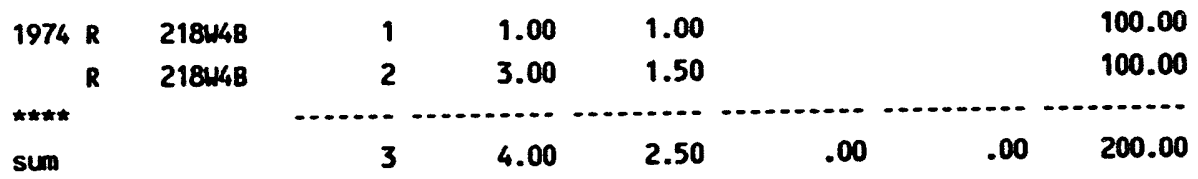

$1969 R \quad 21844 \mathrm{~B} \quad 33 \quad 19.05 \quad 19.05 \quad 10.00$

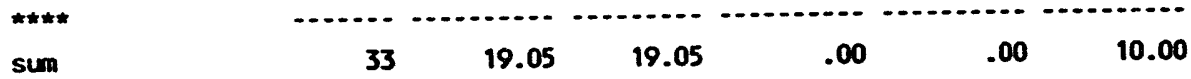

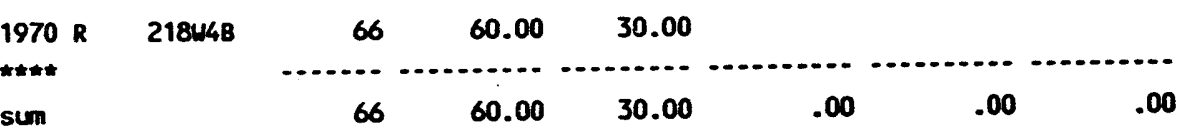

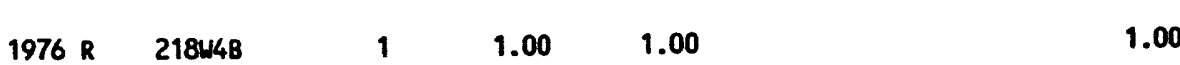

$2 * 2 * 6$

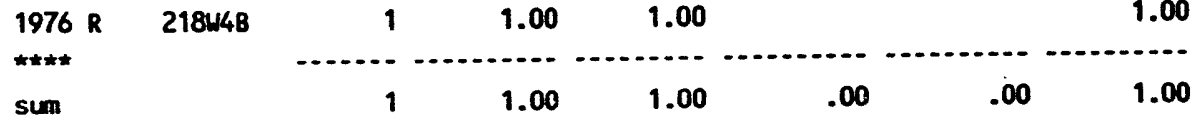

$5.6 * 6.5 * 9.3$

1992 R 2402WE

\begin{tabular}{rrrrrr}
5 & 47.51 & 9.50 & 12.58 & 2.52 & 2.50 \\
\hline 5 & 47.51 & 9.50 & 12.58 & 2.52 & 2.50
\end{tabular}

30 GALLON

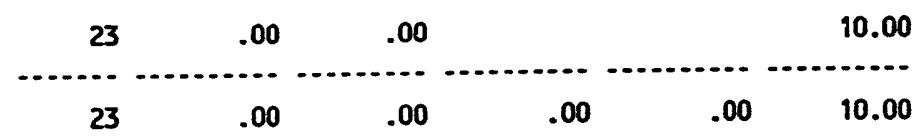

55 GALLON

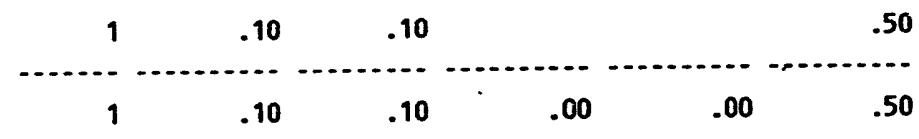

55 GALLON

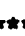

sum

1970 R 21843A
$\star \star \star \star \star$
sum

1989 M $224 \mathrm{~T}$

sum

1990 M $224 \mathrm{~T}$ 


\section{Container Description}

\section{.........................}

METAL DRUMS, BARRELS, KEGS

METAL DRUMS, BARRELS, KEGS

METAL DRUMS, BARRELS, KEGS

METAL DRUMS, BARRELS, KEGS DMETAL DRUMS, BARRELS, KEGS ì

METAL DRUMS, BARRELS, KEGS

METAL DRUMS, BARRELS, KEGS METAL ORUMS, BARRELS, KEGS

METAL DRUMS, BARRELS, KEGS

METAL DRUMS, BARRELS, KEGS METAL DRUMS, BARRELS, KEGS

METAL DRUMS, BARRELS, KEGS
Pri

Total

Avg

Total

Avg

Max

Container Size Date Type Location Count

TRU (g) TRU (g) Alpha (ci) Alpha (ci) Alpha (ci)

sum

55 GALLON

$1991 M \quad 224 T$
$\sin$

1

$\begin{array}{lllll}.23 & .23 & .00 & .00 & .50\end{array}$

\section{R 218146

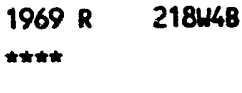

55 GALLON

55 GALLON

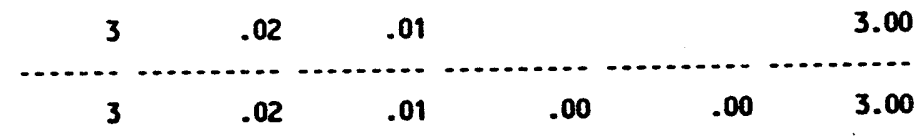

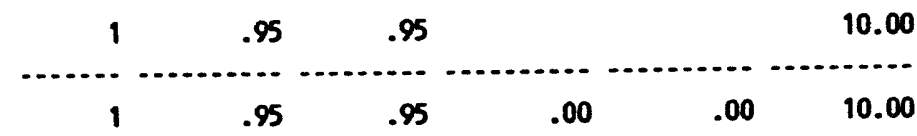

55 GALLON

55 GALLON

55 GALLON

55 GALLON

55 GALLON

55 GALLON

55 GALLON

55 GALLON

55 GALLON

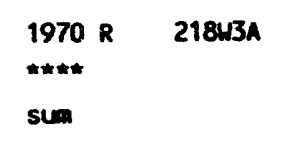

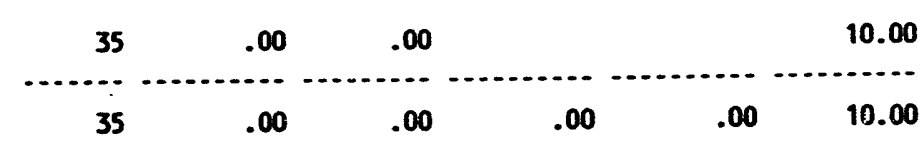

$1971 R \quad 21843 A$

$R \quad 218448$

$\star * \star * *$

sum

\begin{tabular}{rrrrrr}
22 & .00 & .00 & & & 10.00 \\
305 & 162.03 & .53 & & & 11.00 \\
\hline 327 & 162.03 & .53 & .00 & .00 & 21.00
\end{tabular}

1972 R 218448

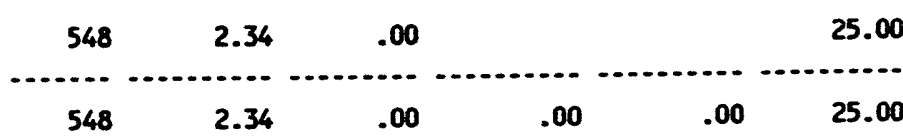

$1973 R \quad 21843 A$

\begin{tabular}{llllll}
$R$ & 218448 & 14 & .14 & .01 & 1.00 \\
\hline & 65 & 1.17 & .02 & 200.00
\end{tabular}

R 218448

sim

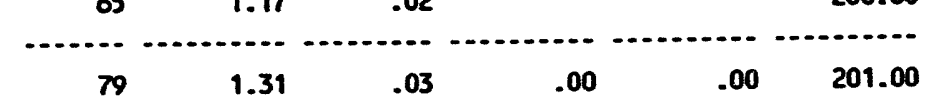

$1974 R \quad 218 W 48$
sum

\begin{tabular}{|c|c|c|c|}
\hline 52 & 16.90 & .33 & \\
\hline 52 & & .33 & .00 \\
\hline
\end{tabular}

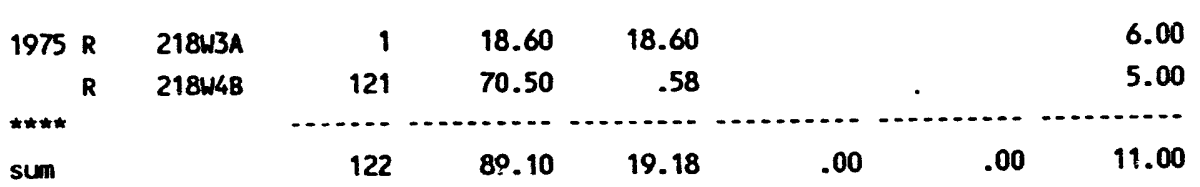

1976 R $\quad 218448$

$53 \quad 16.91 \quad .32$

5.00 
Container Description

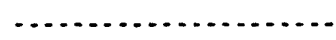

METAL DRUMS, BARRELS, KEGS

METAL DRUMS, BARRELS, KEGS

METAL DRUMS, BARRELS, KEGS

METAL DRUMS, BARRELS, KEGS

i

i

METAL DRUMS, BARRELS, KEGS

METAL DRUMS, BARRELS, KEGS METAL DRUMS, BARRELS, KEGS

METAL DRUMS, BARRELS, KEGS

METAL DRUMS, BARRELS, KEGS

METAL DRUMS, BARRELS, KEGS METAL DRUMS, BARRELS, KEGS Pri

Wast Total Avg Total Avg Max Container Size Date Type Location Count TRU (g) IRU (g) Alpha (ci) Alpha (ci) Alpha (ci)

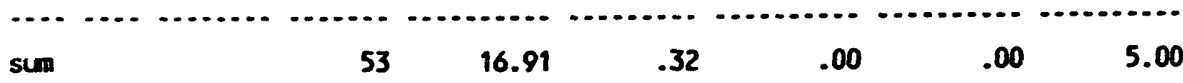

\begin{tabular}{|c|c|c|c|c|c|}
\hline 1977 R & $218 w 48$ & 33 & 47.99 & 1.45 & 117.00 \\
\hline & & 33 & 47.99 & 1.45 & 117.00 \\
\hline
\end{tabular}

55 GALLON

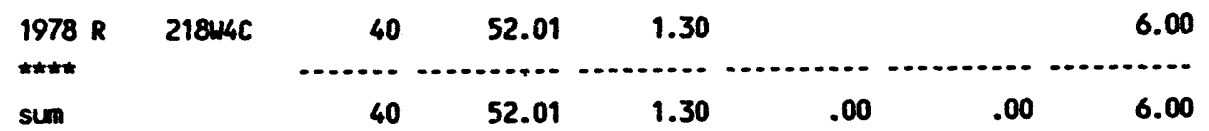

55 GALLON

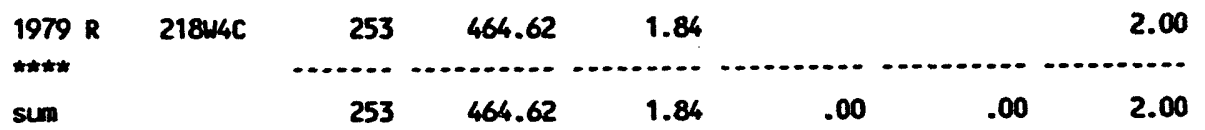

55 GALLON

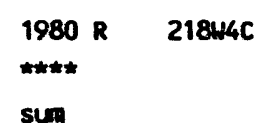

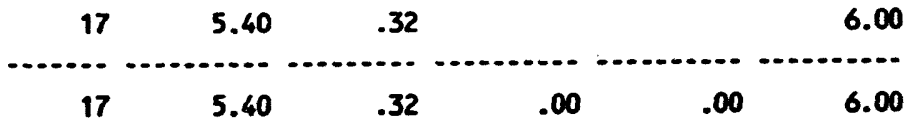

55 GALLON

$1985 R \quad 218$ R $R$

$\begin{array}{rrrr}4 & 7.21 & 1.80 & \end{array} \quad 35.00$

sum

$\begin{array}{lllll}7.21 & 1.80 & .00 & .00 & 35.00\end{array}$

55 GALLON

55 GALLON

$\begin{array}{rl}1987 R & 21844 C \\ R & 224 T\end{array}$

\begin{tabular}{rrrrrr}
2 & 32.94 & 16.47 & & & $\begin{array}{r}7.00 \\
120.00\end{array}$ \\
19 & 292.16 & 15.38 & &. & 120.0 \\
\hline 21 & 325.10 & 31.85 & .00 & .00 & 127.00
\end{tabular}

55 GALLON

sum

$\underset{\substack{1989 \\ \star \star \star \star \star}}{2247}$

\begin{tabular}{|c|c|c|}
\hline 22 & 114.18 & 5.19 \\
\hline 22 & 114.18 & 5.19 \\
\hline
\end{tabular}

55 GALLON

$1990 R \quad 224 T$
sum

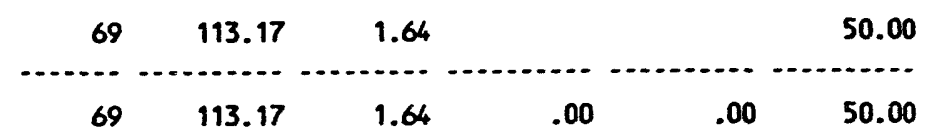

55 GALLON 55 GALLON

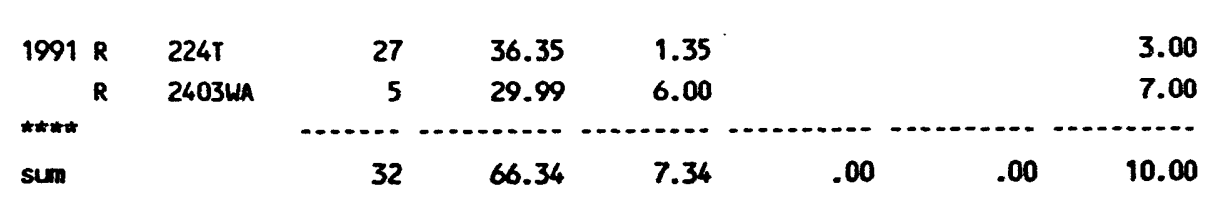




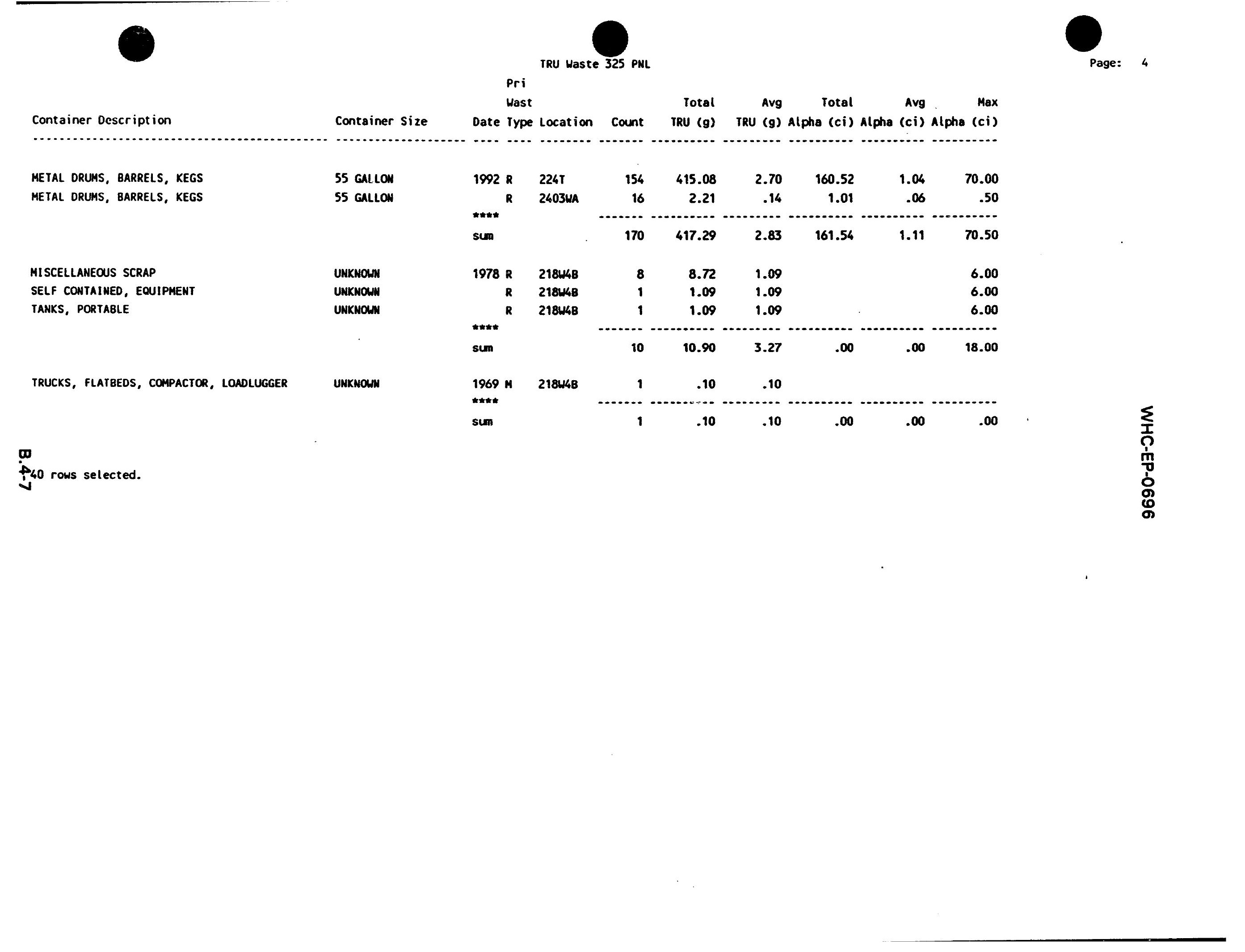


WHC-EP-0696

This page intentionally left blank.

\section{B.4-8}
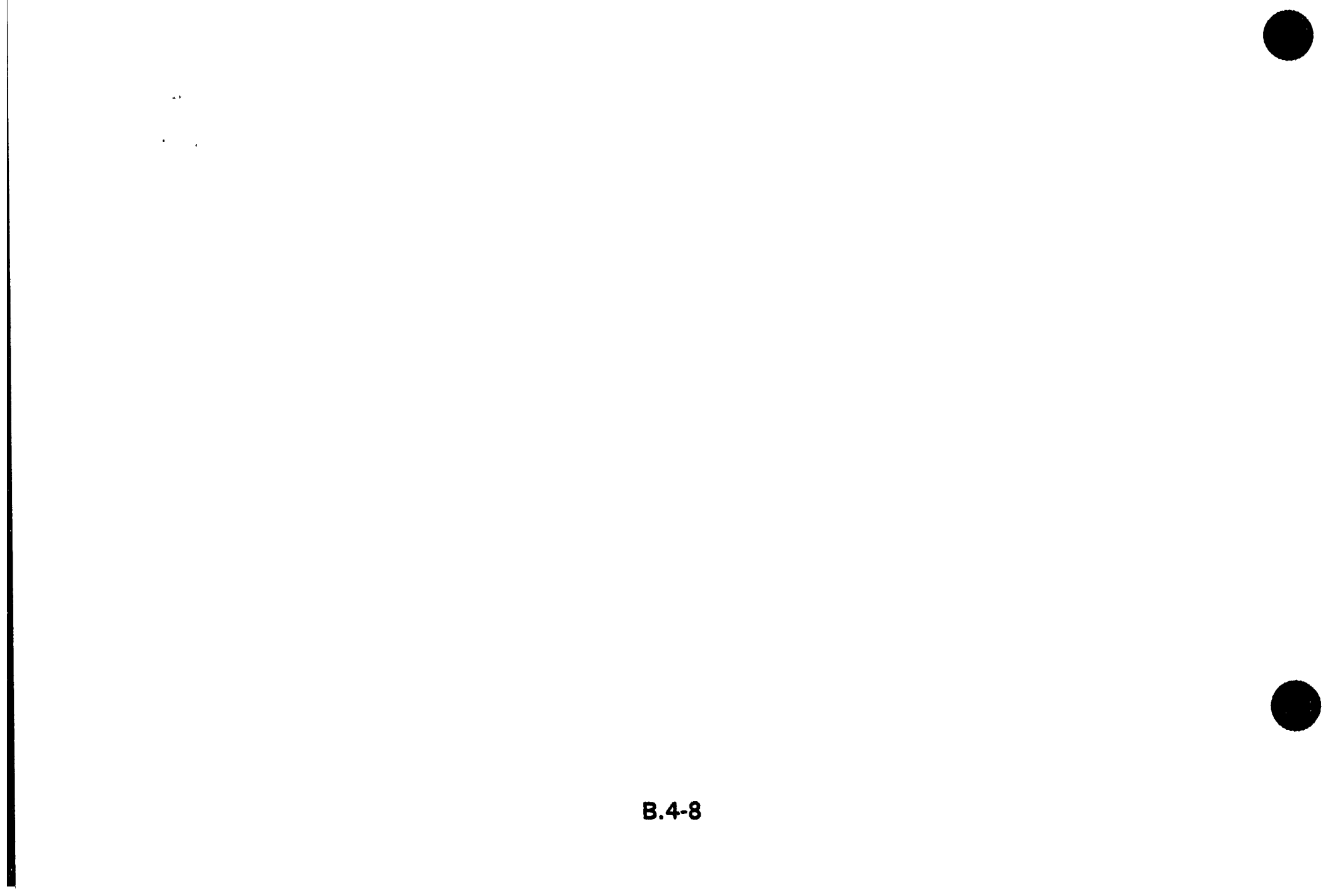
WHC-EP-0696

\section{APPENDIX 8.5}

PHYSICAL CONTENTS DESCRIPTION FOR BUILDING 325 PNL 55-GALLON DRUMS CONTAINING TRU WASTE SORTED BY DATE, PRIMARY WASTE TYPE, AND STORAGE FACILITY 


\section{WHC-EP-0696}

This subappendix describes the physical contents of the 55-gal drums of TRU waste generated by Building 325 PNL. Prior to 1978, physical contents were not required on the burial records, so a great many of the early records list the contents of the drum as "Miscellaneous."

The printout for this computer run sorts the contents data by storage facility, date and primary waste type. The introduction to Appendix B contains a table of waste codes and their meanings. 


\section{WHC-EP-0696}

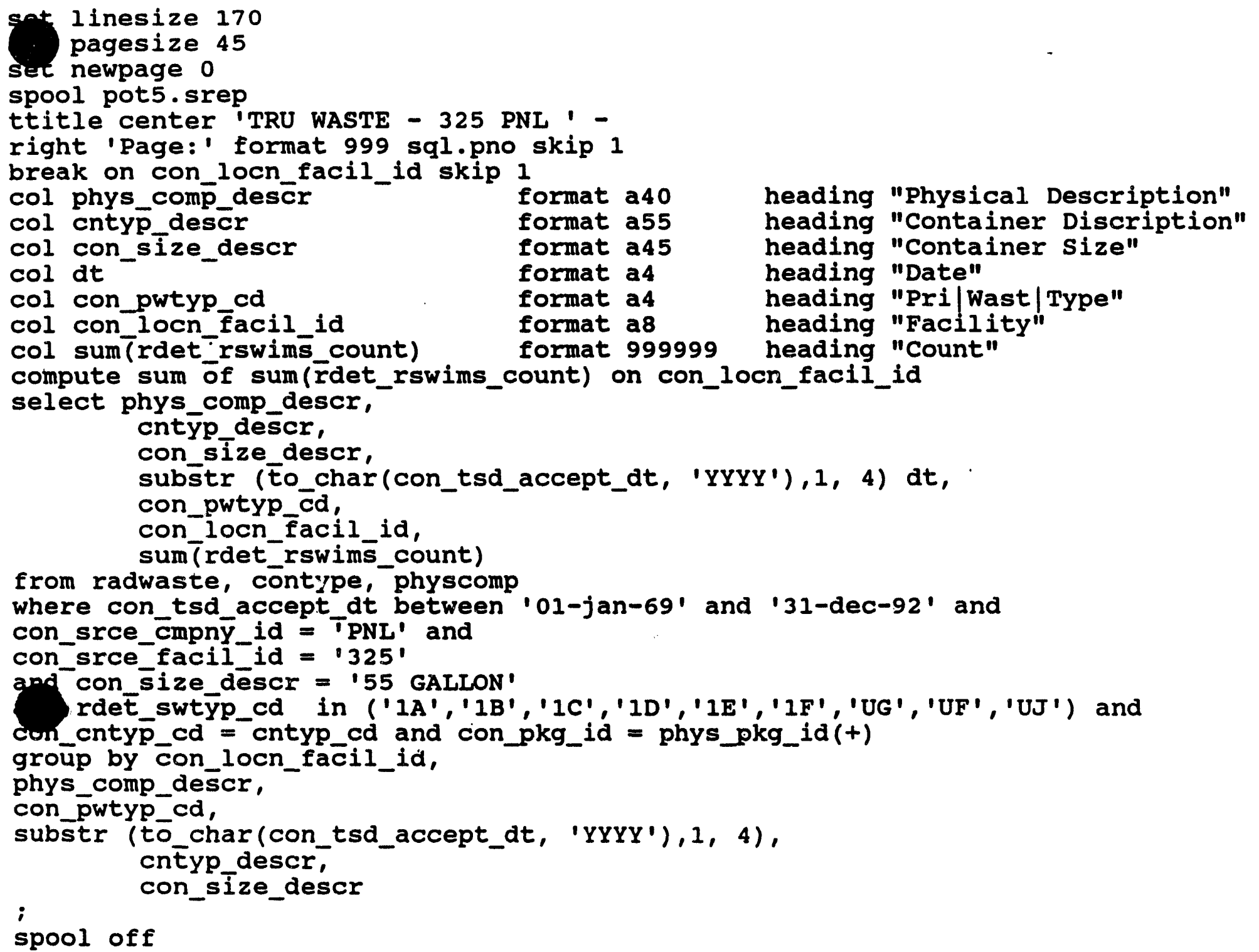




\section{ANIMAL WASTE}

MISCELLAMEOUS/UNKMOWN/OTHER MI SCELLANEOUS/UNKNOWN/OTHER MISCELLANEOUS/UNKNOWN/OTHER MISCELLANEOUS/UNKNOWN/OTHER

DIRT/SOIL/DIATOMACEOUS EARTH MI SCELLANEOUS/UNKNOWN/OTHER MISCELLANEOUS/UNKNOWN/OTHER MISCELLAMEOUS/UNKMOUM/OTHER

\section{w \\ cis}

ABSORBANT/KITY LTR/VERMICULITE ABSORBAMT/KITY LTR/VERMICULITE ANIMAL UASTE

BRICK/FIREBRICK

CLOTH/RAGS/MYLOW

CLOTH/RAGS/WYLON

CLOTH/RAGS/MYLON

CLOTH/RAGS/NYLON

COTTON/KOTEX

DIRT/SOIL/DIATOMACEOUS EARTH

FLOOR SUEEPS

GLASS

GLASS

GLASS

GLASS

INSULATION NON-ASBESTOS

LEAD

METAL/IRON/GALVANIZED/SHEET
METAL DRUAS, BARRELS, KEGS METAL DRUHS, BARRELS, KEGS METAL DRUMS, BARRELS, KEGS METAL DRUHS, BARRELS, KEGS METAL DRUAS, BARRELS, KEGS

METAL DRUMS, BARRELS, KEGS METAL DRLMS, BARRELS, KEGS METAL DRUMS, BARRELS, KEGS METAL DRUNS, BARRELS, KEGS METAL DRUMS, BARRELS, KEGS METAL DRUMS, BARRELS, KEGS METAL DRUAS, BARRELS, KEGS METAL DRUAS, BARRELS, KEGS METAL DRUNS, BARRELS, KEGS METAL DRLMIS, BARRELS, KEGS METAL DRUMS, BARRELS, KEGS

METAL DRUMS, BARRELS, KEGS METAL DRUAS, BARRELS, KEGS METAL DRUAS, BARRELS, KEGS METAL DRUAS, BARRELS, KEGS METAL DRUAS, BARRELS, KEGS METAL DRUMS, BARRELS, KEGS METAL DRUMS, BARRELS, KEGS METAL DRUMS, BARRELS, KEGS METAL DRUMS, BARRELS, KEGS METAL DRUAS, BARRELS, KEGS METAL DRUNS, BARRELS, KEGS METAL DRUMS, BARRELS, KEGS METAL DRUAS, BARRELS, KEGS METAL DRUAS, BARRELS, KEGS METAL DRUMS, BARRELS, KEGS METAL DRUAS, BARRELS, KEGS METAL DRUNS, BARRELS, KEGS METAL DRUAS, BARRELS, KEGS
55 Gallon

55 Gallon

55 Gallow

55 GaLLON

55 Gallon

55 GALLON

55 eallon

55 EALLON

55 GALLON

55 callon

55 GALLOM

55 GALLON

55 Gallow

55 GALLOM

55 GALLON

55 GALLON

55 GALLON

55 GALLON

55 GALLON

55 Gallon

55 GALLON

55 GALLON

55 GALLOM

55 Gallow

55 GALLON

55 GALLON

55 GALLOW

55 GALLON

55 GaLlon

55 GALLON

55 Gallon

55 GALLON

55 GALLON

55 GALLOW
Wast Date Type Facility Count

$\begin{array}{llr}1973 \text { R } & 21843 A & 7 \\ 1970 \text { R } & & 35 \\ 1971 \text { R } & & 22 \\ 1973 \text { R } & & 7 \\ 1975 \text { R } & & 1 \\ & & 1\end{array}$

1970

$1969 R$

$1971 R$

$1972 R$

$1970 R$

$1972 R$

$1973 R$

$1974 R$

1975 R

$1976 R$

$1977 R$

8

115

305

524

65

52

20

53

338

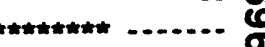

sum

1307

$1985 \mathrm{R}$

$1987 R$

$1980 R$

$1987 R$

$1978 R$

$1979 \mathrm{R}$

$1985 R$

$1987 R$

$1979 R$

$1979 R$

$1987 R$

$1979 R$

$1980 R$

$1985 R$

$1987 R$

$1987 \mathrm{R}$

$1987 R$

$1979 R$

21844

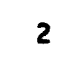

2 
Wast

Container Size Date Iype facility count

Physical Description

METAL/IRON/GaL VANIZED/SHEET METAL/IRON/GALVANIZED/SHEET METAL/IRON/GALVANIZED/SHEET MISCELLANEOUS/UMKNOWM/OTHER MISCELLANEOUS/UNKNOUN/OTHER PAPER/CARDBOARO PAPER/CAROBOARD PAPER/CARDBOARD PAPER/CARDBOARO PLASTIC/POL YURATHANE PLASTIC/POL YURATHANE PLASTIC/POL YURATHANE PLASTIC/POL YURATHANE PLASTIC/POLYURATHANE RUBBER

RUGBER

DRRUBBER

Ü RUBBER

Ó STAINLESS STEEL WOOO/LLMBER/PLYWOOO

ABSORBANT/KITY LTR/VERMICULITE ABSORBANT/KITY LTR/VERMICULITE ABSORBANT/KITY LTR/VERMICULITE ABSORBANT/KITY LTR/VERMICULITE ABSORBANT/KITY LTR/VERMICULITE ABSORBENT/KITY LTR/VERMICULITE ASBESTOS

ASBESTOS

CEMENT

CERAMICS

CERAMICS

CERAMICS

CERAMICS

CHARCOAL

CLOTH/RAGS/HYLOW
Container Discription

METAL DRUMS, BarRELS, KEGS

METAL DRUUS, BARRELS, KEGS

METAL DRUMS, BARRELS, KEGS

METAL DRUMS, BaRRELS, KEGS

METAL DRUMS, BARRELS, KEGS

METAL DRUWS, BARRELS, KEGS

METAL DRUMS, BARRELS, KEGS

METAL DRUMS, BARRELS, KEGS

METAL DRLUS, BARRELS, KEGS

METAL DRUAMS, BARRELS, KEGS

METAL DRLUMS, BARRELS, KEGS

METAL DRUAS, BARRELS, KEGS

METAL DRUMS, BARRELS, KEGS

METAL DRLWS, BARRELS, KEGS

METAL DRLUAS, BARRELS, KEGS

METAL DRLUS, BARRELS, KEGS

METAL DRUNS, BARRELS, KEGS

METAL DRUWS, BARRELS, KEGS

METAL DRLMS, BARRELS, KEGS

METAL DRUMS, BARRELS, KEGS

METAL DRUMS, BARRELS, KEGS

METAL ORUWS, BARRELS, KEGS

METAL DRUNS, BARRELS, KEGS METAL DRLUMS, BARRELS, KEGS METAL DRLUS, BARRELS, KEGS METAL DRLUS, BARRELS, KEGS METAL DRUMS, BARRELS, KEGS METAL DRUWS, BARRELS, KEGS METAL DRLUES, BARRELS, KEGS METAL DRUMS, BARRELS, KEGS METAL DRUWS, BARRELS, KEGS METAL DRUWS, BARRELS, KEGS METAL DRUUS, BARRELS, KEGS METAL DRUMS, BARRELS, KEGS METAL DRLWS, BARRELS, KEGS METAL DRLMS, BaRRELS, KEGS mETAL DRUUS, BARRELS, KEGS

55 GaLLON

55 GALLON

55 GRloN

55 GALLON

55 GALLON

55 GALLON

55 Gallow

55 GALLON

55 Gallow

55 GRLLON

55 GALLOW

55 Gallow

55 GALLON

55 GRLLON

55 GALLON

55 GALLON

55 GALLON

55 GALLOW

55 GALLON

55 GALLON

55 Gallow

55 GaLLON

55 GALLON

55 GALLON

55 GALLON

55 GALLON

55 GALLON

55 GALLON

55 GALLON

55 GALLON

55 GALLON

55 GALLOW

55 GALLON

55 GALLON

55 GALLON

55 GALLON

55 GALLOW

.... ..... .................

\begin{tabular}{|c|c|c|}
\hline $1980 R$ & $2181 \times 4 C$ & 17 \\
\hline $1985 R$ & & 3 \\
\hline 1987 R & & 1 \\
\hline 1978 R & & 1 \\
\hline 1979 : & & 6 \\
\hline 1978 R & & 1 \\
\hline 1979 R & & 162 \\
\hline $1980 R$ & & 17 \\
\hline $1985 R$ & & 3 \\
\hline 1978 R & & 1 \\
\hline 1979 R & & 162 \\
\hline $1980 R$ & & 17 \\
\hline $1985 R$ & & 3 \\
\hline $1987 R$ & & $2<$ \\
\hline 1978 R & & $1 \sum$ \\
\hline 1979 R & & 157 ? \\
\hline 1985 & & 2 䎡 \\
\hline $1987 R$ & & 20 \\
\hline 1987 & & \\
\hline 1979 & & $5 \%$ \\
\hline $1978 R$ & & 38 \\
\hline \multirow[t]{3}{*}{$1979 R$} & & 82 \\
\hline & 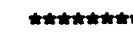 & $\cdots$ \\
\hline & sin & 958 \\
\hline
\end{tabular}

$1991 \mathrm{n}$

2241

1987 R

$1989 R$

$1990 R$

$1991 R$

$1992 R$

$1991 R$

$1992 R$

$1992 R$

$1987 R$

$1989 R$

$1991 R$

$1992 \mathrm{R}$

1990 R

1987 R

8


Physical Description

\section{CLOTH/RAGS/HYLOA}

CLOTH/RAGS/NYLON

CLOTH/RAGS/WYLOW

CLOTH/RAGS/NYLON

COMUEB PADS

COTTON/KOTEX

DIRT/SOIL/DIATOMACEOUS EARTA

EQUIPMENT

FLOOR SWEEPS

FLOOR SUEEPS

FLOOR SLEEPS

FLOOR SLEEPS

FOAN/STYROFOAY

GLASS

GLASS

GLASS

m GLASS

on GLASS

ógLASS

GLASS

INSULATION NON-ASBESTOS

IHSULATION NON-ASBESTOS

LEAD

LEAO

LEATHER

LEATHER

LIOUID

LIOUID

METAL/IRON/GALVAMIZED/SHEET

METAL/IRON/GALVANIZED/SHEET

METAL/IROW/GALVANIZED/SHEET

METAL/IROW/GALVANIZED/SHEET

METAL/IROW/GALVANIZED/SHEET

METAL/IROM/GALVANIZED/SHEET

MISCELLANEOUS/UMKNOWN/OTHER

OXIDES/PONERS

PAPER/CARDBOARD

PAPER/CARDBOARD

PAPER/CARDBOARD

PAPER/CARDBOARD
Container Discription

METAL DRUMS, BARRELS, KEGS

METAL DRLMS, BARRELS, KEGS

METAL DRLAS, BARRELS, KEGS

METAL DRUAS, BARRELS, KEGS

METAL DRLMS, BARRELS, KEGS

METAL DRUANS, BARRELS, KEGS

METAL DRLAHS, BARRELS, KEGS

METAL DRLULS, BARRELS, KEGS

METAL DRUMS, BARRELS, KEGS

METAL DRUNE, BARRELS, KEGS

METAL DRUMS, BARRELS, KEGS

METAL DRUAS, BARRELS, KEGS

METAL DRUAS, BARRELS, KEGS

METAL DRUNLS, BARRELS, KEGS

METAL DRUAS, BARRELS, KEGS

METAL DRLHS, BARRELS, KEGS

METAL DRLUHS, BARRELS, KEGS

METAL DRUAS, BARRELS, KEGS

METAL DRUNS, BARRELS, KEGS

METAL DRUMS, BARRELS, KEGS

METAL DRUHS, BARRELS, KEGS

METAL DRUMS, BARRELS, KEGS

METAL DRUAS, BARRELS, KEGS

METAL DRLAS, BARRELS, KEGS

METAL DRUMLS, BARRELS, KEGS

METAL DRLAS, BARRELS, KEGS

METAL DRLAS, BARRELS, KEGS

METAL DRUMS, BARRELS, KEGS

METAL DRLUIS, BARRELS, KEGS

METAL DRUMS, BARRELS, KEGS

METAL DRUAL, BARRELS, KEGS

METAL DRLWS, BARRELS, KEGS

METAL DRUNS, BARRELS, KEGS

METAL DRLUAS, BARRELS, KEGS

METAL DRUAS, BARRELS, KEGS

METAL DRLWS, BARRELS, KEGS

METAL DRLAS, BARRELS, KEGS

METAL DRUMS, BARRELS, KEGS

METAL DRLULS, BARRELS, KEGS

METAL DRLAS, BARRELS, KEGS
Pri

Hast

Container Size

Date Type Facility Count

55 GALLON

55 GALLOM

55 GalloN

55 Gallon

55 Gallow

55 GALLON

55 GALLON

55 GALLON

55 GALLON

55 GALLON

55 Gallon

55 callon

55 GALLON

55 GALLON

55 Gallon

55 GallOW

55 Gallor

55 GALLON

55 Gallow

55 GAlLON

55 GALLON

55 GALLON

55 GALLON

55 GALLON

55 GALLON

55 GALLON

55 GALLON

55 GALLON

55 GALLON

55 GALLON

55 GALLON

55 GALLON

55 Gallon

55 GALLON

55 GALLON

55 GAlloN

55 GALLON

55 GALLON

55 GALOM

55 GALLO

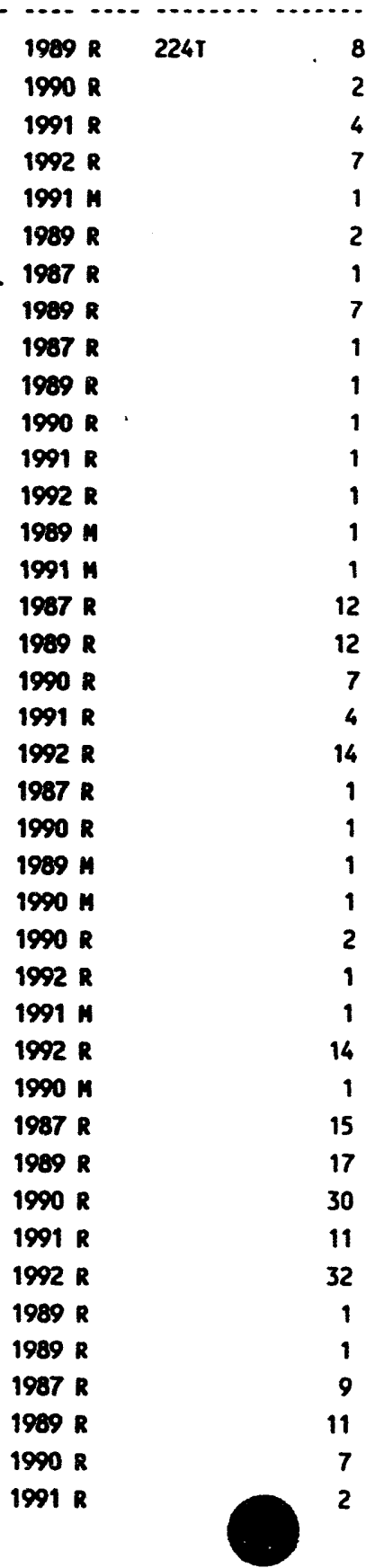


Pri

Wast

Container Size

Date Type Facility count

Physical Description

Container Discription

55 gallon

55 gallon

55 Gallon

55 GALLON

55 GALLON

55 GALLON

55 GALLOH

55 GALLON

55 GALLON

55 GALLON

55 GALLON

55 GALLON

55 GALLON

55 Gallon

55 Gallon

55 Gallon

55 GALLON

55 Gallon

55 GALLON

55 GALLON

55 GalLON

55 GALLOW

55 GALLON

55 GAllon

55 GALLON

55 Gallon

55 GALLON

55 GALLON

55 GALLON

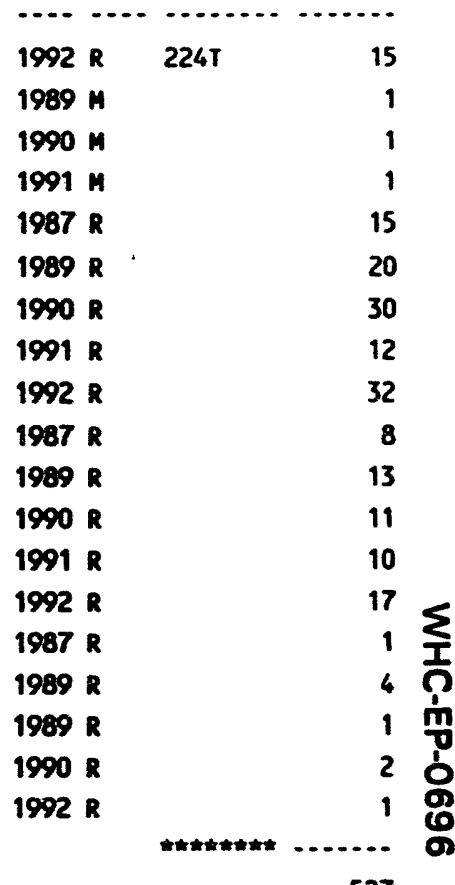

sum

527

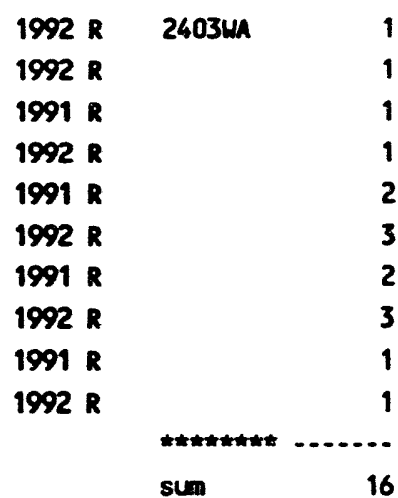




\section{WHC-EP-0696}

This page intentionally left blank.

\section{B.5-8}


WHC-EP-0696

APPENDIX B.6

PHYSICAL CONTENTS DESCRIPTION FOR BUILDING 325 PNL TRU WASTE CONTAINERS OTHER THAN

55-GALLON DRUMS SORTED BY DATE,

PRIMARY WASTE TYPE, AND

STORAGE FACILITY

\section{B.6-1}




\section{WHC-EP-0696}

This subappendix describes the physical contents of the TRU waste containers other than 55-gal drums generated at Building 325 by PNL. Prior to 1978, physical contents were not required on the burial records, so a great many of the early records list the contents of the drum as "Miscellaneous."

The printout for this computer run sorts the contents data by storage facility, date and primary waste type. The introduction to Appendix B contains a table of waste codes and their meanings. 
et linesize 170

pagesize 45

newpage 0

spool pot6.srep

ttitle center 'TRU WASTE - 325 PNL' -

right 'Page:' format 999 sql.pno skip 1

break on con_locn_facil_id skip 1

col phys_comp_descr

col con_size_descr

format a 40

format a 47

col cntȳp_des̄cr

format a 45

col dt

col con_pwtyp_cd

col con locn facil id

col sum(rdet_rswims_count)

col rdet_swtȳp_cd

format a 4

format a 4

format a8

heading "Physical Description"

heading "Container Description"

heading "Container Size"

heading "Date"

heading "Pri/Wast/Type"

heading "Facility"

format 999999

heading "Count"

format a4

compute sum of sum(rdet_rswims_count) on con_locn_facil_id

select phys_comp_descr,

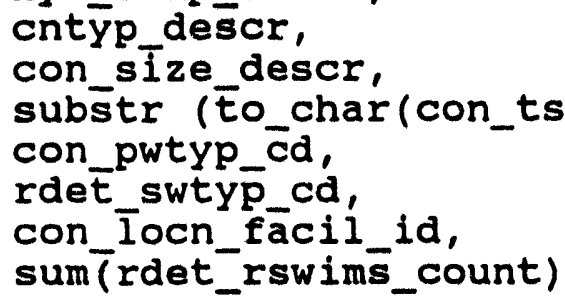

from radwaste, contype, physcomp

where con_tsd_accept_dt between '01-jan-69' and '31-dec-92' and

con_srce_cmpny $i d=\bar{T}_{\mathrm{PNL}}$ ' and

srcé faciI id $=1325^{\prime}$

con size descr 4155 GALLON'

and rdet_swtȳp_cd in ('IA', 'IB', 'IC','ID', 'IE', 'IF', 'UG', 'UF', 'UJ') and con_cntyp_cd $=$ cntyp_cd and con_pkg_id = phys_pkg_id(t)

groūp by con_locn_facil_id,

phys_comp_descr,

con_pwtyp_cd,

rde $\bar{t}$ swty $\bar{p} \_c d$,

subsĒr (to_char(con_tsd_accept_dt, 'YYYY'), 1, 4),

cnËyp_descr,

con_size_descr

;

spool off 
-.........................

\section{CLOTH/RAGS/NYLON}

GLASS

METAL/IRON/GALYANIZED/SHEET

PAPER/CARDBOARD

PLASTIC/POL YURATHANE

RUBBER

WOOD/LUMBER/PL YWOOD

44 rows selected.
METAL BOXES, CARTONS, CASES

METAL BOXES, Cartons, Cases

METAL BOXEs, cartows, cases

METAL BOXES, CARTOWS, CASES

METAL BOXEs, Cartons, Cases

metal bOXEs, cartons, Cases

metal boXes, cartons, cases
$5.6 * 6.5 * 9.3$

$5.6 * 6.5 * 9.3$

$5.6 * 6.5 * 9.3$

$5.6 * 6.5 * 9.3$

$5.6 * 6.5 * 9.3$

$5.6 * 6.5 * 9.3$

$5.6 * 6.5 * 9.3$ t*t=

\begin{tabular}{|c|c|c|}
\hline $1992 R$ & $1 A$ & 240ZUE \\
\hline $1992 R$ & 1A & \\
\hline $1992 \mathrm{R}$ & $1 A$ & \\
\hline $1992 R$ & $1 A$ & \\
\hline $1992 R$ & $1 \mathrm{~A}$ & \\
\hline $1992 \mathrm{R}$ & $1 A$ & \\
\hline 19928 & $1 A$ & \\
\hline
\end{tabular}


WHC-EP-0696

This page intentionally left blank.

B. 6-6 
WHC-EP-0696

APPENDIX $\mathrm{B.7}$

\section{HAZARDOUS CONSTITUENTS OF 55-GALLON}

DRUMS CONTAINING TRU WASTE

B.7-1 
The presence of hazardous constituents in a radioactive waste drum designates the waste as "Mixed" (primary waste code "M"). In this subappendix the hazardous contents of 55-gal drums generated at Building 325 PNL are sorted by storage facility.

Information concerning the hazardous constituents of waste containers was not required prior to 1986. During the R-SWIMS data reentry program in the mid-1980's an attempt was made to add any available information on the hazardous materials present, however, this information was limited. 


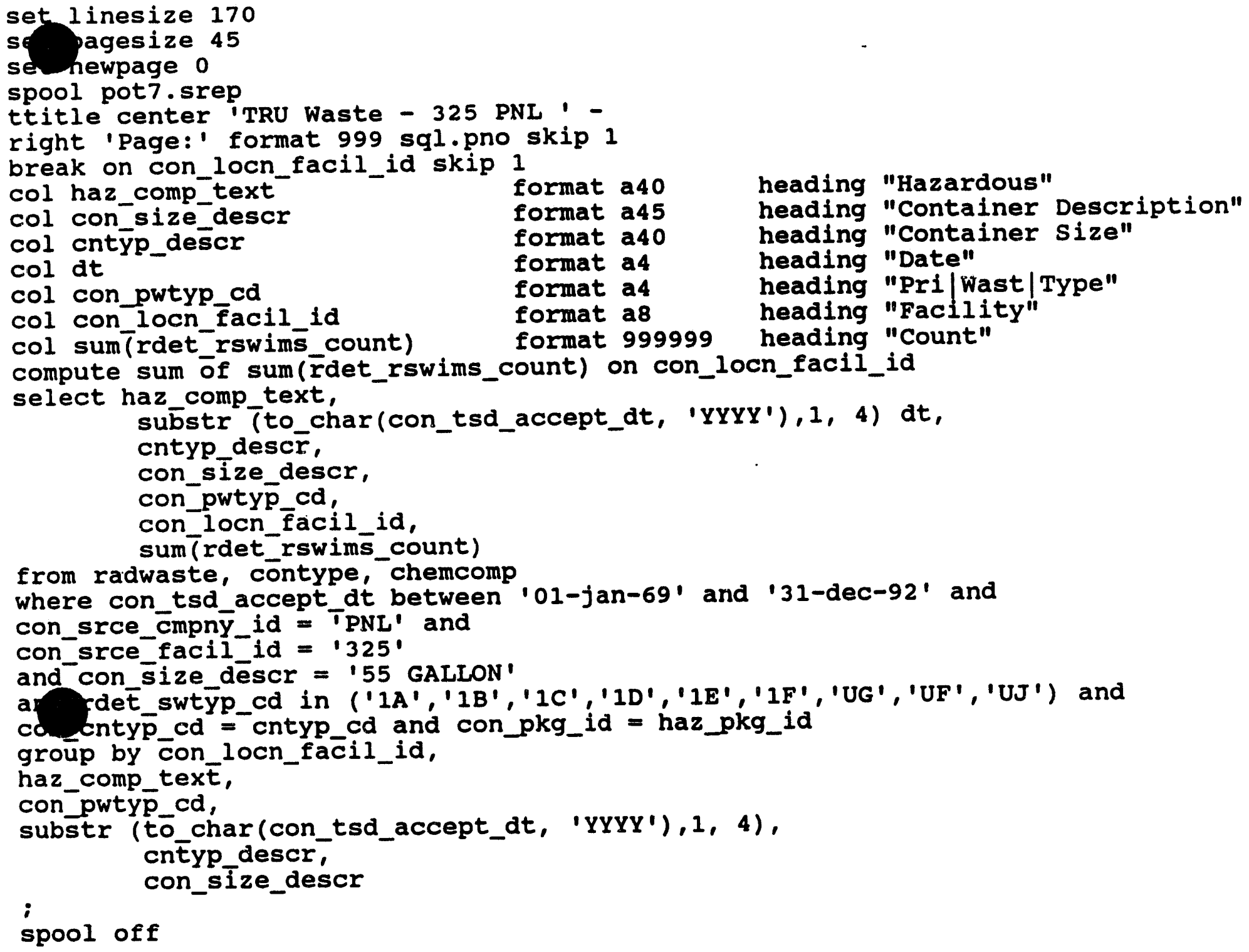




\begin{tabular}{|c|c|c|}
\hline Hazardous & Date Container Size & Container \\
\hline ASBESTIOS & 1991 METAL DRUMS, BARRELS, KEGS & 55 GALLON \\
\hline ASBESTOS & 1992 METAL DRUMS, BARRELS, KEGS & 55 GALLON \\
\hline LEAD & 1989 METAL DRLMS, BARRELS, KEGS & 55 GALLON \\
\hline LEAD & 1990 METAL DRUMS, BARRELS, KEGS & 55 GALLON \\
\hline MERCURY & 1989 METAL DRUHS, BARRELS, KEGS & 55 GALLON \\
\hline NITRIC ACID & 1991 METAL DRLMS, BARRELS, KEGS & 55 GALLON \\
\hline
\end{tabular}

Pri

Wast

Type facility count

.... .................

$\begin{array}{llr}R & 2241 & 1 \\ R & & 4 \\ M & & 1 \\ M & & 1 \\ M & & 1 \\ M & & 1 \\ & & \\ & \text { Sum } & 9\end{array}$

6 rows selected. 
WHC-EP-0696

APPENDIX 8.8

HAZARDOUS CONSTITUENTS OF TRU WASTE CONTAINERS OTHER THAN 55-GALLON DRUMS

B.8-1 


\section{WHC-EP-0696}

The presence of hazardous constituents in a radioactive waste drum designates the waste as "Mixed" (primary waste code "M"). No hazardous constituents are listed in containers other than 55-gal drums generated at Building 325 PNL; therefore this run did not reveal any hazardous information. 


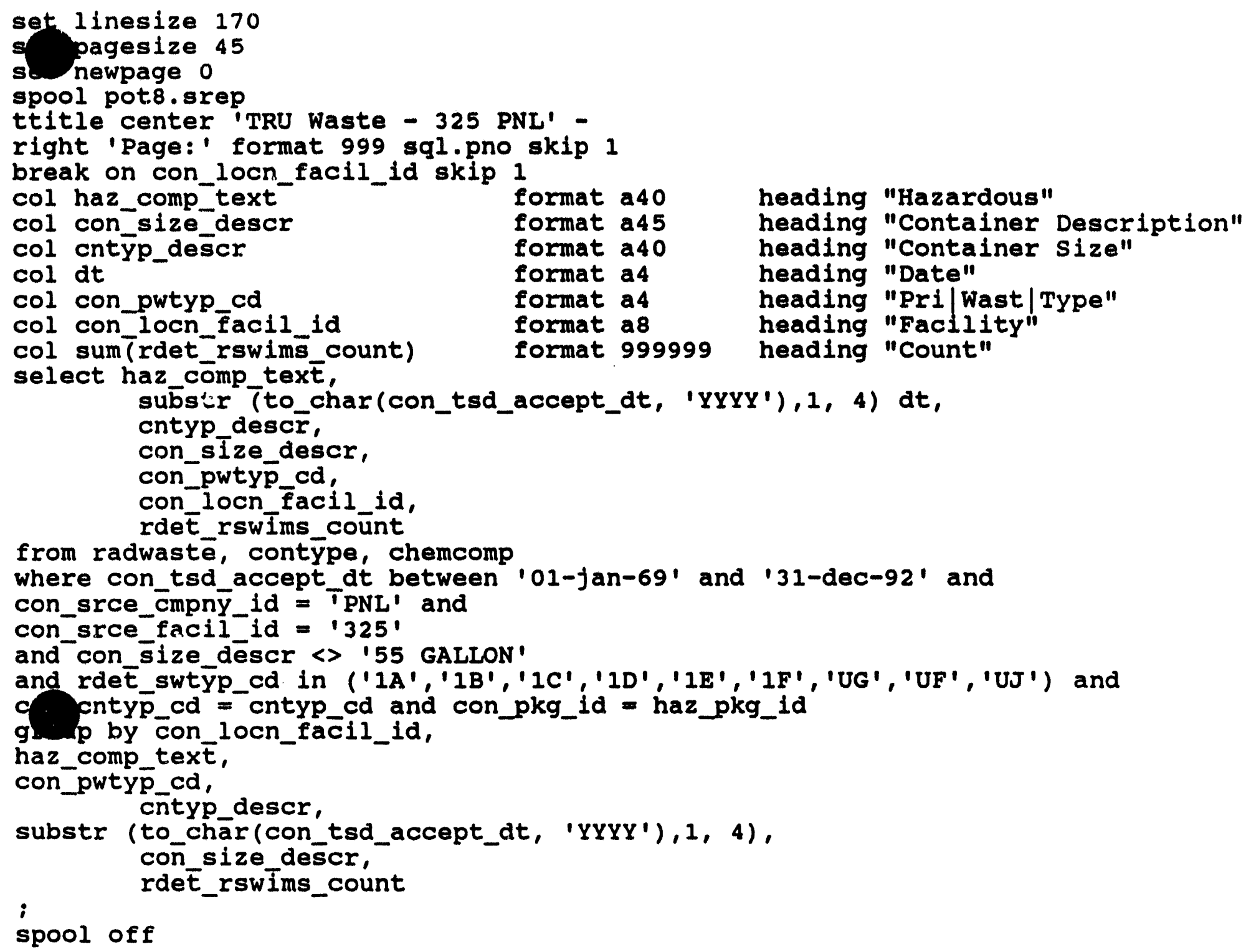


Pri

Wast

Type facility ROET_RSUInS_COWT Date Container size

Container Description

1969 tRUCKS, FLATBEDS, COMPACTOR, LOADLUGGER UMKMOM

M 218 ars 
WHC-EP-0696

APPENDIX 8.8

ISOTOPES LISTED IN BS-CALLON DAUMS CONTAINING TRU WASTE8

B.9-1 
This subappendix contains a listing of the SWITS isotope information for TAU waste from Bullding 325 PNL stored in 58-gal drums. The isotope field in SWITS contains several of the following types of information:

- Speciflc isotope (e.9.. Am-241, Np-237, U-233, Pu-238)

- Generic isotope llatings (0.9.. Pu, uranium-enriched, uranium-depleted)

- Plutonium-239 fiesile gram equivalents (Pu239 FSL GR equiv)

- Total - alpha

- Total bota/gamma

- $\mathrm{PE}-\mathrm{Cl}$ (plutonium equivalent curies).

This computer run contains the isotope field listing along with the number of TRU waste drums with that listing. The run is sorted by storage facility and year. 


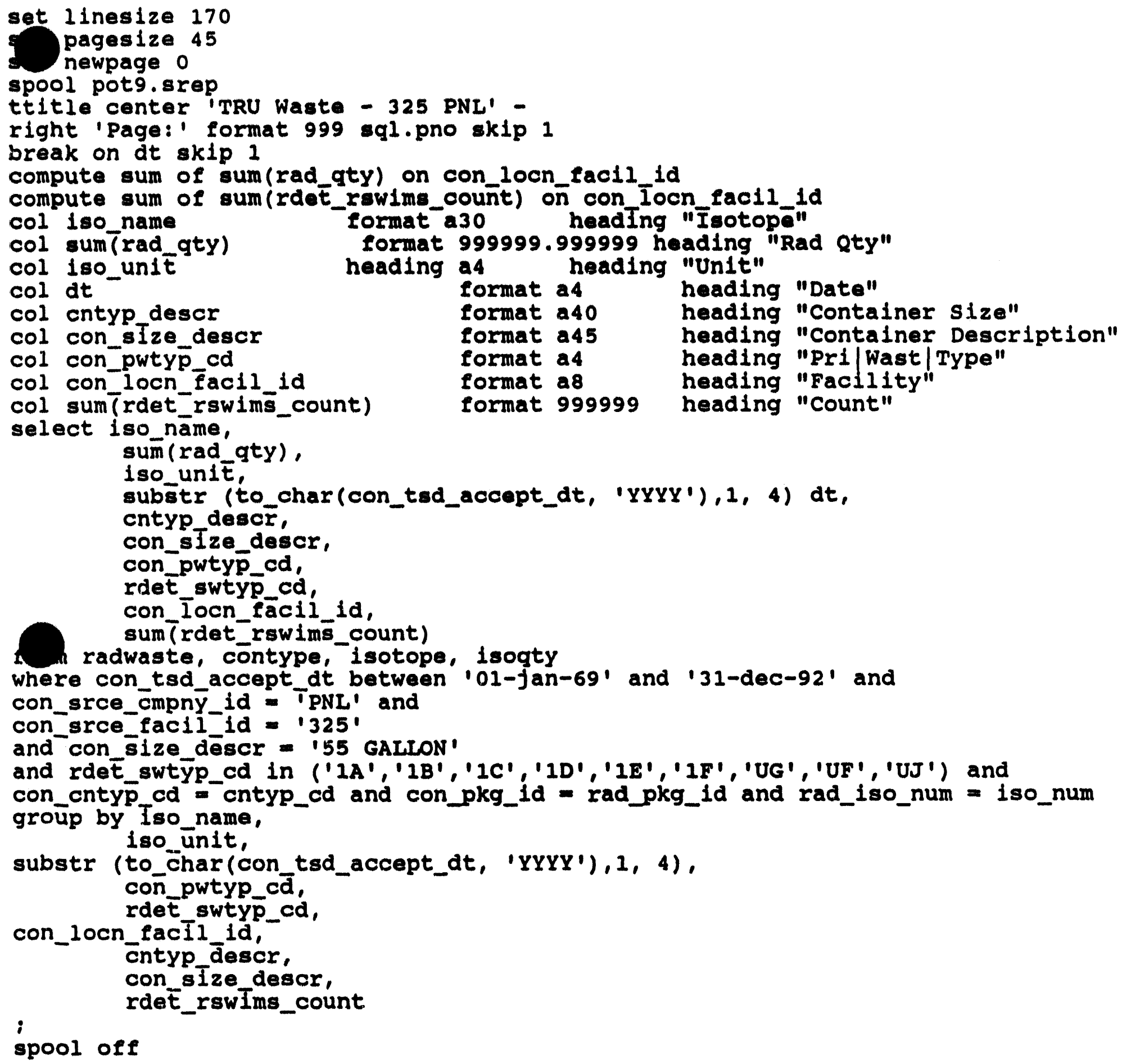

spool off 


\section{1 sotope}

AM-241

PE-CI

PM- 147

PU

Pu

PU

$\infty$
0
1
0

PU

PU

PU
Rad aty Un Date Container size

2.200000 EN 1992 METAL BOKES, CARTOWS, CASES $.000000 \mathrm{CI}$ metal BoXes, Cartous, cases

$$
\text { thet. }
$$

sin

8572.500000 CI 1969 FIBERBOARO/PLASTIC BOXES, CARTOUS, CASES $.100000 \mathrm{GH}$ TRUCKS, FLATBEDS, COMPACTOR, LOADLUGGER sinte

.002300 en 1970 metal Doums, BARRELS, KEGS

the

$\sin$

19.050000 EN 1969 FIBERBOARD/PLASTIC BOXKES, CARTOUS, CASES \#+4.

sin

5.000000 EI 1970 FIBERBOARO/PLASTIC BOXES, CARTOUS, CASES ntat

sin

1.000000 en 1974 CONCRETE BOXES

$3.000000 \mathrm{Gr}$ COUCRETE BOXES

ous

.000100 en 1975 CONCRETE BOXKES

๓tat

sum

$1.000000 \mathrm{GH} 1976$ METAL BOXES, cartous, cases

that

$\sin$

1.090000 en 1978 8URLAP, CLOTH, PAPER OR PLASTIC BAOS

$8.720000 \mathrm{EN}$ MISCELLAMEOUS SCRAP

$1.090000 \mathrm{es}$ SELF COMTAIMED, EOUIPMENT

$1.090000 \mathrm{~cm}$ TAMKS, PORTABLE
Container Description

Type facility count

$5.6 * 6.5 * 9.3$

$5.6 * 6.5 * 9.3$

R 240215

R 24021E

unkenom

unavon

30 callow

uncionat

uncuorim

$4 * 4 * 8$

$4 * 7 \div 9$

$3 * 3 * 3$

$2 * 2 * 6$

$\begin{array}{rrr}R & 218 \times 48 & 33 \\ n & 218468 & 1 \\ & & \ldots . . . \\ & & 34\end{array}$

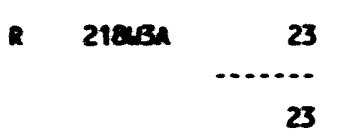

R 21846833

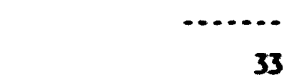

R 218me

33

33

2 218468

R $218 \mathrm{k}$

3

R $218 \times 48$

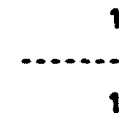

R $218 \times 48$ 1
$\cdots$
$\cdots$

Unxuan

unirnoun

unxioum

unkoun 
Pri

Hest

$5.6 * 6.5 * 9.3$

$5.6 * 6.5 * 9.3$

$5.6 * 6.5 * 9.3$

$5.6 * 6.5 * 9.3$

$5.6 * 6.5 * 9.3$

$1.140000 \mathrm{CH}$ METAL BOXES, CARTOUS, CASES

METAL BOXES, CARTONS, CASES

sum

4.000000 CI 1969 TRUCKS, FLATBEDS, COAPACTOR, LOADLUGGER matto

sum

.115000 CI 1970 METAL DRUMS, BARRELS, KEGS ․t.t.

30 GALLON

TOTAL BETA/GAMMA

TOTAL BETA/GAMAA

TOTAL BETA/GAMMA

TOTAL BETA/GAMMA

TOTAL BETA/GAMHA

TOTAL BETA/GAMAA

TOTAL BETA/GAMYA

TOTAL BETA/GAMA

TOTAL BETA/GAHA

IOTAL BETA/GAIAIA

TOTAL BETA/GAMHA

TOTAL BETA/GAMMA

TOTAL BETA/GAMAR

TOTAL BETA/gamara

TOTAL BETA/GAMMA

TOTAL BETA/GAMMA

TOTAL BETA/GAMA

TOTAL BETA/GAMM

TOTAL BETA/GAMMA
84

.002352 CI 1969 FIBERBOARD/PLASTIC BOXES, CARTOWS, CASES $8580.863171 \mathrm{CI}$ FIBERBOARD/PLASTIC BOXES, CARTOUS, CASES

$1.600000 \mathrm{Cl}$

$.007975 \mathrm{Cl}$

$.331199 \mathrm{Cl}$

$.321308 \mathrm{CI}$

$1.432535 \mathrm{Cl}$

$.018710 \mathrm{Cl}$

$.033265 \mathrm{Cl}$

$.041665 \mathrm{Cl}$

$.007780 \mathrm{cl}$

$.036365 \mathrm{CI}$

$.149785 \mathrm{CI}$ HEPA FILTERS

METAL ORUMS, BARRELS, KEGS

MISCELLAMEOUS SCRAP

SELF CONTAINED, EQUIPMEMT

WOODEN BOXES, CARTOUS, CASES

WOOOEN BOXES, CARTONS, CASES

WOOOEN BOXES, CARTONS, CASES

WOODEN BOXES, CARTOUS, CASES

WOOOEN BOXES, CARTOUS, CASES

WOODEN BOXES, CARTOUS, CASES

sin

.022825 CI 1970 BURLAP, CLOTH, PAPER OR PLASTIC BAGS

$.441885 \mathrm{Cl}$

$.031450 \mathrm{cl}$

$.014040 \mathrm{Cl}$

$.025000 \mathrm{CI}$

$.050000 \mathrm{CI}$
FIBERBOARD/PLASTIC BOXES, CARTONS, CASES MEPA FILTERS

METAL DRLARS, BARRELS, KEGS

METAL DRLAH, BARRELS, KEGS

TRUCKS, FLATBEDS, COAPACTOR, LOADLUGGER
2 CU FT

UnKmow

UNKNOW

30 GALLON

UnKMONM

Unxwown

$1 * 3 * 3$

$2 * 2 * 4$

2*4*10

$3 * 3 * 3$

4*5*6

UnKHown

UnKNOW

UNKNOAN

UnKMOWm

30 GALLON

5 GALLON

UNKMOM
11

\begin{tabular}{|c|c|}
\hline R & 24021E \\
\hline $\mathbf{R}$ & 240ZUE \\
\hline R & 2402UE \\
\hline $\mathbf{R}$ & 240ZUE \\
\hline R & $24021 \mathrm{CE}$ \\
\hline
\end{tabular}

M 218448

1

1

R 21843A

3

218ukB

21844B

218448

2184kB

218448

218448

218448

218448

218448

218448

218448

218448

21844B

6

2017

27

5

23

16

1

1

10

2136

21844B

21844B

21844B

218448

218448

21844B 


Isotope Rad oty Un Date Container Size

$$
\text { thim }
$$

3.000000 CI 1974 CONCRETE BOXES CONCRETE BOXES

TOTAL BETA/GAMMA

TOTAL BETA/GAMHA

TOTAL BETA/GAMAM

Wrotal beta/gama

Ótotal Beta/gama

total BETA/gama

TOTAL BETA/GAMAM

TOTAL BETA/GAMMA

$u-233$

URANIUM-DEPLETED URANIUM-DEPLETED

URANIUN-DEPLETED

$\sin$

.007000 CI 1975 CONCRETE BOXES

thtt

8

$.010000 \mathrm{CI} 1976$ METAL BOXES, CARTONS, CASES

trat:

sum

$.001000 \mathrm{CI} 1978$ BURLAP, ClOTH, PAPER OR PLASTIC baGS

$.008000 \mathrm{Cl}$ MISCELLAMEOUS SCRAP

$.001000 \mathrm{CI}$ SELF CONTAINED, ECUIPMENT

$.001000 \mathrm{Cl}$ TAMKS, PORTABLE

that

$\sin$

atot:

$\sin$

55.000000 GI 1970 FIBERBOARD/PLASTIC BOXES, CARTOWS, CASES *t**

sum

10593.018600 GH 1969 FIBER8OARO/PLASTIC 8OXES, CARTONS, CASES $39.965180 \mathrm{cH}$ SELF CONTAINED, EQUIPMENT

sum

800.000000 EN 1975 CONCRETE BOXES $6.000000 \mathrm{CI}$

Wast

Type facility count

Container Description

-.........

305

$4 * 4 * 8$

$4 * 7 * 9$

$3 * 3 * 3$

218448

218468

,.......

$2 * 2 * 6$

R 2184k

1

(.......

218448

21846

umiram

Unkwow

unknow

$5.6 * 6.5 * 9.3$

UnKNom

unkmon

unknown

$3 \star 3 \star 3$
R 240ZUE 1

R 218 WB 33

33

R 218448 26

218448

28

R $2184 \mathrm{u}_{1}$

$$
\text { twat }
$$

sin 
Red aty Un Date Container Size

$95.000000 \mathrm{CM} 1970$ FIBERBOARD/PLASTIC BOXES, CARTONS, CASES atith

sun

URANIUM-ENRICHED

$80.000000 \mathrm{GA} 1975$ CONCRETE BOXES

timat

$\sin$

URANIUM-NATURAL

1.000000 ON 1969 TRUCKS, FLATBEDS, COMPACTOR, LOAOLUGGER

that

sum
Container Description

UnKMOMN

$3 * 3 * 3$

UNKHOWN
Type facility count

R 218448

........

R 218468

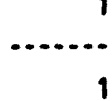

ค 21844B

57 rows selected.

$\infty$
$\varphi$
1 
WHC-EP-0696

This page intentionally left blank.

B.9-8 
WHC-EP-0696

APPENDIX 8.10

ISOTOPES LISTED IN TRU CONTAINERS

OTHER THAN 55-GALLON DRUMS

B.10-1 


\section{WHC-EP-0696}

This subappendix contains a listing of the SWITS isotope information for TRU waste from Building 325 PNL stored in containers other than 55-gal drums. The isotope field in SWITS contains several of the following types of iriformation:

- Specific isotope (e.g., Am-241, Np-237, U-233, Pu-238)

- Generic isotope listings (e.g., Pu, uranium-enriched, uranium-depleted)

- Plutonium-239 equivalent curies (PE-CI)

- Total-alpha

- Total beta/gamma

- PE-Ci (plutonium equivalent curies).

This computer run contains the isotope field listing along with the number of TRU waste containers with that listing. The run is sorted by storage facility and year. 


\section{WHC-EP-0696}

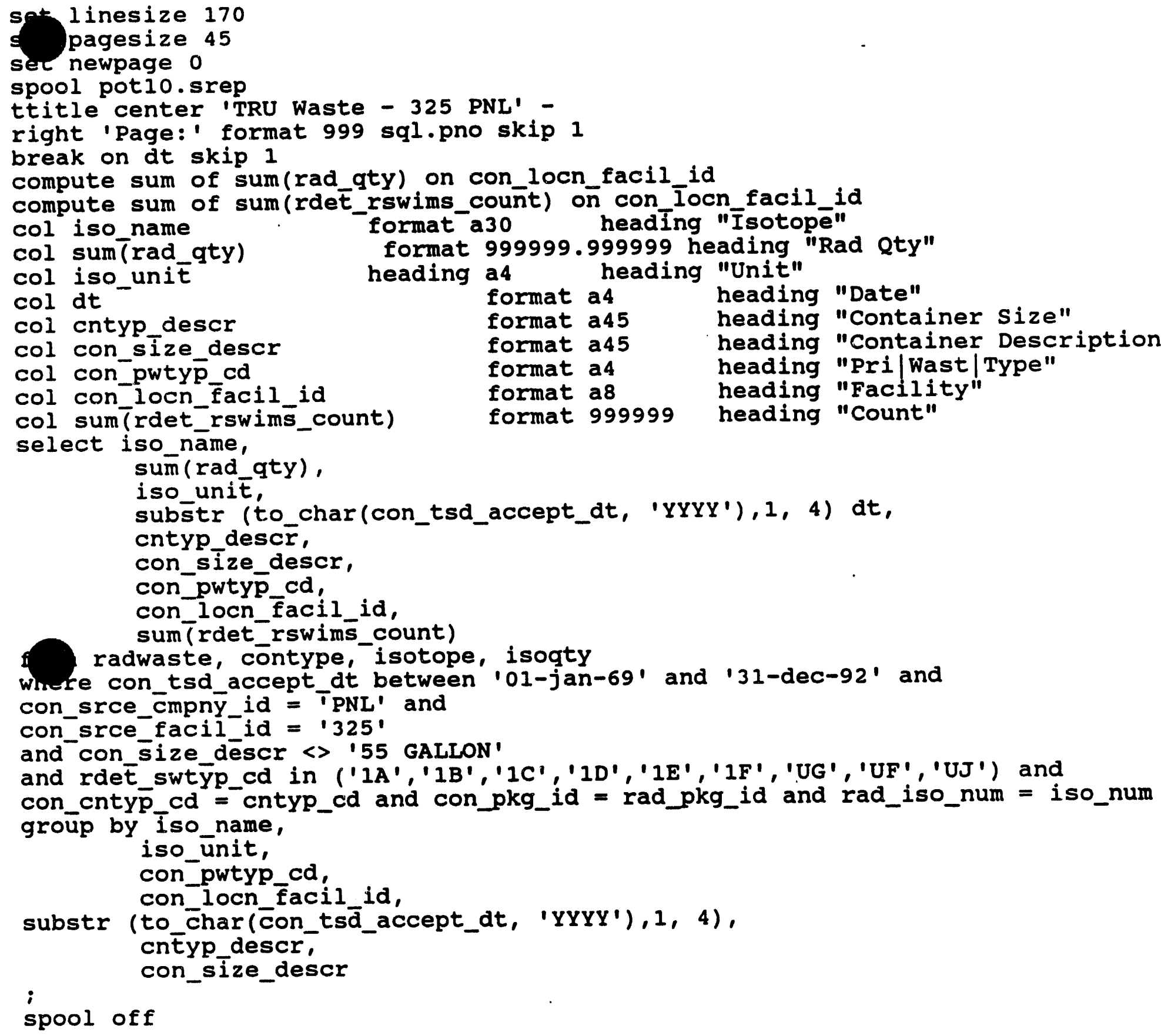




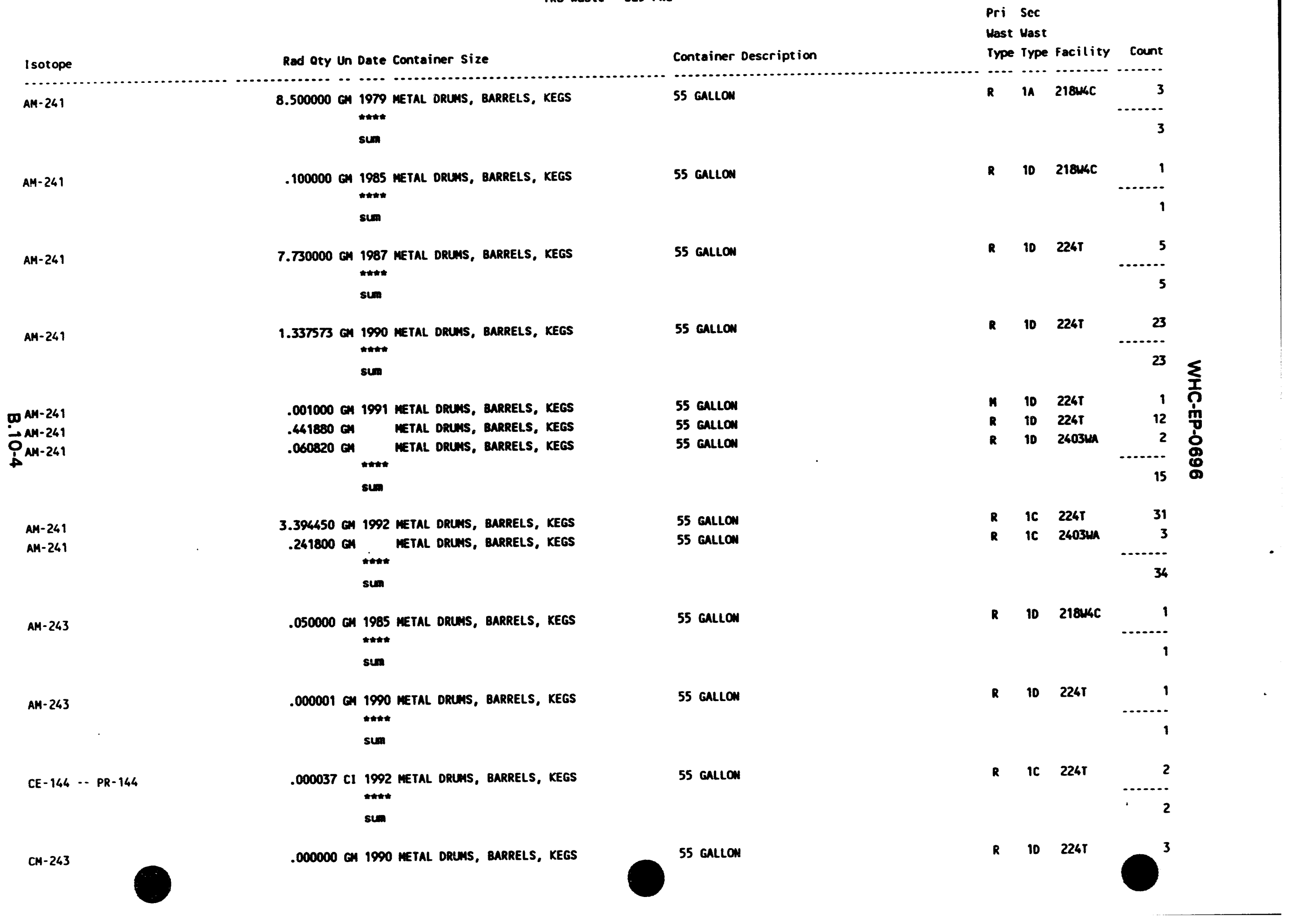




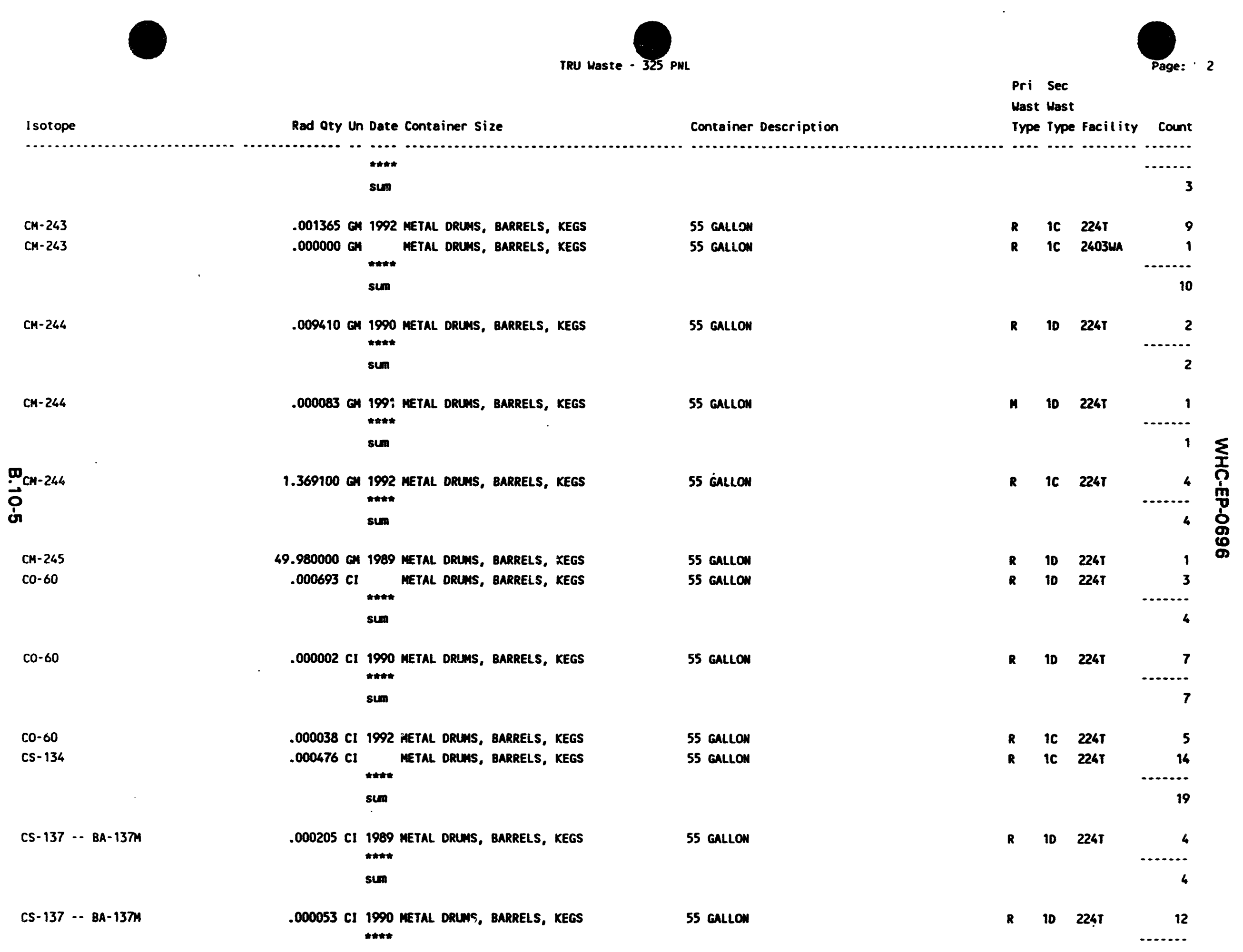




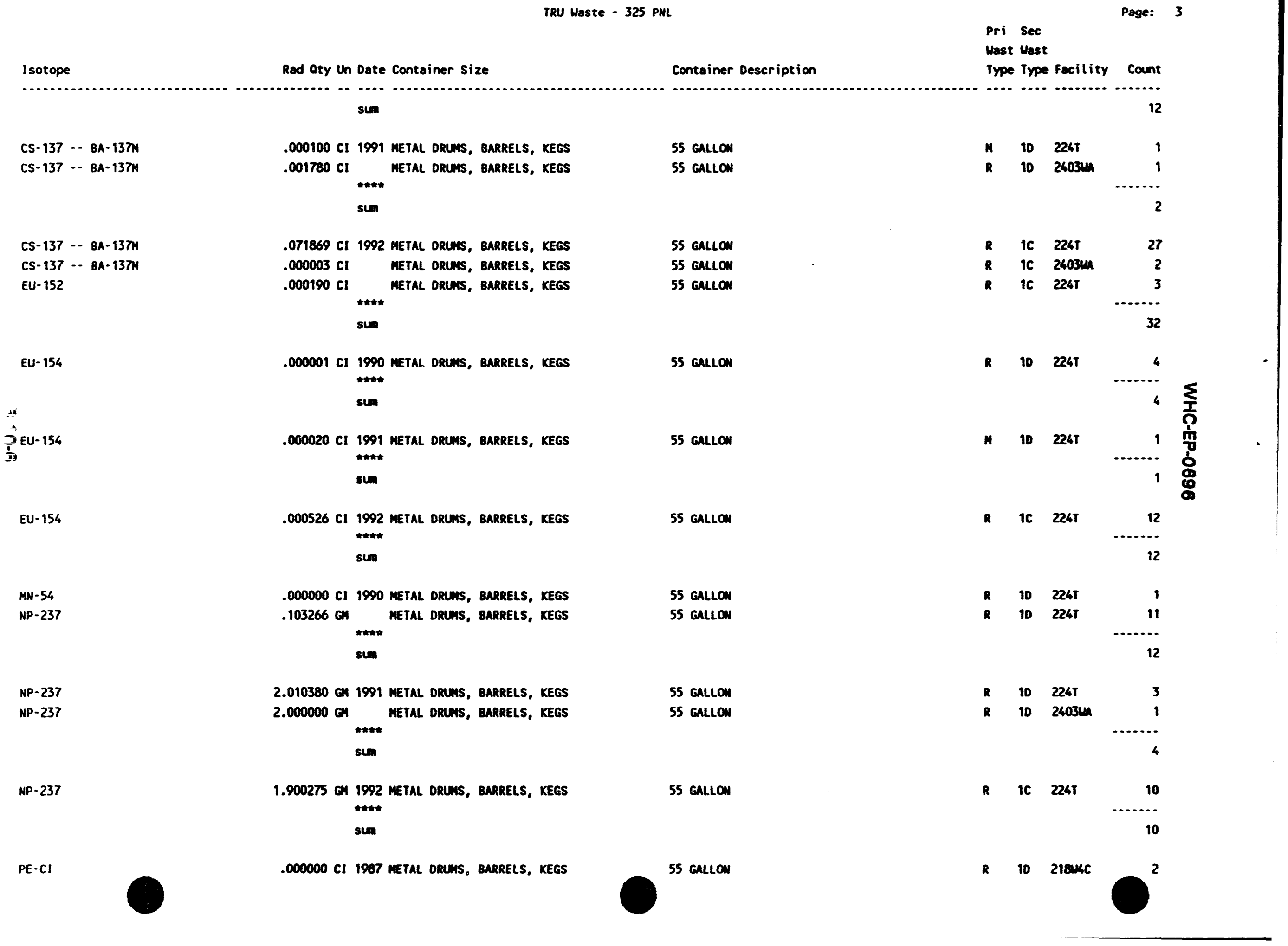


$.000000 \mathrm{Cl} 1987$ METAL DRUMS, BARRELS, KEGS twat

$$
\text { sim }
$$

.000000 Cl 1989 METAL DRUAS, BARRELS, KEGS $.000000 \mathrm{Cl}$ METAL DRLHS, BarReLS, Kegs t*t.

sum

$.000000 \mathrm{CI} 1990$ metal DRUNS, BarRels, KEGS $.000000 \mathrm{Cl}$ MetAl dRUAS, BarRELS, KEGS \#*

sum

.000000 Cl 1991 METAL DRUAS, BARRELS, KEGS $.000000 \mathrm{CI}$ METAL DRLAS, BARRELS, KEGS $.000000 \mathrm{Cl}$ METAL DRUNS, BARRELS, KEGS

$\sin$

427.230000 Cl 1969 METAL DRLAS, BARRELS, KEGS $.949400 \mathrm{CH}$ metal dRLAS, BarReLS, KEGS

sum

.003500 eN 1970 METAL DRUNS, BARRELS, KEGS tom

sem

.002200 GH 1971 METAL DRLAS, BARRELS, KEGS $162.030100 \mathrm{GH}$ METAL DRUNS, BARRELS, KEGS

sum

2.338200 GI 1972 METAL DRUMS, BARRELS, KEGS mithe

sum

.140000 GI 1973 metAL DRUNS, BARRELS, KEGS $1.173400 \mathrm{GH}$ neTAL DREMS, BarReLS, KEGS
55 Gallo

R 2241

55 callow

55 GALLOW

55 Gallon

55 GALLON

55 Gullow

55 callon

55 callon

55 GALLON

55 GALLON

55 GALLON

55 callow

55 Gallo:

55 callow

55 Gallow

55 callo:
* $10 \quad 2245$

$10226 \mathrm{~T}$

R 10 24034

UG 218448

vo 218 was

1

a 1 2103a

35

R IA 218usa 22

R in 218uke 305

327

R 1 a 218aro

548

548

R IA 218Ba

16 
WHC-EP-0696
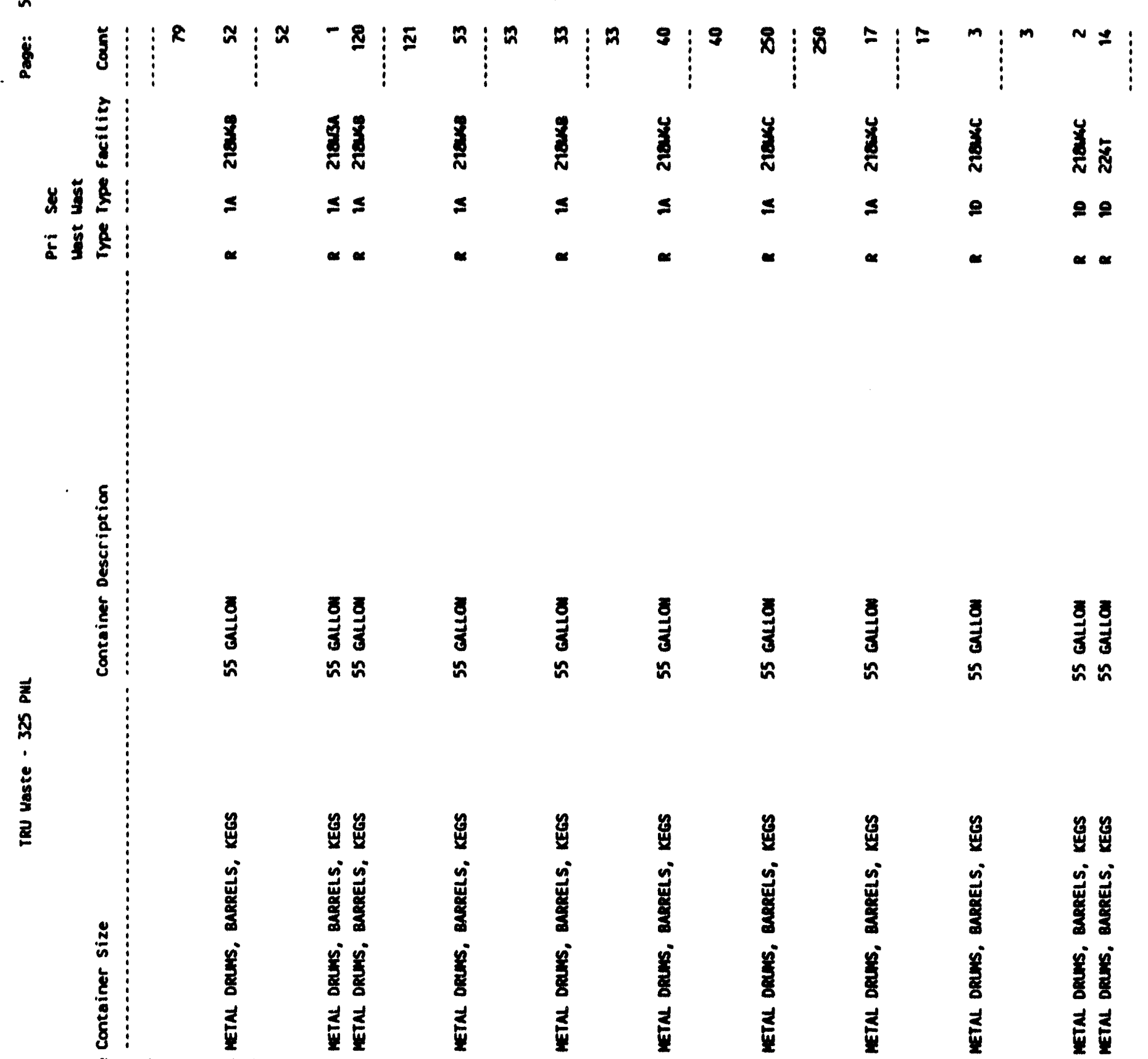

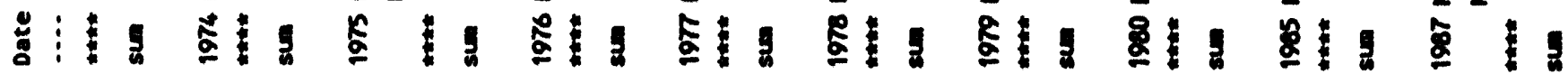

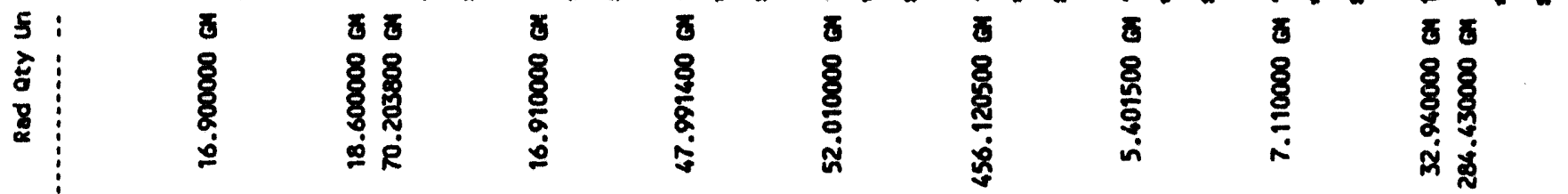

总

$\vec{a} \quad \vec{a} a \quad z$

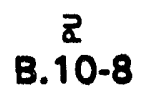

z

a

$\mathfrak{z} a$ 
100000 GH 1909 METAL DRUAS, BARRELS, KEGS $64.200000 \mathrm{OC}$ METAL dauns, Barrels, cregs sate

55 GAllon

55 callon

55 callon

234000 GI 1990 METAL DAUNS, BAREELS, KECS $111.723120 \mathrm{er}$ metal DRtins, Barrels, KECS sin

.016500 on 1991 metal DRUES, BAREELS, KECS $33.895918 \mathrm{GH}$ METAL Dems, enmels, KEOS 27.926400 GM METAL DAUNS, BAREELS, KECS .t.

$\sin$

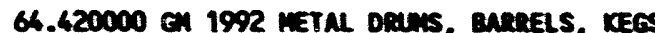
s.m.

sun

.300000 en 1975 meTAL DeUns, BARRELS, MEGS atio

sin

.170863 en 1992 metal Daurs, Burets, KEES $.001533 \mathrm{cH}$ METAL Douns, endeeELs, KECOS $304.368700 \mathrm{CH}$ $1.717000 \mathrm{ES}$ $36.590240 \mathrm{cH}$ $.232900 \mathrm{EA}$ $3.153500 \mathrm{GH}$ $.012345 \mathrm{En}$ $.849767 \mathrm{GS}$ $.002648 \mathrm{GH}$ METAL DRAMS, BARerELS, KEES METAL DeUNS, EARRELS, KECS METAL Douns, andrels, KECS METAL Deans, Barerels, KEES METAL DRUMS, BaBRELS, KECS metal Dewns, Bariels, recs METAL DRUMS, BareeELS, KEES min METAL Douns, Barrels, KEOS $\operatorname{sen}$

.000000 GA 1907 METAL Danns, Bareets, KECS

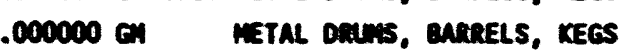

55 cullow

55 callo

55 cula

55 culom

55 callom

55 encon

55 encom

55 cullow

55 enlo

55 culcom

55 callon

55 encion

55 cullon

55 enclom

55 enllom

55 eallon

55 Gnlla

55 cula $\begin{array}{lll}10 & 2261\end{array}$

R 102267

1
21
$\therefore \quad .$.
22

$\begin{array}{rrrr}n & 10 & 2261 & 1 \\ 2 & 10 & 2261 & 30 \\ & & & \ldots . \\ & & & 31\end{array}$

$\begin{array}{rrrr}m & 10 & 2261 & 1 \\ 2 & 10 & 2261 & 12 \\ 1 & \text { 10 } & 26034 & 2 \\ & & & \ldots . . .\end{array}$

R $1 C 2261$

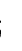

R In 21 ans 1

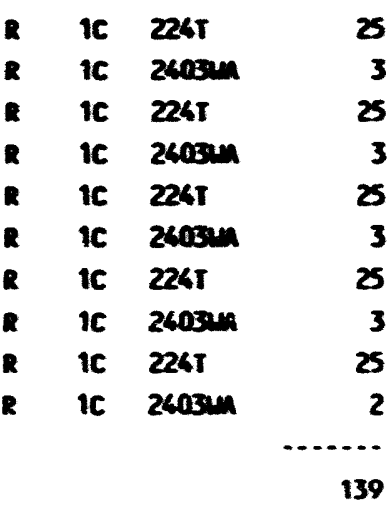

210 218nc 2 


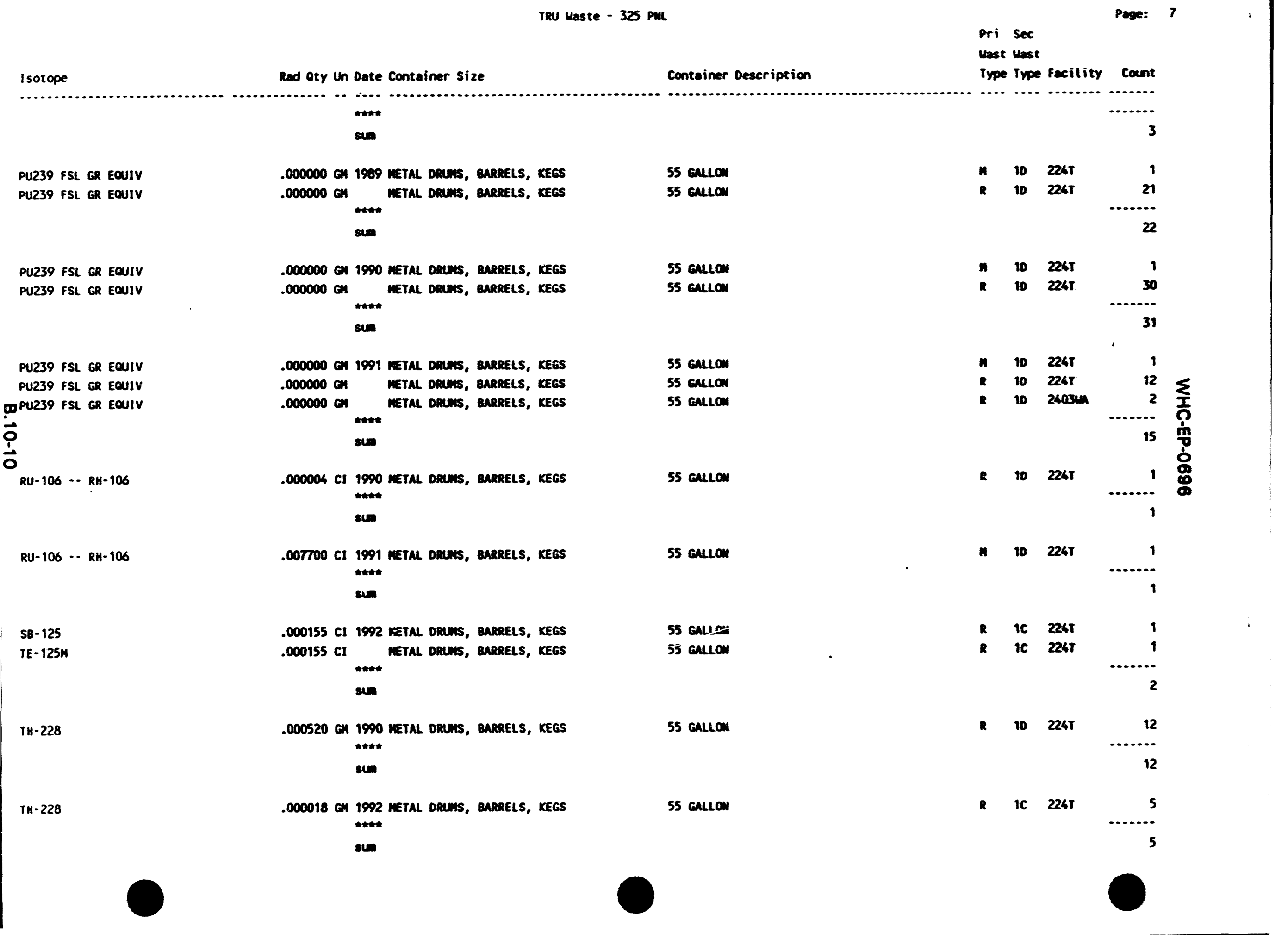


I sotope

Rad Oty Un Date Container size

TH-232

$T H-232$

$T H-232$

TOTAL BETA/GAMM

TOTAL BETA/GAMAa

TOTAL BETA/GAHAM

TOTAL BETA/GAMHA

D TOTAL BETA/gamea

$\overrightarrow{0}$ Total beta/gama

TOTAL BETA/gama

- total beta/gama

TOTAL BETA/gaMma

TOTAL BETA/GAMAM

TOTAL BETA/GAMMA

TOTAL BETA/GAMMA

TOTAL BETA/GAMAA

TOTAL BETA/GAMMA

TOTAL BETA/GAMHA

TOTAL BETA/GAMAA

$$
\text { tat* }
$$

sin

sta

tt+4: sin $.064800 \mathrm{cl}$ $.773000 \mathrm{Cl}$

sim

tat:

sin
9344.000000 GN 1971 METAL DRUNS, BARRELS, KEGS

15000.000000 GN 1972 METAL DRUNS, BARRELS, KEGS

100.000000 EM 1992 METAL DRUMS, BARRELS, KEGS

427.234631 Cl 1969 METAL DRUAS, BARRELS, KEGS $077185 \mathrm{CI}$ METAL DRUMS, BARRELS, KEGS $.047100 \mathrm{Cl}$ METAL DRUNS, BARRELS, KEGS $040785 \mathrm{Cl}$ METAL DRUAS, BARRELS, KEGS .201025 Cl METAL DRUAS, BARRELS, KEGS $.013635 \mathrm{Cl}$ METAL DRUNS, BAERELS, KEGS $028620 \mathrm{Cl}$ METAL DREMS, BARRELS, KEGS $.522050 \mathrm{CI}$ METAL DRLATS, BARRELS, KEGS $.023440 \mathrm{Cl}$ METAL DRUAS, BARRELS, KEGS $.171500 \mathrm{CI}$ metal ORUMS, Barrels, KEGS

.181000 CI 1970 METAL DRLAS, BarRELS, KEGS METAL DRUWS, BARRELS, KEGS

.094000 Cl 1971 METAL dRLAS, BARRELS, KEGS

METAL DRUMS, BARRELS, KEGS

4.016100 CI 1972 METAL ORLAS, BARRELS, KEGS

.014000 CI 1973 METAL DRUNS, BARRELS, KEGS
Container Description

Type Type Facility Count

55 GALLOW

R $14 \quad 218$ ave

55 GALLOW

55 GALLON

Gallon

55 GALLON

55 GALLON

55 GALLON

55 GAllOA

55 Gallon

55 callon

55 GALLOW

55 Gallon

55 GAllon

55 GALLON

55 GAllon

55 GALLON

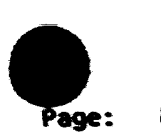

R IC $224 \pi \quad \ldots$.

R UG 218448

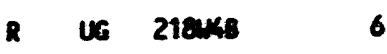

R 16 210468 6

UG 218448

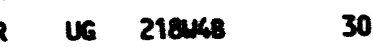

If 219uks

v6 218448

$16 \quad 21848$

UG 218 4.

ut 21846

55 GALLO

$\begin{array}{rrrr}R & 1 A & 21843 A & 22 \\ R & 11 & 218448 & 305 \\ & & & \ldots . . . \\ & & & 327\end{array}$

55 GALLON

55 Gallon
R IA 21848

540
540

U 218468

51

540

R IA 218u3a
R 1 I 218 sa 35 


\section{I sotope}

Rad Oty Un Date Container Size

$1.326000 \mathrm{Cl} 1973$ METAL DRUMS, BARRELS, KEGS motat

sum

TOTAL BETA/GAMYA

TOTAL BETA/GANA

TOTAL BETA/GAMMA

TOTAL BETA/GNAMA

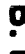

$\stackrel{\overrightarrow{0}}{\vec{N}}$ rotal beta/gama

TOTAL BETA/GAMEA

TOTAL BETA/GAMu

TOTAL BETA/GAMAA

TOTAL BETA/GAMHA

TOTAL BETA/GANA TOTAL BETA/GAM
2.288000 CI 1974 METAL DRUNS, BARRELS, KEGS ationt

sin

.060000 Cl 1975 mETAL DRUNS, BARRELS, KEGS $.721000 \mathrm{Cl}$ METAL DRUNS, BARRELS, KEGS stitit

8

.486000 CI 1976 METAL DRUMS, BARRELS, KEGS thtw

sum

3.874000 CI 1977 METAL DRUNS, BARRELS, KEGS trative

s.

.522000 CI 1978 METAL DRUMS, BARRELS, KEGS m.t.

$\sin$

2.251000 CI 1979 METAL DRUNS, BARRELS, KEGS mitit

sin

1.020000 CI 1990 METAL DRUMS, BARRELS, KEGS (1900

8

.150000 CI 1965 METAL DRLAN, BARRELS, KEGS titit

sin

$.100000 \mathrm{CI} 1987$ METAL DRLUS, BARRELS, KEGS $.005000 \mathrm{Cl}$ metal dRuAs, BarReLS, KEgS
Container Description

55 Gallon

Pri Sec

Wast Mast

Type Type facility count

-..................

R $1 A \quad 218448$ B 55

69

55 Gallon

$\quad .$.
50

55 Gallom

55 GaLLOW

R IA 21843n 1

R IA $218 \mathrm{ka} \quad 120$

121

55 GALLON

R 1 A 218 MB $\quad 53$

53

55 GALLON

R 1 1A 218uks:

32

32

55 callow

R 1 218ukc

40

55 GALLOW

R 1 A 218ukC 250

250

55 GAllon

R 1 218uac 17

17

55 GALLOW

R 10 218u4C 3

55 callon

55 Gallow 
Red Oty Un Date Container size

Pri Sec

Wast Mast

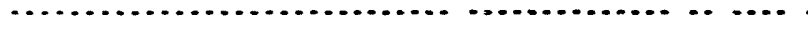

sum

TOTAL BETA/GAMMA

TOTAL BETA/GAMMA

TOTAL BETA/GAMAMA

TOTAL BETA/GAMAMA

TOTAL BETA/gAMAAA

TOTAL BETA/GAMMA

IOTAL BETA/GAMMA

m

$\overrightarrow{\mathbf{0}}$

$\vec{\omega}$ total beta/gamma

TOTAL BETA/GAMEA

TOTAL-ALPHA

TOTAL-ALPHA

TOTAL-ALPHA

TOTAL-ALPHA

TOTAL - ALFHA

TOTAL-ALPHA

TOTAL-ALPHA

TOTAL - ALPHA $2.903091 \mathrm{CI}$ sen $.101419 \mathrm{Cl}$ twat

sum $.004060 \mathrm{CI}$

sun $.000057 \mathrm{Cl}$

sun $.000000 \mathrm{CI}$ thin

sin $.000000 \mathrm{Cl}$ \#*

sun $.000000 \mathrm{Cl}$

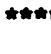

sim
.000500 CI 1989 METAL DRLMS, BARRELS, KEGS METAL DRUMS, BARRELS, KEGS

.000500 CI 1990 METAL DRLAS, BARRELS, KEGS METAL DRUNS, BARRELS, KEGS

.015620 CI 1991 metal DrLUS, BARRELS, KEGS $.014700 \mathrm{Cl}$ METAL DRLAMS, BARRELS, KEGS metal oRUMS, BARRELS, KEGS

158522 CI 1992 METAL DRUMS, BARRELS, KEGS metal druns, Barkels, KEgS

.000000 CI 1987 METAL DRLNAS, BARRELS, KEGS METAL DRUMS, BARRELS, KEGS

55 GALLON

55 gallon

55 GAllON

55 Gallon

55 GALLON

55 callon

55 callow

55 GALLON

55 GALLON

55 GaLLON

55 GALLON

.000000 CI 1989 METAL DRLMS, BARRELS, KEGS

METAL DRUNAS, BARRELS, KEGS

55 GALLON

55 GALLON

.000000 CI 1990 METAL DRLMS, BARRELS, KEGS METAL DRLAS, BARRELS, KEGS

.000000 CI 1991 METAL DRUMS, BARRELS, KEGS $.000000 \mathrm{CI}$ METAL DRUMS, BARRELS, KEGS

55 GALLON

55 GALLOW

55 Gallon

55 Gallo
Container Description

Tyoe Type facility count

$\begin{array}{rrrr}n & 10 & 224 T & 1 \\ R & 10 & 224 T & 21 \\ & & & \ldots \ldots . . \\ & & & 22\end{array}$

H $10 \quad 226 \mathrm{~T}$

1

31

N $10 \quad 2245$

$\begin{array}{llll}M & 10 & 224 \mathrm{~T} & 12\end{array}$

R $10 \quad 2603$ M 2
15

R IC 2241

R IC 2403MA 39

35

R 10 218ukC

R 10 2265

3

M $10 \quad 224 T$

1

R 10

21

H $10 \quad 2245$

R $10 \quad 224$

1

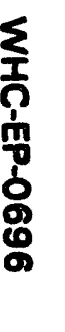

$\begin{array}{rrrr}M & 10 & 224 T & 1 \\ R & 10 & 224 T & 12\end{array}$ 


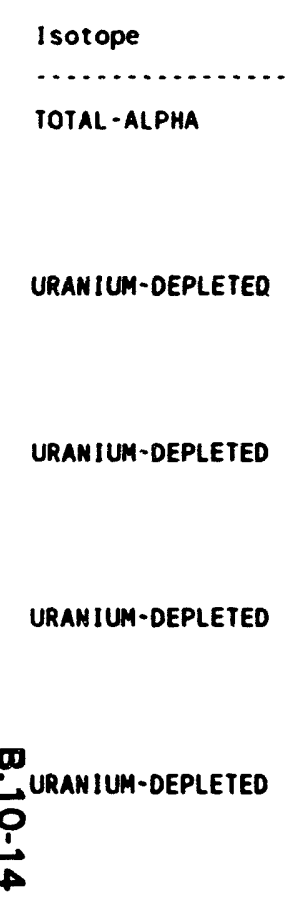

URANIUM-DEPLETED

URANIUM-DEPLETED

URANIUM-DEPLETED

URANIUM-ENRICHED

URANIUM-ENRICHED
Red oty Un Date Container size

.000000 Cl 1991 MTTAL DRUMS, BARRELS, KEGS

thath

sum

3501.014000 GH 1969 METAL DRLMS, BARRELS, KEGS

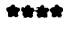

sin

303000.000000 GI 1972 METAL DRUMS, BARRELS, KEGS thithe

sum

.300000 GM 1975 METAL DRUMS, BARRELS, KEGS

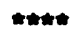

sum

23000.000000 GN 1976 METAL DRUMS, BARRELS, KEGS

*tक:

$\sin$

455.000000 on 1979 METAL DRUMS, BARRELS, KEGS

$\sin$

3400.000000 aN 1989 METAL DRUNS, BARRELS, KEGS thit

84

1297.500000 EI 1992 METAL DRUMS, BARRELS, KEGS that

sum

1821.500000 GH 1971 METAL ORUMS, BARRELS, KEGS ntatim

$\sin$

194.000000 1972 METAL DRUMS, BARRELS, KEGS

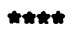

sum
Container Description

Pri Sec

Wast Wast

Type Type facility Count

55 GALLON

R $10 \quad 2603$ A

2

15

55 GALLON

55 Gallow

55 GALLOM

55 GALLON

55 GALLON

55 GALLON

55 GALLOM

55 Gallon

55 GALLON

R 1 I 218w4C 1

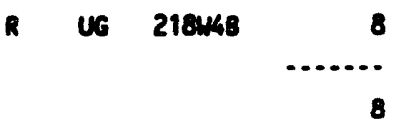

R $1 \mathrm{~A} 218 \mathrm{kB}$

3

R 1 1A 21844

1

(.......

R In 218448

(........

$\cdots \cdots$
1

R $10 \quad 224 \mathrm{~T} \quad 1$

R IC 224 $\begin{array}{r}5 \\ \end{array}$

R In $218448 \quad \begin{aligned} & 4 \\ & \end{aligned}$

R 1 IA 21844B $\begin{array}{r}\text {...... } \\ \end{array}$ 
I sotope Rad Qty Un Date Container Size

Container Description

Type Type Facility count

URANIUM-ENRICHED

URANIUM-ENRICHEO

URANIUM-ENRICHED

URANIUH -ENRICHED

URANIUH-ENRICHED

$\boldsymbol{\infty}$

Q̈URANIUH-NATURAL

ज्

URANIUA-MATURAL

URANIUM-NATURAL

URANIUM-NATURAL

URANIUM-NATURAL

URANIUM-NATURAL
2466.000000 G 1975 METAL DRUMS, BARRELS, KEGS $44.000000 \mathrm{GH}$

METAL DRUMS, BARRELS, KEGS

sum

3530.200000 GH 1977 METAL DRUNS, BARRELS, KEGS

tom

sum

698.980000 GN 1979 METAL DRUMS, BARRELS, KEGS totit*

sum

1273.010000 GH 1992 METAL DRLMS, BARRELS, KEGS t*tw

$\sin$

.006000 GN 1970 METAL DRUMS, BARRELS, KEGS *a*t*

$\sin$

38.600000 OM 1971 METAL DRUMS, BARRELS, KEGS tottit

sua

402000.000000 GA 1972 METAL DRUNS, BARRELS, KEGS แtนm

sum

1.000000 GM 1978 METAL DRUMS, BARRELS, KEGS

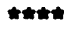

sum

6260.000000 GW 1980 METAL DRLMS, BARRELS, KEGS *t*t

sum

200.000000 GI 1989 METAL DRUAS, BARRELS, KEGS twat
55 GALLON

55 GALLON

55 GALLON

55 callon

55 GALLON

55 GALLON

55 GALLON

55 GaLLON

55 GALLON

55 GALLON

55 GALLOW
R IA 21843A

R IA 218144

R IA 218 448 5

5

R 1 A 218 Mac 7

7

R IC $224 \mathrm{~T}$

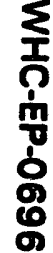

R $1 A \quad 218$ 3a

21813a

.......

R IA 218468

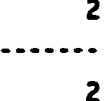

R IA 21844

8
8

R $1 A \quad 218$ WC

1

R IA 218u4C

4

4

$10 \quad 224 \mathrm{~T}$ 12 


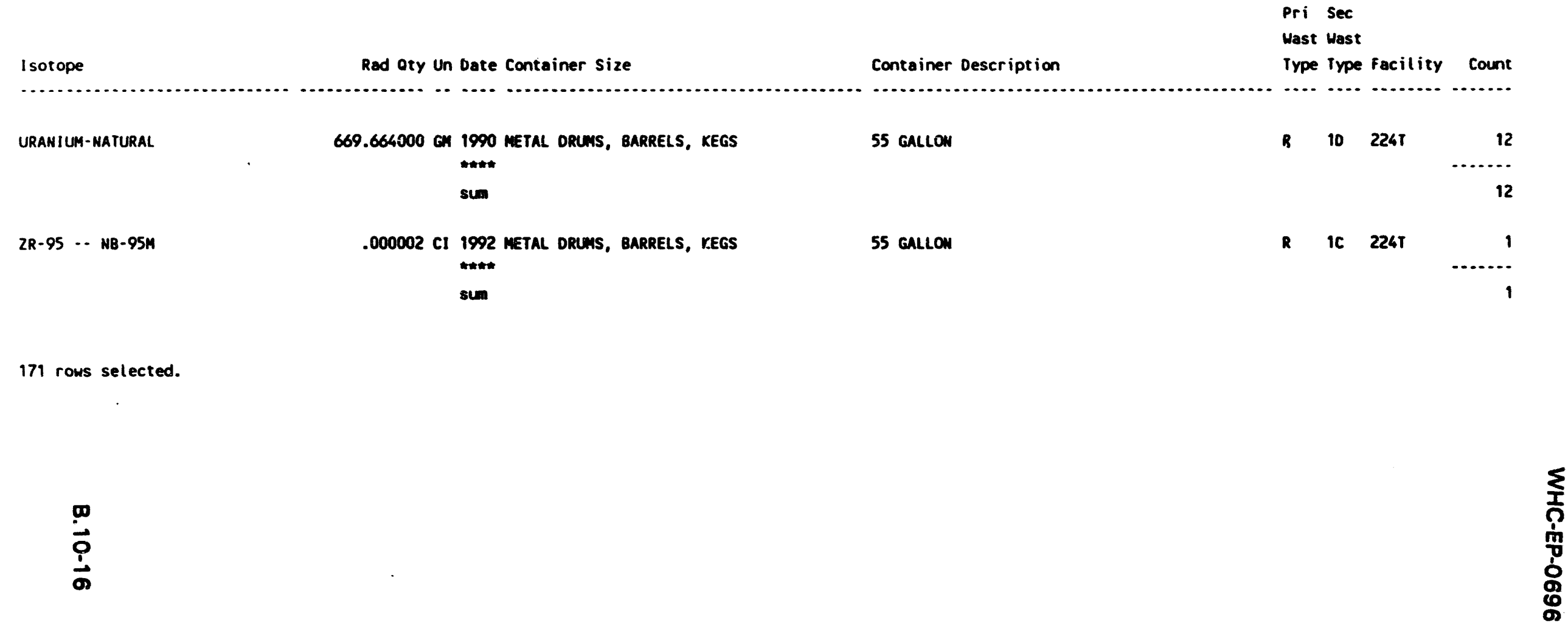




\section{WHC-EP-0696}

\section{APPENDIX C}

\section{DATA ON BUILDING 325A WASTE GENERATION BY PNL FROM}

THE SOLID WASTE INFORMATION TRACKING SYSTEM 
The information found in this appendix is from the SWITS database. This database incorporates the older R-SWIMS database and is used to track information on radioactive and other wastes stored or disposed at the Hanford Site.

Each SWITS data run in this appendix is preceded by the query used to generate the data. A brief explanation of the run and any additional information needed to understand the data presented is also included.

The bulk of the data provided is limited to information about TRU waste generated at Building 325A by PNL; however, some general information on the non-TRU waste is included for completeness. The term non-TRU is used, instead of LLW, because a small percentage of the waste has been designated only as not TRU. It is believed that most, if not all, of the non-TRU waste is LLW.

The data runs in this appendix are further segregated by waste container type. Since initial retrieval efforts and WRAP 1 will focus on 55-gallon drums, these container types are considered separately.

Some general information about SWITS database and the codes used follows. Please note the disclaimer found on the next page. 
Date<smiles>CC(C)OC1(C)CCCC1</smiles>

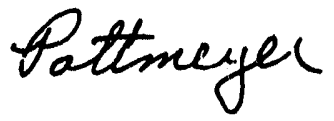

From: Solld Waste Engineering Data Management Group N3-11 $6-4394 / 6-4020$

Re: SWITS DATA REQUEST

Attached for your information and use is the data which you requested. This data represents best available information regarding wastes currently in storage at the Hanford site. I trust the information will be suitable to your needs.

Requests for information from the solid Waste Information and Tracking system (SWITS) are normally relatively limited in scope, requesting specific data fields or summary data. The responses to these requests undergo review during data collection, summary and response preparation.

The response to this request represents a simple reproduction of the swITs Database. Transmittal of this information is made with the following disclaimers:

1) The information contained in this transmittal is raw data, and represents information provided to solid Waste Engineering (SWE) on burlal records or other documents. This data has not been validated.

2) The information contained in this transmittal is subject to change without notice. Continual update of swITS information and improvement of the software system make it impossible to ensure consistency of this data with the database after transmittal.

3) This information is current as of

If I can be of further assistance to you, do not hesitate to call me. 


\section{WHC-EP-0696}

This page intentionally left blank. 
WHC-EP-0696

Primary Waste Type Code Table

Primary Waste Type Code

AH
$\mathbf{C H}$
$\mathbf{H P}$
$\mathbf{M}$
$\mathbf{M P}$
$\mathbf{P}$
$\mathbf{R}$
$\mathbf{R P}$
$\mathbf{U}$

Primary Waste Type Description

\section{HAZ, ADMIN HOTD}

MORATORIUM CONIROIID

HAZARDOUS

IIAZARDOUS WASTE PCB

MIXED

MIXED PCB

NON-REGULATED

PCB

RADIOACTIVE

RADIOACTIVE PCB

UNKNOWN
Ship

Time Rad

IIm - Rad

89 N

89 N

89 N

29 N

89

29

29

29 
WHC-EP-0696

SWIR402

Solid Waste Information and Tracking system

$05 / 01 / 92$

$13: 26$

Page: 1

Secondary Waste Type Code Table

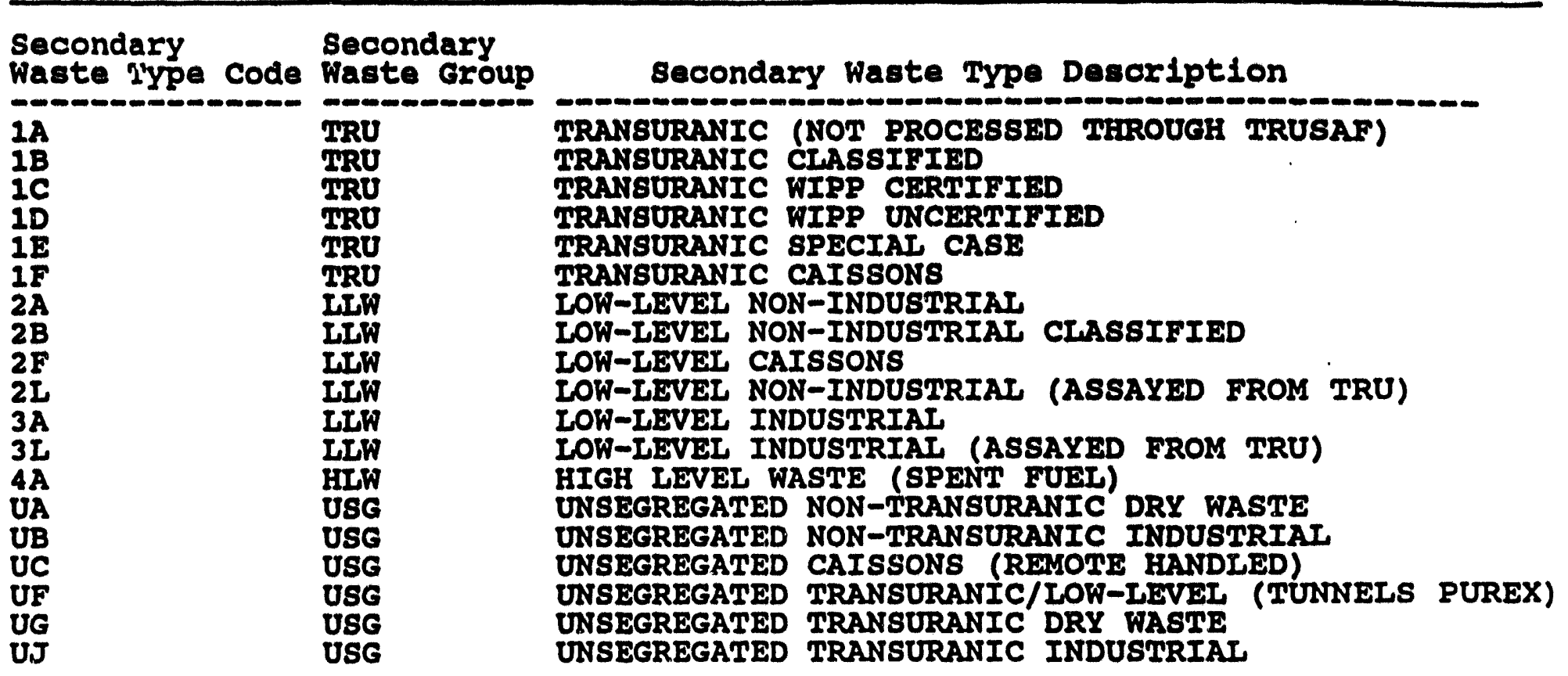




\section{WHC-EP-0696}

\section{APPENDIX C.1}

CONTAINER NUMBER AND WEIGHT OF TRU WASTE IN 55-GALLON DRUMS BY WASTE TYPE

C. $1-1$ 
This data run gives an overall look at the waste types, container numbers and total weight (in kilograms) of the radioactive wastes generated at Building 325A-PNL on a yearly basis. These data are for wastes stored in 55-gallon drums only. The meanings of primary and secondary waste codes can be found in the introductory materials for this appendix.

The date in the average weight column should be taken cautiously, especially for LLW codes. Concern is warranted because LLW records are still done on a batch basis. This means that one weight datum may be the composite weight for a group of drums. The computer program used simply divided the sum of all weight values by the number of values used to compute the total weight, net the actual number of containers that that value represents.

From 1970 until 1978 individual container weights were not required for TRU drums. During the data re-entry program in the mid-1980's standard weights were assigned for all container types; 55-gallon drums were given a standard weight of $68 \mathrm{~kg}$. This is why the average weight/drum for TRU is so consistent during this period. 


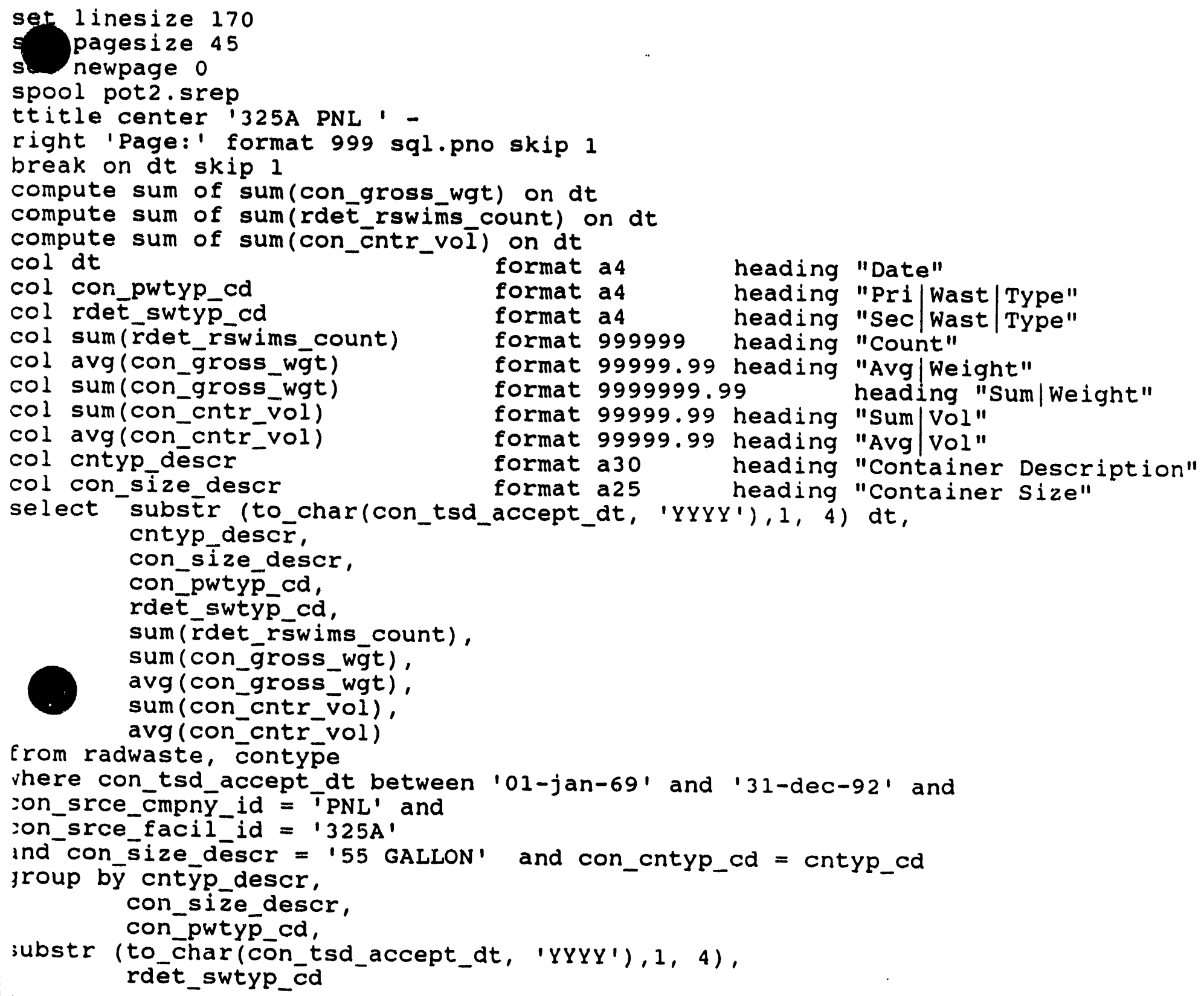




\begin{tabular}{|c|c|c|c|c|c|c|c|c|}
\hline Date Container Description & Container Size & $\begin{array}{l}\text { Pri } \\
\text { Hast } \\
\text { Type }\end{array}$ & $\begin{array}{l}\text { Sec } \\
\text { Wost } \\
\text { Type }\end{array}$ & count & $\begin{array}{l}\text { Sum } \\
\text { Weight }\end{array}$ & $\begin{array}{r}\text { Avg } \\
\text { Weight }\end{array}$ & $\begin{array}{l}\text { Sum } \\
\text { vol }\end{array}$ & $\begin{array}{l}\text { Avg } \\
\text { vol }\end{array}$ \\
\hline 1978 METAL DRUMS, BARRELS, KEGS & 55 callow & M & $2 A$ & 15 & 2477.97 & 825.99 & $\begin{array}{l}3.19 \\
\cdots\end{array}$ & 1.06 \\
\hline sum & & & & 15 & 2477.97 & & 3.19 & \\
\hline 1976 MEIAI DRUMS, BARRELS, KEGS & 55 GALLON & $\mathbf{R}$ & IA & 10 & 3855.50 & 385.55 & 2.10 & .21 \\
\hline sum & & & & 10 & 3855.50 & & 2.10 & \\
\hline $\begin{array}{l}1977 \text { METAL DRUMS, BARRELS, KEGS } \\
* \approx * *\end{array}$ & 55 GALLON & F & $2 \mathrm{~A}$ & 10 & 3016.37 & 1508.19 & $\begin{array}{ll}2.12 \\
\cdots\end{array}$ & 1.06 \\
\hline sum & & & & 10 & 3016.37 & & 2.12 & \\
\hline 1978 METAL DRUMS， BARRELS，KEGS & 55 Gallon & R & $2 \mathrm{~A}$ & 14 & 3097.11 & 3097.11 & 2.97 & 2.97 \\
\hline sum & & & & 14 & 3097.11 & & 2.97 & \\
\hline $\begin{array}{l}1979 \text { METAL DRUMS, BARRELS, KEGS } \\
* * * \star\end{array}$ & 55 GALLOW & $\mathbf{R}$ & $1 \mathrm{~A}$ & 5 & 340.20 & 68.04 & 1.05 & .21 \\
\hline sum & & & & 5 & 340.20 & & 1.05 & \\
\hline
\end{tabular}


WHC-EP-0696

APPENDIX C.2

BUILDING 325A-PNL TRU WASTE CONTAINERS SORTED BY CONTAINER DESCRIPTION, SIZE, YEAR,

AND PRIMARY WASTE TYPE 
This subappendix provides information on Building 325A-PNL TRU waste containers, weight, volume, and primary waste type stored at various TRU waste storage facilities on the Hanford Site. The data provides the TRU waste generation from the Building 325A-PNL Facility by year. 


\section{WHC-EP-0696}

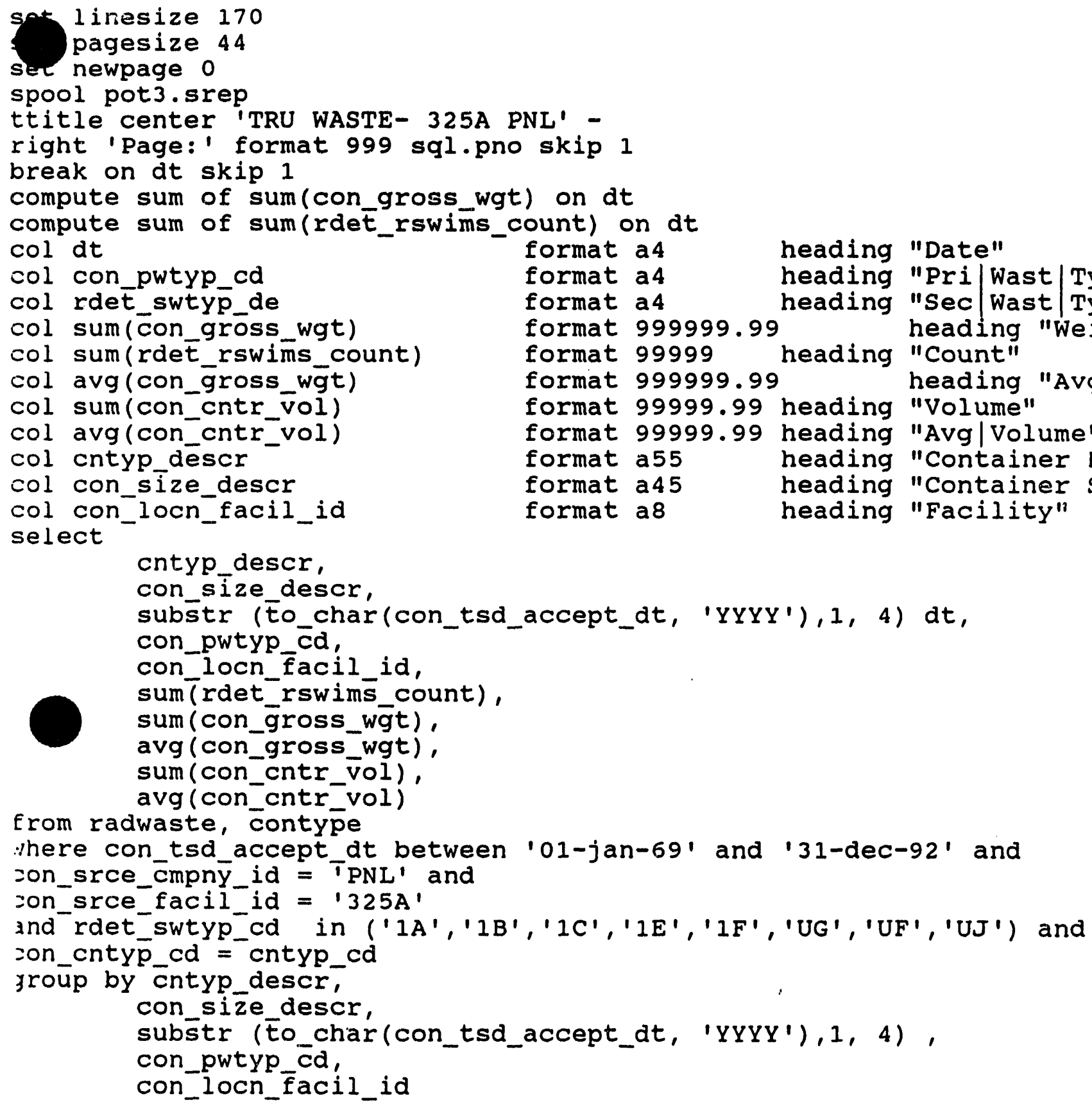

ipool of $f$ 
Container Discription

METAL CYLINDER, CASKS

METAL DRUMS, BARRELS, KEGS

METAL DRUMS, BARRELS, KEGS

METAL ORUMS, BARRELS, KEGS

METAL DRUMS, BARRELS, KEGS

$\stackrel{0}{i}$

metal orums, barrels, kegs

METAL DRUMS, BARRELS, KEGS

METAL DRUMS, BARRELS, KEGS

WOODEN BOXES, CARTONS, CASES

WOCOEN BOXES, CARTONS, CASES

WOOOEN BOXES, CARTONS, CASES

WOOOEN BOXES, CARTONS, CASES
Container Size

UNKNOWN

5 GALLON

5 GALLON

5 GALLON

5 GALLON

5 GALLON

55 GALLON

55 GALLON

$1.5 * 1.5 * 10$

$2 \star 2 * 3$

$3 * 3 * 3$

$3 * 3 * 4$
Date Type facility Count Weight

1975 R 218W3A $\quad 11 \quad 30481.24$

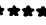

sum

$11 \quad 30481.24$

$\underset{\pi \neq * \pm}{1970 R \quad 218 w 48 B}$

$17 \quad 179.64$

\begin{tabular}{rr}
17 & 179.64 \\
\hline 17 & 179.64
\end{tabular}

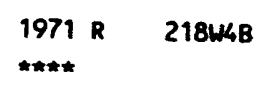

$38 \quad 379.24$

$38 \quad 379.24$

sum

1972 R 218W4B

$* * * *$

$\operatorname{sun}$

$1973 R \quad 218 w 48$

sum

$1983 R \quad 218 w 4 B$

**\#**

sum

1976 R 218W3A

sum

$1979 k \quad 21844 C$

sum

$1969 \mathrm{R}$

$\begin{array}{rr}1969 R & 218 w 4 B \\ R & 218 w 4 B \\ R & 218 w 4 B \\ R & 218 w 48\end{array}$

sum

\begin{tabular}{rr}
10 & 3855.50 \\
\hline 10 & 3855.50
\end{tabular}

\begin{tabular}{|c|c|c|}
\hline $\begin{array}{r}\text { Avg } \\
\text { A }\end{array}$ & & Avg \\
\hline & Volume & Volume \\
\hline \multirow[t]{2}{*}{2771.02} & 67.96 & 6.18 \\
\hline & 67.96 & \\
\hline \multirow[t]{2}{*}{19.96} & .25 & .03 \\
\hline & .25 & \\
\hline \multirow[t]{3}{*}{19.96} & .53 & .03 \\
\hline & $\cdots$ & \\
\hline & .53 & \\
\hline \multirow[t]{3}{*}{19.96} & .50 & .03 \\
\hline & $\cdots$ & \\
\hline & .50 & 5 \\
\hline \multirow[t]{3}{*}{19.96} & .14 & $.03 \bar{?}$ \\
\hline & $\cdots$ & गु \\
\hline & .04 & $\begin{array}{r}\text { O } \\
\text { \& } \\
.04\end{array}$ \\
\hline
\end{tabular}

.04

385.55 2.10

68.04

$\begin{array}{rr}5 & 340.20 \\ 5 & 340.20\end{array}$
1.05
1.05

.21

$200.03 \quad .45 \quad .45$

$338.83 \quad .76 \quad .76$

$\begin{array}{lll}390.55 & 1.74 & .87\end{array}$

$914.44 \quad 2.04 \quad 2.04$

.21

\begin{tabular}{rr}
1 & 200.03 \\
2 & 338.83 \\
2 & 781.09 \\
2 & 914.44 \\
\hline 7 & 2234.39
\end{tabular}


WHC-EP-0696

APPENDIX C.3

BUILDING 325A-PNL NON-TRU WASTE CONTAINERS SORTED BY CONTAINER DESCRIPTION, SIZE, YEAR, AND PRIMARY WASTE TYPE

C.3-1 
This subappendix provides information on PNL Building 325A non-TRU waste containers, weight, volume, and primary waste type stored at various waste storage facilities on the Hanford Site. The data provides the non-TRU waste generation from the PNL Building 325A Facility by year. 


\section{WHC-EP-0696}

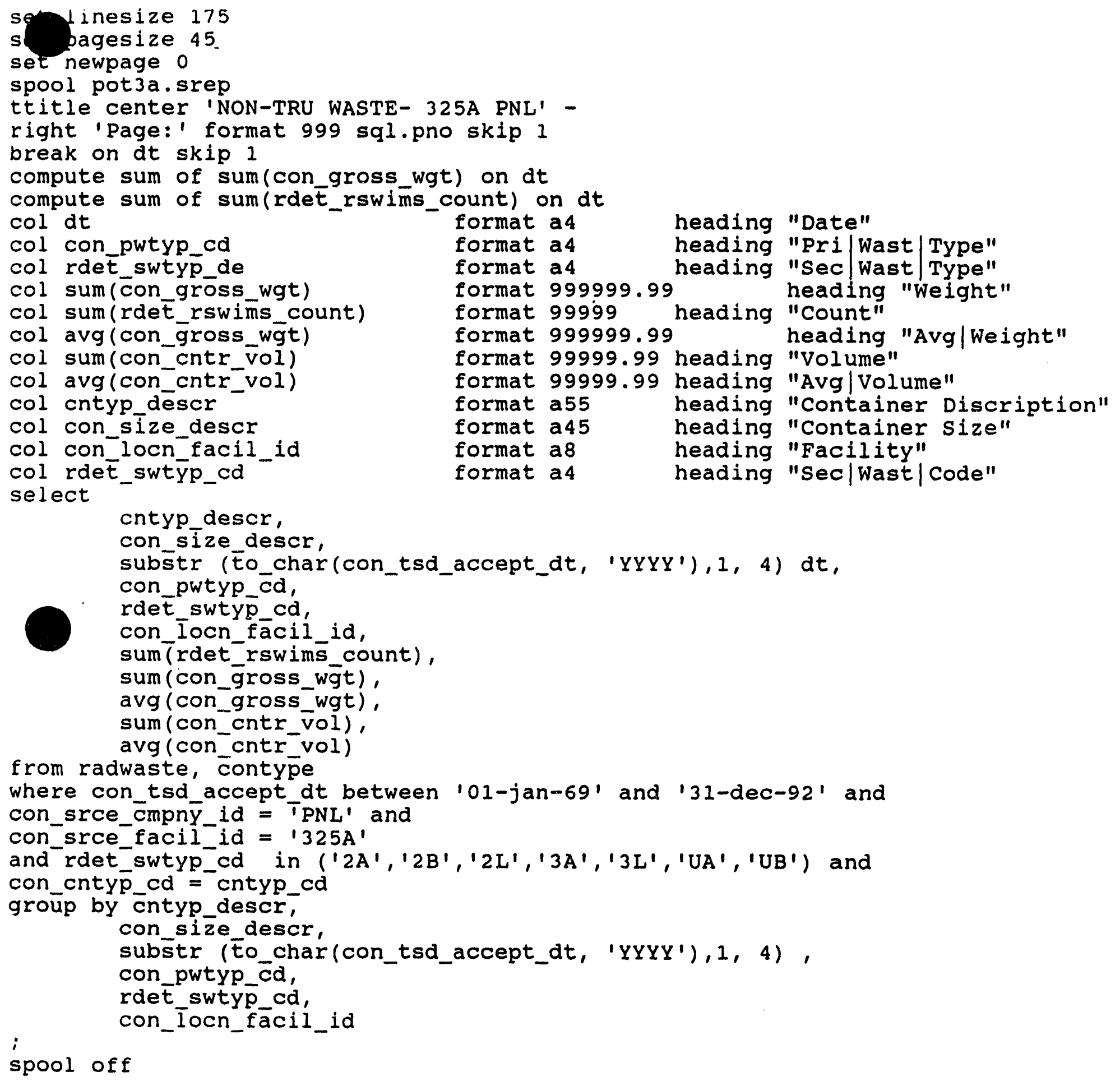




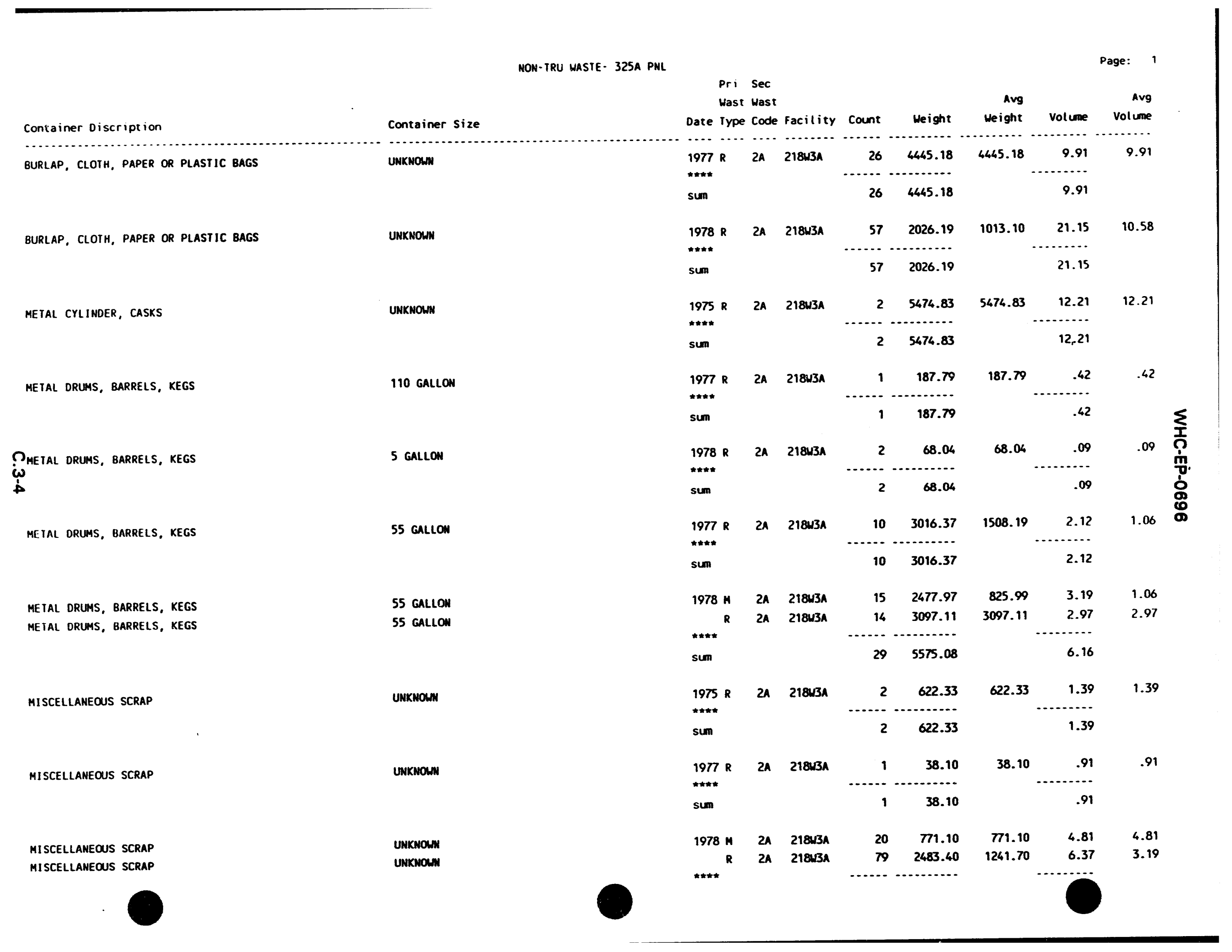




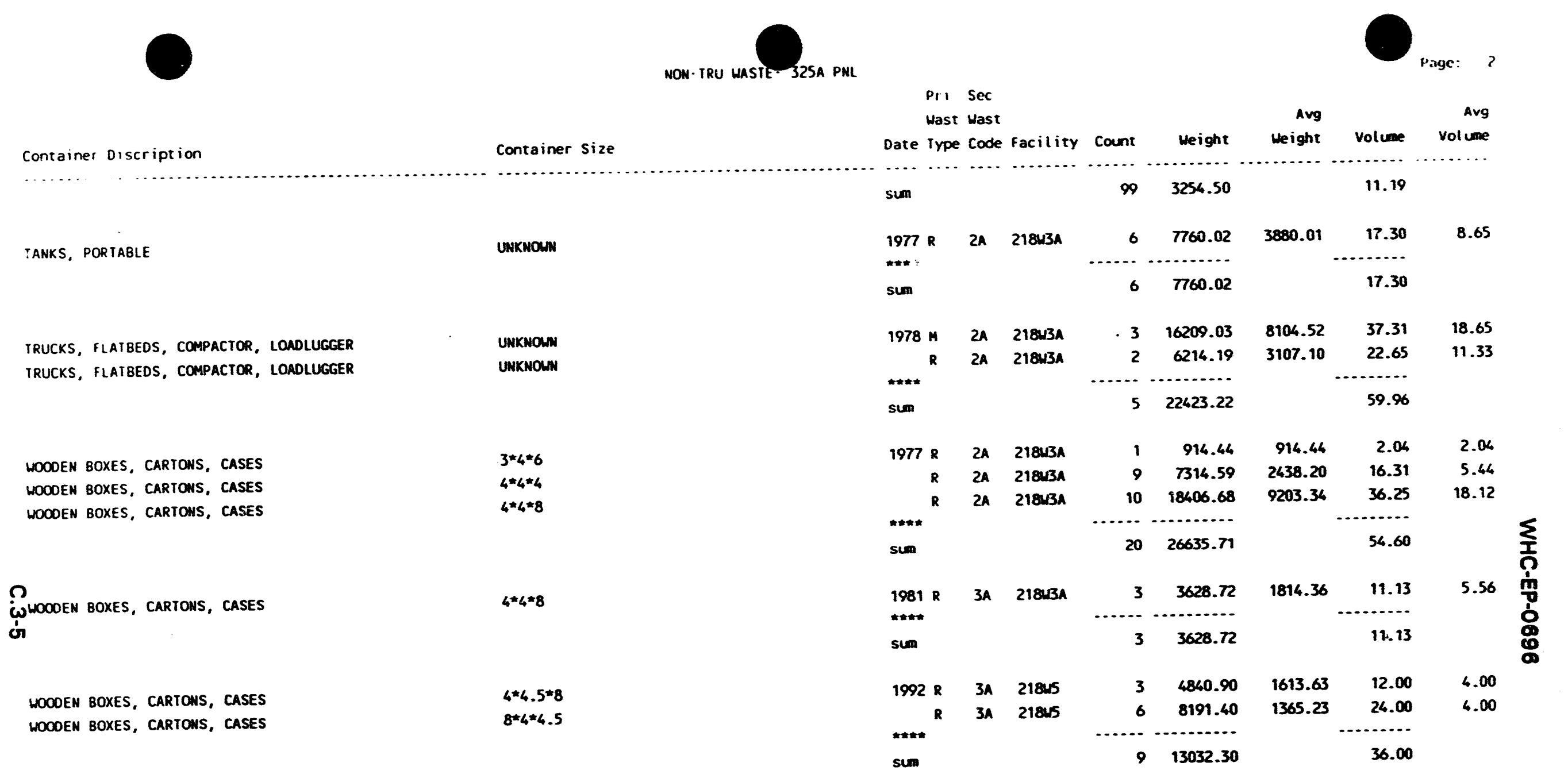


WHC-EP-0696

This page intentionally left blank.

C.3-6 
WHC-EP-0696

APPENDIX C.4

RADIOLOGICAL DATA FOR TRU WASTE CONTAINERS SORTED BY DATE, PRIMARY WASTE TYPE, AND STORAGE FACILITY

C.4-1 
This subappendix summarizes the radiological data for all TRU waste containers generated at Building 325A-PNL. Specifically, this computer run provides the total grams of TRU elements in a given container type in a given year. In addition, the average gram loading for a given container type in a given year has been calculated.

In 1991 and 1992 the number of curies (Ci) of alpha radiation was included on the SWBRs. Since the Building 325A-PNL waste generation data is all from 1970 to 1988, these columns contain blank entries. The "Max Dose" column contains the highest value recorded for a given container in a given year, in $\mathrm{mrem} / \mathrm{hr}$.

Additional information about the isotopes present in Building 325A-PNL waste containers can be found in sub-appendices C.9 and C.10. 


\section{WHC-EP-0696}

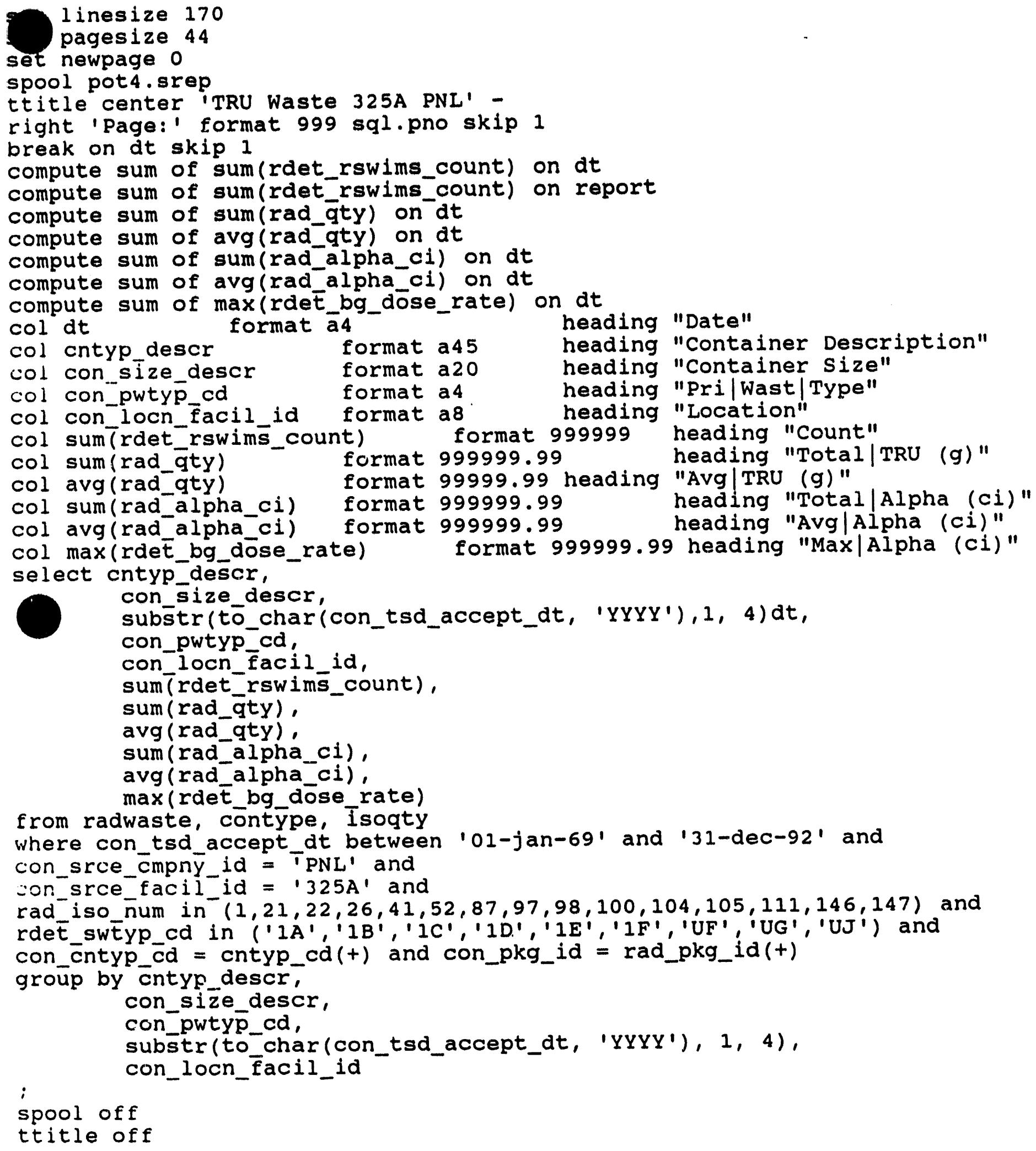




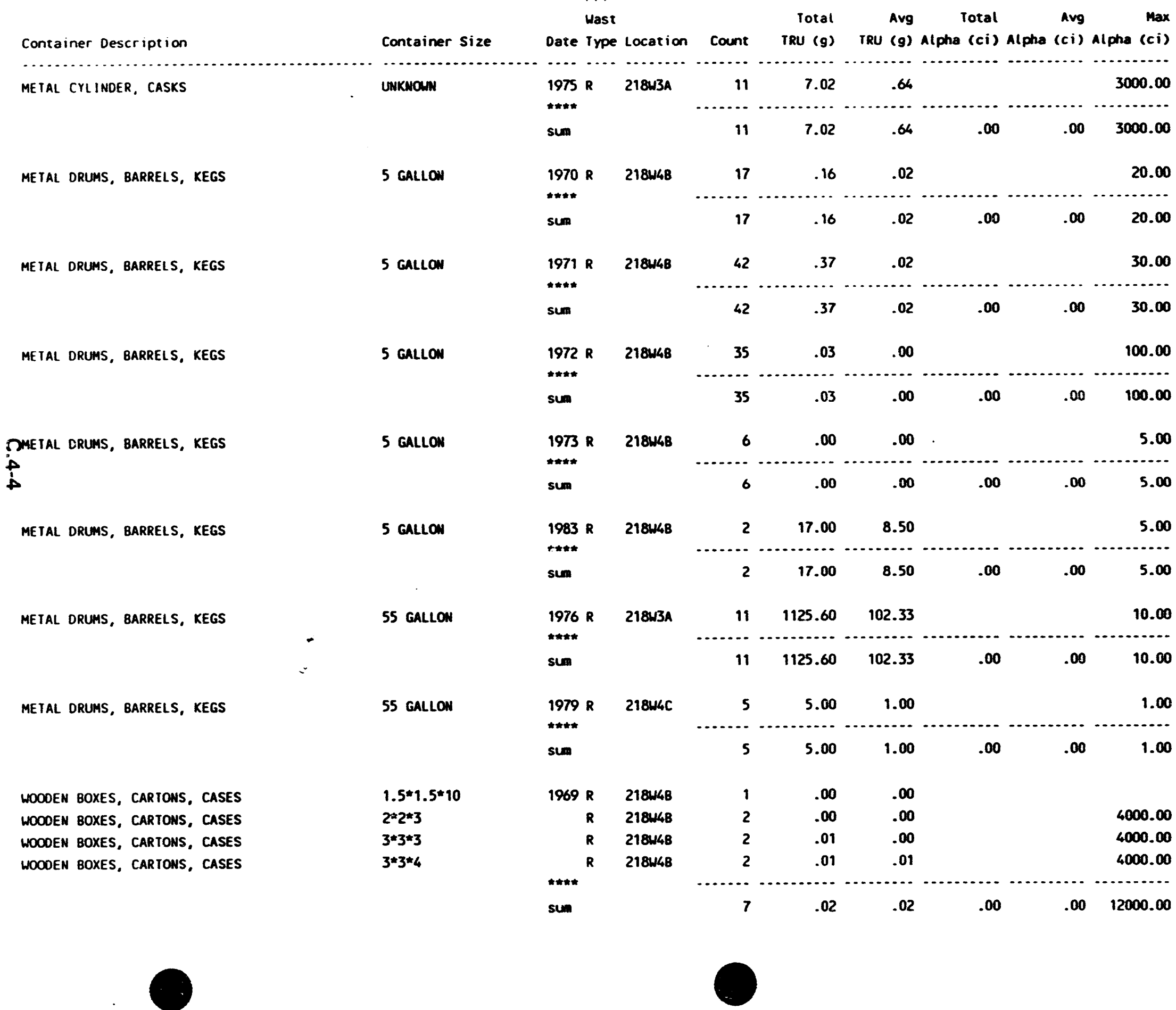


WHC-EP-0696

APPENDIX C.5

PHYSICAL CONTENTS DESCRIPTION FOR BUILDING 325A-PNL 55-GALLON DRUMS CONTAINING TRU WASTE SORTED BY DATE, PRIMARY WASTE TYPE, AND STORAGE FACILITY 


\section{WHC-EP-0696}

This subappendix describes the physical contents of the 55-gallon drums of TRU waste generated by Building 325A-PNL. Prior to 1978, physical contents were not required on the burial records, so a great many of the early records list the contents of the drum as "Miscellaneous."

The printout for this computer run sorts the contents data by storage facility, date and primary waste type. The introduction to Appendix $\mathrm{C}$ contains a table of waste codes and their meanings. 


\section{WHC-EP-0696}

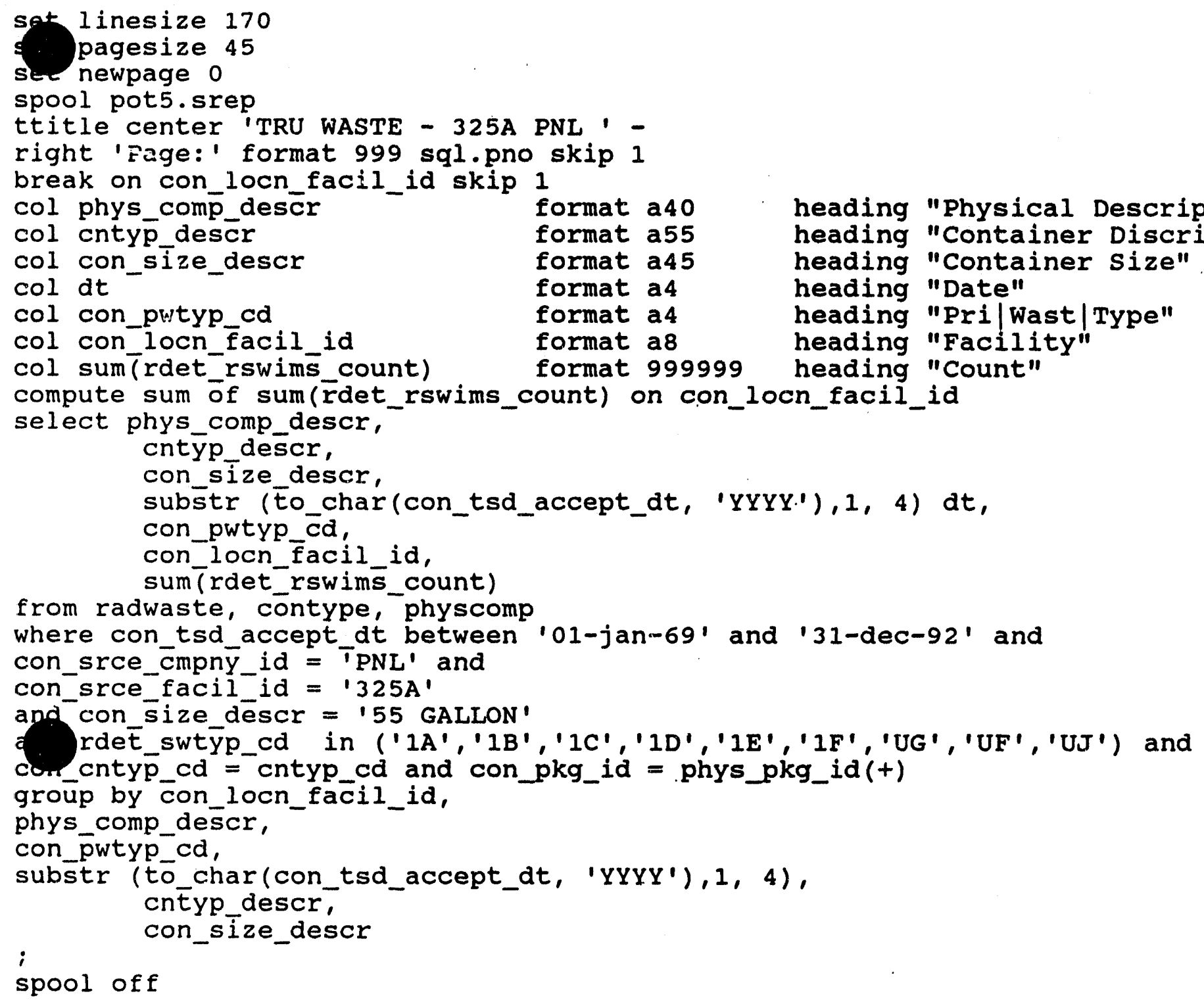




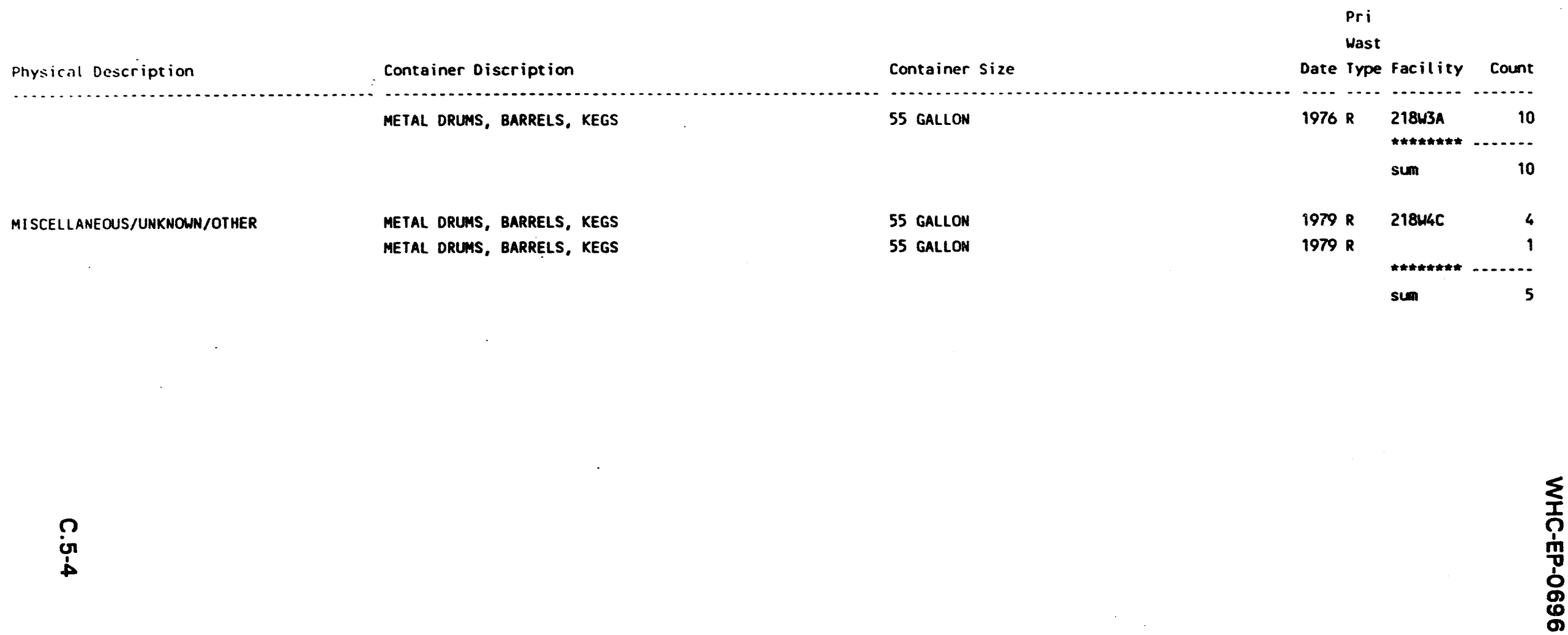


WHC-EP-0696

APPENDIX C.6

PHYSICAL CONTENTS DESCRIPTION FOR BUILDING 325A-PNL TRU WASTE CONTAINERS OTHER THAN 55-GALLON DRUMS SORTED BY DATE, PRIMARY WASTE TYPE, AND STORAGE FACILITY 
This subappendix describes the physical contents of the TRU waste containers other than 55-gallon drums generated at Building 325A-PNL. Prior to 1978, physical contents were not required on the burial records, so a great many of the early records list the contents of the drum as "Miscellaneous."

The printout for this computer run sorts the contents data by storage facility, date and primary waste type. The introduction to Appendix $\mathrm{C}$ contains a table of waste codes and their meanings. 


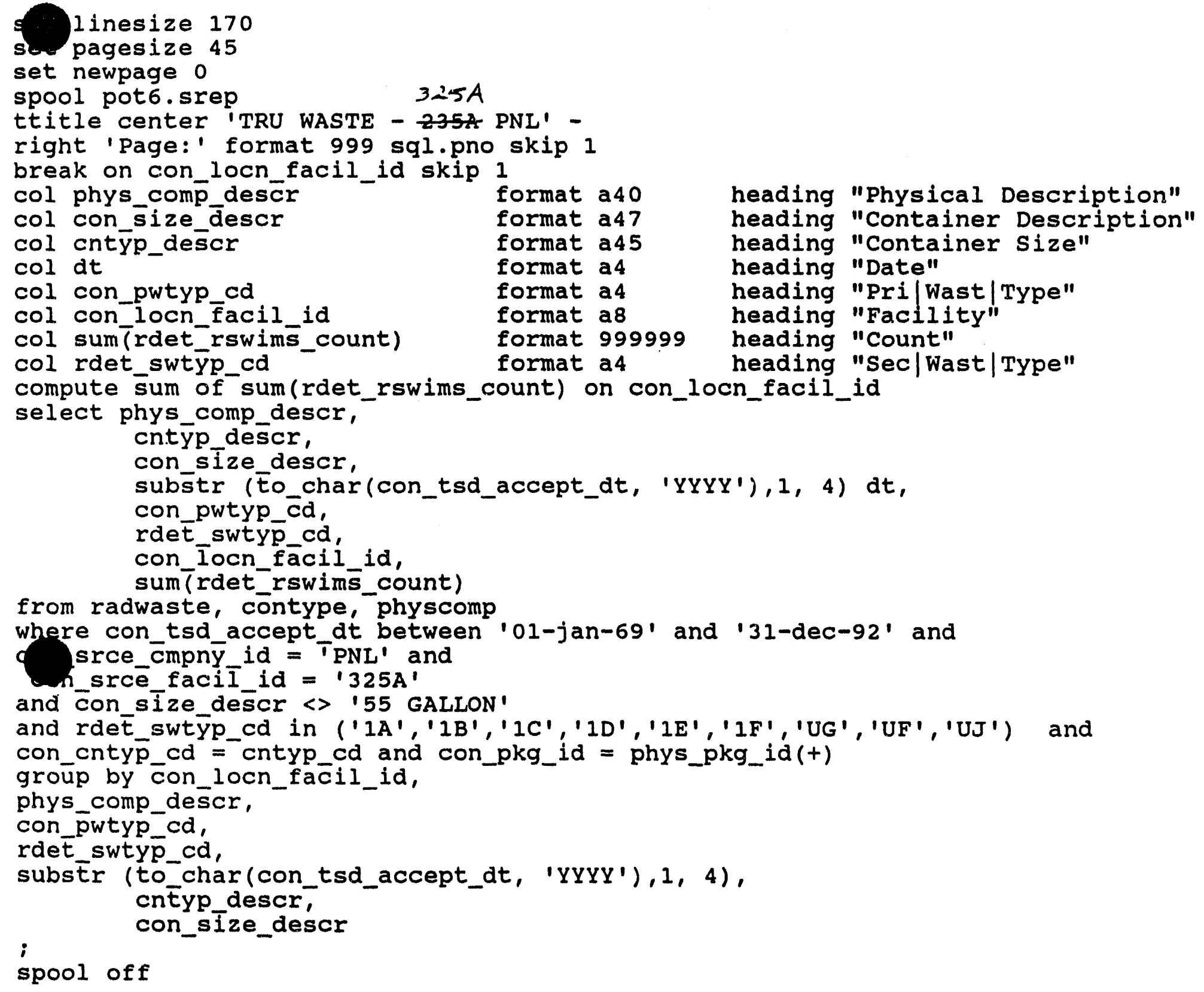

heading "Physical Description" heading "Container Description" heading "Container Size" heading "Date" heading "Pri/Wast/Type" heading "Facility" heading "Count" heading "Sec/Wast/Type" 
Physical Description

Container size

MISCELLANEOUS/UNKNOWN/OTHER

METAL/IRON/GALVANIZED/SHEET MISCELLANEOUS/UNKNOWN/OTHER MISCELLANEOUS/UNKNOWN/OTHER MISCELLANEOUS/UNKNOWN/OTHER MISCELLLANEOUS/UNKNOWM/OTHER OXIDES/POWDERS

$\Omega$
0
$\dot{P}$

12 rows selected.
Container Description

Pei

Wast Wast Date Type Type facility Count

METAL CYLINDER, CASKS

METAL CYLINDER, CASKS

UNKNOWN

UNKNOWN

1975 R $1 A$

1975 R IA

METAL ORUMS, BARRELS, KEGS

WOCOEN BOXES, CARTONS, CASES

WOODEN BOXES, CARTONS, CASES

WOOOEN BOXES, CARTOWS, CASES

WOODEN BOXES, CARTOWS, CASES

METAL DRUMS, BARRELS, KEGS

METAL DRLAMS, BARRELS, KEGS

METAL DRLNS, BARRELS, KEGS

METAL DRUNS, Barrels, KegS

METAL DRUMS, BARRELS, KEGS
5 GALLON
$1.5 * 1.5 * 10$
$2 \star 2 * 3$
$3 * 3 * 3$
$3 * 3 * 4$
5 GALLON
5 GALLON
5 GALLON
5 GALLON
5 GALLOW

\begin{tabular}{|c|c|c|c|}
\hline 1975 R & $1 A$ & $21843 A$ & 8 \\
\hline \multirow[t]{3}{*}{1975 R } & 1A & & 3 \\
\hline & & \multicolumn{2}{|c|}{ 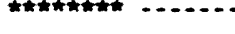 } \\
\hline & & sum & 11 \\
\hline $1983 \mathrm{R}$ & IF & 2181448 & 1 \\
\hline 1969 R & UG & & 1 \\
\hline 1969 R & UG & & 2 \\
\hline $1969 \mathrm{R}$ & UG & & 2 \\
\hline 1969 R & UG & & 2 \\
\hline $1983 R$ & 1F & & 1 \\
\hline $1970 \mathrm{R}$ & If & & 17 \\
\hline $1971 R$ & If & & 38 \\
\hline 1972 R & If & & 35 \\
\hline 1973 R & If & & 6 \\
\hline & & then & \\
\hline & & sum & 105 \\
\hline
\end{tabular}

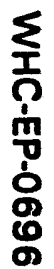


WHC-EP-0696

APPENDIX C.7

HAZARDOUS CONSTITUENTS OF 55-GALLON DRUMS CONTAINING TRU WASTE

C.7-1 
The presence of hazardous constituents in a radioactive waste drum designates the waste as "Mixed" (primary waste code "M"). In this subappendix the hazardous contents of 55-gallon drums generated at Building 325A-PNL are sorted by storage facility.

Information concerning the hazardous constituents of waste containers was not required prior to 1986. During the R-SWIMS data reentry program in the mid-1980's an attempt was made to add any available information on the hazardous materials present, however, this information was limited. 


\section{WHC-EP-0696}

set 1 inesize 170

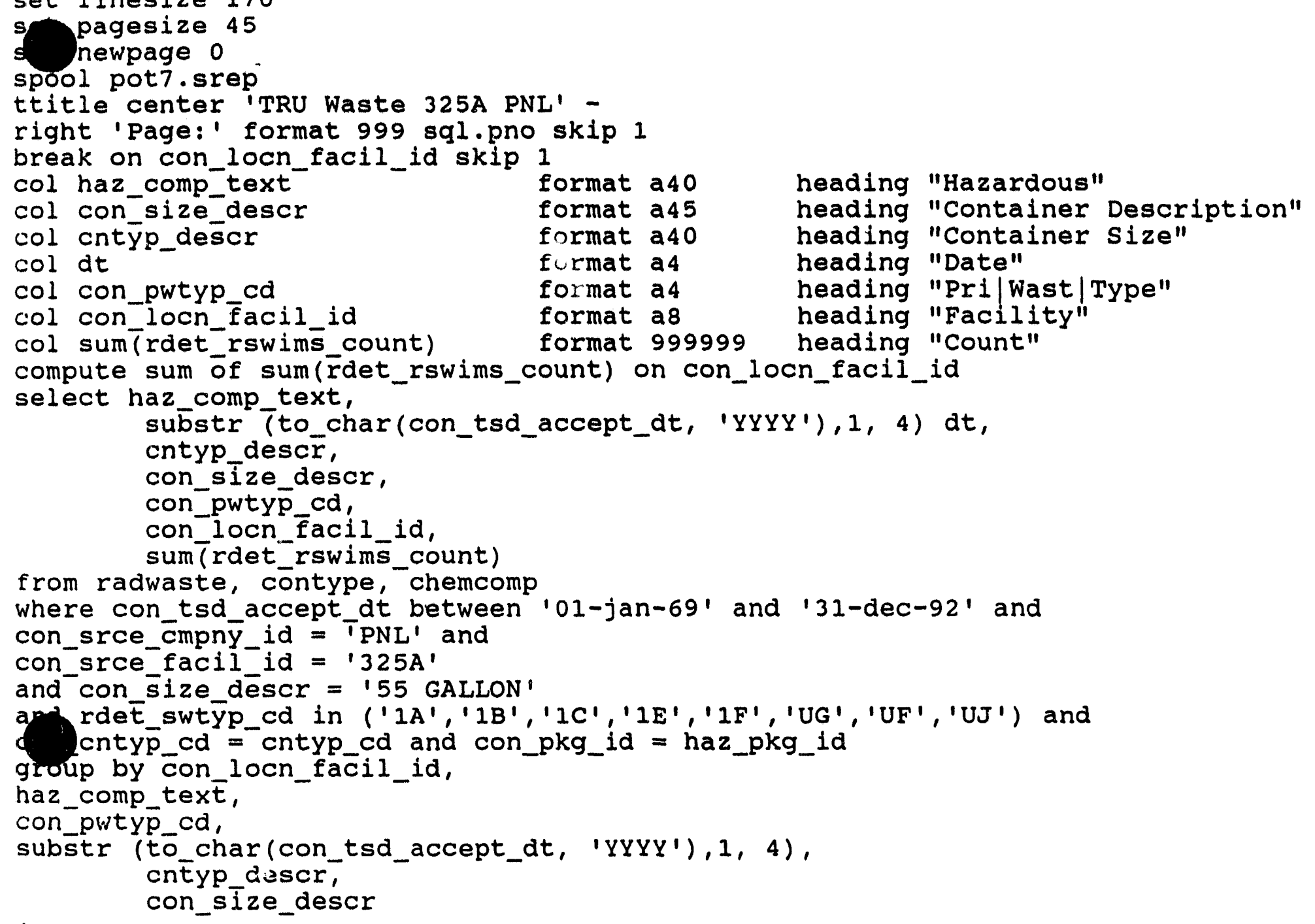


WHC-EP-0696

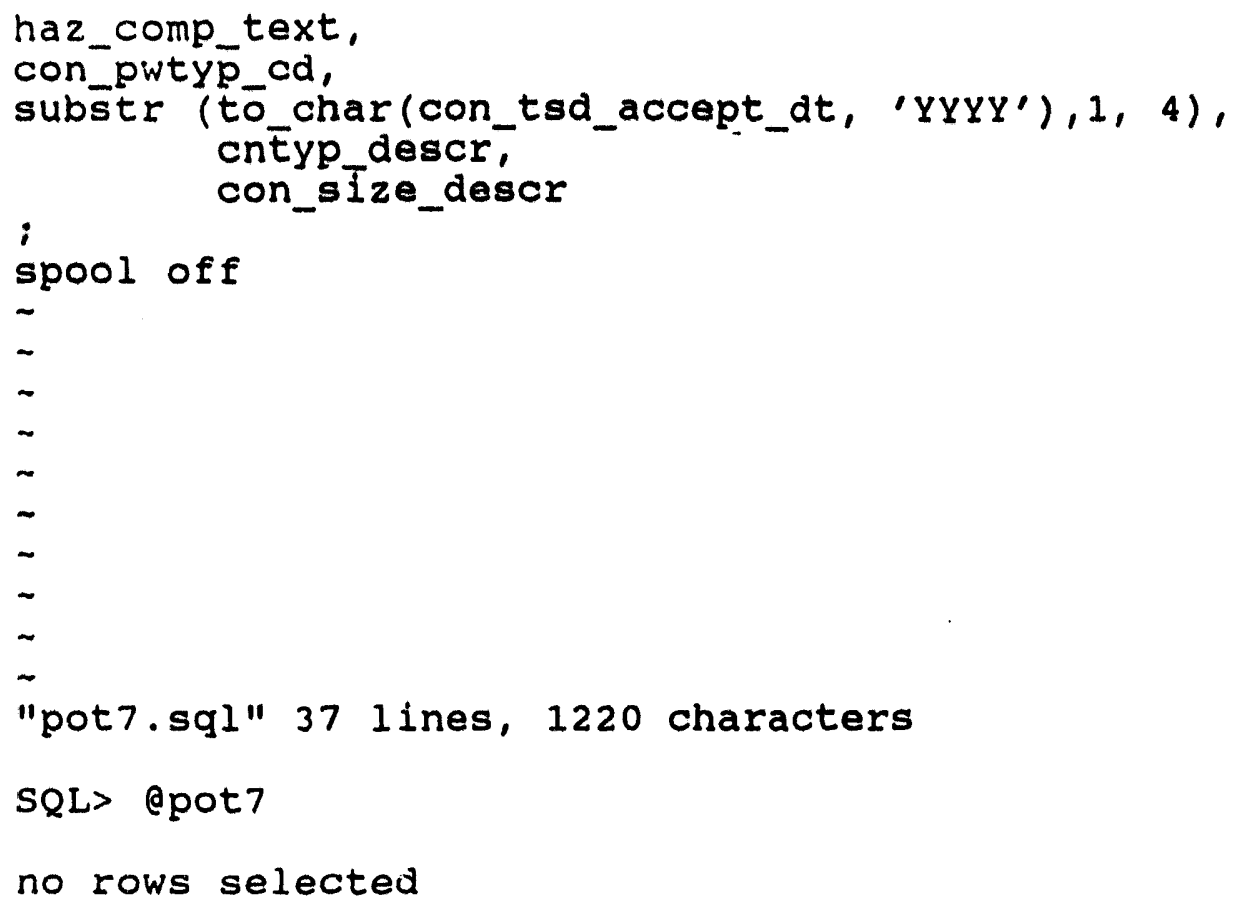




\section{WHC-EP-0696}

APPENDIX C.8

HAZARDOUS CONSTITUENTS OF TRU WASTE CONTAINERS OTHER THAN 55-GALLON DRUMS 
The presence of hazardous constituents in a radioactive waste drum designates the waste as "Mixed" (primary waste code "M"). No hazardous constituents are listed in containers other than 55-gallon drums generated at Building 325A-PNL; therefore this run did not reveal any hazardous information. 

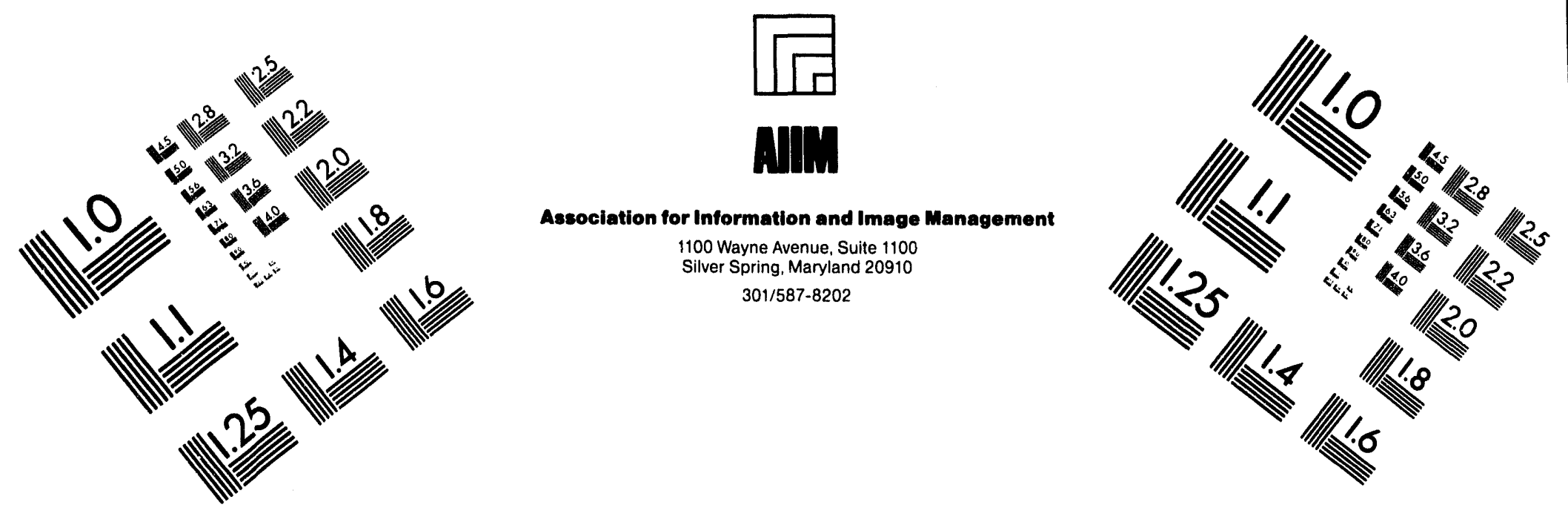

\section{Centimeter}

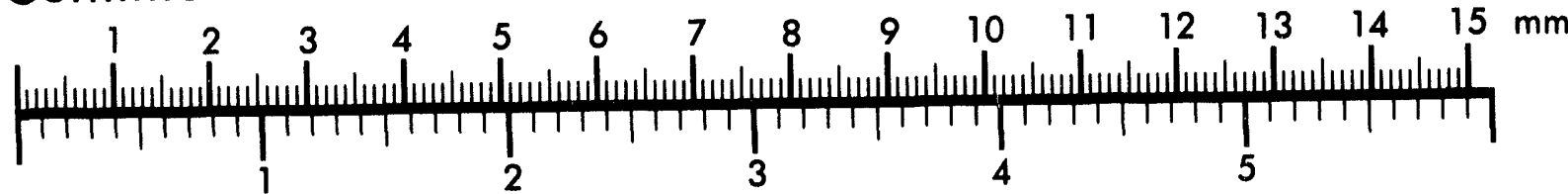
Inches
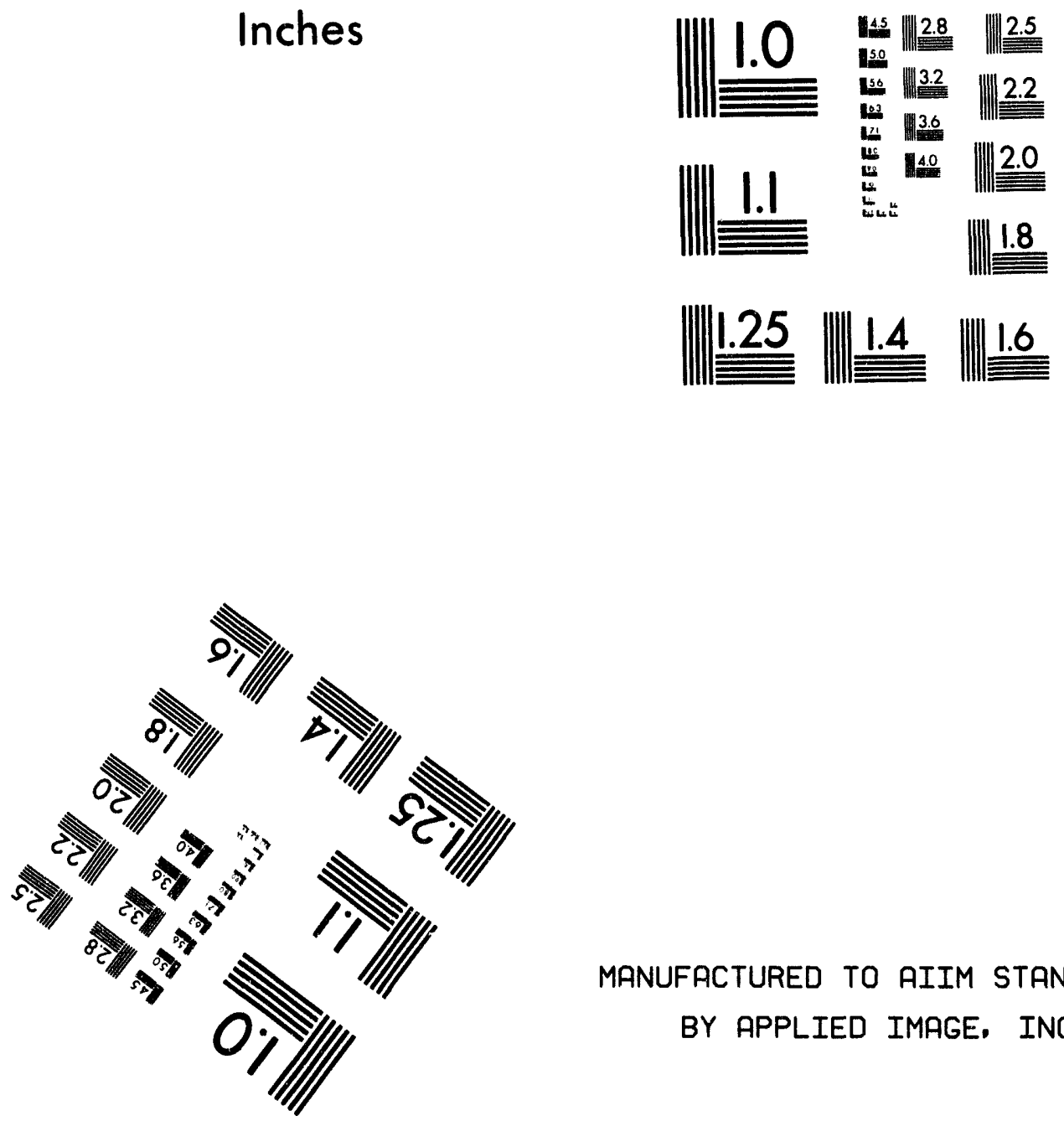

MANUFACTURED TO AIIM STANDARDS BY APPLIED IMAGE, INC.

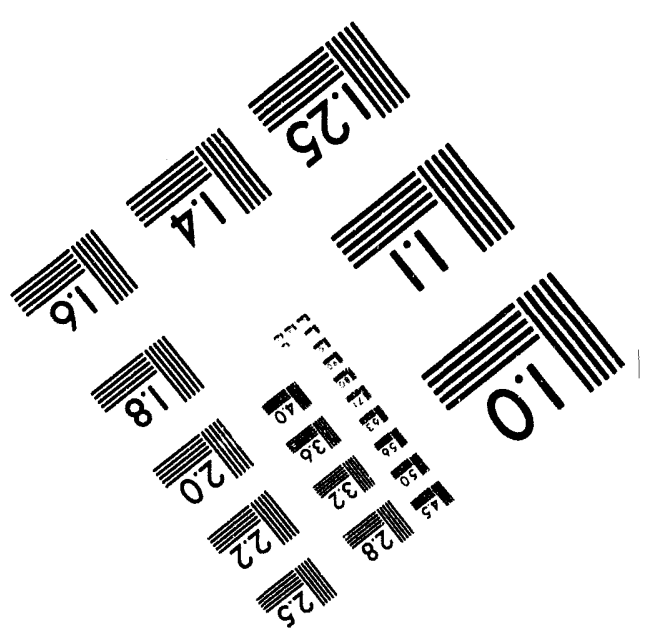



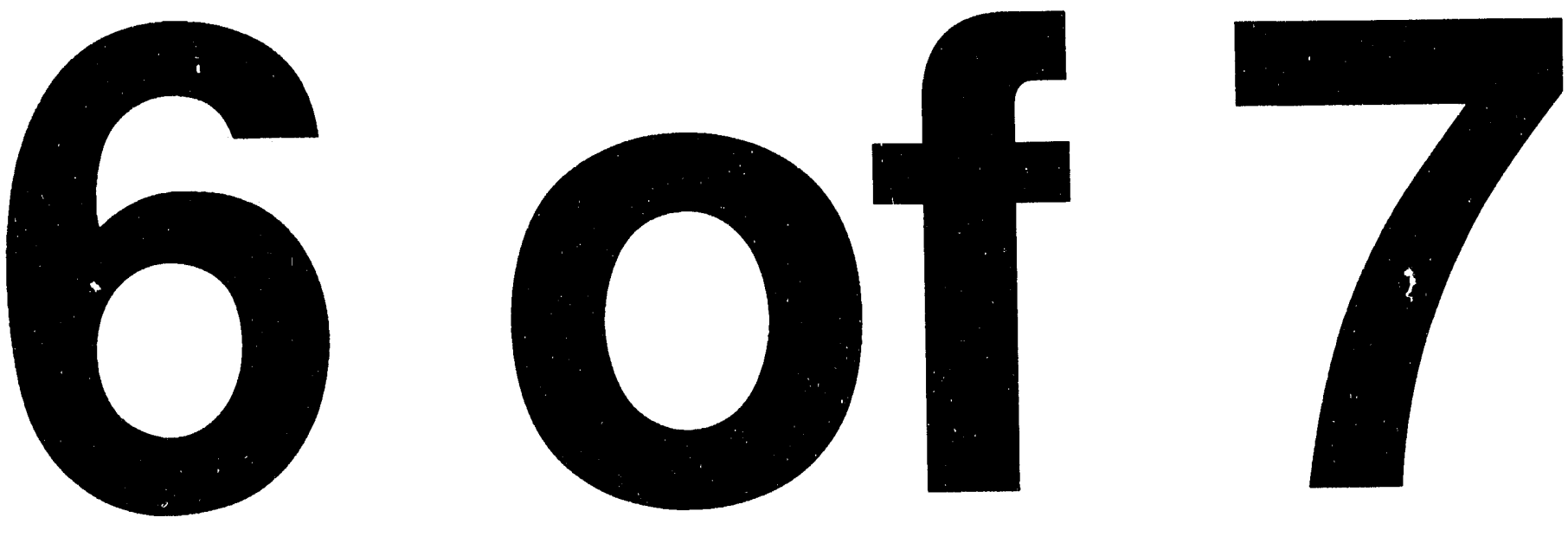


\section{WHC-EP-0696}

set 1 inesize 170

pagesize 45

newpage 0

spool pot8.srep

ttitle center 'TRU waste 325A PNL' -

right 'Page:' format 999 sql.pno skip 1

break on con_locn_facil_id skip 1

col haz_comp_text

col con_size_descr

format a 40

format a 45

col cntȳp descr

col dt

format a 40

col con_pwtyp_cd

format a 4

col con locn facil id

format a 4

col sum(rdet_rswims_count)

format a 8

select haz cömp_texE,

format 999999

heading "Hazardous"
heading "Container Description"
heading "Container Size"
heading "Date"
heading "Pri|Wast|Type"
heading "Facility"
heading "Count"

substr (to_char(con_tsd_accept_dt, 'YYYY'), 1, 4) dt,

cntyp_descr,

consize descr,

con_pwtyp_cd,

con_locn_facil_id,

rdet_rswims_coünt

from radwastēe, contȳpe, chemcomp

where con_tsd_accept_dt between '01-jan-69' and ' 31 -dec-92' and

con_srce_cmpnȳ_id = 'TPL' and

con_srce_facil-id $=$ ' $325 \mathrm{~A}$ '

and con_size_déscr $\longleftrightarrow 155$ GALLON'

and rde $\bar{t}_{\text {swt }}$ swp_cd in ('IA', 'IB', 'IC','IE','IF', 'UG', 'UF', 'UJ') and

9 cntyp_cd $=-c n t y p \_c d$ and con_pkg_id = haz_pkg_id

ip by con_locn_facil_id,

har_comp_text,

con_pwtyp_cd,

cñtyp_descr,

substr (to_chār(con_tsd_accept_dt, 'YYYY'), 1, 4), con size dešcr, rdeE_rswíms_count

i

spool off 


\section{WHC-EP-0696}

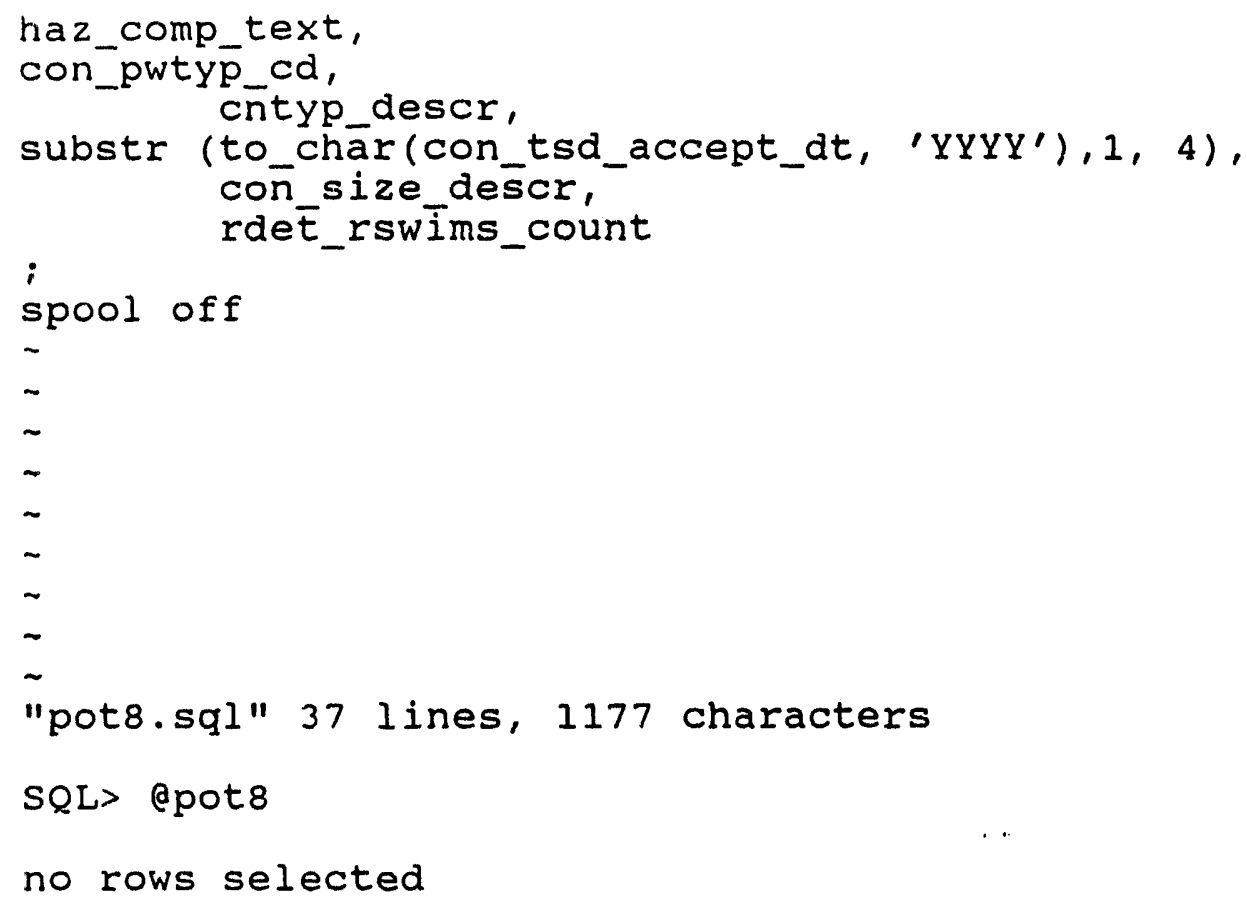


WHC-EP-0696

APPENDIX C.9

ISOTOPES LISTED IN 55-GALLON DRUMS CONTAINING TRU WASTES

C.9-1 
This subappendix contains a listing of the SWITS isotope information for TRU waste from Building 325A-PNL stored in 55-gallon drums. The isotope field in SWITS contains several types of the following information:

- Specific isotope (e.g., Am-241, Np-237, U-233, Pu-238)

- Generic isotope listings (e.g., Pu, uranium-enriched, uranium-depleted.)

- $\quad$ Plutonium-239 fissile gram equivalents (Pu239 FSL GR equiv).

- Total - alpha

- Total beta/gamma

- $\quad$ PE-Ci (plutonium equivalent curies).

This computer run contains the isotope field listing along with the number of TRU waste drums with that listing. The run is sorted by storage facility and year. 


\section{WHC-EP-0696}

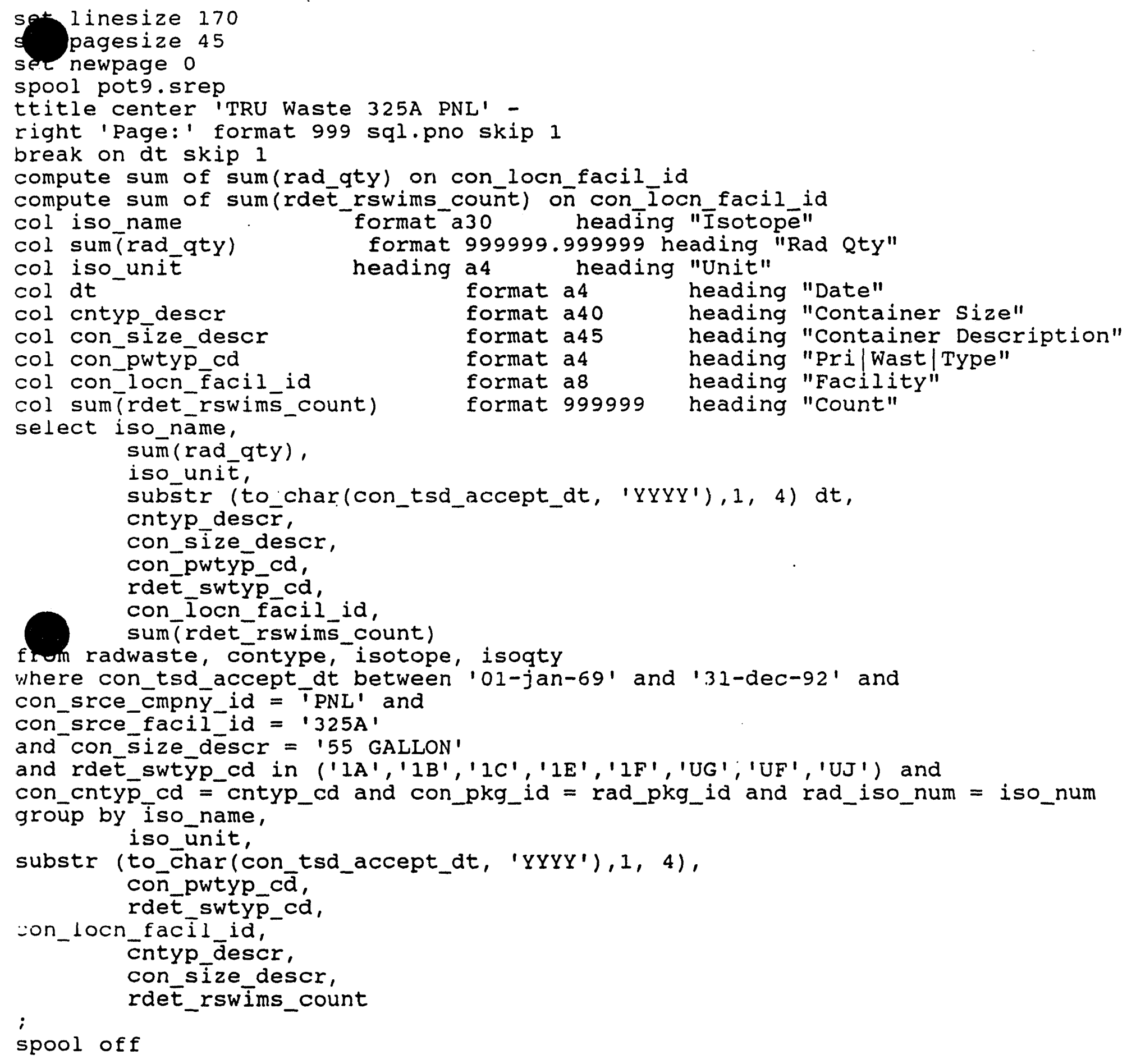


PU

Rad oty Un Date Container Size

1121.600000 GM 1976 METAL DRUMS, BARRELS, KEGS 1121.600000 ***

sum

PU

TH-232

TOTAL BETA/GAMMA

TOTAL BETA/GAMMA

0

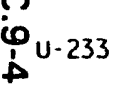

5.000000 GM 1979 METAL DRUMS, BARRELS, KEGS -...............

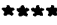

$5.000000 \mathrm{sum}$

22600.000000 GM 1976 METAL DRUMS, BARRELS, KEGS $.070000 \mathrm{Cl}$

METAL DRUMS, BARRELS, KEGS

$$
22600.070000
$$

sum

.005000 CI 1979 METAL DRUMS, BARRELS, KEGS

.005000

4.000000 GM 1976 METAL DRUMS, BARRELS, KEGS

4.000000

4.000000

sum
Container Description

55 GALLON

55 GALLON

55 GALLON

55 GALLON
Pri Sec

Wast Wast

Type Type Facility Count

R 1A 218W3A 10

r...

R $\quad 218 W 4 \mathrm{C}$

R IA 218W3A 10

R IA 218W3A 10

20

R IA 218W3A

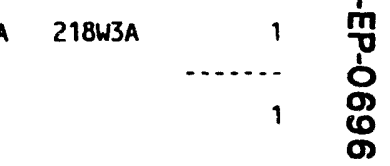

6 rows selected. 
WHC-EP-0696

APPENDIX C.10

ISOTOPES LISTED IN TRU CONTAINERS

OTHER THAN 55-GALLON DRUMS

C. 10-1 


\section{WHC-EP-0696}

This subappendix contains a listing of the SWITS isotope information for TRU waste from Building 325A-PNL stored in containers other than 55-gallon drums. The isotope field in SWITS contains several types of the following information:

- $\quad$ Specific isotope (e.g., Am-241, Np-237, U-233, Pu-238)

- Generic isotope listings (e.g., Pu, uranium-enriched, uranium-depleted)

- Plutonium-239 equivalent curies (PE-CI)

- Total-alpha

- Total beta/gamma

- PE-Ci (plutonium equivalent curies).

This computer run contains the isotope field listing along with the number of TRU waste containers with that listing. The run is sorted by storage facility and year. 


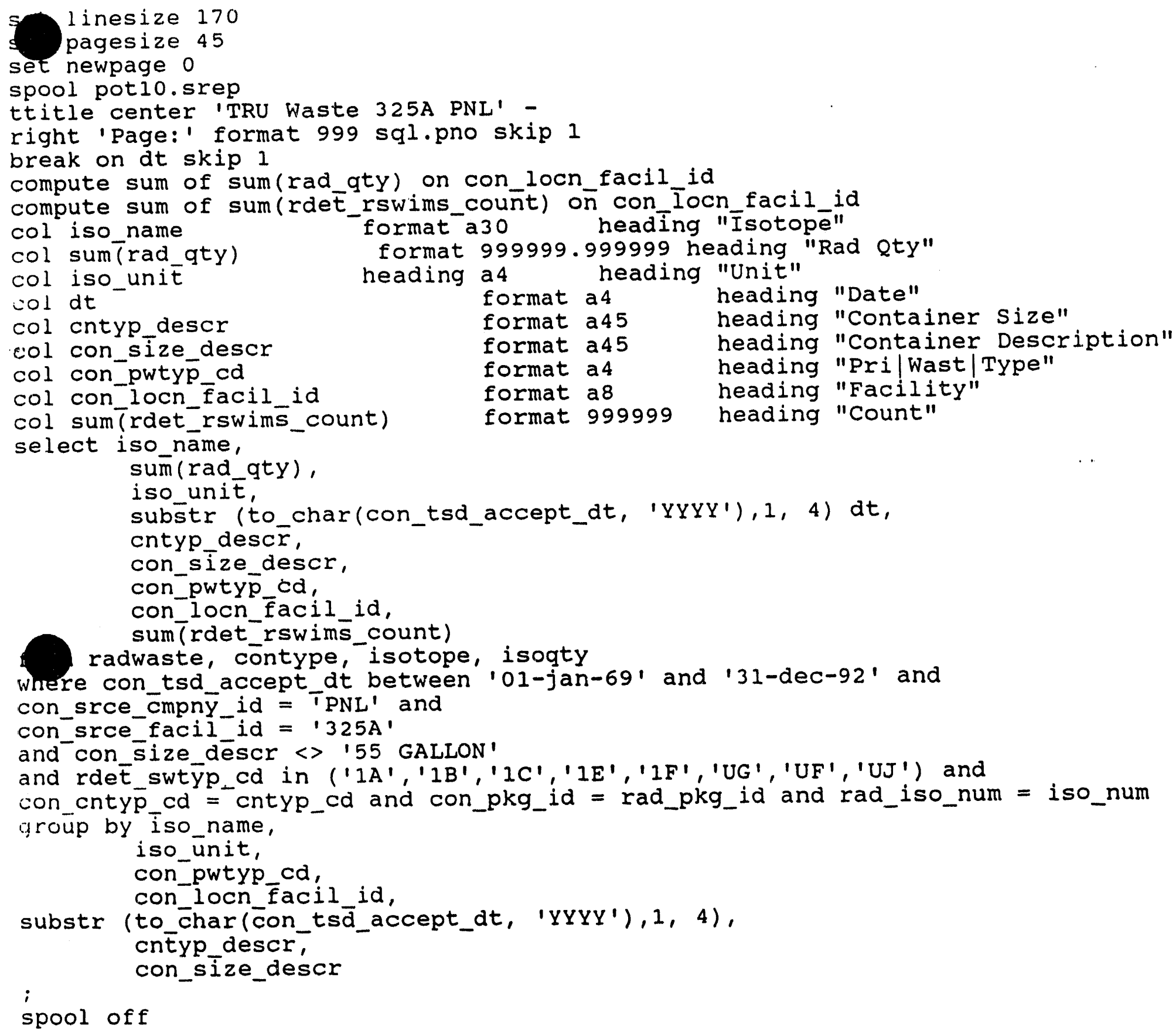


Wast

Container Description

Type facility Count

$.103750 \mathrm{Cl} 1969$ WOODEN BOXES, CARTONS, CASES $.116550 \mathrm{cl}$ WOODEN BOXES, CARTONS, CASES $.279700 \mathrm{Cl}$ WOOOEN BOXES, CARTONS, CASES

$2 * 2 * 3$

$3 \star 3 \star 3$

$3 * 3 * 4$

.500000

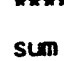

.011000 GM 1971 METAL DRUMS, BARRELS, KEGS

.011000

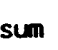

7.019000 GM 1975 METAL CYLINDER, CASKS

7.019000

$.003340 \mathrm{GM} 1969$ WOOOEN BOXES, CARTONS, CASES $.002075 \mathrm{GM}$ WOODEN BOXES, CARTONS, CASES $.008991 \mathrm{GM}$ WOOOEN BOXES, CARTONS, CASES $.005594 \mathrm{GM}$ WOODEN BOXES, CARTONS, CASES

.020000 sum

$.160000 \mathrm{GM} 1970$ METAL DRUMS, BARRELS, KEGS

160000 sum

.361000 GM 1971 METAL DRUMS, BARRELS, KEGS

361000

$.033300 \mathrm{GM} 1972$ METAL DRUMS, BARRELS, KEGS 033300

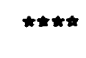

\section{GALLON \\ UNKNOWN \\ $1.5 * 1.5 * 10$ \\ $2 \star 2 \star 3$ \\ $3 \star 3 * 3$ \\ $3 \star 3 \star 4$ \\ 5 GALLON \\ 5 GALLON \\ 5 GALLON}

R 218W4B

R $218 W 48$

R 218W4B

R 218W4B $\quad \begin{array}{r}\ldots \\ \\ \end{array}$

R 218W3A

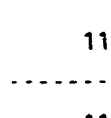

R 218W4B

R 218W4B

R 218W48

R $218 W 4 \mathrm{~B}$

1

2

R $218 W 48$

17

R 218 W4B

38
$\ldots$

R 218W4B 35 
Wast

Container Description
.000500 GM 1973 METAL DRUMS, BARRELS, KEGS

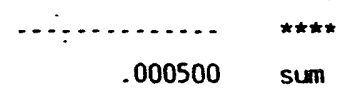

PU

RU-106:-RH-106
SR-90-Y90

TH -232

.7

?̦

TOTAL BETA/GAMMA

TOTAL BETA/GAMMA

TOTAL BETA/GAMMA

TOTAL BETA/GAMMA

TOTAL BETA/GAMMA

TOTAL BETA/GAMMA

TOTAL BETA/GAMMA
.000100 GM 1983 METAL DRUMS, BARRELS, KEGS

.000100

1.010000 CI 1971 METAL DRUMS, BARRELS, KEGS

$30.000000 \mathrm{Cl}$

METAL DRUMS, BARRELS, KEGS

31.010000 sum

8200.000000 GM 1983 METAL DRUMS, BARRELS, KEGS

8200.000000

sum

41.130000 CI 1975 METAL CYLINDER, CASKS

.............

41.130000 sum

$.016700 \mathrm{CI} 1969$ WOODEN BOXES, CARTONS, CASES

$.103750 \mathrm{Cl}$ WOOOEN BOXES, CARTONS, CASES

$.149850 \mathrm{Cl}$ WOODEN BOXES, CARTONS, CASES

$.279700 \mathrm{CI}$ WOODEN BOXES, CARTONS, CASES *ネ*

.550000 sum

$720.174000 \mathrm{Cl} 1970$ METAL DRUMS, BARRELS, KEGS -.............

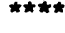

720.174000

sum

$800.006200 \mathrm{CI} 1971$ METAL DRUMS, BARRELS, KEGS

800.006200 sum

5 GALLON

5 GALLON

5 GALLON

5 GALLON

5 GALLON

UNKNOWN

$2 * 2 * 3$

$3 * 3 \star 3$

$3 \star 3 \star 4$

5 GALLON
5 GALLON

$1.5 * 1.5 * 10$

$218 W 4$

$218 W 4 B$

218W3A

$218 W 48$

R 218W4B

R $218 W 48$

218W4B

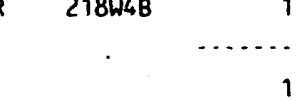

11
11

R $218 W 4 B$

R $218 W 4 B$

R $218 W 4 B$

R $218 \mathrm{W4B}$

1

2

2

7

17

R 218W4B 38

38

R 218W4B 33 
Wast

Container Description

Type facility

Count

I sotope

Rad aty Un Date Container Size

GALLON

R

$218 W 4 B$

010000

$161500 \mathrm{CI} 1983$ METAL DRUMS, BARRELS, KEGS $17.000000 \mathrm{GM}$ METAL DRUMS, BARRELS, KEGS

5 GALLON

5 GALLON

$\geq 0$ rows selected.

$\stackrel{?}{\overrightarrow{0}}$

$U-233$

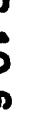




\section{WHC-EP-0696}

\section{APPENDIX D}

DATA ON BUILDING 325B WASTE GENERATION BY WESTINGHOUSE HANFORD FROM THE SOLID WASTE INFORMATION TRACKING SYSTEM 
The information found in this appendix is from the SWITS database. This database incorporates the older R-SWIMS database and is used to track information on radioactive and other wastes stored or disposed at the Hanford Site.

Each SWITS data run in this appendix is preceded by the query used to generate the data. A brief explanation of the run and any additional information needed to understand the data presented is also included.

The bulk of the data provided is limited to information about TRU waste generated at Building 325B-Westinghouse Hanford; however, some general information on the non-TRU waste is included for completeness. The term non-TRU is used, instead of LLW, because a small percentage of the waste has been designated only as not TRU. It is believed that most, if not all, of the non-TRU waste is LLW.

The data runs in this appendix are further segregated by waste container type. Since initial retrieval efforts and WRAP 1 will focus on 55-gallon drums, these container types are considered separately.

Some general information about SWITS database and the codes used follows. Please note the disclaimer found on the next page. 


\section{WHC-EP-0696}

DON'T SAY IT - Write It I

Date:

To:<smiles>CC(Cl)C1CCCCC1C(Cl)Cl</smiles><smiles>C1CCC(C2CCCC2)CC1</smiles>

From: Solld Waste Engineering Data Management Group N3-11 6-4394/6-4020

Re: SWITS DATA REQUEST

Attached for your information and use is the data which you requested. This data represents best available information regarding wastes currently in storage at the Hanford site. I trust the information will be suitable to your needs.

Requests for information from the Solid Waste Information and Tracking System (SWITS) are normally relatively limited in scope, requesting specific data fields or summary data. The responses to these requests undergo review during data collection, summary and response preparation.

The response to this request represents a simple reproduction of the sWITS Database. Transmittal of this information is made with the following disclaimers:

1) The information contained in this transmittal is raw data, and represents information provided to solid Waste Engineering (SWE) on burial records or other documents. This data has not been validated.

2) The information contained in this transmittal is subject to change without notice. Continual update of swITs information and improvement of the software system make it impossible to ensure consistency of this data with the database after transmittal.

3) This information is current as of $12 / 11 / 22$.

If I can be of further assistance to you, do not hesitate to call me. 
WHC-EP-0696

This page intentionally left blank.

D-4 
WHC-EP-0696

SWIR401

Solid Waste Information and Tracking system

$05 / 01 / 92$

$13: 25$

Páge: 1

Primary Waste Type code Table

Primary Waste Type Code

AH

CM.

H

HP

$M$

MP

N

$\mathbf{P}$

$\boldsymbol{R}$

RP

U
Primary Waste Type Description

HAZ, ADMIN HOLD

MORÁTORIUM CONTROIID

HAZARDOUS

HAZARDOUS WASTE PCB

MIXED

MIXED PCB

NON-REGULATED

PCB

RADIOACTIVE

RADIOACTIVE PCB

UNKNOWN
Ship

Time Rad

-

89

89

89

29

89

29

29

29
$-\frac{1}{N}$

N

N

N

$Y$

$Y$

N

N

$Y$

X 
WHC-EP-0696

Secondary Waste Type code Table

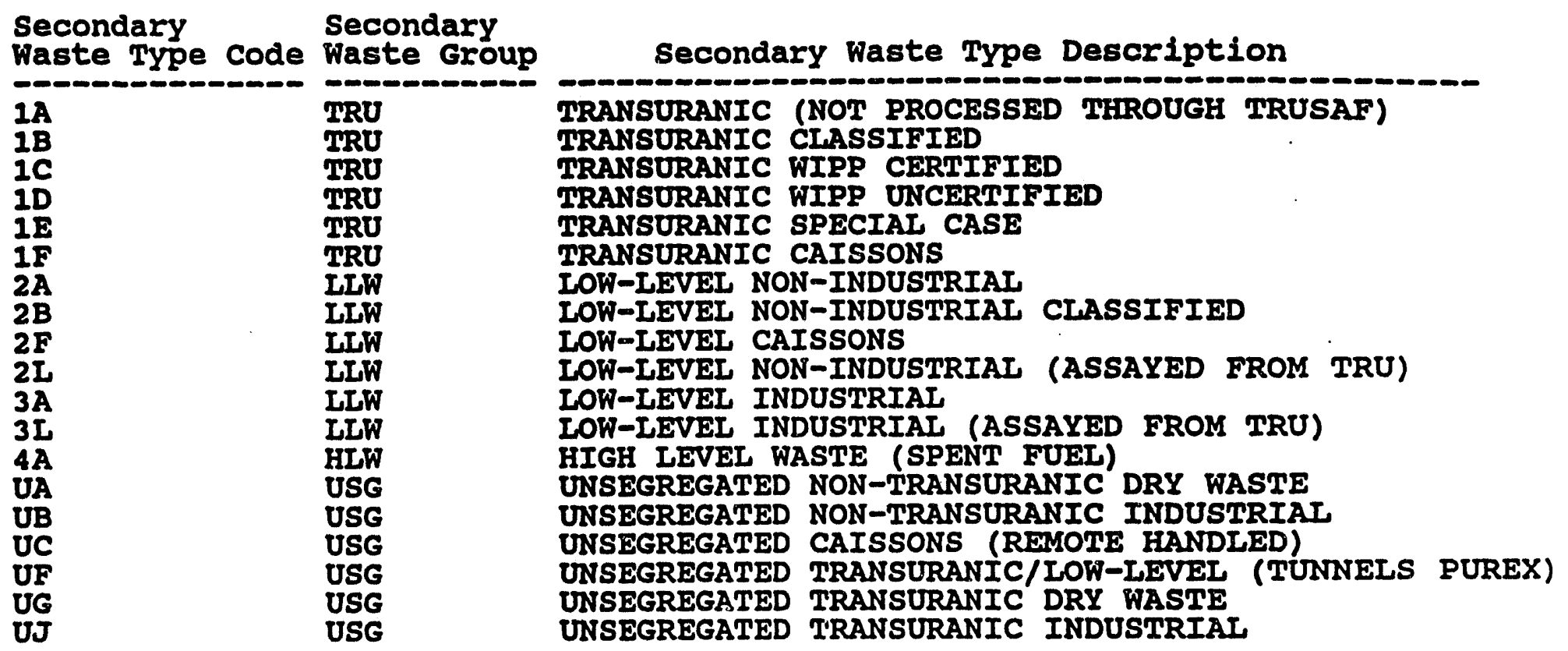


WHC-EP-0696

APPENDIX D.1

CONTAINER NUMBER AND WEIGHT OF TRU WASTE IN 55-GALLON DRUMS BY WASTE TYPE

D.1-1 
This data run gives an overall look at the waste types, container numbers and total weight (in kilograms) of the radioactive wastes generated at Building 325B-Westinghouse Hanford on a yearly basis. These data are for wastes stored in 55-gallon drums only. The meanings of primary and secondary waste codes can be found in the introductory materials for this appendix.

The data in the average weight column should be taken cautiously, especially for LLW codes. Concern is warranted because LLW records are still done on a batch basis. This means that one weight datum may be the composite weight for a group of drums. The computer program used simply divided the sum of all weight values by the number uf values used to compute the total weight, not the actual number of containers that that value represents.

From 1970 until 1978 individual container weights were not required for TRU drums. During the data reentry program in the mid-1980's standard weights were assigned for all container types; 55-gallon drums were given a standard weight of $68 \mathrm{~kg}$. This is why the average weight/drum for TRU is so consistent during this period. 
set linesize 170

set pagesize 45

newpage 0

l pot2.srep

ttitle center '325B WHC ' -

right 'Page:' format 999 sql.pno skip 1

break on dt skip 1

compute sum of sum(con_gross_wgt) on dt

compute sum of sum(rdet_rswims_count) on dt

compute sum of sum(con_cntr_voI) on dt

col dt

col con_pwtyp_cd

col rdet_swtyp_cd

col sum (ŕrdet_rswims_count)

\section{format a 4}

format a 4

format a 4

col avg (con_gross_wgt)

format 999999

format 99999.99 heading

heading "Date"

col sum (con_gross_wgt)

format 9999999.99

col sum(con_cntr_vol)

format 99999.99 he

col avg (con_cntr_vol)

format 99999.99 heading "i.

format a 30

col cntyp_déscr

format a25 heading "Conta
accept_dt, 'YYYY'), 1, 4) dt,

heading "Pri|Wast/Type"

heading "Sec|Wast|Type"

heading "Count"

col con size descr

"Avg|Weight"

heading "sum/Weight"

select cntyp descr $\bar{r}$,

con_size_descr,

con_pwty $\bar{p} \_c d$,

rdet_swtyp_cd,

sum( (rdet_rswims_count),

sum (con_ōross_wgtt),

avg (con_gross_wgt),

sum (con_cntr_vol),

avg (con_cntr_vol)

from radwaste, contype

where con_tsd_accept_dt between '01-jan-69' and '31-dec-92' and

con_srce_cmpny

con srce facil-id $=$ ' $325 \mathrm{~B}^{\prime}$

and con_size_déscr $=155$ GALLON' and con_cntyp_cd $=$ cntyp_cd

group by cntȳp_descr, con_size_descr, con_pwtyp_cd,

substr (to_char(con_tsd_accept_dt, 'YYYY'), 1, 4), rdèt_swtyp_cd

;

spool off 


\section{WHC-EP-0696}

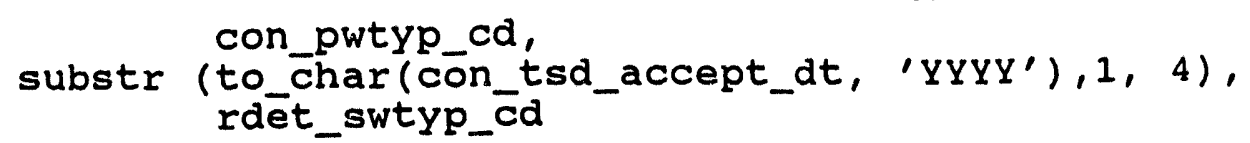


WHC-EP-0696

APPENDIX 0.2

BUILDING 325B-WESTINGHOUSE HANFORD TRU WASTE CONTAINERS SORTED BY CONTAINER DESCRIPTION, SIZE,

YEAR, AND PRIMARY WASTE TYPE 


\section{WHC-EP-0696}

This subappendix provides information on Building 325B-Westinghouse Hanford TRU waste containers, weight, volume, and primary waste type stored at various TRU waste storage facilities on the Hanford Site. The data provides the TRU waste generation from the Building 325B-Westinghouse Hanford Facility by year. 


\section{WHC-EP-0696}

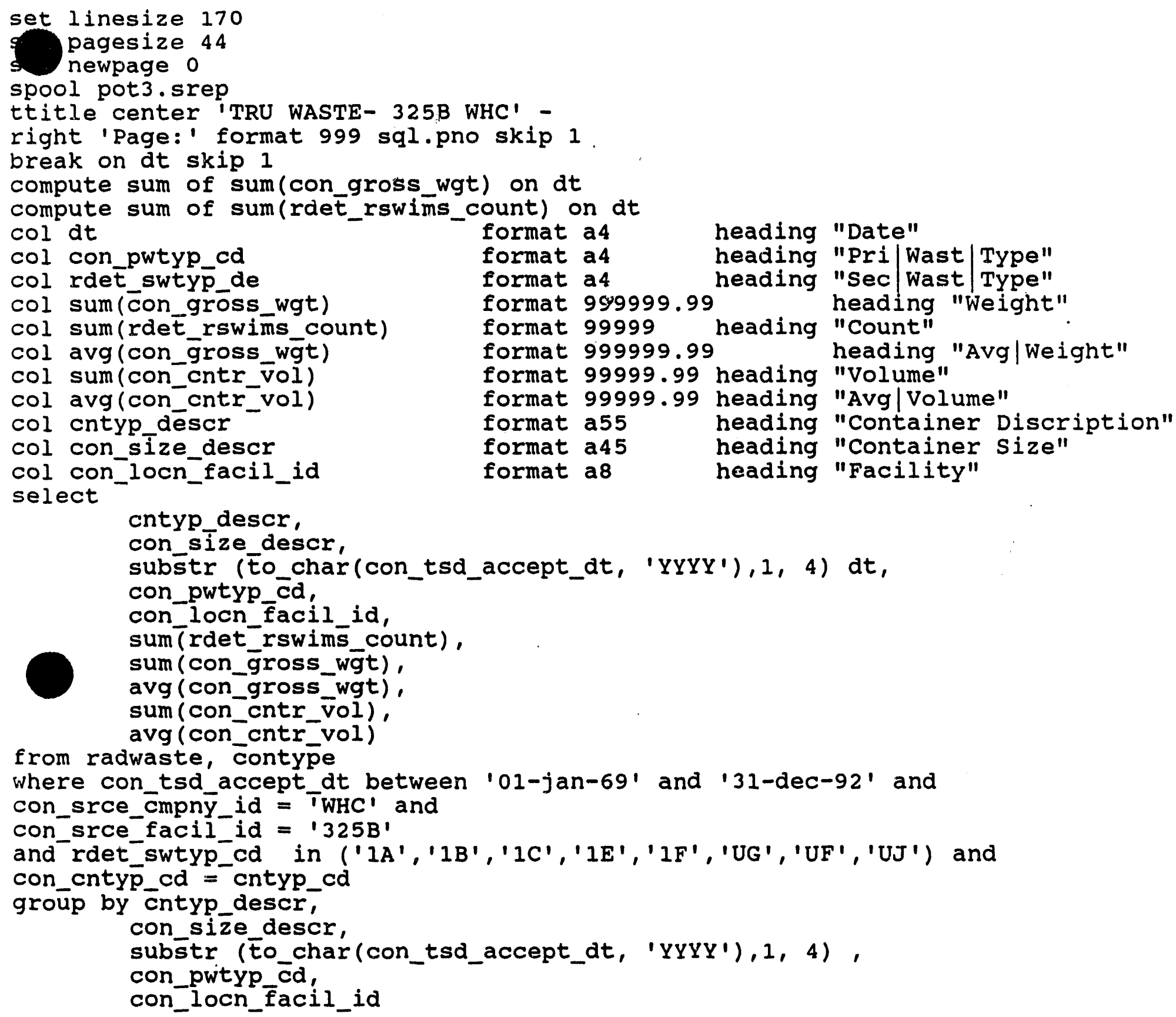


TRU WASTE- 325B WHC

Page:

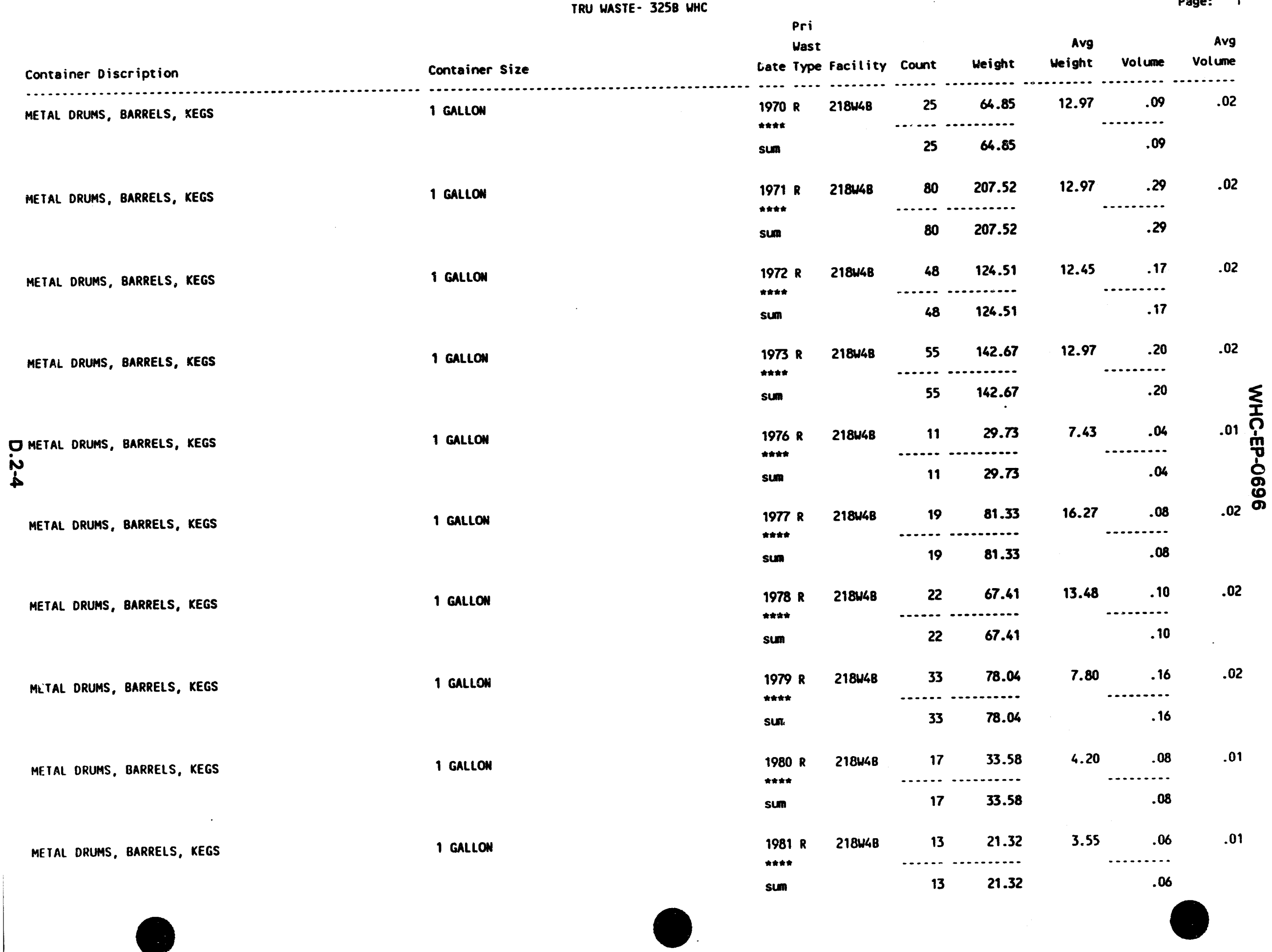




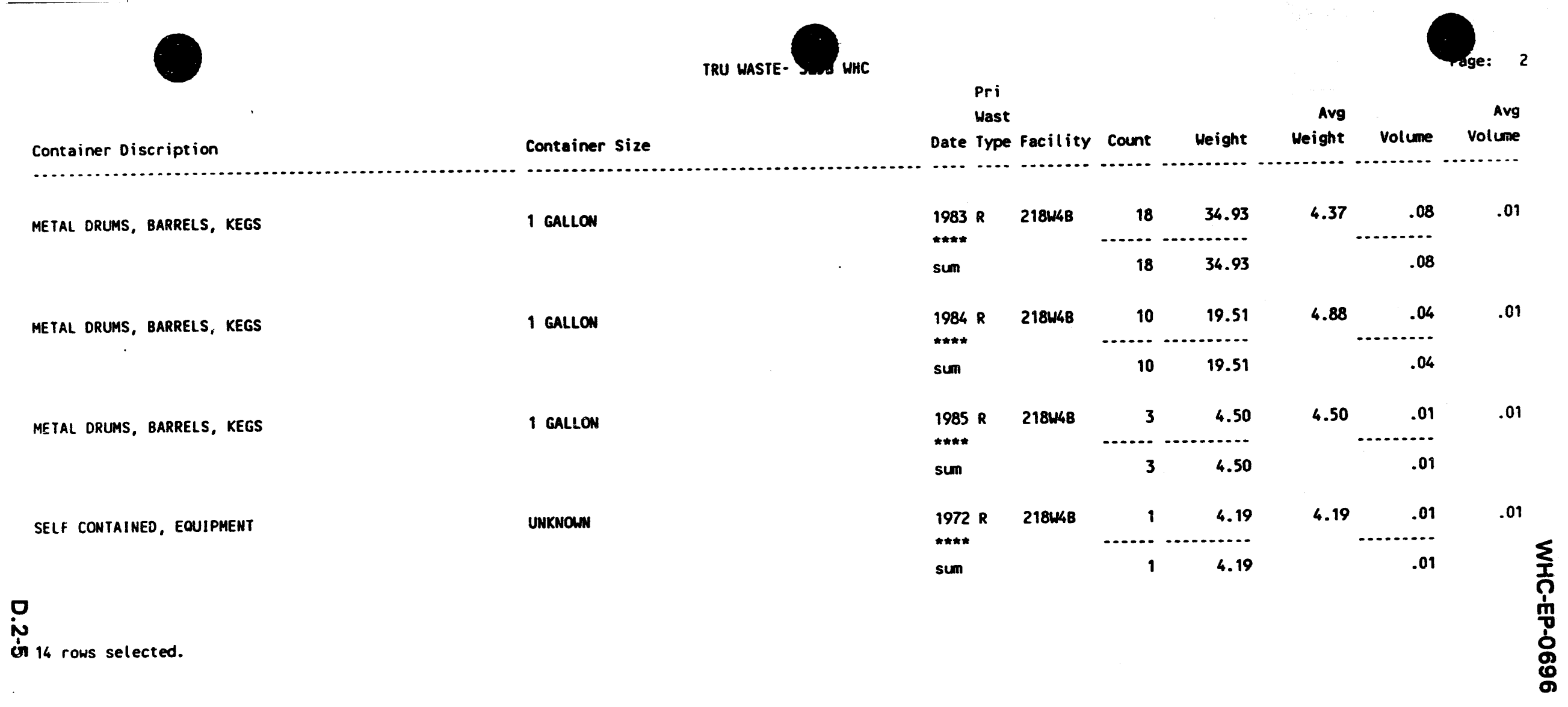


WHC-EP-0696

This page intentionally left blank.

D.2-6 
WHC-EP-0696

APPENDIX 0.3

BUILDING 325B-WESTINGHOUSE HANFORD NON-TRU WASTE CONTAINERS SORTED BY CONTAINER DESCRIPTION, SIZE,

YEAR, AND PRIMARY WASTE TYPE

D.3-1 
This subappendix provides information on Building 325B-Westinghouse Hanford nonTRU waste containers, weight, volume, and primary waste type stored at various waste storage facilities on the Hanford Site. The data provides the non-TRU waste generation from the Building 325B-Westinghouse Hanford Facility by year. 
set 1 inesize 175

set pagesize 45

newpage 0

bl pot3a.srep

ttitle center 'NON-TRU WASTE- 325B WHC' -

right 'Page:' format 999 sql.pno skip 1

break on dt skip 1

compute sum of sum(con gross wgt) on dt

compute sum of sum(rdet_rswims_count) on dt

col dt

col con_pwtyp_cd

col rdet_swtyp_de

col sum( $\overline{c o n}$ gross_wgt)

col sum (rdeE rswims count)

col avg (con_gross_wgt)

col sum (con_cntr vol)

col avg (con_cntr_vol)

col cntyp déscr

col con size descr

col con_locn_facil_id

format a4

format a 4

format a 4

format 999999.99

format 99999

format 999999.99

format 99999.99 heading

format 99999.99 heading

format a55 heading

format a 45

heading

format a 8

col rde't_swtȳp_cd

format a4

heading

heading

"Date"

heading "Pri|Wast/Type"

heading "Sec Wast Type"

heading "Weight"

select

cntyp_descr,

con_size descr,

subs̄tr (Ẽo_char(con_tsd_accept_dt, 'YYYY'), 1, 4) dt,

con_pwtyp_cd,

rdet_swtyp̄_cd,

con_Iocn_facil_id,

sum (rdet_rswims_count),

sum (con_ōross_wḡt),

avg (con_gross_wgt),

sum (con cntr vol),

avg (con_cntr_vol)

from radwaste, contype

where con_tsd_accept_dt between '01-jan-69' and '31-dec-92' and

con_srce_cmpny

con_srce_facil_id $=$ ' $325 \mathrm{~B}$ '

and rdet_swtyp_cd in $\left(' 2 A^{\prime},{ }^{\prime} 2 B^{\prime}, L^{\prime} 2 L^{\prime}, ' 3 A^{\prime},{ }^{\prime}, 3 L^{\prime}, ' U A^{\prime},{ }^{\prime} U B^{\prime}\right)$ and

con_cntyp̄od $=$ cntyp_cd

groüp by cntyp_descr,

con_size_descr,

subs̄tr (Ēo_char(con_tsd_accept_dt, 'YYYY'), 1, 4),

con_pwtyp_cd,

rdet_swtyp_cd,

con_Iocn_facil_id

spool off 
WHC-EP-0696

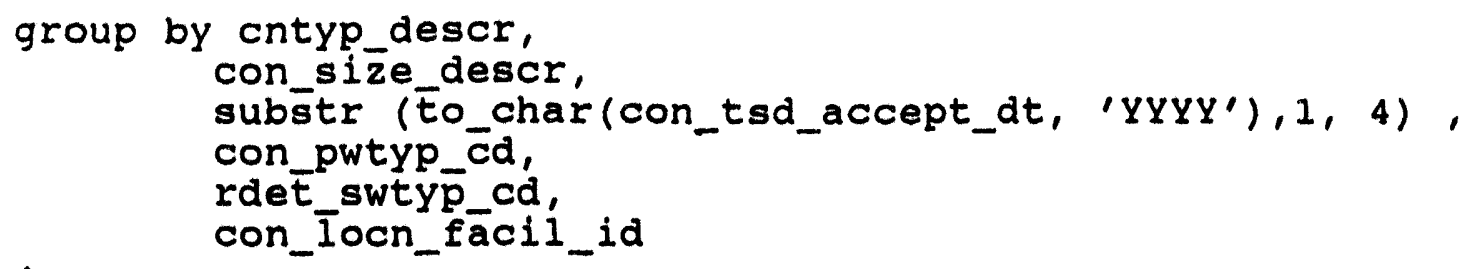


WHC-EP-0696

APPENDIX D.4

RADIOLOGICAL DATA FOR TRU WASTE CONTAINERS

SORTED BY DATE, PRIMARY WASTE TYPE, AND STORAGE FACILITY 
This subappendix summarizes the radiological data for all TRU waste containers generated at Bullding 325B-Westinghouse Hanford. Specifically, this computer run provides the total grams of TRU elements in a given container type in a given year. In addition, the average gram loading for a given container type in a given year has been calculated.

In 1991 and 1992 the number of curies (C) of alpha radiation was included on the SWBRs. Since the Building 3258-Westinghouse Hanford waste generation data is all from 1970 to 1988, these columns contain blank entries. The "Max Dose" column contains the highest value recorded for a given container in a given year, in $\mathrm{mrem} / \mathrm{hr}$.

Additional information about the isotopes present in Building 325B-Westinghouse Hanford waste containers can be found in sub-appendices D.9 and D.10. 


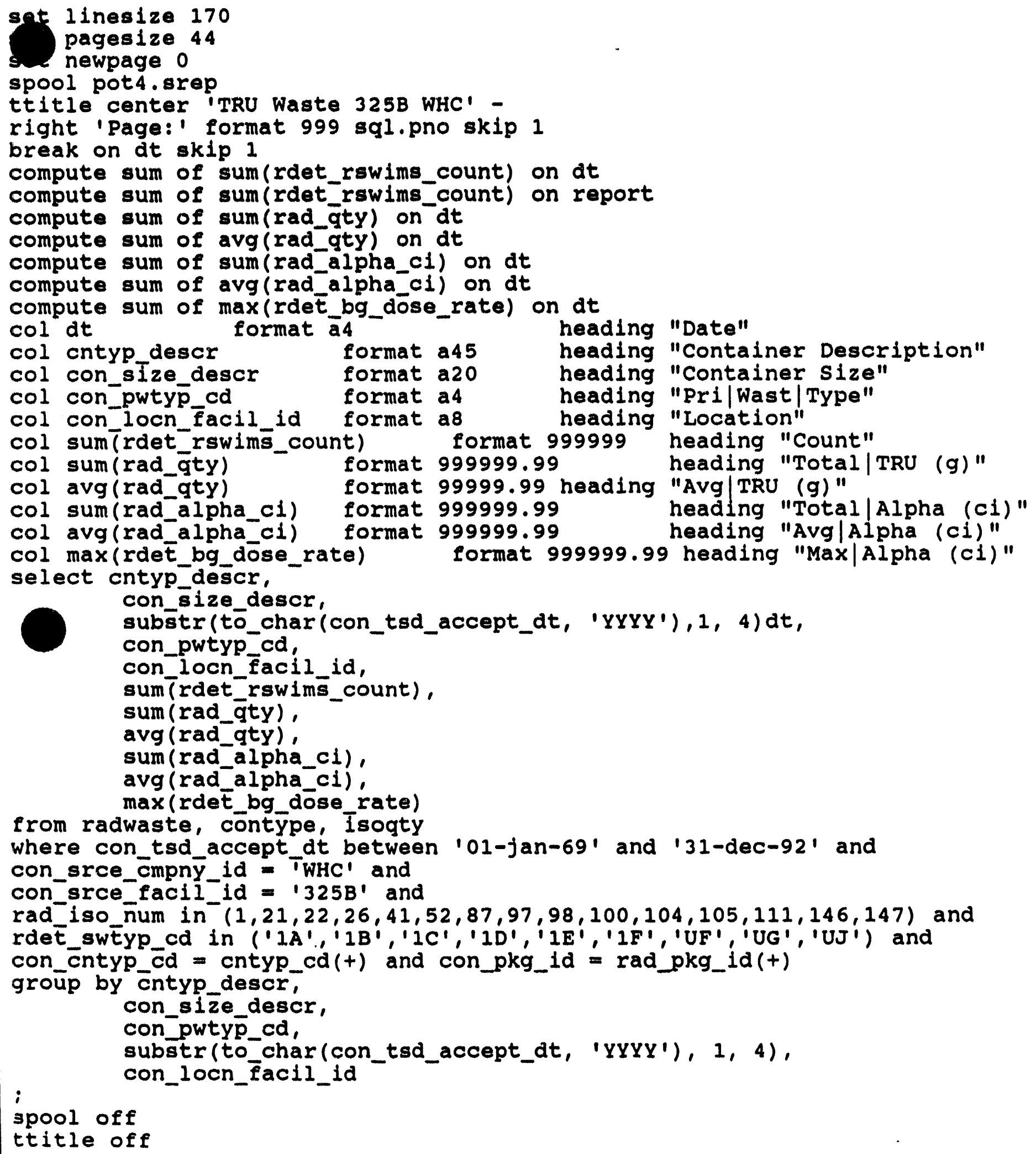




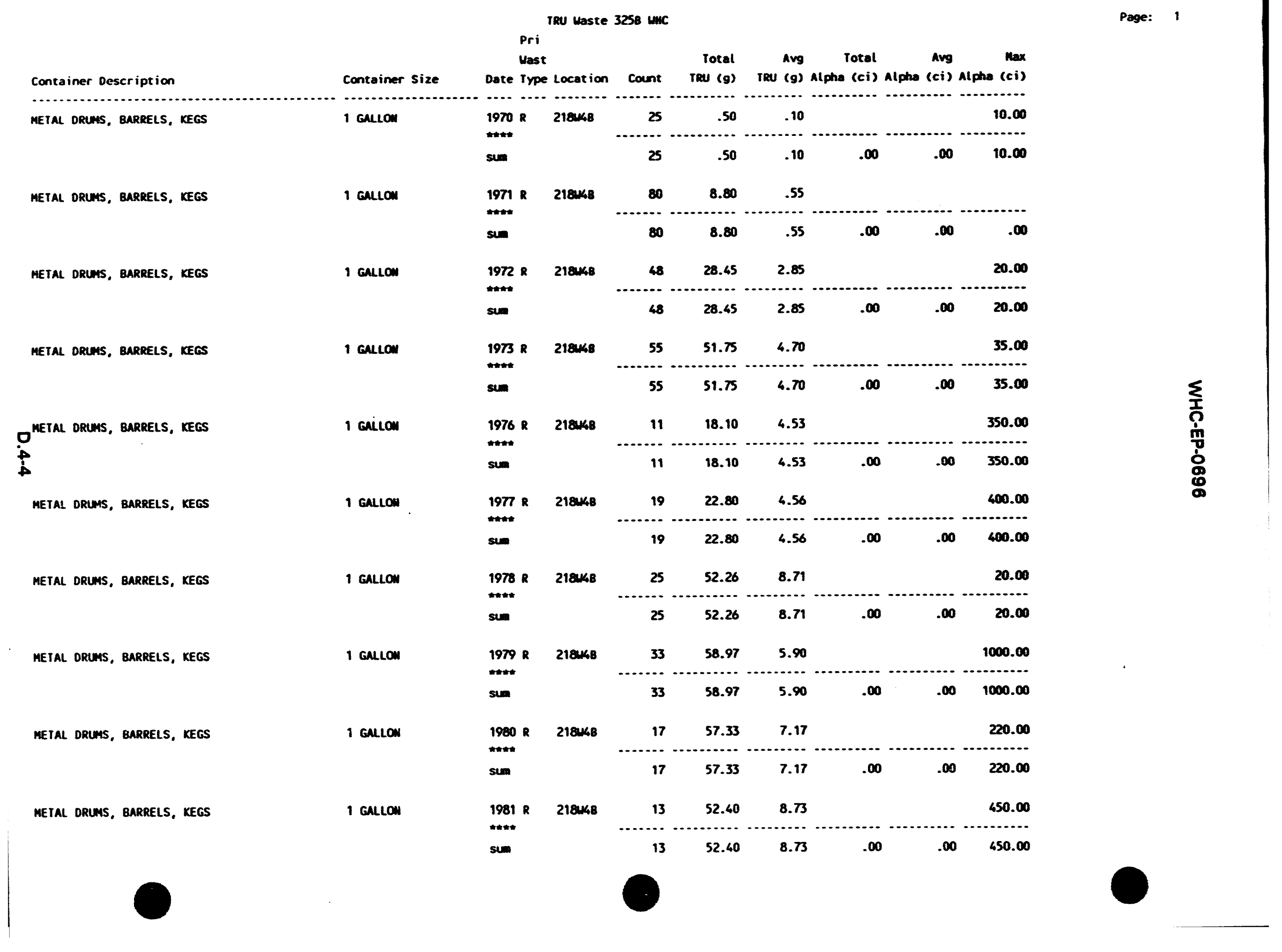




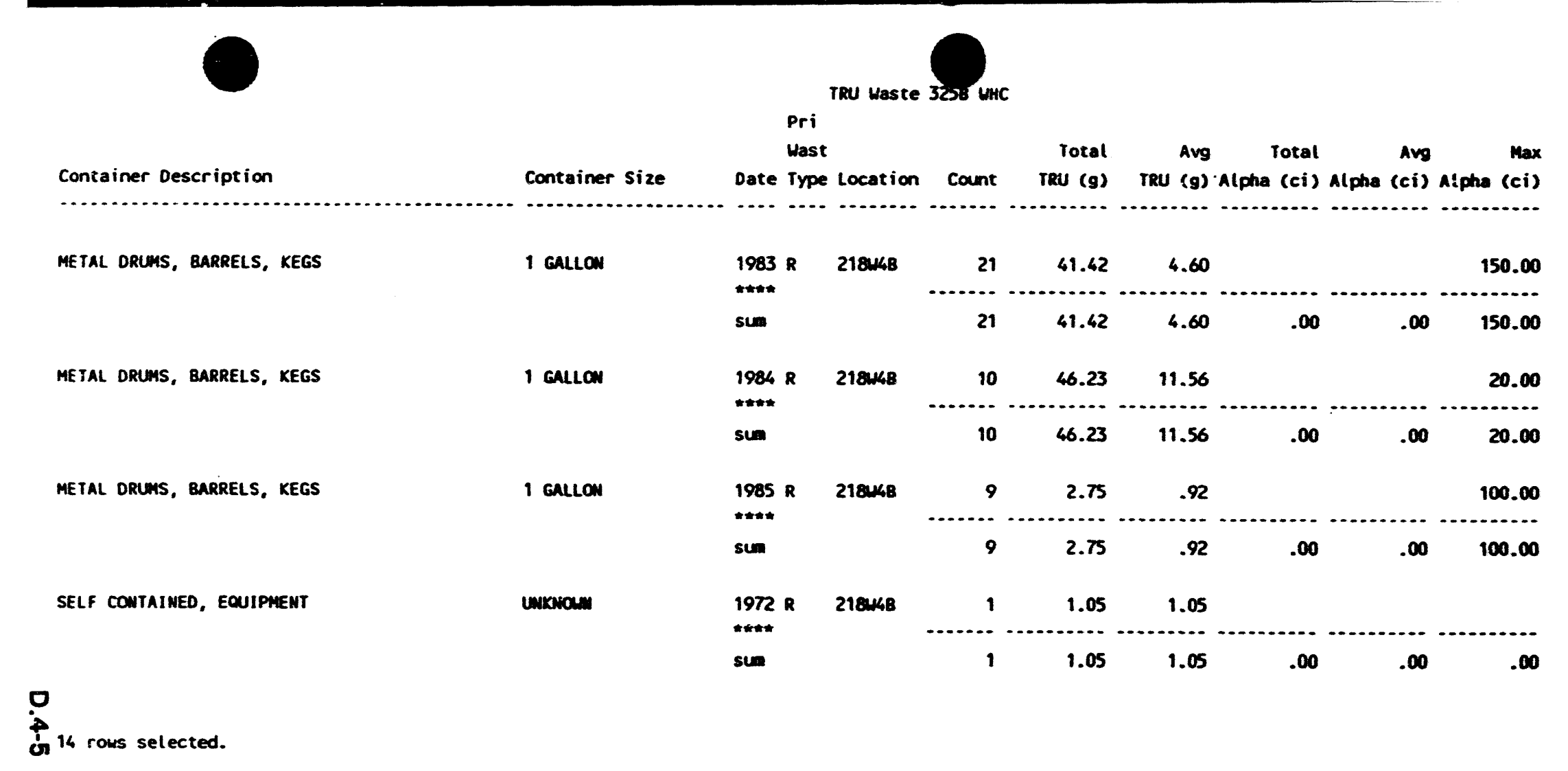


WHC-EP-0696

This page intentionally left blank.

D.4-6 
WHC-EP-0696

APPENDIX 0.5

PHYSICAL CONTENTS DESCRIPTION FOR BUILDING 325B-WESTINOHOUSE HANFORD 55-GALLON DRUMS CONTAININO TRU WASTE SORTED BY DATE, PRIMARY WASTE TYPE, AND STORAGE FACILITY

D.5-1 
This subappendix describes the physical contents of the 55-gallon drums of TRU waste generated by Building 325B-Westinghouse Hanford. Prior to 1978, physical contents were not required on the burial records, so a great many of the early records list the contents of the drum as "Miscellaneous."

The printout for this computer run sorts the contents data by storage facility, date and primary waste type. The introduction to Appendix D contains a table of waste codes and their meanings. 
set linesize 170

WHC-EP-0696

set pagesize 45

sp newpage 0

s. 1 pot5.srep

32513

ttItle center 'TRU WASTE- 2J5B WHC' -

right 'Page:' format 999 sql.pno skip 1

break on con_locn_facil_id skip 1

col phys_comp_des̄̄r

col cntyp_descr

col con_size_descr

col dt

col con_pwtyp_cd

col con locn facil_id

col sum (rdet_rswims_count) format 999999 heading "C
compute sum of sum(rdet_rswims_count) on con_locn_facil_id

format a40

format a55

format $a 45$

format a4

format a4

format a8

heading "Physical Description"

heading "Container Discription"

heading "Container size"

heading "Date"

select phys_comp_descr,

cntȳp_descr,

con_size_descr,

substr (Ẽ_char(con_tsd_accept_dt, 'YYYY'), 1, 4) dt, con_pwtyp_cd,

con_locn_facil_id,

sum (rdet_rswims_count)

from radwaste, contype, physcomp

where con_tsd_accept_dt between '01-jan-69' and '31-dec-92' and

con_srce_cmpny_id = 'WHC' and

con_srce_facil_id $=1325 \mathrm{~B}^{\prime}$

and con_size_descr $=155$ GALLON'

and rdet_swtȳp_cd in ('IA', 'IB', 'IC', 'IE', 'IF', 'UG', 'UF', 'UJ') and

ppun_comp_desc $\bar{r}$ is null and

cntyp_cd = cntyp_cd and con_pkg_id = phys_pkg_id

group by con_locn_facil_id,

phys_comp_des̄cr,

con_pwtyp_cd,

substr (tó_char(con_tsd_accept_dt, 'YYYY'), 1, 4),

cnEyp_descr,

con_size_descr

spool off 


\section{WHC-EP-0696}

con_cntyp_cd $=$ cntyp_cd and con_pkg_id $=$ phys_pkg_id groūp by con_locn_facil_id, phys_comp_descr, con_pwtyp_cd, substr (tō_char(con_tsd_accept_dt, 'YYYY'), 1, 4), cntyp descr, con_size_descr

; spool off

$\sim$

$\sim$

$\sim$

$\sim$

$\sim$

$\sim$

$\sim$

"pot5.sql" 38 lines, 1269 characters

SQL> epot5

no rows selected

SQL> $: \&$

'VT220 80 COL" $^{\circ} 7$ BIT MODE: 
WHC-EP-0696

APPENDIX D.6

PHYSICAL CONTENTS DESCRIPTION FOR BUILDING 325B-WESTINGHOUSE HANFORD TRU WASTE CONTAINERS OTHER THAN 55-GALLON DRUMS SORTED BY DATE, PRIMARY WASTE TYPE, AND STORAGE FACILITY

D.6-1 
This subappendix describes the physical contents of the TRU waste containers other than 55-gallon drums generated at Building 325B-Westinghouse Hanford. Prior to 1978, physical contents were not required on the burial records, so a great many of the early records list the contents of the drum as "Miscellaneous."

The printout for this computer run sorts the contents data by storage facility, date and primary waste type. The introduction to Appendix D contains a table of waste codes and their meanings. 
set linesize 170

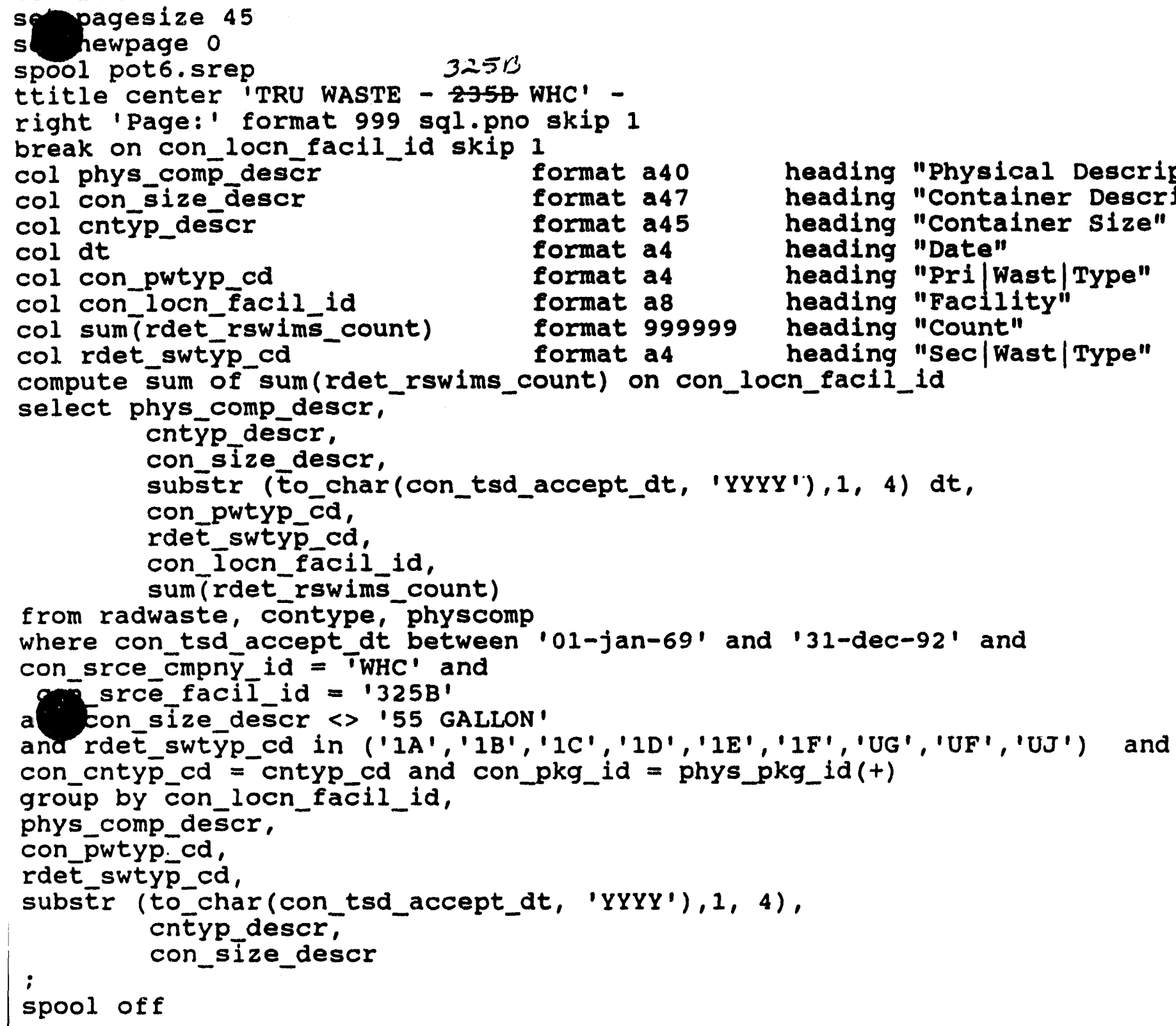


Wast Mast

\section{CLOTH/RAGS/NYLON}

CLOTH/RAGS/HYLON

CLOTH/RAGS/NYLON

CLOTH/RAGS/NYLON

COTION/KOTEX

FUEL/PINS OR ROOS

GLASS

GLASS

GLASS

GLASS

GRAPHITE

METAL/IRON/GALVANIZED/SHEET

METAL/IRON/GALVANIZED/SHEE

METAL/IRON/GALVANIZED/SHEET

METAL/IRON/GALVANIZED/SHEE

METAL/IRON/GALVANIZED/SHEET

O METAL/IROM/galvanIZED/SHEET

of MISCELLANEOUS/UHKNOMN/OTHER

miscellaneOUS/UnKnOWN/OTHER

PAPER/CARDBOARD

PAPER/CARDBOARO

PAPER/CARDBOARD

PAPER/CARDBOARD

PAPER/CARDBOARD

PLASTIC/POL YURATHANE

PLASTIC/POL YURATHANE

PLASTIC/POL YURATHANE

PLASTIC/POL YURATHANE

PLASTIC/POL YURATHANE

PLASTIC/PGL YURATHANE

RUBBER

RUBBER

WOOO/LUMBER/PLYWOOO
METAL DRUMS, BARRELS, KEGS

METAL DRUMS, BARRELS, KEGS

METAL DRUAS, BARRELS, KEGS

METAL DRUAS, BARRELS, KEGS

METAL DRUMS, BARRELS, KEGS

METAL DRUMS, BARRELS, KEGS

METAL DRUMS, BARRELS, KEGS

METAL DRUMS, BARRELS, KEGS

METAL DRUMS, BARRELS, KEGS

METAL DRUMS, BARRELS, KEGS

METAL ORUMS, BARRELS, KEGS

METAL DRUNS, BARRELS, KEGS

METAL ORUMS, BARRELS, KEGS

METAL DRUNS, BARRELS, KEGS

METAL DRUMS, BARRELS, KEGS

METAL DRUMS, BARRELS, KEGS

METAL DRUMS, BARRELS, KEGS

METAL DRUNS, BARRELS, KEGS

SELF CONTAIMED, EQUIPMENT

METAL ORUMA, BARRELS, KEGS

METAL DRLAMS, BARRELS, KEGS

METAL DRUMS, BARRELS, KEGS

METAL DRUMS, BARRELS, KEGS

METAL DRUMS, BARRELS, KEGS

METAL DRUAS, BARRELS, KEGS

METAL DRZMS, BARRELS, KEGS

METAL DRUANS, BARRELS, KEGS

METAL DRUNS, BARRELS, KEGS

METAL DRLMS, BARRELS, KEGS

METAL DRUMS, BARRELS, KEGS

METAL DRUMS, BARRELS, KEGS

METAL DRUMS, BARRELS, KEGS

METAL DRUMS, BARRELS, KEGS

METAL DRUMS, BARRELS, KEGS

METAL DRLMS, BARRELS, KEGS

METAL DRLMS, BARRELS, KEGS

METAL ORUMS, BARRELS, KEGS

METAL DRUMS, BARRELS, KEGS

METAL DRUNS, BARRELS, KEGS

METAL DRLMS, BARRELS, KEGS

\section{GaLLON}

1 gallon

1 Gallow

1 Gallon

1 GaLLOA

1 Gallon

1 GalloN

1 gallon

1 GALLON

1 Gallom

1 GALLON

1 Gallow

1 gallon

1 Gallow

1 gallon

1 gallon

1 GalloN

1 GALLON

UNKMOWN

1 Gallon

1 Gallow

1 Galloy

1 Gallon

1 Gallon

1 gallon

1 callon

1 Gallon

1 GALLON

1 Gallon

1 GALLON

1 gallon

1 Gallon

1 Gallon

1 Gallon

1 Gallon

1 Gallon

1 Gallow

1 Gallon

1 gallon

1 gallon
Date Type Type facility count

\begin{tabular}{|c|c|c|c|}
\hline $1979 \mathrm{R}$ & If & 2181148 & 31 \\
\hline $1980 R$ & If & & 11 \\
\hline $1981 \mathrm{R}$ & If & & 2 \\
\hline $1983 \mathrm{R}$ & If & & 16 \\
\hline $1985 R$ & If & & 3 \\
\hline $1983 \mathrm{R}$ & $1 F$ & & 2 \\
\hline $1979 R$ & If & & 31 \\
\hline $1980 R$ & If & & 17 \\
\hline $1981 \mathrm{R}$ & IF & & 11 \\
\hline $1985 R$ & If & & 3 \\
\hline $1980 R$ & if & & 2 \\
\hline $1979 R$ & If & & 31 \\
\hline $1980 R$ & IF & & 15 \\
\hline $1981 R$ & IF & & 10 \\
\hline 1983 R & If & & 18 \\
\hline $1984 R$ & IF & & 10 \\
\hline $1985 \mathrm{R}$ & If & & 3 \\
\hline 1972 R & If & & 3 \\
\hline 1972 R & IF & & 1 \\
\hline 1979 R & If & & 31 \\
\hline $1980 R$ & If & & 17 \\
\hline
\end{tabular}

$1983 R \quad$ IF -16

$1984 R$ If

1979 R IF 31

$1980 R$ IF 17

$1981 R$ IF

$1983 R \quad 1 F \quad 16$

$1944 R$ If 10

$1985 R$ IF 3

$1983 R$ If 14

$1984 R$ If 2

$1980 R$ If

$1970 R$ If 25

$1971 R$ IF 80

$1972 \mathrm{R}$ IF 45

$1973 R \quad$ IF $\quad 55$

$1976 \mathrm{R}$ IF 11

1977 R IF 19

$1978 \mathrm{R}$ IF 
IRU UASTE - PSB UHC

Pri Sec

Hast Hast

Physical Description

Container Size

Container Description

METAL DRUMS, BARRELS, KEGS

GaLLON
Date Irpe Iype facility Count

.... ..... .... ......... ........

$1979 R$ if 218048

sen

sum

41 rows selected.

סִ 


\section{WHC-EP-0696}

This page intentionally left blank.

\section{D.6-6}


WHC-EP-0698

APPENDIX 0.7

HAZARDOUS CONSTITUENTS OF BB-GALLON

DRUMS CONTAINING TRU WASTE

D.7-1 
The presence of hazardous constituents in a radioactive waste drum designates the waste as "Mixed" (primary waste code "M"). In this subappendix the hazardous contents of 85-gallon drums generated at Building 3258-Westinghouse Hanford are sorted by storage facility.

Information concerning the hazardous constituents of waste containers was not required prior to 1986. During the R-SWIMS data reentry program in the mid-1980's an attempt was made to add any available information on the hazardous materials present, however, this information was limited. 


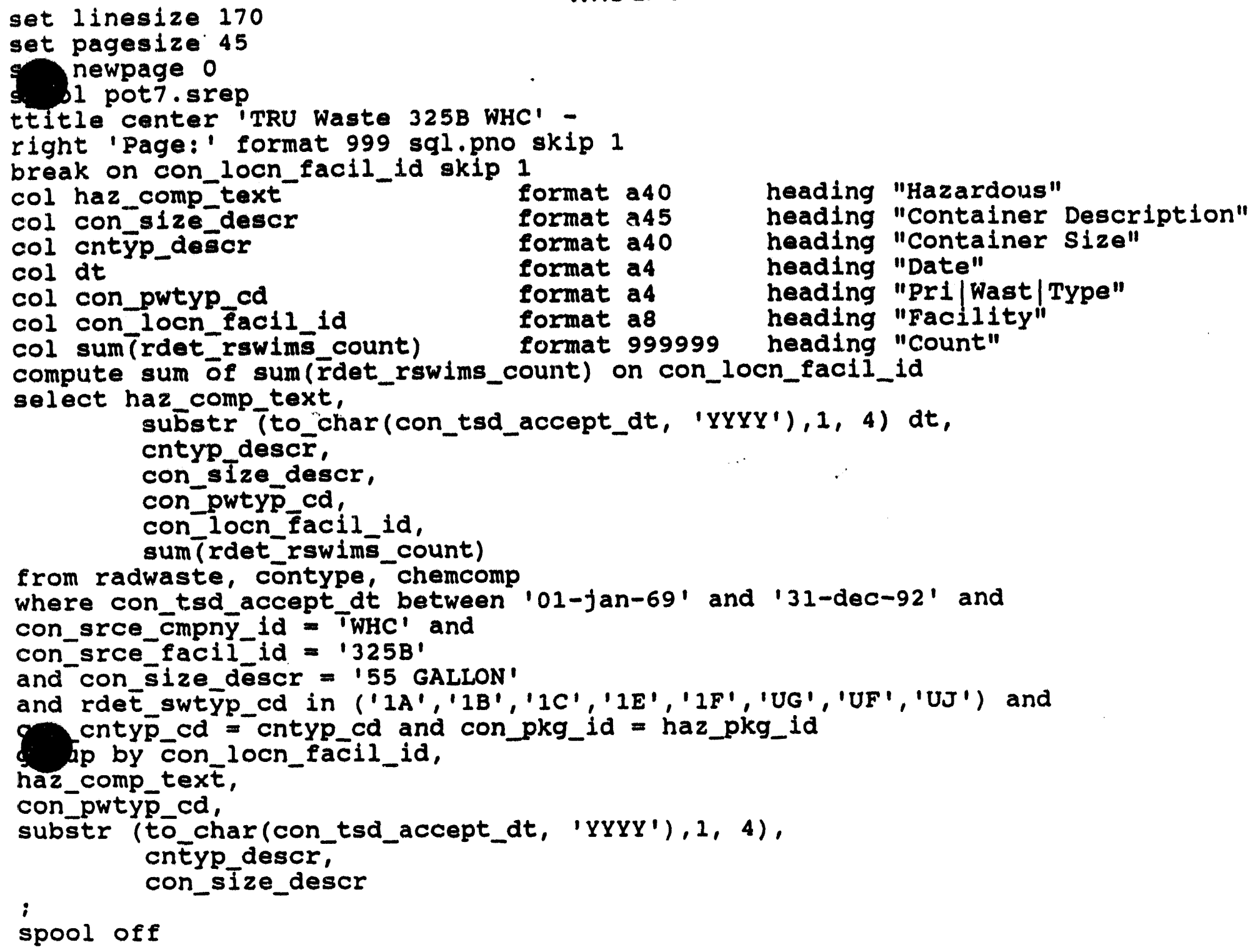

spool off 


\section{WHC-EP-0696}

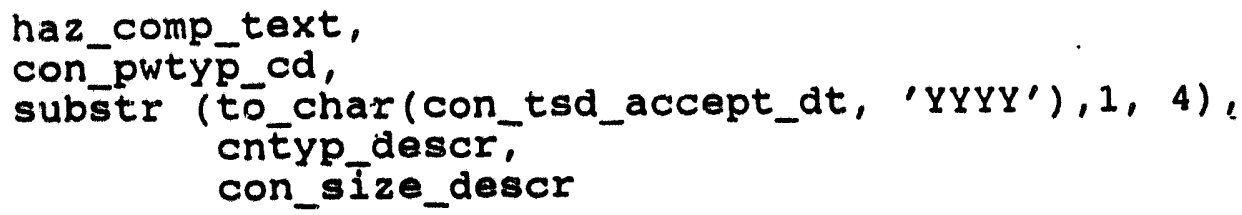

$\sim$

$\sim$

$\sim$

$\sim$

"pot7.sql" 37 lines, 1220 characters

SQL> epot7

no rows selected

SQL> $: \circ$

¿VT220.80 COL' 7 BIT MODE。 
WHC-EP-0696

APPENDIX D.8

HAZARDOUS CONSTITUENTS OF TRU WASTE CONTAINERS OTHER THAN 55-GALLON DRUMS

D.8-1 


\section{WHC-EP-0696}

The presence of hazardous constituents in a radioactive waste drum designates the waste as "Mixed" (primary waste code "M"). No hazardous constituents are listed in containers other than 55-gallon drums generated at Building 325B-Westinghouse Hanford; therefore this run did not reveal any hazardous information. 
set linesize 170

set pagesize 45

newpage 0

1 pot8.srep

ttitle center 'TRU Waste 325B WHC' -

right 'Page:' format 9.99 sql.pno skip 1

break on con_locn_facil_id skip 1

col haz_comp_text

format a 40

col con_size_descr

format a 45

col cntȳp_descr

col dt

col con_pwtyp_cd

format a40

format a4

col con locn facil id

format a 4

col sum(rdet_rswims_count)

format a8

select haz_comp_text.

format 999999

heading "Hazardous"

heading "Container Description"

heading "Container Size"

heading "Date"

heading "Pri|Wast/Type"

heading "Facility"

heading "Count"

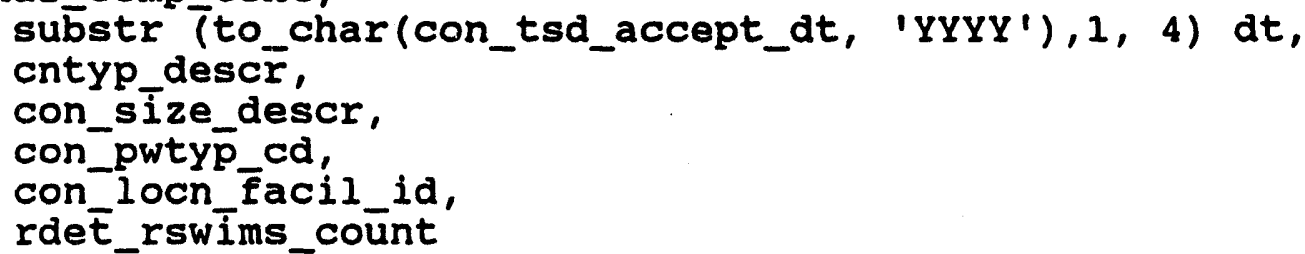

from radwasté, contȳpe, chemcomp

where con_tsd_accept_dt between '01-jan-69' and '31-dec-92' and

con_srce_cmpny

con_srce_facil_id $=$ '325B'

and con size_descr $\longleftrightarrow$ '55 GALLON'

and rdet_swt $\bar{y} p_{-} c d$ in ('IA', 'IB','1C','IE','IF','UG','UF', 'UJ') and

con_cntyp_cd $=$ - cntyp_cd and con_pkg_id = haz_pkg_id

up by con_locn_facil_id, comp_tex $\bar{t}$,

con_pwtyp_cd,

cntyp_descr,

substr (to_chār(con_tsd_accept_dt, 'YYYY'), 1, 4),

consize descr,

rdet_rswims_count

spool off 


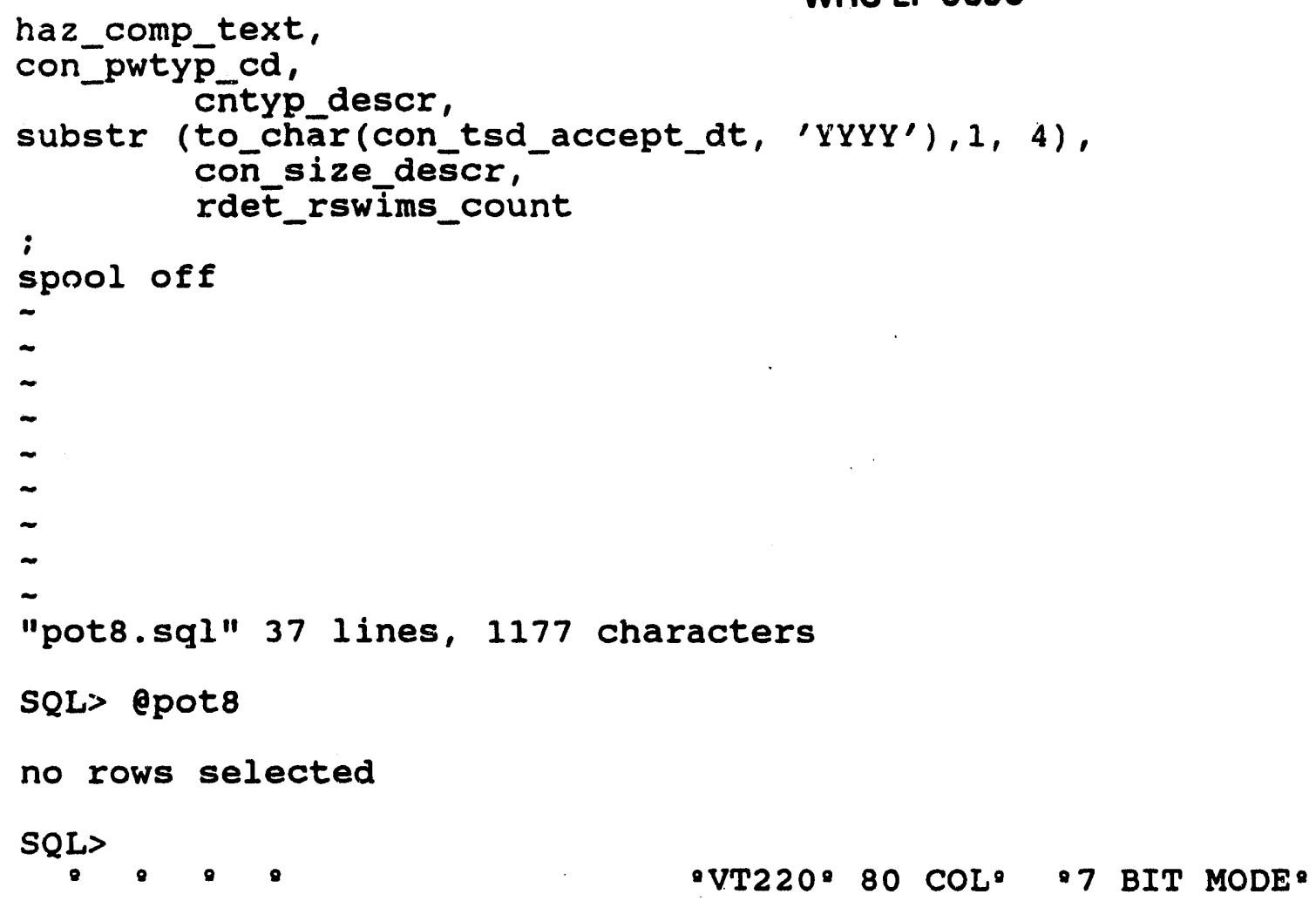


WHC-EP-0696

APPENDIX D.9

ISOTOPES LISTED IN 55-GALLON DRUMS CONTAINING TRU WASTES

D.9-1 
This subappendix contains a listing of the SWITS isotope information for TRU waste from Building 325B-Westinghouse Hanford stored in 55-gallon drums. The isotope field in SWITS contains several types of the following information:

- Specific isotope (e.g., Am-241, Np-237, U-233, Pu-238)

- Generic isotope listings (e.g., Pu, uranium-enriched, uranium-depleted.)

- $\quad$ Plutonium-239 fissile gram equivalents (Pu239 FSL GR equiv).

- Total - alpha

- Total beta/gamma

- $\quad$ PE-Ci (plutonium equivalent curies).

This computer run contains the isotope field listing along with the number of TRU waste drums with that listing. The run is sorted by storage facility and year. 
WHC-EP-0696

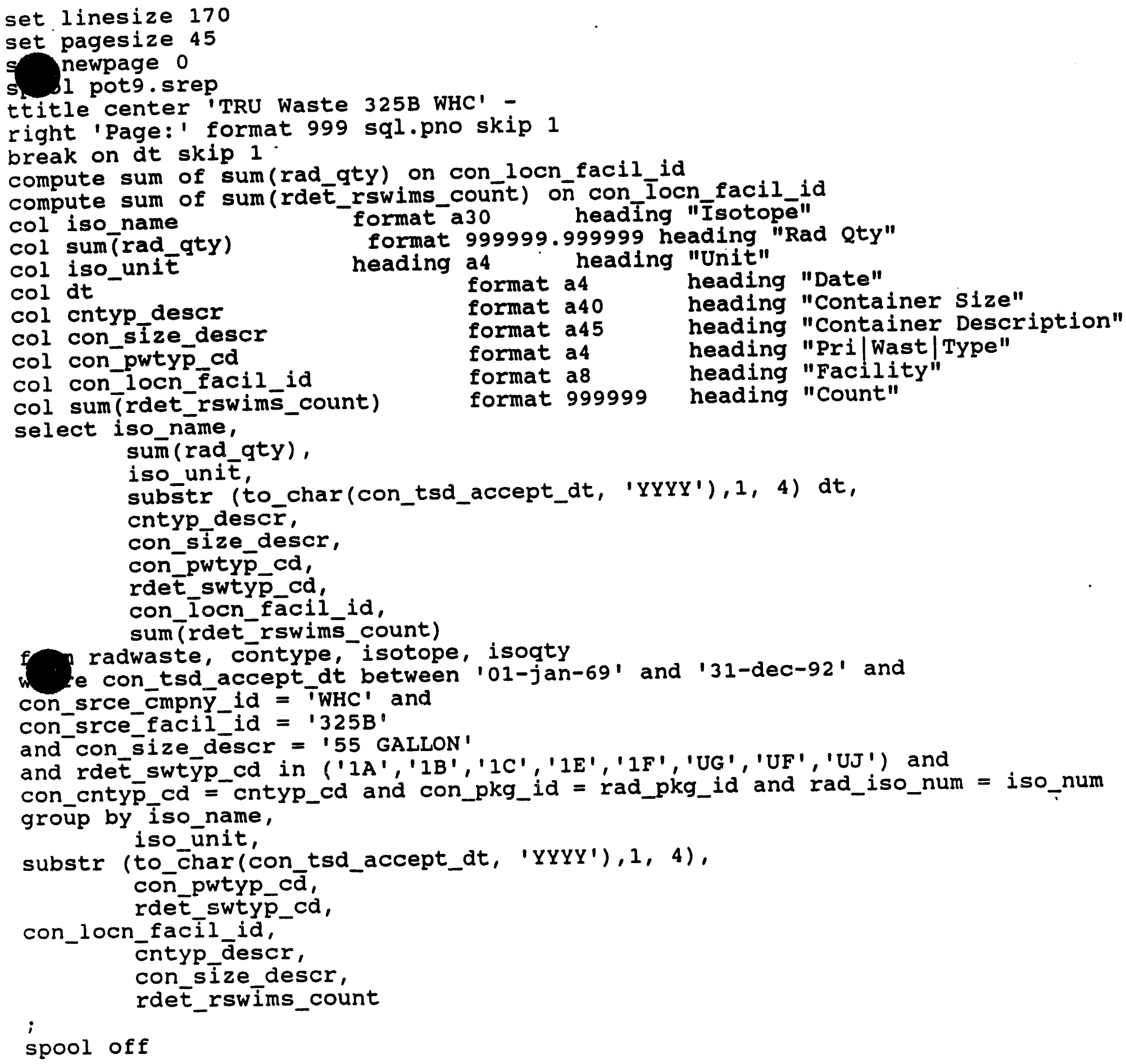

format a4

format a 40

format a 45

format a4

format 999999

heading "Container size"

heading "Container Description" heading "Pri|Wast/Type" heading "Facility" heading "Count" 
WHC-EP-0696

con_cntyp_cd $=$ cntyp_cd and con_pkg_id $=$ rad_pkg_id and rad_iso_num $=$ iso_num groūp by iso name,

substr (to_char(con_tsd_accept_dt, 'YYYY'),1,4),

con_pwtyp_cá, rdet swtyp_cd,

con_locn_facillid,

cntyp descr,

con size descr,

rdeÉtrswims_count

;

spool off

$\sim$

$\sim$

$\sim$

$\sim$

"pot9.sql" 46 lines, 1458 characters

SQL $>$ epot9

no rows selected

SQL,$:$.

-VT220: $80 \mathrm{COL}^{2} \cdot 7$ BIT MODE" 
WHC-EP-0696

\section{APPENDIX D.10}

ISOTOPES LISTED IN TRU CONTAINERS

OTHER THAN 55-GALLON DRUMS

D.10-1 
This subappendix contains a listing of the SWITS isotope information for TRU waste from Building 325B-Westinghouse Hanford stored in containers other than 55-gallon drums. The isotope field in SWITS contains several types of the following information:

- Specific isotope (e.9., Am-241, Np-237, U-233, Pu-238)

- Generic isotope listings (e.g., Pu, uranium-enriched, uranium-depleted)

- Plutonium-239 equivalent curies (PE-CI)

- Total-alpha

- Total beta/gamma

- $\quad$ PE-Ci (plutonium equivalent curies).

This computer run contains the isotope field listing along with the number of TRU waste containers with that listing. The run is sorted by storage facility and year. 


\section{WHC-EP-0696}

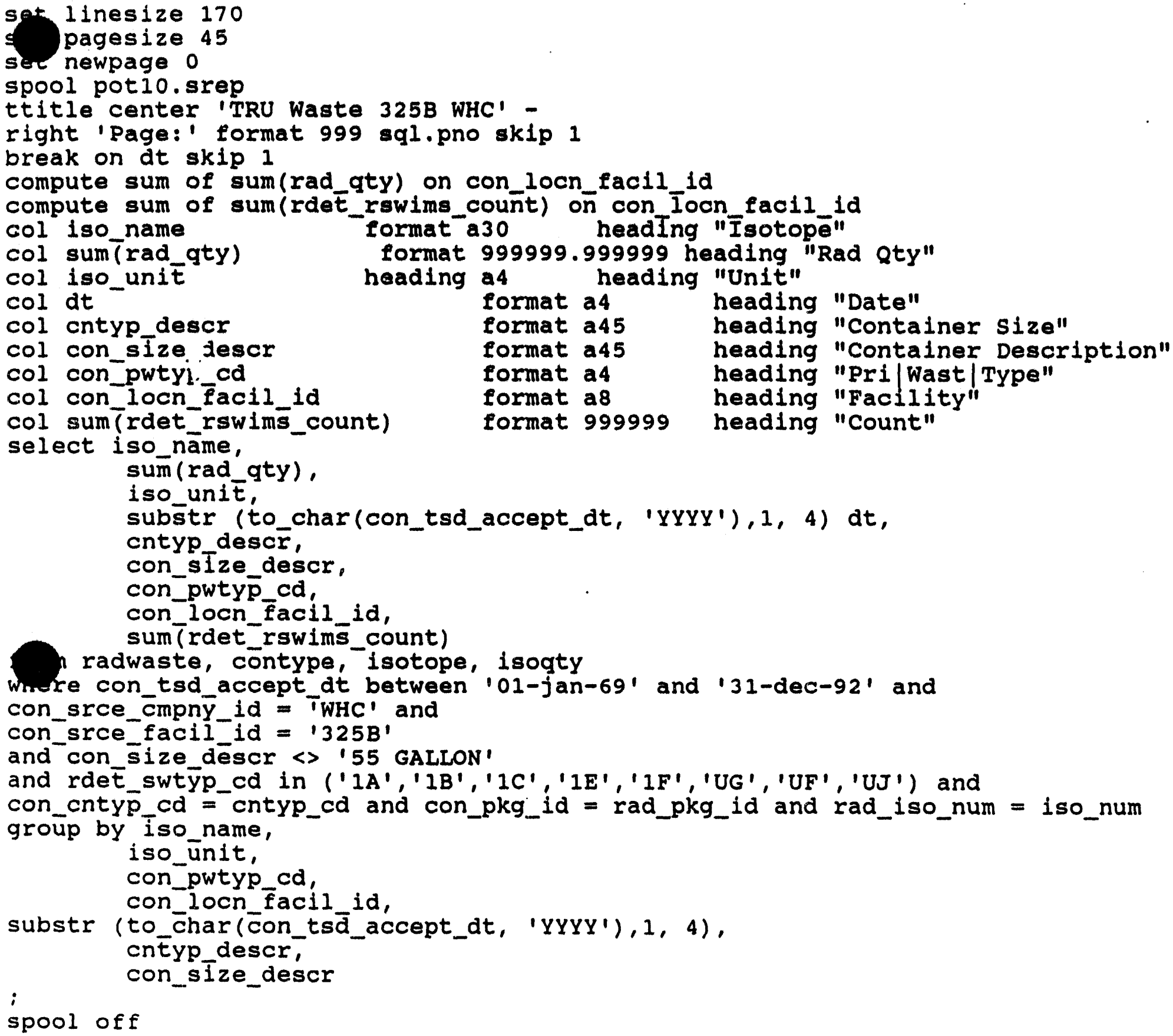

spool off 


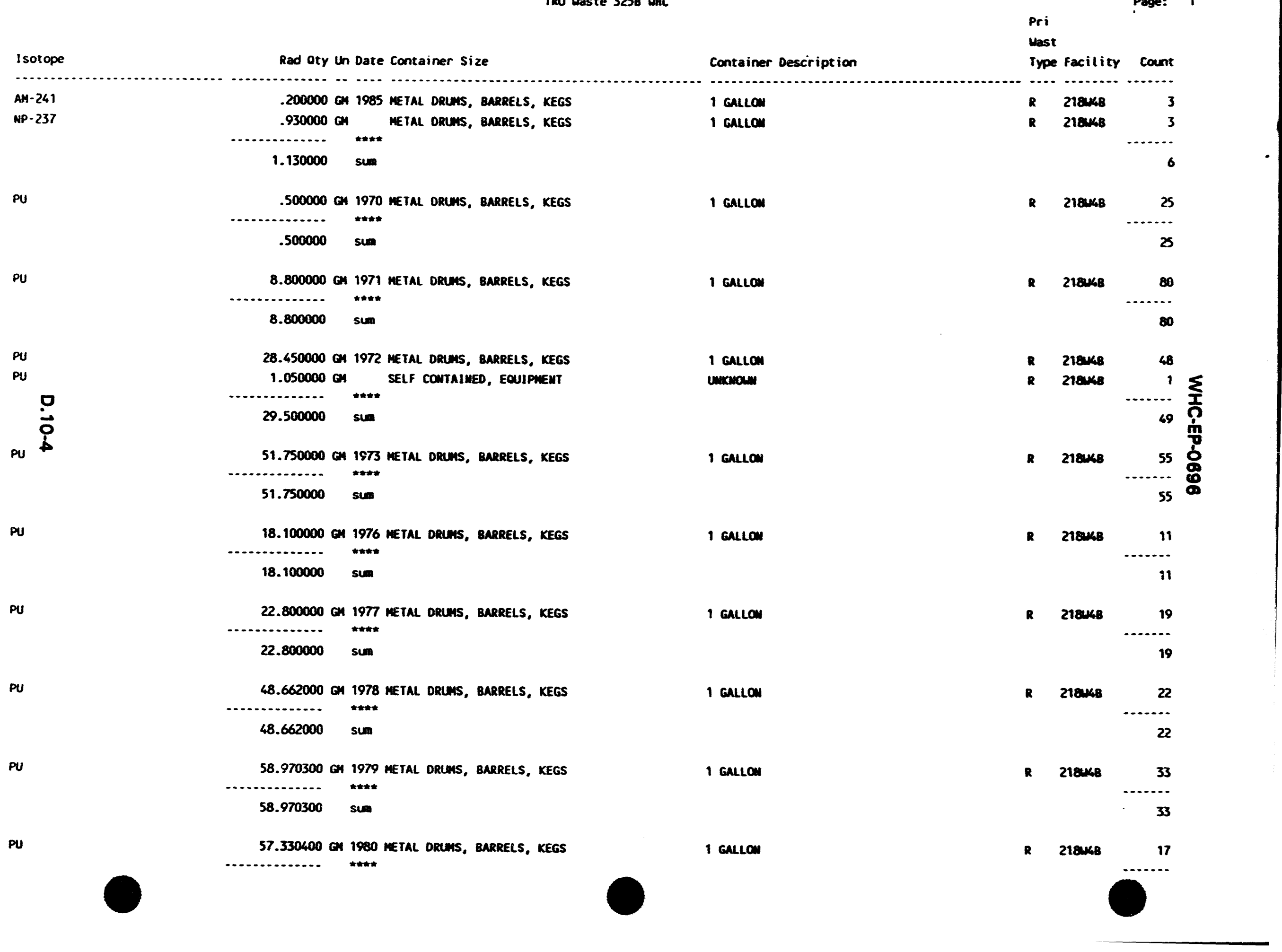


IRU Waste 3258 unc

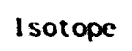

Rad Oty Un Date Container Size

57.330400

sum

PU

52.400300 GM 1981 METAL DRUNS, BARRELS, KEGS

***:

52.400300 sin

PU

41.380100 GM 1983 METAL DRUMS, BARRELS, KECS

41.380100 sum

PU

46.230000 GM 1986 METAL ORUNS, BARRELS, KEGS

46.230000

PU

TH-232

TH -232

$T H-232$

$\mathrm{rH}-232$

TH-232

TOTAL BETA/GAMMA
1.620000 on 1985 METAL DRUNS, BARRELS, KEGS

1.620000

1.350000 GM 1978 METAL DRUAS, BARRELS, KEGS 1.350000 sum

1.350000 an 1979 METAL ORLAS, BARRELS, KEGS

1.350000 t*t*

$\sin$

20.970000 GM 1980 METAL DRLAS, BARRELS, KEGS

20.970000

51.140000 G 1983 mETAL DRLUS, BARRELS, KEES

51.140000

tht:

108.600000 OH 1984 METAL DRUML, BARRELS, KEGS

-............ twat

$108.600000 \mathrm{sum}$

$50.000000 \mathrm{CI} 1970$ MeTAL DRUNS, BARRELS, KEgS

-.............

Container Description

Past

Type facility count

17

1 Gallow

R 218uk

13

1 Grilon

$218 \times 48 \quad 18$

18

1 GRLON

R $218448 \quad 10$

10

1 Gallo

R 218468

1 callon

R 218 4 2.2

1 GrLou

r.a. 2

1 Gallon

1 GALLON

2184a

5

5

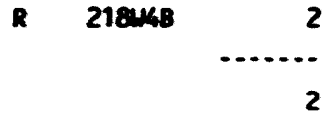

1 Gallon:

R $218468 \quad 25$ 
TOTAL BETA/GAMMA 850.000000 CI 1971 metal DRLAS, BARRELS, KEGS

$203.000000 \mathrm{Cl} 1972$ metal DRUAS, BARRELS, KEGS $7.000000 \mathrm{Cl}$ SELF CONTAINED, EQUIPHEMT

TOTAL BETA/GAMEA

TOTAL BETA/GAMHA

TOTAL BETA/GAMHA

210.000000

155.000000 Cl 1973 metal DRUMS, BarReLS, KEGS .... *t*t

155.000000 sum

TOTAL BETA/GAMMa

$\dot{0}$

TOTAL Beta/gamia

TOTAL BETA/GAMMA

TOTAL BETA/GAMMA

TOTAL BETA/GAMMA

TOTAL BETA/gAMma

TOTAL BETA/GAMma
30.001000 CI 1976 METAL DrLUAS, BARRELS, KEGS ............

30.001000 sum

$50.000000 \mathrm{Cl} 1977$ METAL DRLMS, BARRELS, KEGS -............. the

50.000000 sin

80.100000 CI 1978 METAL DRUUS, BARRELS, KEGS

80.100000

6150.000000 CI 1979 METAL DRUMS, BARRELS, KEGS 6150.000000 t*a........

$40.000000 \mathrm{CI} 1980$ METAL DRUWS, BARRELS, KEGS [.............

40.000000 sum

75.402000 CI 1981 METAL DRUMS, BARRELS, KEGS .... *\#*

75.402000 sim

.220000 Cl 1983 METAL DRUMS, BARRELS, KEGS
1 gallow

1 gallon

unxuoum

1 gallon

1 Gallon

1 Gallon

1 GALLOU

1 GALLON

1 GALLON

1 gallow
B 218468

$\begin{array}{llr}R & 218 \times 48 & 48 \\ R & 218468 & 1\end{array}$

49

R 218468 55

55

R $2184 \times 68 \quad 11$

R 218448

R 2184468 $\begin{array}{r}7 \\ \\ \end{array}$

R 218446 B 33

R 2184kB

R 218448 13

R 2184kB 
I sotope

Rad oty Un Date Container Size

Container Description

. .220000
sim

20000 sim

TOTAL BETA/GAMMA

$.200000 \mathrm{CI} 1984$ METAL DRUMS, BARRELS, KEGS

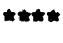

.200000

sum

TOTAL BETA/GAMma

.050000 CI 1985 METAL DRUMS, BarRELS, KEGS

tht:

.050000

sum

$u-233$

3.600000 G 1978 METAL DRUMS, BARRELS, KEGS ......

.040000 GM 1983 METAL DRUMS, BARRELS, KEGS

$u-233$
0
$\dot{0}$

URANIUA-DEPLETED

URANIUM-DEPLETED

URANIUM-DEPLETED

URANIUR-DEPLETED

URANIUH-DEPLETED

URANIUA-DEPLETED
3 METAL DRUMS, BARRELS, KEGS I GALLON

.040000 sum

$4.000000 \mathrm{GM} 1970$ METAL DRUMS, BARRELS, KEGS

4.000000 sum

40.350000 GM 1978 METAL DRUMS, BARRELS, KEGS

40.350000 sum

270.950000 GM 1979 METAL DRUMS, BARRELS, KEGS

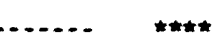

270.950000 sum

55.990000 GM 1980 METAL DRUMS, BARRELS, KEGS

$55.990000 \mathrm{sum}$

11.960000 GH 1981 METAL DRUNS, BARRELS, KEGS

11.960000 sim

739.140000 GM 1983 METAL DRLMS, EARRELS, KEGS
1 Gallow

1 GALLON

1 GALLON

1 Gallow

1 eallon

1 GALLON

1 GaLLON

1 GALlO:

1 Gallon
Pri

Nast

Type facility count

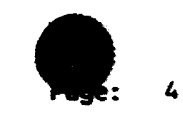

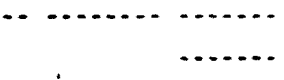

R 218448 10

10

R 218 N4B 3

3

R 2184483

3

R 2180463

.

3

R 218 48 20

20

20

R 218448 ' 8

8

R 2184486

7

R. 218448 7

218148

R 2184488

2 


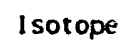

URANIUH-ENRICHED
2.520000 GH 1985 METAL DRUMS, BARRELS, KEGS

2.520000

sum

88.000000 GI 1971 METAL DRUMS, BARRELS, KEGS

.......

88.000000

sum

97.150000 GM 1972 METAL DRLMS, BARRELS, KEGS $3.850000 \mathrm{GM}$ SELF CONTAIMED, EQUIPMENT

................
t\#柿

101.000000

127.000000 GM 1973 METAL DRUMS, BARRELS, KEGS ........... t*atut

127.000000 sum

39.350000 GH 1976 METAL ORUHS, BARRELS, KEGS ............

39.350000 sum

68.200000 GH 1977 METAL DRUMS, BARRELS, KEGS 68.200000 sum

81.700000 GM 1978 METAL DRUMS, BARRELS, KEGS

81.700000

****

849.660000 GH 1979 METAL DRUMS, BARRELS, KEGS

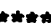

849.660000 sum
Pri

Container Description

Type facility count

1 GALLON

1 gallon

1 Gallow

1 Gallon

UNKMON

1 Gallon

1 Gallon

1 Gallon

1 GALLON
14

R 218448

10

10

R 218 46B 3

R 218468

80

$\begin{array}{llr}R & 218448 & 48 \\ R & 218448 & 1\end{array}$

...

49

R 21844835

35

R 218448 11

11

R $218 \times 486 \quad 19$

19

R 218 4B $\quad 18$

18

R $212448 \quad 24$ 

287.840000 GM 1980 METAL ORUMS, BARRELS, KEGS \#\#\#*

287.840000 sum

URANIUM-ENRICHED

120.760000 GM 1981 METAL DRUMS, BARRELS, KEGS

120.760000 sum

URANIUM-ENRICHED

1418.750000 GM 1983 METAL DRUMS, BARRELS, KEGS

1418.750000

405.480000 GM 1984 METAL DRUMS, BARRELS, KEGS 1984 ME

405.480000 sum

501.700000 GM I985 METAL DRUMS, BARRELS, KEGS

............... *t*t*

501.700000 sum

URANIUM - NATURAL

1.000000 GH 1970 METAL DRUMS, BARRELS, KEGS

1.000000

17.300000 GM 1973 METAL DRUHS, BARRELS, KEGS

URANIUM-NATURAL

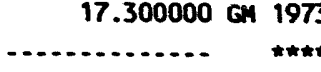

17.300000

sun

URANIUM-NATURAL

54.450000 GM 1978 METAL DRUMS, BARRELS, KEGS .............

54.450000 sum

URANIUM-NATURAL

URANIUM-NATURAL
Pri

Wast

Type facility Count

Container Description

1 Gallon

218448

1 Gallon

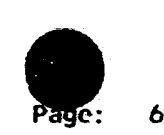

6

R 218 448 $\quad$....

9

R 218W48 7

7

$\begin{array}{lll}218448 & 18\end{array}$

18

R 218448 8

-..

8

R $218948 \quad 3$

1 Gaclon

1 GALLON

218146

3

1 GalloN

R Zi844B 20

1 GaLLON

R 218448

17
$-\ldots$.
17

1 GALLON

R 218448

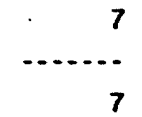

1 GALLON
29.110000 GM 1979 METAL DRUMS, BARRELS, KEGS

.............

29.110000 sum

26.580000 GM 1980 METAL DRUMS, BARRELS, KEGS

.........

26.580000 sum 
eri

Container Description

Type facility count

1 Gallon

R 21844

5.250000 GM 1981 METAL DRUNS, BARRELS, KEGS

5.250000

sum

URANIUM-HATURAL

21.580000 GM 1983 METAL DRUMS, BARRELS, KEGS \#\#**

1 GALLON

$$
21.580000 \text { sum }
$$

URANIUM-NATURAL

9.150000 GM 1984 METAL DRUMS, BARRELS, KEGS \#***

9.150000

1 GALLON

218448

10

10

66 rows selected.

$\stackrel{0}{\dot{0}}$ 
WHC-EP-0696

APPENDIX E

DATA ON BUILDING 325B WASTE GENERATION BY PNL FROM THE SOLID WASTE INFORMATION TRACKING SYSTEM 
The information found in this appendix is from the SWITS database. This database incorporates the older R-SWIMS database and is used to track information on radioactive and other wastes stored or disposed at the Hanford Site.

Each SWITS data run in this appendix is preceded by the query used to generate the data. A brief explanation of the run and any additional information needed to understand the data presented is also included.

The bulk of the data provided is limited to information about TRU waste generated at Building 325B by PNL; however, some general information on the non-TRU waste is included for completeness. The term non-TRU is used, instead of LLW, because a small percentage of the waste has been designated only as not TRU. It is believed that most, if not all, of the non-TRU waste is LLW.

The data runs in this appendix are further segregated by waste container type. Since initial retrieval efforts and WRAP 1 will focus on 55-gallon drums, these container types are considered separately.

Some general information about SWITS database and the codes used follows. Please note the disclaimer found on the next page. 


\section{WHC-EP-0696}

DON'T SAY IT -- Write It 1

-

Date:

то: Yhedey

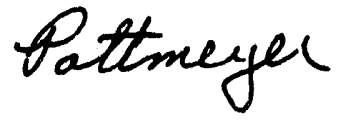

From:

Solld Waste Englneering Data Management Group N3-11 $6-4394 / 6-4020$

Re: SWITS DATA REQUEST

Attached for your information and use is the data which you requested. This data represents best avaliable information regarding wastes currently in storage at the Hanford site. I trust the information will be suitable to your needs.

Requests for information from the solid Waste Information and Tracking System (SWITS) are normally relatively 1 imited in scope, requesting specific data fields or summary data. The responses to these requests undergo review during data collection: summary and response preparation.

The response to this request represents a simple reproduction of the swITS Database. Transmittal of this information is made with the following disclaimers :

1) The information contained in this transmittal is raw data, and represents information provided to Solid Waste Engineering (SWE) on burial records or other documents. This data has not been validated.

2) The information contained in this transmittal is subject to change without notice. Continual update of swITs information and improvement of the software system make it impossible to ensure consistency of this data with the database after transmittal.

3) This information is current as of

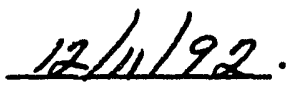

If I can be of further assistance to you, do not hesitate to call me. 
WHC-EP-0696

This page intentionally loft blank.

E-4 
WHC-EP-0696

Solid Waste Information and Tracking system

$05 / 01 / 92$

$13: 25$

Páge: 1

Primary Waste Type Code Table

Primary Waste Type Code

AR
$\mathbf{C H}$
$\mathbf{H}$
$\mathbf{H P}$
$\mathbf{M P}$
$\mathbf{N}$
$\mathbf{R}$
$\mathbf{R P}$
$\mathbf{U}$

Primary Waste Type Description

haz ADETr Bord

MORATORIUY CONYROIID

HAZARDOUS

FIAZARDOUS WASTE PCB

MIXED

MIXED PCB

NON-REGULATED

PCB

RADIOACIIVE

RADIOACTIVE PCB

UNKNOWN ship

Time Rad

89 N

89 N

89

29

89

29

29

29

N

N

$\mathbf{Y}$

$\mathbf{Y}$

$\mathbf{N}$

9

N

$\mathbf{Y}$

$\mathbf{Y}$

N 
WHC-EP-0696

Solid Waste Information and Tracking system

$05 / 01 / 92$

$13: 26$

Page: 1

Secondary Waste Type Code Table

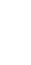

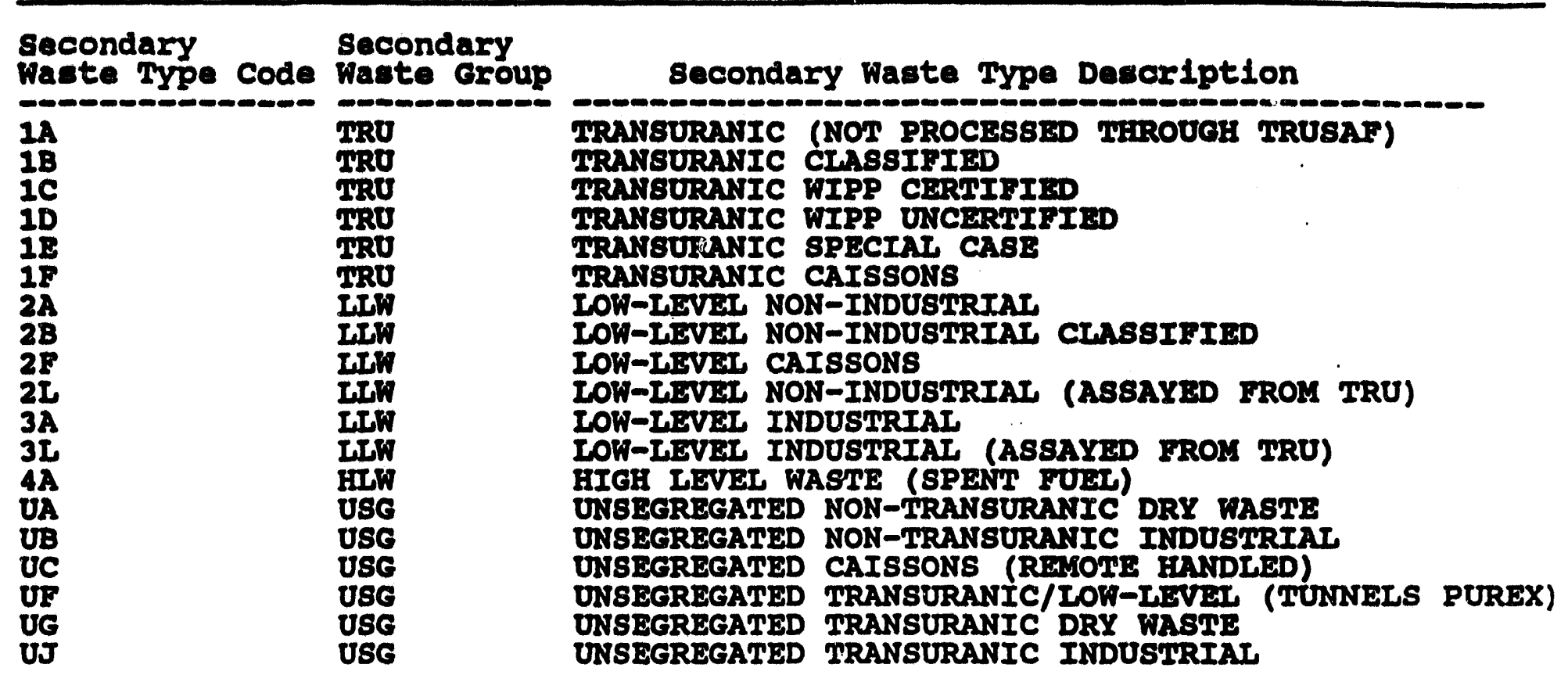


WHC-EP-0698

APPENDIX E.1

CONTAINER NUMBER AND WEIGHT OF TRU WASTE IN B5-GALLON DRUMS BY WASTE TYPE

E.1-1 
This data run gives an overall look at the waste types, container numbers and total weight (in kilograms) of the radioactive wastes generated at Building 325B-PNL on a yearly basis. These data are for wastes atored in 55-gallon drums only. The meanings of primary and secondary waste codes can be found in the introductory materials for this appendix.

The data in the average weight column should be taken cautiously, especially for LLW codes. Concern is warranted because LLW records are still done on a batch basis. This means that one weight datum may be the composite weight for a group of drums. The computer program used simply divided the sum of all woight values by the number of values used to compute the total weight, not the actual number of containers that that value represents.

From 1970 until 1978 individual container weights were not required for TRU drums. During the data reentry program in the mid-1980's standard weights were assigned for all container types; 55-gallon drums were given a standard weight of $68 \mathrm{~kg}$. This is why the average weight/drum for TRU is so consistent during this period. 
set Inesize 170

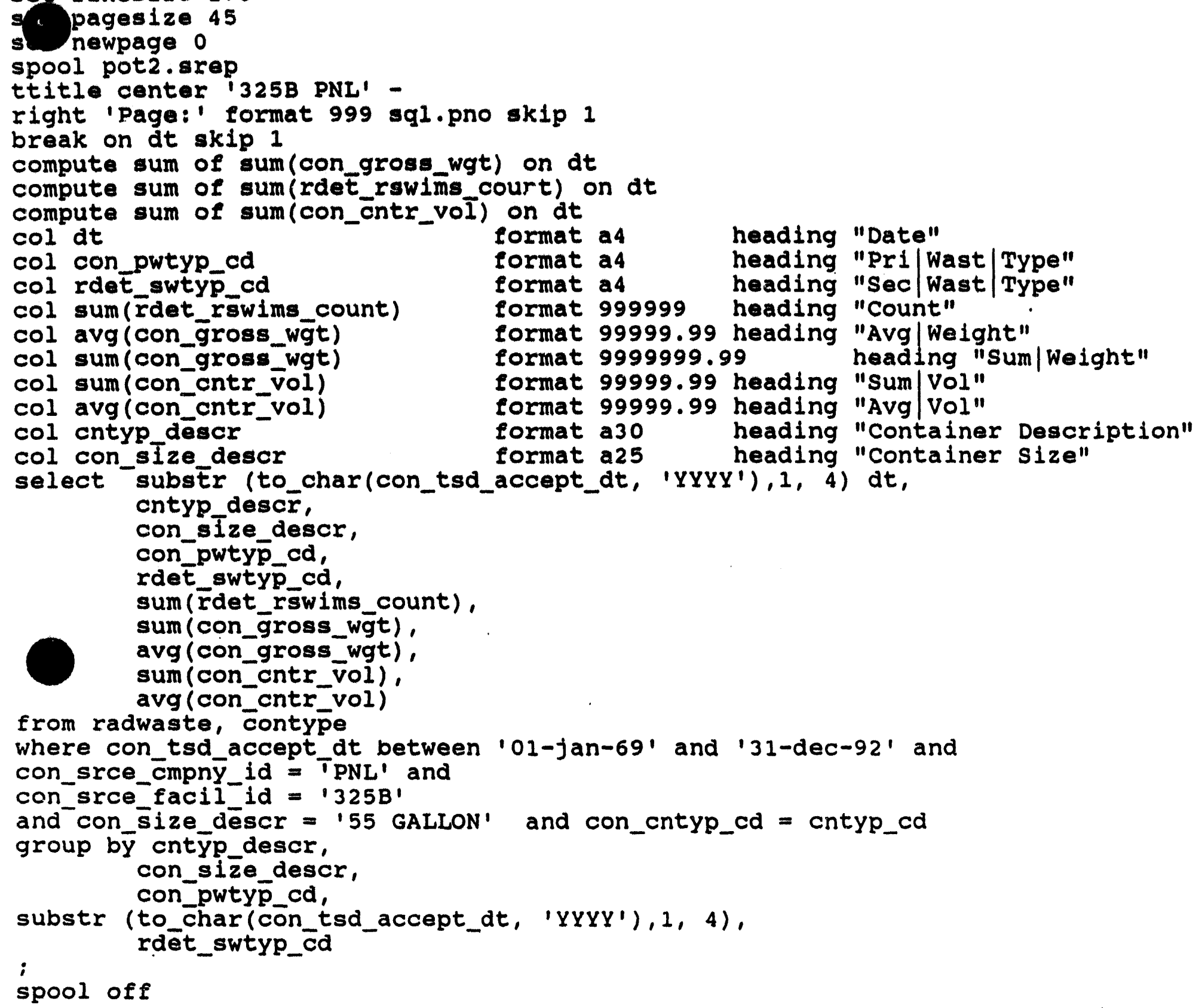


con_size_descr,

WHC-EP-0696

con pwtyp cd,

rdet_swtyp_cd,

sum ( $\bar{r}$ det_rswims_count),

sum (con_gross_wgt),

avg (con_gross_wgt),

sum (con_cntr_vol),

avg (con_cntr_vol)

from radwaste, contype

where con_tsd_accept_dt between '01-jan-69' and '31-dec-92' and con_srce_cmpny

con-srce-facilidd $=1325 \mathrm{~B}^{\prime}$

and con_size_descr $=155$ GALLON' and con_cntyp_cd = cntyp_cd

group by entȳp_descr,

"pot2.sql" 42 IInes, 1393 characters

SQL> A [ :

unknown command "" - rest of line ignored.

SQL>

SQL> epot2

no rows selected

SQL>

QVT220. 80 COL' 9 BIT MODE" 
WHC-EP-0696

\section{APPENDIX E.2}

BUILDINO 325B-PNL TRU WASTE CONTAINERS SORTED BY CONTAINER DESCRIPTION, SIZE, YEAR,

AND PRIMARY WASTE TYPE 


\section{WHC-EP-0696}

This subappendix provides information on Building 325B-PNL TRU waste containers, weight, volume, and primary waste type stored at various TRU waste storage facilities on the Hanford Site. The data provides the TRU waste generation from the Building 325B-PNL Facility by year. 


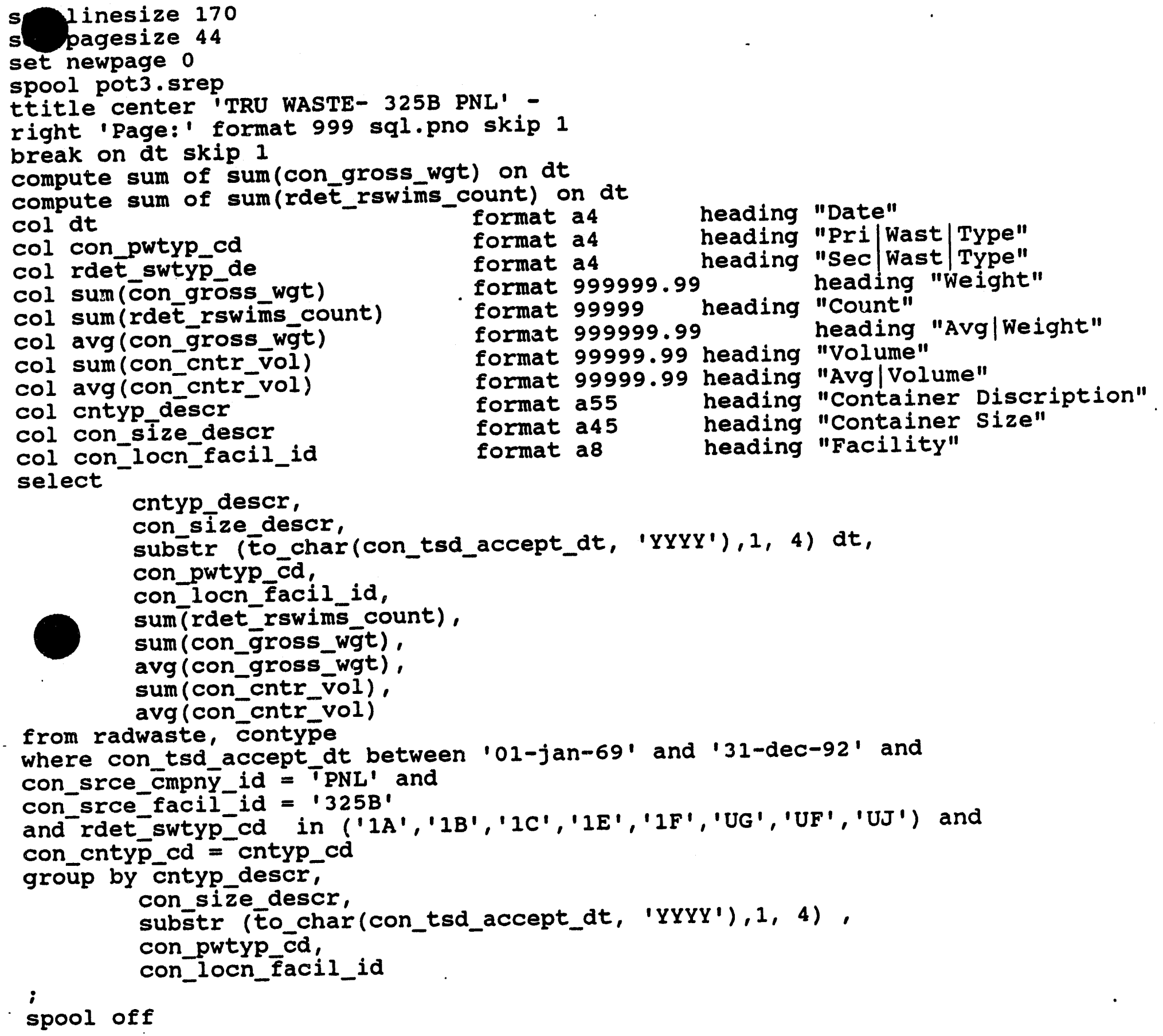


TRU WASTE- 325B PNL

Page: 1

Pri

Nast

Date Type Facility Cant Meignt

.... .... ...............................................

$\begin{array}{lllllll}1970 R & 218448 & 25 & 74.85 & 14.97 & .11 & .02\end{array}$

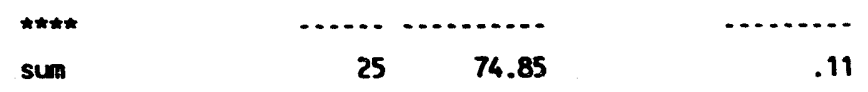

Container Size

1 GALLON
METAL DRUMS, BARRELS, KEGS

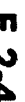


WHC-EP-0696

APPENDIX E.3

BUILDING 325B-PNL NON-TRU WASTE CONTAINERS SORTED BY CONTAINER DESCRIPTION, SIZE, YEAR, AND PRIMARY WASTE TYPE 
This subappendix provides information on Building 325B-PNL non-TRU waste containers, weight, volume, and primary waste type stored at various waste storage facilities on the Hanford Site. The date provides the non-TRU waste generation from the Building 325B-PNL Facility by year. 


\section{WHC-EP-0696}

set 1 inesize 175

set pagesize 45

newpage 0

spool pot3a.srep

ttitle center 'NON-TRU WASTE- 325B PNL' -

right 'Page:' format 999 sql.pno skip 1

break on dt skip 1

compute sum of sum(con_gross_wgt) on dt

compute sum of sum (rdet_rswims_count) on dt

col dt

col con_pwtyp_cd

col rdet_swtyp_de

col sum(con_gross_wgt)

col sum (rde $\bar{t}$ rswims_count)

coil avg (con_gross_wḡt)

col sum (con_ontr_vol)

col avg (con_crtr_vol)

col cntyp_descr

col con_size_descr

col con_locn_facil_id

col rdeE_swtýp_cd

format a4

\begin{tabular}{ll|l|l|} 
format a4 hype" & heading "Pri|Wast \\
format a4 & heading "Sec & Wast & Type"
\end{tabular}

heading "Date"

format 999999.99

format 99999

format 999999.99

format 99999.99 heading

format 99999.99 heading

format a55

format a 45

heading

format $a 8$

heading

heading

format a4

heading

heading "Weight"

select

cntyp_descr, con_size_descr,

subs̄tr (Ẽo_char(con_tsd_accept_dt, 'YYYY'),1,4) dt,

con_pwtyp_cd,

rdet_swty $\bar{p} \_c d$,

con Iocn facil id,

$\operatorname{sum}\left(r d e t \_r s w i m \bar{s} \_c o u n t\right)$,

sum (con_gross_wgt),

avg (con_gross_wgt),

sum (con cntr vol),

avg (con cntr vol)

from radwaste, contype

where con_tsd_accept_dt between '01-jan-69' and '31-dec-92' and

con_srce_cmpny id $=T_{\mathrm{PNL}}{ }^{\prime}$ and

con_srce_facil_id $=1.325 \mathrm{~B}$ '

and rdet_swtyp_cd in $\left(' 2 A^{\prime},{ }^{\prime} 2 B^{\prime}, L^{\prime} 2 L^{\prime},{ }^{\prime} 3 A^{\prime},{ }^{\prime} 3 L^{\prime},{ }^{\prime} U A^{\prime},{ }^{\prime} U B^{\prime}\right)$ and

con_cntyp_cd = cntyp_cd

groüp by cntyp_descr,

con_size_descr,

substtr (É_char(con_tsd_accept_dt, 'YYYY'), 1, 4),

con_pwtyp_cd,

rdet_swtyp_cd,

con_Iocn_facil_id

;

spool off 


\section{WHC-EP-0696}

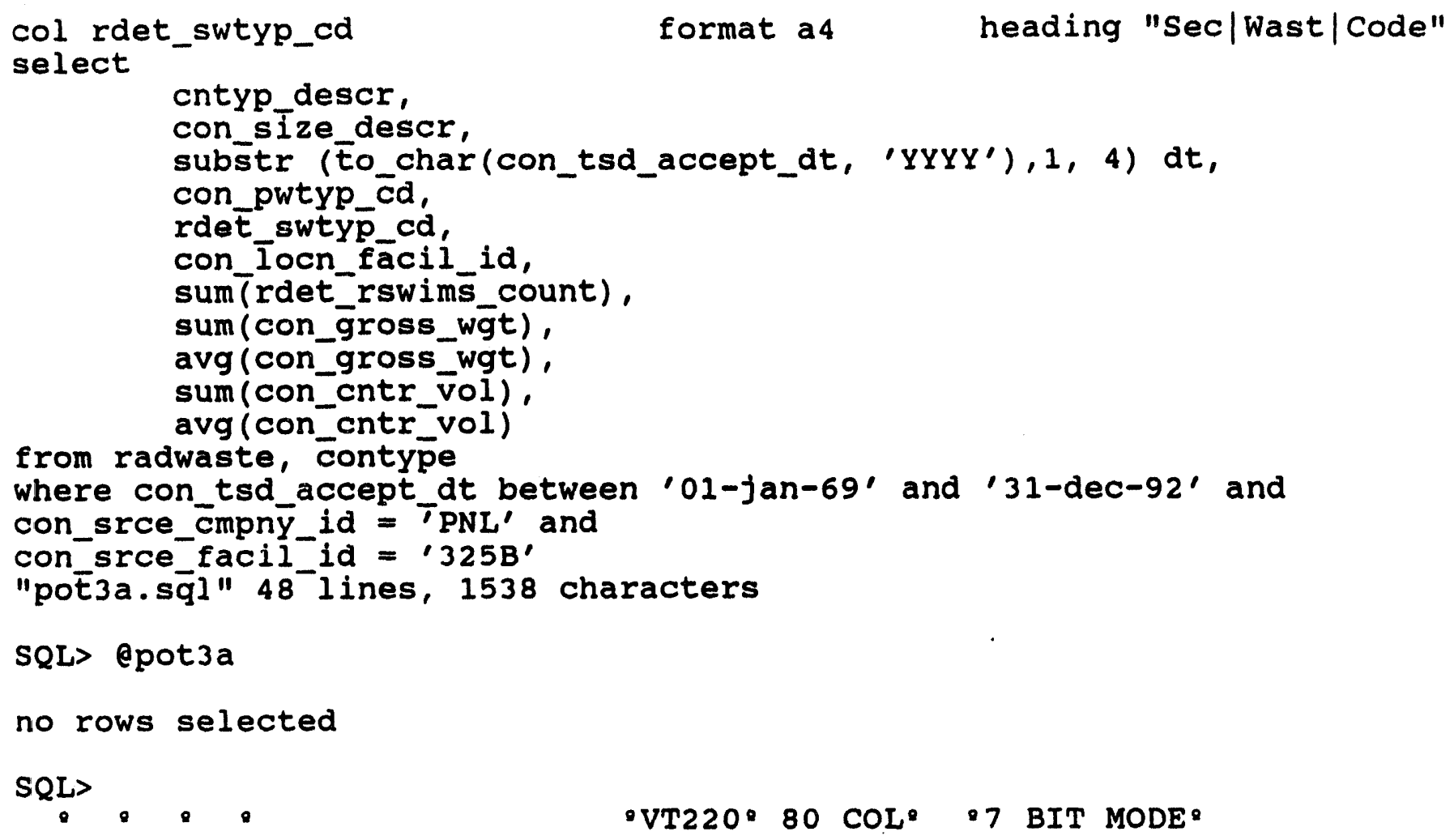




\title{
WHC-EP-0696
}

APPENDIX E.4

\section{RADIOLOGICAL DATA FOR TRU WASTE CONTAINERS}

SORTED BY DATE, PRIMARY WASTE TYPE, AND STORAGE FACILITY

\author{
E.4-1
}


This subappendix summarizes the radiological data for all TRU waste containers generated at Building 325B-PNL. Specifically, this computer run provides the total grams of TRU elements in a given container type in a given year. In addition, the average gram loading for a given container type in a given year has been calculated.

In 1991 and 1992 the number of curies (Ci) of alpha radiation was included on the SWBRs. Since the Building 325B-PNL waste generation data is all from 1970 to 1988 , these columns contain blank entries. The "Max Dose" column contains the highest value recorded for a given container in a given year, in $\mathrm{mrem} / \mathrm{hr}$.

Additional information about the isotopes present in Building 325B-PNL waste containers can be found in subappendices E.9 and E.10. 


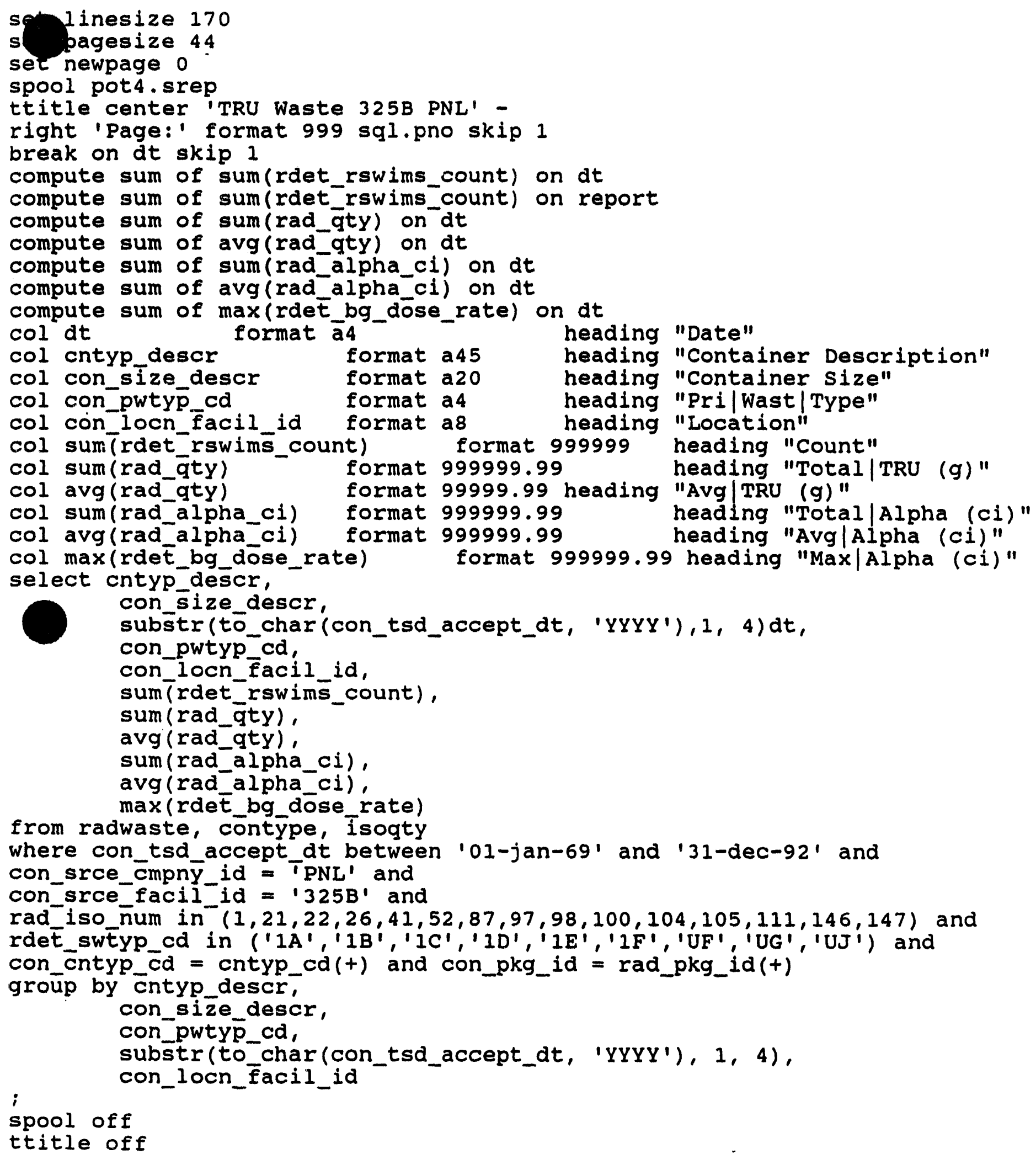



Pri

Container size

Container Description

Wast

Date Irpe Location Count

Total

Avg

$\max$

1 GALLON

METAL DRUMS, BARRELS, KEGS

1970 R 21844B

25

.......

(g) Alpha (ci) Alpha (ci) Alpha (ci)

$01 \quad 00$

.00

1.00

$* * * *$

25

sum

.01

$.01 \quad .00$

$00 \quad .00 \quad 1.00$


WHC-EP-0696

APPENDIX E.5

PHYSICAL CONTENTS DESCRIPTION FOR BUILDINO 325B-PNL 55-GALLON DRUMS CONTAINING TRU WASTE SORTED BY DATE, PRIMARY WASTE TYPE, AND STORAGE FACILITY 
This subappendix describes the physical contents of the 55-gallon drums of TAU waste generated by Building 325B-PNL. Prior to 1978, physical contents were not required on the burial records, so a great many of the early records list the contents of the drum as "Miscellaneous."

The printout for this computer run sorts the contents data by storage facility, date, and primary waste type. The introduction to Appendix E contains a table of waste codes and their meanings. 


\section{WHC-EP-0696}

set 1 inesize 170

set-pagesize 45

hewpage 0

spodil pot5.srep

ttitle c ter 'TRU WASTE- 325B PNL ' -

right 'Page:' format 999 sql.pno skip 1

break on con_locn_facil_id skip 1

col phys_comp_desc̄r

col cntyp descr

col con_size_descr

col dt

col con_pwtyp_cd

col con_locn_facil_id

format a 40 format a55

format a 45

format a 4

format a 4

format as

format 999999

heading "Physical Description"

heading "Container Discription"

heading "Container Size"

heading "Date"

heading "Pri/Wast/Type"

heading "Facility"

heading "Count"

compute sum of sum(rdet_rswims_count) on con_locn_facil_id

select phys_comp_descr,

cntȳp_descr,

con_size_descr,

subs̄tr (Eo_char(con_tsd_accept_dt, 'YYYY'),1,4) dt, con_pwtyp_cd, con locn facil id, sum (rdet_rswims_count)

from radwaste, contype, physcomp

where con_tsd_accept_dt between $101-j a n-691$ and '31-dec-92' and

cun_srce_cmpny

con_srce-facilid $=1325^{\prime}$

and con size descr $=155$ GALLON'

and rdet_swtȳp_cd in ('IA', 'IB', 'IC','IE','IF', 'UG', 'UF', 'UJ') and

f comp_descr is null and

con_cntyp_cd = cntyp_cd and con_pkg_id = phys_pkg_id

groūp by con_locn_facil_id,

phys_comp_descr,

con_pwtyp_cd,

substr (tō_char(con_tsd_accept_dt, 'YYYY'), 1, 4),

cntyp_descr,

con_size_descr

;

spool off 

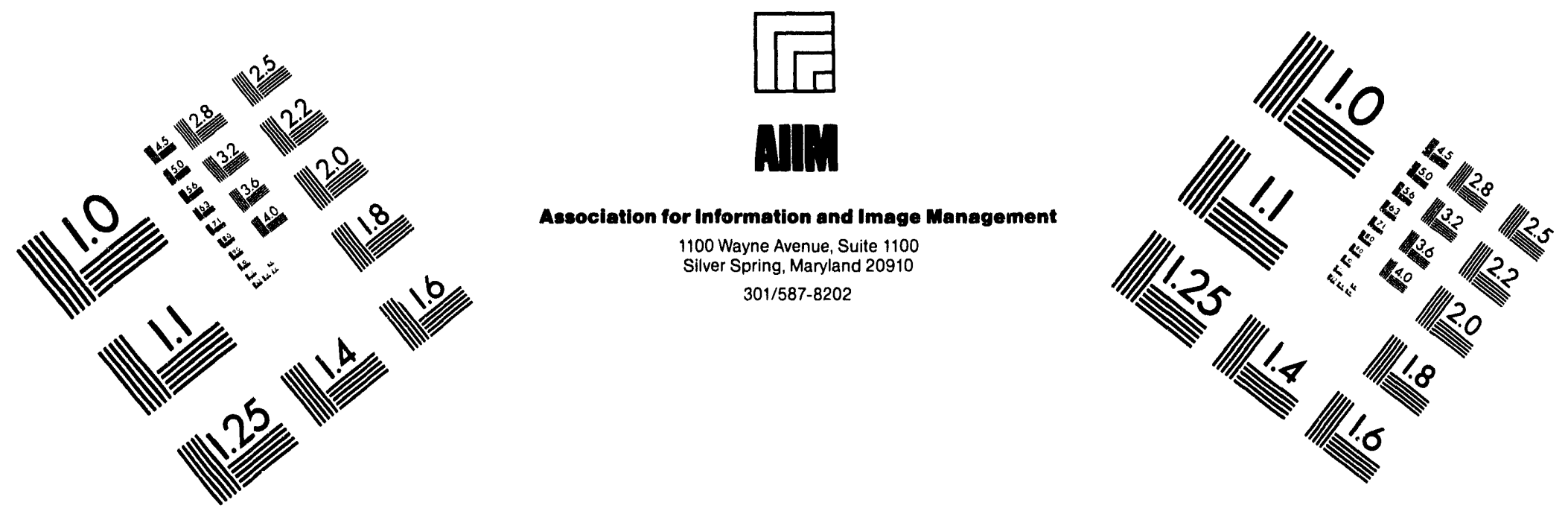

\section{Centimeter}

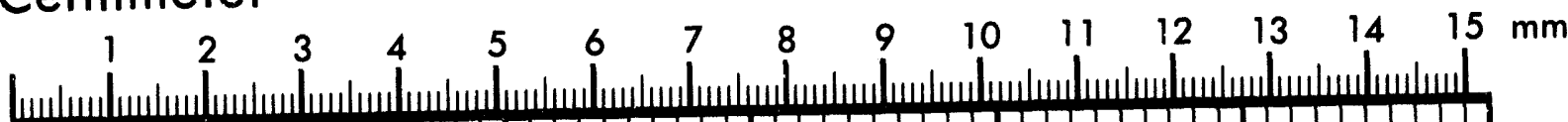

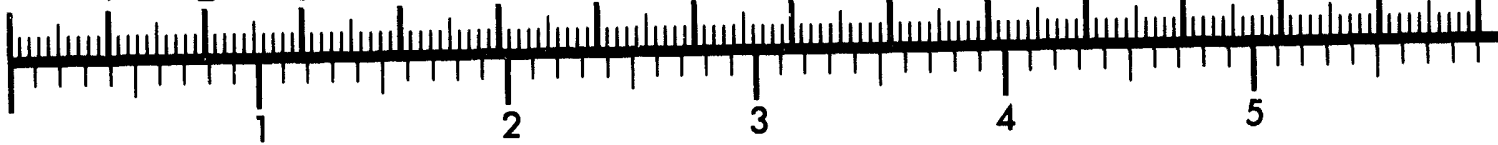
Inches
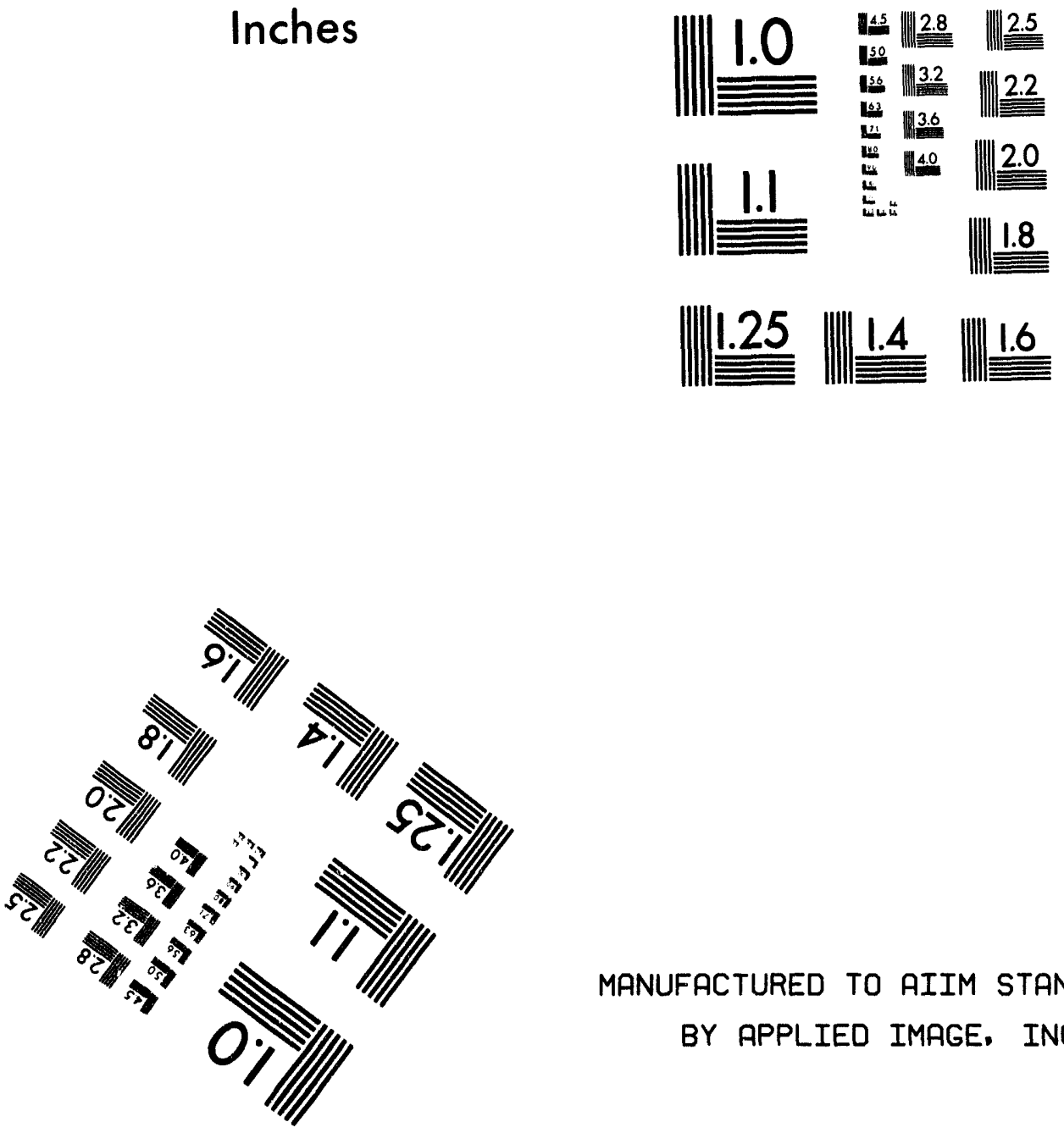

MANUFACTURED TO AIIM STANDARDS

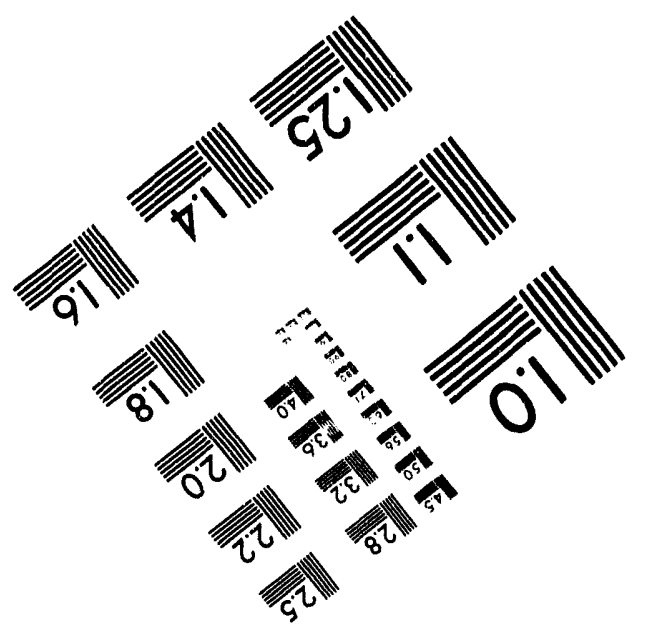



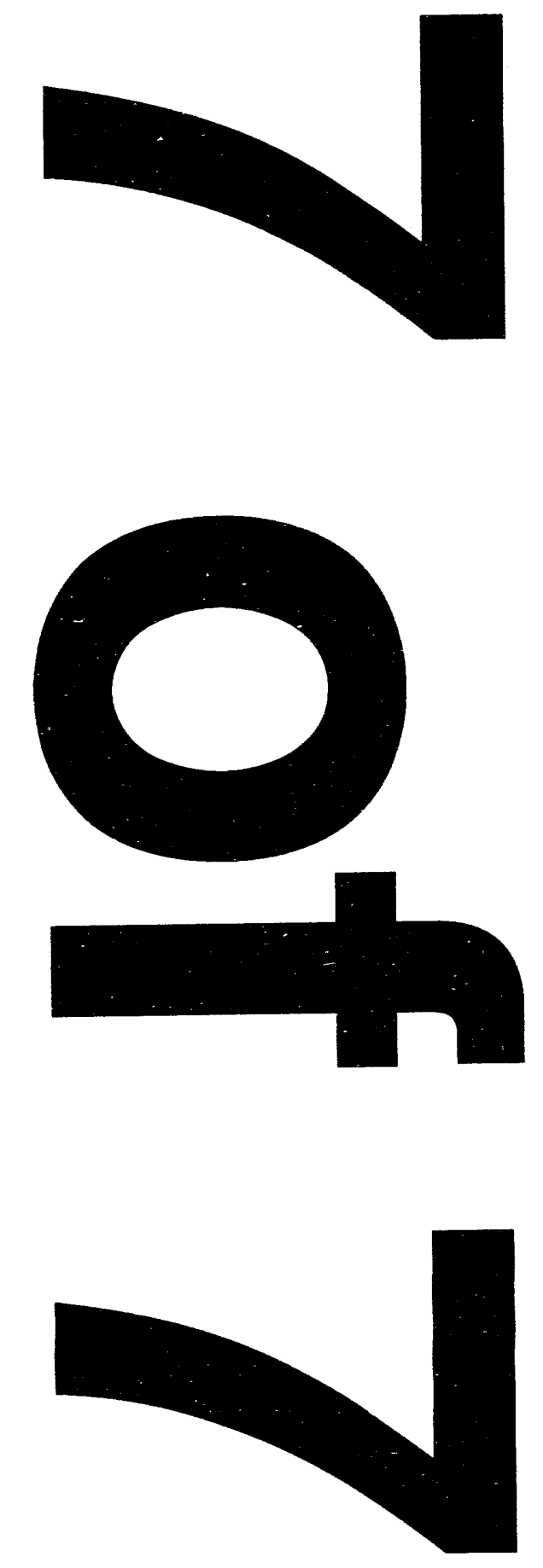
WHC-EP-0696

son_cntyp_cd $=$ cntyp_cd and con_pkg_id $=$ phys_pkg_id groūp by con_locn_facil_id, ohys_comp_descr, zon_pwtyp_cd,

substr (to_char(con_tsd_accept_dt, 'YYYY'), 1, 4), cntyp_descr, con_size_descr

;

spool off

$\sim$

$\sim$

$\sim$

$\sim$

$\sim$

$\sim$

$\sim$

"pot5.sql" 38 lines, 1269 characters

SQL> epot5

no rows selected

$\underset{8}{\text { SQL }>~: ~}:$

${ }^{\circ} \mathrm{VT220} 8 \mathrm{COL}^{2}$

27 BIT MODE: 
WHC-EP-0696

APPENDIX E.6

PHYSICAL CONTENTS DESCRIPTION FOR BUILDING 325B-PNL TRU WASTE CONTAINERS OTHER THAN 55-GALLON DRUMS SORTED BY DATE, PRIMARY WASTE TYPE, AND STORAGE FACILITY 
This subappendix describes the physical contents of the TRU waste containers other than 55-gallon drums generaced at Building 325B-PNL. Prior to 1978, physical contents were not required on the burial records, so a great many of the early records list the contents of the drum as "Miscellaneous."

The printout for this computer run sorts the contents data by storage facility, date and primary waste type. The introduction to Appendix E contains a table of waste codes and their mẹanings. 


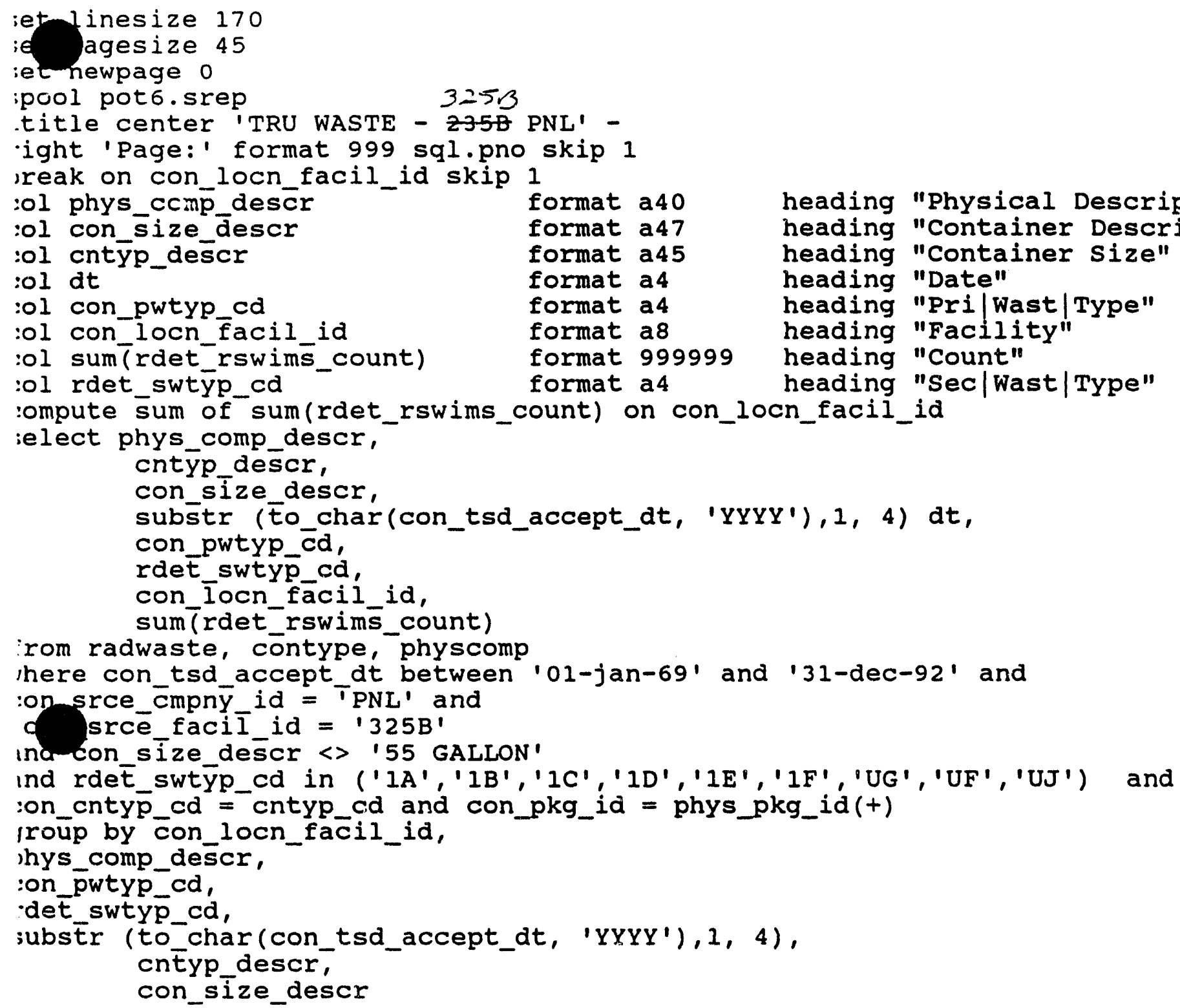

spool off 
Pri Sec

Wast Wast

Container Size

Container Description

Date Type Type facility Count

METAL DRUMS, BARRELS, KEGS

1 GALLON

$1970 \mathrm{R}$

if $218 W 48$

sum 
WHC-EP-0696

APPENDIX E.7

HAZARDOUS CONSTITUENTS OF 55-GALLON DRUMS CONTAINING TRU WASTE

E.7-1 
The presence of hazardous constituents in a radioactive waste drum designates the waste as "Mixed" (primary waste code "M"). In this sub-appendix the hazardous contents of 55-gallon drums generated at Building 325B-PNL are sorted by storage facility.

Information concerning the hazardous constituents of waste containers was not required prior to 1986. During the R-SWIMS data reentry program in the mid-1980's an attempt was made to add any available information on the hazardous materials present, however, this information was limited. 


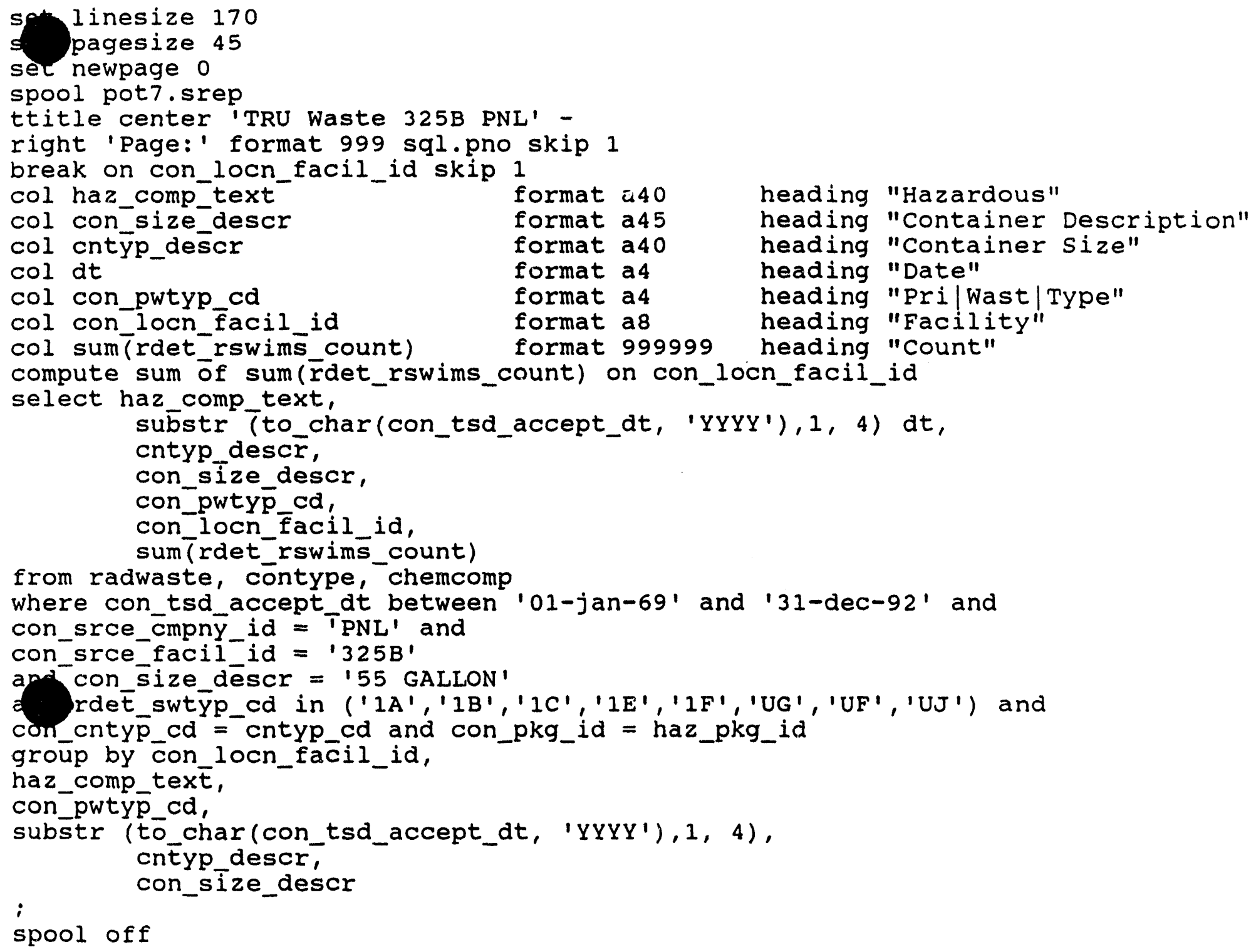




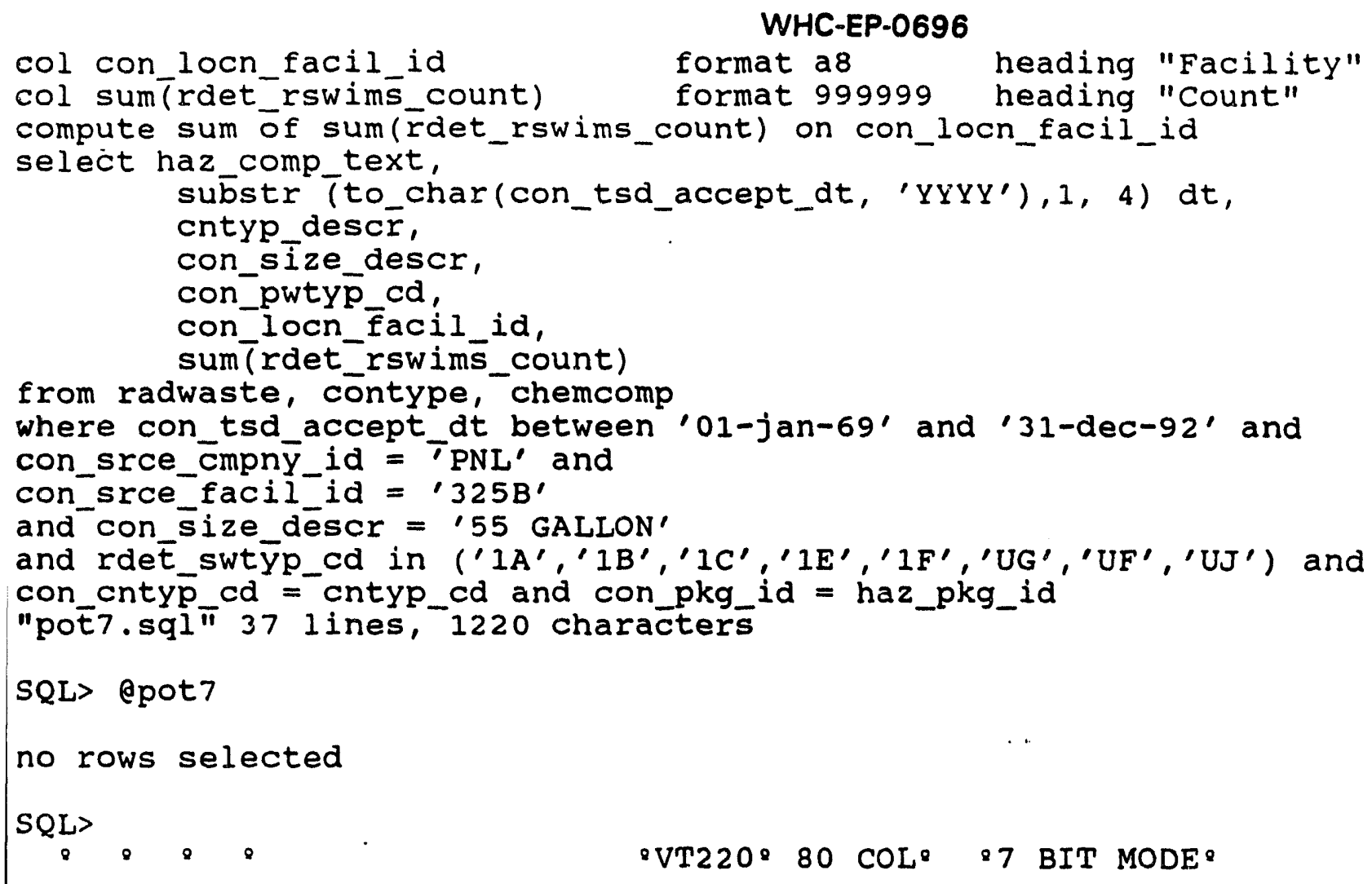


WHC-EP-0696

APPENDIX E.8

HAZARDOUS CONSTITUENTS OF TRU WASTE CONTAINERS OTHER THAN 55-GALLON DRUMS

\section{E.8-1}


The presence of hazardous constituents in a radioactive waste drum designates the waste as "Mixed" (primary waste code " $\mathrm{M}$ ). No hazardous constituents are listed in containers other than 55-gallon drums generated at Building 325B-PNL; therefore this run did not reveal any hazardous information. 


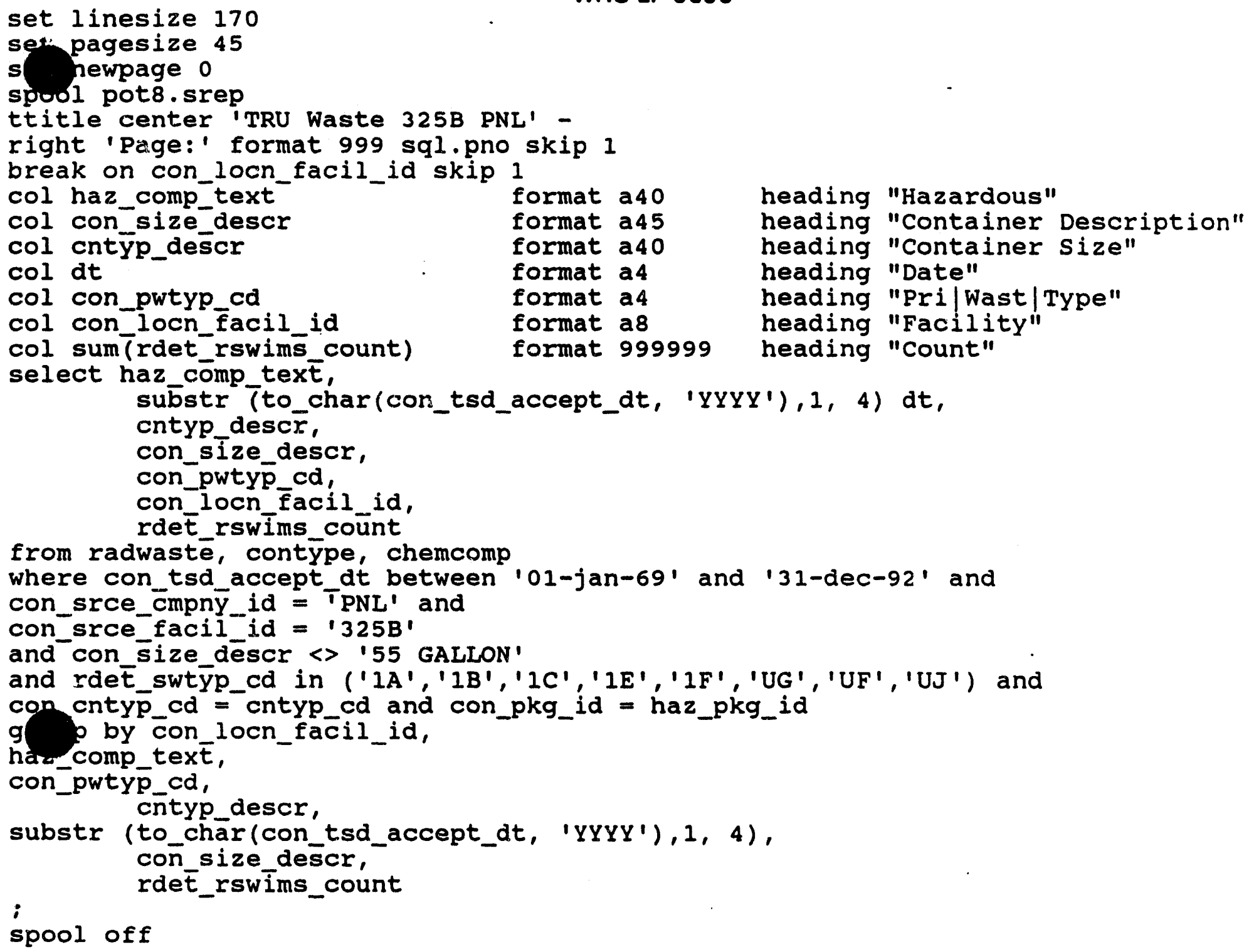




\section{WHC-EP-0696}

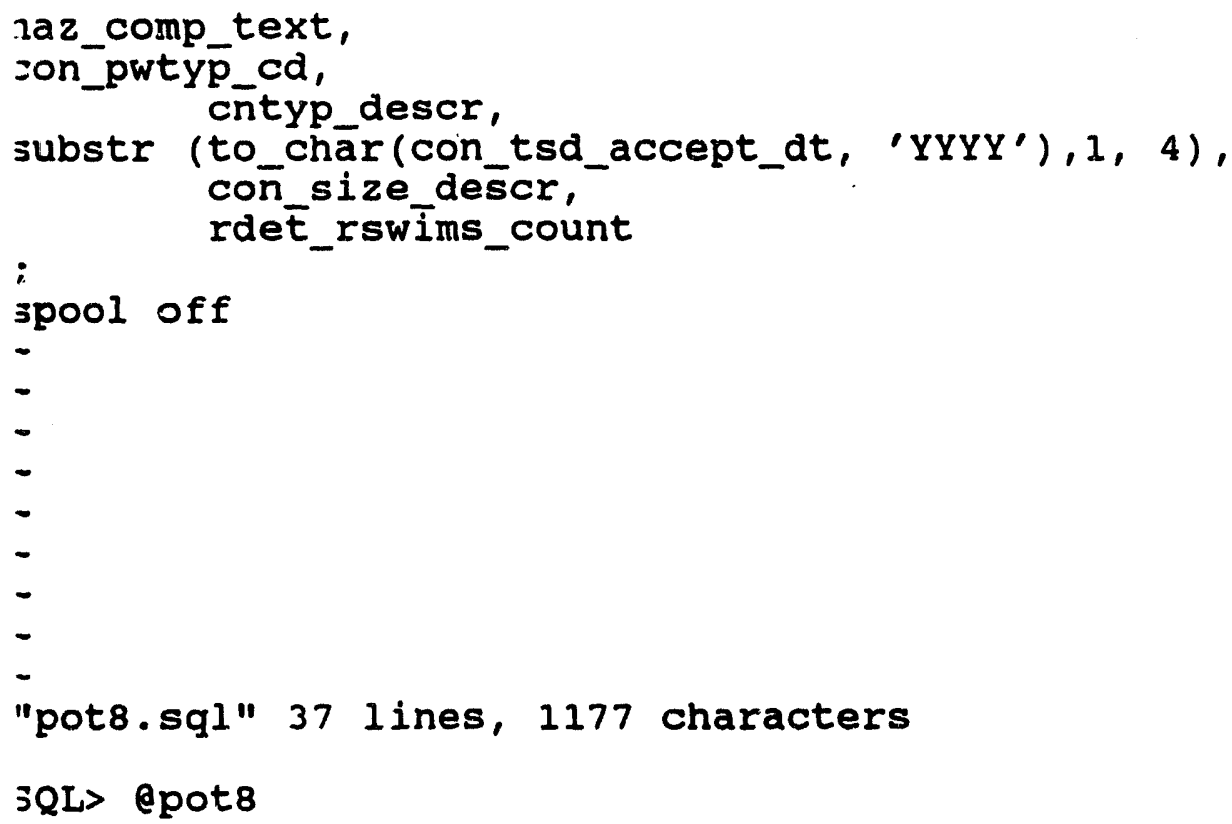


WHC-EP-0696

APPENDIX E.9

ISOTOPES LISTED IN 55-GALLON DRUMS CONTAINING TRU WASTES

E.9-1 
This subappendix contains a listing of the SWITS isotope information for TRU waste from Building 325B-PNL stored in 55-gallon drums. The isotope field in SWITS contains several types of the following information:

- Specific isotope (e.g., Am-241, Np-237, U-233, Pu-238)

- Generic isotope listings (e.g., Pu, uranium-enriched, uranium-depleted.)

- $\quad$ Plutonium-239 fissile gram equivalents (Pu239 FSL GR equiv).

- Total - alpha

- Total beta/gamma

- $\quad$ PE-Ci (plutonium equivalent curies).

This computer run contains the isotope field listing along with the number of TRU waste drums with that listing. The run is sorted by storage facility and year. 


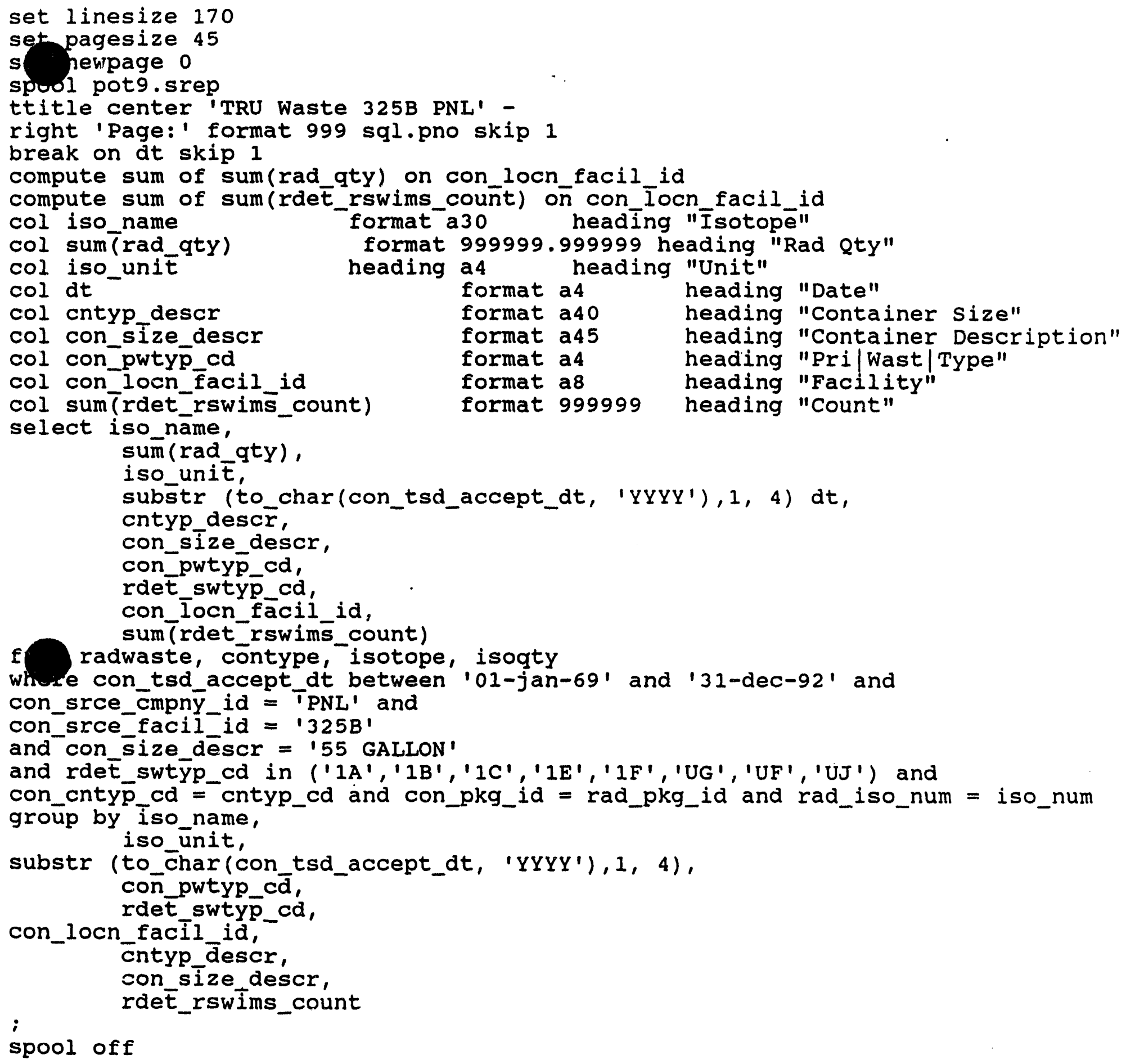




\section{WHC-EP-0696}

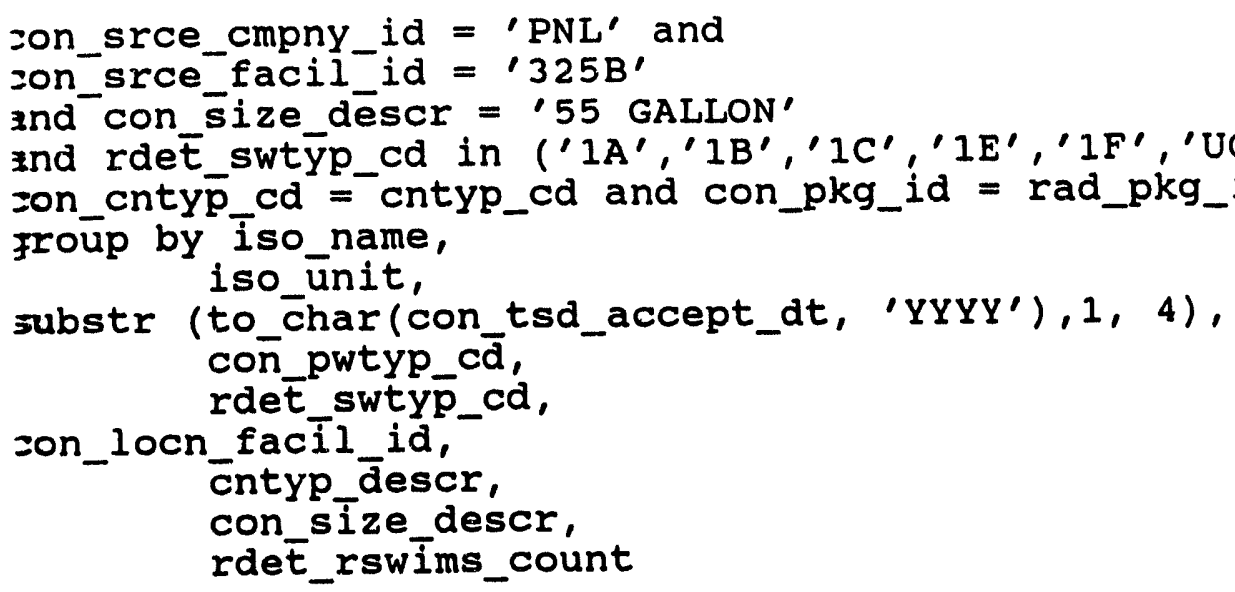


WHC-EP-0696

\section{APPENDIX E.10}

ISOTOPES LISTED IN TRU CONTAINERS

OTHER THAN 55-GALLON DRUMS

E.10-1 
This subappendix contains a listing of the SWITS isotope information for TRU waste from Building 325B-PNL stored in containers other than 55-gallon drums. The isotope field in SWITS contains several types of the following information:

- Specific isotope (e.g., Am-241, Np-237, U-233, Pu-238)

- Generic isotope listings (e.g., Pu, uranium-enriched, uranium-depleted)

- Plutonium-239 equivalent curies (PE-Cl)

- Total-alpha

- Total beta/gamma

- $\quad$ PE-Ci (plutonium equivalent curies).

This computer run contains the isotope field listing along with the number of TRU waste containers with that listing. The run is sorted by storage facility and year. 


\section{WHC-EP-0696}

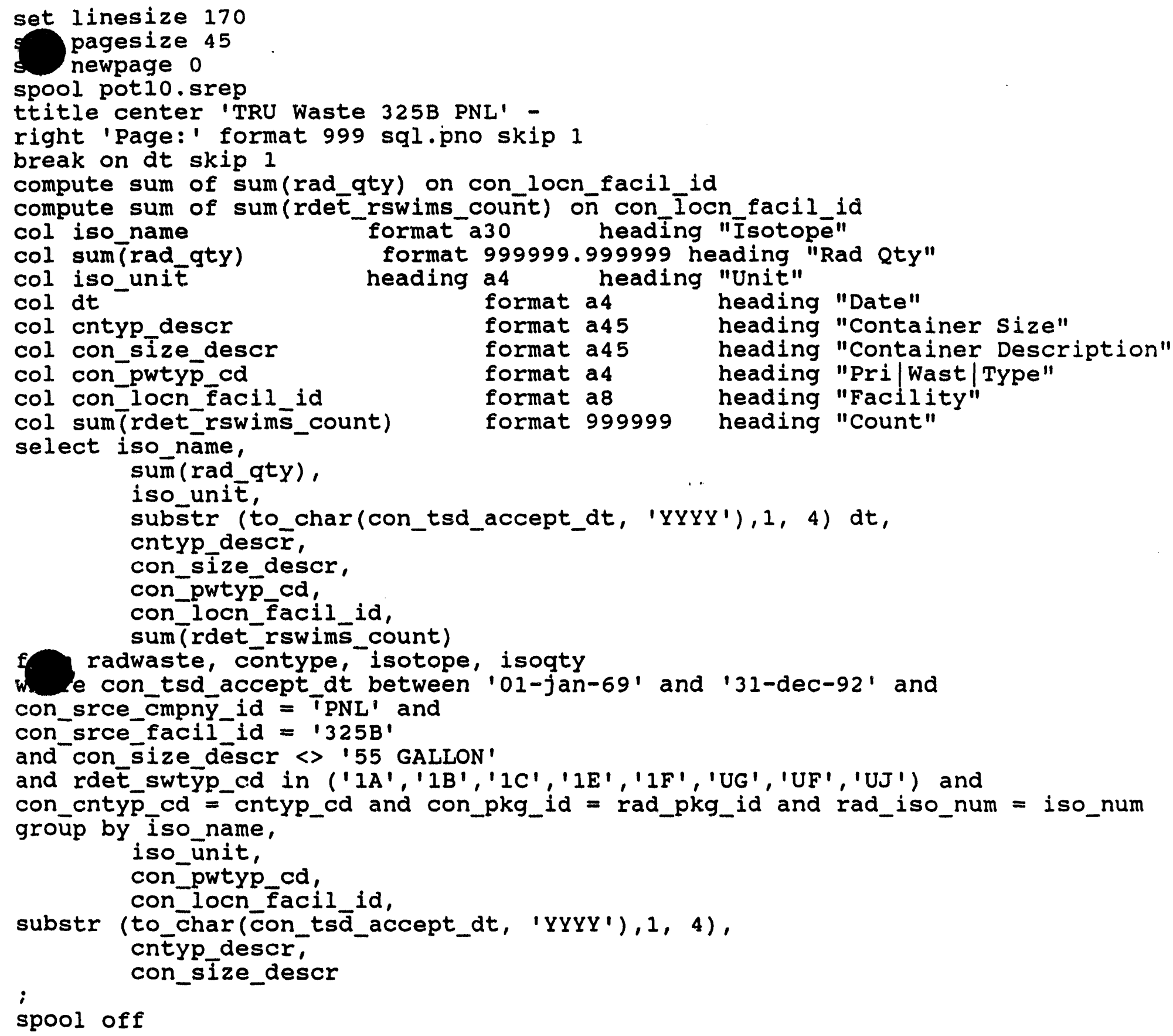

spool off 
I sotope

Rad Oty Un Date Container Size

Container Description

O05000 GY 1970 METAL DRUMS, BARRELS, KEGS $70.001000 \mathrm{CI}$ METAL DRUMS, BARRELS, KEGS 5.000000 GH METAL DRUNS, BARRELS, KEGS *

75.006000 sum

1 gallon

1 GALLON

1 GALLON

75.006000
Pri

Wast

Type facility count

218448

218448

218448

25

25

25

TOTAL BETA/GAMAM 
WHC-EP-0696

\section{DISTRIBUTION}

Number of copies

OFFSITE

3

Los Alamos Technical Associates, Inc.

8633 W. Gage Boulevard

Kennewick, Washington 99336

J. Pottmeyer (3)

ONSITE

4

29
U.S. Department of Eneray. Richland Operations Office

R. M. Gordon

R. F. Guercia (2)

Public Reading Room

R3-80

A5-21

A 1-65

Westinohouse Hanford Company

J. D. Anderson

N3-13

M. C. Burt

J. A. Demiter

P7-22

L5-31

D. J. Deschane

D. R. Duncan (5)

W. O. Greenhalgh

K. L. Hladek

D. M. Johnson

J. P. Joyce

W. B. Larson

B. A. Mayancsik

D. C. McCain

M. M. McCarthy

W. W. Olson

J. G. Riddelle

R. J. Roberts

D. E. Roohr

R. T. Steele

J. A. Swenson (2)

H. H. VanTuyl

Central Files

Document Processing and

Distribution (2)

Information Release

Administration
P8-24

H5-33

L5-31

N3-13

$\mathrm{H} 5-33$

H4-68

P7-25

H5-33

B5-25

G1-19

H4-70

H5-33

N3-13

L6-40

T6-22

G6-45

L8-04

L8-04

L8-07 
WHC-EP-0696

This page intentionally left blank. 

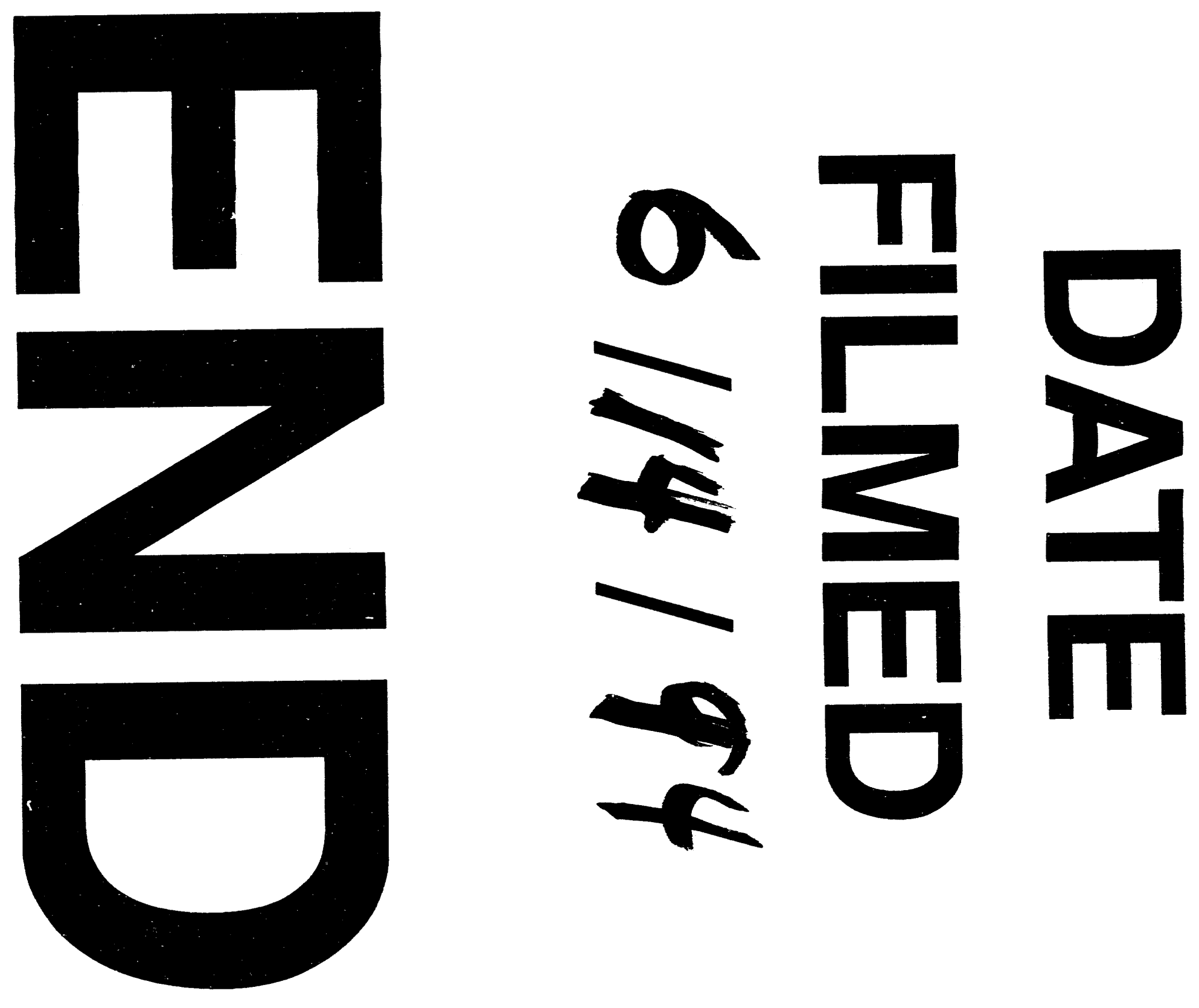


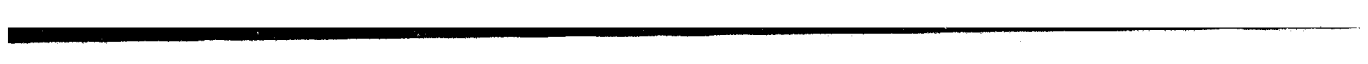

